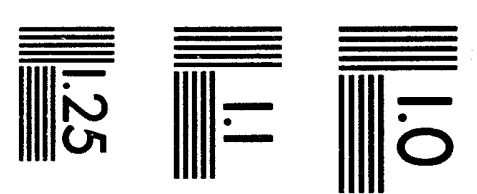

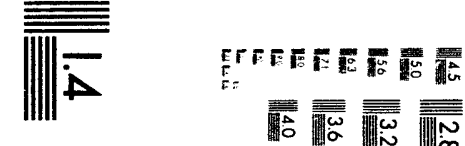

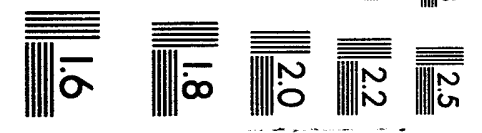



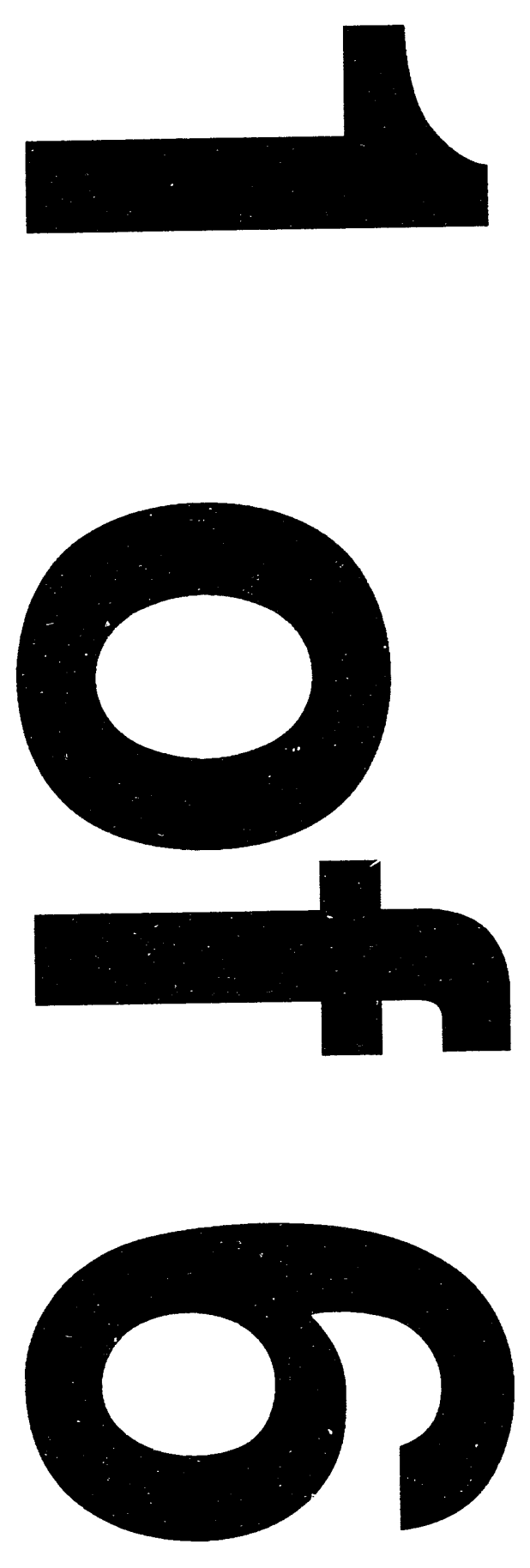
PNL-8370

Ver. 2.0

INTEGRATED BASELINE SYSTEM (IBS)

Version 2.0

\section{UTILITIES GUIDE}

M. J. Burford

T. R. Downing

J. R. Williams

J. C. Bower (a)

March 1994

Prepared for the

U.S. Army Nuclear and Chemical Agency under a Related Services Agreement with the U.S. Department of Energy Contract DE-AC06-76RLO 1830

Pacific Northwest Laboratory

Richland, Washington 99352

(a) Bower Software Services Kennewick, Washington 


\section{NOTICE}

The Integrated Baseline System (IBS) is an emergency planning and training tool intended to supplement existing resources for emergency planning, training, and management. IBS results must NOT be used as the sole basis for a decision regarding public safety. Although IBS is operational, not all IBS software/models have been verified. The assumptions of the IBS software should be reviewed to ensure its appropriateness for an intended use. 


\section{Preface}

The Integrated Baseline System (IBS) is an emergency management planning and analysis tool being developed under the direction of the U.S. Army Nuclear and Chemical Agency. The rollowing documents were developed to support system users. The audience for each is identified.

This IBS Utilities Guide explains how you can use IBS utility programs to manage and manipulate various kinds of IBS data. These programs include utilities for creating, editing, and displaying maps and other data that are referenced to geographic location. Audience: chiefly data managers but also system managers and some emergency management planners and analysts.

The IBS User Guide explains how to start and use the IBS program, which is designed to help civilian emergency management personnel to plan for and support their responses to a chemical-releasing event at a military chemical stockpile. $^{(a)}$ Audience: all users of the IBS, especially emergency management planners and analysts.

The IBS Models Guide summarizes the IBS use of several computer models for predicting the results of emergency situations. These include models for predicting dispersion/doses of airborne contaminants, traffic evacuation, explosion effects, heat radiation from a fire, and siren sound transmission. The guide references additional technical documentation on the models when such documentation is available from other sources. Audience: chiefly emergency management planners and analysts, but also data managers and system managers.

The IBS Data Management Guide provides the information needed to manage the data files and database used to support the administrative, user-environment, database management, and operational capabilities of the IBS. Audience: chiefly database administrators and system managers, but also emergency management planners and analysts who want to know details of the emergency management data.

The IBS System Management Guide defines IBS hardware and software requirements and gives instructions for installing, upgrading, or transferring the IBS software package. Audience: system managers.

(a) The IBS program was developed as part of the U.S. Army's Chemical Stockpile Emergency Preparedness Program (CSEPP). 


\section{About This Guide}

\section{Purpose}

The Integrated Baseline System (IBS) is an emergency management planning and analysis tool that was developed under the direction of the U.S. Army Nuclear and Chemical Agency (USANCA). ${ }^{\text {(a) }}$ This Utilities Guide explains how to operate individual utility programs that are supplied as a part of the IBS. These utility programs are chiefly for managing and manipulating various kinds of IBS data and system administration files. Many of the utilities are for creating, editing, converting, or displaying map data and other data that are related to geographic location. Only a few are for activities directly related to emergency management planning.

\section{Scope}

This guide is a reference document that provides an operational description for each IBS utility program. Procedures in other documents (such as the IBI' System Management Guide and the IBS Data Management Guide) sometimes refer you to this guide for specific instructions about accomplishing system management and data management tasks.

Although the utility program descriptions vary in detail, each description tells you how to start and interact with the utility to achieve the desired results. The graphic map display and editing utilities refer to the IBS User Guide for general concepts you should know about using IBS graphic map displays and function menus.

This guide often refers to standard IBS files and file formats. In general these files and formats are not described in detail here, although the guide does include an overview of IBS map data. For detailed descriptions of IBS data files, refer to the IBS Data Management Guide. Some system administration files are explained in the IBS System Management Guide.

(a) The IBS is being developed by the Pacific Northwest Laboratory (PNL). PNL is operated for the U.S. Department of Energy by Battelle Memorial Institute under contract DE-ACO6-76RLO 1830. 


\section{Audience}

Because this reference guide describes a host of IBS utility programs that are designed for many different purposes, it is addressed to a wide range of IBS usersfrom emergency management analysts and planners to IBS system managers and database administrators. Almost all the utilities require some knowledge of VAX/VMS computer disk directory and file conventions, if only to locate and use the files on which the utilities operate. Utilities aimed specifically at system managers and database administrators require a thorough knowledge of the VAX/VMS operating system, directory structures, and files.

\section{Organization}

This guide consists of the following two sections and six appendixes:

\section{Section 1. Introduction}

What you need to know before using the IBS utilities, and how to find the utilities that you need.

\section{Section 2. Utility Program Descriptions}

Instructions for how to operate each utility-ordered alphabetically by the utility names.

\section{Appendix A. IBS Topographies (Map Layers)}

A list of the names of the standard IBS topographies and the two-character topographic codes associated with each topography.

\section{Appendix B. IBS Attribute Dictionary}

A list of the contents of the IBS attribute dictionary-the current master set of attributes values that are used to assign physical or other descriptive features to spatial data objects in the IBS.

\section{Appendix C. IBS Icon Dictionary (Icon Control File)}

A list of the contents of the IBS icon dictionary or icon control file-the current master list of IBS icons, icon attributes, and DMS icon file names used in displaying graphic icons on maps.

\section{Appendix D. Map Projections in IBS Map Displays}

Background about choosing how the earth's curved surface should be displayed when translated to a two-dimensional map on your terminal screen. 


\section{Appendix E. IBS Privilege Listings}

Tables that cross-reference IBS programs and program options with IBS user privileges. An IBS system manager or database administrator assigns specific user privileges to control which IBS programs and program options can be used by each user.

\section{Appendix F. Undocumented Utility Programs}

Iists of utilities included in the system but which are NOT documented and NOT directly used by IBS users. These are either utilities designed to be called from other IBS programs or special utilities used by system developers.

\section{How to Use This Guide}

If you are looking for the operating instructions to a specific utility, look up the utility in Section 2, which is organized alphabetically by the utility names.

If you are browsing to find a desired function or a utility name, use the capsule utility descriptions in Section 1 to find out what the utilities can do. The capsule descriptions are grouped in overlapping task categories that can help you isolate the function that you need.

If you are working with IBS map data or with the IBS map database, you can use the Overview of IBS Map Data at the end of Section 1 as a brief reference on IBS map data terms and concepts. If those topics are unfamiliar to you, refer to the IBS Data Management Guide for detailed information. 


\section{Visual Conventions}

You will find the following typographic conventions in this guide.

Type Style

bold

italic

bold italic

ALL CAPITALS

screen text typeface

GRAPHIC MENU TYPEFACE
Meaning

Text that you must type at the keyboard.

Example: S NEWUSER

Placehoiders for information that you must provide. Example: $\$$ DELETE filename

Here you would type the actual name of a file instead of the word shown in italics.

Sometimes highlights IBS special terms

Directory names, file names, program names, and acronyms

Nongraphic text that appears on the terminal screen, such as in menus and prompts.

Example: Enter a file name $\Rightarrow$

Menu options on graphic screen menus 


\section{Contents}

Preface $\ldots \ldots \ldots \ldots \ldots \ldots \ldots \ldots \ldots \ldots \ldots \ldots \ldots \ldots \ldots \ldots$ iii

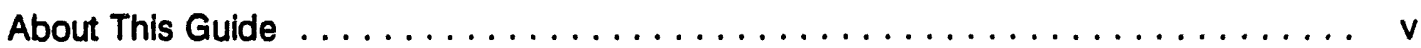

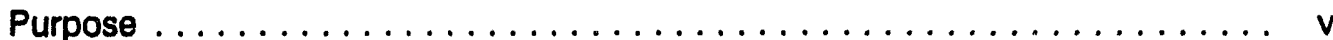

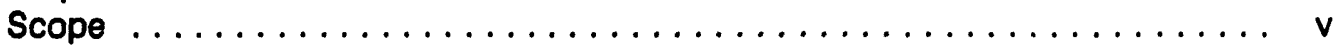

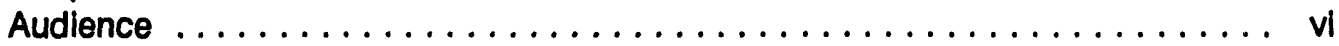

Organization ........................... vi

How to Use This Guide $\ldots \ldots \ldots \ldots \ldots \ldots \ldots \ldots \ldots \ldots$ vill

Visual Conventions $\ldots \ldots \ldots \ldots \ldots \ldots \ldots \ldots \ldots \ldots \ldots$ vill

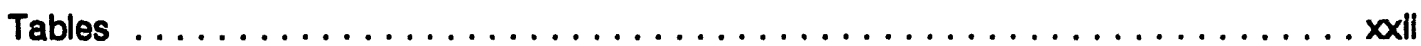

\section{Section 1. Introduction}

What You Need to Know Before Using the IBS Utility Programs $\ldots \ldots \ldots \ldots 1.2$

Finding the Utilities that You Need . . . . . . . . . . . . . . . . 1.3

Emergency Management and Planning Utilities $\ldots \ldots \ldots \ldots \ldots \ldots 1.4$

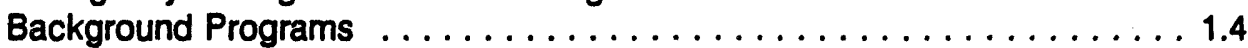

General System Utilities $\ldots \ldots \ldots \ldots \ldots \ldots \ldots \ldots \ldots \ldots \ldots \ldots \ldots$

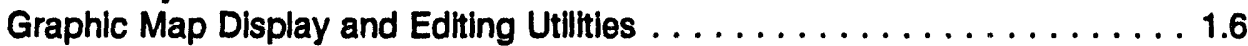

Data File Modlfication Utillties $\ldots \ldots \ldots \ldots \ldots \ldots \ldots \ldots \ldots \ldots \ldots .7$

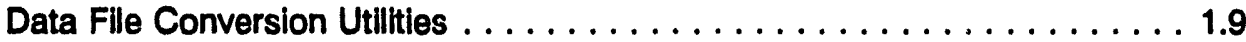

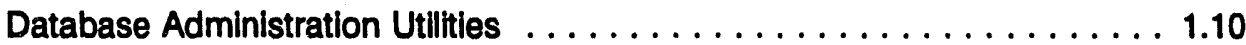

Database Quality Assurance (QA) Utilities . . . . . . . . . . . . . 1.12

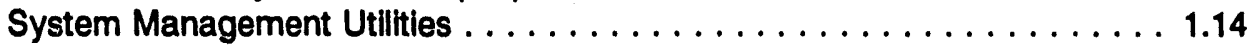

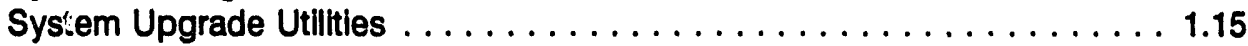

Diagnostic and Reporting Utillities $\ldots \ldots \ldots \ldots \ldots \ldots \ldots \ldots \ldots \ldots \ldots$

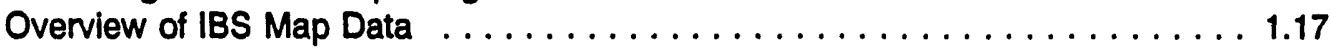

Map Data Terminology . . . . . . . . . . . . . . . . . 1.17

IBS Map Databases and Database Components . . . . . . . . . . 1.18

Map Database Directories and Files . . . . . . . . . . . . 1.19

Attributes and Attribute Dictionary ................. 1.21

\section{Section 2. Utility Program Descriptions}

How the Program Descriptions are Organized $\ldots \ldots \ldots \ldots \ldots \ldots \ldots \ldots \ldots$

Starting and Using the Utility Programs $\ldots \ldots \ldots \ldots \ldots \ldots \ldots \ldots \ldots \ldots \ldots .1$

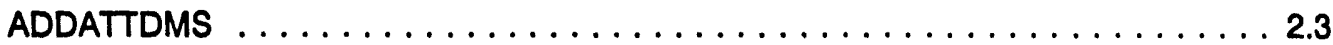

What You Need to Know Before Using ADDATTDMS . . . . . . . . 2.3

Adding New Attributes to a DMS File with ADDATTDMS . . . . . . . . 2.4 


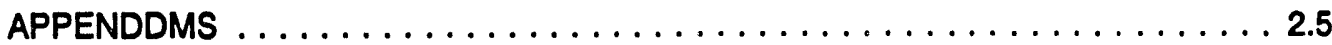

Appending One DMS File to Another with APPENDDMS $\ldots \ldots \ldots \ldots 2.5$

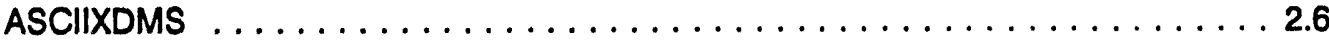

What You Need to Know Before Using ASCIIXDMS . . . . . . . . 2.6

Creating a DMS File from a DMX ASCll File with ASCIIXDMS . . . . . . 2.7

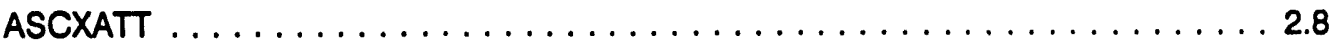

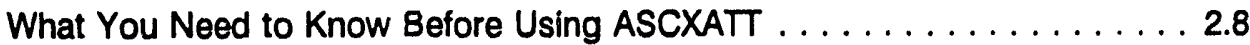

Creating a Binary Attribute Dictionary File from an ASCII File $\ldots \ldots \ldots 2.8$

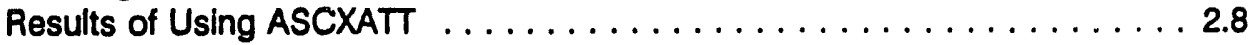

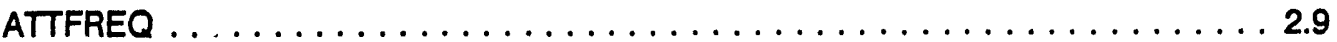

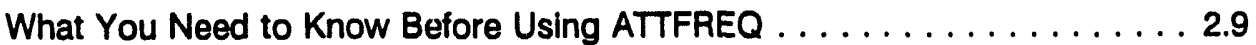

Reporting the Frequency of Attributes in DMS File(s) $\ldots \ldots \ldots \ldots \ldots 2.9$

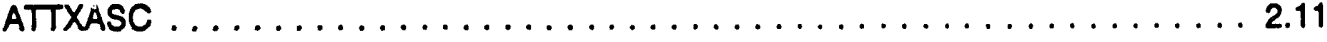

What You Need to Know Before Using ATTXASC . . . . . . . . . . . 2.11

Creating an ASCII Version of a Binary Attribute Dictionary $\ldots \ldots \ldots .2 .11$

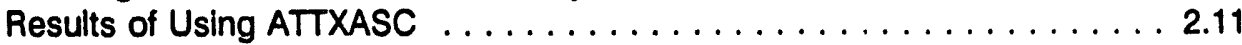

CHKICONS ............................... 2.12

Validating the Icon Dictionary with CHKICONS ............. 2.12

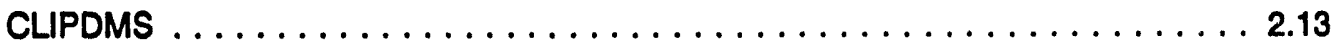

What You Need to Know Before Using CLIPDMS . . . . . . . . 2.13

Creating Area Component Files from a DMS File with CLIPDMS $\ldots \ldots \ldots 2.13$

Clipping a Single Area File from a DMS File . . . . . . . . 2.13

Clipping Multiple Area Files from a DMS File $\ldots \ldots \ldots \ldots \ldots .14$

COLORDMS ................................. 2.15

Converting DMS File Features to a Single Color with COLORDMS $\ldots \ldots 2.15$

CONTOUR ................................. 2.16

What You Should Know Before Using CONTOUR . . . . . . . . . 2.16

Creating a Vector Contour File from a TAC File with CONTOUR . . . . 2.16

Results of Using CONTOUR ..................... 2.16

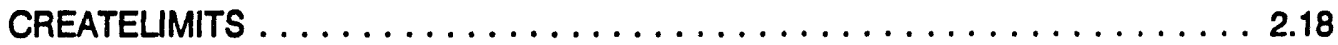

What You Need to Know Before Using CREATELIMITS . . . . . . . . 2.18

Creating a Binary Limit file with CREATELIMITS $\ldots \ldots \ldots \ldots \ldots \ldots . .18$

Entering Data File Names Directly . . . . . . . . . . . . 2.19

Using a List of Data File Names . . . . . . . . . . . . . 2.19

Results of Using CREATELIMITS $\ldots \ldots \ldots \ldots \ldots \ldots \ldots \ldots \ldots \ldots \ldots$

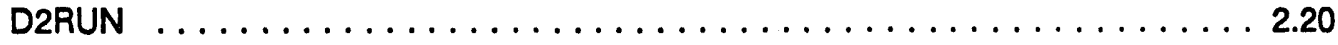

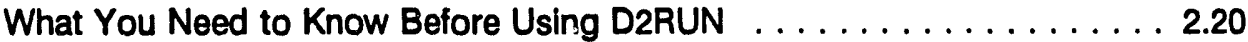

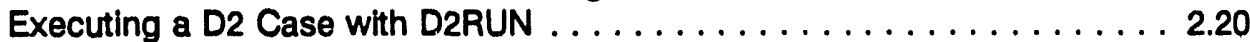

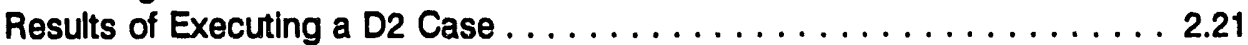

Executing Multiple D2 Cases $\ldots \ldots \ldots \ldots \ldots \ldots \ldots \ldots \ldots \ldots \ldots \ldots \ldots$ 


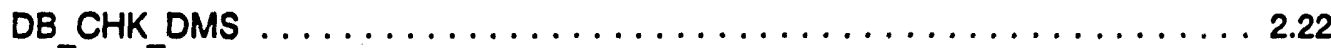

What You Need to Know Before Using DB CHK DMS . . . . . . . . 2.22

Checking a Topography or Database for DMS File Problems . . . . . . 2.22

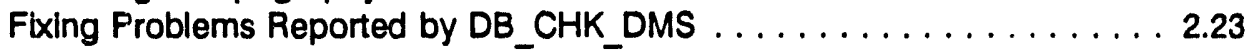

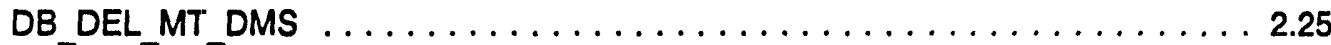

What You Need to Know Before Using DB DEL MT DMS $\ldots \ldots \ldots \ldots 2.25$

Checking a Topography or Database for Empty DMS Files . . . . . . 2.25

Consolidating DMS Files Found to Contain No Data . . . . . . . . . . 2.26

DB FIX DMS LIMITS . . . . . . . . . . . . . . . . . 2.27

What You Need to Know Before Using DB_FIX DMS LIMITS . . . . . . 2.27

Matching the DMS File Header Limit Information to the File Data . . . . 2.27

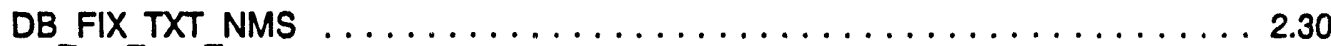

What You Need to Know Before Using DB_FIX_TXT_DMS $\ldots \ldots \ldots 2.30$

Finding Incorrect Text File Names in DMS Files . . . . . . . . . . . . . 2.30

Fixing DMS Files Found to Contain Incorrect Text File Names $\ldots \ldots \ldots 2.31$

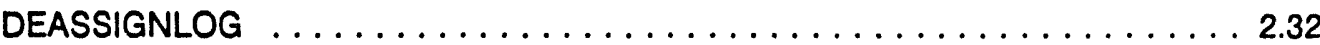

Deassigning Unnecessary Map Database Logical Names . . . . . . . . . 2.32

DECLUTTER . . . . . . . . . . . . . . . . . . . . . 2.33

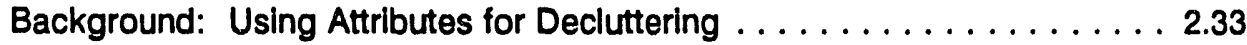

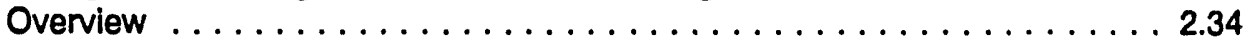

Procedure for Applying Decluttering Attributes in a DMS File $\ldots \ldots \ldots 2.34$

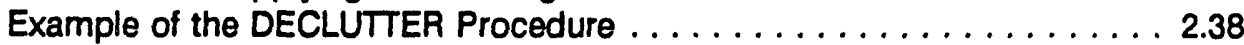

Using Decluttering Numbers . . . . . . . . . . . . . . . . . . . 2.41

Altering the Procedure for a Different Range of Decluttering Numbers . . . 2.42

DELPTDB . . . . . . . . . . . . . . . . . . . . . . 2.45

What You Need to Know Before Using DELPTDB . . . . . . . . . . . 2.45

Deleting People Tracking Information with DELPTDB . . . . . . . . 2.45

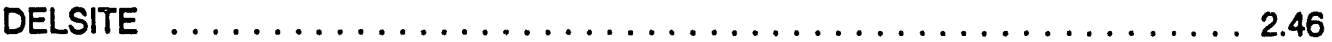

Why delete a site database? . . . . . . . . . . . . . . . 246

What You Need to Know Before Using DELSITE ............ 2.46

Deleting a Site Database with DELSITE . . . . . . . . . . . . 2.47

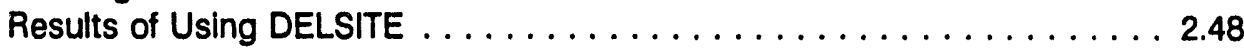

DELTREE . . . . . . . . . . . . . . . . . . . . . . 2.49

Deleting All Files and Directories in a Directory Tree with DELTREE $\ldots .2 .49$

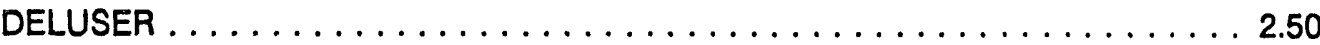

Why delete user accounts? . . . . . . . . . . . . . . . 2.50

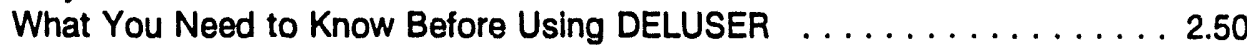

Deleting a User Account with DELUSER $\ldots \ldots \ldots \ldots \ldots \ldots \ldots \ldots \ldots . \ldots \ldots$

Resillts of Using DELUSER $\ldots \ldots \ldots \ldots \ldots \ldots \ldots \ldots \ldots \ldots \ldots \ldots \ldots$ 


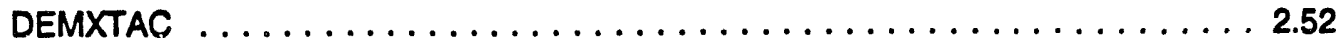

What You Need to Know Before Using DEMXTAC . . . . . . . . 2.52

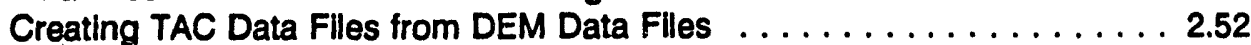

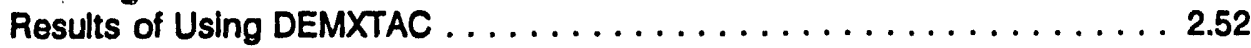

DLGXDMS ................................. 2.53

What You Need to Know Before Using DLGXDMS . . . . . . . . . 2.53

Converting DLG Data to DMS Format with DLGXDMS . . . . . . . . 2.54

Converting a DLG File with an Attribute (.ATT) File . . . . . . 2.54

Converting a DLG File without an Attribute (.ATT) File . . . . . 2.55

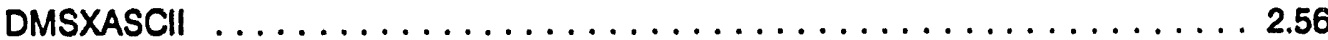

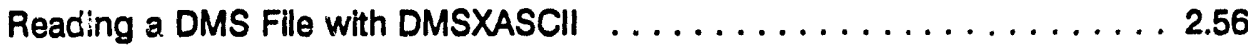

Creating an ASCII Information File from a DMS File . . . . . . 2.56

Displaying DMS File Information on Your Screen $\ldots \ldots \ldots \ldots 2.58$

DMSXDLG ............................... 2.59

What You Need to Know Before Using DMSXDLG . . . . . . . . . . 2.59

Converting DMS Data to DLG Format with DMSXDLG . . . . . . . . 2.59

DMSXDTX ................................ 2.60

Listing Location and Text Labels for Objects in a DMS File . . . . . . . 2.60

DOLIMITXX .................................. 2.61

What You Neigd to Know Before Using DOUMITXX . . . . . . . . . . 2.61

Creating Limit Files for a Current Database $\ldots \ldots \ldots \ldots \ldots \ldots \ldots \ldots . \ldots \ldots$

DOSEEUMITXX .................................. 2.62

Creating ASCII Limit Files for a Current Database . . . . . . . . . 2.62

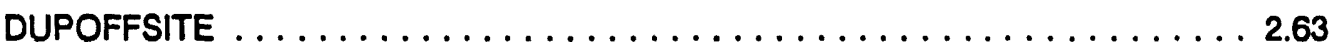

What You Need to Know Before Using DUPOFFSITE . . . . . . . . . 2.63

Duplicating an OFFSITE\$DIR Site with DUPOFFSITE $\ldots \ldots \ldots \ldots \ldots 2.63$

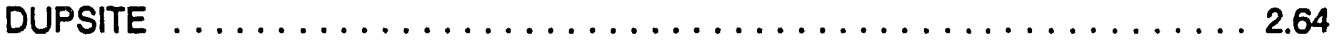

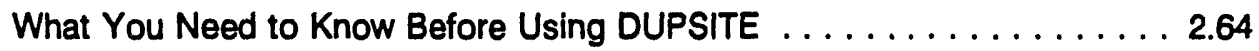

Duplicating a Site with DUPSITE . . . . . . . . . . . . . . . . 2.64

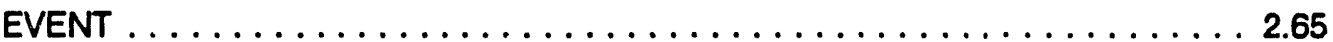

What You Should Know About Using EVENT . . . . . . . . . . . . . . 2.65

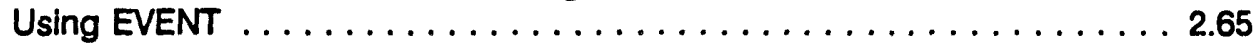

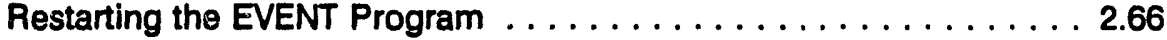

Stopping the EVENT Program . . . . . . . . . . . . . 2.66

Getting Information about EVENT Transactions: Log and Flag Files . 2.66

EXTRACT . . . . . . . . . . . . . . . . . . . . . . . . 2.67

Extracting Data from a DMS File or Site Database . . . . . . . . 2.67

Output DMS File Name Prefix . . . . . . . . . . . . 2.67

Selecting an Area to Extract - from a File or from a Database . . . . . 2.68

Select Data Based on Decluttering ..................... 2.69

Select Data Based on Attribute(s) . . . . . . . . . . . . . . . 2.70 
Select Data Based on Object Type $\ldots \ldots \ldots \ldots \ldots \ldots \ldots \ldots .71$

Extracting from a Single File ................... 2.71

Extracting from the Current Site Database $\ldots \ldots \ldots \ldots \ldots \ldots \ldots . . \ldots 2$.

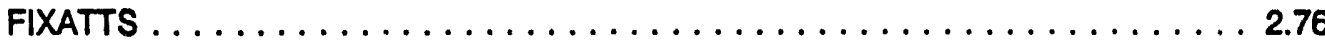

What You Need to Know Before Using FIXATTS . . . . . . . . . . . 2.76

Merging Two Attribute Dictionary Files with FIXATTS . . . . . . . . 2.76

FIXCNX and FIXCNX.BIS . . . . . . . . . . . . . . . 2.78

Creating a New Case Index File for a Site .............. 2.78

Creating New Case Index Flles for All Sites on the System . . . . . . . 2.78

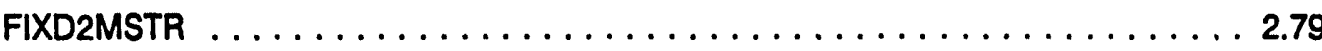

What You Need to Know Before Using FIXD2MSTR . . . . . . . . 2.79

Creating a D2 Master File for a User Site . . . . . . . . . . . . . . . 2.79

Creating D2 Master Files for All User Sites . . . . . . . . . . . . 2.79

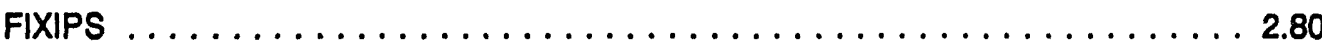

What You Need to Know Before Using FIXIPS . . . . . . . . . . 2.80

Upgrading IP Data Files for the Current Version of IBS with FIXIPS . . . 2.80

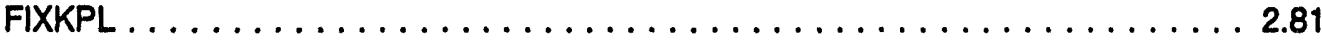

Upgrading the Known Polygons File with FIXKPL $\ldots \ldots \ldots \ldots \ldots \ldots 2.81$

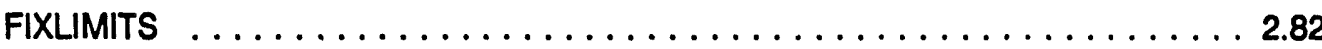

Updating the Limits in a DMS File Header . . . . . . . . . . . . 2.82

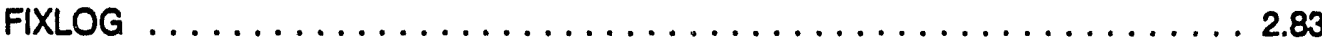

Fixing the Log Journal Files with FIXLOG . . . . . . . . . . . 2.83

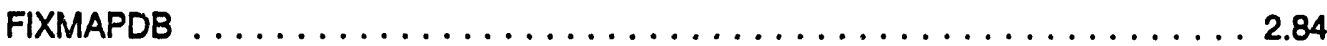

FIXMSTRDIR $\ldots \ldots \ldots \ldots \ldots \ldots \ldots \ldots \ldots \ldots \ldots \ldots \ldots \ldots . . \ldots \ldots$

What You Should Know Before Using FIXMSTRDIR . . . . . . . 2.86

Bullding a Master Directory List of Disk., User, and Site Names with

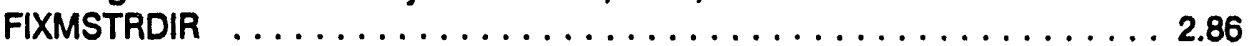

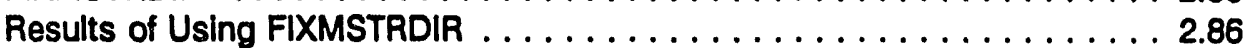

FIXOFFON . . . . . . . . . . . . . . . . . . . . . . . . . 2.87

What You Need to Know Before Using FIXOFFON . . . . . . . . . . 2.87

Flxing the OFF_TO_ON.DAT File $\ldots \ldots \ldots \ldots \ldots \ldots \ldots \ldots \ldots \ldots 2.87$

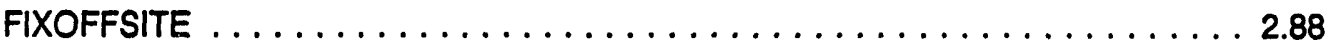

Updating [OFFSITEXox] Directories for the Current Version of IBS $\ldots 2.88$

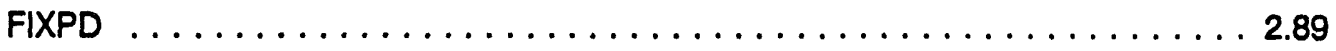

Upgrading the IBS Permissions File with FIXPD $\ldots \ldots \ldots \ldots \ldots \ldots 2.89$

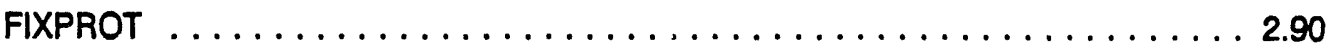

Upgrading IBS File and Directory Protections with FIXPROT . . . . 2.90 


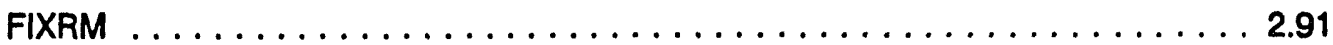

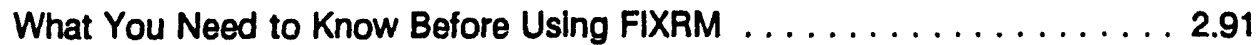

Preparing an Update Batch File with FIXRM . . . . . . . . . . . . 2.91

Updating Facility and Resource Data to Use Correct Icons . . . . . . . 2.92

FIXRSP and FIXRSP BIS $\ldots \ldots \ldots \ldots \ldots \ldots \ldots \ldots \ldots \ldots . . \ldots \ldots$

Updating MESŌRAD .RSP Files for One User Site with FIXRSP . . . . . 2.93

Updating MESORAD .RSP Files for All Users on the System . . . . . . . 2.93

FIXSETUP . . . . . . . . . . . . . . . . . . . . . . 2.94

Updating the SETUP.COM Files for the Current Version of IBS . . . . . 2.94

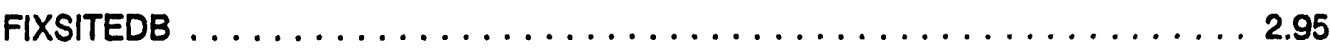

Converting Pre-IBS 1.0 Databases to IBS Format $\ldots \ldots \ldots \ldots \ldots \ldots 6$

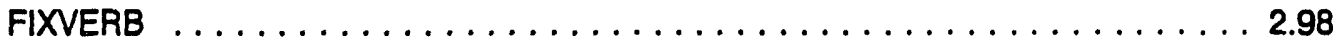

Upgrading the System DCLTABLES with FIXVERB $\ldots \ldots \ldots \ldots \ldots . . \ldots 8$

FTPSTART and FTPSTOP . . . . . . . . . . . . . . . . . . . 2.99

What You Need to Know Before Using FTPSTART and FTPSTOP . . . . . 2.99

Starting the Offpost/Onpost Modem Link with FTPSTART . . . . . . . . 2.99

Stopping the Offpost/Onpost Modem Link with FTPSTOP . . . . . . 2.100

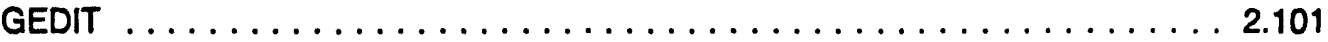

Description of the GEDIT Sottware . . . . . . . . . . . . . . . 2.101

What You Need to Know to Before Using GEDIT . . . . . . . . . . . . . . . 2.101

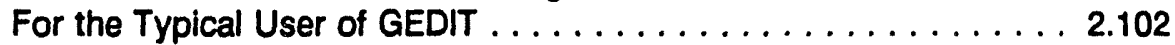

For the In-Depth User of GEDIT . . . . . . . . . . . . . . . . . 2.102

Terminology for Graphic Data Structures . . . . . . . . . . . . . . 2.102

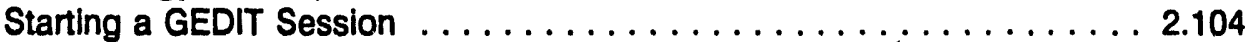

Using an Icon Control File . . . . . . . . . . . . . . . . . 2.104

Specifying Input Files . . . . . . . . . . . . . . . . . . . . . . 2.104

Background: Screen Coordinates of Input Files . . . . . . . 2.105

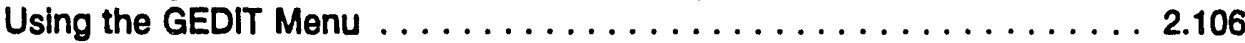

Extt (Save Changes) . . . . . . . . . . . . . . . . . 2.107

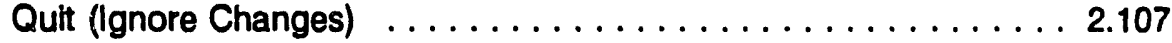

Help . . . . . . . . . . . . . . . . . . . . . . . . 2.107

Cancel ..................................... 2.107

Continue ............................ 2.107

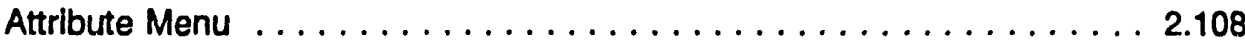

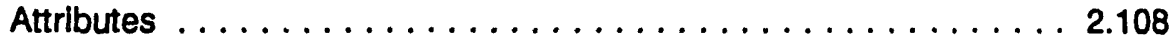

Using Attribute Menu Functions . . . . . . . . . . . . . 2.109

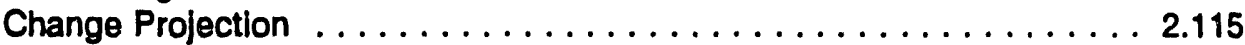

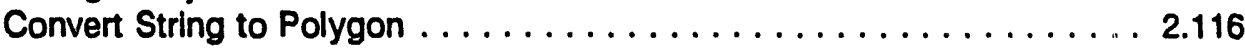

Delete Composite String . . . . . . . . . . . . . . . . . . . . . . 2.117

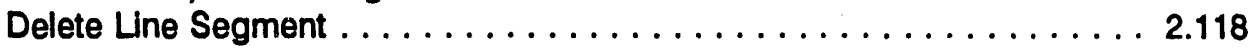

Delete Sub Polygon . . . . . . . . . . . . . . . . . . . . 2.119

Display Coordinates .......................... 2.120

Form Complex Polygon $\ldots \ldots \ldots \ldots \ldots \ldots \ldots \ldots \ldots \ldots \ldots \ldots . \ldots \ldots$

Geometric Menu . . . . . . . . . . . . . . . . . . . . . . . . . . 2.122

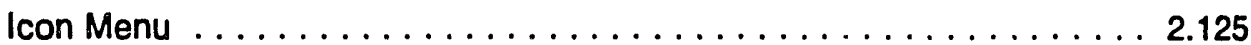




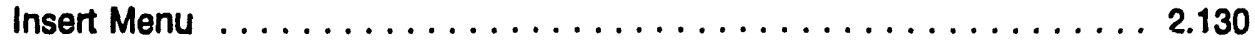

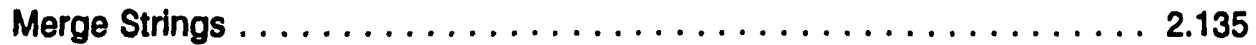

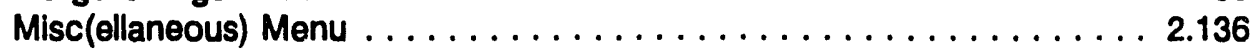

Modify Defaults . . . . . . . . . . . . . . . . . . . . . 2.148

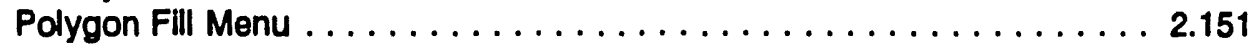

Re-Color-Texture String . . . . . . . . . . . . . . . . . . . 2.154

Refresh Screen . . . . . . . . . . . . . . . . . . . 2.155

Restore Base Plot $\ldots \ldots \ldots \ldots \ldots \ldots \ldots \ldots \ldots \ldots \ldots \ldots \ldots \ldots \ldots$

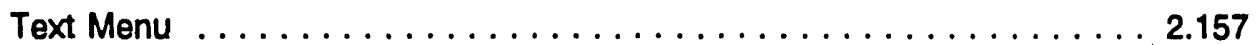

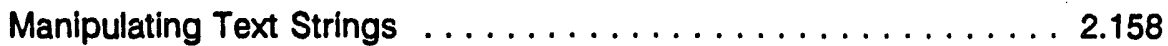

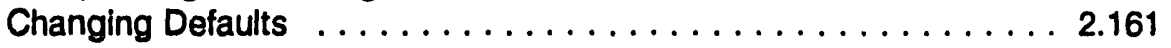

Controlling the Display . . . . . . . . . . . . . 2.163

Text String Menu ................................ 2.164

Unsave Composite String .................... 2.166

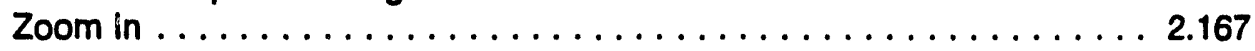

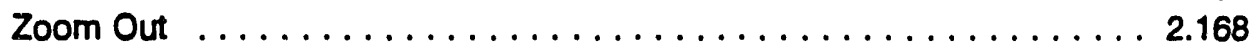

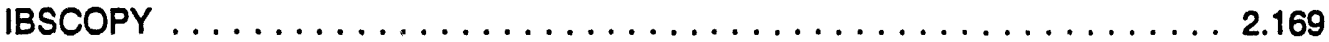

What You Need to Know Before Using IBSCOPY . . . . . . . . . . 2.169

Copying a File Between IBS Systems or Users . . . . . . . . . . . 2.169

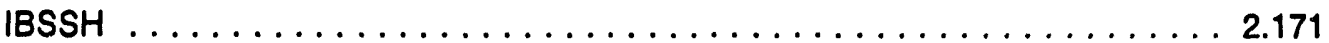

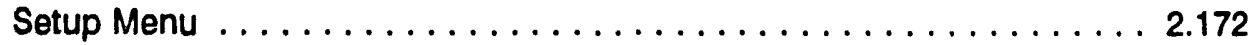

IBS Programs Menu . . . . . . . . . . . . . . . . . 2.172

System Administration Menu . . . . . . . . . . . . . . . . 2.173

Conversion Utilities Menu . . . . . . . . . . . . . . . . 2.173

Miscellaneous Utilties Menu . . . . . . . . . . . . . . . . . . . . 2.174

Graphic Models Menu . . . . . . . . . . . . . . . . . . . . . . . . 2.174

Non-Graphic Models Menu . . . . . . . . . . . . . . . . . . . . 2.175

Graphic GIS Utilities Menu . . . . . . . . . . . . . . . . . . . . . . . . 2.175

Non-Graphic GIS Utilities Menu . . . . . . . . . . . . . . . 2.176

IMPORT . . . . . . . . . . . . . . . . . . . . . . . . . . . . 2.177

What You Need to Know Before Using IMPORT . . . . . . . . . . . . . . . . . 2.177

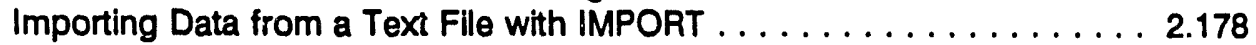

Verifying and Correcting Imported Data . . . . . . . . . . . . . . . 2.183

For Advanced Users: Modifying the Input FORMATs . . . . . . . . 2.184

Special Notes on Importing Formatted Personnel Data . . . . . . . . . 2.186

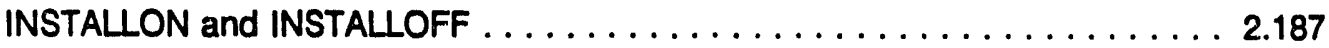

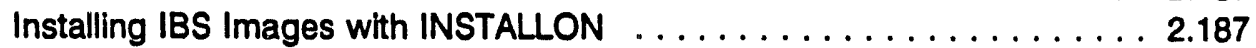

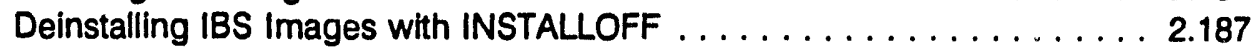

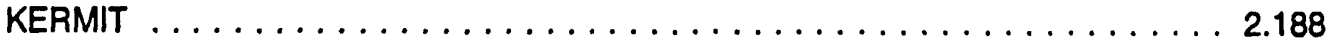

Transferring Files with KERMIT $\ldots \ldots \ldots \ldots \ldots \ldots \ldots \ldots \ldots \ldots 2.189$

LIMITXX . . . . . . . . . . . . . . . . . . . . . . . . . . . . . . 2.190

What You Need to Know Before Using LIMITXX . . . . . . . . . . . . . . . 2.190

Creating a Limit File for a Topography . . . . . . . . . . . . . . . . . 2.190 
LINEAR JOIN . . . . . . . . . . . . . . . . . . . . . . . . 2.191

What You Need to Know Before Using LINEAR JOIN . . . . . . . . . . . . 2.191

Criteria for Joining Strings . . . . . . . . . . . . . . . 2.191

Strategies for Joining Strings . . . . . . . . . . . . . . . . . . 2.192

Reducing the Number of Strings in a DMS File with LINEAR_JOIN . . . 2.193

Results of Using LINEAR JOIN . . . . . . . . . . . . . . . . . . . 2.194

LISTCNX . . . . . . . . . . . . . . . . . . . . . . . . . . . . . . . . 2.195

Listing Model Cases Found in the Case Index File . . . . . . . . 2.195

LJ TIGER . . . . . . . . . . . . . . . . . . . . . . . . . . . 2.196

What You Need to Know Before Using LJ TIGER . . . . . . . . . . . 2.196

Joining Discrete Strings in a DMS File with L_TIGER . . . . . . . . . 2.197

LOOKTXT . . . . . . . . . . . . . . . . . . . . . . . . . . . . . . . . . . 2.198

What You Need to Know Before Using LOOKTXT . . . . . . . . . . . . 2.198

Looking at the Contents of a DMS Text (.TXT) File . . . . . . . . . . 2.198

MAIL C ....................................... 2.199

What You Need to Know Before Using MAIL_C . . . . . . . . . . . 2.199

Restarting the Mail Checking Process . . . . . . . . . . . . . . . . . . . 2.199

Results of Using MAlL_C . . . . . . . . . . . . . . . . . 2.199

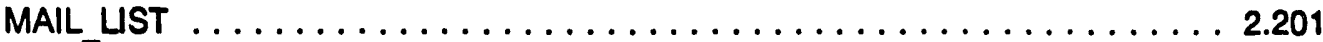

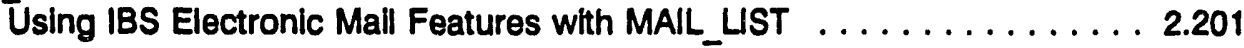

MAKEDMS . . . . . . . . . . . . . . . . . . . . . . . . . . . . . . . 2.202

Creating DMS Files from ASCII Input Files with MAKEDMS . . . . . . . 2.202

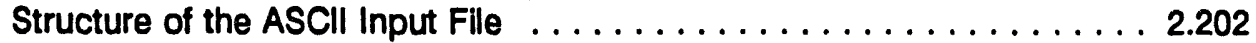

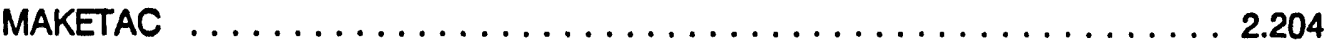

What You Need to Know Before Using MAKETAC . . . . . . . . . . . . 2.204

Creating a New TAC File with MAKETAC . . . . . . . . . . . . . . . . . 2.204

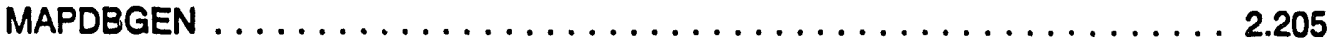

Why create a "user-defined" map database? . . . . . . . . . . . . 2.205

What You Need to Know Before Using MAPDBGEN . . . . . . . . . . . 2.205

Creating a User-Defined Map Database with MAPDBGEN . . . . . . . . . 2.207

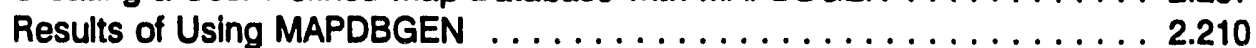

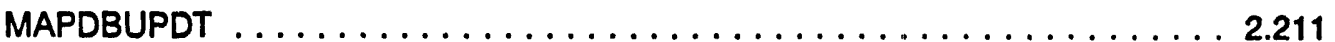

Where Does the Update Data Come From? . . . . . . . . . . . . . 2.211

What Are the Requirements of Database Updating? . . . . . . . . 2.211

What Happens During an Update? . . . . . . . . . . . . . 2.213

A Procedure for Updating a DMS Database . . . . . . . . . . . . . . . 2.214

Example of Updating a Database Topography . . . . . . . . . . . . . . 2.217

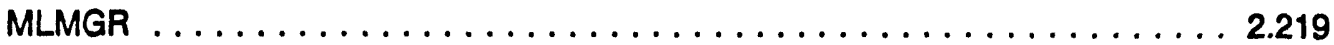

What You Need to Know Before Using MLMGR . . . . . . . . . 2.219

Updating the IBS Mail List . . . . . . . . . . . . . . . . . . . 2.220

1 : Adding New Names to the Mail List . . . . . . . . . 2.221 
2: Modifying Information about Users on the Mail List . . . . . 2.222

3 : Deleting Users from the Mall List ............... 2.223

4 : Updating Agency/Position Information on the Mall List . . . . . 2.224

5 : Updating Mail Address Information on the Mail List . . . . . . 2.225

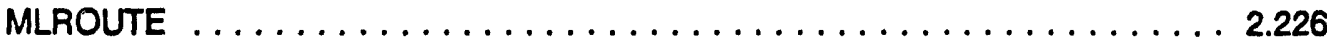

What You Need to Know Before Using MLROUTE . . . . . . . . . 2.226

Updating the Mail Lists of Networked Systems with MLROUTE . . . . . 2.226

MODATTDMS . . . . . . . . . . . . . . . . . . . . . . . . . . . 2.227

What You Need to Know Before Using MODATTDMS . . . . . . . . . . . 2.227

Replacing Attribute Codes in a DMS File Using MODATTDMS . . . . . . . 2.227

MODTEXTNM . . . . . . . . . . . . . . . . . . . . . . . . . . . . 2.228

Changing the Text File Referenced in a DMS File Header . . . . . . . . 2.228

Results of Using MODTEXTNM . . . . . . . . . . . . . . . . . . . . 2.229

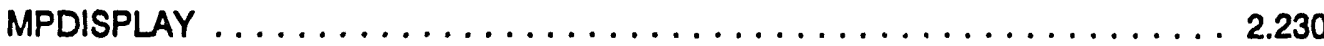

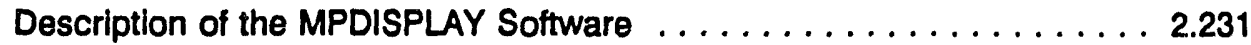

What You Need to Know to Use the MPDISPLAY Software . . . . . . . 2.231

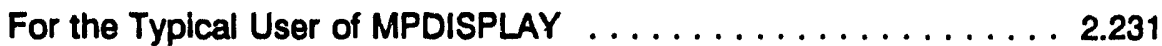

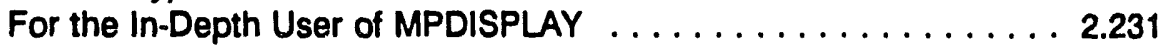

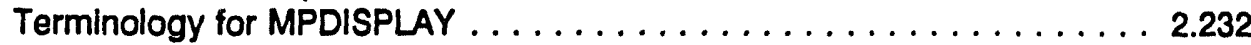

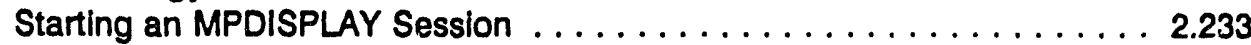

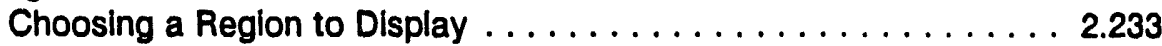

Choosing the Type of Map Projection . . . . . . . . . . . . . . 2.237

Choosing Topographic Features . . . . . . . . . . . . 2.239

Entering the Name of an Edit File . . . . . . . . . . . . . . 2.241

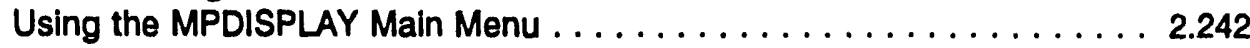

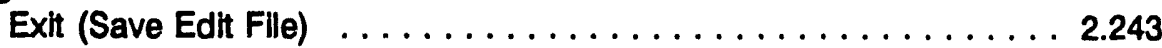

Quit (Delete Edit File) . . . . . . . . . . . . . . . . . . . 2.243

Help . . . . . . . . . . . . . . . . . . . . . . . . . . . . 2.243

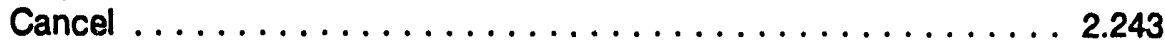

Continue ...........................2.243

Attribute Functions . . . . . . . . . . . . . . . . . . . . . 2.244

Edit Functions . . . . . . . . . . . . . . . . . . . . . . . . . . 2.252

Elevation Functions . . . . . . . . . . . . . . . . . . . . 2.253

Icon Functions . . . . . . . . . . . . . . . . . . . . . . . . . . . . . . 2.257

Item Display Functions . . . . . . . . . . . . . . . . . . . 2.260

Screen View Functions . . . . . . . . . . . . . . . . . . . . . 2.263

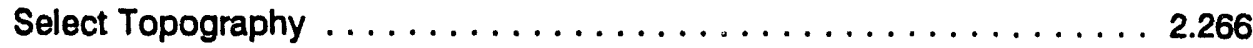

Displaying the List of Topographies . . . . . . . . . . . . 2.267

Picking Topographies from the Menu . . . . . . . . . . . . . . . 2.267

Topographic Codes ....................... 2.268

Store Region . . . . . . . . . . . . . . . . . . . . . . 2.269

Results of using STORE REGION $\ldots \ldots \ldots \ldots \ldots \ldots \ldots \ldots 2.271$

Names of Map Display Data Files . . . . . . . . . . . . . 2.271

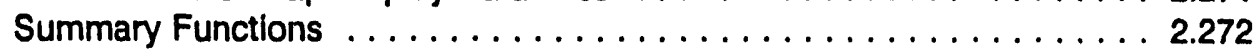

MYTAPE . . . . . . . . . . . . . . . . . . . . . . . . . . . 2.279

Processing Foreign Tapes with MYTAPE $\ldots \ldots \ldots \ldots \ldots \ldots \ldots \ldots .2 .279$

IBS Utilities Guide - 3/1/94 XVii 
NEWLOG $\ldots \ldots \ldots \ldots \ldots \ldots \ldots \ldots \ldots \ldots \ldots \ldots \ldots \ldots \ldots \ldots \ldots .280$

Starting New IBS Log Journals . . . . . . . . . . . . . 2.280

NEWSITE . . . . . . . . . . . . . . . . . . . . . . . . . 2.281

Why create a new site database? $\ldots \ldots \ldots \ldots \ldots \ldots \ldots \ldots \ldots .28 . \ldots \ldots$

What You Need to Know Before Using NEWSITE . . . . . . . . . . 2.282

Using NEWSITE to Define an IBS Site Database . . . . . . . . . . . 2.284

Creating Directories for the New Site . . . . . . . . . . . . . 2.284

Copying Topographic Data to the New Site . . . . . . . . . 2.285

Results of Using NEWSITE . . . . . . . . . . . . . . . 2.288

NEWUSER $\ldots \ldots \ldots \ldots \ldots \ldots \ldots \ldots \ldots \ldots \ldots \ldots \ldots \ldots \ldots \ldots \ldots .28 . \ldots \ldots 9$

Why create a new user account? . . . . . . . . . . . . . 2.289

What You Need to Know Before Using NEWUSER . . . . . . . . . . 2.289

Creating or Modifying a User Account with NEWUSER . . . . . . . . . 2.291

Results of Using NEWUSER . . . . . . . . . . . . . . . . . . . . . . . . . 2.292

PDMGR . . . . . . . . . . . . . . . . . . . . . . . . . . 2.293

What You Need to Know Before Using PDMGR . . . . . . . . . 2.293

Modifying IBS Privileges with PDMGR . . . . . . . . . . . . . . . . . 2.294

PLOTDMS . . . . . . . . . . . . . . . . . . . . . . . . . . . . . . . . . . 2.299

What You Need to Know Before Using PLOTDMS . . . . . . . . . . 2.299

Plotting the Contents of a DMS File with PLOTDMS . . . . . . . . . . 2.299

PLOTICON . . . . . . . . . . . . . . . . . . . . . . . 2.305

What You Need to Know Before Using PLOTICON . . . . . . . . . . 2.305

Plotting the Avallable IBS Icons . . . . . . . . . . . . . . . . 2.305

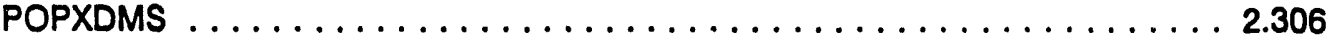

What You Need to Know Before Using POPXDMS . . . . . . . . . 2.306

Creating DMS Population Files from Census Bureau STF1B Files $\ldots \ldots 2.307$

Example of Converting Data Below the State Level . . . . . . . . . 2.307

Example of Converting State-Level Data . . . . . . . . . 2.308

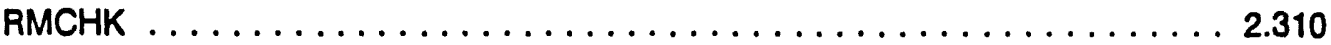

Checking Resource Management Data with RMCHK . . . . . . . 2.310

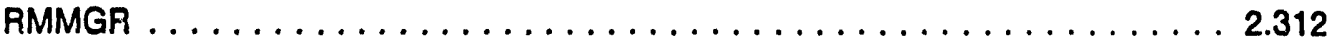

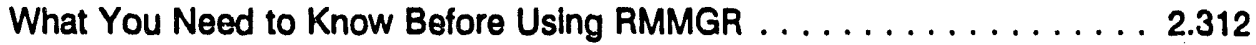

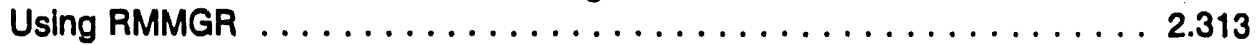

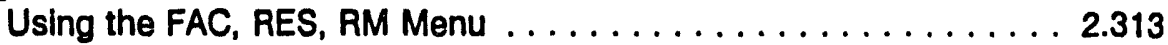

Using the FT, KP Menu . . . . . . . . . . . . . . . . 2.314

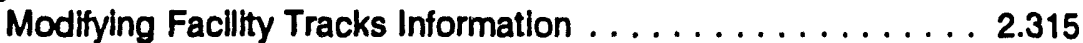

Modifying Known Points Information ............ 2.315

Using the AG, PER, POS Menu . . . . . . . . . . . . . 2.319

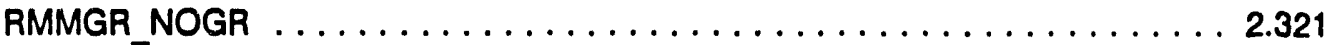

What You Need to Know Before Using RMMGR NOGR . . . . . . . 2.321

Managing Special IBS Data with RMMGR_NOGR $\ldots \ldots \ldots \ldots \ldots \ldots 2.321$

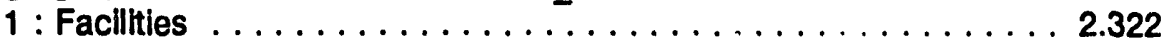


$2:$ Resources ......................... 2.323

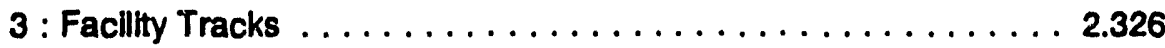

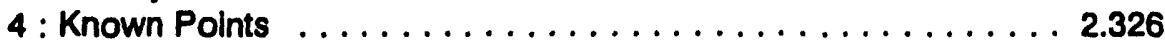

5 : Agencies . . . . . . . . . . . . . . . . . . . . . . . 2.328

6 : Positions . . . . . . 2.328

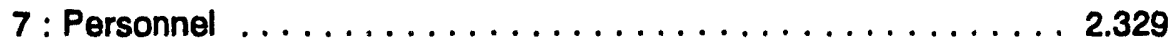

8 : Special Population . . . . . . . . . . . . . . . . . . 2.329

SCALEDMS . . . . . . . . . . . . . . . . . . . . . . . 2.332

What You Need to Know Before Using SCALEDMS $\ldots \ldots \ldots \ldots \ldots . . \ldots 232$

Scaling a DMS File with SCALEDMS $\ldots \ldots \ldots \ldots \ldots \ldots \ldots \ldots \ldots .332$

SEELIMITS . . . . . . . . . . . . . . . . . . . . . 2.333

What You Need to Know Before Using SEELIMITS . . . . . . . . . 2.333

Displaying the Contents of a Limit file with SEELIMITS . . . . . . . . 2.333

Saving the Contents of a Limit file with SEELIMITS . . . . . . . . . . . 2.334

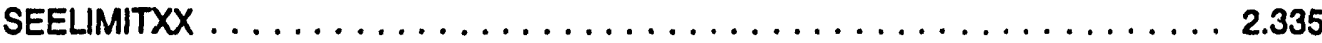

Creating an ASCll Version of a Binary Limit file $\ldots \ldots \ldots \ldots \ldots \ldots . . . \ldots 235$

SEETXTNM ............................... 2.336

Seeing the Name of a DMS File's Text File with SEETXTNM . . . . . 2.336

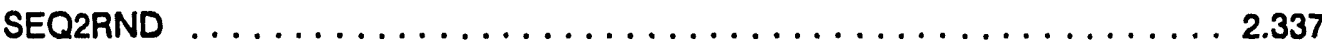

Generating a Random Access File based on a Sequential File . . . . . 2.337

SETUPTEK . . . . . . . . . . . . . . . . . . . . . . . . 2.338

Returning a Tektronix Terminal to Default Settings with SETUPTEK . . . 2.338

SHOWAT . . . . . . . . . . . . . . . . . . . . . . . . . . . . . 2.339

Showing Equivalent Attribute Values in Octal/Hexadecimal/Decimal . . . 2.339

SHOWCNX . . . . . . . . . . . . . . . . . . . . . . . . 2.340

Showing the Model Case Index File for a Site $\ldots \ldots \ldots \ldots \ldots \ldots 2.340$

SHOWCOL . . . . . . . . . . . . . . . . . . . . . . . . . 2.341

Showing Colors Associated with Terminal Pen Numbers . . . . . . . . . . 2.341

SHOWCOVER ............................. 2.342

Creating an Area Coverage Map for a Topography (Map Layer) . . . . 2.342

SHOWENV . . . . . . . . . . . . . . . . . . . . . . . . . . 2.343

Showing User Environment Characteristics with SHOWENV $\ldots \ldots \ldots .2 .343$

SHOWTEK . . . . . . . . . . . . . . . . . . . . . . . . . . . 2.344

Displaying Current Tektronix TRPLOT Settings with SHOWTEK . . . . . 2.344

SITEBKP $\ldots \ldots \ldots \ldots \ldots \ldots \ldots \ldots \ldots \ldots \ldots \ldots \ldots \ldots \ldots \ldots . . \ldots \ldots 45$

Backing Up or Restoring Selected IBS Site Data with SITEBKP . . . . . 2.345 


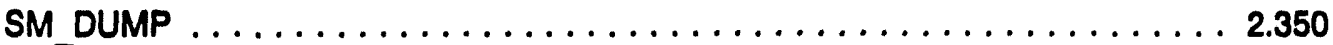

What You Need to Know Before Using SM DUMP . . . . . . . . . . 2.350

Copying Shared Memory to a File or to the Screen with SM_DUMP . . . 2.350

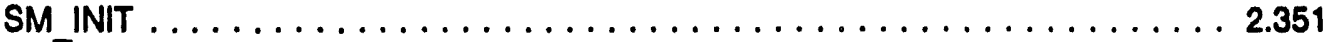

What You Need to Know Before Using SM INIT . . . . . . . . . 2.351

Initiallzing Shared Memory Variables with SM_INIT . . . . . . . . . 2.351

SM SET . . . . . . . . . . . . . . . . . . . . . . . . 2.352

What You Need to Know Before Using SM SET . . . . . . . . . . 2.352

Setting Shared Memory Variables with SM_SET . . . . . . . . . . 2.352

SMOOTHER . . . . . . . . . . . . . . . . . . . . . . . . 2.353

Reducing the Number of Points in a DMS File with SMOOTHER . . . . . 2.353

SPLITDMS . . . . . . . . . . . . . . . . . . . . . 2.354

Splitting Off DMS Objects that Have No Attributes with SPLITDMS $\ldots . .2 .354$

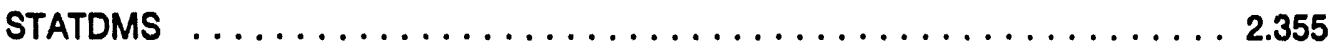

What You Need to Know Before Using STATDMS . . . . . . . . . 2.355

Producing DMS Data Statistics with STATDMS . . . . . . . . . . . . . 2.355

Producing a Statistics Report for One DMS File ... . . . . . 2.355

Producing a Report of DMS File Statistics for a DMS Database . . . 2.356

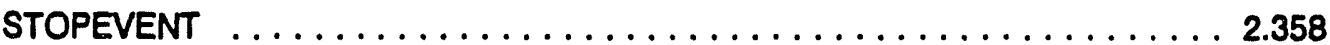

Stopping the EVENT Background Program with STOPEVENT . . . . . 2.358

STOPMAIL C . . . . . . . . . . . . . . . . . . . . . 2.359

Stopping the MAIL_C Background Program with STOPMAIL_C $\ldots \ldots 2.359$

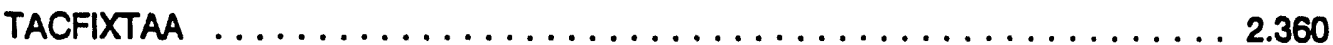

Changing the TAA File Reference in a TAC File ... . . . . . . . 2.360

TACUXUTM . . . . . . . . . . . . . . . . . . . . . . . . . . . . 2.361

What You Need to Know Before Using TACLXUTM . . . . . . . . . 2.361

Converting TAC File Coordinate Data with TACLLXUTM . . . . . . . 2.362

Results of Using TACLXUTM . . . . . . . . . . . . . . . . . . . 2.362

TACXASCII . . . . . . . . . . . . . . . . . . . . . . . . . 2.363

Displaying the Contents of a TAC Header Flle with TACXASCII . . . . . 2.363

Displaying TAC Data with TACXASCII ................. 2.364

Capturing the Cell Data in an ASCll File ................... 2.365

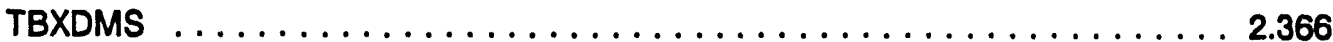

What You Need to Know Before Using TBXDMS ............ 2.366

Creating DMS Boundary Data Files from TIGER/LIne Data Files . . . . . 2.366

TEKSETUP . . . . . . . . . . . . . . . . . . . . . . . . . . . 2.369

Changing Screen Color Assignments with TEKSETUP . . . . . . . 2.369 
THINNER . . . . . . . . . . . . . . . . . . . . . . 2.370

Reducing the Number of Points in a DMS File with THINNER . . . . . 2.370

TIGERCVT . . . . . . . . . . . . . . . . . . . . . . . . 2.371

What You Need to Know Before Using TIGERCVT . . . . . . . . . . . . 2.371

Preparing for TIGER-to-DMS Data Conversion with TIGERCVT . . . . 2.372

Results of Using TIGERCVT . . . . . . . . . . . . . . . . . 2.375

Converting Census Bureau Data Using TIGERXDMS.COM,

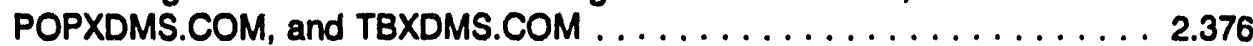

TIGERCVT Program Messages $\ldots \ldots \ldots \ldots \ldots \ldots \ldots \ldots \ldots \ldots .377$

TIGERXDMS . . . . . . . . . . . . . . . . . . . . . . . 2.379

What You Need to Know Before Using TIGERXDMS . . . . . . . . . 2.379

Creating DMS Data Files from TIGER/Line Data Files . . . . . . . . 2.380

TIGERXDMS Program Messages $\ldots \ldots \ldots \ldots \ldots \ldots \ldots \ldots \ldots \ldots .381$

UPDATEATT $\ldots \ldots \ldots \ldots \ldots \ldots \ldots \ldots \ldots \ldots \ldots \ldots \ldots \ldots \ldots \ldots \ldots .383$

What You Need to Know Before Using UPDATEATT . . . . . . . . 2.383

Updating the IBS Attribute Dictionary with UPDATEATT . . . . . . . 2.384

A - Adding a New Attribute Record . . . . . . . . . . . . . 2.384

C - Changing an Attribute Record $\ldots \ldots \ldots \ldots \ldots \ldots \ldots \ldots 2.385$

D - Deleting an Attribute Record ................. 2.385

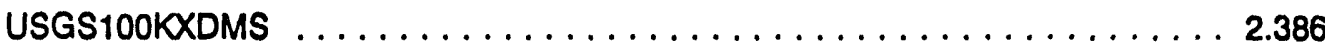

Converting USGS Data Files for a Special-Purpose DMS Database $\ldots \ldots 2.386$

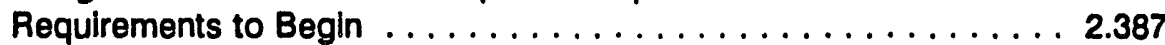

Procedure . . . . . . . . . . . . . . . . . . . . . . . . 2.387

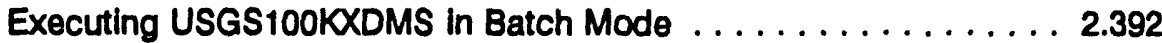

Errors Caused by Missing Attribute Codes . . . . . . . . . . . 2.393

Results of Using USGS100KXDMS . . . . . . . . . . . . . . . . . 2.393

USGSXDMS . . . . . . . . . . . . . . . . . . . . . . . . . . 2.394

Converting USGS Format Data to DMS Format with USGSXDMS $\ldots \ldots 2.394$

UTMXLL . . . . . . . . . . . . . . . . . . . . . . . . . . . . . . . . 2.395

Converting Between UTM and Longitude/Latitude with UTMXLL . . . . . . 2.395

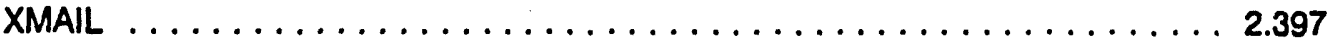

Using Mail Enhancements in XMAlL . . . . . . . . . . . . . . 2.397

\section{Appendix A. IBS Topographles (Map Layers)}

IBS Topographies (Map Layers) Listing $\ldots \ldots \ldots \ldots \ldots \ldots \ldots \ldots \ldots$ A.1

\section{Appendix B. IBS Attribute Dictionary}

IBS Attribute Dictionary Listing $\ldots \ldots \ldots \ldots \ldots \ldots \ldots \ldots \ldots$ B.1 


\section{Appendix C. IBS Icon Dictionary (Icon Control File)}

IBS Icon Dictionary Listing

\section{Appendix D. Map Projections In IBS Map Displays}

Why use different map projections? $\ldots \ldots \ldots \ldots \ldots \ldots \ldots \ldots \ldots \ldots$ D.1

Choosing a Map Projection Type . . . . . . . . . . . . . . . . . . D.1

\section{Appendix E. IBS Privilege Listings}

\section{Appendix F. Undocumented Utility Programs}

Utilities Called by other IBS Programs $\ldots \ldots \ldots \ldots \ldots \ldots \ldots \ldots \ldots \ldots$.1

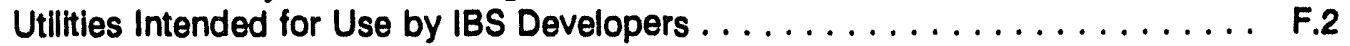

Index

\section{Tables}

Section 2. Utility Descriptions

2.1. Correspondence Between Decluttering Numbers and Attribute

Parameter Values (for the DECLUTTER procedure) $\ldots \ldots \ldots \ldots 2.42$

2.2. Attributes Resulting from Insert Menu Functions . . . . . . . . . . . . 2.134

Appendix E. IBS Privilege Listings

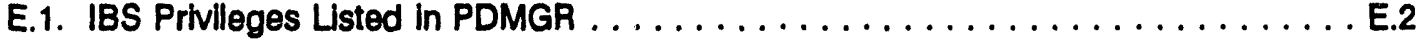

E.2. Privileges Listed by Program \& by Menu Options in the IBS Program . . . . . . E.3

E.3. IBS Program Menu Options Listed by Privilege E.8 


\section{Section 1 Introduction}

The Integrated Baseline System (IBS) is being developed by the Pacific Northwest Laboratory (PNL) under the direction of the U.S. Army Nuclear and Chemical Agency (USANCA). This IBS Utilities Guide explains how you can use the IBS utilities-the utility programs included in the system. With a few exceptions, these utilities are programs that support the system by managing and manipulating various kinds of IBS files-rather than by performing tasks directly related to emergency management.

This section explains what you need to know before using the utilities and summarizes the tasks that the utilities were designed to accomplish. To help you find which utilities may be useful for your tasks, this section supplies capsule descriptions of each utility within several overlapping categories:

Tasks Accomplished Using IBS Utilities

Use independent programs for risk area analysis, resource management, message tracking, and executing the D2 dispersion hazard assessment model.

Use and monitor background programs that must be running before the system will function properly.

Use electronic mail; assist tape file transfers; send files to other IBS users

Display IBS maps on a graphic terminal screen and edit the map data or associated data.

Create individual map data files or modify their contents.

Convert map data files to and from formats that can be used by the IBS.

Create, examine, and modify database files and database administration files.

Perform quality assurance procedures on IBS map, site, and offsite databases.

Support system management tasks (such as creating user accounts, deleting user accounts, and creating system management files).

Modify certain system and data files to make earlier systems compatible with a new version of the IBS.
In this section see ...

Emergency Management and

Planning Utilities

Background Programs

General System Utilities

Graphic Map Display and Editing Utilities

Data File Modification Utilities

Data File Conversion Utilities

Database Administration Utilities

Database Quality Assurance (QA) Utilities

System Management Utilities

System Upgrade Utilities 
Tasks Accomplished Using IBS Utilities

Display and report information about various data files, database support files, system management files, and shared memory variables.
In this section see ...

Diagnostic and Reporting Utilities

If you are an IBS system manager or database administrator, you can find many utilities that will help you maintain the IBS and keep it running correctly.

If you are an emergency management planner, analyst, or information manager, you are probably most interested in the utilities that monitor or manipulate IBS data: the emergency management and planning utilities, the graphic map display and editing utilities, the data file modification utilities, and the database administration utilities.

The rest of this section is divided into three parts:

- What You Need to Know Before Using the IBS Utility Programs

- Finding the Utilities that You Need (capsule descriptions)

- Overview of IBS Map Data.

\section{What You Need to Know Before Using the IBS Utility Programs}

Before using the utility programs, you must be an authorized user with an IBS user account set up by the system manager. Then when you $\log$ in, the system automatically sets up your computer environment so that you have the proper access to IBS programs, directories, and files.

IBS Privileges. You must also have the proper authorizations or IBS privileges to use a specific utility program. Because the IBS utilities are designed for a wide range of users, they require a wide range of IBS user privileges. Some utilities are for generai use and require no special privilege; others are for use only by system managers or database administrators and may also require specific VAX/VMS privileges. If you find that you cannot use a specific utility program because you do not have the necessary privileges, contact your IBS system manager.

Using Your Equipment. You should be familiar with the operation of your computer terminal and with basic IBS interactions, both as described in Section 1 of the IBS User Guide. If you plan to use utilities that display graphic maps, you must have a supported graphics terminal with an attached graphic input device such as a mouse, joydisk, or bit pad. 
IBS Map Data. Many of the IBS utilities concern the use of the IBS map database and map data. For this reason, the Overview of IBS Map Data later in this section summarizes terms and key concepts about IBS map databases and the Digital Mapping Structure (DMS) map data used by the IBS. For complete information about IBS map data, refer to the IBS Data Management Guide.

\section{Finding the Utilities that You Need}

The operational descriptions of the IBS utilities are listed in alphabetical order by utility name in Section 2 of this guide. To point your way into Section 2, the following sections furnish capsule descriptions of the IBS utilities within several functional or task categories:

- Emergency Management and Planning

- Background Programs

- General System Utilities

- Graphic Map Display and Editing Utilities

- Data File Modification Utilities

- Data File Conversion Utilities

- Database Administration Utilities

- Database Quality Assurance (QA) Utilities

- System Management Utilities

- System Upgrade Utilities

- Diagnostic and Reporting Utilities.

You can browse the capsule descriptions to find out what the utilities can do and then refer to Section 2 for more detail.

Some utilities occur in more than one category. For example, the Diagnostic and Reporting Utilities include many utilities found in other categories. Other utilities (such as the Data File Conversion Utilities) are restricted to a single category when they could be considered a special subset of a broader category (such as the Database Administration Utilities).

Undocumented Utility Programs. For completeness, Appendix F lists other utilities included in the system but which are NOT documented and NOT directly used by IBS users. These are either utilities designed to be called from other IBS programs or special utilities used by system developers. 


\section{Emergency Management and Planning Utilities}

These programs duplicate or enhance emergency management support functions found in the IBS program. For people who are using only those functions, these utilities may be faster and more convenient to use than the full IBS program.

$\begin{array}{ll}\text { Utility Name } & \begin{array}{l}\text { Purpose / Description } \\ \text { D2RUN }\end{array} \\ \text { RMecutes IBS's dispersion hazard assessment model for a } \\ \text { specified case of the current planning site } \\ \text { (Resource Management Manager) displays maps with resource } \\ \text { and facility icons; enables you to update the contents of the } \\ \text { personnel, resources, and facilities databases for the entire } \\ \text { system; duplicates features of RESOURCE MANAGEMENT } \\ \text { and PERSONNEL MANAGEMENT in the IBS program. For } \\ \text { many details about the functions supported by RMMGR, see } \\ \text { Resource Management in the IBS User Guide. } \\ \text { (Nongraphic version of RMMGR) enables you to update the } \\ \text { contents of the personnel, resources, and facilities databases } \\ \text { from a text terminal; duplicates features of RESOURCE } \\ \text { MANAGEMENT and PERSONNEL MANAGEMENT in } \\ \text { RMMGR and in the IBS program. For many details about } \\ \text { the functions supported by RMMGR_NOGR, see Resource } \\ \text { Management in the IBS User Guide. }\end{array}$

\section{Background Programs}

The following programs include batch processes that normally start automatically when the computer system is started. These programs should always be running: they are necessary for other system functions to work correctly. Only the system manager or someone with appropriate system privileges can stop or restart these programs. (Sec also FTPSTART and FTPSTOP in System Management Utilities.)

Utziity Name

EVENT

STOPEVENT

MAIL_C

STOPMAIl_C
Purpose / Description

detects data sent from the onpost system and then informs IBS users or takes other appropriate actions

stops the EVENT batch job

(Mail Checker) checks the mail file of each IBS user once per minute and displays mail status messages

stops the Mail Checker batch job 


\section{General System Utilities}

The following general system utilities are of use to all IBS users and require no special privileges. The MYTAPE utility has been added to the system as an operating system command.

Utility Name

MAIL_LIST

XMAIL

IBSCOPY

IBSSH

KERMIT

MYTAPE

SETUPTEK

TEKSETUP

UTMXLL

\section{Purpose / Description}

enables you to use electronic mail features; duplicates the mail features of Message Board in the IBS program

enhances the system's electronic mail capabilities

transfers files between users over a network between computer systems

IBS "shell" program that provides menu access to many IBS utilities and other programs (Some of these programs may require special IBS privileges.)

permits transfer and exchanges of sequential files over ordinary telecommunication lines between computers

simplifies tape file transfers between VAX systems and between VAX systems and other systems

resets a Tektronix $41 \times x$ terminal to default characteristics

enables you to set Tektronix terminal screen background and foreground colors

converts between Universal Transverse Mercator (UTM) coordinates and longitude-latitude coordinates 


\section{Graphic Map Display and Editing Utilities}

These utility programs all display IBS graphic map data and require a graphic terminal. Three of the programs-GEDIT, MPDISPLAY, and RMMGR-are interactive programs that enable you to display, modify, and save map data or associated information. (See also PLOTICON under Diagnostic and Reporting Utilities.)

The RMMGR program duplicates or enhances resource management support functions found in the IBS program. For people who are using only those functions, these utilities may be faster and more convenient to use than the full IBS program. (See also RMMGR_NOGR, a version of RMMGR that can be used on nongraphic terminals.)

Utility Name

GEDIT

MPDISPLAY

PLOTDMS

RMMGR

\section{Purpose / Description}

provides tools for interactive graphic display and editing of DMS files

graphically displays and stores map information from the IBS map database

displays graphic data from DMS file(s)

(Resource Management Manager) displays maps with resource and facility icons; enables you to update the contents of the personnel, resources, and facilities databases for the entire system; duplicates features of RESOURCE MANAGEMENT and PERSONNEL MANAGEMENT in the IBS program. For many details about the functions supported by RMMGR, please see Resource Management in the IBS User Guide. 


\section{Data File Modification Utilities}

You can use the following utilities to produce individual map data files and to examine or modify their contents. Most of these utilities operate on DMS data files; a few operate on Terrain Analysis Cell (TAC) data files. These programs are all text-based programs: graphic map display and editing utilities are listed separately. Data file conversion utilities are also listed in a separate category.

Utility Name

MAKETAC

TACLLXUTM

TACFIXTAA

APPENDDMS

SPLITDMS

CLIPDMS

EXTRACT

FIXLIMITS

COLORDMS

MODTEXTNM

MODATTDMS

ADDATTDMS

DECLUTTER

LINEAR_JOIN

L_TIGER

SCALEDMS

(continued)
Purpose / Description

TAC File Utility: creates a TAC file with specified extents and uniform cell values

TAC File Utility: converts TAC files both ways between the longitude-latitude coordinate system and the universal transverse mercator (UTM) coordinate system

TAC File Utility: enables you to change the TAA filename reference in a TAC file

appends one DMS file to another

splits a DMS file into two parts: one containing objects that have attributes and one containing objects that have no attributes

clips a specific area of data from a DMS file to create one or more new DMS files; subdivides a DMS file into new DMS files based on longitude-latitude steps

extracts selected data from a DMS file or database to create one or more new DMS files

enables you to change the area limits specified in the header of a DMS file

modifies a DMS file so that all features have one color

enables you to modify the name of the text file referenced in the header of a DMS file

changes an attribute in a DMS file to a different attribute

adds an attribute to all objects in a DMS file

adds a decluttering attribute to a DMS file

reads a single DMS file and joins any vector strings that have closely matching end points and common attributes

functions similar to LINEAR JOIN, but the program is optimized for use with DMS files base on TIGER data

scales objects larger or smaller in a DMS file 
Utility Name

SEQ2RND

SMOOTHER

THINNER

\section{Purpose / Description}

converts a sequential file to a random access file

reduces the number of coordinate points that compose strings in a DMS file by deleting co-linear intermediate points

reduces the number of coordinate points that compose strings in a DMS file by deleting points that would be displayed at the same locations 


\section{Data File Conversion Utilities}

The data conversion utilities convert map data files from one format to another, preserving the original files and creating new files in the second format. Reciprocal utilities for converting to and from DMS format are paired in the following list.

Utility Name

IMPORT

ASCIIXDMS

$\&$

DMSXASCII

DLGXDMS

\&

DMSXDLG

MAKEDMS

TIGERCVT

TIGERXDMS

POPXDMS

TBXDMS

USGS100KXDMS

USGSXDMS

DEMXTAC

CONTOUR
Purpose / Description

imports data from special text files to IBS databases for agencies, facilities, known points, job positions, or resources

reads a "DMX" file (a specially formatted ASCII data file) and creates a new DMS file

reads and reports the contents of DMS files; can create a "DMX" formatted ASCII file

reads a map data file in the USGS Digital Line Graph (DLG) optional format and creates one or more DMS files

reads a DMS file and creates a new file in the USGS DLG optional format

creates a DMS file from a specially formatted sequential ASCII file

For TIGER data: creates command files for executing the POPXDMS, TIGERXDMS, and TBXDMS utilities

For TIGER data: creates DMS data files from TIGER/Line data files provided by the Bureau of the Census

For TIGER data: creates DMS data files from STF1B population data files provided by the Bureau of the Census

For TIGER data: creates DMS boundary data files from TIGER/Line data files provided by the Bureau of the Census

creates a DMS file from a USGS DLG standard format file of 1:100,000-scale data (with some attribute conversion)

reads data files in USGS DLG standard format and creates new DMS files (without attribute conversion)

creates TAC files from USGS Digital Elevation Mapping (DEM) data

produces a DMS file of vector contours based on cell data from a TAC file 


\section{Database Administration Utilities}

The following utilities are for database administration tasks associated with IBS site databases and functional databases. These include programs for creating and modifying data-level control files and for creating, modifying, archiving, and deleting databases.

\begin{tabular}{|c|c|}
\hline Utility Name & Purpose / Description \\
\hline $\begin{array}{l}\text { ASCXATT } \\
\&\end{array}$ & $\begin{array}{l}\text { creates an IBS attribute dictionary from a specially formatted } \\
\text { ASCII file (reverses ATTXASC) }\end{array}$ \\
\hline ATTXASC & $\begin{array}{l}\text { creates a specially formatted ASCII file from an IBS attribute } \\
\text { dictionary file (see ASCXATT) }\end{array}$ \\
\hline UPDATEATT & $\begin{array}{l}\text { enables an authorized user to update the IBS attribute } \\
\text { dictionary }\end{array}$ \\
\hline CREATELIMITS & $\begin{array}{l}\text { creates a binary limit file to support one topography in a DMS } \\
\text { database }\end{array}$ \\
\hline SEELIMITS & reads and reports the contents of a binary limit file \\
\hline SEELIMITXX & creates an ASCII text version of a binary limit file \\
\hline DOSEELIMITXX & runs SEELIMITXX for all topographies of an entire database \\
\hline LIMITXX & $\begin{array}{l}\text { creates a binary limit file for one topography of a DMS } \\
\text { database }\end{array}$ \\
\hline DOLIMITXX & runs LIMITXX for all topographies of a database \\
\hline CHKICONS & $\begin{array}{l}\text { checks to make sure that all the icons listed in the icon control } \\
\text { file (MAPSICONSCTL) are valid }\end{array}$ \\
\hline $\begin{array}{l}\text { FIXCNX } \\
\& \\
\text { FIXCNX.BIS }\end{array}$ & $\begin{array}{l}\text { fixes a CASEINDEX.DAT file to match the existing case files } \\
\text { for one user (FIXCNX) or for all sites on the system } \\
\text { (FIXCNX.BIS) }\end{array}$ \\
\hline FIXLOG & fixes corrupted log journals (or creates new ones) \\
\hline NEWLOG & renames the current log journals and creates new ones \\
\hline MAPDBGEN & $\begin{array}{l}\text { extracts a portion of a map database to create a separate } \\
\text { "user-defined" map database }\end{array}$ \\
\hline SHOWCOVER & $\begin{array}{l}\text { creates a DMS file showing the area covered by all files of a } \\
\text { topography (all files listed in a limit file) }\end{array}$ \\
\hline MAPDBUPDT & $\begin{array}{l}\text { enables you to update a DMS database by adding new or } \\
\text { modified files of topography data }\end{array}$ \\
\hline DUPSITE & copy a site database ([INFOMANAGER.SITES.site]) \\
\hline $\begin{array}{l}\text { DUPOFFSITE } \\
\text { (continued) }\end{array}$ & copy an offsite database ([OFFSITE.site]) \\
\hline
\end{tabular}


Utility Name

DELPTDB

RMMGR

RMIMR_NOGR

NEWSITE

DELSITE

SITEBKP

\section{Purpose / Description}

removes old data and resets startup values in the people tracking database used by the LOCATE program

displays maps with resource and facility icons; enables you to update the contents of the personnel, resources, and facilities databases

enables you to update the contents of the personnel, resources, and facilities databases from a nongraphic terminal

creates directories and assembles topographic, population, and other data files for a user's site database

deletes an entire site database from a user's directory

save or restore different sets of site data 


\section{Database Quality Assurance (QA) Utilities}

The following utilities are for database QA tasks associated with IBS map, site, and offsite databases. These QA utilities include programs for checking, correcting, and otherwise modifying data files or database support files. The .COM utilities are meant to be run interactively; the .BIS and .DRV utilities are meant to be run in batch mode. Some of these utilities run other utilities several times to cover the directories and files of a database. Some of the command file utilities generate a new .BIS file (in the top-level directory of the map database), which are meant to be run in batch mode.

Many of these special-purpose utilities are NOT documented in detail in Section 2. Those that ARE documented in Section 2 are marked with an asterisk ( $\left.{ }^{*}\right)$ here.

Utility Name

DB_CHK_DMS.BIS
DB_DOLIMTTXX.BIS
DB_FIX_DMS_LIMITS.BIS
FIXLIMITS_SN_XX.BIS
DB_DEL_MT_DMS.DRV
DB_FIX_TXT_NMS.DRV
DB_CHK_DMS.EXE *
DB_DEL_MT_DMS.EXE *
DB_FIX_DMS_LIMITS.EXE *
DB_FIX_TXT_NMS.EXE*
DB_ASCIIXDMS.COM
DB_CHK_DMS_DOC.COM
DB_CHK_TAC.COM
DB_FIX_TAC.COM
DB_DEL_TXT.COM
DB_DIFF.COM
DB_DMSXASCII.COM
DB_DXAXD.COM
DB_ATTFREQ.COM
DB_JUNK.COM
(COntinued)

Purpose / Description

checks database for DMS file problems

runs DOLIMITXX for a DB

runs DB_FIX_DMS_LIMITS (auto)

Example: Set Sn limits to state boundary

generates a .BIS file to delete empty DMS files

generates a .BIS file to fix TXT names

checks a topography or database for DMS file problems

generates a .BIS file to delete empty DMS files

fixes DMS limits for topo(s)

generates a .BIS file to fix TXT names

generates a .BIS file to convert entire DB from DMX

p.ocesses DB_CHK_DMS.LOG to create .DOC-\% files

generates a .BIS file to check TAA references

generates a .BIS file to delete unused TXT files

Do DIFF on .BIN, .DAT, or T.DAT files

generates a .BIS file to dump entire DB to DMX/NL:

generates a .BIS file to fix RT/TT attributes

generates a .BIS file to run ATTFREQ

generates a .BIS file to delete junk files 
Utility Name

DB_L_TIGER.COM

DB_LOOKTXT.COM

DB_MAKE_CHK_DIRS.COM

DB_MODTEXTNM.COM

DB_OFFSITEDIFF.COM

DB_SEETXTNM.COM

DB_SEETXTNM_CHK.COM

DB_SEETXTNM_DOC.COM

DB_SHOWCOVER.COM

DB_SITEDIFF.COM

DB_STATDMS.COM

DB_WRONGTOPO.COM
Purpose / Description

generates a .BIS file to do L__TIGER

generates a .BIS file to dump TXT files

creates dirs under [.CHK] for all topos

generates a .BIS file to do MODTEXTNM

shows different files in $\mathbf{2}$ offsites

generates a .BIS file to do SEETXTNM

finds DMS files with weirdly named TXT files

creates .DOC from .LOG file

generates a .BIS file to do SHOWCOVER

shows different files in 2 sites

generates a .BIS file to do STATDMS

shows DMS in wrong topo (not $[x x]^{*} x x$.DMS) 


\section{System Management Utillities}

The following utilities support miscellaneous system management tasks for the IBS. For contexts concerning the use of these utilities, refer to the IBS System Management Guide and the IBS Data Management Guide.

Utility Name

NEWUSER

DELUSER

DELTREE

DEASSIGNLOG

INSTALLON

INSTALLOFF

PDMGR

FIXIMSTRDIR

SM_DUMP

SM_INIT

SM_SET

MLMGR

MLROUTE

FTPSTART

FTPSTOP
Purpose / Description

creates new IBS user accounts

deletes an IBS user account along with all the user's directories and files

-Caution 4: deletes (prunes) a directory tree and ALL its files deassigns unused map logical names installs IBS images (CHGOSITE, IBS_SM, XMAIL) de-installs IBS images from the system changes IBS program privileges for IBS users

builds a master directory list of the valid disk names, user names, and site names on the system

reads and reports the current contents of the IBS shared memory block

initializes the variables stored in the IBS shared memory block

shows the current values of some of the shared memory variables and enables you to change them

enables an authorized person to add or delete names from the system master mailing list

enables an authorized person to update the mail list files of IBS systems connected by a network

establishes the modem link between compatible onpost and offpost systems supporting the Chemical Stockpile Emergency Preparedness Program (CSEPP)

stops the modem link established by FTPSTART 


\section{System Upgrade Utilities}

The system upgrade utilities are designed for use on systems that are being upgraded for a new version of the IBS. These programs modify certain system and data files to make the files compatible with the new version of IBS. These programs are generally intended for one-time use at the time of system upgrade or installation: once you have upgraded the necessary files, the utilities are no longer necessary. If your system is a new installation, you can ignore these programs because they are used automatically during installation procedures (refer to the IBS System Management Guide).

Utility Name

FIXMAPDB

FIXSITEDB

FIXATTS

FIXD2MSTR

FIXIPS

FTXKPL

FIXOFFON

FIXOFFSITE

FIXPD

FIXPROT

FIXRM

FIXRSP

$\&$

FIXRSP_BIS

FIXSETUP

FIXVERB
Purpose / Description

converts map databases to the current format

converts site databases to the current format

combines two attribute dictionaries into a single dictionary

creates an index file of D2 data for a site and upgrades D2 input files to the current format

upgrades Implementing Procedure (IP) files to the current format

fixes the Known Polygons file to ensure that all user-drawn polygons, Emergency Planning Zone polygons, and D2 plume polygons are included

fixes the file that maps the onpost site names to the operational offpost site names

upgrades all [OFFSITExocox] directories to the current setup upgrades the IBS permissions file to the current format and content

sets file and directory protections for all IBS files and current map database

fixes Resource Management data for an offsite database so that correct icons are used

fixes the Mesorad model .RSP files for one user (FIXRSP) or for all users on the system (FIXRSP_BIS)

fixes database setup filc in all your own sites or in all sites on the system

adds/deletes verbs to/from the systems DCLTABLES 


\section{Diagnostic and Reporting Utilities}

These utilities display and/or report information about various parts of the IBS: data files, database support files, system management information, and variables in shared memory.

Utility Name

RMCHK

DMSXASCII

DMSXDTX

SEETXTNM

LOOKTXT

STATDMS

ATTFREQ

SHOWATT

ATTXASC

SEELIMITS

CHKICONS

PLOTICON

SHOWCOL

SHOWTEK

LISTCNX

SHOWCNX

SHOWENV

SM_DUMP

SM_SET

\section{Purpose / Description}

checks and reports on the completeness of Resource Management data

reads and reports the contents of DMS files (see ASCIIXDMS)

reads and reports the text labels and longitude-latitude coordinates of objects in a DMS file

reports the text (.TXT) file name referenced in a DMS file

lists the contents of a DMS text file

computes and displays summary statistics for attributes and objects in DMS file(s)

calculates and displays shows the frequency of each type of attribute in DMS file(s)

converts attribute values (major, minor, parameter) between decimal, hexadecimal, and octal numbers

creates a specially formatted ASCII file from an IBS attribute dictionary file (see ASCXATT)

reads and reports the contents of a binary limit file

checks to make sure that all the icons listed in the icon control file (icon dictionary MAP\$ICONSCTL) are valid

displays all icons specified in the icon control file

displays pen numbers (Tektronix terminal colors) and their associated colors

shows Tektronix terminal characteristics

lists all cases for all models of the current site and how each case is used

list the case numbers for each model for a site

shows current user environment characteristics for an IBS user reads and reports the current contents of the IBS shared memory block

shows the current values of some of the shared memory variables and enables you to change them 


\section{Overview of IBS Map Data}

The Integrated Baseline System (IBS) includes a geographic database and computer programs for the storage, graphic display, and editing of map-related data. These programs and database are the basic elements of the IBS's geographic information system (GIS), which is used to manage and manipulate data that are related to geographic location. These GIS functions extend into the emergency management modeling portion of the system, where they are used to graphically depict modeling results overlaid on site maps.

This section summarizes IBS map data concepts and terms used in many of the utility program descriptions in Section 2 of this guide. For complete details on IBS data and data management, please refer to the IBS Data Management Guide.

This overview includes the following topics:

- Map Data Terminology

- IBS Map Databases and Database Components

\section{Map Data Terminology}

The following paragraphs define IBS map data terms that you should understand.

- Digital Mapping Structure (DMS) -- Digital Mapping Structure is the data file format used to store graphic data for use by the IBS mapping functions (such as the MPDISPLAY map display program and the GEDIT graphic editing program). DMS files typically contain line or irregular point data depicting topographic features (such as boundaries, railroads, or hospitals) at particular longitude-latitude locations. DMS files have ".DMS" as the file name extension. For more information on DMS files and format, see the IBS Data Management Guide.

- Graphic data structures -- DMS files can describe several kinds of point-vector graphic data structures for depicting map data items. These include:

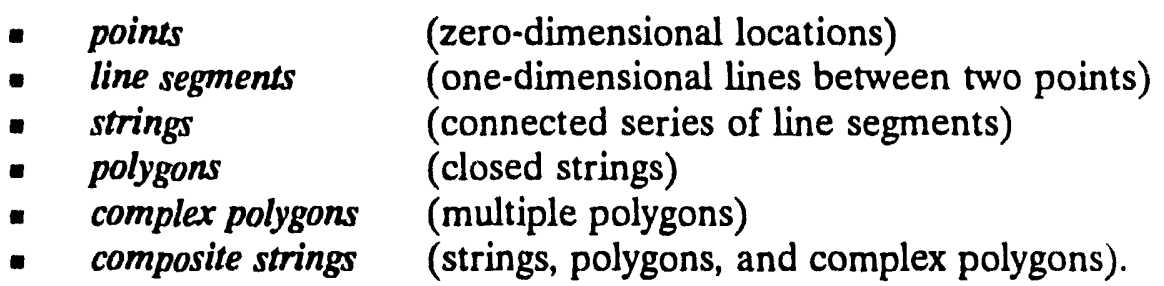

- IBS Map Database -- The IBS map database for your site is a DMS database that contains site geographic information for shared use by all users. The 
databise is generated by consolidating geographic information from various national, state, and local sources. For detailed information on the IBS map database, please refer to the IBS Data Management Guide.

- Icon -- Icons are graphic figures that can be associated with a particular point on a map. They always appear at a constant viewing size, regardless of the map scale. They provide a consistent means of representing facilities, resources, or any repeating topographic features that occur at point locations. A standard set of icons is available for use in the IBS.

- Terrain Analysis Cell (TAC) data -- This is the name of the data file format used to store cell data, which are numeric values associated with the cells of a regularly spaced grid. The only TAC data in the IBS map database is elevation data. The utilities that support the use of TAC elevation files are MPDISPLAY, GEDIT, DEMXTAC, and CONTOUR. TAC data sets (.TAC and .TAA files) are defined in the IBS Data Management Guide.

- Topography -- In general terms, a topography is a graphic description of a geographic region. In the IBS, a topography (or map layer) is a set of DMS data illes for depicting map features that have a common theme. For example, "railroads," "streams," and "water bodies" are all different topographies used by the IBS map database.

\section{IBS Map Databases and Database Components}

IBS geographic map databases store graphic data that represent geographic features at specific longitude-latitude locations. These databases use the Digital Mapping Structure (DMS) file format and software developed by the Pacific Northwest Laboratory.

You do NOT need to know about the detailed structure of an IBS map database to display maps or map-related data. However, if you plan to examine, copy, or edit specific data, refer to the following summary of DMS database components. Details on actual DMS file and data structures are described in the IBS Data Management Guide. The rest of this section includes the following topics:

- Map Database Directories and Files

- Attributes and Attribute Dictionary. 


\section{Map Database Directories and Files}

A map database consists of special support files and data files distributed within various subdirectories of an overall database directory. Figure 1.1 illustrates the basic directory structures and files in an IBS map database. (Although several other structures and files support a map database, these are the basic ones.) Specific directory and file names are shown in upper-case letters. Variable parts of file names and directory names are shown in lower-case letters.
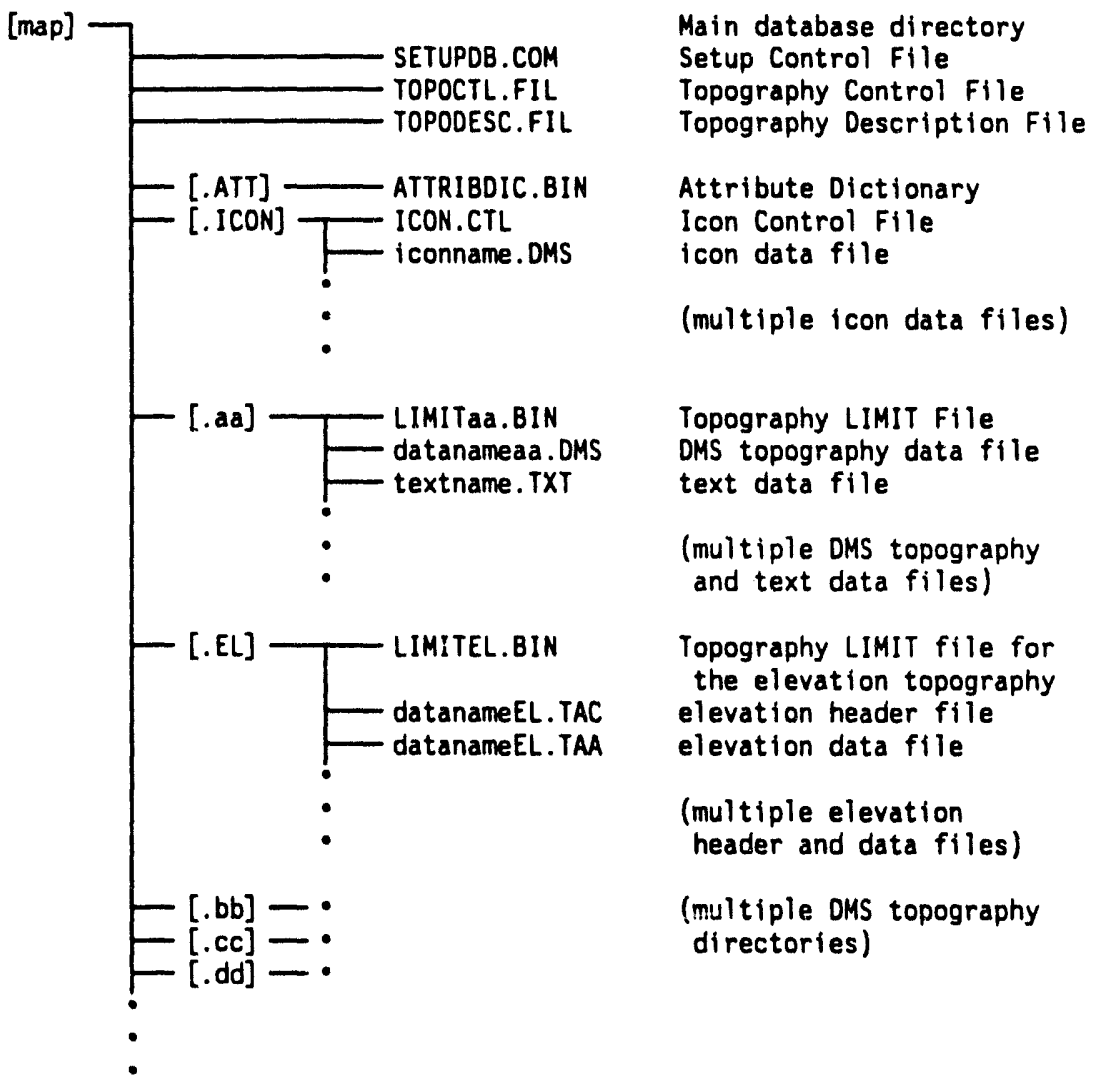

Figure 1.1. IBS Map Database Directories and Files

The components of a map database (as depicted in Figure 1.1) are the following:

- $[$ map $]$

The main (top-level) directory of the database. This may be a root directory or any subdirectory.

- SETUPDB.COM Setup control file. This is a command file that assigns logical names so that the files of the DMS database can be accessed by the IBS software. 
- TOPOCTL.FIL Topography control file. This control file contains the number and names of DMS file "cover maps" that depict one or more geographic areas within the database.

- TOPODESC.FIL Topography description file. This control file lists the different topographies and the database directories where the topographic data reside.

- [map.ATT]

Attribute dictionary directory. This directory contains the attribute dictionary referenced by the database.

- ATTRIBDIC.BIN Attribute dictionary. This is the binary file that defines the attributes that can be assigned to objects in a DMS file.

- [map.ICON]

Icon directory. This directory contains the icon control file referenced by the database.

- ICON.CTL

Icon dictionary or icon control file. This ASCII file contains a list of the available icons and the names of the icon data files needed to draw each icon.

- iconname.DMS Icon data file. This is a DMS file that contains the graphic data for one icon figure. Only the graphic description is contained in an icon data file. Specific location information is associated with icon seed points, which are stored in the topography data files.

- [map.xx]

- LIMITxx.BIN

Topography subdirectories. Each of these subdirectories contains all the data for one of the topographies listed in the topography description file. The name of the subdirectory is a two-letter topographic code assigned to that topography (see Topographic Codes on the next page).

Topography Limit file. This binary file specifies the names and geographic extents of all the DMS files (or TAC files) that make up the topography in that subdirectory.

- datanamexx.DMS Topography data file (one or more files of DMS topographic data in the subdirectory). The front part of the file name may be any descriptive name, but most file names end in "xx.DMS" where the two-letter topographic code is substituted for $x x$.

- dataname.TXT Text data file. These files contain text labels or supplementary text information about features within DMS files. The DMS files contain no text but do contain the name of a separate text data file. 
- datanameEL.TAC TAC header file. This file is the first part of a TAC data set. It contains information about the cell data stored in the .TAA file.

- datanameEL.TAA TAC data file. This file is the second part of a TAC data set. It contains the cell data described in the .TAC file.

More detailed descriptions and examples of these database components are supplied in the IBS Data Management Guide.

Topographic Codes. Each topography is associated with a two-letter topographic code that is used in the topography subdirectory and data file names. For example, $A B$ signifies administrative boundary data. The administrative boundary data would reside in the [map.AB] subdirectory and consist of files named filenameAB.DMS. (The exception to this rule is the elevation topography, which would reside in the [map.EL] subdirectory and consist of files named filenameEL.TAC and filenameEL.TAA.) The topographic codes for standard IBS topographies are listed in Appendix A.

\section{Attributes and Attribute Dictionary}

Topographic data in DMS files are geographically defined by longitude-latitude and topologically defined as points or composite strings. Further database description of DMS data items is accomplished through attributes. Attributes are numeric values used to describe data items or some characteristic of data items. The main attribute values are the following three numbers:

\section{- a Major attribute code \\ - a Minor attribute code \\ - a Parameter value}

(to indicate a major topographic group) (to indicate a feature within the group)

(which may supply data about the specific feature).

These attribute values are often depicted as three numbers separated by semicolons: Major;Minor;Parameter. For example, 1;560;100 indicates a Major code of 1, a Minor code of 560, and a Parameter value of 100 .

A special file called an attribute dictionary defines the meaning of each Major;Minor pair of attribute codes by associating the codes with a text description. For example, the attribute dictionary defines the following attribute code pairs:

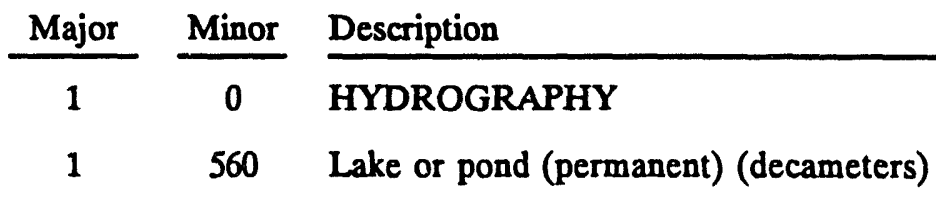

The Major code of 1 indicates the major topographic group, hydrography. Notice in the second example that the parameter value is defined in parentheses at the 
end of the text description. Attribute codes may be specified without a parameter value; some attribute codes do not even have a parameter value defined. For example, the attribute dictionary defines $1 ; 500$ as just "Aqueduct."

The attribute dictionary for the standard IBS map database is listed in Appendix B.

Up to 15 attributes (Major;Minor;Parameter sets) can be assigned to a particular composite string or point in a DMS file. The attributes of a composite string usually identify the string as representing a topographic feature. The attributes of a point can identify that point as:

- an icon seed point: the location of an icon

- a text seed point: the location of a piece of text

- an item point: a point location that has only attributes without being associated with particular graphics or text.

Text attributes point to the location of text in a text data file. Any DMS data item can have text associated with it through its attributes. The name of the text data file is stored in the DMS file header, the initial portion of the DMS graphics file.

The MPDISPLAY documentation in Section 2 explains how you can view the attributes of items on the screen or use attributes as selection criteria for items to be displayed. For example, you can choose to display all lakes, only permanent lakes, or just permanent lakes that are smaller than 100 decameters across (see Attribute Functions in the description of MPDISPLAY).

The GEDIT documentation in Section 2 explains how you can assign attribute codes to data items (see Attribute Menu in the description of GEDIT). 


\section{Section 2 \\ Utility Program Descriptions}

This section explains the operation of individual IBS utility programs.

\section{How the Program Descriptions are Organized}

The program descriptions in this section appear in alphabetical order by utility name. We expect that you are looking up utilities named in other documents or in Section 1 of this guide. If you are browsing to find a desired function or a utility name, refer to the capsule descriptions of each utility, in Section 1. The capsule descriptions there are grouped in general task categories.

\section{Starting and Using the Utility Programs}

Startup commands are always specified in the utility program description. To start one of the utilities (in general), type the name of the utility at the VAX system prompt (which is often the dollar sign, \$) and then press the RETURN key:

\section{\$ utilityname <RETURN>}

In this guide the $\$$ symbol is used to represent the VAX system prompt.

A few of the utilities require that you enter a complete command string instead of just the utility name. For example:

$$
\begin{array}{llc}
\text { \$ RUN IEMISSEXE:utilityname } & <\text { RETURN> } & \text { (for some program files) } \\
\text { or \$ RUN IEMIS\$QA:utilityname } & <\text { RETURN> } & \text { (for database QA programs) } \\
\text { or \$ @IEMIS\$EXE:utilityname } & <\text { RETURN> } & \text { (for some command files) }
\end{array}
$$

IBS Shell. Many IBS utilities are available as options on menus presented by the IBS Shell utility (IBSSH). The menus supply short descriptions of the included utilities, and you can start the utilities by typing option numbers rather than the utility names. Because it requires time to start the IBS Shell and use the menus, you may find it faster to use individual utilities if you already know the utility names. See the description of IBSSH in this document. 
Examples of Interactions. The program descriptions usually include examples of program prompts and user responses. A sequence of interactions may be boxed like this with simulated responses shown in bold typeface:

Example:

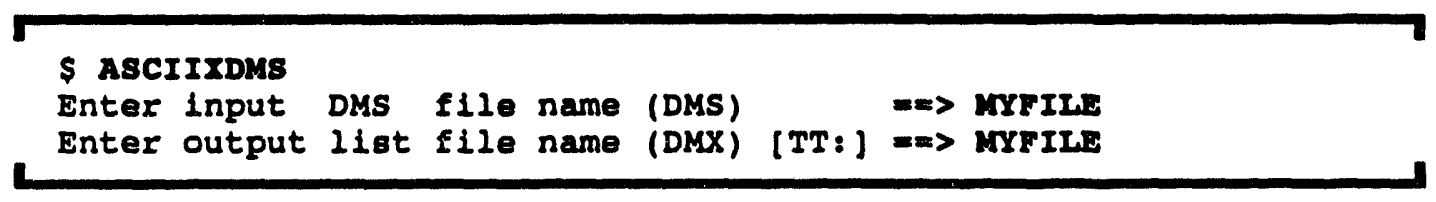

Default Values and File Names. Default responses to program prompts may appear in brackets at the end of the prompt. To use this default, just press RETURN instead of entering the value. When a utility asks you to enter the name of a DMS file (or any other file that has a specific file name extension in parentheses-.DMS, .TXT, .BIN, .DLG, etc.), you can enter the file name without the extension: the appropriate extension is assumed or appended to the file name automatically. 


\section{ADDATTDMS}

The ADDATTDMS utility is a data file modification utility that enables you to add attributes to selected objects in a DMS data file. A new DMS file with the attribute additions will be generated.

\section{What You Need to Know Before Using ADDATTDMS}

The general process is to specify the name of the input DMS file, a name for the output DMS file, a Boolean logical expression that specifies DMS records in the old DMS file, and new attributes to be added to these records in the new DMS file.

The Boolean expression specifies a combination of attributes. All objects having those attributes are selected to have the new attributes in the new DMS file. The Boolean expression can be formed from the following list of operators and symbols:

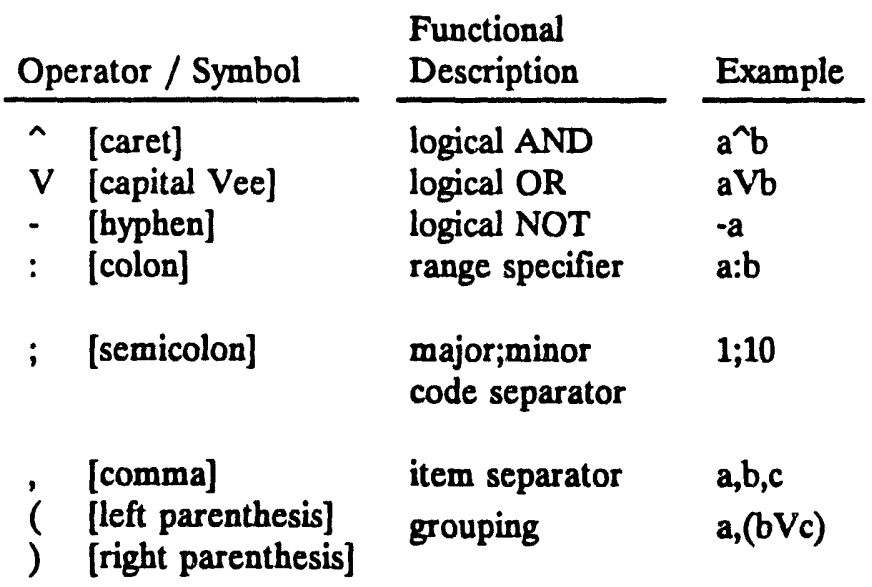

The attribute codes are combined with these symbols to create Boolean logical expressions.

Examples:

4;5:4;100 indicates all data items having an attribute with a Major code of 4 and a Minor code of 5 to 100 (inclusive)

$6 ; 13^{\wedge}(4 ; 5: 4 ; 100) \quad$ indicates all data items having an attribute with a Major code of 6 and a Minor code of 13 AND (as well as) having an attribute with a Major code of 4 and a Minor code of 5 to 100 (inclusive)

(examples continued) 
$5 ; 8 \mathrm{~V}\left(6 ; 13^{\wedge}(4 ; 5: 4 ; 100)\right) \quad$ indicates any data items having an attribute with EITHER a Major code of 5 and a Minor code of 8 OR the same code specifications as the previous example.

\section{Adding Now Attributes to a DMS File with ADDATTDMS}

To start the utility, enter ADDATTDMS at the system prompt. The following is an example of using this utility. In the example, the Boolean expression selects all data items that have attributes with a major code of 6 and a minor code of 13 but NOT a major code of 4 and minor codes of 5 or 6 . To these data items in a new file, the utility adds attributes with a major code of 4 , minor codes of 5 and 6 , and parameter values of 0 .

Example:

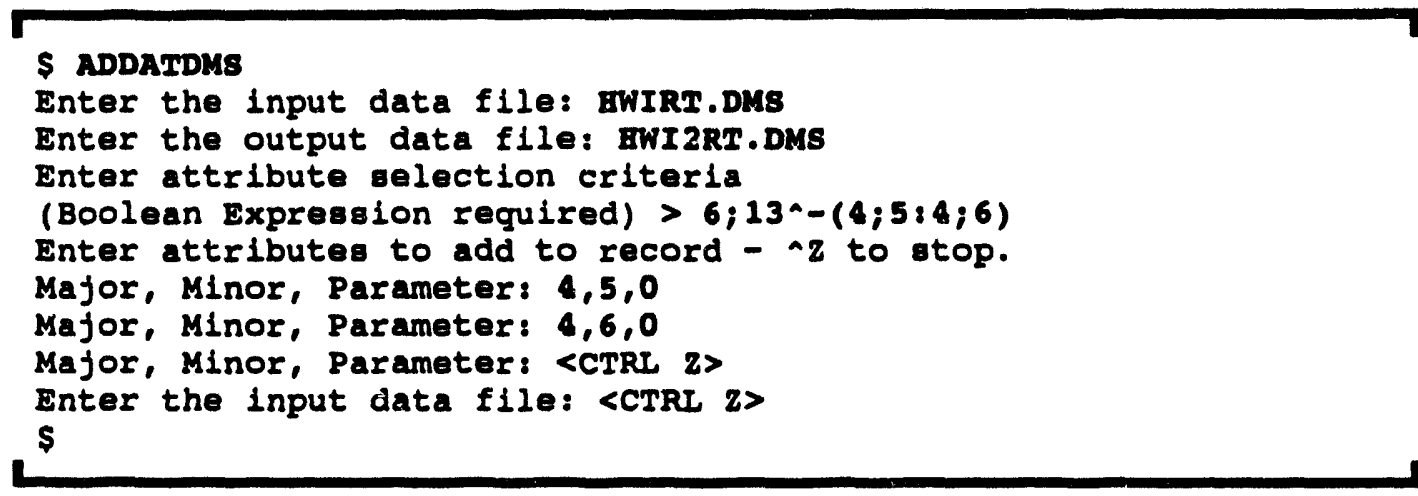

You are prompted for major, minor, and parameter values for the attributes to be added until you press CTRL-Z. When you press CTRL-Z to quit adding those values, ADDATTDMS begins another iteration of the same process, prompting for an input data file. Continue through as many iterations as you wish.

To exit, press CTRL-Z at the "Enter input data file: "prompt. 


\section{APPENDDMS}

The APPENDDMS utility appends one DMS file to another to create a new DMS file that contains the data from both files. You can use APPENDDMS to merge a set of small DMS files together to create a single file. It is most often used to consolidate a group of common DMS files to create a new topography that can be output using IBS map display software, such as MPDISPLAY.

\section{Appending One DMS File to Another with APPENDDMS}

To start the utility, just enter APPENDDMS at the system prompt. A new version of the "appended to" file is always created. For example:

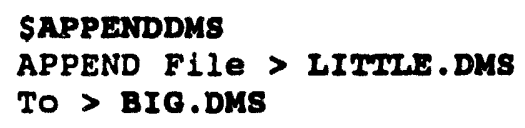

This interaction causes a new version of BIG.DMS to be created with the data from LITTLE.DMS appended to it.

An example of how you can display the combined file is to change the file's name to match a topography of a "stored region" that was created using MPDISPLAY or GEDIT. You can then use MPDISPLAY to retrieve and display the stored region and the new topography. 


\section{ASCIIXDMS}

The ASCIIXDMS utility creates a DMS file from a standard ASCII data file when the ASCII file has the proper "DMX" file format. Such an ASCII file could be created by a query from a DMS database, by a data conversion program (such as DMSXASCII), or by entering the data manually. For example, you can use the DMSXASCII utility to create an editable ASCII file from a DMS data file. After editing the ASCII file, you can use ASCIIXDMS to create a DMS data file from the edited ASCII file.

\section{What You Need to Know Before Using ASCIIXDMS}

The key to using ASCIXDMS is being certain that the ASCI source file is properly formatted. To see the format of a DMX-formatted ASCII file, you can use the DMSXASCII utility to read a DMS file and produce its ASCII equivalent: a DMX file (see DMSXASCII). To understand what information is included in a DMX-formatted ASCII file, you can also use the DMSXASCII utility to produce a commented ASCII file that identifies the included numeric data.

The following paragraphs summarize the contents of a DMX file. For detailed information on the DMX file format, refer to the IBS Data Management Guide.

The first record of the DMX file contains the path and name of the text file that will be associated with the new DMS file if DMS text files are created. The name of the text file must have 12 characters or less, including a .TXT extension. An example of a valid text file name is NEWDATA.TXT: it is 11 characters long and has the correct extension. If the text file is associated with a map database topography file in MAPSDATASxx, then the path and name should be specified as TSx:textfilename where $x x$ is the two-character topographic code.

The rest of the DMX file contains information that describes the graphical data types and attributes to be converted to DMS format. The information for one data type (point, line, or polygon), its associated attributes, and location (longitudelatitude) requires more than one record. These records are ordered as follows:

1. data type header

2. attributes

3. text information

4. polygon descriptors

5. data points (always)

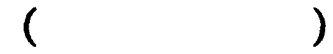

(if the data type is text)

(if the data type is a complex polygon)

(always). 
The commented output from the DMSXASCII utility lists the data type definition required by the ASCIIXDMS utility.

Note: You must NOT use commented output from DMSXASCII as input to the ASCIIXDMS utility. If you wish to modify a DMS file by converting it to DMX-formatted ASCII form, editing the DMX file, and then converting it back to DMS form, use only uncommented output from DMSXASCII.

\section{Creating a DMS File from a DMX ASCII File with ASCIIXDMS}

The following example sequence would convert the ASCII data stored in the file called "MYFILE.DAT" to DMS format, creating a file called "NEWFILE.DMS":

Example:

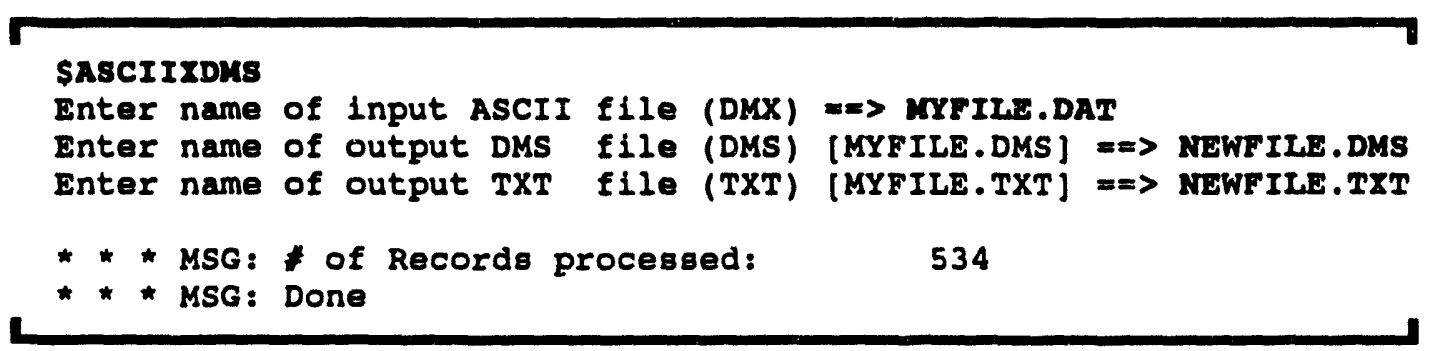

The ASCIXDMS utility asks you to specify the following items:

name of input ASCII file

name of output DMS file

name of output TXT file
This is the name of the special DMX-formatted ASCII file that ASCIIXDMS will read. If you do not specify a file name extension, the .DMX file extension is assumed. CTRL- $Z$ cancels the program at this point.

This is a name for the new DMS file. If you do not specify a file name extension, the .DMS extension is used. Instead of entering the output file name, you can also give the following keyboard responses:

$\begin{array}{ll}\text { Your Response } & \text { Result / Comments } \\ \text { RETURN } & \begin{array}{l}\text { Uses the same name as the ASCII } \\ \text { file but with the .DMS extension. }\end{array} \\ \text { CTRL-Z } & \text { Cancels the program at this point. }\end{array}$

This is a name for the new TXT file. If you do not specify a file name extension, the .TXT extension is used. As above, you have options either to use the ASCII file name with a .TXT extension or to cancel. 


\section{ASCXATT}

The ASCXATT utility reads an ASCII text file version of an IBS attribute dictionary file and creates the binary attribute dictionary file. The binary form of the file is used to assign physical or other descriptive features to topographic data in the IBS. A complementary utility, ATTXASC, reverses the process to produce an ASCII version of the binary file. A database administrator can modify the ASCII attribute dictionary file and then convert the ASCII file back into binary form by using the ASCXATT utility. This is a means of modifying the attribute dictionary.

\section{What You Need to Know Before Using ASCXATT}

The utility prompts you to specify only two items:

input ASCII file name

output Dictionary file name
This is the name of the ASCII attribute dictionary file. The extension. BIX is the IBS convention for an ASCII file translated from a binary attribute dictionary.

This is the name of the binary attribute dictionary file to be created. The IBS master attribute dictionary is ATTRIBDIC.BIN, which is located in the MAP\$ATTRIB directory.

\section{Creating a Binary Attribute Dictionary File from an ASCII File}

To start the utility, enter ASCXATT at the system prompt. In the following sequence, ASCXATT produces a binary attribute dictionary file, ATTRIBDIC.BIN.

\$ ASCXAIT

Enter input ASCII file name (ATTRIBDIC.BIX] $\Rightarrow \Rightarrow$ ASCIIfilename

Enter output Dictionary file name [MAP\$ATT...] $==>$ <RETURN>

* * MSG: * Attribute日 : nnnn

\section{Results of Using ASCXATT}

This produces a binary attribute dictionary file that contains the attribute codes (major code, minor code, parameter value) and other data for each item in the specified attribute dictionary. For details of the attribute dictionary file formats, see the descriptions of ATTRIBDIC.BIX and ATTRIBDIC.BIN in the IBS Data Management Guide. 


\section{ATTFREQ}

The ATTFREQ utility is a diagnostic and reporting program that writes a report listing the frequency of attributes in one or more DMS files.

\section{What You Need to Know Before Using ATTFREQ}

You may analyze either a single DMS file or specify a binary limit file containing multiple DMS filenames. Limit files are found in the map database directories, which are named by the convention MAP\$DATASxx, where $x x$ is a two-character code that identifies a topography (map layer). The limit file is named LIMITxx.BIN, where $x x$ is the topographic code.

\section{Reporting the Frequency of Attributes in DMS File(s)}

Before you start, move to the directory that contains the DMS file(s) you wish to analyze: S SET DEFAULT [directoryname]

To start the utility, enter ATTFREQ at the system prompt. The following interactions are an example of analyzing one DMS file.

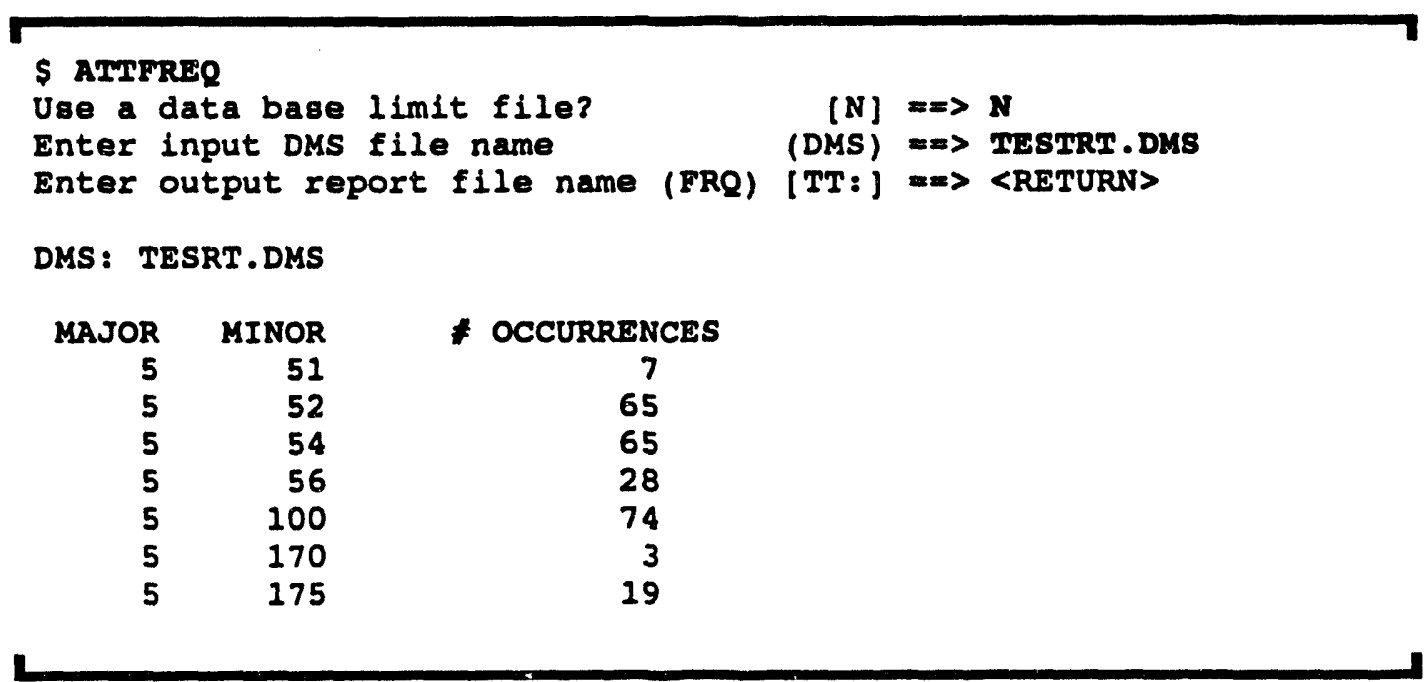

If you answer $Y$, to use a database limit file, ATTFREQ displays the following prompt instead of "Enter input DMS file name":

Enter data base limito file name

$(B I N)==>$

Enter the name of the binary limit file that contains the names of the DMS files for which you want attribute frequency reports. 
When asked for the output file name, you can display the report on the terminal screen by pressing RETURN instead of entering the file name.

The attribute frequency report contains the major and minor attribute codes and the number of occurrences for each combination. 


\section{ATTXASC}

The ATTXASC utility reads a binary IBS attribute dictionary file and creates a ASCII text file version of the same information, which you easily read or edit. The attribute dictionary, ATTRIBDIC.BIN, identifies and interprets the attributes of objects in a DMS database. A database administrator can modify the ASCII attribute dictionary file and then convert the ASCII file back into binary form by using the ASCXATT utility. This is a means of modifying the attribute dictionary.

\section{What You Need to Know Before Using ATTXASC}

The utility prompts you to specify only two items:

input dictionary file name

output file name
This is the name of the binary attribute dictionary file. The IBS master attribute dictionary is ATTRIBDIC.BIN, which is located in the MAP\$ATTRIB directory.

This is the name of the ASCII file to be created. If you do not specify a file name, the name ATTRIEDIC.BIX is used. The extension .BIX is the IBS convention for an ASCII file translated from a binary attribute dictionary.

\section{Creating an ASCII Version of a Binary Attribute Dictionary}

To start the utility, enter ATTXASC at the system prompt. The following example sequence produces an ASCII file named ATTCODES.BIX.

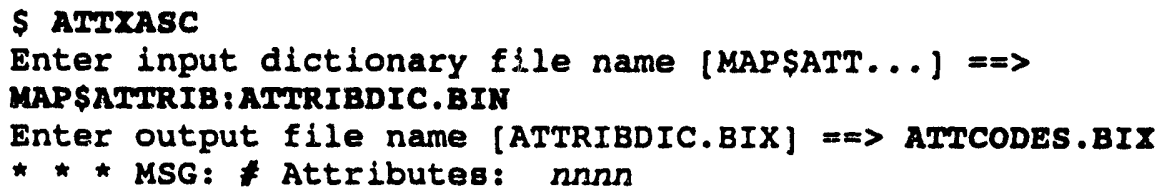

\section{Results of Using ATTXASC}

This produces an ASCII file that lists the attribute codes (major code, minor code, parameter value) for each item in the specified attribute dictionary. Also listed are the default pen type, default line type, decluttering code, and decluttering scale factor for each item. You can edit this ASCII file so that it can be used as input to the ASCXATT or DECLUTTER utilities. For details of the ASCII attribute dictionary file format, see ATTRIBDIC.BIX in the IBS Data Management Guide. 


\section{CHKICONS}

The CHKICONS utility checks to make sure that all the icons listed in the icon dictionary (MAPSICON\$CTL) are valid. The icon dictionary is also referred to as the icon control file.

\section{Validating the Icon Dictionary with CHKICONS}

To execute this program, enter CHKICONS at the system prompt.

If an icon specified in the icon control file does not exist, CHKICONS displays the code of the error that occurred, the major and minor codes listed for the icon, the icon description, and the name of the icon file listed. When CHKICONS completes its check of the entire icon control file, it prints out the number of icons processed. 


\section{CLIPDMS}

The CLIPDMS utility enables you to subdivide a single DMS data file based on fixed longitude-latitude step sizes, creating one or more new DMS files that each contain part of the data from the original DMS file. This utility is useful when you need to create a DMS database from a single large data file, or when you wish to extract a particular area from within a given file. CLIPDMS is a text program and can be executed from a nongraphic terminal.

\section{What You Need to Know Before Using CLIPDMS}

You can use CLIPDMS in two ways: 1) you can create a single DMS file that is "clipped" from a larger DMS file, or 2) you can create multiple DMS files, each clipped to the same area extents. In the first case you must know the longitudelatitude extents of the area that you want to clip. In the second case, you must know a starting longitude-latitude and decide on a fixed longitude-latitude step size for subdividing the original DMS file.

\section{Creating Area Component Files from a DMS File with CLIPDMS}

To start the utility, enter CLIPDMS at the system prompt. The utility begins by asking whether a single area is to be selected:

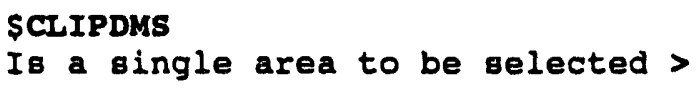

If you want to create a single file, answer $Y$. If you want to create several files, answer $\mathbf{N}$.

\section{Clipping a Single Area File from a DMS File}

If you choose to create a single file, CLIPDMS prompts you for input and output file names followed by the longitude-latitude extents of the area to be selected. The following example illustrates the creation of a file called NEWMAP.DMS covering longitude -110 to -108 and latitude 42 to 43.5 from a file called BIGMAP.DMS. 


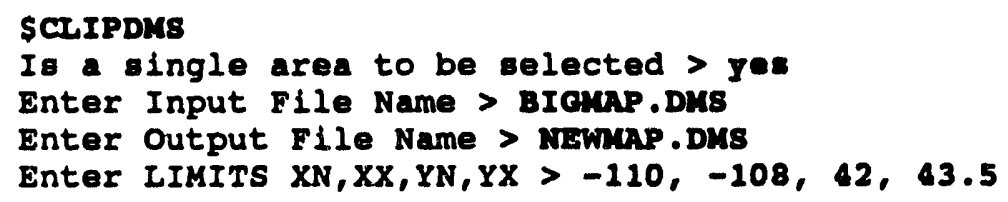

\section{Clipping Multiple Area Files from a DMS File}

If you choose to cut the DMS file into several smaller area files, CLIPDMS prompts you for the input filename, a five-character designator that all new files will use as the basis for their names, the starting longitude-latitude for file creation, and the longitude-latitude step sizes to be used when determining the limits of the new files. For example, the following sequence subdivides the file BIGMAP.DMS (spanning the area of -105 to -90 longitude and 34 to 45 latitude) into multiple files, each containing data for 1 degree of longitude and $1 / 2$ degree of latitude.

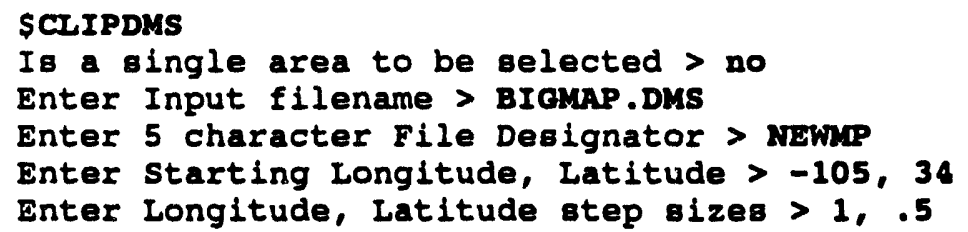

The first file created (NEWMPOOO1.DMS) will then contain data clipped to $(-105,-104)$ degrees longitude and $(34,34.5)$ degrees latitude. Subsequent files are created until the complete extent of BIGMAP.DMS is covered. When CLIPDMS creates multiple files in this way, it also creates a binary limit file (LIMIT.BIN) that can be used if the new files are intended to be topography files for a DMS database. The limit file is a support file that lists the names and extents of a set of DMS topography files. (See CREATELIMITS.) 


\section{COLORDMS}

The COLORDMS utility modifies all features within a DMS file such that they will be displayed using a single color.

\section{Converting DMS File Features to a Single Color with COLORDMS}

To start the utility, enter COLORDMS at the system prompt. The program requests that you enter the input filename, output filename, and pen number (color) to be used during display. For a list of pen numbers and their colors, use the SHOWCOL utility.

\section{Example:}

SCOLORDMS

Enter Input Fileame > OLD1.DMs

Enter Output Filename $>$ NEW1.DMS

Enter New Pen Number $>6$

Enter Input Filename > OID2.Dus

Enter Output Filename > NEW2.DMS

Enter New Pen Number $>7$

Enter Input Filename $>\langle C T R I-Z\rangle$

In this example, COLORDMS creates NEW1.DMS and NEW2.DMS from OLD1.DMS and OLD2.DMS. All pen numbers in NEW1.DMS are set equal to 6 and all pen numbers in NEW2.DMS are set equal to 7.

When you are finished, press CTRL-Z to end the repeating sequence of prompts. 


\section{CONTOUR}

The CONTOUR utility produces a DMS-format file of vector contours based on cell data from a Terrain Analysis Cell (TAC) file. The purpose of the program is to provide displayable vector contours that represent isopleths of the cell data for use by models in the system. At present this utility is used only for the contouring of elevation data as provided by the USGS.

\section{What You Should Know Before Using CONTOUR}

Before starting the utility, set the default directory to the directory where the TAC data reside:

\$ SET DEFAULT directoryname

CONTOUR must be executed from the directory where the TAC data resides or else the TAC data may not be read correctly. Usually this directory will be MAPSDATASEL: where the IBS elevation data resides.

Program Input. The primary input to CONTOUR is a TAC data set (a .TAC file and a .TAA file). The TAC format is described in the Data Management Guide.

\section{Creating a Vector Contour File from a TAC File with CONTOUR}

To start the utility, just enter CONTOUR at the system prompt. The program then prompts you to enter several items:

- the name of an input TAC file (including the .TAC file name extension)

- the name of an output file (including the .DMS file name extension)

- the elevation intervals (changes in elevation) between contour lines

- the color of contour lines.

See the following page for an example of using CONTOUR

\section{Results of Using CONTOUR}

CONTOUR produces one DMS-format file of vectors representing elevation contours. The utility also displays statistics on the minimum and maximum cell values (elevations) found in the input file, and on the size of the vector strings produced in the output file. The longest vector string currently allowable in the system is 15000 points. If the longest string is more than 15000 , CONTOUR breaks the contour into separate strings of 15000 points (or fewer) and displays a message that tells you this. 
The following is an example of using CONTOUR. See the notes below for information about special places in the sequence.

Example:

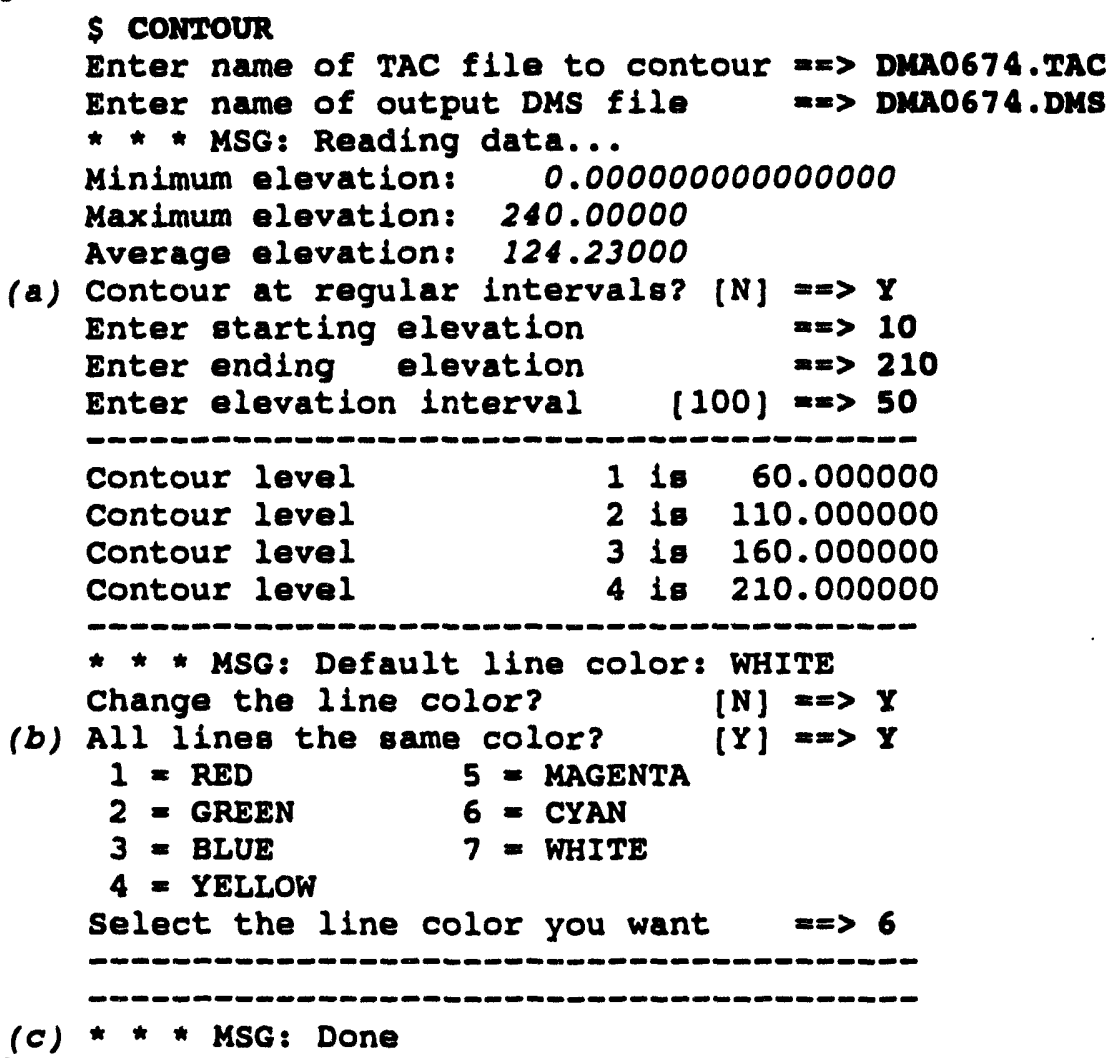

(a) If you enter $\mathbf{N}$ to choose irregular intervals, the program asks you to enter "contour levels" (elevations) one at a time:

Enter contour level $1(<C R>=$ Done) $=>$

After you enter a contour level value, the program prompts for the next contour level $(2,3$, etc.) until you finish by pressing RETURN.

(b) If you enter $\mathbf{N}$ to choose differing line colors, the program lists your color options and asks you to select a line color for each interval, one at a time:

Select line color for interval $1 \Rightarrow m$ (and so on)

(c) If for some reason the program creates an object that is NOT a polygon, you will see a message that identifies the object number $(n n n)$ in the DMS file:

* * MSG: Not a polygon: nnn 


\section{CREATELIMITS}

The CREATELIMITS utility creates a binary limit file (LIMITxx.BIN), which is a database support file that must be located in each topography (map layer) subdirectory of a DMS geographic database. It specifies the names and geographic extents of all the topographic data files in the subdirectory (where " $x x^{n}$ is the twocharacter code for that topography and also the subdirectory name). In general, CREATELIMITS is used only when you are creating a special user-defined topography for an existing DMS database.

To see what information is included in a particular binary limit file, you can use the SEELIMITS utility.

\section{What You Need to Know Before Using CREATELIMITS}

The utility creates the binary limit file in your default directory. Before using CREATELIMITS, set the default directory to the topography subdirectory where the DMS (or TAC) topography files are located. For example, the following command sets the default directory to the WB or "water bodies" topography subdirectory of the current DMS database:

\section{\$ SET DEFAULT MAP\$DATA\$WB}

For CREATELIMITS to do its job, you must also supply the utility program with the names of the topography data files. You can do this in one of two ways:

- Specify the names of the data files individually at the keyboard.

OR

- Specify the name of a separate file that contains a list of the data file names.

If there are only a few data files, you may prefer to enter the file names directly. If there are many data files, it might be more practical to create a list of the data file names, which CREATELIMITS would then read. The list file is just an ASCII text list of the data file names with one file name on each line record of the list.

\section{Creating a Binary Limit file with CREATELIMITS}

The following examples show how to create a binary limit file in the default "water bodies" topography subdirectory (MAP\$DATASWB), which contains two data files, LAKE.DMS and RIVER.DMS. 


\section{Entering Data Flle Names Directly}

This example shows how to create a binary limit file, LIMITWB.BIN, by directly entering the names of the topography data files at the keyboard.

Example:

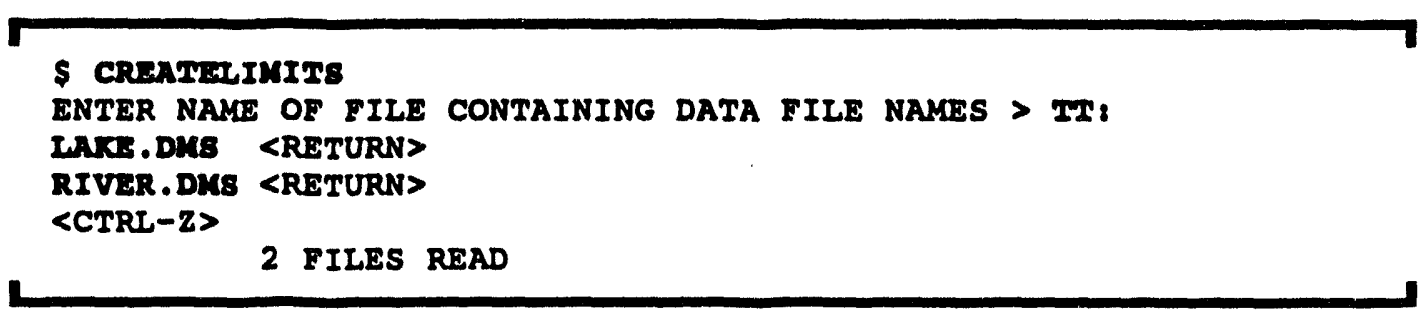

1. To start the utility, just enter CREATELIMITS at the system prompt.

2. When CREATELIMITS prompts for the name of a file, enter TT: instead. CREATELIMITS then accepts entries from the keyboard.

3. Enter the names of each data file, following each name with a carriage return.

4. To finish the list of file names, press CTRL-Z.

\section{Using a List of Data File Names}

This example shows how to create a binary limit file, LIMITWB.BIN, given a file (T.DAT) containing the list of DMS (or TAC) files in the topography. In this example T.DAT contains the names of two files, LAKE.DMS and RIVER.DMS.

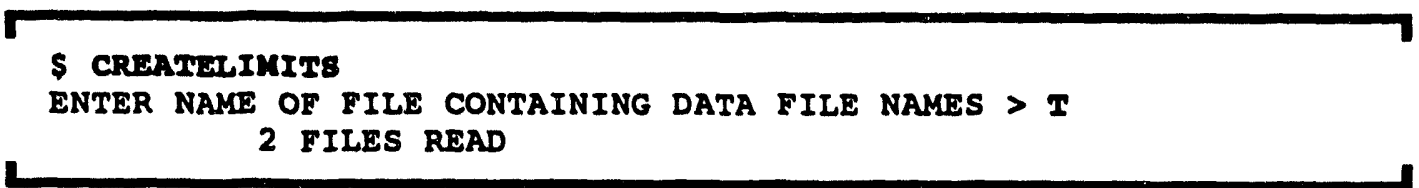

\section{Results of Using CREATELIMITS}

For these examples, CREATELIMITS would create a binary file, LIMITWB.BIN, that specifies the names and geographic extents of LAKE.DMS and RIVER.DMS. To see what information is included in a particular binary limit file, you can use the SEELIMITS utility. 


\section{D2RUN}

The D2RUN utility supports the use of D2, the Army's modeling program for predicting the downwind hazard associated with the release of a chemical agent. The D2RUN utility runs a specified D2 case in the current planning site, producing several supporting files in addition to the model output files. You can examine these files to follow D2 case execution or to diagnose case modeling problems.

\section{What You Need to Know Before Using D2RUN}

This utility works only with D2 cases for the current planning site. It es NOT work for operational sites. If you wish to use D2RUN on a shared site (one that the information manager can select as the operational site by using the CHANGE SITE option in the IBS program), then the information manager must run the utility on the given site in planning mode.

You must supply the number of the D2 case that you wish to execute. To see a list of case numbers for the given site, use either the LISTCNX or SHOWCNX utilities.

\section{Executing a D2 Case with D2RUN}

To execute this utility, enter D2RUN nnn at the system prompt, where $n n n$ is the case number.

No further interaction is necessary. The specified D2 case for the current site will be executed. As the case is executed, you will see status messages similar to those in the following example for case 008 .

Example:

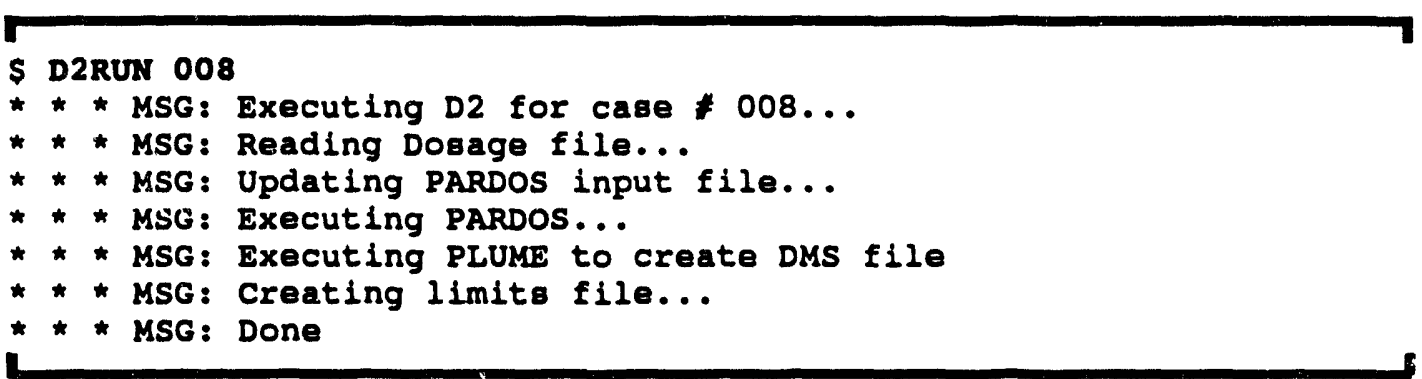




\section{Results of Executing a D2 Case}

The output files created by the utility and the D2 model are as follows:

SITESOSD2:CnnnD2LOG.DAT *

SITESOSD2:CnnnDOSAGE.DAT *

SITESOSD2:CnnnPLOT.DAT

SITESOSD2:CnnnPOSINP.DAT

SITESOSD2:CnnnSCRIPT.DAT

SITESOSD2:CnnnSUICE.DAT *
Log of the D2 run as would be seen if D2PC were run interactively. ${ }^{(a)}$ This is the best file to view if something unexpected happens during model execution.

Output file used to create the graphical outline of the D2 track. This file is based on CnnnPLOT.DAT and SITESISD2:CnnnD2INP.DAT.

Plot file written directly from D2PC used to create the ConnnDOSAGE.DAT file.

Output file from D2PC used as input to PARDOS.

List of inputs into D2PC without any explanations. If you want to see a more verbose version of this file, see CnnnD2LOG.DAT.

PARDOS output file which contains track time information.

- All files marked with an asterisk can be viewed from the PRINT D2 REPORT option within the IBS program

(a) See Innovative Emergency Management, Inc. and M.M. Myirski. 1993. Reference Manual: D2PC and Hazard Analysis. DAHC35-92-D-0018. Chemical Division, U.S. Army Nuclear and Chemical Agency (USANCA), 7500 Backlick Road, Building 5073, Springfield, Virginia 22150-3198.

Error Messages about the Dosage File. Watch closely for error messages. If there is an error message regarding the dosage file, it is unlikely that D2 produced any usable output. The ConnD2LOG.DAT file can often provide good insight into what went wrong. After checking the CnnnD2LOG.DAT file, check the D2 inputs by using the IBS or IBS_NOGR programs (see the IBS User Guide) or by using the D2_GR or D2_NOGR programs (see the IBS Models Guide or the IBSSH utility).

Error Messages about PARDOS. Some D2 tracks will not produce PARDOS output. If you receive an error message regarding PARDOS but no other error messages, the application has probably created the track itself but omitted the track time information.

\section{Executing Multiple D2 Cases}

If you want to run many cases, it might be best to run them as a batch job. If you plan to run these in batch mode, be sure to specify a log file so that you can look at the el ror messages after execution. 


\section{DB_CHK_DMS}

The DB_CHK_DMS utility is a quality assurance utility that checks either a map database topography or an entire map database for some common DMS file problems:

- directories that contain no DMS files

- empty DMS files (files that contain no data points)

- DMS files that have objects with no attributes

- DMS files that have objects with points located at 0.0

- DMS files with incorrect limits

- DMS files that contain objects with more that 5000 points (the maximum)

- attributes not found in the attribute dictionary.

This utility does NOT change any DMS files: it just reports errors. Other quality assurance utilities (noted later) can fix most (but not all) of the problems reported by DB_CHK_DMS.

\section{What You Need to Know Before Using DB_CHK_DMS}

DB_CHK_DMS operates on your current map database: unless you specify a different map database, both by disk name and by database name. To specify a specific topography, you must know the two-character topography code associated with the topography. See Appendix A, IBS Topographies for a list of IBS topographies and their two-character codes.

\section{Checking a Topography or Database for DMS File Problems}

To start the utility, enter RUN IEMIS\$QA:DB_CHK_DMS at the system prompt. In the following example, DB_CHK_DMS checks for DMS file problems in an entire map database named TESTDB located on disk device DISK2. 
Example:

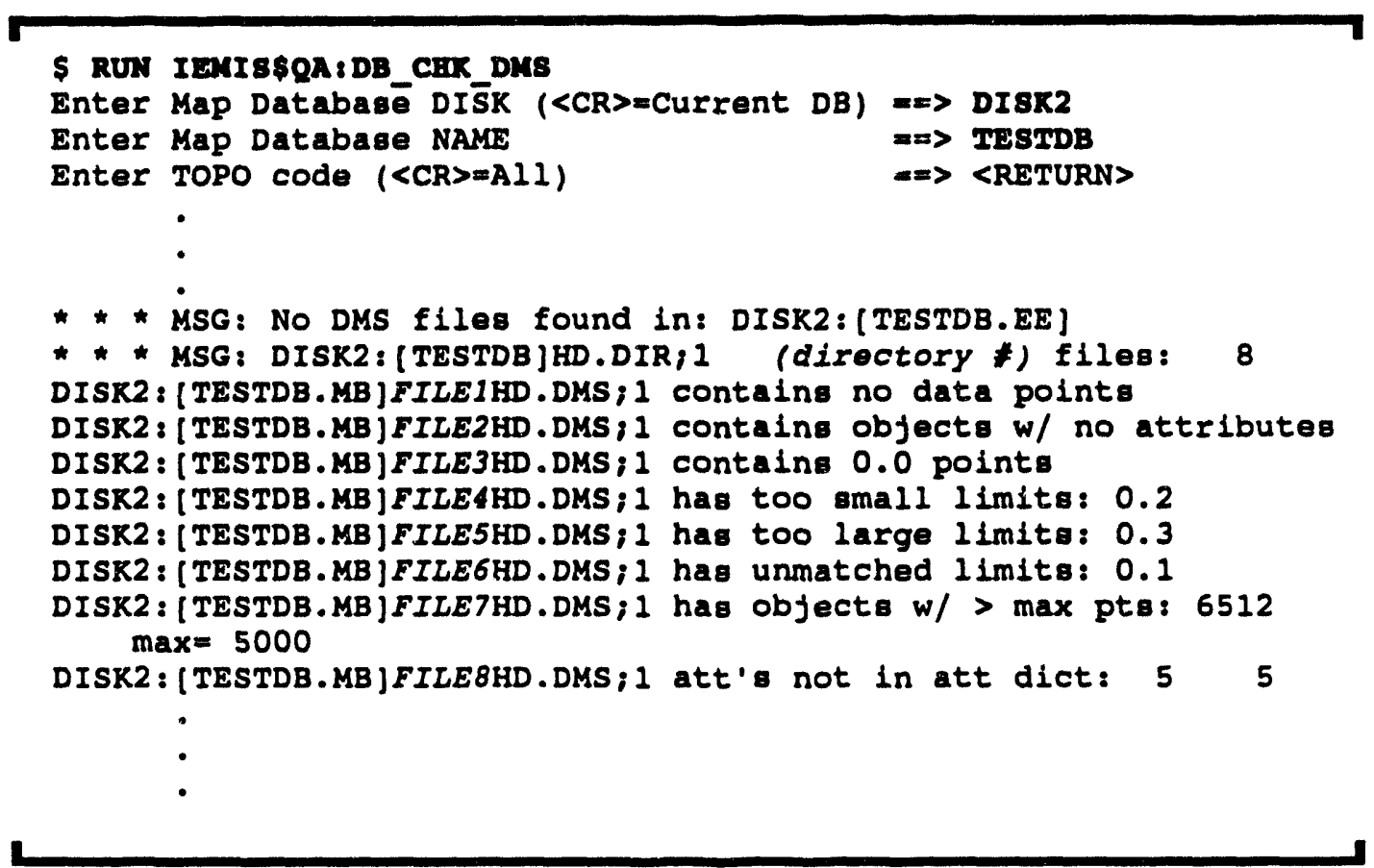

If you press RETURN instead of entering a disk name, the utility uses the current map database and does not prompt for a map database name.

If you press RETURN instead of entering a topographic code, the utility checks ALL topographies of the specified database.

\section{Fixing Problems Reported by DB_CHK_DMS}

As the program checks the topography or database, it presents a series of messages to indicate the progress of the checking. The example here shows one each of the kind of errors that DB_CHK_DMS detects. The following table indicates the problems reported by $\bar{D} B \_C \bar{H} K$ DMS and which other IBS quality assurance (QA) utilities might be used to correct them. 
Table. Fixing Problems Reported by DB_CHK_DMS

Message

No DMS files found in...

file contains no data points

file contains objects w no attributes

file contains 0.0 points

file contains too small limits

file contains too large limits

file has unmatched limits

file has objects w/ $>$ max pts

file att's not in att dict
Problem

Topography directory is empty of supporting topograpiny files.

DMS file is empty of data.

Some file objects have no identifying attributes.

Bad data: some points registered to 0.0 long or lat instead of the correct locations.

The data covers more area than specified in DMS file header. ${ }^{(a)}$

The data covers less area than specified in DMS file header. ${ }^{(a)}$

The data area is within tolerance limits of the check but does not match DMS file header limits. ${ }^{(a)}$

The file has more than the 5000-point maximum for IBS map data files.

Attributes used for an object are not found in the system attribute dictionary.
Solution - QA Utility

Update database to delete directory or add the necessary DMS topography files.

Delete files DB_DEL_MT_DMS

Add attributes - GEDIT

no solution without more information

Change DMS file DB_FIX_DMS_LIMITS

Change DMS file DB_FIX_DMS_LIMITS

Change DMS file DB_FIX_DMS_LIMITS

Remove excess points -

THINNER or SMOOTHER

(b) Modify all instances of the attributes MODATTDMS or

Modify attributes for single objects - GEDIT or

Add attribute (numbers) to the attribute dictionary

- UPDATEATT

(a) The value noted is the largest discontinuity, either in longitude or latitude (degrees).

(b) In all cases, knowing what the attributes should be is impossible without further information. 


\section{DB_DEL_MT_DMS}

The DB_DEL_MT_DMS utility is a quality assurance utility that checks either a map database topography or an entire map database for DMS files that contain no data (DataBase DELete eMpTy DMS). This utility does NOT change any DMS files, but it creates a batch command (.BIS) file that you can use to move all the empty files found into a $[\ldots \mathrm{CHK} x \mathrm{x}]$ directory.

\section{What You Need to Know Before Using DB_DEL_MT_DMS}

DB DEL MT DMS operates on your current map database unless you specify a different map database, both by disk name and by database name. To specify a specific topography, you must know the two-character topography code associated with the topography. See Appendix A, IBS Topographies for a list of IBS topographies and their two-character codes.

\section{Checking a Topography or Database for Empty DMS Files}

To start the utility, enter RUN IEMIS\$QA:DB DEL MT DMS at the system prompt. In the following example, DB_DEL_ $\bar{M} T$ DMS checks for empty DMS files in an entire map database named TESTD $B$ located on disk device DISK2, creating a .BIS file (DELALLMT.BIS) that could be used to delete all DMS files found to contain no data.

Example:

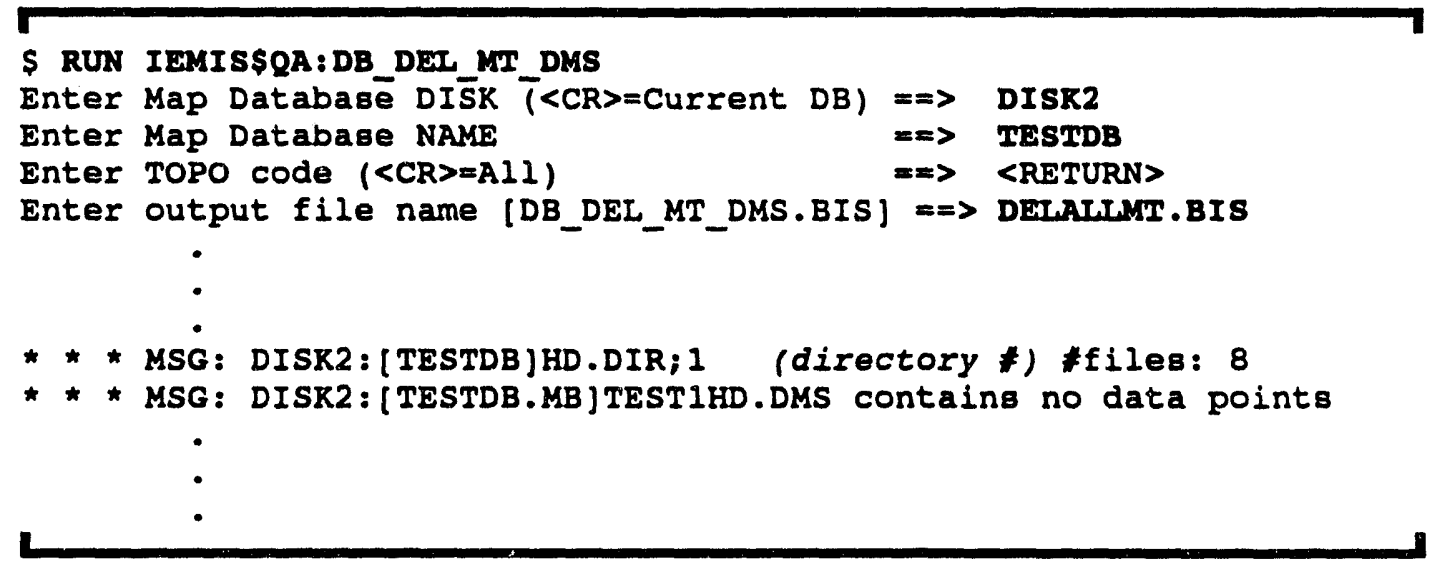

If you press RETURN instead of entering a disk name, the utility uses the current map database and does not prompt for a map database name.

If you press RETURN instead of entering a topographic code, the utility checks ALL topographies of the specified database. 
If you press RETURN instead of entering an output file name, the batch command file is named DB_DEL_MT DMS.BIS by default. The .BIS file is created in your current directory.

\section{Consolidating DMS Files Found to Contain No Data}

To move all the empty DMS files into the $[\ldots \mathrm{CHK} x \mathrm{x}]$ directories, enter

SUBMIT/NOPRINI/NOTIFY filename.BIS

at the system prompt in your login directory, where filename is whatever you entered as the output file name (or DB_DEL_MT_DMS.BIS if you accepted the default output file name). The [...CHK...] directory is created in the top-level directory of the map database being checked, and a [...CHK.xx] subdirectory is created for each topography where $x x$ is the two-character topographic code. 


\section{DB_FIX_DMS_LIMITS}

Every DMS file contains some file header information that contains the limits for that DMS file. In some cases, these limits in the DMS header are incorrect.

The DB_FIX_DMS_LIMITS utility is a quality assurance utility that checks either a map database topography or an entire map database for DMS files that have incorrect limits specified in the DMS file header. DB_FIX_DMS_LIMITS automatically changes the file header limits according to either the actual values or values that you specify.

\section{What You Need to Know Before Using DB_FIX_DMS_LIMITS}

DB FIX_DMS_LIMITS operates on your current map database unless you specify a different map database, both by disk name and by database name. To specify a specific topography, you must know the two-character topography code associated with the topography. See Appendix A, IBS Topographies for a list of IBS topographies and their two-character codes.

\section{Matching the DMS File Header Limit Information to the File Data}

To start the utility, enter RUN IEMIS\$QA:DB_FIX_DMS_LIMITS at the system prompt. In the following example,

Example:

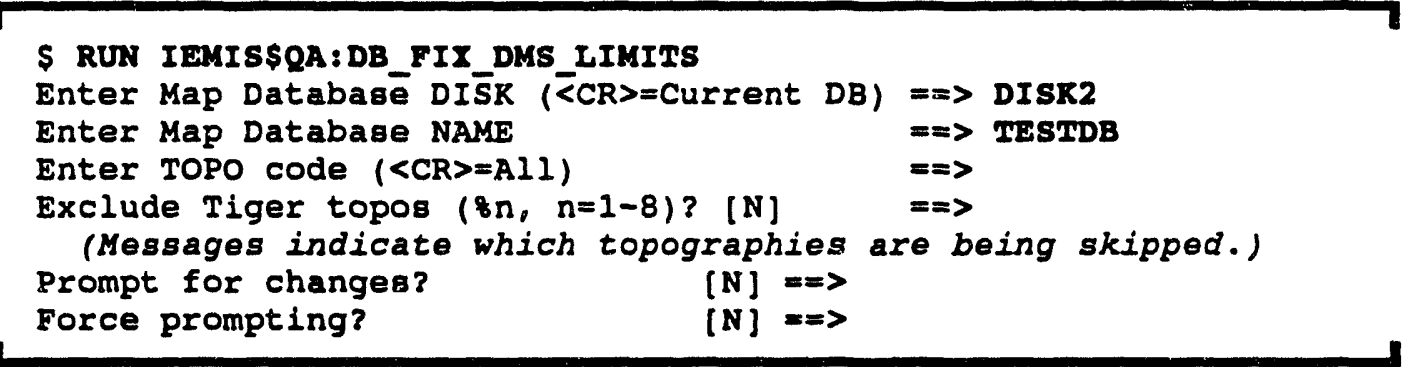

Map Database DISK If you press RETURN instead of entering a disk name, the utility uses the current map database and does not prompt for a map database name.

TOPO code If you press RETURN instead of entering a topographic code, the utility checks ALL topographies of the specified database. If you enter a specific topographic code, the utility skips the following prompt about Tiger topographies. 
Exclude Tiger topos? Changing the limits on Tiger DMS Population files may cause problems: because each file contains only a single point, the limits would be set to include only that point. The Tiger topographies are currently identified by two-character topographic codes. The first character varies but the second character is a numeral (1-8).

- Y means "Yes, exclude Tiger topographies." Messages indicate which topographies are skipped.

- $\quad$ or RETURN means "No, include Tiger topographies."

Prompt for changes? - Y means "Prompt for a choice of limit values only when a DMS file is determined to have incorrect limit values."

- N or RETURN means "Automatically use the actual limit values of the data."

Force Prompting? - Y means "Prompt for a choice of limit values for every DMS file." You might want to do this to check a few files before switching to automatically accepting the actual limit values.

- $\quad \mathrm{N}$ means "Do NOT prompt for every DMS file."

If you chose either to be prompted for changes or to force prompting, a menu similar to the following appears for an individual DMS file:

Example:

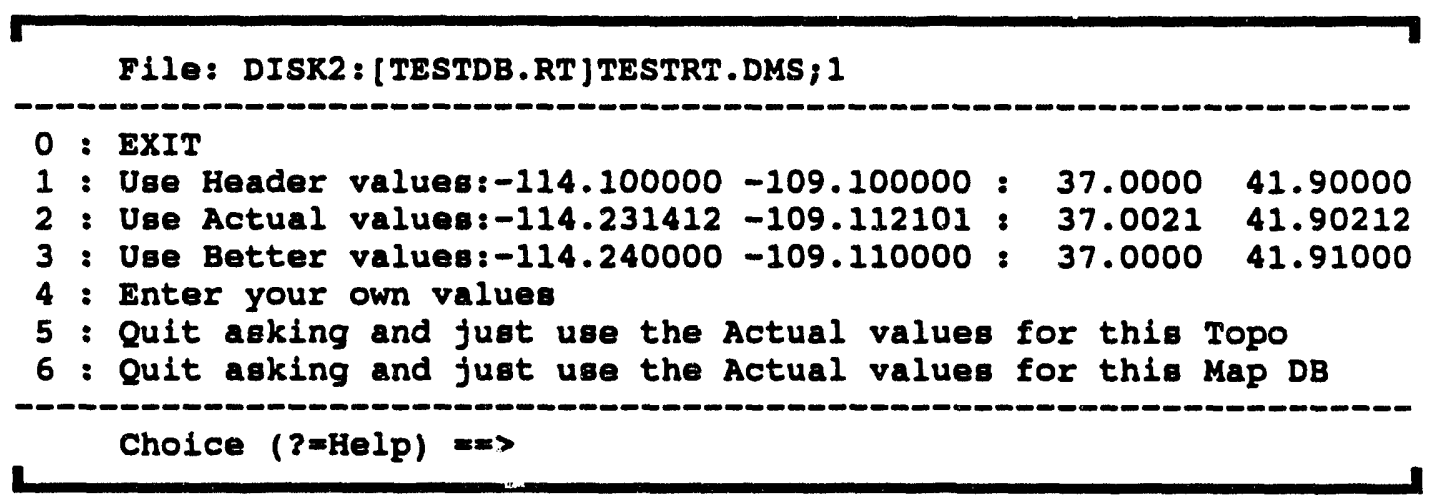

Use the menu above to choose how you want to fix the limit values: 
Menu Choice

o EXIT

1 Use Header Values

2 Use Actual Values

3 Use Better Values

4 Enter your own values

5 ..Actual values for this Topo

6 ..Actual values for this Map DB
Result

Leave the current DMS file unchanged and quit the utility.

Leave the current DMS file unchanged and continue.

Use the actual limits of the data.

Use values rounded from actual.

Show prompts for minimum and maximum longitude and latitude.

Cancel prompting and use actual limits for the rest of this topography. Return to prompting at the next topography.

Cancel prompting and use actual limits for the rest of the database. 


\section{DB_FIX_TXT_NMS}

Many DMS files contain a reference to an associated text file (.TXT), which contains textual data that supports the DMS graphic data. In some cases, the name of the associated.TXT file referenced in the DMS file may be blank, refer to a non-existing TXT file, or contain an incorrect logical name.

The DB FIX TXT NMS utility is a quality assurance utility that checks either a map database topography or an entire map database for DMS files that contain incorrect.TXT file names. This utility does not change any DMS files, but it creates a .BIS file that you can execute to correct the .TXT file names.

\section{What You Need to Know Before Using DB_FIX_TXT_DMS}

DB FIX TXT DMS operates on your current map database unless you specify a different map đatabase, both by disk name and by database name. To specify a specific topography, you must know the two-character topography code associated with the topography. See Appendix A, IBS Topographies for a list of IBS topographies and their two-character codes.

\section{Finding Incorrect Text File Names in DMS Files}

To start the utility, enter RUN IEMIS\$QA:DB_FIX_TXT_NMS at the system prompt. In the following example, the utility fixes only the water bodies (WB) topography files in a database named TESTDB on disk device DISK2.

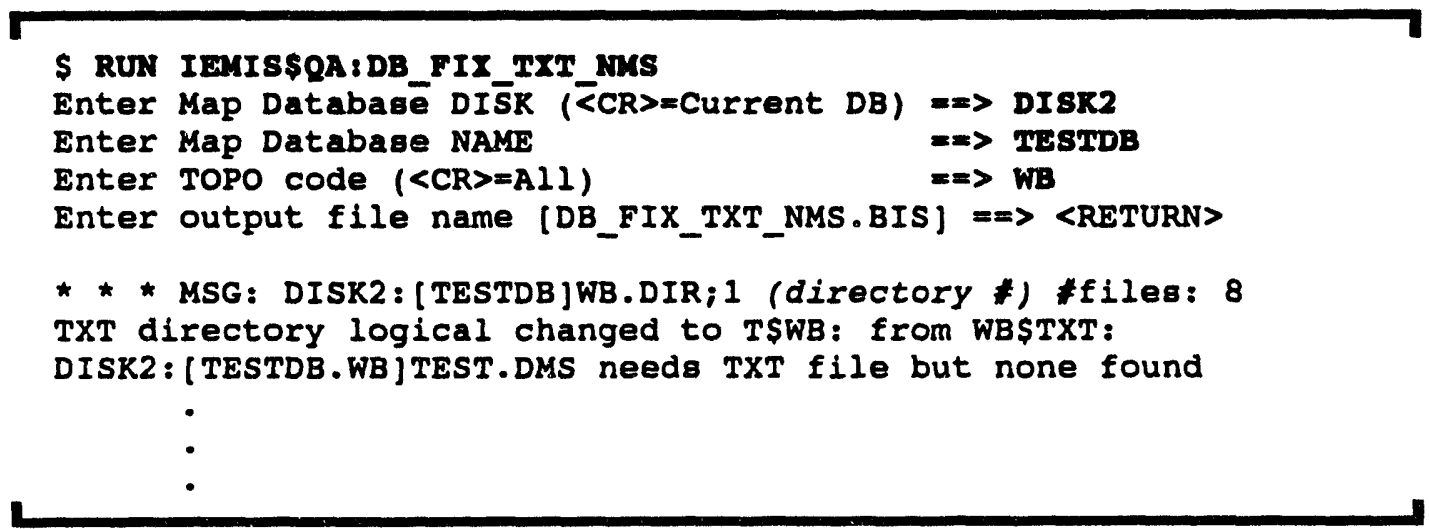

If you press RETURN instead of entering a disk name, the utility uses the current map database and does not prompt for a map database name.

If you press RETURN instead of entering a topographic code, the utility checks ALL topographies of the specified database. 
If you press RETURN instead of entering an output file name, the batch command file is named DB_FIX_TXT_NMS.BIS by default. The .BIS file is created in your current directory.

Messages indicate the progress of DB_FIX_TXT_NMS, identifying the DMS files that have a problem with the .TXT file name.

\section{Fixing DMS Files Found to Contain Incorrect Text File Names}

To fix the DMS files found to have problems with the .TXT file name, enter

SUBMIT/NOPRINT/NOTIFY filename.BIS

at the system prompt from your login directory, where filename is whatever you entered as the output filename when using DB_FIX_TXT_NMS. If possible, the DMS files are matched with the correct text files, and the text file names or logical names are fixed in the DMS files. 


\section{DEASSIGNLOG}

The DEASSIGNLOG utility is a system management program that helps you deassign all unnecessary map database (mapdb) logical names. Although deassigning these logical names might free up some system resources, it serves mostly a housekeeping function: it deassigns all logical names that point to topographies (map layers) that do NOT exist in your current map database.

\section{Deassigning Unnecessary Map Database Logical Names}

To start this utility, enter DEASSIGNLOG at the system prompt.

The utility creates a command file, DEASSIGN.COM in your login directory, and instructs you to execute this command file to deassign the unused logical names.

You may wish to look at the contents of DEASSIGN.COM first to check what logical names are unused (or what topographies are missing).

To deassign the unused logical names, enter @SYS\$LOGIN:DEASSIGN.COM at the system prompt, as indicated in the following example. The unused map logical names are then deassigned.

Example:

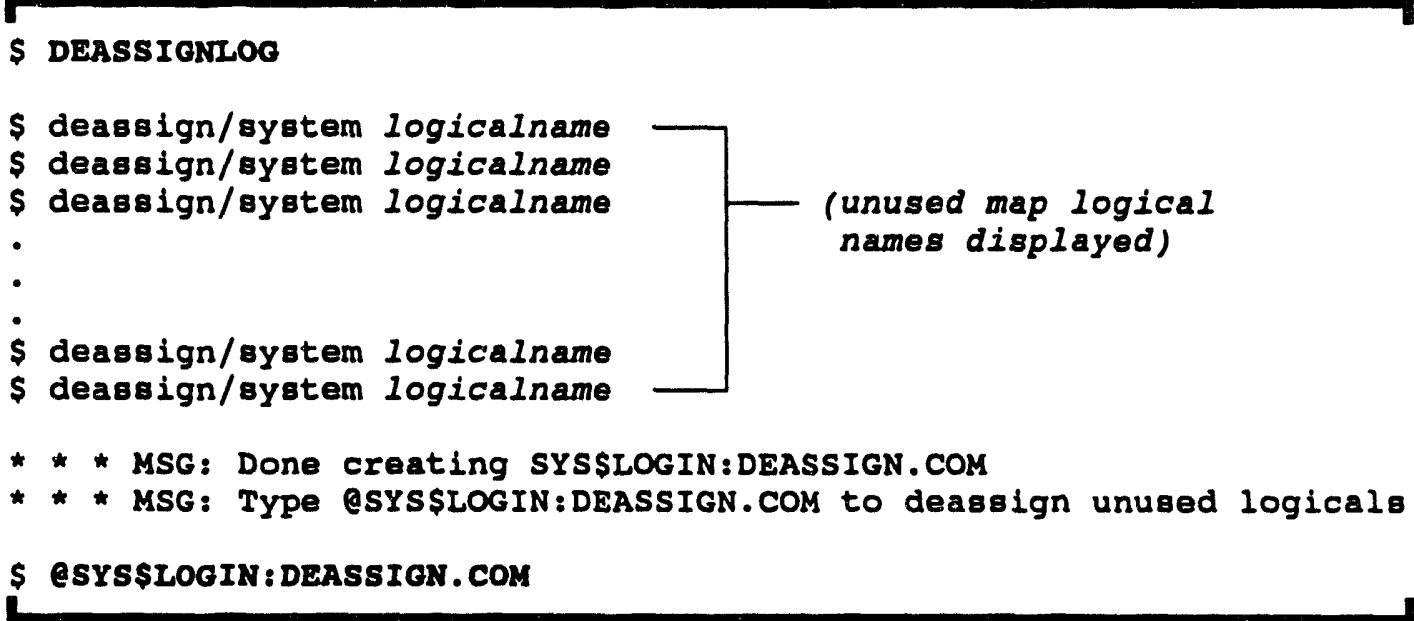




\section{DECLUTTER}

The system map display software includes a function for decluttering-reducing the detail and number of objects shown in a graphic display. Decluttering may be based on an absolute level or on the scale of the area displayed. The decluttering function depends on the attribute parameter values of the objects, but some objects do not have usable (nonzero) attribute parameter values.

The DECLUTTER utility enables you to add a special decluttering attribute to objects in a DMS file; this allows the screen display of that file to be decluttered when using MPDISPLAY. You can find more information about decluttering attributes in this discussion and in Appendix B, IBS Attribute Dictionary.

\section{Background: Using Attributes for Decluttering}

Attributes may be assigned to any data item (object) of any topography and can be either categorical or numeric. All attributes consists of a major code, minor code, parameter value, and a text string description.

Categorical attributes have a parameter value of zero and are used to classify an object. For example:

\section{MAJOR MINOR PARAM DESCRIPTION}

$$
5 \quad 150 \quad 0 \quad \text { Road or Street, Class } 4
$$

Numeric attributes have a numeric parameter value that is associated with the object (such as a road number or a stream's rate of flow). For example:

$\begin{array}{cccl}\text { MAJOR } & \text { MINOR } & \text { PARAM } & \text { DESCRIPTION } \\ 5 & 650 & 90 & \text { Interstate Route Number }\end{array}$

See Appendix B for a list of the attributes in the standard IBS attribute dictionary.

Since decluttering is based on parameter values, there is no means to directly declutter based on categorical attributes. The following instructions explain how to use DECLUTTER within an overall process of adding and using decluttering attributes within a DMS file. 


\section{Overview}

There are several steps in setting up a DMS file for screen display decluttering:

1. Create an ASCII Attribute Dictionary File using the ATTXASC utility.

2. Edit the ASCII file to create a specialized input dictionary file for decluttering.

3. Run DECLUTTER. (Inputs: the input dictionary file and a DMS file)

4. Add a decluttering attribute to the binary Attribute Dictionary using the UPDATEATT utility.

\section{Procedure for Applying Decluttering Attributes in a DMS File}

1. Create an ASCII Attribute Dictionary file using the ATTXASC utility:

At the prompt, enter the name of the attribute dictionary binary file. Enter ATTRIBDIC.BIX when prompted for the output file name.

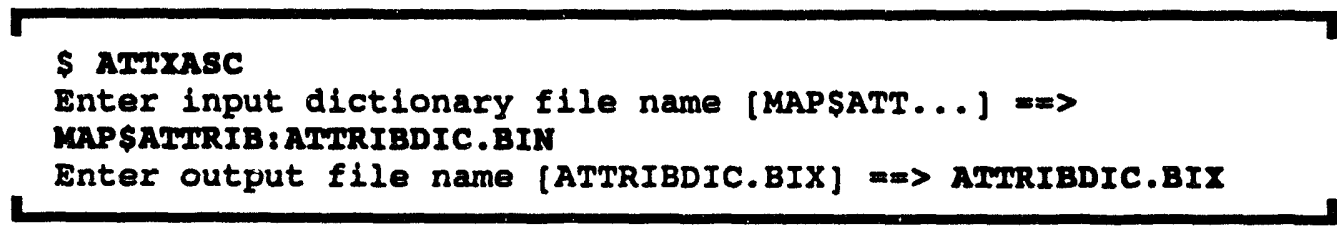

This produces an ASCII file that contains the attribute codes (Major code, Minor code, parameter value) for each item in the current attribute dictionary. The name of the file will be ATTRIBDIC.BIX. You will next edit this file so that it can be used as input to DECLUTTER.

2. Edit the ASCII file (ATTRIBDIC.BIX) to create a Declutter Input File: You can use the VAX/VMS editor (EDT) to edit the ATTRIBDIC.BIX file.

a. Delete all records not in the selected category.

That is, delete all records that do not apply to the topography to which you are adding decluttering attributes. For example, if you are adding decluttering attributes to objects in a ROADS AND TRAIlS topographic file, then you would delete all records except those that have a Major code of 5--ROADS AND TRAILS. 
Further, you may delete any attributes that are NOT used by objects in the DMS file.

b. For each remaining record (set of attributes), change the parameter value to one of the values shown in the following range:

Parameter Value
32,000
16,000
8,000
4,000
2,000
1,000
500
250
125
63
32
16
8
4
2

A higher parameter value represents an attribute of an object that is more likely to be displayed when decluttering is applied. That is, an object with attributes having a parameter value of 1 will be removed from the display before an object with attributes having a parameter value of 32,000 .

c. Save the edited copy of ATTRIBDIC.BIX for use as an input dictionary file for DECLUTTER.

3. Run DECLUTTER.

a. Enter RUN IEMIS\$EXE:DECLUTTER at the system prompt.

In steps $3 \mathrm{~b}$ through $3 \mathrm{f}$, you will see the following series of prompts, one at a time. (Sample responses are included here.) 


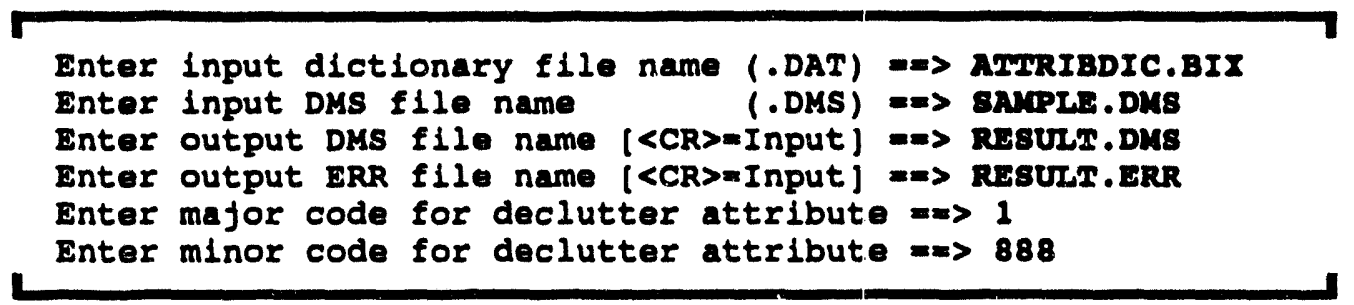

b. Enter ATTRIBDIC.BIX as the input dictionary file name.

c. Enter the name of the DMS file to which you wish to add decluttering attributes.

d. Enter the name of the new DMS file that will contain the decluttering attributes. (The input file name is the default name for the new DMS file.)

e. Enter the name of an output error (ERR) file: (see Error File below).

f. Enter the Major code of the decluttering attribute: Use a Major code number corresponding to the major topographic category.

g. Enter the Minor code of the decluttering attribute: Type 888 as the minor code to be used for the decluttering attribute.

After DECLUTTER has finished, you will have two new files:

- A new DMS file: This file will be identical to the original DMS file except that each object that has (one or more of the) attributes listed in the Declutter Input File will also have an additional attribute-a decluttering attribute:

(Major code); 888; (parameter value)

where the parameter value is the (highest) value listed in the input dictionary file for any attributes of the object. (See the example.)

- Error File: This output error (ERR) file contains special error messages and information for situations when DECLUTTER cannot correctly add a decluttering attribute to an object. 
For example, messages will be recorded for objects that already have the maximum number of attributes allowed (15) and for objects that either have no attributes or have only attributes with parameter values of zero. (In the latter cases, the parameter values of the new decluttering attributes will be zero, which is not usable.)

4. Add a decluttering attribute to the binary Attribute Dictionary using the UPDATEATT utility: Before you can use decluttering with the new DMS file, you must add a corresponding decluttering attribute to the system Attribute Dictionary.

a. Enter RUN IEMIS\$EXE:UPDATEATT at the system prompt.

You will see a prompt that asks you to select an option for updating the Attribute Dictionary.

b. Enter $\mathbf{A}$ to "Add a new attribute record."

You will see a data form that contains default values in six data fields. You will alter some of these fields to add the decluttering attribute.

Conventions for entering data on the form: Use the arrow keys to move from one field to another on the form. When you are finished, press GOLD Z to accept the entries--or press GOLD Y to quit and restore the original contents of the form. Pressing RETURN also advances from one field to the next. In the last field of the form, pressing RETURN (like GOLD Z) accepts the current form data and then exits the form. (See the description of UPDATEATT for more information.)

c. Enter values in the following fields:

\section{Attribute Code:}

- Type the "Major Coden number that corresponds to the topographic category used earlier as input to DECLUTTER.

- Type 888 as the "Minor Code" for the decluttering attribute.

- Type 500 as the "parameter value."

Attribute Description: Enter Decluttering attribute (Range 12-27) as the text description. (See Using Decluttering Numbers, page 2.41)

Decluttering Code: Enter 21 as the decluttering code. 
Parameter-Decluttering Scale Factor: Enter 1 as the scale factor.

d. Press CTRL-Z io accept the changes and exit the data form.

Again you will see the prompt that asks you to select an option for updating the Attribute Dictionary.

e. Enter $\mathbf{E}$ to "Exit" the program and update the Attribute Dictionary.

The Attribute Dictionary will be updated to include the decluttering attribute for the selected topographic category.

\section{Example of the DECLUTTER Procedure}

The following example creates an output DMS file that has the decluttering attributes added, allowing the decluttering option to be used in MPDISPLAY. With decluttering "on," MPDISPLAY will display the most significant roads in the new file when you are viewing a large area and more secondary roads when you zoom in on a smaller area.

Starting DMS File: AFTRD01RD.DMS, a topographic file of "Roads \& Trails" data.

Product DMS File: NEWFILE.DMS, th $\quad$ loads \& Trails" data with decluttering attribut.

1. Create an ASCII Attribute Dictionary file using the ATTXASC utility:

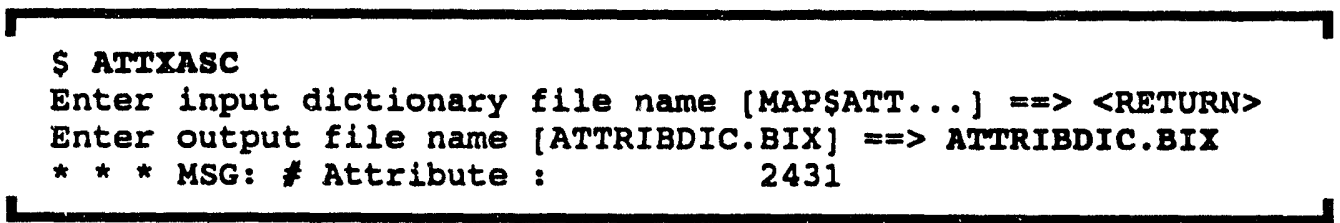

This produces the ASCII attribute dictionary file ATTRIBDIC.BIX in your current directory.

2. Edit the ASCII file (ATTRIBDIC.BIX) to create a Declutter Input File: The following portion of ATTRIBDIC.BIX is shown for the ROADS AND TRAIIS category.

The furmat of ATTRIBDIC.BIX should be as follows (headings added): 


$\begin{array}{cccl}\text { MAJOR } & \text { MINOR } & \text { PARAM } & \text { DESCRIPTION } \\ 5 & 0 & 0 & \text { ROAD AND TRAIIS } \\ 5 & 10 & 0 & \text { Bridge Abutment } \\ 5 & 20 & 0 & \text { Tunnel Portal } \\ 5 & 30 & 0 & \text { Gate } \\ 5 & 40 & 0 & \text { Cul-de-sac } \\ 5 & 50 & 0 & \text { Dead End } \\ 5 & 51 & 0 & \text { Interstate (Proposed) }\end{array}$

It is necessary to edit these parameter values, replacing them with values that range from 1 (detail that is removed first when large-area displays are decluttered) to 32,000 (objects that are removed last when decluttering is on). That is, the larger the parameter value, the more likely it is that the object will remain displayed when decluttering is applied. (See page 2.35 for a full list of the parameter values.)

The following is a partial sample of our edited ATTRIBDIC.BIX file:

5

5

5

5

5

5

5

5

5

5

5

5

5

5

5

5
0

10

20

30

40

50

51

52

53

$$
54
$$

55

56

57

58

59

60
0 Roads and Trails

0 Bridge Abutment

o Tunnel Portal

0 Gate

0 Cul-de-sac

0 Dead End

32,000 Interstate (Proposed)

32,000 Interstate

32,000 Interstate (Under Construction)

16,000 U.S. Highway

16,000

U.S. Highway (Under Construction)

8,000 State Highway

8,000 State Highway (Under Construction)

4,000

4,000

2,000

Secondary State and County

Secondary State and County (UC)

Primary Route, Class 1, Symbol, Undiv. 


\section{Run DECLUTTER}

The following sequence of DECLUTTER prompts and responses will create the NEWFILE.DMS with a declutter attribute added to every object in the DMS file AFTRD01RD.DMS.

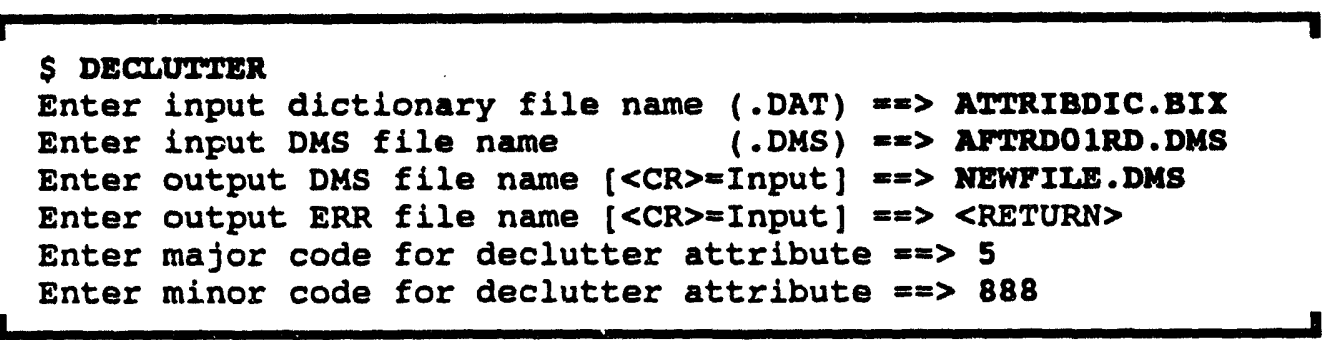

New DMS File. The DMS output file, NEWFILE.DMS, contains all the objects from AFTRD01RD.DMS. Each object contains an additional new decluttering attribute. The major and minor codes of this attribute are 5 and 888 , respectively. The parameter value assigned to the attribute 5,888 is the largest parameter value (from ATTRIBDIC.BIX) corresponding to the attributes of the object.

For example, if the attributes of an object in AFTRD01RD.DMS have the attributes:

$\begin{array}{ccc}\text { MAJOR } & \text { MINOR } & \text { PARAMETER } \\ 5 & 51 & 0 \\ 5 & 60 & 0\end{array}$

The same object in NEWFILE.DMS will have the attributes:

$\begin{array}{crr}\text { MAJOR } & \text { MINOR } & \text { PARAMETER } \\ 5 & 51 & 0 \\ 5 & 60 & 0 \\ 5 & 888 & 32,000\end{array}$

Error File. In the example, DECLUTTER creates an error file named AFTRD01RD.ERR. This file contains any error messages that might have been displayed during execution and additional diagnostic information. 


\section{Using Decluttering Numbers}

MPDISPLAY uses the attribute parameter values and the Attribute Dictionary's decluttering attribute to calculate a decluttering number that is applied in decluttering a graphics display:

decluttering \# = decluttering code $+\log \left(\mathrm{PV}^{\prime} / \mathrm{PV}\right) /[(\log 2) *$ scale factor $]$

where

decluttering code $=$ the "Decluttering Code" entered in the Attribute Dictionary. (This number is approximately 6 less than the maximum decluttering number.)

$P^{\prime}=$ the parameter value (obtained from the decluttering attribute attached to every object in the DMS file).

PV = the parameter value of the attribute in the Attribute Dictionary (which is $\mathbf{5 0 0}$ for the decluttering attribute as shown in the procedure).

scale factor $=$ the "Parameter-Decluttering Scale Factor" of the decluttering attribute in the attribute dictionary (which is 1 for the decluttering attribute as shown in the procedure.)

The procedure for adding decluttering attributes creates decluttering attributes whose parameter values will be used in the formula above to calculate decluttering numbers in the range from 12 to 27 (as shown in Table 2.1).

The selection of the "declutter code" and parameter values in the DECLUTTER procedure to achieve this range of program-calculable decluttering numbers was somewhat arbitrary. (See the following discussion, Altering the Procedure for a Different Range of Decluttering Numbers.)

The "Item Display Functions" menu of the MPDISPLAY software includes a DECLUTTER ON/OFF option that enables you to 1) turn decluttering on or off and 2) enter an optional "decluttering level" (decluttering number). If you enter a decluttering number, then the decluttering level remains constant, regardless of display scale, and all objects evaluated (with the formula) at LESS THAN the selected decluttering number will NOT be displayed. 


\section{Altering the Procedure for a Different Range of Decluttering Numbers}

Although the procedure arbitrarily provides parameter numbers in the range from 12 to 27, you could alter the procedure to give a higher or lower range. It may be that the automatic decluttering (according to scale) does not work the way you want it to work with a specific topography. Or you might wish to do this if you need to coordinate the decluttering of two or more topographies. Then each topography might use a different range of decluttering numbers and a different decluttering attribute in the attribute dictionary.

Table 2.1. Correspondence Between Decluttering Numbers and Attribute Parameter Values (for the DECLUTTER procedure)

$\begin{array}{crr}\begin{array}{cr}\text { Decluttering } \\ \text { Number }\end{array} & & \begin{array}{c}\text { Parameter } \\ \text { Value }\end{array} \\ 27 & & 32,000 \\ 26 & & 16,000 \\ 25 & & 8,000 \\ 24 & & 4,000 \\ 23 & & 2,000 \\ 22 & & 1,000 \\ 21 & 500 \\ 20 & 250 \\ 19 & 125 \\ 18 & 63 \\ 17 & 32 \\ 16 & 16 \\ 15 & 8 \\ 14 & 4 \\ 13 & 2 \\ 12 & 1\end{array}$

To use a different range of decluttering numbers, you would need to figure what parameter values should be entered in ATTRIBDIC.BIX to give the desired range: 
1. Decide what range of declutter numbers is necessary to obtain the desired output:

- The declutter code $=($ maximum declutter number -6$)$. For any given "declutter code", there is a maximum declutter number range of about 16.

- It may be necessary to experiment with several sets of parameter values to obtain the desired output.

- Example: In the procedure, we chose a maximum declutter level of 27 for all objects. This level results in a declutter value range of 12 to 27 . The declutter code should be 6 less than the maximum declutter level:

$$
27 \cdot 6=21
$$

2. Use the decluttering number equation to back-calculate the parameter value (PV) that must be entered in the attribute dictionary.

- A parameter value may vary in value from -32768 to 32767 . Use 32,000 for the attribute parameter value of the objects associated with largest decluttering number selected.

- Use a scale factor of 1.

- Example: Substituting these values into the decluttering number equation gives:

$$
27=21+\log (32,000 / \mathrm{PV}) /[(\log 2) * 1]
$$

which reduces to

$$
\mathrm{PV}=500
$$

where 500 is the parameter value for the new decluttering attribute that should be added to the attribute dictionary.

3. Solve the equation for PV' to generate a table of parameter values corresponding to the remaining decluttering numbers in the chosen range.

- Example: Substituting the next decluttering value (26) and the PV value of 500 in the equation gives:

$$
26=21+\log \left(P V^{\prime} / 500\right) /[(\log 2) * 1]
$$


which reduces to

$$
P V^{\prime}=16,000
$$

4. Enter these parameter values (PV') in the parameter column of the Declutter Input File (ATTRIBDIC.BIX) and then run DECLUTTER.

5. Add the decluttering attribute to the Attribute Dictionary.

- Use the chosen "Declutter Code" and the calculated parameter value (PV). 


\section{DELPTDB}

The DELPTDB (DELete People Tracking DataBase data) utility clears old data and resets startup values in the people tracking database used by the LOCATE program. DELPTDB should be used to set up initial values in the database under these circumstances:

- when the system is first installed

- after an event is over and all post-event analysis of the people tracking data has been completed (for either a real or exercise event).

LOCATE is a people-tracking program that supports the Chemical Stockpile Emergency Preparedness mission of the IBS program. Refer to the IBS User Guide for specific information about the LOCATE and IBS programs.

\section{What You Need to Know Before Using DELPTDB}

You must have "Locate Manager" privilege before you can use DELPTDB.

The DELPTDB utility

- deletes from the database all the people entered by the LOCATE program

- sets all the reception facilities to "Inactiven

- inserts zeros for the numbers of people received, released, and remaining at all facilities.

Unless you make a backup of the data before using DELPTDB, all the information in the people tracking database is gone.

\section{Deleting People Tracking Information with DELPTDB}

To use the utility, enter DELPTDB at the system prompt. No other interaction is necessary. 


\section{DELSITE}

The DELSITE utility deletes an entire IBS site database from the system, removing all the directories, subdirectories, and files associated with the site database. Anyone with the appropriate IBS privilege can delete a site database by using DELSITE. DELSITE is a stand-alone utility but is also accessible as the IBS program's "Delete Site" option under SETUP-COPY CASE OR SITE FILES.

\section{Caution}

DELSITE permanently removes a site database. Once deleted, a site database cannot be recovered unless it is available on system backups.

This discussion covers the following topics:

- Why delete a site database?

- What You Need to Know Before Using DELSITE

- Deleting a Site Database with DELSITE

- Results of Using DELSITE.

\section{Why delete a site database?}

If for some reason a site database becomes obsolete and is no longer needed, it can be removed by any user with the appropriate IBS privilege. The disk space used by the site database then becomes available for system use.

\section{What You Need to Know Before Using DELSITE}

Before using DELSITE, you must know the following information, which DELSITE will request as input:

disk This is the name of the disk device on which the user account currently resides.

Example: DISK1

user This is the name of the user account under which the site database is located. All IBS site databases are located as subdirectories under a user account directory. 
site This refers to the four-character site code that serves as the subdirectory name of the site database to be deleted.

Examples: $A B C D$ or TEST

Before using the DELSITE utility, make sure that this information is correct. If this information is wrong, DELSITE could remove the wrong site database. You could expect the owners of a wrongfully wiped database to be unhappy.

\section{Deleting a Site Database with DELSITE}

The following is an example of how to delete a site database using the DELSITE utility.

To start the utility, just enter DELSITE at the system prompt. The program then prompts you for the information outlined in the previous section. The series of prompts will look like the following (simulated answers are in bold):

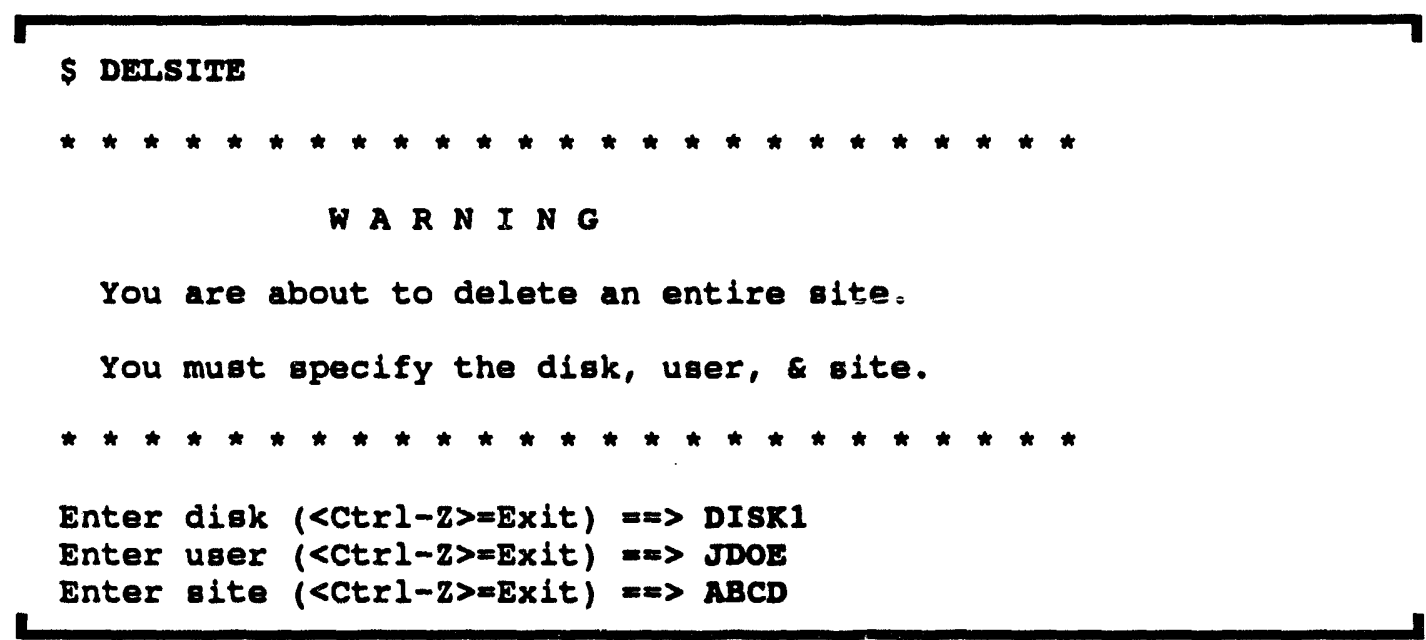

To cancel the deletion process at any of these prompts and exit, press CTRL-Z.

To delete a specific database, ente the appropriate disk, user, and site names as requested. Once you have supplied this information, the utility deletes the site database, displaying a sequence of messages similar to the following: 


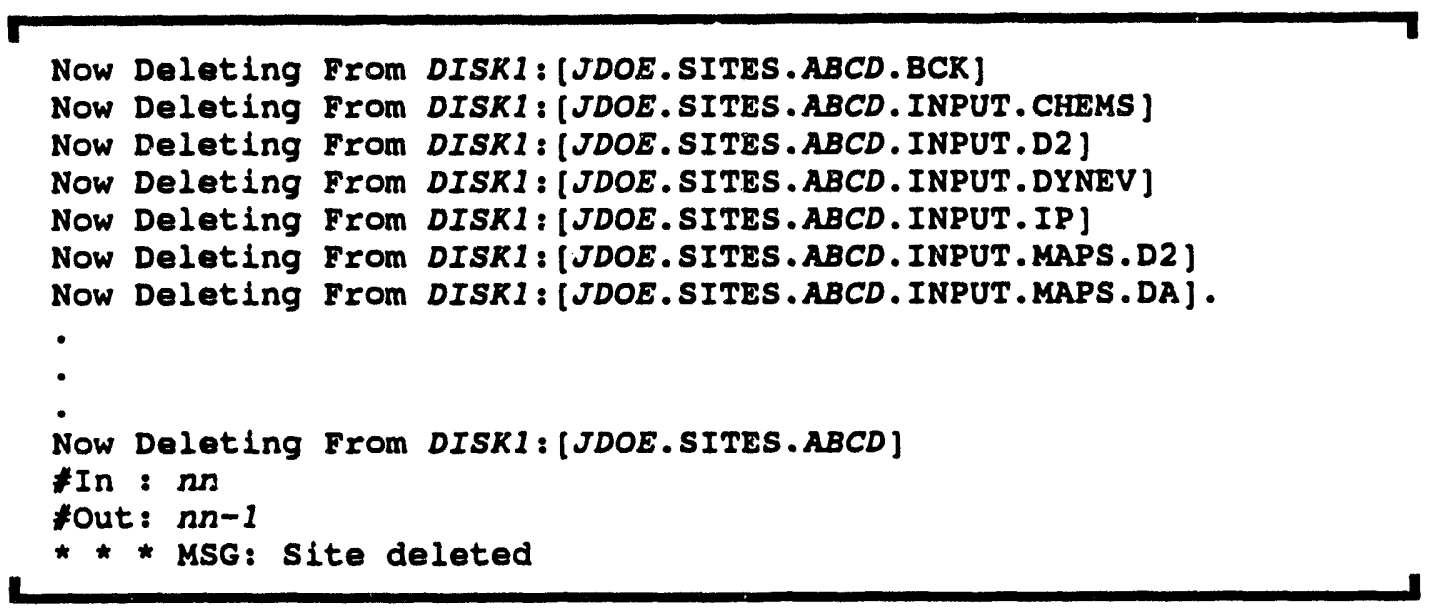

\section{Results of Using DELSITE}

DELSITE removes all the directories and files associated with a site database. A message indicates the number of sites before site deletion (\#In) and after site deletion (\#Out). Once removed, the site files cannot be restored through any IBS utilities. If a mistake is made, the only possible way you could recover the data would be to retrieve it from system backups made earlier. 


\section{DELTREE}

The DELTREE utility is a system management program that deletes all files and directories in a directory tree, including the root.

\section{Caution}

This utility deletes an entire directory tree with ALL its files: it is advisable to have a complete system backup stored away before DELTREE is used.

\section{Deleting All Files and Directories in a Directory Tree with DELTREE}

To start the utility, enter DELTREE at the system prompt. DELTREE prompts for the directory root to be deleted, as shown below. This root and all files and directories below it will be deleted.

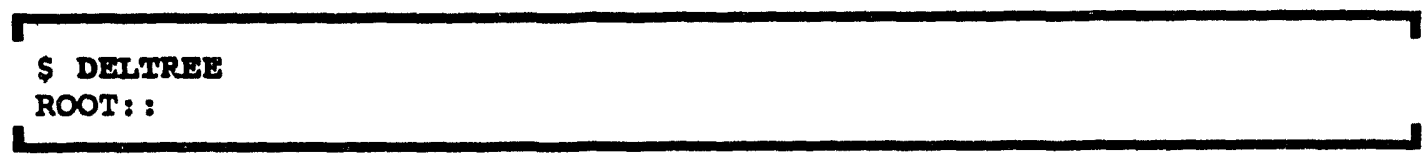




\section{DELUSER}

The DELUSER utility is used by the system manager to delete user accounts.

\section{Caution}

Once deleted, accounts cannot be recovered unless they are avallable on system backups. This utllity should be used with extreme caution.

This section covers the following topics:

- Why delete user accounts?

- What You Need to Know Before Using DELUSEF:

- Deleting a User Account with DELUSER

- Results of Using DELUSER

\section{Why delete user accounts?}

When a user is no longer with a project, or has been denied access to your system, you will want to remove his or her account to ensure that he or she cannot access your system. Deleting a user account removes all the information from the account and prevents the user from logging in. Once deleted, this information cannot be retrieved unless it is stored on system backups.

\section{What You Need to Know Before Using DELUSiER}

Before using DELUSER, there are certain pieces of information you need to know.

username The username is the name under which the user will log in.

The user enters this name at the system login prompt.

userdisk The userdisk is the name of the disk on which the account currently resides.

Example: DISR1

Before using the DELUSER utility, make sure that this information is correct. If this information is wrong, DELUSER could remove the wrong user by accident. 


\section{Deleting a User Account with DELUSER}

To run the utility, just type DELUSER at the system prompt. In the following example, simulated answers are in bold.

S DETUSER

USERNAME? : tost

USERDISK? : DI8K1

Once you have entered this information, the utility will prompt you for confirmation with the following message:

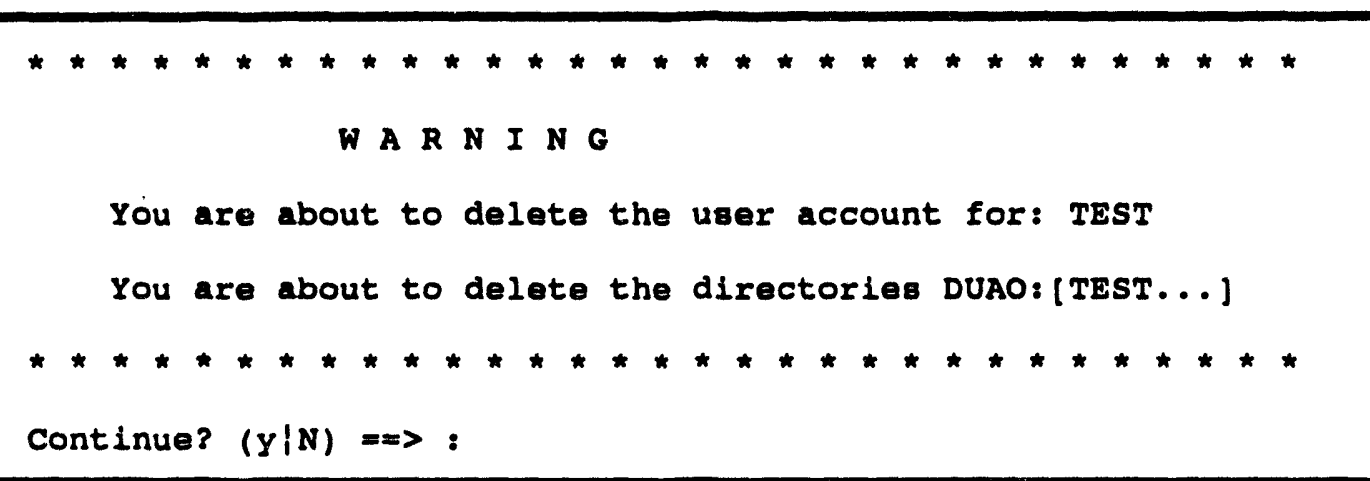

Entering $Y$ at this prompt indicates to the system that you want to delete the account; entering $\mathbf{N}$ aborts the deletion process. This is the last confirmation prompt before the account is deleted.

Once you confirm a deletion, the DELUSER utility automatically removes all the files and data related to the specified user account.

\section{Results of Using DELUSER}

DELUSER removes all the files associated with a user account. Once this is done, the files cannot be restored through any IBS utilities.

DELUSER also automatically removes the user's record from the system master mail list file. This same deletion is propagated to other systems on the network according to the contents of the IEMIS\$SYSF:MAIL SYSTEMS.DAT file (see the IBS Data Management Guide for information about this file). The EVENT program must be executing to update the mail list file. If EVENT is not executing, then changes to the mail list remain unprocessed until EVENT is started. When EVENT is restarted, it processes the waiting changes, so the changes made while EVENT is not running are NOT lost. 


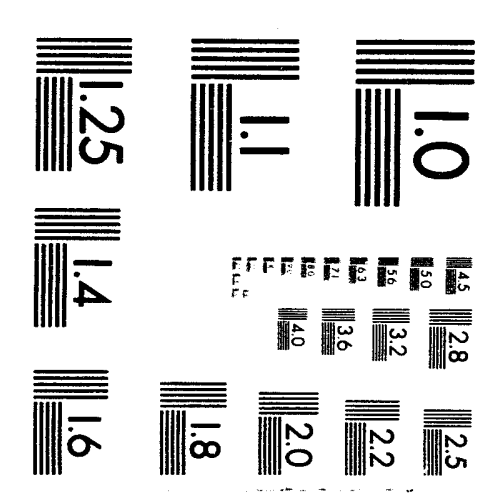



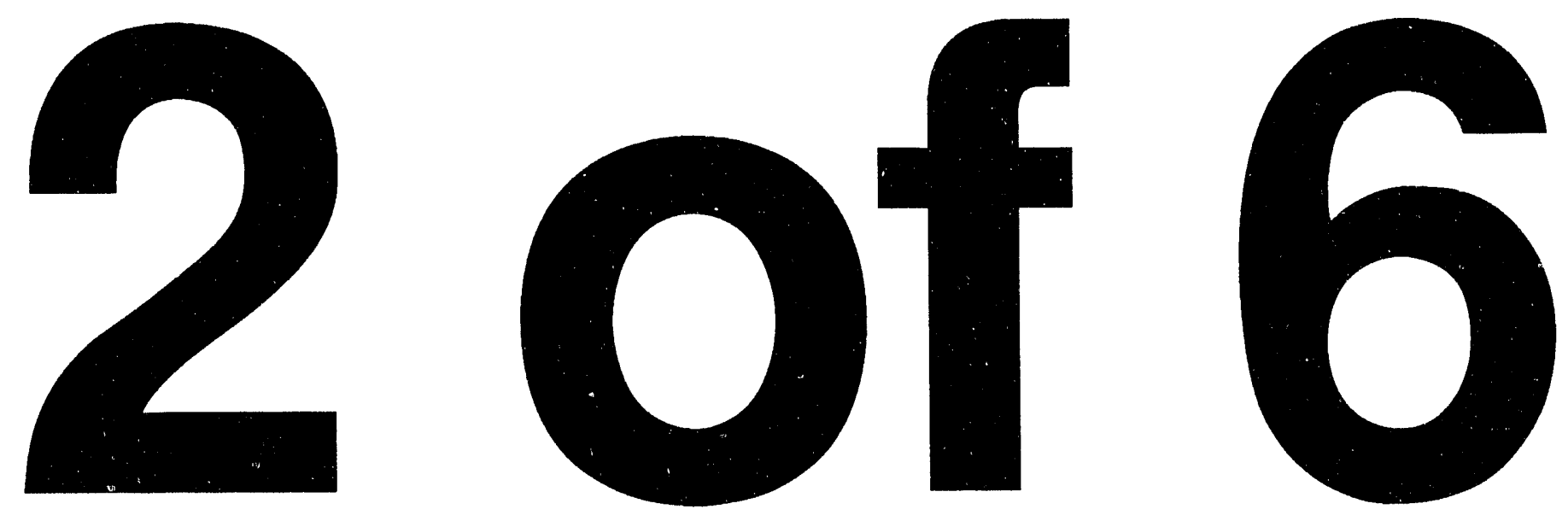


\section{DEMXTAC}

The DEMXTAC utility converts Digital Elevation Mapping (DEM) data provided by the U.S. Geological Survey (USGS) into the Terrain Analysis Cell (TAC3) format used by some models in IBS. The DEM data must be for 1:250,000-scale, 1-degree by 1-degree map shects. The purpose of the program is to provide elevation data files for model and utility use in IBS.

\section{What You Need to Know Before Using DEMXTAC}

The inputs to DEMXTAC are ASCII format DEM files provided on tapes by the USGS. The files are structured with a record size of 1024 bytes. One file (product) is provided for each 1-degree by 1-degree map sheet for which data is requested. Further information on file content is available in the USGS document, Digital Elevation Models, Data Users Guide 5.

The files should be read with the MYTAPE utility provided with the system.

\section{Creating TAC Data Files from DEM Data Files}

To s'art the utility, just enter DEMXTAC at the system prompt.

The program then prompts you for an input DEM file name; if you do not specify a file name extension, an extension of .DEM is assumed. The output file name will have the same name as this input file but with a .TAC extension. An example of its use follows:

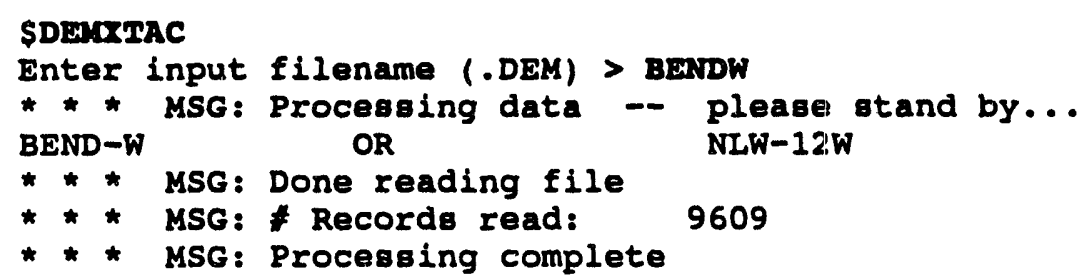

\section{Results of Using DEMXTAC}

DEMXTAC produces TAC3 data files, which have a binary format. One TAC data set (a .TAC file and a .TAA file) is produced for each input file. The TAC formats are described in the IBS Data Management Guide. 


\section{DLGXDMS}

The DLGXDMS utility reads a map data file in the USGS Digital Line Graph (DLG) optional distribution format (1:100,000-scale or 1:24,000-scale map format) and then creates one or more files in the IBS Digital Mapping Structure (DMS) format. These DMS files can then be used by IBS mapping functions. DLGXDMS and its complement, the DMSXDLG utility, can be used to convert back and forth between formats.

\section{What You Need to Know Before Using DLGXDMS}

The DLGXDMS utility asks you to specify at least three items:

input $D L G$ file name

attribute file name
This is the name of the DLG file to be read.

This is the name of an ancillary attribute file (ATT file name extension) associated with the DLG file. Only DLG files created by the DMSXDLG utility will have an separate ancillary attribute file. This ancillary attribute file stores the DMS attributes, pen numbers, and line types when DMSXDLG is used to convert DMS data to the DLG format (because this information is not directly transferable to the DLG format). DLG files produced by DMSXDLG contain no attributes.

output DMS file name This is the name of the DMS file to be created.

If the DLG file is not associated with an attribute (ATT) file, then the DLGXDMS utility also asks you to specify the following information for each category of DLG data contained in the DLG file:

2 character code

DMS pen number (0-15)
This is a two-character that indicates what type of topography (map layer) is to be created. DLGXDMS appends this code to the name of the resulting DMS file. If you wish to follow IBS topographic code conventions, see Appendix A for a list of codes.

This is a number that indicates which color is used to draw the map layer. If you wish to follow the color conventions used in the IBS geographic databases, you will need to know the pen numbers associated with your topographies. You can examine an existing DMS topography file and identify the pen number by using the utility DMSXASCII. 
DMS line type (0.7)

This is a number that indicates which line type is used to draw the map layer. DLGXDMS lists your choices.

\section{Converting DLG Data to DMS Format with DLGXDMS}

To start the DLGXDMS utility, just enter DLGXDMS at the systcm prompt. The following sections are two example sequences for reading a $I L G$ file to create corresponding file(s) in DMS format.

- The first example creates a DMS file from a DLG file with an associated attribute file. This means that the DLG file must have been generated by the DMSXDLG utility in an earlier data conversion.

- The second example creates a DMS file from a DLG file without an associated attribute file. The source of the DLG file in this example could be the U.S. Geological Survey.

The utility prompts you for the information outlined in the previous section.

\section{Converting a DLG File with an Attribute (.ATT) File}

The following sequence illustrates the conversion of a DLG file with an ancillary attribute file.

Example:

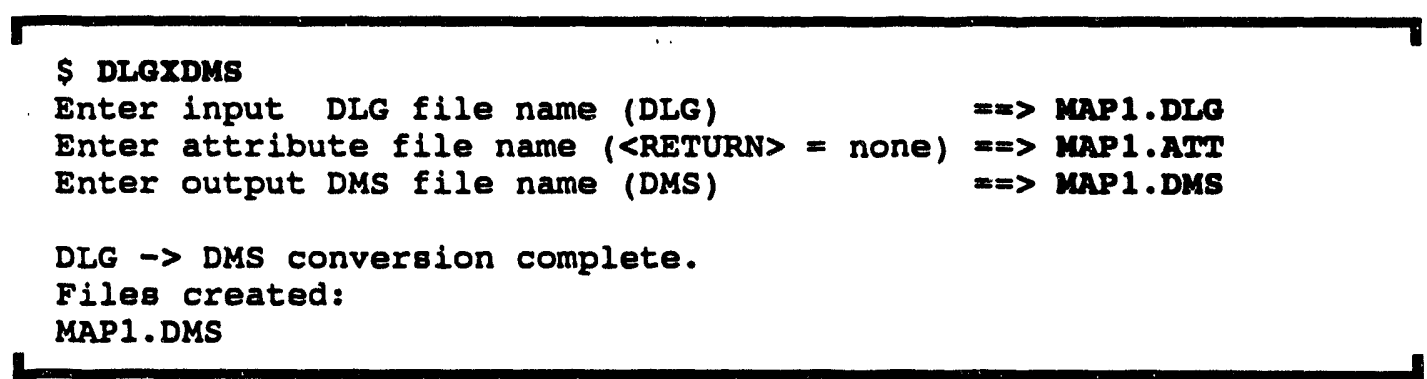

If the DLG file has an associated ancillary attribute file, enter the name of the attribute file at the program prompt.

Result: DLGXDMS creates a single DMS file, using the DMS attribute, pen number, and line type information from the ancillary attribute file. 


\section{Converting a DLG File without an Attribute (.ATT) File}

The following sequence illustrates the conversion of a DLG file that contains a single data category (Transportation, Roads, and Trails) with no ancillary attribute file.

Example:

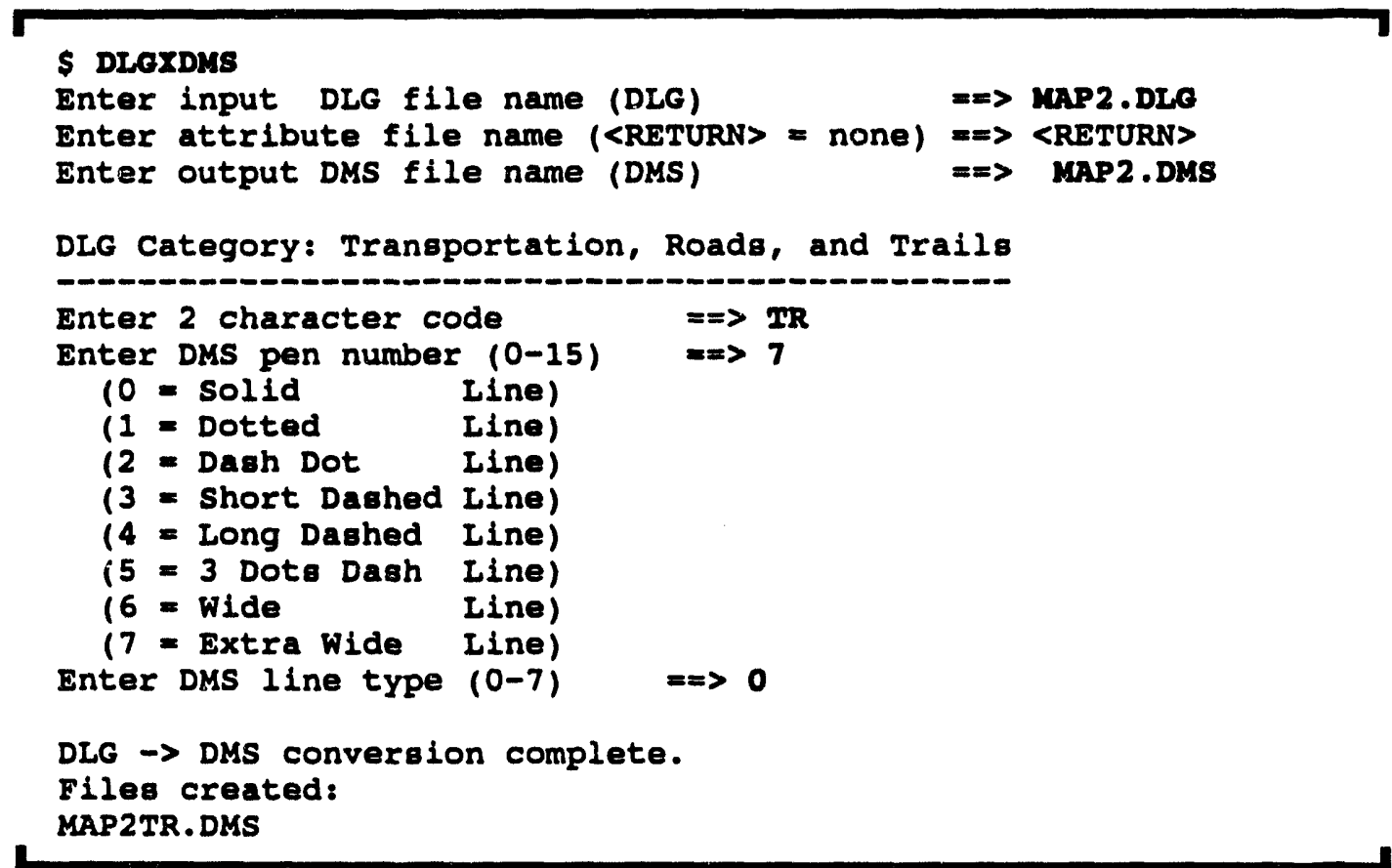

If the DLG file does not have an ancillary attribute file, just press the RETURN key when the utility prompts you for the attribute file name.

Then for each DLG data category within the DLG file, DLGXDMS prompts you to enter the two-character code, a DMS pen number, and a line type to be used for DMS data within that category. USGS $1: 100,000$-scale map attributes that have a corresponding DMS attribute are converted to the equivalent DMS attributes. If there is no equivalent DMS attribute, the utility assigns a default DMS attribute ( major $=0$, minor $=0$, parameter $=0$ ), which indicates that an attribute is present for the data object but that there was no DMS equivalent.

Result: DLGXDMS creates one DMS file for each category of data in the DLG file. This has the effect of decomposing the DLG data into separate map layers, one DMS file for each map layer. Each DMS file created will have the twocharacter code appended to the file name. 


\section{DMSXASCII}

The DMSXASCII utility reads DMS files and creates ASCII records suitable for display at a terminal or for storage within an ASCII sequential file. This enables you to see the DMS file header information, to see when the file was created, and to determine the data's area extents. You can also produce a formatted ASCII data file (.DMX extension) for use in other programs (such as ASCIIXDMS) or examine a file for the points used to create map objects. DMSXASCII can produce information about the file header, record header, and object coordinates.

In earlier versions of the software, this utility was named READDMS. You can still start the software by entering READDMS at the system prompt.

\section{Reading a DMS File with DMSXASCII}

To start the utility, just enter DMSXASCII at the system prompt.

The following sections are two example sequences for creating ASCII information from a DMS file:

- The first example creates an ASCII file that contains information from the DMS file.

- The second example displays the information on the terminal screen.

\section{Creating an ASCII Information File from a DMS File}

In the following example, DMSXASCII reads the contents of MYFILE.DMS and creates an ASCII version of the information in MYFILE.DMX:

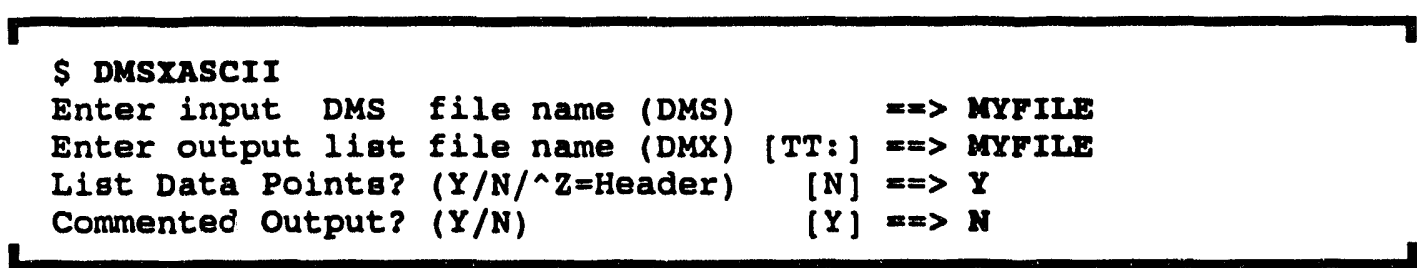

The DMSXASCII utility asks you to specify the following items:

input DMS file name This is the name of the DMS file that DMSXASCII will read. If you do not specify an extension, the .DMS file extension is used. CTRL- $Z$ cancels the program at this point. 
output list file name

List Data Points?

\section{Commented Output?}

$\mathbf{N}$

CTRL-Z
This is a name for the ASCII file. If you do not specify a file name extension, the .DMX extension is used. You can also do the following instead of entering the output file name:

$\begin{array}{ll}\text { Your Response } & \begin{array}{l}\text { Result / Comments } \\ \text { CTRL-Z }\end{array} \\ \text { RETURN the same name as the DMS } \\ \text { file but with the .DMX extension. }\end{array}$

Here you must indicate what information you wish to extract from the DMS file:

\begin{tabular}{|c|c|}
\hline Your Response & Result / Comments \\
\hline $\mathbf{Y}$ & Includes all information. \\
\hline $\mathbf{N}$ or RETURN & $\begin{array}{l}\text { Excludes point coordinates, text } \\
\text { strings, arc/polygon extents, and } \\
\text { complex polygon information. }\end{array}$ \\
\hline CTRL-Z & $\begin{array}{l}\text { Includes only information from } \\
\text { the DMS file header: extents, } \\
\text { scale, dates, and text file name. }\end{array}$ \\
\hline
\end{tabular}

Here you can choose whether or not to include automatic comments that identify the information in the ASCII text. Your response depends on how you plan to use the information:

\begin{tabular}{|c|c|}
\hline Your Response & Result / Comments \\
\hline Y or RETURN & $\begin{array}{l}\text { Comments are inserted in the } \\
\text { ASCII text to identify the } \\
\text { information. }\end{array}$ \\
\hline $\mathbf{N}$ & $\begin{array}{l}\text { Produces only numeric } \\
\text { information without comments. } \\
\text { Do this if you wish to edit the } \\
\text { resulting ASCII file and later } \\
\text { convert it back to a DMS file } \\
\text { (with the ASCIIXDMS utility). }\end{array}$ \\
\hline CTRL-Z & CTRL- $\mathrm{Z}$ is ignored here. \\
\hline
\end{tabular}




\section{Displaying DMS File Information on Your Screen}

In the following example, DMSXASCII reads the contents of MYFILE.DMS and displays only information from the file's header on the terminal screen:

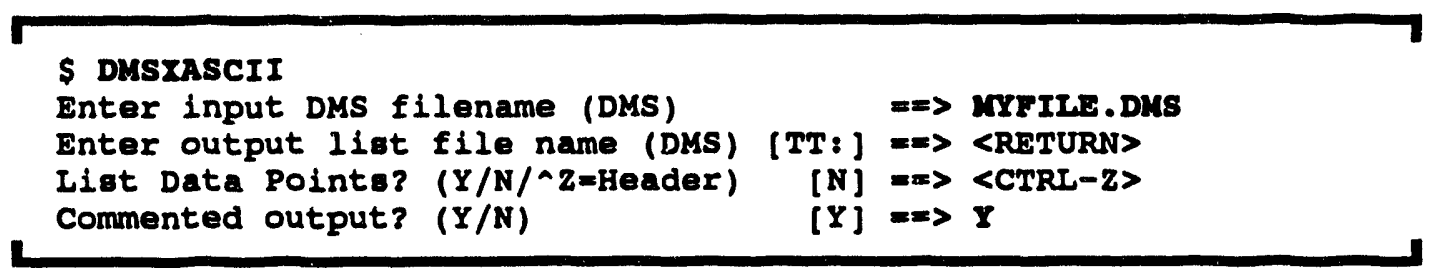

TT: designates your terminal, so the output is displayed on the screen. 


\section{DMSXDLG}

The DMSXDLG utility reads a DMS file and creates a new file in the USGS Digital Line Graph (DLG) optional distribution format (1:100,000-scale map format). DMSXDLG and its complement, the DLGXDMS utility, can be used to convert back and forth between formats.

\section{What You Need to Know Before Using DMSXDLG}

The DMSXDLG utility asks you to specify two items:

input DMS file name This is the name of the DMS file to be read.

output DLG file name This is the name of the DLG file to be created.

\section{Converting DMS Data to DLG Format with DMSXDLG}

To start the DMSXDLG utility, just enter DMSXDLG at the system prompt. The following is an example of the sequence used to convert a DMS file, MAP.DMS, to DLG format. The utility prompts you for the information outlined in the previous section.

Example:

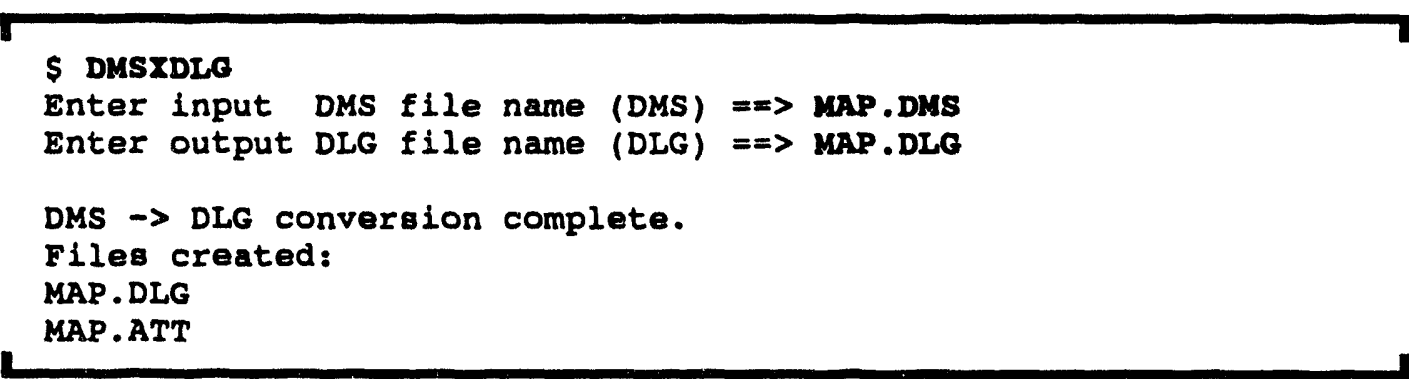

Result: DMSXDLG creates two files--a DLG file and an ancillary attribute file. For the DLG file, the utility converts DMS point data to DLG node data, DMS line data to DLG line data, and DMS polygon and complex polygon data to DLG line data. The DLG file is in level 2 format, so that "data linkage" information (such as which lines are adjacent to a given area) is not present.

The ancillary attribute file (ATT file name extension) stores the DMS attributes, pen numbers and line types for the DMS data (because this information is not directly transferable to the DLG format). No attributes are contained within the DLG file. See the IBS Data Management Guide for more detailed information on the format of the DLG and ATT file formats. 


\section{DMSXDTX}

The DMSXDTX utility is a diagnostic and reporting program that scans a DMS file for objects having text labels associated with them and dumps the text to a output file (DTX). The generated DTX output file lists each object's corresponding latitude, longitude, and text label.

\section{Listing Location and Text Labels for Objects in a DMS File}

To start the utility, enter DMSXDTX at the system prompt as shown in the example here.

Example:

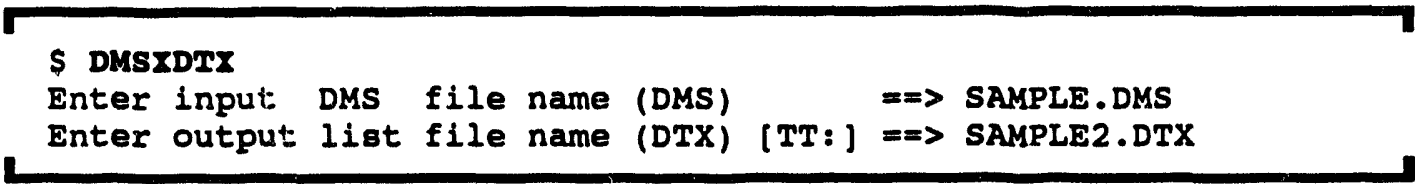

To list the output on the terminal screen, press RETURN instead of entering a file name. This is an example of the DTX output information:

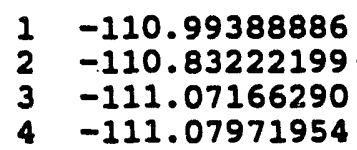

$4-111.07971954$
40.45194626 A Hollow

40.76805496 A-1 Peak

40.97249985 Aagard Ranch

39.88944626 Aagard Ridge 


\section{DOLIMITXX}

The DOLIMITXX utility is a database administration program that runs the LIMITXX utility for all topographies in one of the current databases: the map database, the site database, or the offsite database. In other words, in each of the directories MAPSTOPDIR:[xx], SITE\$MAPDIR:[xx], or OFF\$MAPDIR:[xx], LIMITXX can create a limit file that lists all of the DMS (or TAC) files in that directory. The $x x$ in the directory names represents the two-character code of a topography (map layer).

\section{What You Need to Know Before Using DOLIMITXX}

Although DOLIMITXX can be used on the current site or offsite databases, there is little reason to use DOLIMITXX for anything other than the current map database. In fact, the program can create invalid limit files for any topographies that contain model output files (D2, EX, FI, VA, and WD) because all the DMS files with be put in the limit file, not just the one associated with the current case.

DOLIMITXX is also called by the database QA utility, DB_DOLIMITXX.BIS, which runs DOLIMITXX in batch mode for the current map database. See also the DB_DOLIMITXX, LIMITXX, CREATELIMITS, and SEELIMITS utilities.

\section{Creating Limit Files for a Current Database}

To start the utility, enter DOLIMITXX $X$ at the system prompt, where $X$ is one of $\mathbf{M}$ (map), S (site), or $\mathbf{O}$ (offsite). If you do not initially specify the type of current database on which to operate, DOLIMITXX prompts you to specify one as shown in this example.

Example:

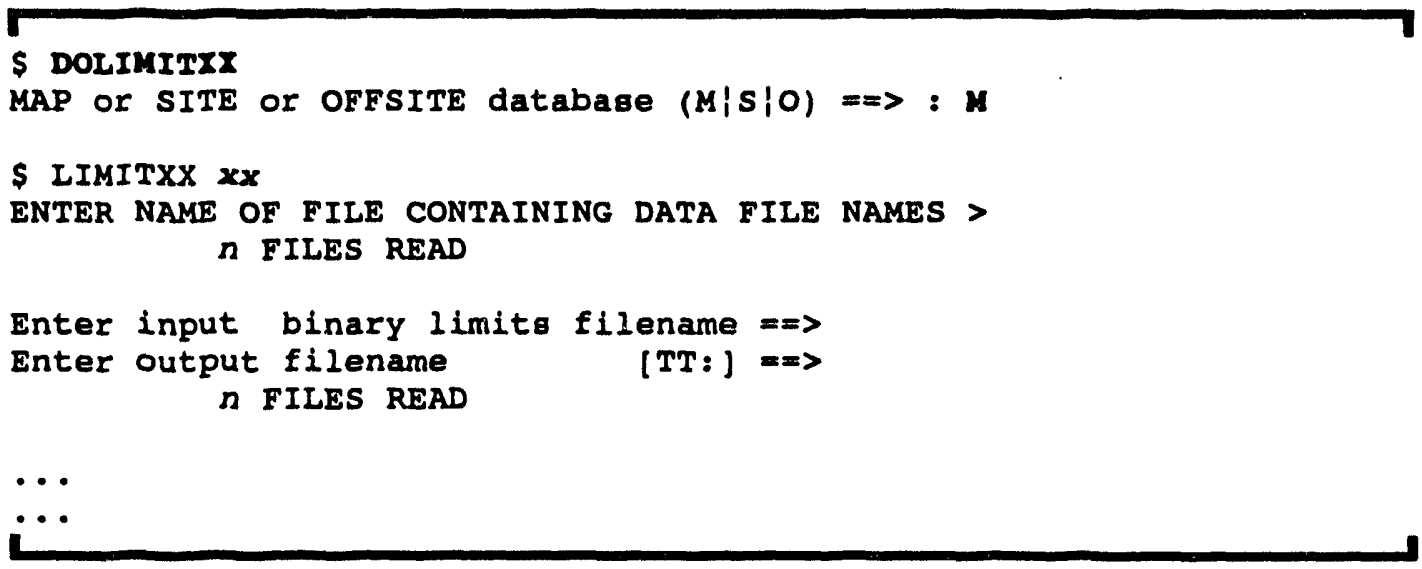




\section{DOSEELIMITXX}

The DOSEELIMITXX utility is a database administration utility that runs the SEELIMITXX utility for all topographies in one of the current databases: the map database, the site database, or the offsite database. In other words, in each of the directories MAP\$TOPDIR:[xx], SITE\$MAPDIR:[xx], or OFF\$MAPDIR:[xx], DOSEELIMITXX can create an ASCII version of the limit file that lists all of the DMS (or TAC) files in that directory. The $x x$ in the directory names represents the two-character code of a topography (map layer).

See also the SEELIMITXX utility.

\section{Creating ASCII Limit Files for a Current Database}

To start the utility, enter DOSEELIMITXX $X$ at the system prompt, where $X$ is one of $\mathbf{M}$ (map), $\mathbf{S}$ (site), or $\mathbf{O}$ (offsite). If you do not initially specify the type of current database on which to operate, DOSEELIMITXX prompts you to specify one, as shown in the following sequence:

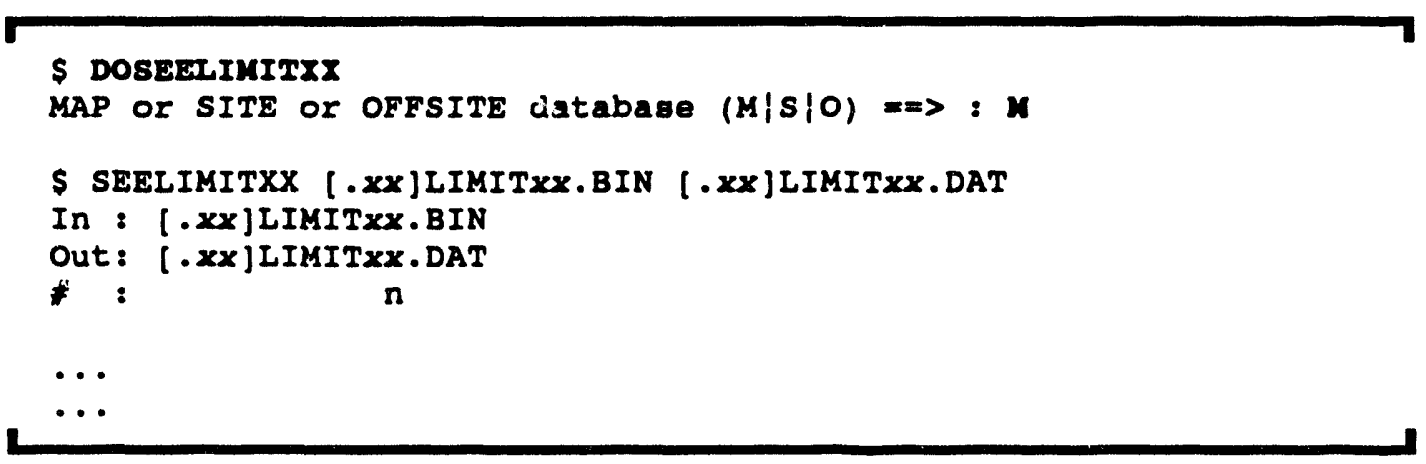




\section{DUPOFFSITE}

The DUPOFFSITE utility is a database administration utility that duplicates sites found under OFFSITESDIR.

\section{What You Need to Know Before Using DUPOFFSITE}

The DUPOFFSITE utility asks you to specify the following items:

Old site

New site

Old directory

New directory
This is the four-character site code of the site that you want to duplicate (used in the directory name).

This is the four-character site code of the new site. This is the top-level directory for the old site. The default is [EVNDB:[OFFSITE]].

This is the top-level directory for the new site.

\section{Duplicating an OFFSITE\$DIR Site with DUPOFFSITE}

To begin the utility, enter DUPOFFSITE at the system prompt. The utility displays the series of prompts shown below.

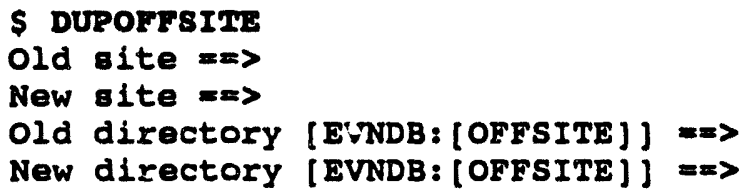

If the directory you wish to specify is EVNDB:[OFFSITE], you may simply press RETURN to accept the default. If you wish to specify another directory for either one, enter the name.

Status messages will scroll by as the site files are copied and/or updated. 


\section{DUPSITE}

The DUPSITE utility is a database administration utility that duplicates a site by copying all the site's subdirectories and updating files which need to be changed.

\section{What You Need to Know Before Using DUPSITE}

The DUPSITE utility asks you to specify the following items:

Old site

New site

Old directory

New directory
This is the four-character site code of the site that you want to duplicate (used in the directory name).

This is the four-character site code of the new site.

This is the top-level directory for the old site. The default is [EVNDB:[INFOMANAGER]].

This is the top-level directory for the new site.

\section{Duplicating a Site with DUPSITE}

To begin the utility, enter DUPSITE at the system prompt. The utility displays the series of prompts shown below.

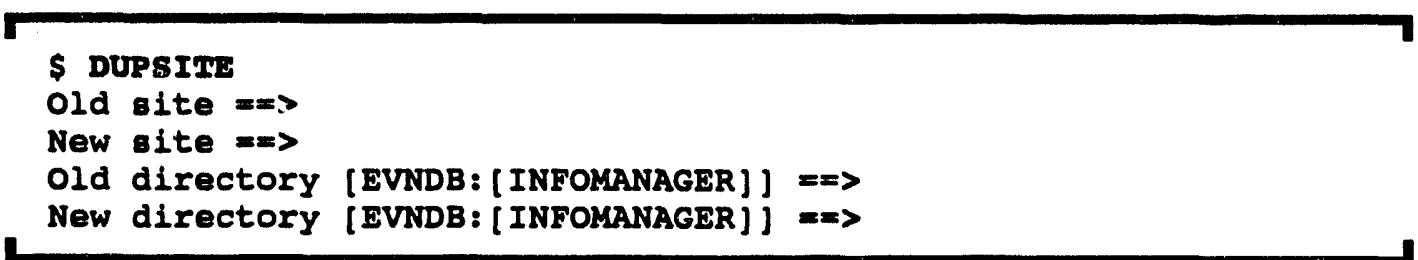

If the directory you wish to specify is EVNDB:[INFOMANAGER], you may simply press RETURN to accept the default. If you wish to specify another directory for either one, enter the name.

Status messages will scroll by as the site files are copied and/or updated.

Note: This utility does not update any SITE.DAT file. 


\section{EVENT}

The EVENT program is responsible for processing data files sent from the onpost system or from the people registration program, LOCATE. Depending on the contents of the files, EVENT can notify IBS users of new data, update shared memory (variables that identify the current operational environment and are accessible to many users simultaneously), and/or declare an event. EVENT also detects and processes ONSITESDIR:MLUPDATE.DAT files to update information in the system master mail list file.

\section{What You Should Know About Using EVENT}

The EVENT program runs in background (batch) mode on the VAX computer, and should be kept running at all times. Under normal conditions, this batch process is already running and does not need to be started independently: the process is started whenever the computer is rebooted via the following path:

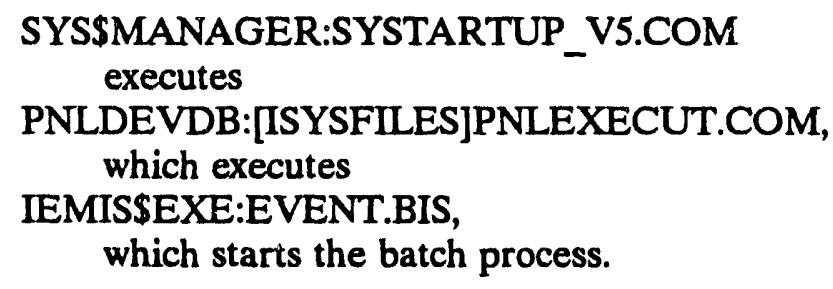

It is important that EVENT always be running. If it is stopped for some reason, the IBS will NOT be notified of any new files from the onpost system. Only a database administrator or system manager is authorized to restart EVENT. To restart it, you must have BYPASS, CMKRNL, OPER, and WORLD privileges.

The EVENT program must be running in the background in order to process several types of files. If EVENT is not running, these files will remain unprocessed until EVENT is started. When EVENT restarts, it processes the waiting files, so changes made while EVENT is not running will NOT be lost.

For a listing of the onpost files that are detected by EVENT, see the description of XFERLIST.DAT in the IBS Data Management Guide. EVENT also detects SITESOFF:P_T_UPDATE.DAT from the LOCATE program.

\section{Using EVENT}

The following instructions explain how to restart the program if it has stopped, how to stop the program, and how to check what the program is doing. 


\section{Restarting the EVENT Program}

To restart the program, just enter EVENT or @IEMIS\$EXE:EVENT.COM at the system prompt. If the program is already running, this has no effect.

\section{Stopping the EVENT Program}

To see if the program is running, check the batch queue for the EVENT job:

\section{\$ SHOW QUEUE/ALL SYS\$BATCH}

If you need to stop the program for some reason, you must remove the job from the batch queue, using the entry number listed with the previous command:

\section{\$ DELETE SYS\$BATCH/ENTRY = en'ry \#}

\section{Getting Information about EVENT Transactions: Log and Flag Files}

The program creates a log file in the INFOSDIR directory. To look at the log file, enter:

\section{\$ TYPE/PAGE INFOSDIR:EVENT.LOG}

However, since EVENT normally just chugs along, saying nothing unless it runs into problems, you will not be able to see what is really happening. You can force the program to be more verbose by creating a flag file:

\section{\$ CREATE IEMISSSYSF:EVENT.DBG <CTRL-Z>}

The program will then record in the log file an informative message about each file received from the onpost system (or the LOCATE program).

Note: You can create the flag file while the program is running-the messages begin when the next onpost or LOCATE file is processed.

Deleting the Flag File. If the program is in verbose mode and many files are sent from LOCATE or the onpost system, then the log file could become quite large. You can either restart the program and delete the log file, or you can turn off verbose mode (while the program is still running) by deleting the flag file:

\section{\$ DELETE IEMIS\$SYSF:EVENT.DBG;*}

Deleting the Log File. If you stop the program, you may want to delete the log file because it just takes up valuable disk space. Enter:

\section{\$ DELETE INFO\$DIR:EVENT.LOG;*}




\section{EXTRACT}

The EXTRACT utility is a nongraphic, text-based program that enables you to select DMS data from your current site database or from an individual DMS data file. Within an area of interest that you specify, you can restrict the extracted data based on decluttering, attributes, object types, and (for databases) topographies. The extracted data are deposited into files within your current default directory. The new DMS files can then be used with other IBS programs.

\section{Extracting Data from a DMS File or Site Database}

To start the utility, enter EXTRACT at the system prompt. The basic interactions of the utility are shown in the following sequence:

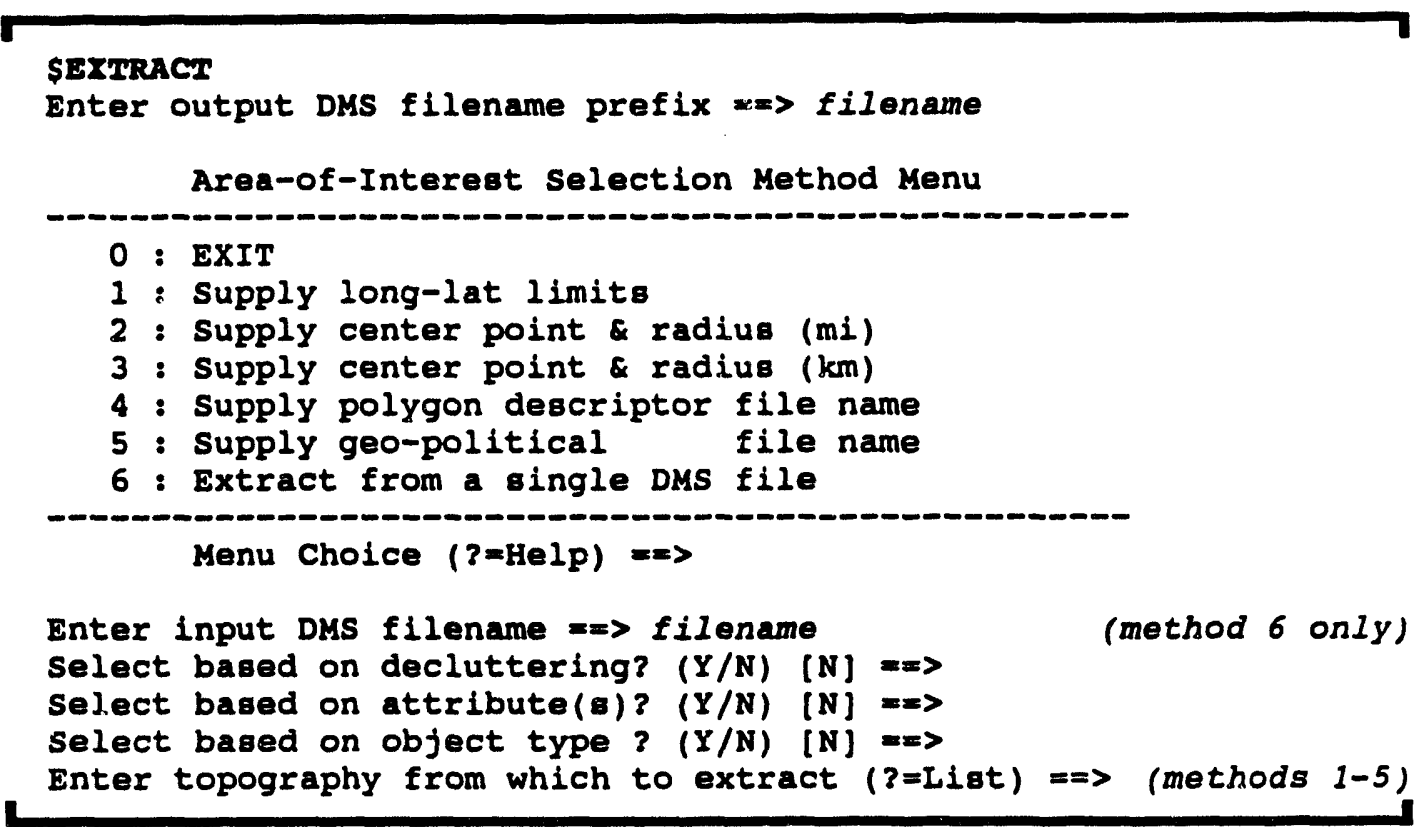

Depending on how you respond to these prompts, EXTRACT asks you for further information, as explained here.

\section{Output DMS File Name Prefix}

EXTRACT begins by asking for an output file name "prefix" for the file to be created (omit the .DMS extension):

$$
\text { Enter output DMS filename prefix }=\Rightarrow
$$

In most cases EXTRACT appends a two-character topographic code to this prefix to construct the final output file name(s). For example, if you enter MYFILE as 
the prefix and then extract roads information (topographic code $=\mathrm{rt}$ ) from the current database, then one resulting data file will be MYFILERT.DMS. When you extract from a single DMS data file, EXTRACT uses the output file name exactly as you enter it and does not append the topographic code.

\section{Selecting an Area to Extract - from a Flle or from a Database}

EXTRACT then displays a menu that offers six basic ways of selecting a geographic area for extraction:

Area-of-Interest Selection Method Menu

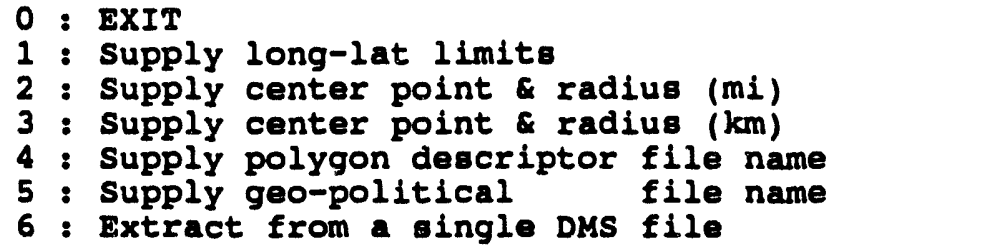

Menu Choice $(z=$ Help $) \Rightarrow$

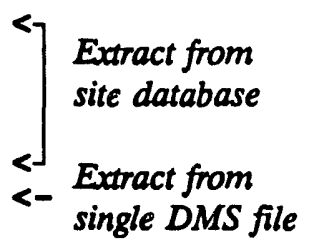

Options 1 through 5 select an area from the current site database. Option 6 selects the data from a single DMS file. Depending on which type of extraction you select, EXTRACT prompts you for the necessary information:

1: long-lat limits: EXTRACT prompts for the minimum and maximum longitudes and latitudes of the area to be extracted.

Enter minimum long, $\max$ long, min lat, $\max$ lat $=>$

2: center point \& radius (mi): EXTRACT prompts for the radius and the longitude-latitude of the center of the area. Give the radius in miles.

Enter center point (long, lat) and radius $==>$

3 : center point \& radius (km): EXTRACT gives the same prompt for the radius and the center of the area. Give the radius in kilometers.

4: polygon descriptor file name: EXTRACT prompts for the name of the file containing the polygon to be used for setting the limits of the extraction:

Enter polygon descriptor filename (.DMS) $\Rightarrow$

The data extracted are based on a rectangle which surrounds the polygon and are therefore not limited precisely to the polygon boundary. The STORE REGION function within MPDISPLAY or GEDIT creates a polygon clip file that could be used as the polygon descriptor file for EXTRACT. 
5: geo-political file name: This type of extraction may be used if you have previously created a geo-political master fle containing Federal Information Processing Standard (FIPS) codes, area names, and area limits. EXTRACT will first asks for the name of the geo-political master file and then whether the selection is to be performed by FIPS code or by area name:

Enter geo-political filename $\Rightarrow$

select by area name? ( $N=$ FIPS code) $(Y / N)[N]=m N$

EXTRACT then prompts for either the FIPS code or the area name for the region to be extracted.

Geo-political master files are sequential ASCII files that are best created or modified by a text editor. Two records exist for each region to be defined within the file. The first record contains the FIPS code in columns 1-10 and the region name in columns 11-30. The second record contains the minimum and maximum longitude and latitude in free field format (separated by commas or spaces).

For example if Washington State and Oregon were to be defined within a geo-political master file, they could appear as follows:

$\checkmark$ column 1

O000327651WASHINGTON STATE

$-125.0,-116.5,45.0,49.0$

O000327652OREGON STATE

$-125.0,-116.5,41.5,46.0$

6 : single DMS file: EXTRACT prompts for the name of the file.

Enter input DMS filename $=>$

What Next? Regardless of how you specify the area, EXTRACT then enables you to further refine the data selection criteria based on any combination of decluttering values, attribute values, and object types.

\section{Select Data Based on Decluttering}

Within the chosen area of interest EXTRACT then enables you to restrict the data extraction based on decluttering:

Select based on decluttering? $(Y / N)[N]=x$

If you answer $\mathbf{Y}$ to base data selection on decluttering, EXTRACT prompts for the decluttering criterion to be used throughout this process:

Enter declutter value $(-1=$ belect by area) $[-1]==>$ 


\begin{tabular}{|c|c|c|}
\hline & $\begin{array}{l}\text { Entry } \\
\text { Values }\end{array}$ & Results \\
\hline $\begin{array}{l}\text { Normal decluttering } \\
\text { range: }\end{array}$ & $1-30$ & $\begin{aligned} 1 & =\text { no decluttering (considers all data) } \\
30 & =\text { maximum decluttering (considers least amount of data) }\end{aligned}$ \\
\hline Default entry: & -1 & $\begin{array}{l}\text { EXTRACT automatically calculates a declutter value based } \\
\text { on the size of the area being extracted. The calculated value } \\
\text { is displayed in a screen message. }\end{array}$ \\
\hline
\end{tabular}

Typically, a value of 23 or 24 is appropriate for an area the size of an average state or values of 26 or 27 is appropriate for multiple states or for the entire United States. To use the value calculated by EXTRACT, just press RETURN.

\section{Select Data Based on Attribute(s)}

EXTRACT then enables you to restrict the extracted data items to those with specific attribute values:

Select based on attribute(8)? (Y/N) [N] $=>$

If you answer $\mathbf{Y}$ to base data selection on attributes, EXTRACT prompts for the attribute selection criteria:

Enter attribute selection command $(z=\mathrm{Help}) \Rightarrow$

For the "attribute selection command," enter a Boolean logical expression that combines attribute codes and the following operators and symbols:

\begin{tabular}{|c|c|c|}
\hline Operator / Symbol & $\begin{array}{l}\text { Functional } \\
\text { Description }\end{array}$ & Example \\
\hline $\begin{array}{ll}\text { A } & \text { [caret] } \\
\text { V } & \text { [capital Vee] } \\
- & \text { [hyphen] } \\
: & \text { [colon] }\end{array}$ & $\begin{array}{l}\text { logical AND } \\
\text { logical OR } \\
\text { logical NOT } \\
\text { range specifier }\end{array}$ & $\begin{array}{l}a \hat{a} b \\
a V b \\
-a \\
a: b\end{array}$ \\
\hline ; [semicolon] & $\begin{array}{l}\text { major;minor } \\
\text { code separator }\end{array}$ & $1 ; 10$ \\
\hline $\begin{array}{ll}\text { ' } & \text { [comma] } \\
\text { (left parenthesis] } & \text { [right parenthesis] }\end{array}$ & $\begin{array}{l}\text { item separator } \\
\text { grouping }\end{array}$ & $\begin{array}{l}a, b, c \\
a,(b V c)\end{array}$ \\
\hline
\end{tabular}

For example: $6 ; 13^{\wedge}(4 ; 5: 4 ; 100)$ indicates all data items having an attribute with a Major code of 6 and a Minor code of 13 AND also having an attribute with a Major code of 4 and a Minor code of 5 to 100 (inclusive). 


\section{Select Data Based on Object Type}

EXTRACT then enables you to restrict the extracted data items to certain types of objects:

Select based on object type $?(Y / N)[N]=3$

If you answer $\mathbf{Y}$ to base data selection on object type, EXTRACT displays this menu of object types:

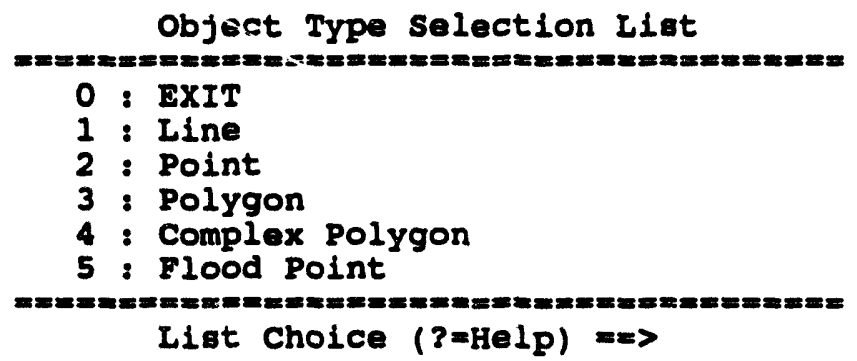

To select one or more object types for extraction:

1. Enter the number corresponding to an object type.

$A$ " = >" symbol marks each selected object type. To cancel and unmark the selection, just enter the number again.

2. After all desired object types are marked, enter 0 to exit.

\section{Extracting from a Single Flle}

If you chose to extract data from a single file (option 6), EXTRACT extracts the data based on any selection criteria you entered for decluttering, attributes, or object types.

For example, if you wanted to extract only current state highway data (attribute $5 ; 56)$ from a single roads-and-trails data file named ALLROADRT.DMS, you would enter the following sequence: 
Example: Extracting from a Single File

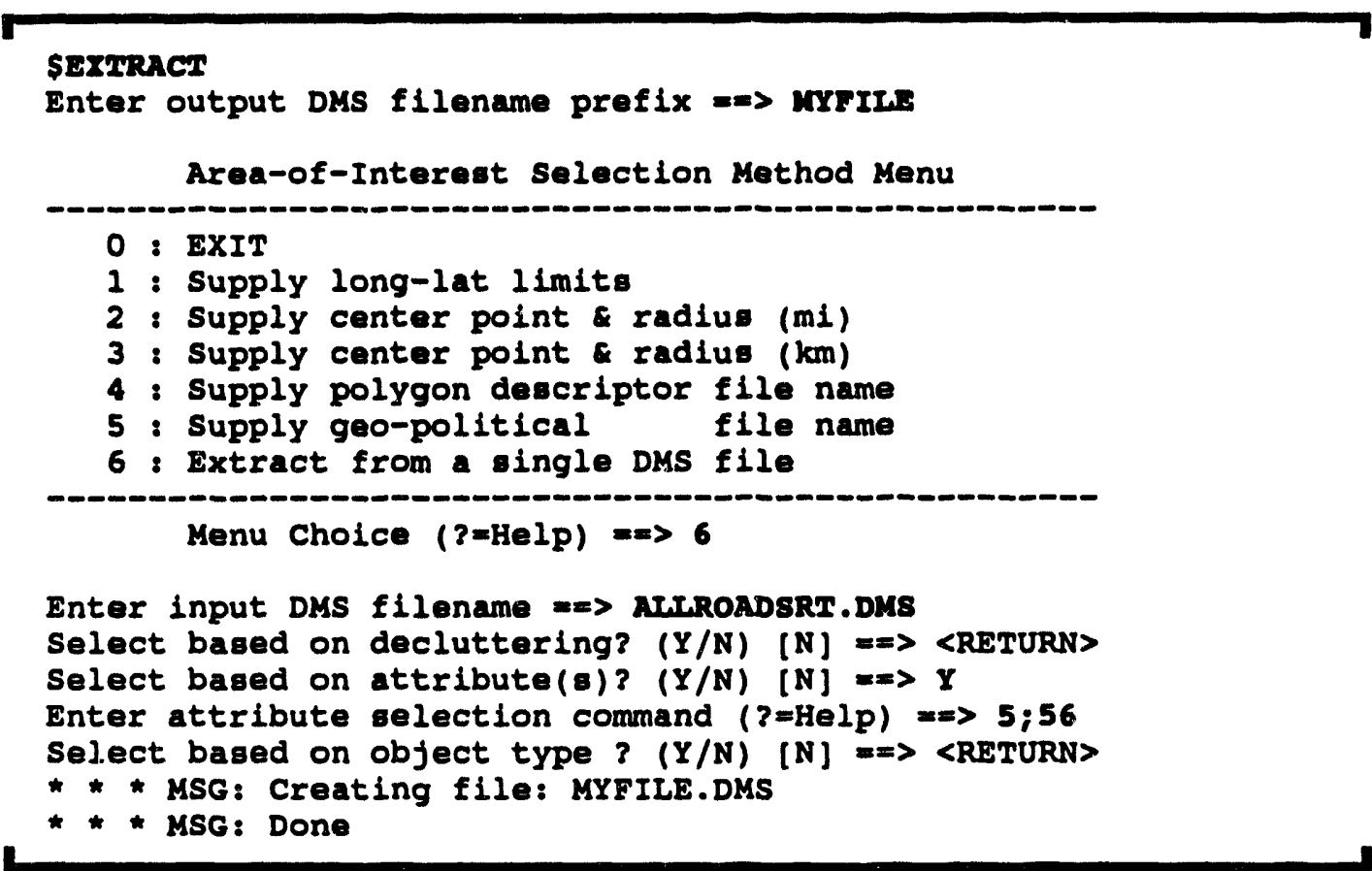

The result would be a single file, MYFILE.DMS, which would contain vector data describing state highways. In this case, EXTRACT does NOT append a twocharacter topographic code to the output file name.

\section{Extracting from the Current Site Database}

If you choose to extract data from the current site DMS database, EXTRACT gives you the opportunity to identify topographies that you want to include:

Enter topography from which to extract $(?=$ List) $=>$

To select one or more topographies for extraction, use one or both of the following methods:

Entering topography codes

1. At the prompt, enter a two-character topographic code.

The prompt repeats. Repeat step 1 for each topography that you want to include.

2. To continue, press RETURN at the prompt without typing any code.

EXTRACT then extracts the specified topographies, limited by any criterion you specified for decluttering, attributes, and object types. 
Selecting from the topography list

1. At the prompt, enter ? to see a list of topographies:

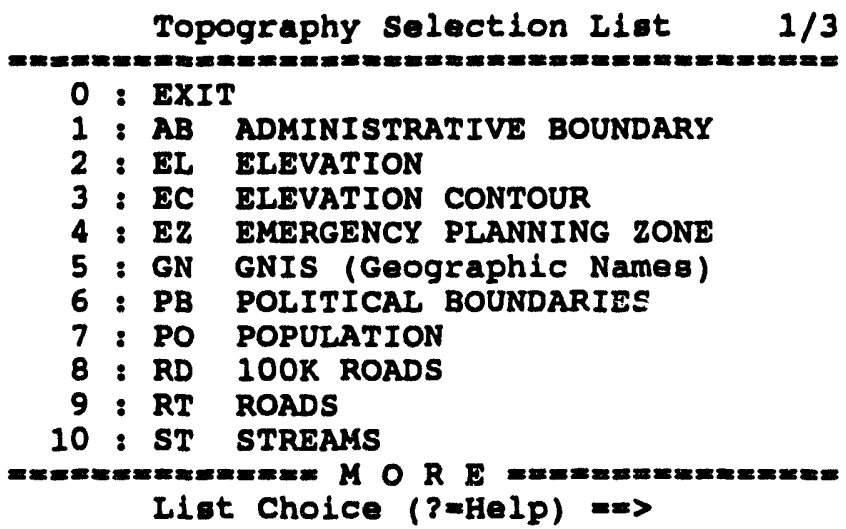

2. Enter the number corresponding to a topography on the list.

$A$ " $=>$ " symbol marks each selected topography. To cancel and unmark the selection, just enter the number again. To see more of the list, enter $\mathbf{D}$ (for down). To get help with list choices, enter ?.

3. After all desired topographies are marked, enter 0 to exit.

The original prompt for entering a topography repeats.

4. To continue, press RETURN at the prompt without typing any code.

EXTRACT then extracts the specified topographies, limited by any criterion you specified for decluttering, attributes, and object types.

For example, you would enter the following sequence if you wanted to extract elevation contour (EC) and roads (RT) data within a 20 -mile radius of 100 degrees west longitude and 42 degrees north latitude, conforming to a decluttering value of 23 (or greater): 
Example: Extracting from the Current Site Database

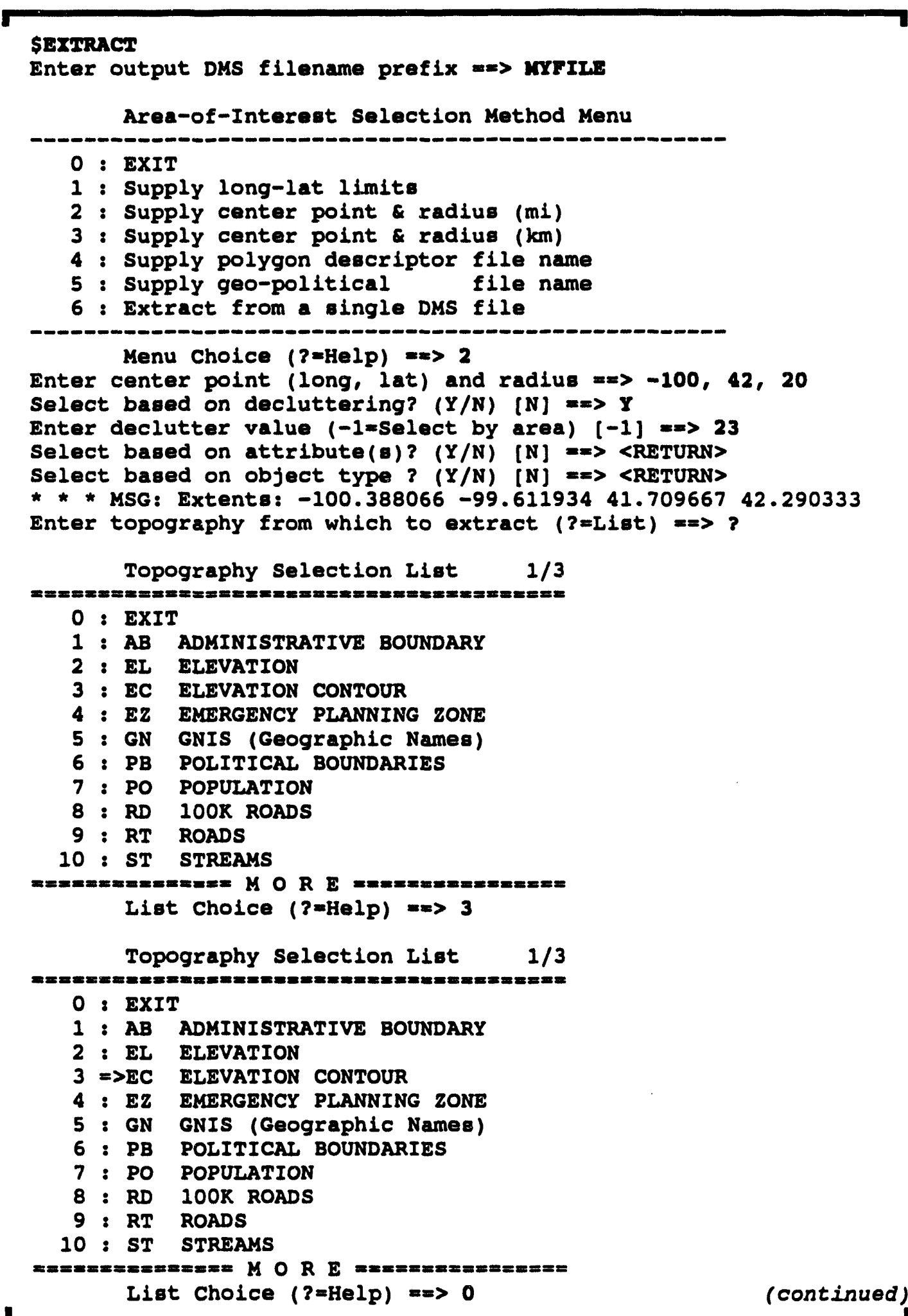


Example: Extracting from the Current Site Database (continued)

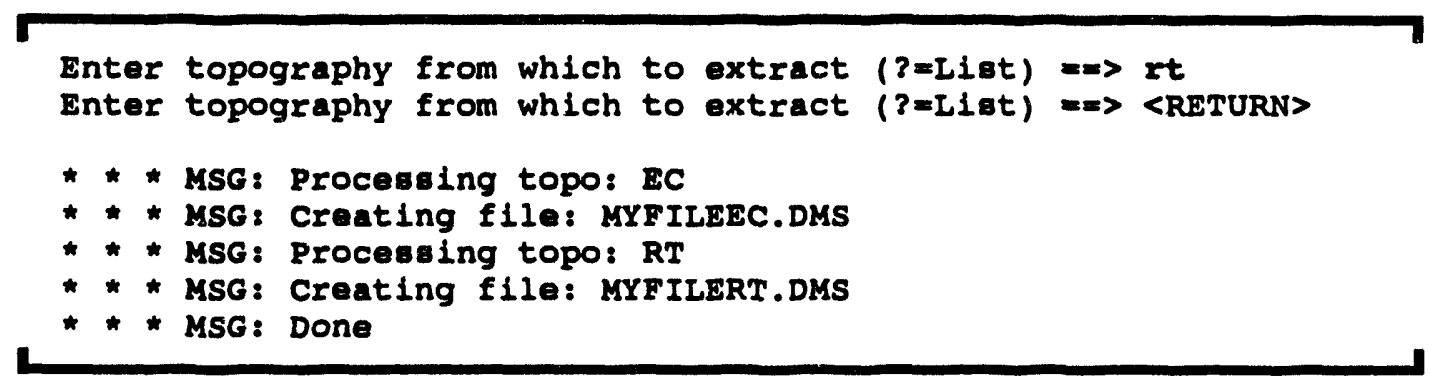

EXTRACT would then create files MYFILEEC.DMS and MYFILERT.DMS, which would contain the elevation contour and roads data for the desired region. Notice that EXTRACT appends the two-character topographic codes to your original output file name prefix. 


\section{FIXATTS}

The FIXATTS utility is a system upgrade program that combines two IBS attribute dictionary files into a single attribute dictionary, flagging any attribute major/minor code combinations that had different descriptions (or other information) in the two original attribute dictionaries. You would then need to edit the resulting file to resolve the differences for duplicated attributes.

\section{What You Need to Know Before Using FIXATTS}

The IBS attribute dictionary files come in two formats: a binary format (.BIN) used by most IBS map-related programs and an ASCII text format (.BIX) used for viewing, editing, and reporting attribute information. You may specify either type of attribute dictionary file, but if you use .BIN files, FIXATTS first translates the .BIN files to .BIX files by calling the ATTXASC utility.

\section{Merging Two Attribute Dictionary Files with FIXATTS}

To start the program, enter FIXATTS at the system prompt. As shown in the following example, FIXATTS prompts you for the input filenames of the two attribute dictionaries to use, and the output filename to use.

Example:

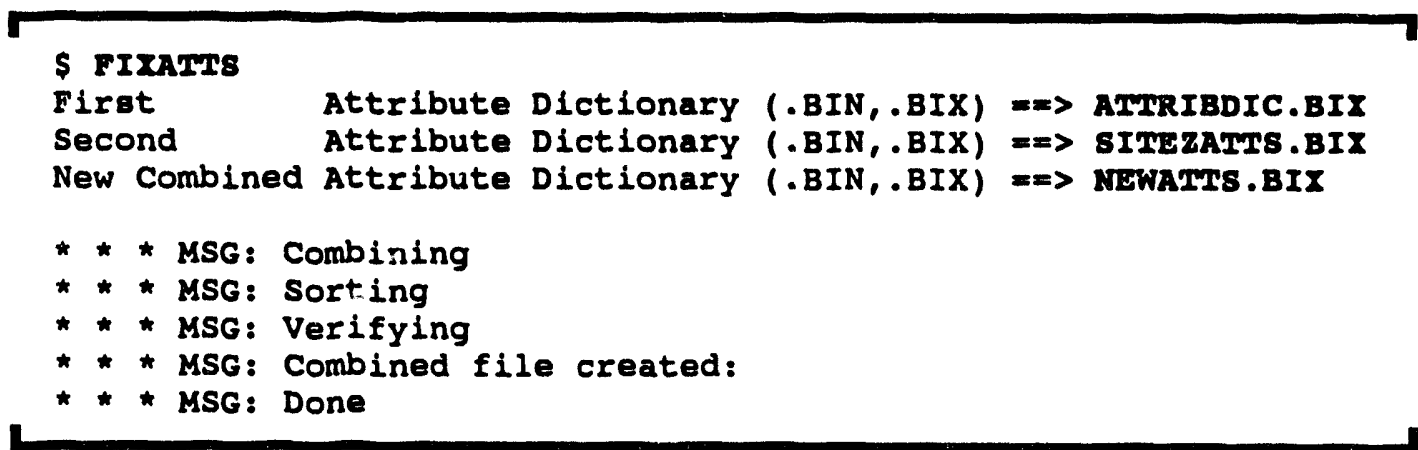

The program then displays a series of processing and status messages such as those shown above. If you use the .BIN files, you will also see several messages from the program ATTXASC as the .BIN files are first converted to .BIX files.

Watch for status messages that resemble this one:

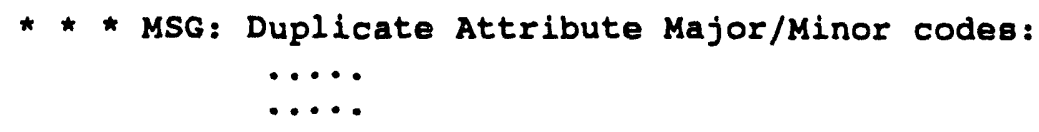


These messages indicate that you will need to edit the resulting attribute dictionary to resolve the conflicting Major/Minor code. You must either remove one of the conflicting codes, or merge the two into a single item by using a text editor such as the VAX ediior, EDT. If you need to convert the final corrected attribute dictionary from ASCII back into binary format for use by IBS, use the ASCXATT utility. 


\section{FIXCNX and FIXCNX.BIS}

The FIXCNX utility is a database administration utility that creates a new Case Index file (CASEINDEX.DAT) for one user's site. This utility is also called from FIXSITEDB. FIXCNX.BIS runs FIXCNX for all sites on the system.

\section{Creating a New Case Index File for a Site}

Before starting FIXCNX, move to the site directory to be updated:

$$
\text { \$ SET DEFAULT disk: [user.sITEs.site] }
$$

where disk is the disk device, user is the user name, and site is the four-character code of the site.

To start the utility, enter FIXCNX at the system prompt.

The case numbers found for the site scroll across the screen as the CASEINDEX.DAT is updated. When the process is complete, a new CASEINDEX.DAT in the current directory will accurately reflect the cases found for the site.

\section{Creating New Case Index Files for All Sites on the System}

To run this utility for all sites on the system, enter @IEMIS\$EXE:FIXCNX.BIS at the system prompt. 


\section{FIXD2MSTR}

The FIXD2MSTR utility is a system upgrade utility that creates an updated index file containing useful D2 data for one site (D2_MASTER_FILE.DAT). This file is used for D2 searches within IBS. One side effect of this utility is that it converts the D2 input files from earlier IBS formats to the current IBS format. This utility should be run for each user site containing D2 cases during upgrade installation.

It is recommended that you have a complete system backup before running this or any conversion utility.

\section{What You Need to Know Before Using FIXD2MSTR}

FIXD2MSTR requires that you supply the following information:

disk
username disk device where the site database is located
the username of the person needing the D2 master
file
site
the four-character code of the site that contains the
D2 cases

\section{Creating a D2 Master File for a User Site}

To run the utility, enter

FIXD2MSTR disk uaername site

at the system prompt, supplying the actual values of disk, username, and site.

Once this utility has been run, the converted files can be found in SITE\$I\$D2.

\section{Creating D2 Master Files for All User Sites}

To run FIXD2MSTR for the entire system (all users and all sites), enter

\section{PIEMIS\$EXE \& FIXD2MSIR}

at the system prompt. This command file creates a batch file (FIXD2MSTR.BIS) in your login directory and displays a series of processing and status messages, but no further input is necessary. To actually fix all D2 master files for the system, change to your login directory, and enter this command at the system prompt:

SUBMIT/NOPRINT/NOTIFY/QUE=SYS\$BATCH/LOG=SYS\$LOGIN：FIXD2MSTR . BIS 


\section{FIXIPS}

The FIXIPS utility is a system upgrade utility that converts Implementing Procedure (IP) data files of an earlier version of IBS to the format used by the current version of IBS-for one IBS user and one site. To do this, it merges each OPIP/IP combination into one odd-numbered file. It then updates the CASEINDEX.DAT, SCEN_INDEX.DAT, and SCEN_TABLE.DAT files to point to the newly merged IP files. This utility should be run for each user site containing IPs during an upgrade installation of IBS.

It is recommended that you have a complete system backup before running this or any conversion utility.

\section{What You Need to Know Before Using FIXIPS}

FIXIPS requires that you supply the following information:

disk disk device where the site database is located
username the username of the person who has IPs for
conversion
site
the four-character code of the site that contains IPs
to be converted

\section{Upgrading IP Data Files for the Current Version of IBS with FIXIPS}

To run the utility, enter

pIXIPs disk usernane site

at the system prompt, supplying the actual values of disk, username, and site.

FIXIPS renames Version 1.03 IP files to have an .OLD file extension. The new odd-numbered files will have .DAT extensions. Once this utility has been run, the converted files can be found in SITESIP.

To run this utility for the entire system (for all users and all sites), enter

QIEMI8\$EXE: FIXIPE.CON

at the system prompt. This command file runs FIXIPS for all users and sites on the system. 


\section{FIXKPL}

The FIXKPL utility is a system upgrade utility. It changes the Known Polygons file to ensure that all user-drawn polygons (Risk Polygons, Damage Assessment Areas, Search/Rescue), all Emergency Planning Zones, and all D2 plume polygons are included in the Known Polygons file for the current offsite database.

This utility should be executed as part of the process to upgrade between versions of the IBS software. For IBS 2.00, this adds additional D2 Plume polygons to support the 10 dosage/concentration levels now available.

\section{Upgrading the Known Polygons File with FIXKPL}

To start the utility, enter @IEMIS\$EXE:FIXKPL at the system prompt. (This command file runs a separate FIXKPL program and specifies that the user-drawn polygons be used. You may also enter FIXKPL, and then enter the two-character topographic codes for all the user-drawn polygons: RP, SR, DA.)

Names of Emergency Planning Zones. Each Emergency Planning Zone (EPZ) has a five-character code that is used in this file. FIXKPL attempts to calculate it using the following process:

1) If the old version of the file included the EPZ, use the code from that file.

2) Each EPZ polygon may have a text attribute which is that EPZ's code. This string will be used by FIXKPL.

3) Each EPZ may have a text string that will be drawn inside that EPZ when text display is enabled. FIXKPL will search for such a text string to use as the EPZ name.

4) If none of these exist, a name is created based on the Krown Polygon number: EZ\#\#\# where \#\#\# is the Known Polygon number. 


\section{FIXLIMITS}

The FIXLIMITS utility is a data file modification utility that updates the file limits contained in a DMS file header.

\section{Updating the Limits in a DMS File Header}

To start the utility, enter FIXIIMITS at the system prompt. The utility then prompts for the input file name as shown below.

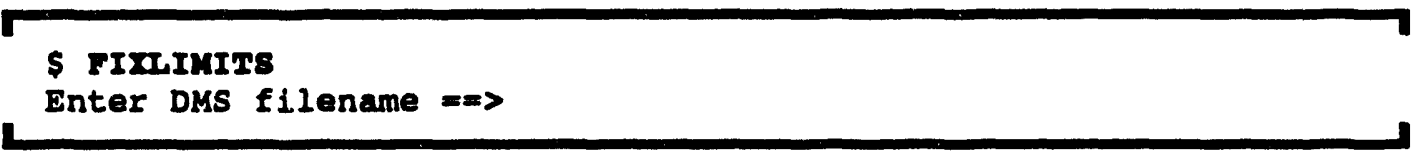

Enter the filename to update. The utility then presents a menu for updating the limits in the header:

Example:

\begin{tabular}{|c|c|c|c|c|c|c|c|}
\hline & & Iimit uI & date menu ( & 226) & & & \\
\hline $\begin{array}{l}0 \\
1 \\
2 \\
3 \\
4\end{array}$ & $\begin{array}{l}: \\
: \\
: \\
:\end{array}$ & $\begin{array}{l}\text { EXIT } \\
\text { Header: } \\
\text { Actual: } \\
\text { Better: } \\
\text { User? : }\end{array}$ & $\begin{array}{l}-112.365131 \\
-112.365131 \\
-112.37\end{array}$ & $\begin{array}{l}-112.340595 \\
-112.340595 \\
-112.34\end{array}$ & $:$ & $\begin{array}{l}40.310147 \\
40.310147 \\
40.31\end{array}$ & $\begin{array}{l}40.363203 \\
40.363203 \\
40.37\end{array}$ \\
\hline
\end{tabular}

You can update the header limits by selecting the data listed in option 2,3, or 4 in the menu above.

\begin{tabular}{lll}
\multicolumn{1}{c}{ Menu Choice } & Result \\
\cline { 1 - 2 } O & EXIT & Leave the current DMS file unchanged and quit the utility. \\
1 & Header & Leave the current DMS file unchanged and end. \\
2 & Actual & Use the actual limits of the data, and end. \\
3 & Better & Use values rounded from actual, and end. \\
4 & User? & Shows prompts for you to enter custom longitude-latitude extents.
\end{tabular}

For IBS Software Developers. The numbers listed in parentheses at the top of the limit update menu represent the number of strings and the number of points, parameters of interest mostly to IBS software developers. 


\section{FIXLOG}

The FIXLOG utility is a database administration utility that fixes corrupted log journal files by setting the record count value in the first record to be the actual number of records in the file plus one. The log files record various IBS reports exchanged by the offpost and onpost systems, messages, and other IBS activities. The IBS program uses the log files to create "Message Board" reports.

\section{Fixing the Log Journal Files with FIXLOG}

To start the utility, enter FIXIOG at the system prompt. No further interaction is necessary.

The log header record counts will be reset to the correct values. 


\section{FIXMAPDB}

The FIXMAPDB (Fix Map Database) utility is a system upgrade program for converting pre-IBS 1.0 map databases to IBS 1.0 format.

Required Privileges:

- System Privilege (SYSPRV)

File input:

- PNLDEVDB:[ISYSFILES]SETUPDB.COM TEMPLATE

- PNLDEVDB:[ISYSFILES]TOPODESC.FIL_TEMPLATE

File input/output:

- PNLDEVDB:[ISYSFILES]ASS DISK LOG.COM

- PNLDEVDB:[ISYSFILES]ASS MAP SYM.COM

- PNLDEVDB:[ISYSFILES]ASS_MAP_LOG.COM

File output:

- SETUPDB.COM

- TOPOCTL.FIL

- TOPODESC.FIL

Interactlve input:

- $\mathbf{Y}$ or $\mathbf{N}$ responses to three prompts asking whether to create files

Example:

1. First, move to the top directory of the map database.

\$ 8ET DEFAULT disk:[directory]

2. Run the FIXMAPDB utility.

\$ FIXMAPDB (or eIEUIS\$EXE:FIXNAPDB)

a. Answer $\mathbf{Y}$ to the prompt,

Create SETUPDB.COM? $(Y \mid n)=>$ : Y

This creates the setup file which assign logical names pointing to the map database. If necessary, it updates PNLDEVDB:[ISYSFILES]ASS DISK_LOG.COM, adding a disk logical to point to the map database disk. If necessary, it also updates PNLDEVDB:[ISYSFILES]ASS_MAP_SYM.COM, adding symbols to setup IBS to use the map database. 
b. Answer $\mathbf{Y}$ to the prompt,

Create TOPOCTL.FIL? $(Y \mid n)=\infty: Y$

This creates the topography control file which points to the icon control file and the cover maps.

c. Answer $\mathbf{Y}$ to the prompc,

Create TOPODESC.FIL? $(Y \mid n) \Rightarrow=$ :

This creates the topography description file which defines the map layers (topographies) available to IBS users. If necessary, it updates PNLDEVDB:[ISYSFILES]ASS_MAP_LOG.COM, adding logical names for topographies in your map database which are not part of normal map databases.

If you answer $\mathbf{Y}$ to create the TOPODESC.FIL, you will see an additional prompt:

Retain order of file? $(Y / n)=\Longrightarrow$

- Answer $\mathbf{Y}$ to have the resulting file in the same order as the original. OR

- Answer $\mathbf{N}$ to have the resulting file sorted alphabetically.

If any of the files in PNLDEVDB:[ISYSFILES] are updated, they will be invoked, making the map database accessible upon completion of FIXMAPDB. 


\section{FIXMSTRDIR}

The FIXMSTRDIR utility is a system management program that builds a master directory list of the valid disk names, user names, and site names on the system (MASTER DIRECTORY.BIN). This list is used as a source of online "help" information for IBS capabilities that transfer or copy model case files and site files between users. These capabilities are available as COPY CASE OR SITE FILE functions under SETUP in the IBS program.

\section{What You Should Know Before Using FIXMSTRDIR}

The FIXMSTRDIR program uses as its primary input a file named ALLSITE.DAT, which is a list of the locations of all user sites known to the system. Therefore the ALLSITE.DAT file must be up to date for FIXMSTRDIR to be successful. The ALLSITE utility creates IEMIS\$SYSF:ALLSITE.DAT.

System managers should use the ALLSITE utility and then FIXMSTRDIR whenever they add a new user to the system so that the user and the user's data will be recognized as options when IBS users copy or transfer case and site files.

\section{Building a Master Directory List of Disk, User, and Site Names with FIXMSTRDIR}

To start the utility, enter RUN IEMISSEXE:FIXMSTRDIR at the system prompt. FIXMSTRDIR displays the master directory list and does not normally require any further interaction. For example:

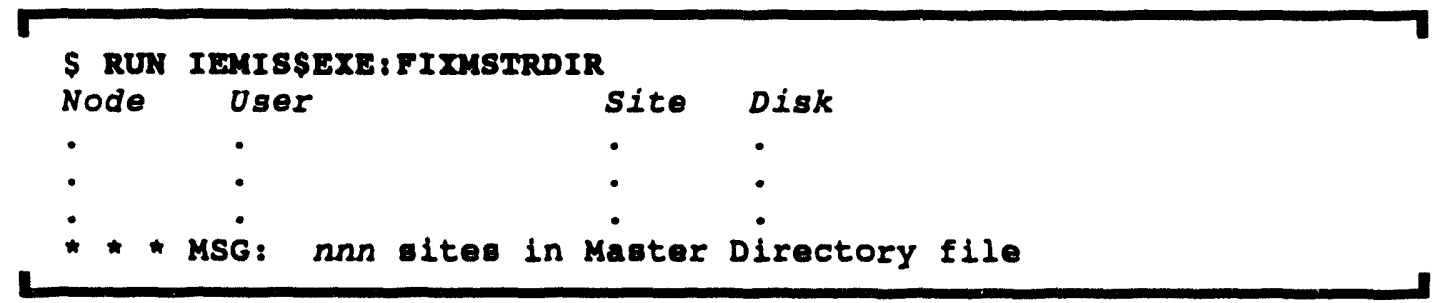

\section{Results of Using FIXMSTRDIR}

FIXMSTRDIR writes a set of six help records into a new version of the master directory list for each site reference found in IEMIS\$SYSF:ALLSITE.DAT. For each site record processed, FIXMSTRDIR examines the contents of the user's site directory. 


\section{FIXOFFON}

The FIXOFFON utility is a system upgrade program that repairs the IEMISSSYSF:OFF_TO_ON.DAT file to match those sites specified in Infomanager's SITE.DAT file. FIXOFFON would be used by an Infomanager or database administrator in the unlikely event that these files do not match. (The OFF_TO_ON.DAT file maps the onpost site names to the operational offpost site names.)

\section{What You Need to Know Before Using FIXOFFON}

FIXOFFON adds any sites that are specified in the SITE.DAT file but not found in the OFF TO ON.DAT file, and removes any extra records that do not pertain to any of Infomanager's sites. If FIXOFFON finds Infomanager sites that have no corresponding record in OFF_TO_ON.DAT, you must be able to enter the correct four-character onsite site code.

If no OFF_TO_ON.DAT file is found, a new one is created.

\section{Fixing the OFF_TO_ON.DAT File}

To start the utility, enter FIXOFFON at the system prompt.

If any sites are found in Infomanager's SITE.DAT that do not have corresponding record in the OFF_TO_ON.DAT file, the program displays a prompt similar to the following:

Enter Onsite code corresponding to INFOMANAGER site [code] $=\Rightarrow$

Enter the four-character "onsite code" that identifies the onpost depot that corresponds to the operational site specified in the prompt.

If any records are found in the OFF_TO_ON.DAT file that do not have a corresponding site in Infomanager's SITE.DAT file, the program displays a prompt similar to the following:

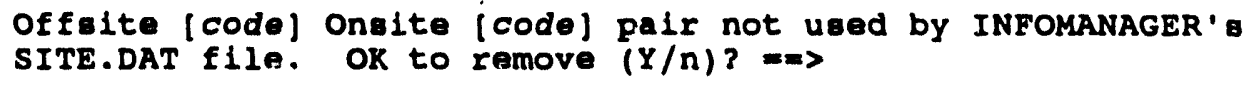

Enter $Y$ or press RETURN to remove the specified record from the OFF_TO_ON.DAT file. Entering $\mathbf{N}$ retains the specified record in the OFF_TO_ON.DAT file. 


\section{FIXOFFSITE}

The FIXOFFSITE utility is a system upgrade utility that supports the upgrade of the IBS from an earlier version to the current version. The program updates the directories under EVNDB:[OFFSITE $x \times x x$ ] to add several new directories: EVNDB:[OFFSITExaxx.SEND] and EVNDB:[OFFSITExacox.FROM]. This automatically updates all OFFSITE directories found.

\section{Updating [OFFSITE.xoxx] Directories for the Current Version of IBS}

To start the utility, enter FIXOFFSITE at the system prompt. No further interaction is necessary.

The program displays a series of processing messages, indicating the progress of the program and which OFFSITE directories are being updated. 
FIXPD

The FIXPD utility is a system upgrade utility that converts the PERMITI.DAT permission file from the format used by a previous version of the IBS to the format used by the current version of the IBS. This utility should be used only at upgrade installation.

\section{Upgrading the IBS Permissions File with FIXPD}

To start this utility, enter FIXPD at the system prompt. No further interaction is necessary.

When the PERMITI.DAT file is converted, a message similar to the following appears:

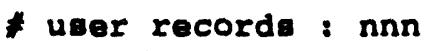




\section{FIXPROT}

The FIXPROT utility is a system upgrade utility that sets file and directory protections for all IBS files and the current map database. This utility requires SYSPRV privilege and is expected to be run only at installation.

\section{Upgrading IBS File and Directory Protections with FIXPROT}

To start this utility, enter FIXPROT at the system prompt. No further interaction is necessary.

The file and directory protections are upgraded for the current version of IBS. 


\section{FIXRM}

The FIXRM utility is a system upgrade program that creates a batch file to update IBS Facility and Resource data based on changes made in the icon dictionary (ICON.CTL) file for a new version of the IBS. The batch file updates the Facility and Resource data so that the correct icons are used from the new ICON.CTL file in the new version of the IBS. The utility is used once during a sequence of system update/installation activities.

\section{What You Need to Know Before Using FIXRM}

You must know the name and location (complete pathname) of the "old" ICON.CTL file being used by the previous (old) versiori of the IBS software. If the old ICON.CTL file is MAPSICONS:ICON.CTL, you must also use the file version number to distinguish the file from the new ICON.CTL file, which is installed as a new version of MAP\$ICONS:ICON.CTL.

\section{Preparing an Update Batch File with FIXRM}

To start the utility, enter @IEMISSEXE:FIXRM at the system prompt. You are then prompted for the name of the "old" ICON.CTL file.

Example:

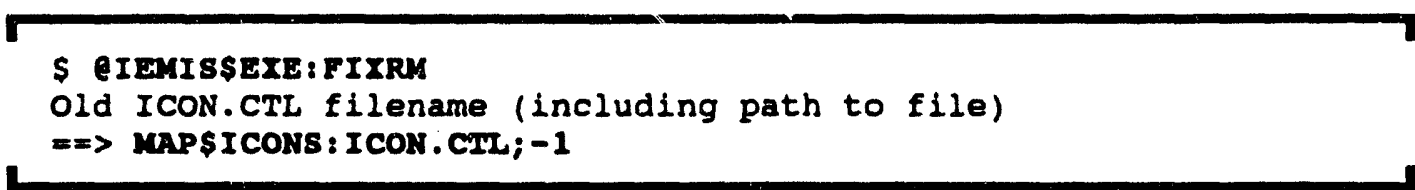

Notice that you must specify the entire path for the file. In the example shown here, the previous icon dictionary file was MAP\$ICONS:ICON.CTL, so the version number -1 was used to signify a file version one less than the most current version, which would be the new icon dictionary file.

The utility then creates the file FIXRM.BIS in your login directory. You may wish to edit this file to remove sites that you do not wish to have updated, or to add additional sites. The format for each command line in FIXRM.BIS is

\$ FIXRM Disk Site Usemame OldIconCtlFilename 


\section{Updating Facility and Resource Data to Use Correct Icons}

To update the Facility and Resource data, enter

$$
\text { SUBMIT/NOPRINT/NOTIFY/QUEUE=SY8\$BATCB/LOG=SYS\$LOGIN: FIXRM.BIS }
$$

at the system prompt from your login directory. 


\section{FIXRSP and FIXRSP_BIS}

The FIXRSP utility is a system upgrade utility that fixes the MESORAD model's .RSP files for one user site. The FIXRSP BIS utility creates a batch file for fixing all the MESORAD .RSP files on the system. The .RSP files are master control files for MESORAD case input.

\section{Updating MESORAD .RSP Files for One User Site with FIXRSP}

To start this utility, enter the following command at the system prompt:

\section{@IEMIS\$EXE:FIXRSP site1 site2 directorypath1 directorypath2}

where site1 and site 2 are (normally) the same four-character code of the site to be fixed, and directorypath1 and directorypath 2 are (normally) the same full directory name including disk and username.

For example, the following would fix the RSP files for the Infomanager site TEST.

S EIEMIS\$EXE:FIXR8P TE8T TEST EVNDB: [ INFOWANAGER] EVNDB \& [ INTOUANACER]

\section{Updating MESORAD .RSP Files for All Users on the System}

To start the utility, enter FIXRSP BIS at the system prompt. The program displays a series of processing and status messages, but no further interaction is necessary.

The program creates the batch file FIXRSP.BIS in your login directory.

To actually fix all the MESORAD .RSP files and complete the job, change to your login directory, and enter this command at the system prompt:

SUBMIT/NOPRINT/NOTIFY/QUE=8YS\$BATCE/LOG=SYS\$LOGIN：FIXRSP.BIS 


\section{FIXSETUP}

The FIXSETUP utility is a system upgrade utility that supports the upgrade from a previous version of IBS to the current version. The program updates the SETUP.COM files in all your own sites or in all sites on the system.

You must have system privilege to fix all sites on the system.

\section{Updating the SETUP.COM Files for the Current Version of IBS}

To start the program, enter FIXSETUP at the system prompt. FIXSETUP displays the following prompt:

Do All ates on eystem? $(y / N)=x$

Enter $\mathbf{Y}$ to fix all sites on the system, or $\mathbf{N}$ to fix only your own sites.

FIXSETUP then displays a series of processing messages, indicating the progress of the program and which sites are being updated. No further interaction is necessary. 


\section{FIXSITEDB}

The FIXSITEDB (Fix Site Database) utility is a system upgrade program for converting pre-IBS 1.0 site databases to IBS format. Each IBS user must run the utility to convert private site databases. The following occurs:

1. Create missing directories. These are the same directories created by the NEWSITE utility.

2. Move TAC files from [.INPUT.MAPS]. They are gridded cell data used by the OSPM and MESORAD models.

3. Delete setup and control files in [.INPUT.MAPS]. The setup file has been completely rewritten and moved, whereas the control files are no longer used.

4. Delete unused directories. These include directories for the old FLOOD and HEAT models, plus the SRFX-91 EMP directory.

5. Delete volatile topographics (DA,EI,RM,RP,SR,WD,WI). These are no longer private but shared topographies under [OFFSITEsite].

6. Delete files in [.INPUT.OFF]. These are no longer private but shared files under [OFFSITE.site], except for the Current Risk Area file, whose format has changed anyway.

7. Create a new SETUP.COM file. This is the replacement for the file deleted earlier (step 3).

8. Create a new CASEINDEX.DAT file. For each model case in the site database, set its corresponding flag in the file. This can be done as a stand. alone activity using the FIXCNX utility (@IEMIS\$EXE:FIXCNX), but you must first be in the correct directory, SITE\$DIR ([user.SITES site]).

\section{Required Privileges:}

- none

\section{File output:}

- [user.SITES site]SETUP.COM

- [user.SITES site]CASEINDEX.DAT

Interactive input:

- Site name

- Map Database name 


\section{Notes:}

- All references to "[." really mean "[user.SITES.SITE.".

- Running the utility on an IBS 1.0 site database should cause no problems, but it will replace your existing SETUP.COM and CASEINDEX.DAT files. The new files should be correct, but you may want to verify this.

- Files and directories are not really deleted, just moved to a temporary directory, [.OLD], from which you can delete them later if the converted site database is correct.

\section{Converting Pre-IBS 1.0 Databases to IBS Format}

To start the utility, enter FIXSITEDB at the system prompt.

Example:

1. Run the FIXSITEDB utility.

$$
\text { S FIXSITEDB (Or EIEMIS\$EXE: FIXSITEDB) }
$$

2. Enter the name of the site to convert.

$$
\text { Enter name of site to convert }=m \text { : site }
$$

3. Enter the name of associated map database.

Enter Map Database name [Map] $\Rightarrow>$ : mapdb

The default is the current map database shown in brackets, Map. This is needed when creating the SETUP.COM file so that it knows which map database SETUPDB.COM file to use.

The utility will then proceed to convert the site database. While doing so, a number of informational messages describing progress will appear. They may include the following: 


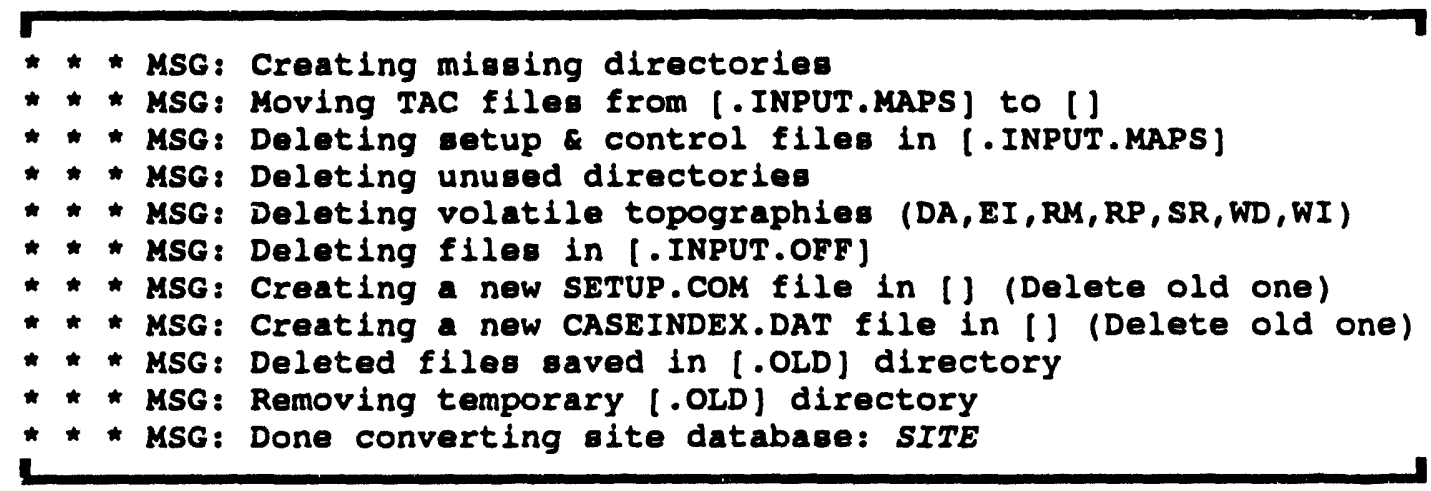

Any error results in one of the following messages:

** ERR: You have not site: SITE
** ERR: Aborting..."




\section{FIXVERB}

The FIXVERB utility is a system upgrade utility that adds necessary verbs to the DCLTABLES, such as MYTAPE. This utility requires SYSPRV and CMKRNL privileges, and is expected to run only at installation.

\section{Upgrading the System DCLTABLES with FIXVERB}

To start this utility, enter FIXVERB at the system prompt. No further interaction is necessary.

Any necessary verbs are added to the DCLTABLES. 


\section{FTPSTART and FTPSTOP}

The FTPSTART utility establishes the modem link tetween some CSEPP onpost and offpost systems (see What You Need to Know... below). IBS users may then send or receive files to or from the onpost system using the local File Transfer Protocol (FTP) software. It is expected that the modem link will be periodically established (perhaps each morning) and disconnected (perhaps each evening). This link must be established before anyone can transfer IBS reports to the onpost system (by using the IBS program's MESSAGE BOARD option, for example). If the link is not established, the reports will NOT be sent between systems.

The FTPSTOP utility disconnects the modem link that was established by FTPSTART. This can be done when the connection is not needed.

\section{What You Need to Know Before Using FTPSTART and FTPSTOP}

FTPSTART and FTPSTOP are specifically set up for using the WIN/tcp software package. If your system has a different transport control protocol (tcp) package, you will NOT be able to use FTPSTART or FTPSTOP. The modem link would have to be established by other means.

You must have special user privileges to use either FTPSTART or FTPSTOP.

\section{Starting the Offpost/Onpost Modem Link with FTPSTART}

To use the utility, just enter FTPSTART at the system prompt. That's all there is to it. You may then see one or more of the following messages:

* * MSG: SLIP IINK running

You will see this message when the link is established (or if the link was already established when you tried to use FTPSTART).

* * MSG: SLIP LINR not running You will see this message before the program attempts to establish the modem link. As it attempts to establish the link, the program also checks every 30 seconds to see whether the link has been established. The program will check up to 20 times over the first 10 minutes, displaying one of the two status messages after each check. If you see 20 lines of the * * MSG: SLIP IINK not running message and never see a * * MSG: SLIP IINK running message, then the link attempt failed.

* * ERR: No privilege to check SLIP IINK You will see this message if you do not have the correct privilege to establish the modem link. 


\section{Stopping the Offpost/Onpost Modem Link with FTPSTOP}

To use the utility, just enter FTPSTOP at the system prompt. That's all there is to it. You may then see one or more of the following messages:

* * MSG: SLIP LINR etopped

You will see this message if the program successfully breaks the link.

* * ERR: SLIP LINR not vtarted

You will see this message if the link has not been established and there is no link to stop.

* * ERR: No privilege to check or stop SIIP IINR

You will see this message if you do not have the correct privilege to break the link. 


\section{GEDIT}

The GEDIT utility is an interactive graphics editor program that enables you to edit and create graphic representations of two-dimensional data. GEDIT requires a graphics workstation. This section covers the following topics.

- Description of the GEDIT Software

- What You Need to Know to Use the GEDIT Software

- Terminology for Graphic Data Structures

- Starting a GEDIT Session

- Using the GEDIT Menu.

\section{Description of the GEDIT Software}

GEDIT is an interactive program that enables you to edit or create graphic representations of two-dimensional data. Graphics input devices are used to manipulate graphic data structures (points, line segments, polygons, etc.) that are stored in and retrieved from the data files of a DMS map database.

GEDIT operates on data files that use Digital Mapping Structure (DMS), the data file structure established for the IBS geo-coded database. GEDIT can be used to customize map data from the IBS site map database to include special information for specific applications.

The GEDIT (graphic map editing) software and the related MPDISPLAY (graphic map display) software were created by Pacific Northwest Laboratory. They contain a library of subprograms that are accessible by other programs for creating, storing, and displaying two-dimensional data.

\section{What You Need to Know to Before Using GEDIT}

This discussion is intended as a guide for new users of the GEDIT software. It contains information on the operation of GEDIT and assumes that you will apply GEDIT to the current IBS database or to another database that is constructed according to IBS standards for graphically represented geographic data. You should be familiar with the graphics input device attached to your terminal. 


\section{For the Typical User of GEDIT}

This documentation covers all the concepts and interactions necessary for using the GEDIT software: terminology for graphic data structures (page 2.102), starting a GEDIT session (page 2.104), and using the GEDIT main menu (page 2.106). The section on terminology defines some terms that are used in the rest of the GEDIT discussion.

Typical users need not be closely concerned with IBS standards and file structures, although review of those standards can provide insight into what and how data is stored.

\section{For the In-Depth User of GEDIT}

In-depth users of GEDIT can find standards and structures for an IBS map database in the IBS Data Management Guide. A procedure for creating icons (graphical figures that always appear the same size) is also described in the IBS Data Management Guide.

\section{Terminology for Graphic Data Structures}

GEDIT operates on data files that are in DMS format. DMS files explicitly associate longitude and latitude values with several kinds of data: points, line segments, strings, polygons, and complex polygons. The data for these structures are stored sequentially, and there is no implicit order in which data appear within the file.

The display of text and graphic icons is also supported in DMS, and attributes may be associated with any data item to further define its characteristics. The following is a description of terms as they are used in this manual:

- A point is a 0-dimensional object that specifies geometric location. One set of coordinates specifies the location of each point.

- A line segment is a 1-dimensional object that is a direct line between two points.

- A string is a connected series of line segments.

- Polygons are areas enclosed by a single geometric boundary that does not cross itself (i.e., a polygon is a closed string).

- Complex polygons are structures that define areas composed of multiple simple polygons. 
The simple polygons that compose a complex polygon are called subpolygons.

- Inclusive subpolygons define areas inside their boundaries.

- Exclusive subpolygons define areas outside their boundaries.

Some GEDIT operations work on the complex polygon as a single entity; other functions allow the manipulation of subpolygons.

- Compasite strings are a series of strings, or X-Y pairs that are connected physically or logically.

For example, a composite string is a series of line segments which may be drawn on a flatbed plotter without removing the pen from the plotting surface. In general, the term composite string refers to the string, polygon, and complex polygon data structures as a set.

- An attribute is a special set of numeric values that describe additional characteristics of data items. For example, an attribute may identify a particular polygon as a lake of a particular kind. Attributes consist of three attribute descriptors: a Major attribute code, a Minor attribute code, and a Parameter value. These values can act as pointers into predefined table files that contain descriptions of each attribute. A major file of this type is the attribute dictionary, which contains a master list of the Major/Minor attribute codes and their definitions in DMS files.

All data structures may have attributes associated with them. For example, the attributes of a point can identify that point as

- an icon seed point: the location of an icon (see Icon, below)

- a text point: the starting location of a piece of text

- a color flood seed point: the starting location of a color flood point

- an item point: a point location that has only attributes without being associated with particular graphics or text.

- Text attributes point to the location of text in a t:ext data file. Any data item can have text associated with it through its attributes. The name of the text data file is stored in the DMS file header, the initial portion of the DMS graphics file.

- Icons are graphic figures that can be associated with a particular data point (called an icon seed point). Icons always appear at a constant viewing size, regardless of the map scale. They provide a consistent means of representing hospitals, airports, schools, or any topographic feature that occurs repeatedly. 
A predefined icon dictionary (icon control file) includes attribute codes, a descriptor name for each icon, and the names of files that contain the graphical descriptions, in standard DMS format, of each icon.

\section{Starting a GEDIT Session}

Start a GEDIT session by entering GEDIT at the system prompt. For example:

\$ GEDIT <RETURN>

You will then specify the input files that are to be edited (or created). All of the files that are to be used or updated during a GEDIT session must be specified at the beginning of the session. The files may include an icon dictionary (icon control file) and one or more data files.

\section{Using an Icon Control File}

GEDIT begins processing by looking for an icon control file. The icon control file contains the current list of available icons and the data files that are needed to plot each icon. Generally, the System Manager will have already defined a system default icon control file that the IBS software uses unless you specify otherwise. If no icon control file has been defined, GEDIT displays a message to let you know and then continues processing.

If you wish to use a different set of icons (for example, icons that are not designated in the system default icon control file), then you must redefine the icon control file.

\section{Specifying Input Flles}

GEDIT now begins prompting for the input files (for example, topography files containing map data that you have stored using the MPDISPLAY software).

Enter DMS flle name $A *=\Rightarrow$

To enter input file names:

1. Type the name of an input file at the prompt, and press RETURN.

If the file you specify does not currently exist, GEDIT will open a new data file for you. If you press CTRL-Z instead of entering a file name, GEDIT will return you to the system prompt.

The first file you specify will be the default file for subsequent GEDIT operations: GEDIT INSERT functions will apply only to this default file (until 
you change the default file through the MODIFY DEFAULTS option on the GEDIT main menu).

2. Repeat step 1 for up to 10 input files.

Up to 10 files may be edited simultaneously; however, insertions only apply to one file at a time, the default file. To edit a region that you have stored using the MPDISPLAY software, enter the name of each topography file that you need to edit. For example:

Enter DMS file name $\quad 1==>$ mawaII2RI <RETURN>

The input files specified are the Roads and Trails (RT) topography file and the Water Bodies (WB) topography file for a region named HAWAIII.

3. After entering the last file name, press CTRL-Z.

This indicates to GEDIT that all files have been specified. GEDIT will display the input file data (if there is any) on the graphics screen. Next to the data display will be the main menu that lists graphic editor options for altering or adding to the displayed data.

\section{Background: Screen Coordinates of Input Files}

If you create a new input file (with no old input files in the same GEDIT session), GEDIT assigns an arbitrary set of X-Y coordinates to the (initially) blank screen display area. The coordinates will be in screen inches with $X=0, Y=0$ at the center of the display area. The approximate extent of the screen will be about -5 to +5 .

Data drawn into the arbitrary coordinate system can be converted into a known coordinate system by using the REGISTER POINTS option, which is selectable from the Misc Menu. If you convert to a known coordinate system and then EXIT, the new file will be saved in that form. When you next use the file as the first input file (default file) of a different GEDIT session, the data will be redrawn into the known coordinate system.

If you draw data into the arbitrary coordinate system of a new file and then EXIT without converting to a known coordinate system, the file will be saved without specific coordinate references. When you next use the file as the first input file (default file) of a different GEDIT session, GEDIT will redraw the data to fill the screen area. Thus the graphic elements of the recalled file may be drawn with proportions different than the original screen display. 


\section{Using the GEDIT Menu}

After reading in the input files, GEDIT presents the following menu:

\begin{tabular}{|l|}
\hline EXIT (SAVE CHANGES) \\
QUIT (IGNORE CHANGES) \\
HELP \\
CANCEL \\
CONTINUE \\
DELETE UNE SEGMENT \\
DELETE SUB POLYGON \\
DELETE COMPOSITE STRING \\
UNSAVE COMPOSITE STRING \\
RE-COLOR-TEXTURE STRING \\
MERGE STRINGS \\
INSERT MENU \\
CONVERT STRING TO POLYGON \\
FORM COMPLEX POLYGON \\
ZOOM IN \\
ZOOM OUT \\
RESTORE BASE PLOT \\
REFRESH SCREEN \\
ATTRIBUTE MENU \\
ICON MENU \\
TEXT MENU \\
TEXT STRING MENU \\
GEOMETRIC MENU \\
MISC MENU \\
DISPLAY COORDINATES \\
MODIFY DEFAULTS \\
POLYGON FIU MENU \\
CHANGE PROJECTION \\
\hline
\end{tabular}

These general options are explained together on the following page.

These specific functions are explained in alphabetical order following the general options.

The selection of some options on the main menu will result in the display of another menu from which further selections must be made.

The options of the GEDIT menu are explained in the following pages. Before continuing, it is important that you understand what happens to the data files at the end of a GEDIT session. 


\section{What Happens to Data Files at the End of a GEDIT Session}

- Pick EXIT to save all your changes. The GEDIT functions change the data displayed on the screen. However, no data files are permanently changed until you end the editing session by picking EXIT (SAVE CHANGES). The data files you specified as input to GEDIT are then updated to reflect the changes made during the editing session.

- Pick QUIT to end without saving your changes. If you do not want to modify the data files in accordance with the changes made during an editing session, pick QUIT instead of EXIT. The data files will NOT be updated.

The following paragraphs describe the general options on the GEDIT menu.

\section{Exit (Save Changes)}

Picking EXIT (SAVE CHANGES) incorporates your changes into new versions of the input files and then ends the session. On other menus, EXIT signals that you are through with the current menu and the operations on that menu. Operational control then returns to the previous or calling menu.

\section{Quit (Ignore Changes)}

Picking QUIT (IGNORE CHANGES) ends the GEDIT session without incorporating any changes in the input files. That is, any changes or insertions made during the session are discarded, and new versions of the input files are NOT created.

Help

Picking HELP displays information about the current menu.

\section{Cancel}

Picking CANCEL terminates the current function. Use this function when it becomes undesirable to complete a function that has been started. If the cursor disappears during the completion of a function, you will be unable to use CANCEL until the cursor reappears.

\section{Continue}

Picking CONTINUE signals the completion of functions such as the selection of points or composite strings. For example, in an operation that requires you to choose a point on the screen, you might pick several points before finding the correct one and then selecting CONTINUE. 


\section{Attribute Menu}

You can use the ATTRIBUTE MENU functions to add, delete, modify, or look up the attributes of a graphic data item. Display programs, such as MPDISPLAY, can use attributes as selection criteria for displaying (or not displaying) specific data items.

Two kinds of data items can have attributes: points and composite strings. Points are specified by a single set of $x, y$ coordinates such as a longitude-latitude location. The attributes of a point can identify that point as

- an icon seed point: the location of an icon

- a text seed point: the location of a piece of text

- a color flood seed point: the starting location of a color flood point

- an item point: a point location that has only attributes without being associated with particular graphics or text.

Composite strings may represent data items such as roads or rivers, which may have attribute information associated with them.

The following sections discuss attributes and how to use the Attribute Menu.

\section{Attributes}

Attributes are numeric values used to describe data items or some characteristic of data items. Attributes consist of three numbers separated by semicolons:

- a Major attribute code (to indicate a major topographic group)

- a Minor attribute code (to indicate a feature within the group)

- a Parameter value (which may give data about the specific feature).

For instance, 1;560;300 indicates

- a Major code of 1 (Hydrography)

- a Minor code of 560 (Permanent lake or pond)

- a Parameter value of 300 (length of the lake in decameters).

A graphic data item within a DMS file may have up to 15 sets of different attributes. GEDIT (and other IBS software) interprets these attributes by using a standardized dictionary file of IBS attributes-the attribute dictionary. The attribute dictionary defines the meaning of each Major;Minor pair of attribute codes. Appendix B lists the attribute codes contained in the IBS attribute dictionary. 
The attribute menu functions enable you to assign attributes to various items. Included on the menu are options for inserting and deleting points with attributes and for displaying the locations of attribute points.

\section{Using Attribute Menu Functions}

Picking ATTRIBUTE MENU results in the following submenu:
EXIT
HELP
CANCEL
CONTINUE
DISPLAY ATTRIBUTES
ADD ATTRIBUTE
DELETE ATTRIBUTE
MODIFY ATTRIBUTE
LOOKUP ATTRIBUTE
INSERT POINT
DELETE POINT
ENABLE POINT
DISABLE POINT
REFRESH SCREEN

The principal options of the submenu are explained here.

DISPLAY ATTRIBUTES: Displays the attributes associated with a particular data item.

\section{Pick DISPLAY ATTRIBUTES.}

2. Pick a point on or near the desired point or composite string.

The data item will blink and, if it has attributes associated with it, they will be displayed in a table. For example, this table displays information on two attributes:

$\begin{array}{rrrrl}* & \text { MAJOR } & \text { MINOR } & \text { PARAM } & \text { DESCRIPTION } \\ 1 & 7 & 150 & 0 & \text { SEAplane ramp Or landing area } \\ 2 & 8 & 7 & 6 & \text { NO ENTRY IN ATTRIEUTE DICTIONARY }\end{array}$


where \# is the index number and the following four columns list each attribute (the Major code, the Minor code, and the Parameter value) plus a text description of the data item (if a description exists in the attribute dictionary). Notice that the second set of attributes has "NO ENTRY IN ATTRIBUTE DICTIONARY"; that is, the attributes $8 ; 7 ; 6$ might have some meaning for the person who created the data item, but they are not in the attribute dictionary (the master list of attributes) normally used by GEDIT.

3. Repeat step 2 for any other data item.

As you continue to pick data items, old attribute information is retained on the text screen. To clear the screen and make it easier to read, press the D ERASE key.

4. Pick any other submenu item to end the DISPLAY ATTRIBUTES function.

- ADD ATTRIBUTE: Allows association of a new attribute with a data item.

\section{Pick ADD ATTRIBUTE.}

2. Pick a point on or near the desired point or composite string.

The data item will blink and any attributes already associated with the data item will be listed in the dialogue area, as shown for the DISPLAY ATTRIBUTES function.

3. Pick CONTINUE.

GEDIT will then prompt for a new attribute to be added.

ENTER ATTRIBUTE VALUE TO BE ADDED AS MAJ MIN PARAMETER >

4. Type a major code (MAJ), minor code (MIN), and parameter; then press RETURN. (Type spaces or commas between items; do not type parentheses.)

DELETE ATTRIBUTE: Allows deletion of an attribute already associated with a data item.

\section{Pick DELETE ATTRIBUTE.}

2. Pick a point on or near the desired point or composite string. 
The data item will blink, and any attributes associated with the data item will be listed in the dialogue area, as shown for the DISPLAY ATTRIBUTES function.

3. Pick CONTINUE.

GEDIT will then prompt for the index number (\#) of the attribute to be deleted:

ENTER INDEX \# OF ATTRIBUTE TO BE DELETED >

4. Type the desired index number and press RETURN.

GEDIT will delete the selected attribute from the data item's attributes. You can use the DISPLAY ATTRIBUTES function to verify that a particular attribute has been deleted.

MODIFY ATTRIBUTE: Allows modification of an attribute already associated with a data item.

1. Pick MODIFY ATTRIBUTE.

2. Pick a point on or near the desired point or composite string.

The data item will blink, and any attributes already associated with the data item will be listed in the dialogue area, as shown for the DISPLAY ATTRIBUTES function.

3. Pick CONTINUE.

GEDIT will prompt you for the index number of the attribute to modify and the new attribute values:

ENTER INDEX * AND NEW ATTRIBUTE VALUE AS MAJ MIN PARAMETER >

4. Type an index number (INDEX \#), major code (MAJ), minor code (MIN), and parameter; then press RETURN. (Type spaces or commas between items.)

GEDIT will modify the attribute as specified.

LOOKUP ATTRIBUTE: Enables you to look up an attribute in the attribute dictionary. You can reference an attribute by attribute codes or by the description in the attribute dictionary. 
Picking LOOKUP ATTRIBUTE displays the following submenu:

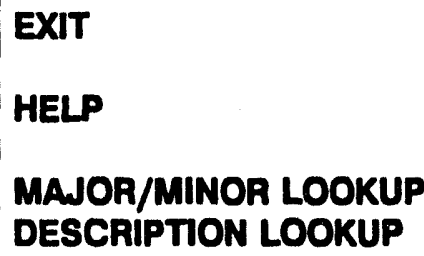

The principal options of this submenu are explained here.

MANOR/MINOR LOOKUP: Enables you to display the attribute associated with a particular pairing of Major and Minor attribute codes in the attribute dictionary. A master list of the attributes in the attribute dictionary is presented in Appendix B of this guide.

You will see the prompt

ENTER MAJOR MINOR ATTRIBUTE CODE >

Type a Major attribute code (up to two digits), a comma, and a Minor attribute code (up to four digits). Then press RETURN.

Example: 7,150

If the Major/Minor code pairing exists in the attribute dictionary, your entry will be displayed along with a parameter value (PARAM) and a description, as in this example:

$$
\begin{array}{crcl}
\text { MAJOR } & \text { MINOR } & \text { PARAM } & \text { DESCRIPTION } \\
7 & 150 & 0 & \text { SEAplane ramp or landing area }
\end{array}
$$

If no entry exists for the Major/Minor code pairing, the description note will read, "NO ENTRY IN ATTRIBUTE DICTIONARY."

Note: To see a list of all attributes that contain a particular Major code, enter a Minor code of zero. For example, the entry 5,0 will list every attribute that has a Major code of 5 (Roads and Trails) in the attribute dictionary. Be ready to press CTRL-S to "freeze" the listing as it prints on the screen: such listings are usually more than one screen long and can scroll by quickly. Press CTRL-Q to restart the listing.

DESCRIPTION LOOKUP: Enables you to display the attribute associated with a particular character string in the attribute dictionary descriptions. 
You will see the prompt

ENTER DESCRIPTION TO LOOKUP >

Type a string of characters that you are seeking in the attribute dictionary descriptions (Example: car); then press RETURN.

You will see a form of your entry listed under DESCRIPTION, along with the corresponding major and minor codes and parameter value

(PARAM) as in this example:

$\begin{array}{rrcl}\text { MAJOR } & \text { MINOR } & \text { PARAM } & \text { DESCRIPTION } \\ 1 & 280 & 0 & \text { Outline of a Carolina Bay } \\ 6 & 50 & 0 & \text { Carline } \\ 9 & 210 & 0 & \text { Carrying contour } \\ 9 & 560 & 0 & \text { Evacuee Reception/care points }\end{array}$

Note: Any DESCRIPTION that contains your character string in the dictionary will be displayed.

INSERT POINT: Associates a user-entered attribute with a new point location.

1. Pick INSERT POINT.

GEDIT will prompt you for a new attribute to be added:

ENTER ATTRIBUTE VALUE TO BE ADDED AS MAJ MIN PARAMETER >

2. Enter a Major (MAJ) attribute code (up to two digits), a comma, a Minor (MIN) attribute code (up to four digits), a comma, a parameter (up to three digits) and press RETURN. Example: 1,190,0

3. Pick a point in the display where you want the attribute positioned.

DELETE POINT: Enables you to delete a data point and its associated attributes.

1. Pick DELETE POINT.

2. Pick a point on or near the point that you wish to delete.

The data point will blink. 


\section{Pick CONTINUE.}

The data point will be deleted.

- ENABLE POINT: Places an asterisk "** at the location of every point with attributes in each input file.

These points denote item points, icon seed points, text points, and color flood seed points. For example, if you are editing a single file that contains text, this feature will show how many text items there are and where the starting point of each item is located without taking the (possibly long) time to plot the text. ENABLE POINTS is particularly useful for locating text when the decluttering factor does not allow the text to be plotted on the screen.

DISABLE POINT: Disables the display of points with attributes, allowing future plots to be created without showing the locations of attribute/point data.

DISABLE POINT allows you to remove the attribute/point markers from screen when used in conjunction with REFRESH SCREEN: Pick DISABLE POINT; then pick REFRESH SCREEN. The system will redraw the screen without the attribute/point markers.

- REFRESH SCREEN: Redraws the existing display image using the current ENABLE POINT and DISABLE POINT settings. 


\section{Change Projection}

This function enables you to change the map projection used for displaying DMS files in GEDIT. CHANGE PROJECTION begins by presenting a menu of map projection options for displaying maps:

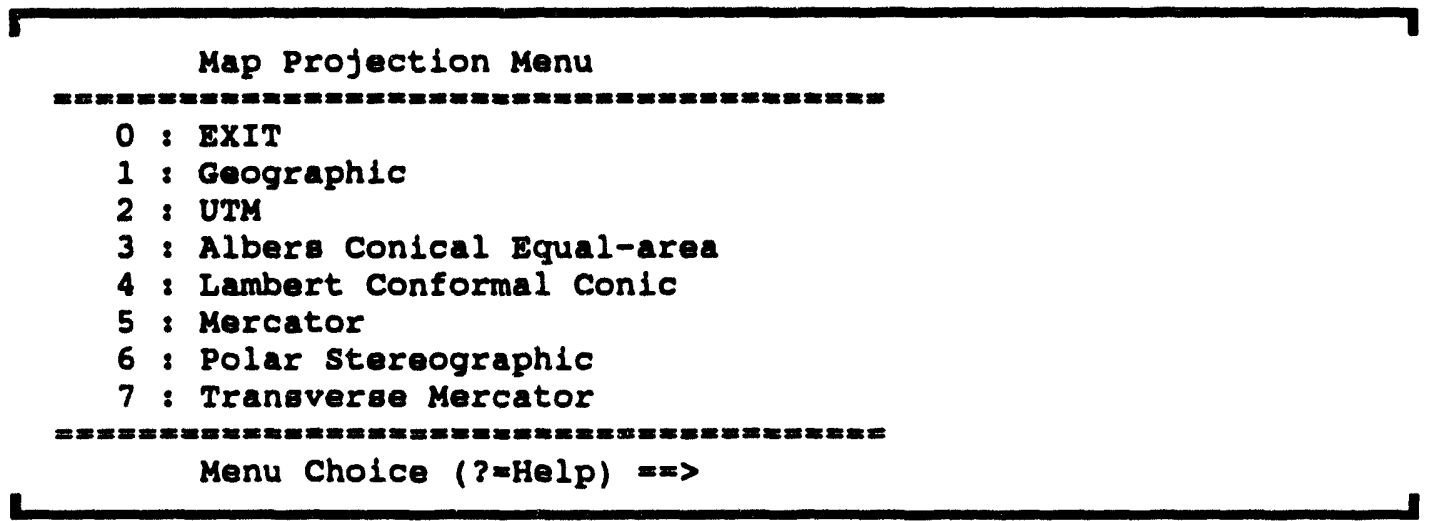

The default map projection used by GEDIT and other IBS mapping software is the "Geographic" projection. The Geographic projection (1) is a straightforward projection onto a regular $x, y$ plane and is a good choice for most cases (up to 100 -mile radius). Enter a different number (2 to 7 ) if you desire a projection other than Geographic. The following table is a simple summary of how the different map projections might be used. See Appendix D for background on the projection types.

Map Projection Type

Geographic

Universal Transverse Mercator (UTM) or Transverse Mercator

Albers Conical Equal-Area or Lambert Conformal Conic

Polar Stereographic
Situations for Use

small areas (up to a 100-mile radius)

medium-sized areas (up to 1000-mile radius) or where the largest extent of the mapped area extends north-south rather than east-west

larger areas, or where the largest extent of the mapped area extends east-west

polar areas of the globe

After you select a projection type, GEDIT redraws the display in the new projection. 


\section{Convert String to Polygon}

This function converts a string to a polygon. The string can be in any input file that was read in at the beginning of the session. A polygon converted from a string will be contained in the same file that contained the string.

1. Pick CONVERT STRING TO POLYGON.

2. Pick a point on or near the desired string.

The selected string will blink.

3. Pick CONTINUE.

If the string was closed (that is, if the end points of the string are identical), then the string will become a polygon without any modifications. If the string was not closed, a line will be drawn between the two end points to form a closed string. 


\section{Delete Composite String}

To delete a composite string, pick the DELETE COMPOSITE STRING menu item and then pick the composite string to be deleted:

\section{Pick DELETE COMPOSITE StRING.}

2. Pick a point on or near composite string to be deleted.

The cursor will disappear while GEDIT searches for a composite string near the point selected. When the cursor reappears, the closest composite string will be blinking. If this is not the correct item to be modified, or if no composite string is blinking, move the cursor closer to the correct composite string and pick another point.

\section{Pick CONTINUE.}

The composite string will disappear from the screen, as will any attributes of the composite string.

When you pick a composite string to be deleted, the composite string is copied into a special holding area of computer memory called the save buffer. This occurs whether or not you actually continue and delete the composite string. Once the copy of the composite string is in the save buffer, you can use the UNSAVE COMPOSITE STRING function to insert a copy (or copies) of the composite string into the screen display. 


\section{Delete Line Segment}

To delete a line segment, pick the DELETE UNE SEGMENT menu item and then pick the line segment to be deleted:

1. Pick DELETE LNe SEGMENT.

2. Pick a point on or near the line segment to be deleted.

The cursor will disappear while GEDIT searches for a line segment near the point selected. When the cursor reappears, the closest line segment will be blinking. If this is not the line segment to be deleted, or if no line segment is blinking, move the cursor closer to the line segment and pick another point.

3. Pick CONTINUE.

The line segment will disappear from the screen, as will any attributes of the line segment. 


\section{Delete Sub_Polygon}

A subpolygon is a simple polygon that has been combined with other polygons to form a complex polygon (see FORM COMPLEX POLYGON, page 2.121). Picking DELETE SUB_POLYGON enables you to delete a subpolygon in the same way as you would delete $\mathbf{a}$ line segment.

\section{Pick DELETE SUB_POLYGON.}

2. Pick a point on or near the subpolygon to be deleted.

The cursor will disappear while GEDIT searches for the subpolygon near the point selected. When the cursor reappears, the closest subpolygon will be blinking. If this is not the correct subpolygon, or if no subpolygon is blinking, move the cursor closer to the subpolygon and pick another point.

3. Pick CONTINUE.

GEDIT will delete the subpolygon. 


\section{Display Coordinates}

Picking DISPLAY COORDINATES and then a point on the display results in the following information on the text screen:

- longitude-latitude of the point

- the Great Circle distance from the point to the center of the display (shortest arc over the surface of the earth)

- the change in distance from one picked point to the next

- the cumulative distance (total miles) from the first point picked for coordinates.

Picking subsequent points will display this information until you terminate the function by picking any item on the main menu.

If you are using GEDIT to create a map or drawing from scratch, the longitude and latitude values will be an arbitrary set of coordinate values. To register points on the display with real longitude-latitude values, refer to the REGISTER POINTS option on the Misc(ellaneous) Menu (page 2.136). 


\section{Form Complex Polygon}

This function combines simple polygons to form a complex polygon or combines an already existing complex polygon with a simple polygon to form a new complex polygon. The polygons involved may be in any of the input files read in at the beginning of the GEDIT session.

\section{Pick FORM COMPLEX POLYGON.}

The menu item will continue to blink until the function is complete.

2. Pick a point on or near a simple or complex polygon.

The selected polygon will blink. If $\mathrm{r}$ o polygon is blinking, or if the wrong polygon is blinking, reposition the cursor and pick another point that is closer to the desired polygon.

\section{Pick CONTINUE.}

If a simple polygon was selected, GEDIT will prompt you to indicate whether the polygon is to define an inclusive or exclusive area:

INCLUSIVE OR EXCLUSIVE>

An inclusive polygon defines the area inside its boundary.

An exclusive polygon defines the area outside its boundary.

(For example, consider a small triangle contained completely within a larger triangle. The small triangle could be specified as defining an exclusive area outside its boundary, while the larger triangle could be specified as defining an inclusive area inside its boundary. The complex polygon would then describe the area between the two boundaries. GEDIT's polygon fill capabilities could be applied to fill that area with a color or pattern (described on page 2.151).

4. If GEDIT prompts you,) type "I" for Inclusive or "E" for Exclusive, and press RETURN.

GEDIT will temporarily remove the selected polygon from the display. The FORM COMPLEX POLYGON menu item will still be blinking, indicating that a second polygon must be selected to form the complex polygon.

5. Repeat steps 2 through 4 (and 5 , as necessary) to select a second polygon that is to be combined with the first to form a complex polygon.

The complex polygon formed by this function will have the same color and reside in the same file as the first polygon picked, in step 2. The second polygon will be deleted from its file. 


\section{Geometric Menu}

The Geometric Menu functions include the capabilities for drawing, scaling, rotating, and horizontally inverting polygons (which are referred to as composite strings). The drawing function enables you to produce $n$-sided regular polygons with different horizontal and vertical dimensions, including circles and ellipses (high-n polygons).

Picking GEOMETRIC MENU results in the following submenu:

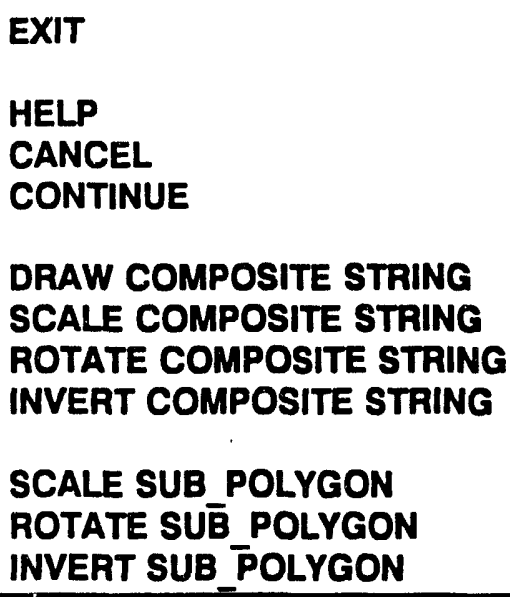

The principal options of this submenu are explained in the following paragraphs.

EXIT: Returns operation to the GEDIT main menu. Any of the draw, scale, rotate, or invert functions may be aborted by picking EXIT or another function before picking CONTINUE.

DRAW COMPOSITE STRING: Used to create a regular $n$-sided polygon with independent $\mathrm{X}$ (horizontal) and $\mathrm{Y}$ (vertical) dimensions. The polygons created are inserted into the default file.

1. Pick DRAW COMPOSITE STRING.

GEDIT will prompt for an $X$ and $Y$ radius (in screen inches) and the number of sides for the geometric figure. Both $\mathrm{X}$ and $\mathrm{Y}$ must be greater than zero. The number of sides must be three or more.

2. Type the three values, separated by commas (or spaces) and followed by RETURN, as in this example:

ENTER X RADIUS, Y RADIUS, AND \# OF SIDES $>1,1,4<$ RETURN>

3. Pick a point on the display screen where the figure is to be drawn. 
The figure (a 1X1 square in the example) will be drawn at that point on the display and inserted into the default file.

- SCALE COMPOSITE STRING: Used to change the size of a composite string.

1. Pick SCALE COMPOSITE StRING.

GEDIT will respond with a prompt for a scale factor.

2. Type a scale factor (decimal or decimal fraction greater than zero), as in this example:

ENTER SCALE FACTOR >.75 <RETURN>

3. Pick a point on or near the string that is to be scaled.

The cursor will disappear while GEDIT searches for the nearest composite string. When the cursor reappears, the closest composite string will be blinking. If no composite string is blinking, or if the wrong composite string is blinking, reposition the cursor and pick a point closer to the desired string.

4. Pick CONTINue.

The selected string will be erased and redrawn, scaled to the desired size (three-quarter size in the example).

ROTATE COMPOSITE STRING: Used to rotate a string on the display.

1. Pick Rotate COMPOSITE STRING.

GEDIT will prompt for the number of degrees of rotation (clockwise positive; counterclockwise negative).

2. Type a rotation angle $(0-360)$, followed by RETURN, as in this example:

ENTER ROTATION FACTOR IN DEGREES >45 <RETURN>

3. Pick a point on or near the string to be rotated.

The string nearest the picked point will blink.

4. Pick CONTINUE.

The selected string will be erased and redrawn at the designated rotation. 
- INVERT COMPOSITE STRING: Used to invert (horizontally flip) a composite string.

1. Pick INVERT_COMPOSITE_STRING.

2. Pick the string to be inverted, using CONTINUE.

The string will be erased, redrawn, and inverted on the screen.

SCALE SUB POLYGON, ROTATE SUB_POLYGON, and INVERT SUB_POLYGON: These functions work the same as the corresponding functions for composite strings but operate on subpolygons instead of composite strings. 


\section{Icon Menu}

An icon is a graphical figure that can be associated with a particular data point (location). Icons always appear at a constant viewing size, regardless of the map scale (which can be changed with the zoom functions). The location of icons is governed by icon seed points, which indicate the type of icon to be drawn and where to center the icon.

The icons available for display are listed in the icon control file. The icon control file also contains the locations of the DMS files that are needed to plot each icon. A system default icon control file is used if you do not specify one.

Picking ICON MENU results in the following submenu:

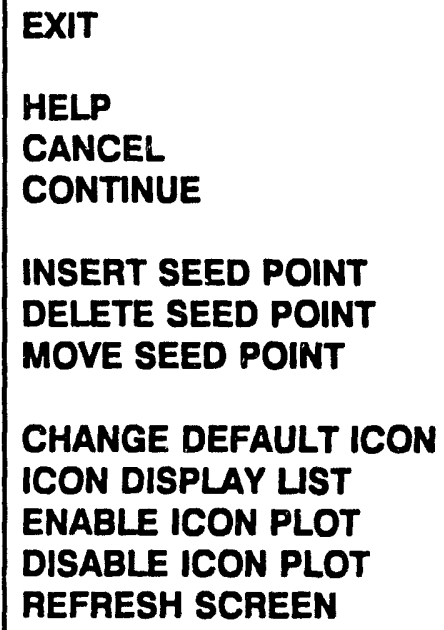

The principal options of the submenu are explained below.

INSERT SEED POINT: Allows insertion of an icon seed point at the next point picked on the display.

\section{Pick INSERT SEED POINT.}

2. Pick a point (seed point) on the display where you want to display the default icon. (Refer to the CHANGE DEFAULT ICON function.)

GEDIT will position an icon seed point at that picked location on the display. The seed point will be inserted into the default file, and the default icon will be displayed at the chosen point. 
- DELETE SEED POINT: Allows removal of an icon seed point from the appropriate file.

1. Pick Delete SeEd POINT.

2. Pick a point near the seed point you want to delete.

Seed points can be located by enabling icon plotting so that the icons are displayed. The seed point nearest to the picked point will blink. If this is not the correct seed point, pick other points on the display until you find the correct seed point.

3. Pick CONTINUE.

GEDIT will delete the blinking seed point and associated icon and remove the seed point from the appropriate file.

MOVE SEED POINT: Allows relocation of an existing icon seed point.

1. Pick MOVE SEED POINT.

2. Pick a point near the seed point you want to move.

Seed points can be located by enabling icon plotting so that the icons are displayed. The seed point nearest to the picked point will blink. If this is not the correct seed point, pick other points on the display until you find the correct seed point.

\section{Pick CONTINUE.}

4. Pick a new point where you want to relocate the icon.

GEDIT will delete the blinking seed point and associated icon and relocate the seed point at the new location.

CHANGE DEFAULT ICON: Changes the default icon, which is the icon associated with any insertion of an icon seed point. Picking CHANGE DEFAULT ICON results in a menu of available icons.

The current default icon is always listed at the top of the menu, as shown: 


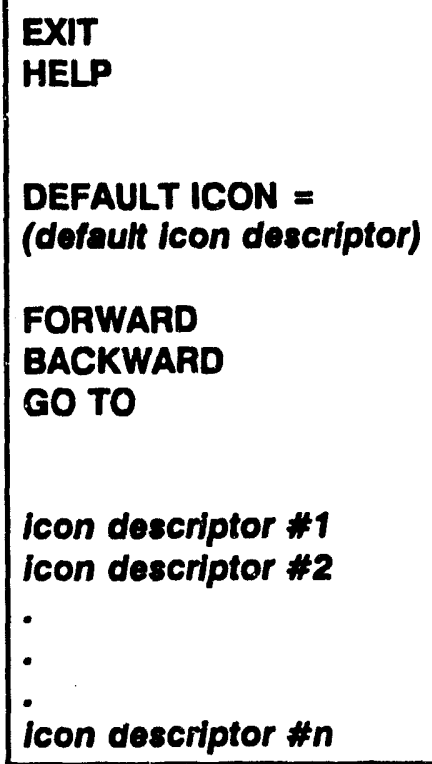

To switch to a different default icon, pick an alternative from the list provided. The new icon will then appear at the top of the menu. Once the desired icon has been selected, pick EXIT to return control to the ICON MENU.

All available icons are in a paged menu with 16 icons per page. To scroll through the icon list, select the menu item (FORWARD, BACKWARD, GO TO) that describes which way you want to go:

- FORWARD: Shows the next page of 16 icons. If you reach the end of the list, the system will return you to the first page of the icon listing.

口 BACKWARD: Shows the previous page of 16 icons. If you reach the first page of the list, the system will return you to the last page of the icon listing.

- GO TO: After you select GO TO, the system will prompt you for the page number desired (ENTER PAGE \# OF ICONS DESIRED >). The system then shows you the icon page requested. If the page number requested is less than 1, the system will display the first page of icons. If the page number requested is greater than the number of icon pages available, the system will send you to the last page of icons.

If you change the default icon (or modify the icon display list), then the next time you use the function, the icon list will start at the page of the last icon changed (or modified). 
- ICON DISPLAY LST: Specifies which of the available icons may be displayed. GEDIT will present a submenu that lists the available icons:

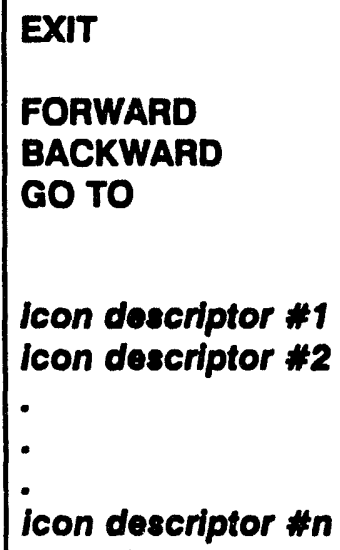

For example, the default IBS icon control file results in this icon display list:

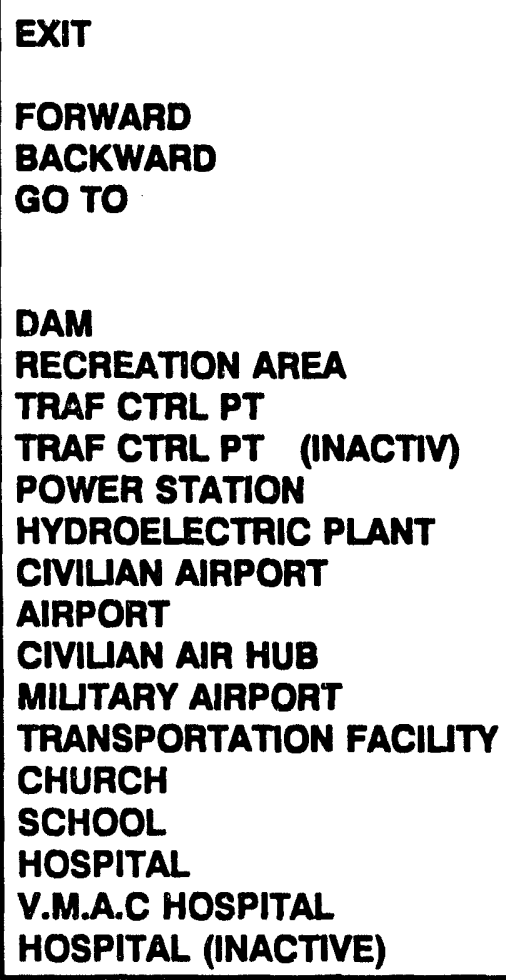


Select icon categories or types to be displayed by picking the appropriate descriptor in the menu. As a descriptor is selected, its color will toggle between the standard menu color (normally orange) and a secondary color (normally green) to indicate that the icon has been selected. By default all icons are set to be displayed.

To cancel a selection, simply pick that item again, and its color will toggle back to the standard color. The selected icons are eligible for display and will be plotted the next time REFRESH SCREEN is selected if ENABLE ICON PLOT has been picked and seed points exist for the selected area.

Picking EXIT in the ICON DISPLAY UST menu returns control to the ICON MENU.

Picking FORWARD, BACKWARD, or GOTO on the menu enables you to page through the icon display list as described for the CHANGE DEFAULT ICONS function. If you modify the icon display list (or change the default icon), then the next time you use the function, the icon list will start at the page of the last icon selected for display (or the last default icon changed).

- ENABLE ICON PLOT: Used (along with DISABLE ICON PLOT) to specify whether the icons selected on the icon display list are actually displayed. When you pick ENABLE ICON PLOT, GEDIT checks to see if any of the selected icon types occur in the display area and draws the icons at seed points on the display.

- DISABLE ICON PLOT: Switches off the capability to display icons (without erasing any icons that are currently on the screen).

Use the REFRESH SCREEN option to subsequently erase disabled icons.

REFRESH SCREEN: Redraws the current display, just as in the main menu. The REFRESH SCREEN function is included in this menu for convenience in using the Icon Menu functions. Use the REFRESH SCREEN option to erase icons after disabling icon plotting or after changing the Icon Display List. 


\section{Insert Menu}

The INSERT MENU functions allows insertion of a string of the default color and line type. Picking INSERT MENU results in the following submenu, which gives you options for how a string is inserted:

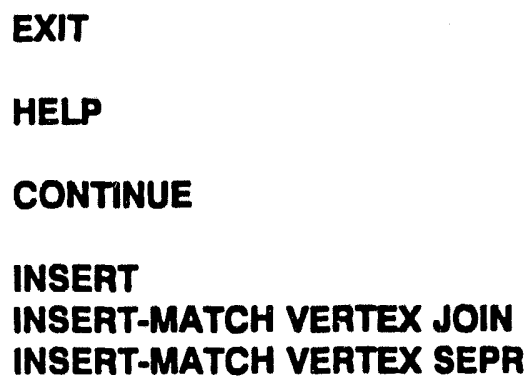

The principal options of the submenu are explained below.

- INSERT: Inserts a string. As you pick points on the display, the INSERT function draws line segments between the points, from the first point to the second, from the second to the third, and so on.

1. Pick INSERT on the Insert Menu.

2. Pick a starting point on the display.

3. Pick another point on the display.

GEDIT will draw a line between this point and the previous point.

4. Repeat step 3, as desired.

GEDIT will continue to build the string as a continuing series of line segments.

\section{Pick CONTINUE.}

This accepts the string as part of the current display and the current default file (although the file is not actually updated until you pick "EXIT" at the end of a GEDIT session). Picking any other menu item will cancel the INSERT function and erase the string from the display.

INSERT-MATCH VERTEX JOIN: Enables you to attach a new string to an existing string by starting the new string at a vertex of the existing string. That is, INSERT-MATCH VERTEX JOIN forces the starting point alignment of a new string at an endpoint or midstring vertex of the existing string. 
1. Pick INSERT-MATCH VERTEX JOIN on the Inisert Menu.

The menu item will blink to indicate selection.

2. Pick a point on or near a vertex at which a new string is to be attached.

The cursor will disappear while GEDIT searches for the nearest vertex. When the cursor reappears, an asterisk (*) vill be blinking on the nearest vertex. (If no vertex is blinking or if the wrong vertex is blinking, reposition the cursor and pick another point that is closer to the desired vertex.)

\section{Pick CONTINUE.}

The INSERT menu item will blink to indicate that you are now in INSERT mode. The selected vertex will serve as the starting point for a new string. You can continue to build the new string by picking points and then picking CONTINUE, as described for the INSERT function.

- If the vertex IS an endpoint of the existing string and the existing string is in the default file, the new string being inserted will be appended to (become part of) the existing string. The new composite string will have the same attributes as the original string (see Attribute Menu, page 2.108).

- If the vertex is NOT an endpoint or if the existing string is NOT in the default file, then the new string does NOT become part of the existing string.

To draw a string that connects two vertices of one or more existing string(s): Continue from step 3 and follow steps 4 through 6 below.

4. Pick INSERT-MATCH VERTEX JOIN again (after step 3).

The menu item will again blink.

5. Pick a point on or near the second desired vertex.

The picked vertex will blink to indicate selection.

6. Pick CONTINUE.

A line segment will be drawn between the two selected vertices. The new string will be appended to the existing string(s) at any vertex that is also the endpoint of a string in the default file. If the two selected vertices were the endpoints of two different strings in the default file, then all the strings will be joined as one composite string. This method can be used 
to merge two strings at endpoints other than their closest endpoints. The MERGE STRINGS function automatically merges two strings at their closest endpoints.

To connect a string in progress to an existing string: Use the INSERT-MATCH VERTEX function from within the insert mode:

1. Pick INSERT on the Insert Menu.

The menu item will blink to indicate selection.

2. Pick points, as desired, to define a string of line segments.

GEDIT will draw segments from one point to the next in the default color.

3. Pick INSERT-MATCH VERTEX JOIN.

The menu item will blink.

4. Pick a point on or near the desired vertex.

The vertex will blink. (If no vertex is blinking or if the wrong vertex is blinking, reposition the cursor and pick another point that is closer to the desired vertex.)

\section{Pick CONTINUE.}

GEDIT will draw a line between the selected vertex and the endpoint (last point) of the string being inserted. The new string is inserted into the display, and the INSERT function ends.

INSERT-MATCH VERTEX SEPR: Enables you to draw a new string, starting from the vertex of an existing string. This function works the same as INSERT-MATCH VERTEX JOIN, except the new string is independent and is NOT stored as a part of the existing string. The new string will have no attributes (unless you add attributes later with ATTRIBUTE MENU functions, page 2.108).

It is possible to use any combination of the Insert Menu items to insert strings that connect in various ways. For example: 
To insert a new string between the endpoints of two existing strings, joining with one string but not the other:

1. Pick INSERT-MATCH VERTEX JOIN.

2. Pick a point on or near the endpoint of the string to be joined.

3. Pick CONTINUE.

The INSERT menu item will blink to indicate that you are now in insert mode. The selected vertex will serve as the starting point for a new string. You can continue to build the new string by picking points and then picking CONTINUE, as described for the INSERT function.

4. To connect the new string to the second existing string, pick INSERT-MATCH VERTEX SEPR.

5. Pick a point on or near the selected endpoint of the second string.

6. Pick CONTINUE.

The new segment is now part of the first string and has all the attributes of the first string. This new composite string "connects" to the endpoint of the second string on the screen but does not contain the second string or share its attributes.

Other options for using the Insert Menu

dude:

- Start with SEPR and end with JOIN.

- Start with JOIN and end with JOIN.

- Start with SEPR and end with SEPR.

Attributes of the Inserted Strings. When a composite string is created by using INSERT-MATCH VERTEX JOIN, the new string will get ALL the attributes of ALL the joined strings. When a string is inserted by using INSERT-MATCH VERTEX SEPR, the new string has no attributes because it is not actually joined to anything. For example, Table 2.2 shows the attributes of strings resulting from connecting "String 1" with "String $2^{n}$ with various combinations of Insert Menu functions. 
Table 22. Attributes Resulting from Insert Menu Functions

Order of Using the INSERT-

MATCH VERTEX Functions

Attributes of the Joined String

JOIN then JOIN Attributes of String 1 AND String 2

JOIN then SEPR Attributes of String 1 ONLY

SEPR then JOIN Attributes of String 2 ONLY

SEPR then SEPR NO attributes 


\section{Merge Strings}

The MERGE STRINGS function joins two line strings into a single element by connecting them at their nearest endpoints. Only lines are processed by this function; attempting to merge points or polygons will result in an error.

1. Pick MERGE STRINGS.

2. Pick a point on or near one of the strings to be merged.

The string will blink.

3. Pick CONTINUE.

4. Repeat steps 2 and 3 for the second string.

GEDIT will connect the two strings at their nearest endpoints. The color and line type of the merged string will be the same as the first string selected, while the attributes of the new string will be a union of those from both strings selected. 


\section{Misc(ellaneous) Menu}

Picking this option results in a submenu containing several unrelated functions:

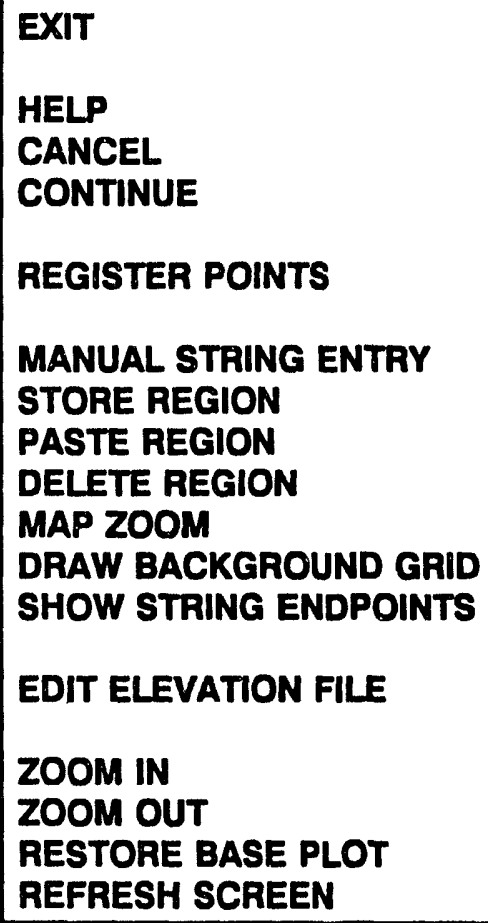

The principal options of this submenu are explained below.

REGISTER POINTS: Converts an existing set of data points into a known coordinate system. For example, points on a map that was digitized in an arbitrary coordinate system may need to be converted to longitude and latitude (long-lat points). This process is referred to as map registration.

To accomplish map registration, location values (e.g., longitude-latitude) of four different locations must be entered and then their positions must be picked on the display. It is best if these four points lay more or less on the four corners of the rectangular area of interest on the display. The four points used in calibrating the registration process are referred to as known points.

Known point registration can be used for maps as well as any other coordinate system. When you choose REGISTER POINTS, the program displays a reminder screen that lists seven steps for registering a map. The program then lead you through each step. 
Seven steps to register a map:

1. Specify coordinate system.

2. If long/lat, specify decimal degrees or degrees-minutes-seconds.

3. Repeat steps 4-5 for the four points needed to register a map.

4. Select a point on the display.

5. Enter coordinate values (for that point).

6. Confirm that the four points are correct.

7. Specify projection (select from list).

GEDIT begins by prompting:

Will known points be in long/lat? [Y] $==>$

1. If the schematic being digitized is not a map, answer $\mathbf{N}$. Coordinates must be input as decimal numbers.

If map coordinates will be specified in longitude/latitude, answer $\mathbf{Y}$ or press RETURN. You must then specify whether long-lat values will be input as degrees-minutes-seconds or as decimal numbers:

Will the long/lat be in degree-min-sec? [N] $\Rightarrow=>$

2. If you will enter long/lat coordinates in degrees, minutes, and seconds, then answer $\mathbf{Y}$. If you will enter only decimal degrees, answer $\mathbf{N}$ or press RETURN. In either case, GEDIT prompts you to select a point on the screen:

* * MSG: Select point * $n$ on the screen

3. Follow steps 4 and 5 four times (once for each point).

4. Pick one of the registration points on the display.

GEDIT then prompts you to enter coordinates for that point.

5. Enter coordinate values for that point.

After you enter coordinate values for the fourth point, GEDIT displays the point coordinates and prompts you to confirm that these points are correct. For example:

$\begin{array}{llllr}\text { Pt: : } 1 & -119.500000 & 47.000000 & 4.6571677 & 3.9255425 \\ \text { Pt: : } 2 & -120.000000 & 47.000000 & -0.8664571 & 3.9255425 \\ \text { Pt: : } 3 & -120.000000 & 46.500000 & -0.8664571 & -1.7003706 \\ \text { Pt*: } 4 & -119.500000 & 46.500000 & 4.5971281 & -1.7003706 \\ \text { Are thege 4 points correct? }(Y / N) & {[Y]=\Rightarrow}\end{array}$

6. To confirm that the point coordinates are correct, enter $\mathbf{Y}$. 
GEDIT then prompts for a map projection type as described on page 2.115 .

7. Specify a map projection by selecting from the map projection list.

This completes the point registration process.

MANUAL STRING ENTRY: Draws line segments between points specified by input of coordinates.

A prompt will appear on the screen asking for entry of the $X$ and $Y$ coordinates. Type $\mathrm{X}$ and $\mathrm{Y}$ coordinates, separated by a comma and followed by RETURN. GEDIT will continue prompting for $\mathrm{X}$ and $\mathrm{Y}$ coordinates until a CTRL-Z is pressed.

- STORE REGION: Allows storage of areas of the display for later use.

You may store the entire region displayed in the current display area or define a smaller area within the region:

- To store the entire region in the current display: Use the graphics cursor to pick STORE REGION. Then pick SELECT CONTINUE.

- To store a region within the current display:

1. Pick STORE REGION.

2. Use the graphics cursor to pick points on the display that define a polygon that surrounds the desired region.

GEDIT will draw lines between the points as they are entered, indicating the boundary of the desired region.

3. After picking the last point, pick CONTINUE to draw a line between the first and the last point, completing the polygon.

The area inside the polygon will then be saved in a file. Files with ".CLP" extensions will be created for each file being edited by GEDIT. Thus, if ROADS.DMS, LAKES.DMS and STREAMS.DMS were currently being edited, three new files (ROADS.CLP, LAKES.CLP, and STREAMS.CLP) would be created by the STORE REGION function. If any of the "clipped" area contained text strings, then GEDIT creates one text file called CLIP.TXT. Each clipped file that contains text will refer to this file in its header, and all text data points will reference text strings within this file. 
The STORE REGION command always creates a file that describes the bounding polygon for the clipped region. This file is provided for use with the PASTE REGION command. The file name is always GEDIT.CLP.

- PASTE REGION: Provides a means of merging an existing file with one currently being modified by GEDIT.

When PASTE REGION is activated, GEDIT solicits, from the terminal, the file names of 1) a file containing the description of a closed polygon defining the region in which clipping is to occur (usually GEDIT.CLP), and 2) the file which will be appended to the current default file.

After entering the second file name, the polygon will be temporarily displayed and the default file clipped to outside the polygon. Then the second file is appended to the default file. Only the default file is affected by the PASTE REGION command.

DELETE REGION: Allows deletion of data within a user-defined polygon. This function can be used to clear an area of the display and quickly delete unwanted elements.

\section{Pick DELETE REGION.}

(If you then immediately pick CONTINUE, all the data within the current screen display will be deleted.)

2. Pick points on the screen that define a polygon surrounding the area that you want to delete.

GEDIT will draw segments between the points as they are entered, indicating the boundary of the desired region.

3. After picking the last point, pick CONTINUE to draw a segment between the first and the last point, completing the polygon.

GEDIT will then delete all the data within the defined polygon. Remember that your original input files are not actually changed to reflect any graphic editing until you leave GEDIT by using the EXIT function.

- DRAW BACKGROUND GRID: Puts a grid of squares or concentric rings (target) as background on the screen.

GEDIT will query whether the grid is to be a square or a target.

1. Type $\mathbf{S}$ for square o: $\mathbf{T}$ for target, followed by RETURN.

2. Pick a point on the display to be the center point of the grid. 
If the grid is to be a target, GEDIT will prompt you for the radius of the target (in miles) and the number of rings. If the grid is to be a square, GEDIT will prompt you for the number of cells and the distance to the edge from the center (in miles). All values must be greater than zero.

3. Type the appropriate values, and press RETURN.

The grid will be drawn over the specified area. This function is a "one-time" function: any screen refresh or redra will redraw the screen without the grid.

SHOW STRING ENDPOINTS: Shows the endpoints of each displayed string by plotting an asterisk(*) to mark each endpoint.

This function is helpful when you want to merge strings (page 2.135) or continue drawing a string from an endpoint (page 2.130). This function is a "one-time" function: any screen refresh or redraw will redraw the screen without the endpoint markers.

aDIT ELEVATION FILE: Picking this option results in the following prompt:

Enter name of elevation file $=>$

You must then enter the name of a TAC elevation file. (Terrain Analysis Cell files are described in the IBS Data Management Guide.) After you enter the name of an elevation file, GEDIT zooms the display so that the elevation data fills the map display area. (To restore the display size after leaving the elevation editing functions, use RESTORE BASE PLOT.)

You will see the following submenu of elevation-related options. 


\begin{tabular}{l}
\hline EXIT (SAVE TAC FILE CHANGES) \\
QUIT (DEL TAC FILE CHANGES) \\
HELP \\
CANCEL \\
CONTINUE \\
MODIFY ELEVATION POINT \\
MODIFY GROUPED ELEV POINTS \\
RETURN ELEVATION \\
RETURN SLOPE \\
ENABLE ELEVATION RESOLUTION \\
DISABLE ELEVATION RESOLUTION \\
DISPLAY ELEVATION STATISTICS \\
DISPLAY ATTRIBUTES \\
ZOOM IN \\
ZOOM OUT \\
RESTORE BASE AREA \\
REFRESH SCREEN
\end{tabular}

The principal options of this submenu are explained below.

a EXIT (SAVE TAC FILE CHANGES): Returns to the GEDIT main menu, saving a new version of the elevation file. This new version will include any changes that you make to the elevation data.

- QUIT (DEL TAC FILE CHANGES): Returns to the GEDIT menu but does NOT save elevation changes and does not create a new version of the elevation file.

- MODIFY ELEVATION POINT: Enables you to find and modify an elevation data value.

1. Pick MODIFY ELEVATION POINT.

2. Pick a point on the screen.

A yellow asterisk (*) will mark the selected location. Depending on the screen density of the elevation data, a box representing the borders of the elevation data cell may also appear. 


\section{Pick CONTINUE.}

You will see a message and prompt such as the following:

CURRENT CELL VALUE: 216 meterB

ENTER NEW CELL VALUE (〈CTRL-Z> TO ABORT) :

4. Enter a new value (or press CTRL- $Z$ to avoid entering a new value).

口 MODIFY GROUPED ELEV(ation) POINTS: Displays a submenu that enables you to modify all cell values within a specified polygon area or within the current screen area:

\section{EXIT \\ HELP \\ CANCEL \\ CONTINUE \\ CURRENT SCREEN \\ DRAW POLYGON \\ SELECT POLYGON \\ EDIT POLYGON CELL VALUES}

The principal options of this submenu are explained below.

- CURRENT SCREEN: Selects the currently displayed screen area as a polygon within which ycu can change elevation cell values. The menu item will blink in green as long as the current screen is the selected polygon. (See EDIT POLYGON CELL VALUES, next page.)

- DRAW POLYGON: Enables you to define a polygon within which you can change elevation values.

1. Pick DRAW POLYGON.

2. Pick three or more points that define the sides of a polygon.

GEDIT will draw segments between each new point and the previous point.

3. Pick CONTINUE to complete the polygon.

GEDIT will draw the final segment between the first and last points picked. The polygon will blink in green to indicate that the polygon is selected. The polygon can then be used for EDIT POLYGON CELL VALUES. 
- SELECT POLYGON: Enables you to pick an existing polygon within which you can change elevation values.

\section{Pick SELECT POLYGON.}

The menu item will blink in green to indicate that further input is required.

2. Pick any point on a polygon displayed on the screen.

The polygon will blink in white to indicate a pick. If the wrong polygon is highlighted, pick again on the desired polygon until it is highlighted.

3. Pick CONTINUE to confirm the polygon selection.

The polygon will blink in green to indicate that the polygon was properly selected. The menu option will stop blinking to indicate that no further input is required for polygon selection. The polygon can then be used with EDIT POLYGON CELL VALUES.

- EDIT POLYGON CELL VALUES: Enables you to enter one elevation value for all cells within the currently selected polygon (which can be the entire screen area).

1. Identify an area by using one of the three menu options: CURRENT SCREEN, DRAW POLYGON, Or SELECT POLYGON.

\section{Pick EDIT POLYGON CELL VALUES.}

You will see this prompt:

ENTER NEW CELI VALUE (<CTRL-Z> TO ABORT) :

3. Enter an elevation value (or press CTRL-Z to cancel the request).

If you enter an elevation value, that value will apply to all cells within the selected polygon. 
- RETURN ELEVATION: Displays the elevation at a selected point.

1. Pick Return elevation.

2. Pick a point on the screen.

A yellow asterisk (*) will mark the selected location. Depending on the screen density of the elevation data, a box representing the borders of the elevation data cell may also appear.

The elevation of the selected point (center point of the cell) will be displayed.

D RETURN SLOPE: Displays the absolute difference between the elevation values at two selected points.

1. Pick RETURN SLOPE.

2. Pick a point on the screen.

A yellow asterisk (*) will mark the selected location. Depending on the screen density of the elevation data, a box representing the borders of the elevation data cell may also appear.

3. Pick CONTINUE.

4. Pick a second point on the screen.

A second yellow asterisk will appear.

5. Pick CONTINUE again.

A line connecting the two points will be drawn. The absolute difference between the elevation values will be displayed on the terminal screen.

ENABLE ELEVATION RESOLUTION: Shows the resolution of the elevation data by drawing white grid lines around the elevation data cells.

To show the elevation data grid, just pick ENABLE ELEVATION RESOLUTION. If the current display screen requires more than 50 rows or columns of cells in the elevation data grid, you will see a warning message and a count of the number of rows and columns: 


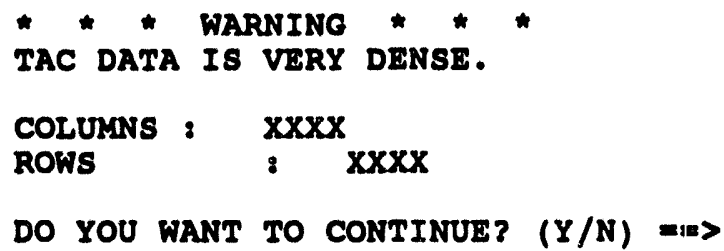

Because displaying a dense elevation data grid can effectively obliterate the other displayed data, you should consider answering "No" and zooming in to a smaller area before displaying the elevation grid.

DISABLE ELEVATION RESOLUTION: Turns off the capability to display a line grid of elevation data cells.

This option does not erase currently marked elevation cells, but the markers will disappear the next time the screen is redrawn.

DISPLAY ELEVATION STATISTICS: Displays information about the elevation data with respect to 1) the base map and 2) the current view (screen display).

EXAMPLE: When the Base Map and the Current View are the Same

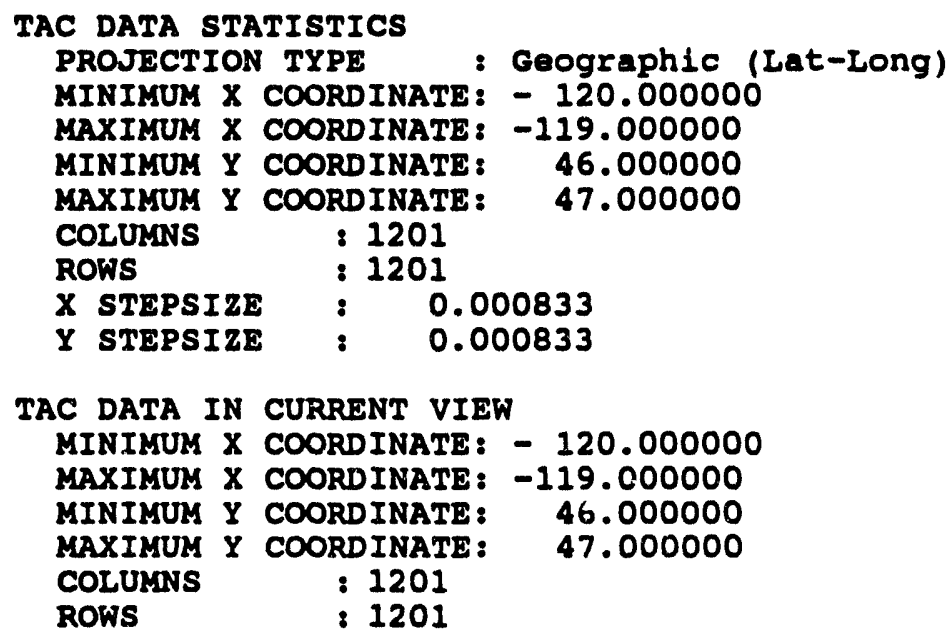

DISPLAY ATTRIBUTES: Displays the attributes associated with a selected data item.

1. Pick DISPLAY ATtRIBUTES. 
2. Pick a point on or near the desired point or composite string.

The data item will blink, and if it has attributes associated with it, they will be displayed in a table. For example, this table displays information on two attributes:

$\begin{array}{ccccc}* & \text { MAJOR } & \text { MINOR } & \text { PARAM } & \text { DESCRIPTION } \\ 1 & 7 & 150 & 0 & \text { Seaplane ramp or landing area } \\ 2 & 8 & 7 & 6 & \text { NO ENTRY IN ATTRIBUTE DICTIONARY }\end{array}$

where \# is the index number and the following four columns list each attribute (the Major code, the Minor code, and the Parameter value) plus a text description of the data item (if a description exists in the attribute dictionary). Notice that the second set of attributes has "NO ENTRY IN ATTRIBUTE DICTIONARY": that is, the attributes $8 ; 7 ; 6$ might have some meaning for the person who created the data item, but they are not in the attribute dictionary (the master list of attributes) normally used by GEDIT. (See Appendix B for a list of attributes in the attribute dictionary.)

3. Repeat step 2 for any other data item.

As you continue to pick data items, old attribute information is retained on the text screen. To clear the screen and make it easier to read, press the D ERASE key.

4. Pick any other submenu item to end the DISPLAY ATTRIBUTES function.

The following four viewing functions are included in the EDIT ELEVATION FILE menu for convenience. Their functions are described in detail as options of the GEDIT main menu.

ZOOM IN: Enlarges a square portion of the displayed area, as described for the same option on the main menu.

ZOOM OUT: Reduces the currently displayed area to a square area within a larger displayed area, as described for the same option on the main menu.

RESTORE BASE PLOT: Redraws the base plot in the display area, as described for the same option on the main menu.

REFRESH SCREEN: Redraws the current display, as described for the same option on the main menu. 


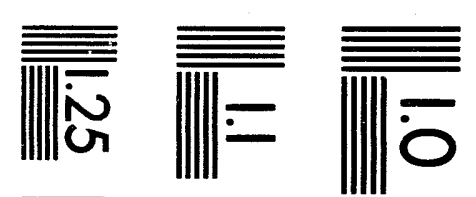

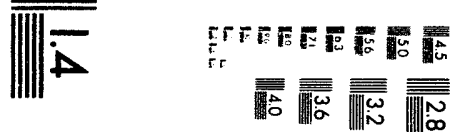

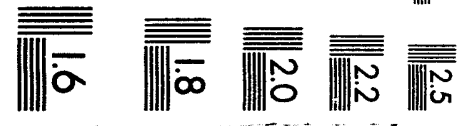



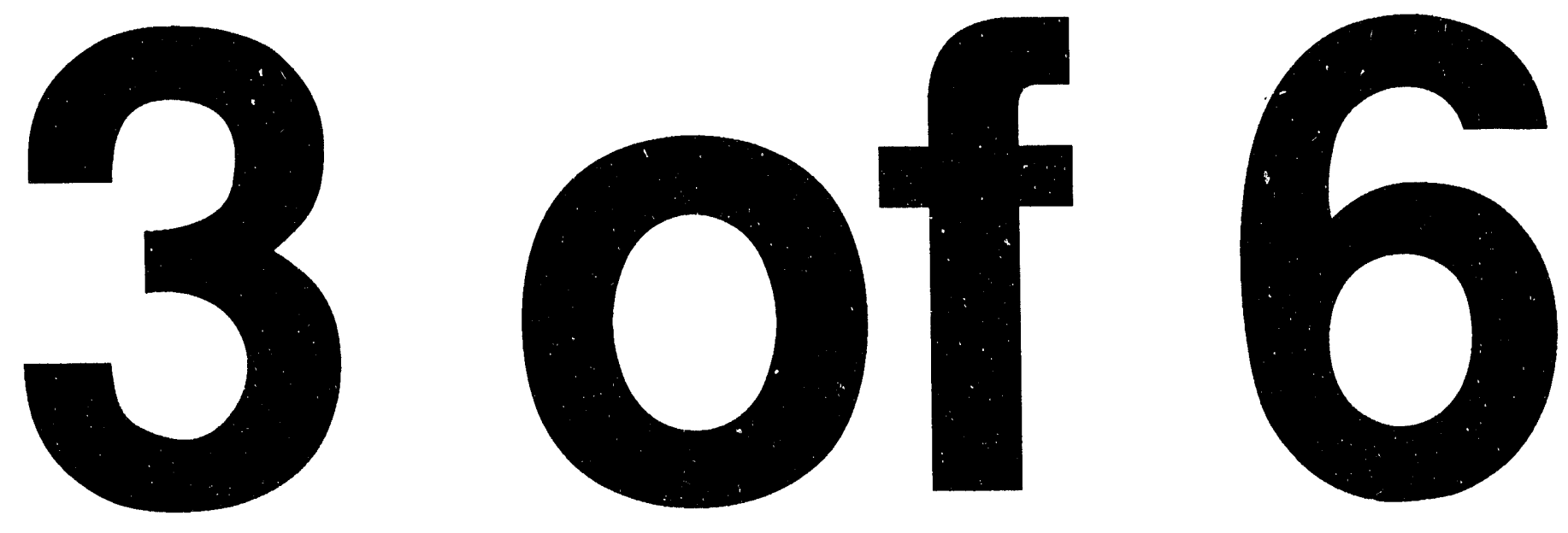
The following viewing functions are included in the Misc(ellaneous) Menu for convenience. All these functions except MAP ZOOM are described in detail as options of the GEDIT main menu.

ZOOM IN: Enlarges a square portion of the displayed area, as described for the same option on the main menu.

ZOOM OUT: Reduces the currently displayed area to a square area within a larger displayed area, as described for the same option on the main menu.

- MAP ZOOM: Centers the displayed area at a specified long-lat point and a specified radius of display. This function should be used only when the input files contain map data in longitude-latitude. Hint: You can use the COORDINATE PICK function to find the longitude-latitude of a point on the display and to determine distances that could be used for a display radius.

\section{Pick MAP ZOOM.}

GEDIT will query for the radius (in miles) and the center point (long-lat) of the area to be displayed. (If you have used the COORDINATE PICK function previously, the longitude-latitude of the last-picked point may be on the text screen, if other functions have not used the screen for other purposes.)

2. Type the radius, the longitude, and the latitude (separated by commas or spaces).

(Pressing CTRL-Z will exit and abort the MAP ZOOM function.)

\section{Press RETURN.}

GEDIT will redraw the displayed area with the specified point at the center. If you do not see what you expected, recheck the radius and the longitude-latitude values.

RESTORE BASE PLOT: Redraws the base plot in the display area, as described for the same option on the main menu.

REFRESH SCREEN: Redraws the current display, as described for the same option on the main menu. 


\section{Modify Defaults}

Picking this option results in a submenu for modifying several default items:

$$
\begin{aligned}
& \text { EXIT } \\
& \text { HELP } \\
& \text { CHANGE DEFAULT COLOR } \\
& \text { CHANGE DEFAULT FILE } \\
& \text { CHANGE DEFAULT LINE TYPE } \\
& \text { MODIFY BASE WINDOW }
\end{aligned}
$$

The principal options of this submenu are explained below.

- CHANGE DEFAULT COLOR: Enables you to change the color that is automatically used for string insertion, color flooding, and polygon definition.

During insertion, flooding, and polygon definition, lines are drawn with the default color (pen \#). Picking CHANGE DEFAULT COLOR enables you to select a new default pen number $(0-255)$ through the following submenu:

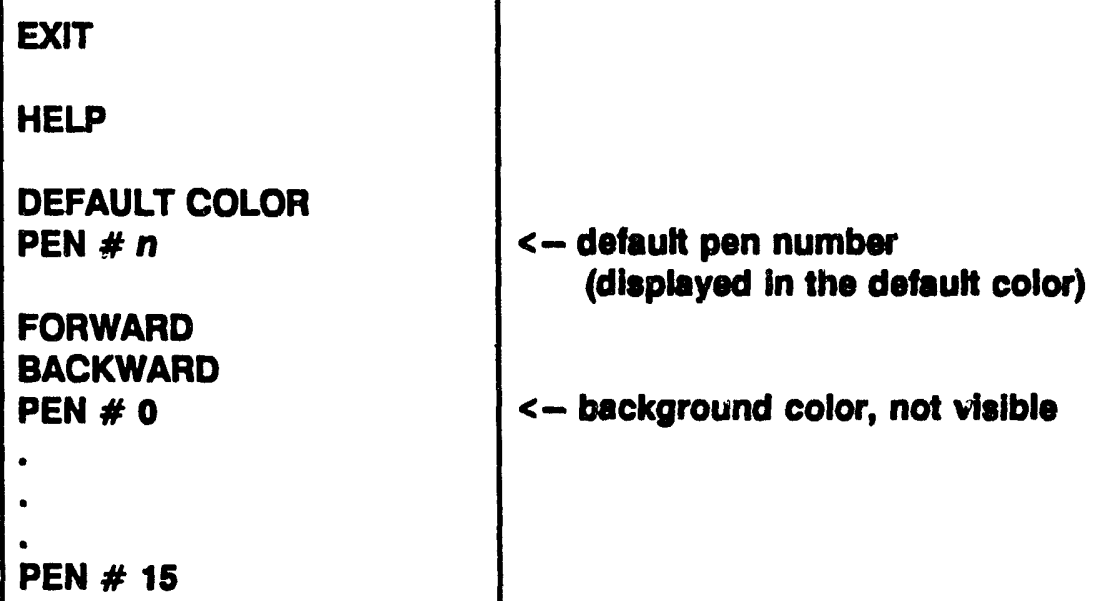

<- default pen number

(dlaplayed in the default color)

<- background color, not visible

The default color entry at the top of the menu is provided for reference and is displayed using the default color. Each PEN \# $x$ item on the menu is displayed in the corresponding color.

- FORWARD (or BACKWARD): Displays the next (or previous) 16 pen colors in the menu area. There are 256 possible colors with pen numbers that range from 0 to 255 . 
D PEN \# $X$ : Causes $X$ to become the default color, and the DEFAULT COLOR item at the top of the menu is redisplayed in that color. (Note that pen \# 0 is the hackground color and will not be visible. However, pen \# 0 can be selected by picking the appropriate area on the menu. To check that pen \#0 has been selected, watch the DEFAULT COLOR item. If it disappears, the default color has become pen \# 0 .

CHANGE DEFAULT FILE: Allows selection of a different file for insertions and pasted regions.

When multiple files are being edited, insertions and pasted regions only modify the current default file. CHANGE DEFAULT FILE enables you to select a new default file so that insertions will be associated with the appropriate file. Picking CHANGE DEFAULT FILE displays the following submenu:

\begin{tabular}{l}
\hline (default file name) \\
EXIT \\
$($ file \# 1) \\
$($ file \# 2) \\
$:$ \\
\hline \\
(file \# n)
\end{tabular}

The name of the current default file is listed at the top of the menu for reference and is displayed in a different color than the rest of the menu. Picking file \# $n$ causes that file to become the Default File, and the first line of the menu will be rewritten with the selected file name.

CHANGE DEFAULT UNE TYPE: Enables you to change the type of line that will be used for subsequent drawing and inserting. Picking CHANGE DEFAULT LINE TYPE displays the following menu. 


EXIT
HELP
DEFAULT LNE TYPE =
(dofaut IInO tYPO)
SOLD
DOTTED
DASH DOT
DASH
DOT DOT DOT DASH
WIDE LINE
EXTRA WIDE LNE

The current default line type is listed at the top of the menu for reference and is displayed in a different color than the rest of the menu.

Picking EXIT terminates the command and returns control to the MODIFY DEFAULTS menu. Picking any other item on the submenu causes the selected line type to become the new default line type, listing it at the top of the menu.

MODIFY BASE WINDOW: Used to alter the minimum and maximum location values for restoring and displaying the base plot.

Picking MODIFY BASE WINDOW results in the following prompt for new base limits:

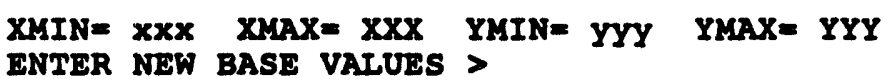

Press CTRL-Z to abort the function, or enter new limits by typing

1. minimum horizontal value (followed by a comma or RETURN)

2. maximum horizontal value (followed by a comma or RETURN)

3. minimum vertical value (followed by a comma or RETURN)

4. maximum vertical value (followed by RETURN).

If the display data has been registered to known longitude-latitude values, then these limits would be longitude ( $y$ and $Y$ ) and latitude ( $x$ and $X)$ values. If the display data has not been registered to a specific coordinate system, then these base window limits refer to an arbitrary default system of horizontal (x) and vertical $(y)$ coordinates. 


\section{Polygon Fill Menu}

The POLYGON FIL MENU enables you to fill the areas inside a polygon with a color or pattern. The pattern type, pattern spacing, and fill color can all be specified. Any polygon can have polygon fill characteristics associated with it.

Picking POLYGON FILL MENU results in the following submenu for controlling the filling of polygon areas with a color pattern:

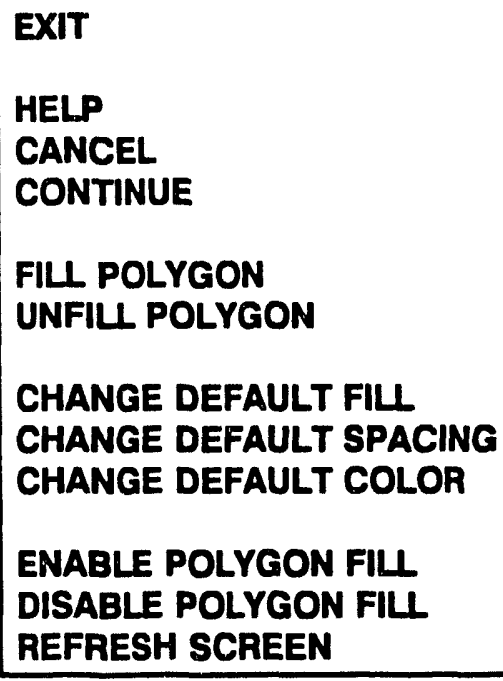

The principal options of this submenu are explained below.

FIL POLYGON: Associates fill characteristics with a polygon.

1. Pick FILL POLYGON.

2. Pick a point on or near the polygon to be filled.

The selected polygon will blink. If no polygon is blinking or if the wrong polygon is blinking, pick another point that is closer to the desired polygon.

3. Pick CONTINUE.

The selected polygon will disappear temporarily. GEDIT will respond with the following prompt for pen (color), fill (pattern), and pattern spacing (space between lines in the fill pattern).

ENTER PEN \#, FILL \#, \& SPACING > 
4. Press CTRL- $Z$ to accept the current default values or type in three numerical values (separated by commas or RETURNs) for

pen \# (0 to 255 -- see Modify Defaults, page 2.148)

fill \# (0 to 6 -- see Change Default Fill, below)

spacing (pattern line spacing in inches)

Example:

ENTER PEN *, FILI * _ SPACING >6,6,.25 <RETURN>

After you press RETURN, GEDIT will fill the polygon, and those fill characteristics will be associated with the polygon. In the example, the polygon fill would be an aqua (6) crosshatch fill (6) with 1/4-inch (0.25) spacing.

UNFIU POLYGON: Cancels the fill characteristics associated with a polygon. Pick a polygon as above; then pick CONTINUE. If the polygon is already filled, the fill will be erased.

Change Default fILL: Used to change the default fill type. Picking CHANGE DEFAULT FILL results in the following submenu:

\begin{tabular}{|l|}
\hline EXIT \\
HELP \\
DEFAULT FIL = \\
(defauIt FII tYPE) \\
O SOLID FIL \\
1 HORIZONTAL FIL \\
2 VERTICAL FIL \\
3 BOX FIL \\
4 45 DEGREE FIL \\
5 135 DEGREE FILL \\
6 CROSSHATCH FIL \\
\hline
\end{tabular}

The top line shows the current default fill. This can be changed by picking any of the entries $0-6$. If a different type is selected, the top line will change to reflect the new default fill type. Picking EXIT returns you to the Polygon Fill Menu. 
CHANGE DEFAULT SPACING: Used to change the spacing between the lines of the fill pattern.

\section{Pick Change default SPACing.}

The current spacing will be printed on the text screen along with a message requesting a new spacing number.

2. Type a new spacing number and press RETURN, as in the example below.

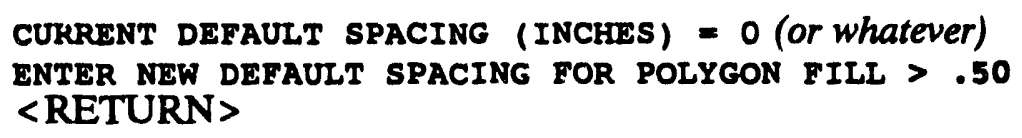

The numbers entered are interpreted as inches relative to the graphics screen coordinates. In this example, the spacing of 0.50 will result in $1 / 2$ inch spacing between the lines in the fill pattern.

CHANGE DEFAULT COLOR: Used to change the default color for further polygon definitions (or other operations). The CHANGE DEFAULT COLOR function is included in this menu for convenience and operates as described in the MODIFY DEFAULTS menu (see page 2.148).

ENABLE POLYGON FIL: Fills all polygons that have fill characteristics. A polygon to be filled must have already been associated with a fill color and pattern.

- DISABLE POLYGON FILL: Disables the automatic filling of polygons, negating the ENABLE POLYGON FIL command. You can still use the FILL POLYGON function. To erase prior polygon fills, pick DISABLE POLYGON FILL; then pick REFRESH SCREEN.

- REFRESH SCREEN: Redraws the current display, just as in the main menu. The REFRESH SCREEN function is included in this menu for convenience in using the Polygon Fill Menu functions. 


\section{Re-Color-Texture String}

This function changes the color and texture (line type) of a composite string to the "default" color and line type. Therefore, it is important to first pick the desired "default color" and the desired "default line type" (see page 2.148, MODIFY DEFAULTS). After setting the desired defaults:

1. Pick RE-COLOR-TeXtuRe String.

2. Pick a point on or near the desired string.

The composite string will then blink. If this is not the desired string, pick a different one.

3. Pick CONTINUE to modify the color and texture of the string to the default values. 


\section{Refresh Screen}

Picking REFRESH SCREEN redraws the current display.

This command will restore display elements that might have been damaged, for instance, when an overlapping string was deleted. This command can also be useful for removing display elements that you no longer wish to display, such as text after text display has been disabled (see Text Menu in this section). 


\section{Restore Base Plot}

Initially the base plot is the area displayed when the main menu is first presented. The base plot is defined by the minimum and maximum values of the input data. These limits can be reset by using the MODIFY BASE WINDOW command (see page 2.148, MODIFY DEFAULTS).

Picking RESTORE BASE PLOT causes the base plot to be redrawn in the display area of the screen. This function might be used after zooming, for instance, to restore the display to its original scale and location. 


\section{Text Menu}

Picking Text Menu results in the following submenu for manipulating text strings:

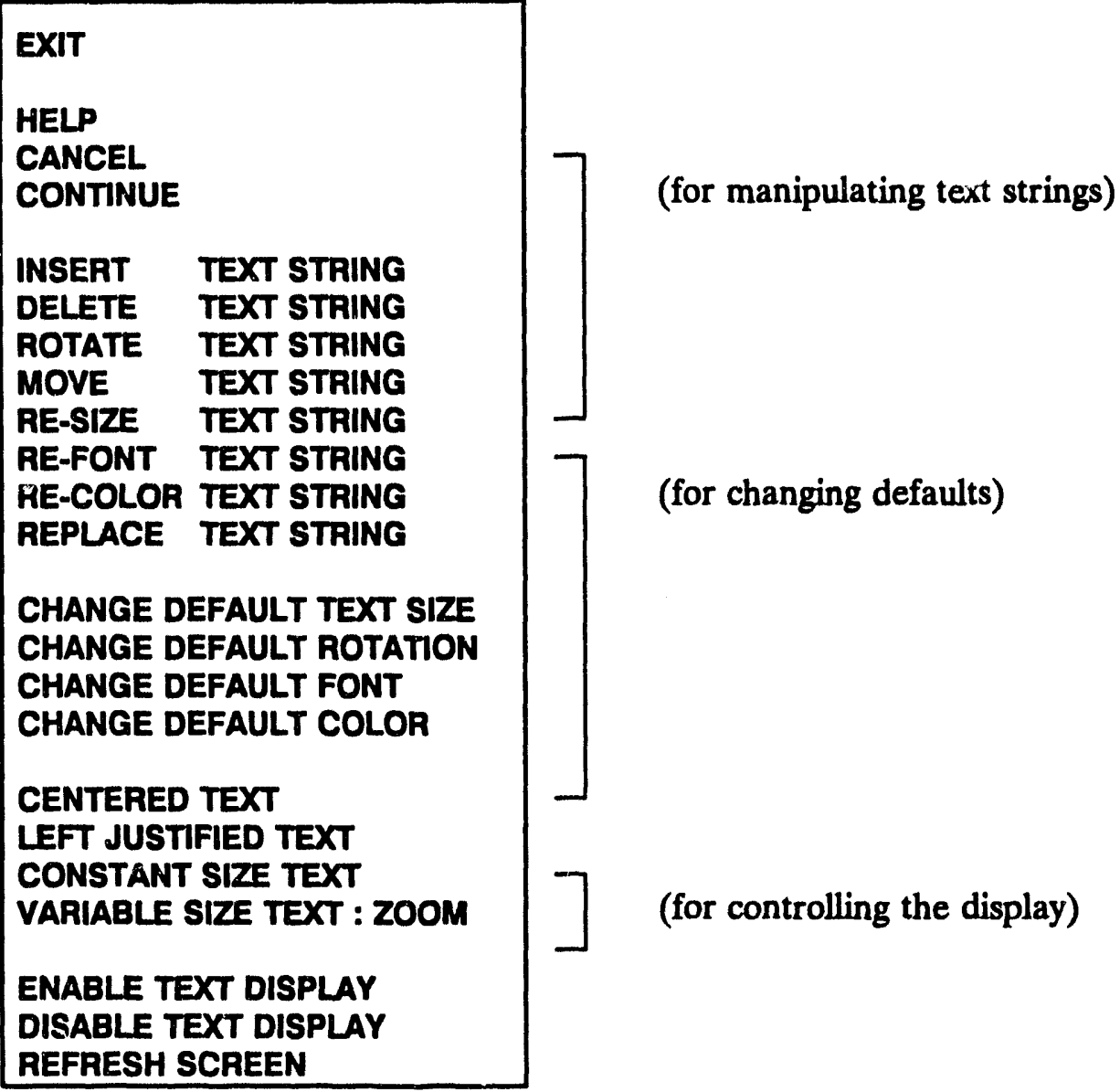

Several functions on this submenu require you to pick a text string (on the display) for subsequent operations. This is the procedure:

To Pick a Text String:

1. Use the graphics cursor to pick a point on the text (usually near the bottom middle of the first line of text, unless the text has been centered.)

A small point indicator will begin blinking to indicate which line of text has been picked.

2. Pick CONTINUE.

GEDIT will then continue with the selected function. 
The principal options of the Text Menu are explained in the following list, which has been grouped according to the annotations on the menu above.

\section{Manipulating Text Strings}

INSERT TEXT STRING: Used to insert text into the display.

1. INSERT TEXT STRING.

GEDIT will display the following prompt:

ENTER TEXT IEN, SIZE, ROTATION, AND FONT >

2. Press CTRL-Z to accept the current default values for text pen (color), size, rotation, and font type, or type new values (separated by commas or spaces) followed by RETURN.

The required parameters are

Text pen: a number indicating the text color ( 0 to 15$)$

Text size: inches or decimal fractions of inches (greater than 0 )

Rotation: 0 - 360 degrees, clockwise from horizontal

Font type: 0 - 4:

0 = Sitick

1 = Enhanced Stick

2 = Script

3 = Roman Triplex

$4=$ Gothic

When you start GEDIT, the default values are 0.1-inch text size, 0 degrees rotation, and 0 (Stick) font type. These default items can be changed using the corresponding CHANGE DEFAULT items in this submenu.

Next GEDIT will displey a prompt for lines of text:

ENTER LINE OF TEXT >

3. Type a line of text, followed by RETURN.

GEDIT will repeat the prompt for text input.

4. Repeat step 3, entering each text line (up to 5 lines) until the text string is complete. 
5. After you are done entering strings of text, press CTRL-Z in response to the text input prompt.

6. Pick a point on the display where the text string is to begin.

Once the position has been selected, GEDIT will determine if a text file already exists for the default data file. If a text file exists, the new text string will be inserted into that file.

If a text file does not exist, GEDIT will prompt you for the name of a text file:

Enter TEXT file name >

7. If you see this prompt, either a) enter a text file name of 7 characters or less (with no filename extension) or b) press RETURN or CTRL-Z to create a text file that has the same name as the current DMS data file but with a ".TXT" extension.

GEDIT puts the tex, file in your default directory and then inserts the name of the text file into the current DMS data file.

DELETE TEXT STRING: Allows deletion of a picked text string.

1. Pick Delete TEXT STRING.

2. Pick the texi string to be deleted. Then pick CONTINUE.

GEDIT will remove the text string from the display (and from the appropriate text file).

ROTATE TEXT STRING: Allows input of a degrees value for rotation of the text string on the display.

1. Pick ROTATE TEXT STRING.

2. Pick the text string to be rotated. Then pick CONTINUE.

GEDIT will display the following prompt:

CURRENT TEXT ROTATION (IN DEGREES) $=0.0$ (Or whatever) ENTER NEW TEXT ROTATION (IN DEGREES) >

3. Type a rotation value, in degrees clockwise from horizontal $(0-360)$, followed by RETURN. (Negative degrees are counterclockwise.)

The text string will be erased, redrawn, and rotated by the desired amount. 
- MOVE TEXT STRING: Allows relocation of a text string within the display area.

1. Pick MOVE TEXT STRING.

2. Pick the text string to be moved. Then pick CONTINUE.

3. Pick a point on the screen where the text is to be relocated.

The text will be erased and redrawn at the new location.

RE-SIZE TEXT STRING: Used to modify the size of an existing text string.

1. Pick RE-SIZE_TEXT_STRING.

2. Pick the text string to be re-sized. Then pick CONTINUE.

GEDIT will display the following prompt:

CURRENT TEXT SIZE (IN INCHES) $=0.1000$ (or whatever)

ENTER NEW TEXT SIZE (IN INCHES) >

3. Type in a new text size value $(0<$ size $\leq 10)$, and press RETURN.

GEDIT will erase the text string and redraw it at the entered size.

RE-FONT TEXT STRING: Used to redraw an existing text string in a different font (typeface).

1. Pick RE-FONT TEXT STRING.

2. Pick the text string to be drawn in a different font. Then pick CONTINUE.

GEDIT will display the following prompt:

ENTER NEW FONT TYPE:

3. Type a new font value $(0-4)$ and press RETURN.

The text string will be redrawn in the new font. (See the description of CHANGE DEFAULT FONT in this section for a list of fonts and their corresponding integer values.)

RE-COLOR TEXT STRING: Used to redraw a text string in a different color.

1. Pick RE-COLOR TEXT STRING. 
2. Pick the text string to be re-colored. Then pick CONTINUE.

Enter text color $[1]=>$

3. Enter a pen number.

The text string will be erased and redrawn in the new color.

REPLACE TEXT STRING: Used to replace one text string with another at the same location.

\section{1. heplace text StRing.}

GEDIT will respond with the prompts to specify a pen (color), size, rotation, font, and new text.

2. Enter text, following the same procedure used for INSERT TEXT STRING.

3. After pressing CTRL-Z to signal the last line of new text, pick the text string to be replaced. Then pick CONTINUE.

The selected text string will be erased and replaced with the new text.

\section{Changing Defaults}

Changing the default values for text size, rotation, font, and color will affect these characteristics for any new text strings. Changing the default color will affect any new data element.

CHANGE DEFAULT TEXT SIZE: Displays the current default text size (in inches) and prompts for a new default value. Type a new value followed by RETURN or press CTRL-Z to abort the function and retain the current value.

ChANGE DEfAULT ROTATION: Displays the current default rotation (in clockwise degrees) and prompts for a new default value. Type a new value followed by RETURN or press CTRL-Z to abort the function and retain the current value.

- CHANGE DEFAULT FONT: Displays the following submenu for selecting an alternative font: 


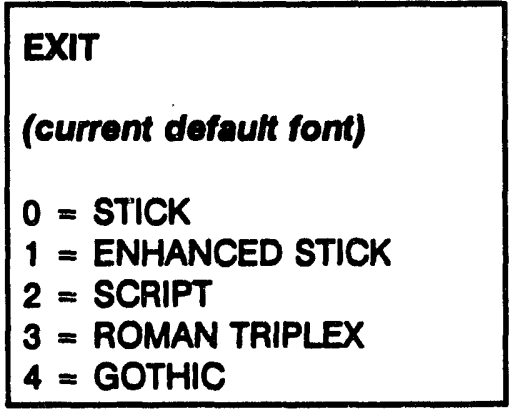

Pick a new value or exit to retain the current value. The new default font will be listed in the submenu. Some fonts may not support all ASCII characters, especially punctuation.

- CHANGE DEFAULT COLOR: Displays a submenu for selecting an alternative default color. This function is included in the Text Menu for convenience and operates as described for the MODIFY DEFAULTS option of the main menu (see page 2.148).

The text that you insert may be left justified (the initial default) or centered. The text may also be variable size (the initial default) or constant size.

CENTERED TEXT: Causes any new text string to be centered about the location selected for the text string.

- LEFT JUSTIFIED TEXT: Causes any new text string to begin at the location selected for the text string, on a line defined by the selected point and the text rotation.

a CONSTANT SIZE TEXT: Causes any new text string to be displayed at the default text size, regardless of any zooming or changes in the scale of the display area.

- VARIABLE SIZE TEXT - ZOOM: Causes any new text to change in size when the size of the display area changes (that is, the text string will be affected by zooming). 


\section{Controlling the Display}

The ENABLE TEXT DISPLAY and DISABLE TEXT DISPLAY functions are on/off switches for telling GEDIT whether or not to display available text.

- ENABLE TEXT DISPLAY: Turns on the capability to display any text information associated with the input files, and draws the text on the display. By default, text display is disabled upon entering GEDIT.

(Text will not be displayed if the currently displayed area is considerably larger than the scale at which the text was originally entered. If expected text is not displayed when you pick ENABLE TEXT DISPLAY, use a zoom function from the main menu to magnify the display to a scale that is close to that used when the text was entered. The text should then appear on the display.)

DISABLE TEXT DISPLAY: Turns off the capability to display text information.

Any text on the screen will remain there until the screen is redrawn (for example, by picking REFRESH SCREEN).

- REFRESH SCREEN: Redraws the current display, just as in the main menu. The REFRESH SCREEN function is included in this menu for convenience in using the Text Menu functions. Use the REFRESH SCREEN option from the main menu to subsequently erase disabled text. 


\section{Text String Menu}

Picking TEXT STRING MENU results in the following submenu for manipulating text strings:

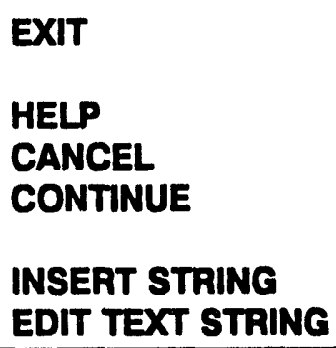

The principal options of this submenu are explained in the following list.

INSERT STRING: Used to insert a text seed point and text associated with that point location. This text is normally invisible and can only be viewed by using attribute selection functions (see ATTRIBUTE MENU, page 2.108). This text will NOT be displayed when you pick ENABLE TEXT DISPLAY on the Text Menu. This can be useful in associating text with certain topographic features, such as different portions of a road or different areas of a lake.

1. Pick INSERT STRING.

GEDIT will display the following prompt:

ENTER Line of string >

2. Type a line of text, followed by RETURN.

3. Repeat step 2, entering up to a total of 5 lines of text.

4. When you are done entering text, press CTRL- $Z$ in response to the text input prompt.

5. Position the cross-hairs to the desired area of the map, and pick a point location.

- If there is no text file associated the current "edit file," the system will inform you and request a TEXT file name:

No TEXT file associated with filename.dms

Enter TEXT file name > 
- If you see this prompt, enter a file name for the text.

A seed-point marker (a white asterisk) will appear at that point, and the function is complete.

EDIT TEXT STRING: Enables you to add or replace the text associated with a text seed point. If no text is associated with the given seed point, the new text will simply be added to the other attributes associated with the point. If on the other hand, there is already text associated with the seed point, the new text will replace the old text. In other words, you may only have one text string associated with a point at a time. For a complete mapping of all seed points for possible editing, you need to first ENABLE POINT data (see ATTRIBUTE MENU, page 2.108).

1. Pick EDIT TEXT STRING.

GEDIT will display the following prompt:

ENTER Line of otring >

2. Type a line of text, followed by RETURN.

3. Repeat step 2, entering up to a total of 5 lines of text.

4. When you are done entering text, press CTRL- $Z$ in response to the text input prompt.

Now you need to choose the seed point to be edited.

5. Position the cross-hairs to pick an existing point.

A seed-point marker will begin blinking.

6. If this is the correct point, pick CONTINUE.

If you wish a different point, repeat step 5 until you are satisfied with your selection. Then pick CONTINUE.

When the seed point marker stops blinking, the function is complete. 


\section{Unsave Composite String}

This option enables you to insert (into the screen display) a composite string that has been previously stored in a special holding area of computer memory called the save buffer.

The DELETE COMPOSITE STRING and RE-COLOR-TEXTURE STRING functions place a copy of a composite string into the save buffer when you pick a composite string to be deleted, recolored, or retextured. Once the picked composite string begins to blink, the copy is in the save buffer, whether or not you continue the deletion or recoloring/retexturing.

Note: Other functions also use the save buffer for temporary storage. If you want to use the string stored in the save buffer, retrieve it as soon as possible after storing it-before using another function that would replace the string with something else.

The following procedure is a typical sequence of functions that uses UNSAVE COMPOSITE STRING to copy a composite string.

To copy a composite string at another location on the screen display:

\section{Pick Delete COMPOSIte StRing.}

The menu item will blink in a second color.

2. Pick a point on or near the composite string to be deleted.

The composite string will be copied into the save buffer and then begin to blink on the screen.

3. Pick CANCEL so that you do not have to continue with the delete function.

The composite string will stop blinking, but the copy will be retained in the save buffer.

4. Pick UnSAVE COMPOSITE StRING.

The menu item will blink in a second color.

5. Pick the location on the display where the string is to be copied.

The composite string will be redrawn at that location. 


\section{Zoom In}

Pick ZOOM IN to enlarge a square portion of the displayed area.

1. Pick ZOOM IN.

2. Pick a point on the display at the center of the area that you want to enlarge.

3. Pick a second point that would lie on one side of the square area that you want to enlarge with the first point at the center.

After you define the area, GEDIT will redraw the display, enlarging the defined area to fill the display area.

If you are not satisfied with the area that you have enlarged, you can pick RESTORE BASE PLOT to redraw the area as it was first displayed (see RESTORE BASE PLOT in this section). 


\section{Zoom Out}

Pick ZOOM OUT to reduce the currently displayed area to a square within a larger area. Define the area to be reduced (scaled down) in the same manner as described for the ZOOM IN command:

1. Pick ZOOM OUT.

2. Pick a point on the map at the center of the area that you want to reduce (scale down).

3. Pick a point on the display that would lie on the edge of a square with the first point at the center.

The currently displayed area will be rescaled to the size of the square, and the center point of the square will be centered in the display area.

It is possible to zoom out beyond the maximum values of the input data, resulting in "blank" areas at the edges of the redrawn display window. You can always use the RESTORE BASE PLOT command to redraw the base plot display. 


\section{IBSCOPY}

The IBSCOPY utility is a general system utility that enables you to transfer files between IBS users on different computer systems over a network.

\section{What You Need to Know Before Using IBSCOPY}

To transfer files between computer systems, you must know the node names of the coinputer systems, the user names of the user(s) on each system, and the name of the source file to be transferred. You can also specify the name of the file at its destination. If you are transferring a file to or from a user other than yourself, you must know and use that user's password. If you are transferring a file to or from a different node other than the current one, you must know and use the appropriate password.

\section{Copying a File Between IBS Systems or Users}

To start the utility, enter IBSCOPY at the system prompt. The program presents the following series of prompts:

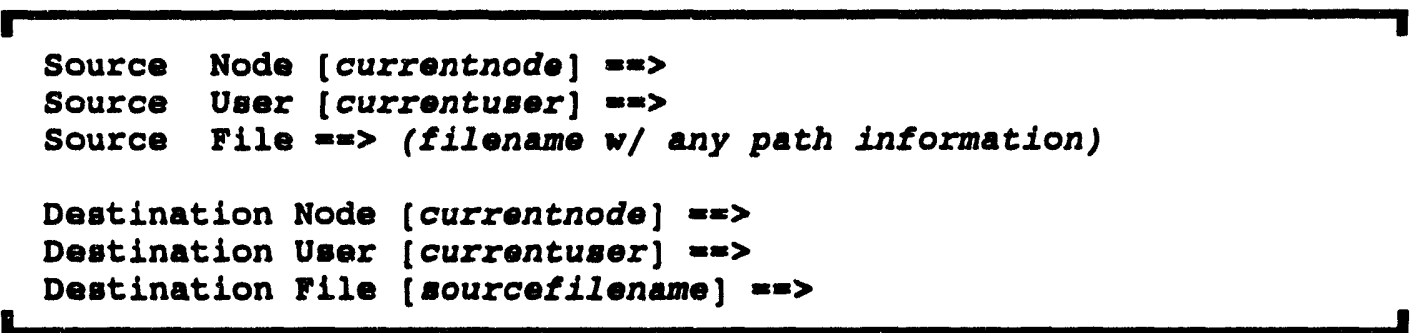

If either of the above nodes are not the current node, or if the users are not the current user, IBSCOPY prompts for the appropriate password, either another user's password or your password on the other node:

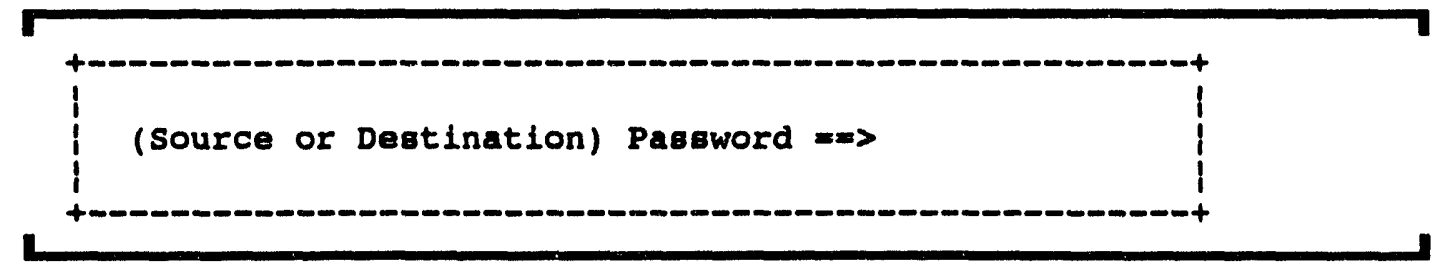


You are then prompted whether you wish to use FTP to copy files:

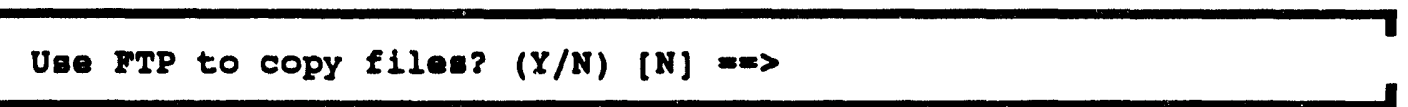

- Y uses FTP to copy files (used for Off-> On post tranfers). You would then see the following message and prompt:

Please Wait...
View FTP.OUT file? (Y/N) $=>$
If you respond Y, then the output of the FTP command is displayed.
N or RETURN uses the VMS COPY command to copy files
(likely used for all Off-> Off post tranfers over a DEC network). You would
then see messages similar to these, followed by the output of the VMS COPY
command:
\$ copY -
(source) -
(destination)
Please wait...
(output of VMS COPY command)




\section{IBSSH}

The IBSSH (IBS Shell) utility enables you to access many of the IBS utilities and other programs by using a menu-driven interface. It has one top-level menu that accesses nine submenus. Except for items in the Setup submenu, all options in all the other submenus run a specific utility or IBS program: the program names appear on the menu along with short descriptions. If you know the name of a program, it is much faster to just type its name at the system prompt because IBSSH does have some overhead associated with it. After running the selected utility, IBSSH starts again at the top-level menu.

To start IBSSH, just enter IBSSH at the system prompt. The IBS Shell then displays it top-level Main Menu:

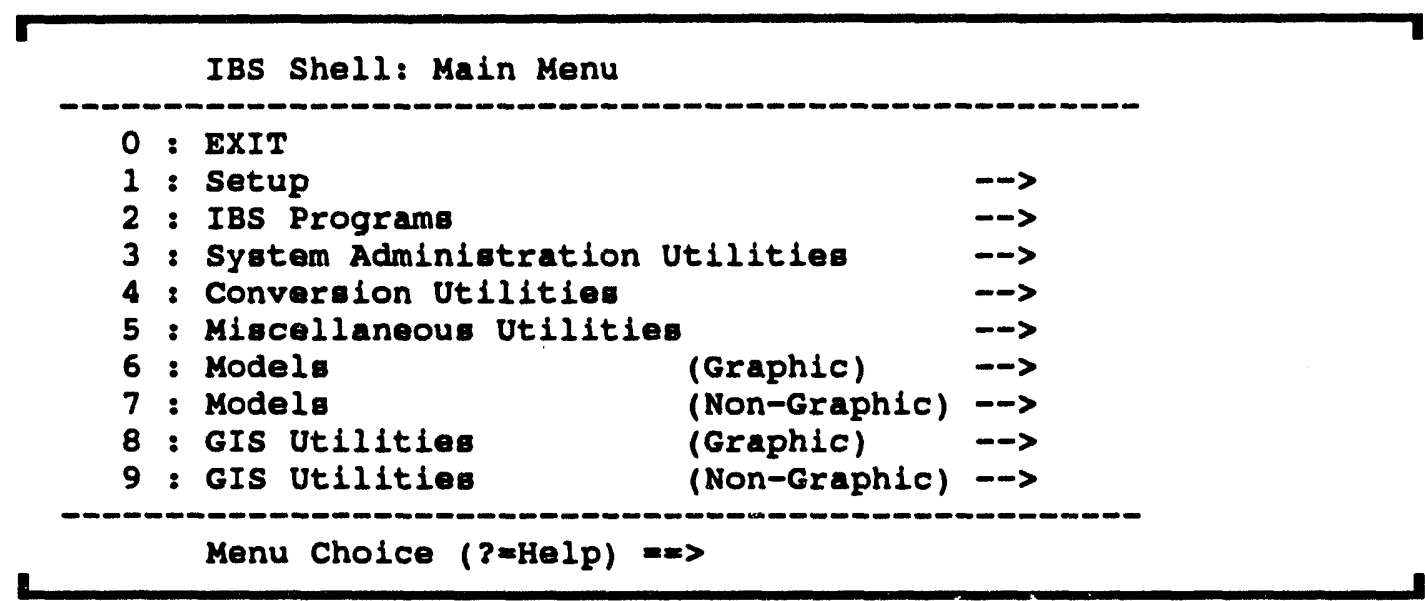

To start a program, choose one of the submenus and then choose the specific utility or program from the submenu. The submenus are illustrated here. In some cases, where the submenus would be split onto separate screens, the following illustrations have been expanded to show all menu options.

The menus labeled "(Graphic)" include programs for which a graphic terminal is required. 


\section{Setup Menu}

Items on this menu are described under SETUP in the IBS User Guide.

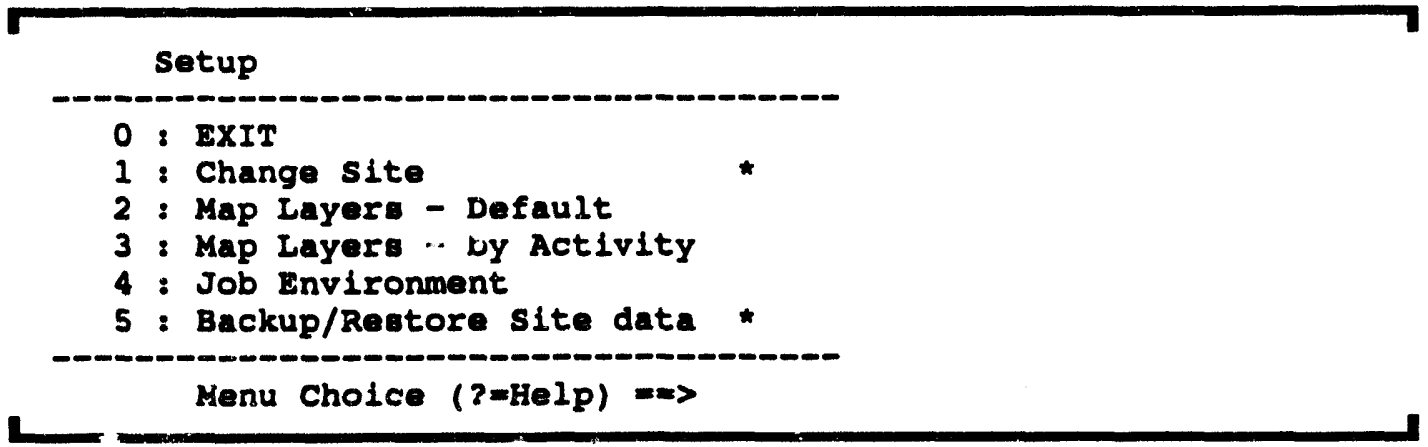

\section{IBS Programs Menu}

Items on this menu are described in the IBS User Guide.

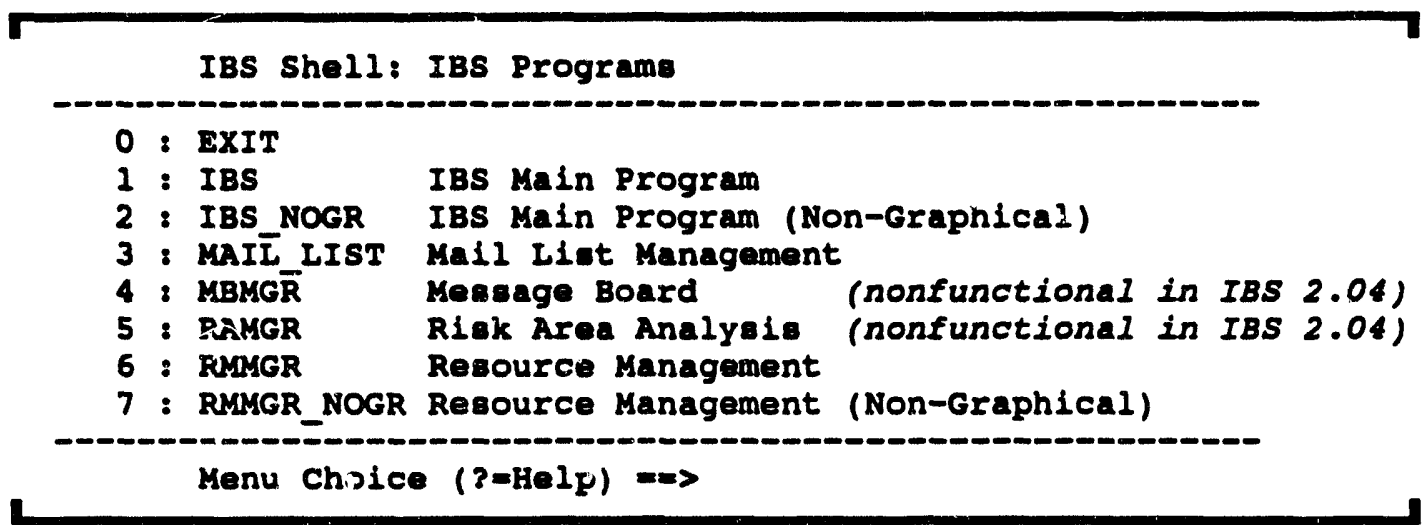




\section{System Administration Menu}

Items on this menu are described individually elsewhere in this Utilities Guide.

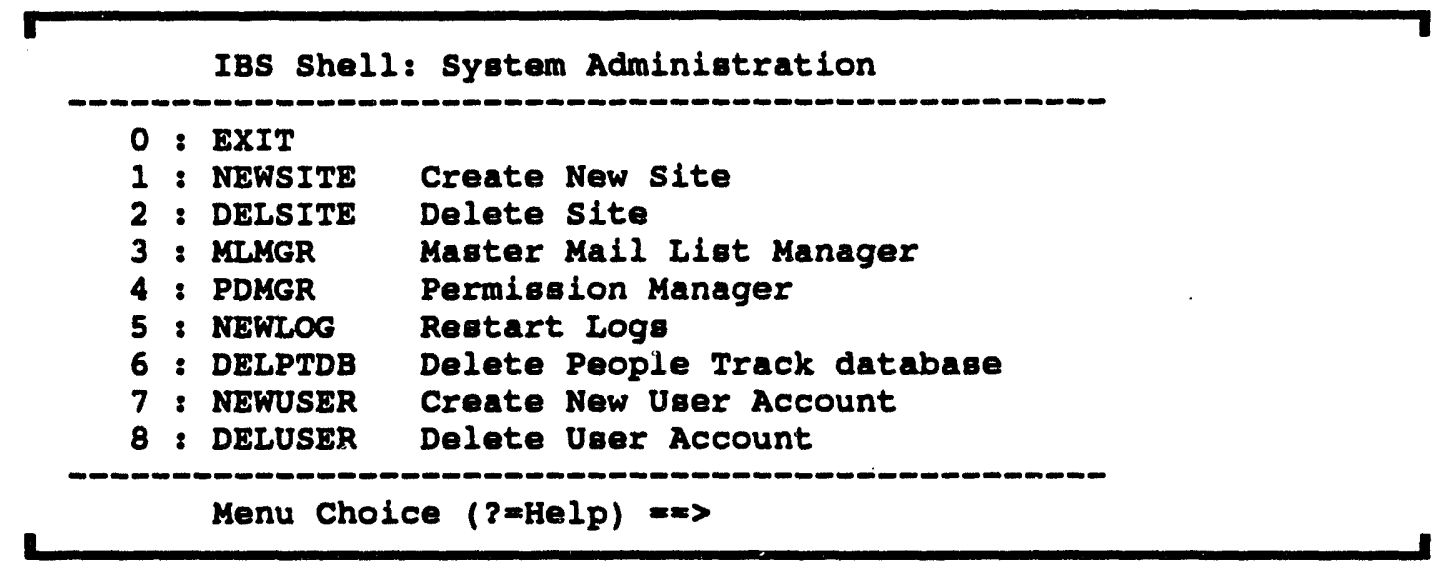

\section{Conversion Utilities Menu}

Items on this menu are described individually elsewhere in this Utilities Guide.

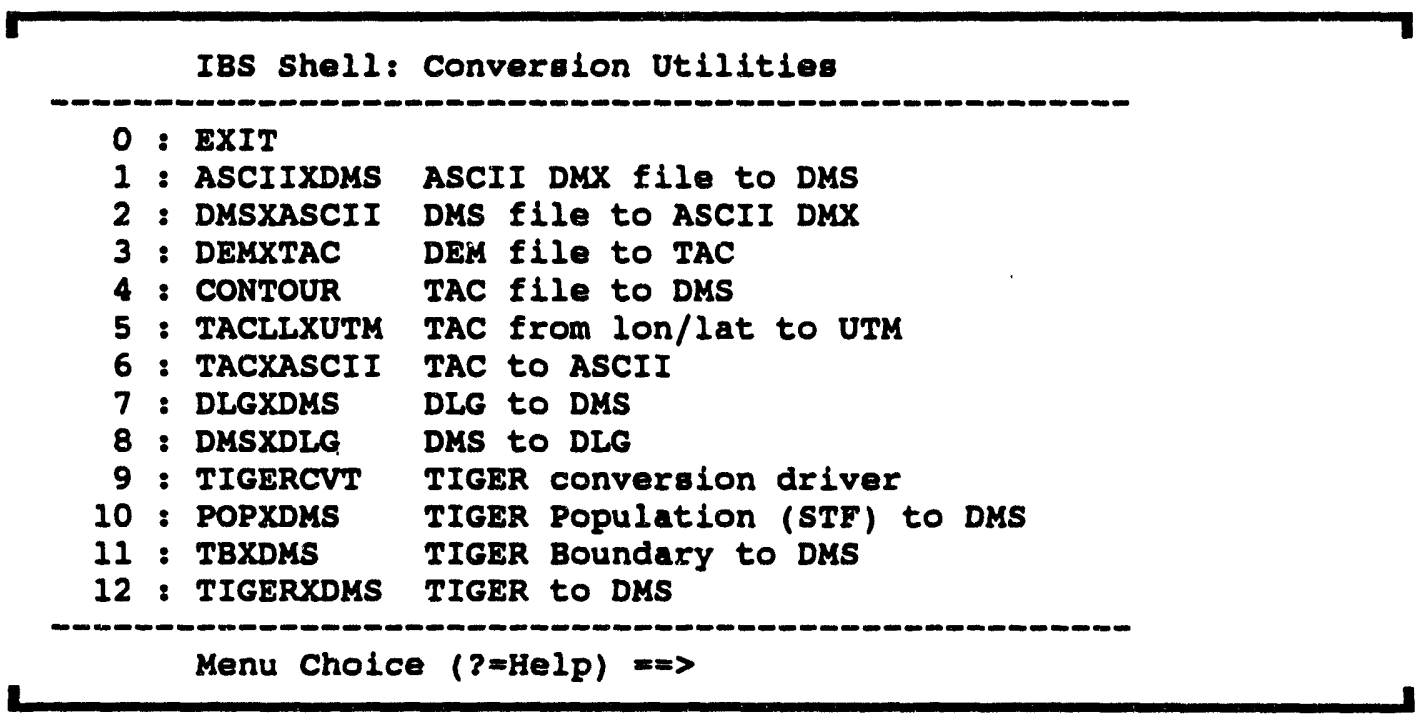




\section{Miscellaneous Utilities Menu}

Items on this menu are described individually elsewhere in this Utilities Guide.

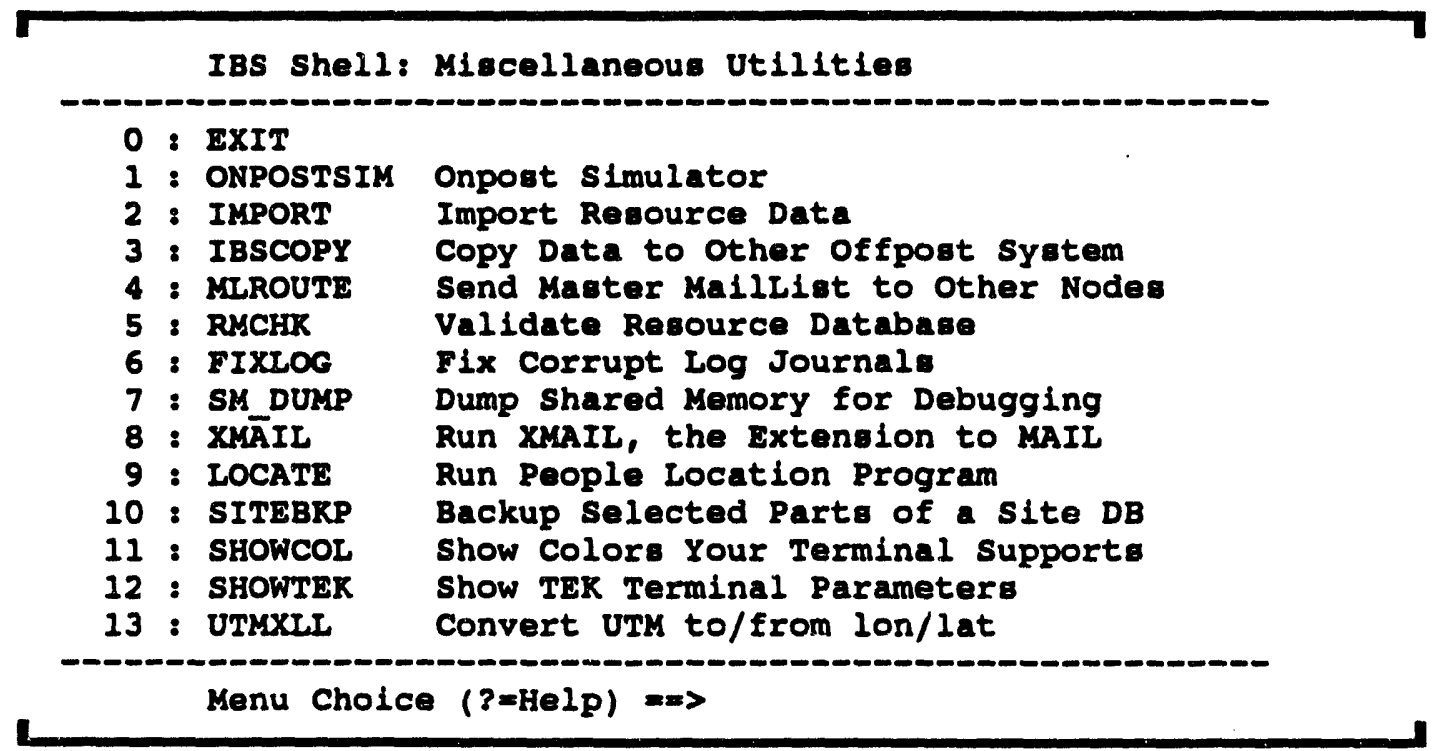

\section{Graphic Models Menu}

Most items on this menu are described in the IBS Models Guide or User Guide.

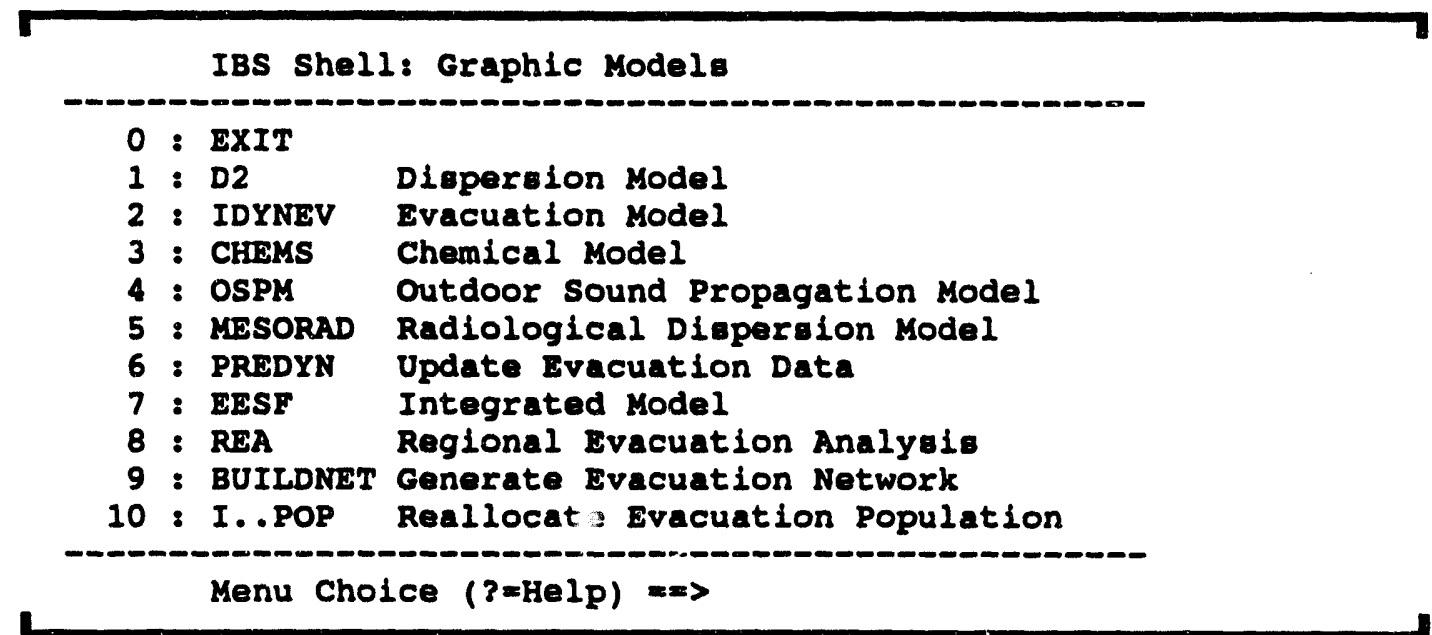




\section{Non-Graphic Models Menu}

Most items on this menu are described in the IBS Models Guide or User Guide.

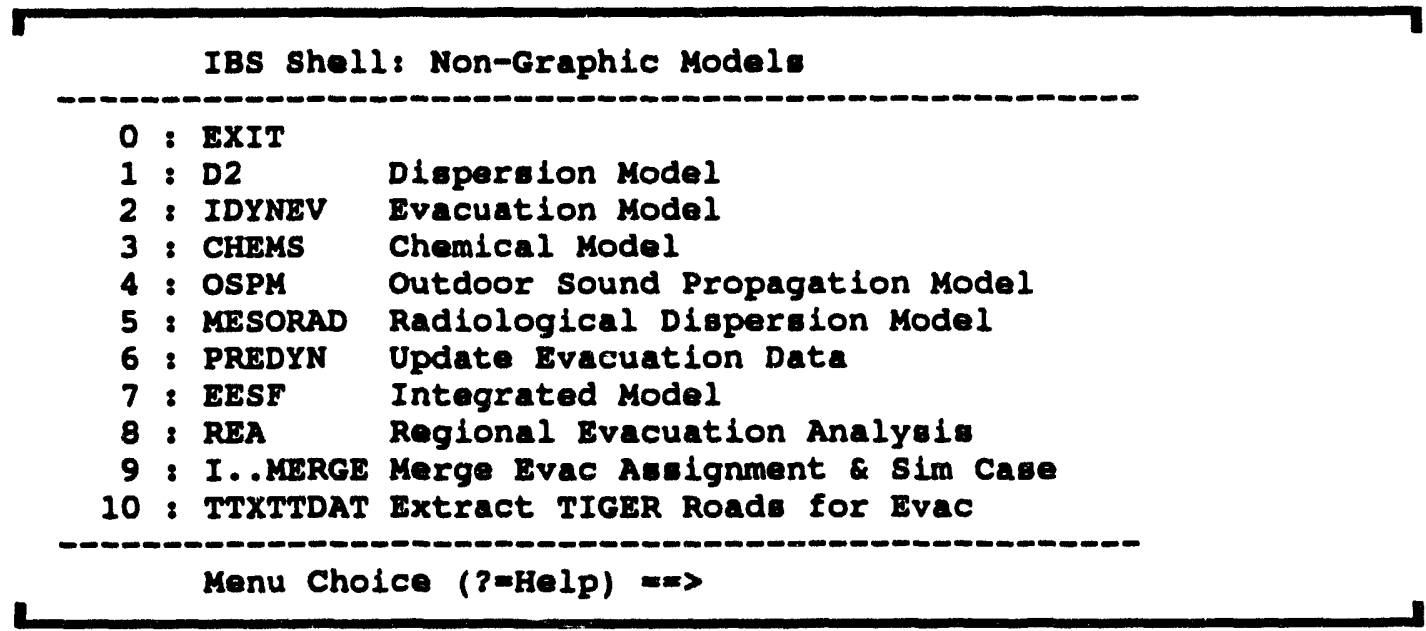

\section{Graphic GIS Utilities Menu}

Items on this Geographic Information System (GIS) Utilities menu are described individually elsewhere in this Utilities Guide.

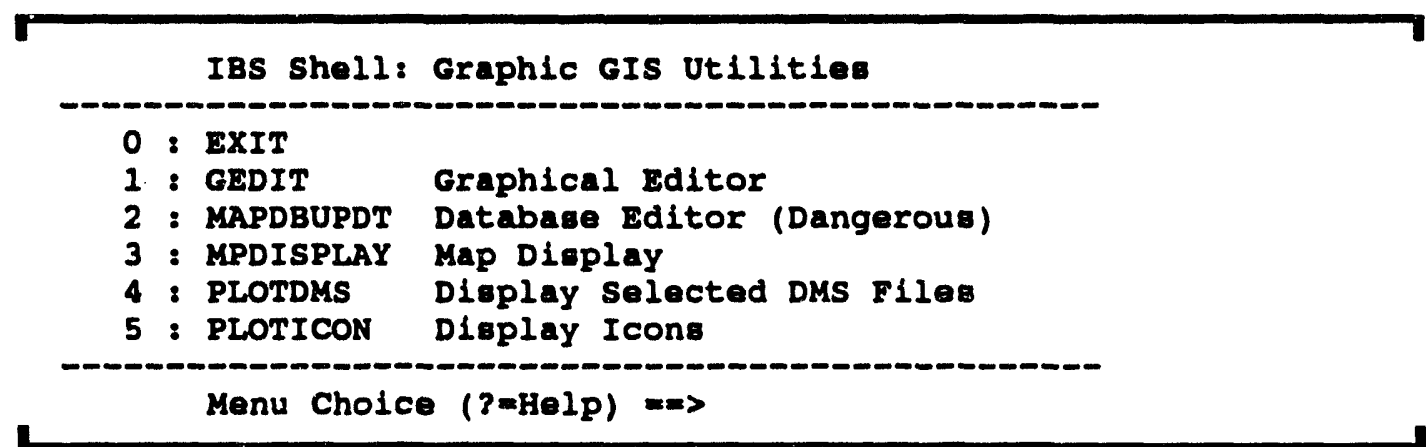




\section{Non-Graphic GIS Utilities Menu}

Items on this Non-Graphic Geographic Information System (GIS) Utilities menu are described individually elsewhere in this Utilities Guide.

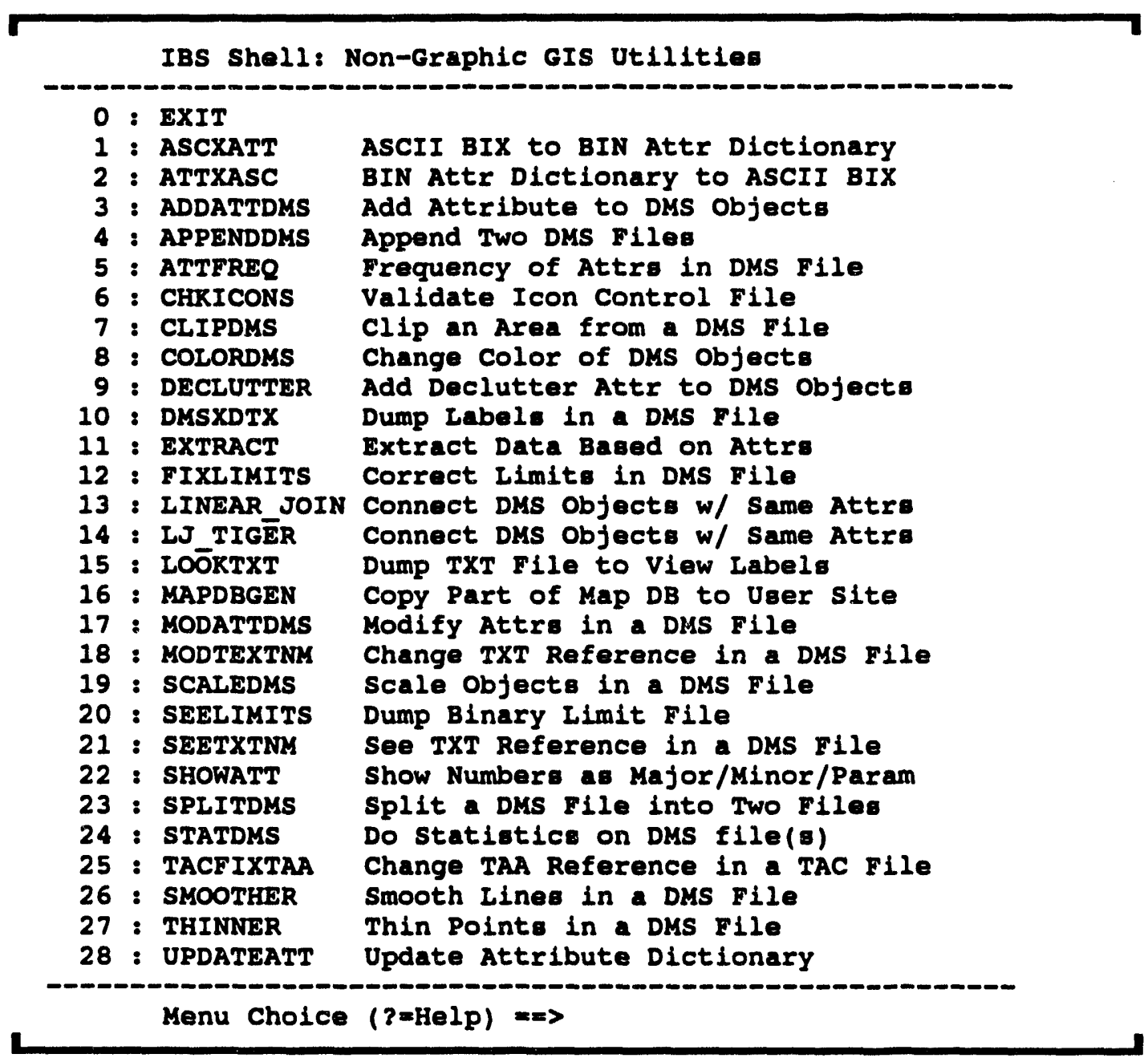




\section{IMPORT}

The IMPORT utility is designed to import data (about agencies, facilities, known points, personnel, job positions, or resources) from a text file to the appropriate IBS database(s) of the current IBS site. The text file can be an external source of information or data transferred from one IBS system to another. The utility imports one type of data at a time. After the utility imports the data, it reports the number of changes made to each type of data in the IBS databases.

IMPORT is an independent utility, but it is also available as a menu option of the RMMGR utility.

\section{What You Need to Know Before Using IMPORT}

To use the IMPORT utility effectively, you must be familiar with the ordering of information within the file you are importing. The IMPORT utility is most useful to IBS data managers and those who are especially knowledgeable about IBS data and data structures.

The IMPORT utility imports two kinds of text data files: either a "formatted" text file that contains data formatted according to certain FORTRAN programming conventions (perhaps transferred from another IBS system) or a "free-form" text file that contains data in some other format. If the text file has a complicated format, some knowledge of FORTRAN could be useful. If you are importing data from a free-form text file, then you must also know what characters separate the data fields in the file. Based on information that you supply about the text file, IMPORT can create a format specification (.FMT) file that IMPORT can use later to import data from the same file or from an identically formatted text file.

The utility imports data to the database(s) of the current IBS site. To switch between sites before importing data, use the SETUP-CHANGE SITE option of the IBS program.

Although the utility imports only one type of IBS data at a time, the data are interrelated, and sometimes one type of data requires another type of data to be complete. For example, you might add a facility whose contact person is not located in the personnel database, or perhaps the imported data is incomplete. In such cases the utility displays messages as it processes the data file and may create related data records that you would need to update later. Importing data is therefore a two-step process:

I. Use the IMPORT utility to transfer the data into the desired database(s).

II. Then verify that the data is complete, adding or updating data records when necessary. 


\section{Importing Data from a Text File with IMPORT}

1. To start the utility, just enter IMPORT at the system prompt (or select IMPORT from the menu of the RMMGR utility). The utility displays the code for the current site:

Import into site: sitecode. Correct? $(Y / N /<C t r l-2>=A b o r t)==>$

2. To confirm the current site and continue, enter $\mathbf{Y}$.

OR

To quit now, enter $\mathbf{N}$ or press CTRL-Z.

Entering $\mathbf{Y}$ confirms the site and displays this menu of importable data types.

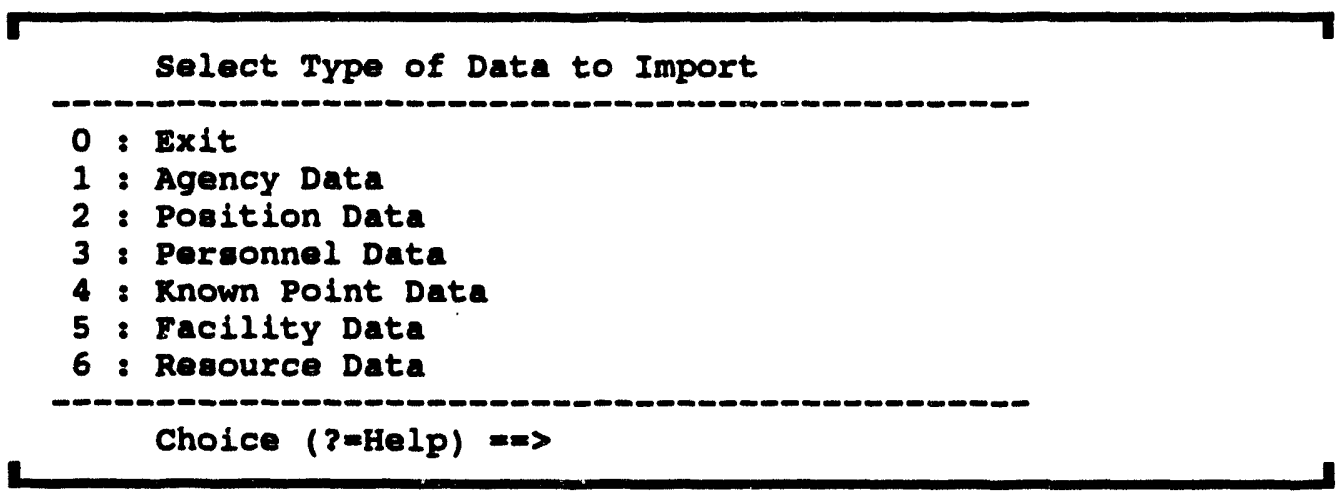

3. Select the type of data contained in the file from which you will be importing.

Note: Importing ALL data from a different "site." If you are importing a full set of IBS data from a different site code, then we recommend that you import the data in the order shown on the menu above: agency data first and resource data last. That sequence results in the fewest program messages about data that is not found or about the replacement of old data records with new ones. The corresponding IBS data files, in order, are the following:

\begin{tabular}{lll} 
Data Type & & File to Import \\
\cline { 1 - 1 } Agencies & & AGENCY LOOKUP.DAT \\
Positions & & POSITION_LOOKUP.DAT \\
Personnel & & PERSONNEL.DAT \\
Known Points & KNOWN_POINTS.DAT \\
Facilities & FACILITY.DAT \\
Resources & RESOURCE.DAT
\end{tabular}


After you choose a data type, the program asks:

Enter Filename to read from $m=>$

4. Enter the name of the text file that contains the data.

The utility then reads the file and attempts to determine whether it is a "formatted" file or a "free-format" file. If the file is not found, you will be prompted again to enter an existing file name.

Next you are prompted whether or not you wish to use a file of import specifications that tell IMPORT how the data file should be read. An import specification file (created during an earlier session of IMPORT) simplifies the import process if you need to read from the same source file several times.

Read Import speciflcations fram flle? (Y/N/<Ctrl-Z>=Abort) $=\Longrightarrow$

5. To use an import specification file, enter $\mathbf{Y}$.

OR

To enter new specifications for reading the data file, enter $\mathbf{N}$.

- If you enter $\mathbf{Y}$, the utility asks for the name of the specification file:

Enter filename containing specifications [.FMT] ==>

-Enter the name of the file that contains the import specifications, and then skip ahead to step 6 . (If the specification file is not found, you are prompted again to enter the file name again.)

Note: Importing data from another IBS site code. Ready-made import specification files are included with the IBS for importing IBS data files from another site code: just enter one of the file names listed here, depending on the type of data you are importing.

\begin{tabular}{lll} 
Data Type & & Specification File \\
\cline { 1 - 1 } Agencies & & IEMISSSYSF:AG.FMT \\
Positions & & IEMISSSYSF:POS.FMT \\
Personnel & IEMISSSYSF:PER.FMT \\
Known Points & IEMISSSYSF:KP.FMT \\
Facilities & IEMISSSYSF:FAC.FMT \\
Resources & IEMISSSYSF:RES.FMT
\end{tabular}


- If you enter $\mathbf{N}$, the utility displays a menu containing all the different fields used by the type of data you are importing:

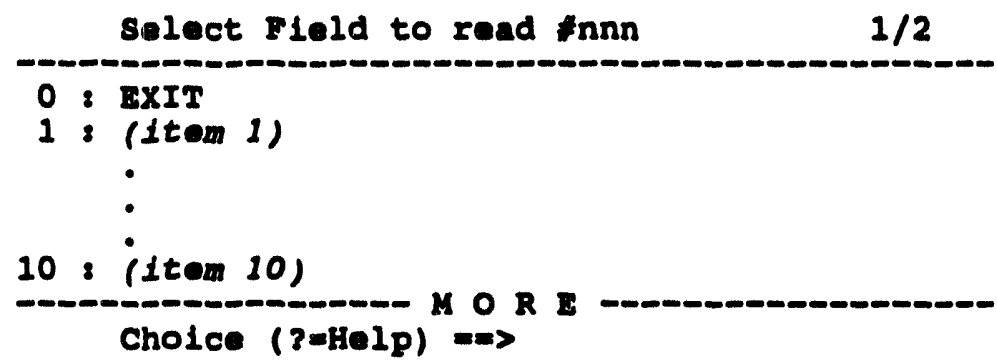

-Select the menu items In The Order They Need To Be Read From The File. Once an item has been selected, it will be removed from the menu, and the menu will reappear, allowing you to select the next item to be read. When all items in the file have been selected, enter 0 to exit. (Note that not all items must be selected from the menu: select only those items that are in the input file.)

If the file you are reading from is determined to be a free-format file, IMPORT then asks you to specify which characters separate the fields in the file:

Enter all character that may separate records in file. $=>$

- Type each character used as a field separator and press RETURN.

For example, this line might be found in a file to be imported:

MEMORIAL HOSPITAL, HOSPITAL, 1900 WEST MAIN ST.

This line contains the Facility name, Type, and Address for a Facility to import. The character separating the fields is the comma ",".

- You must ensure that the characters you say separate the lines into fields are NOT found in the fields themselves. In the above example, you would not be able to specify a space " " as a separating character because several of the fields contain spaces; this would cause the file to be processed incorrectly.

- If the fields in a file you wish to import do NOT contain spaces, spaces could be used as a separating character. However, if you are specifying spaces as separating characters, then you MUST use quotation marks "n around your input at the above prompt. For example if spaces, commas, and dashes separate fields, you would enter ", -" at the prompt shown above.

- If each field to read is on a separate line, you only need to press RETURN at the above prompt. 
You are then given the prompt:

Modify the Input FORMAT's? ( $Y / N /<C t r l-Z>=A b o r t)=m$

6. To use IMPORT normally, enter $\mathbf{N}$.

OR

To change how the data will be read by the IMPORT program, enter $\mathbf{Y}$.

If you are familiar with the FORTRAN programming language, this option can be useful if you want to change how the data will be read in by the IMPORT program. Unless you are familiar with FORTRAN, you should enter N. (See For Advanced Users: Modifying the Input FORMATs later in this discussion.)

If you've either created the Specifications by hand or changed the specifications by changing the FORMAT statements, you will then see the following prompts

Save epecificatione to a flie? ( $(\mathrm{N} /\langle\mathrm{Ct} / \mathrm{I}-\mathrm{Z}>=\mathrm{Ab}$ ort $) \Rightarrow$

7. To decline and proceed to step 8, enter $\mathbf{N}$.

OR

To save the specifications for later use, enter $\mathbf{Y}$.

If you are planning to import data from this same source again, you should enter $Y$, which gives the following prompt:

Enter filename to contain specificatione [.FMT] $=>$

-Enter the filename for the specifications. Later you can easily import data from this same source again without having to respecify the format of the file.

8. In either case, the program then begins to process the file according to all the specifications set or loaded from the specifications file. After reading the first new record of the desired type (Facility, Resource, etc.), the record is printed onscreen, and IMPORT asks you to verify that the record appears to have been read correctly. Here is an example for Resources: 


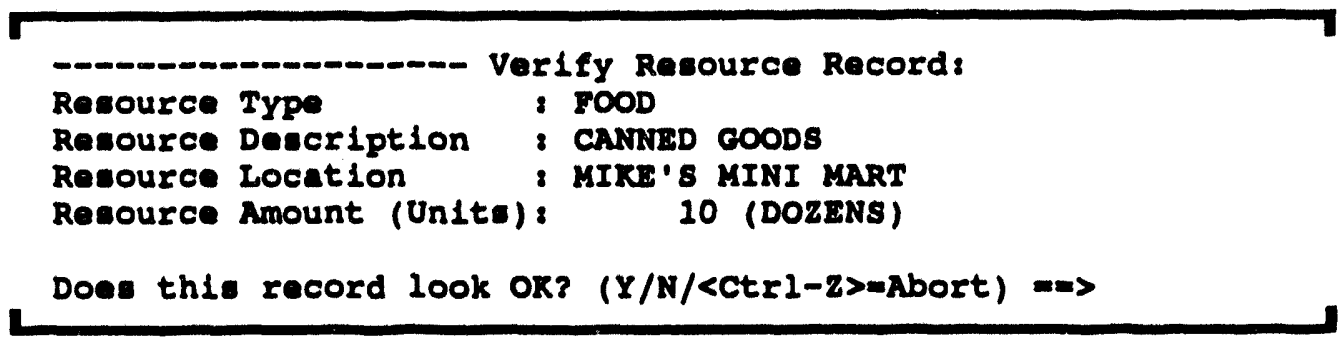

To abort the program without saving ANY information, enter N. You must then restart, changing the specifications to read the file correctly.

OR

To confirm and continue, enter $\mathbf{Y}$.

If you continue, the following prompt displays:

Verify All recorde? $(Y / N /<C t r l-Z>=A b o r t) \Rightarrow$

9. To let the IMPORT process continue without verification, enter $\mathbf{N}$.

OR

To visually check every record read from the file (as in step 8), enter $Y$.

During processing of the file you may receive messages in the following cases:

- A record similar to the one being imported already exists. You are then presented with both records, and prompted whether you wish to use the NEW record.

- A record in a different database is referenced, but not found (for example, a Facility whose contact person is not located in the Personnel Database). A very simple record of the type required is then added.

You may want to note the items for which this occurs, so you will know what needs to be verified and corrected later (for example, which person is being added).

- Required data for a record is incorrect or missing. For example, a personnel record with blank names, or a Facility that uses an unknown Facility Type.

When the program completes, IMPORT displays summary messages about how many records have been processed and the number of changes have been made to the database. 


\section{Example:}

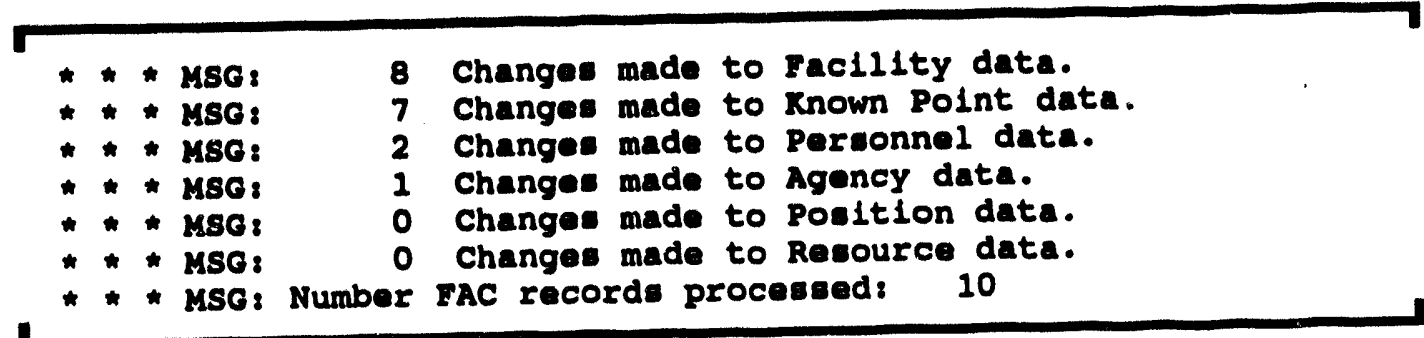

The differences between the number processed and the number of changes to each type of data indicates that some of the data in the imported file already existed in the Database. In the above example, 2 Facilities, 3 Known Points, 8 Personnel, and 9 Agencies referenced in the imported file were found to already exist in the database and were therefore not added.

\section{Verifying and Correcting Imported Data}

You should note what types of data were changed, and use IBS and the RMCHK utility to verify that everything is correct for all of the different data types importec/changed by the IMPORT utility. (Refer to the IBS User Guide.) Some things to specifically check:

- Use RESOURCE MANAGEMENT-MODIFY FACILTIES to check that every facility has a Type.

- If facilities are not drawn in the correct location (or are not on the screen at all), this is probably either because there was no Known Point data or the Facility Type has not been specified (see the first bullet above).

Use RESOURCE MANAGEMENT-MODIFY FACIUTIES-MODIFY KNOWN POINT to select the Facility that is not located correctly. (If you are given a crosshairs to select a Known Point and the Facility isn't on the screen, select CONTINUE so you can select the Facility to move by using a List or by entering its name.]

- Agencies that were added by anything other than Importing Agencies will not have had an Agency Name. The Name would have been set to "AgencyCode AGENCY NAME" where the code is correct but the agency name is not. These should be changed to use the correct Agency Name using PERSONNEL MANAGEMENT-MODIFY AGENCIES.

- Personnel added from Importing Facilities will have blanks for many fields. These should be filled with the correct data using PERSONNEL MANAGEMENT-MODIFY PEOPLE.

- Facilities added from Importing Resources will have blanks for nearly every 
field. These should be filled with the correct data using RESOURCE MANAGEMENT-MODIFY FACILTIES. (These facilities also fall into categories identified by the first two bullets in this list.)

\section{For Advanced Users: Modifying the Input FORMATs}

To modify the input FORMAT statements used by the IMPORT utility, you MUST have knowledge of the FORTRAN programming language. If you answered $Y$ in step 6 of the IMPORT sequence, then IMPORT displays a menu that lists the FORMATs used. The example shown here is for Resource Data.

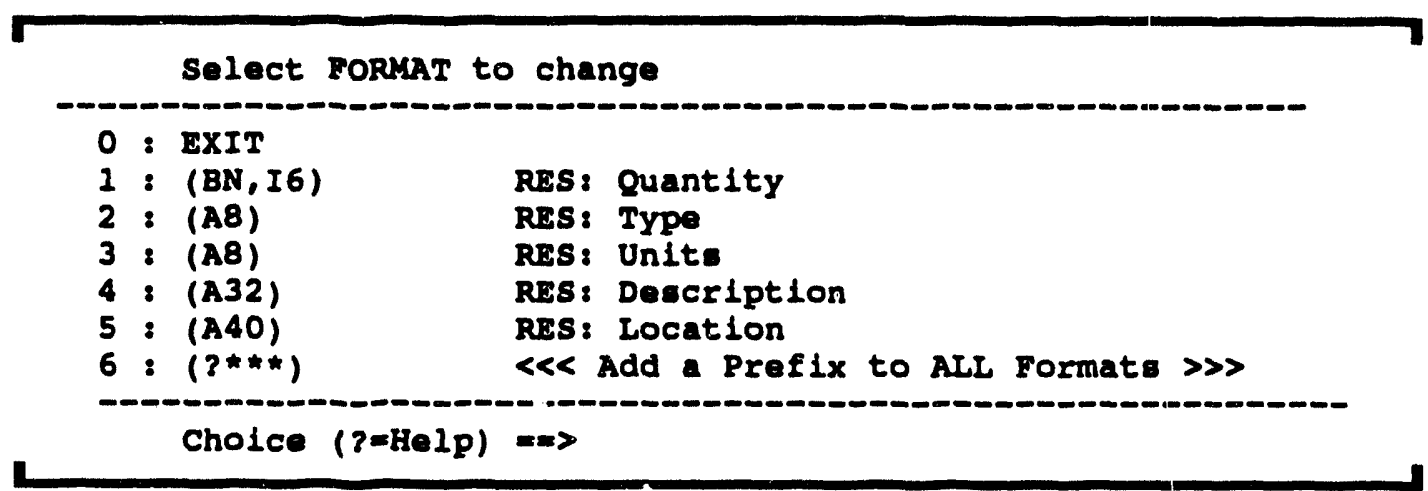

If you have read the specifications for a formatted file from a specifications file, the FORMAT statements shown may be long and complex, with lots of $n n X$ 's. They may also be too long to fit in the menu with descriptions.

6a. Select the number of the FORMAT statement you wish to modify.

OR

Select the final menu option to add a prefix to ALL formats.

- If you select a FORMAT statement, you are given the prompt:

(Field Description) Old FORMAT: (Format statement) Enter now FORMAT statement $=m$

-Enter the FORMAT statement you wish to use instead of the selected one.

- Some features of the standard FORTRAN Format are not supported including: Repeat fields "2A10" and Nested formats "(2(1X,A5))").

- Numeric fields may need to be prefixed with (BN...) [see example in menu item \# 1 above] to correctly ignore spaces. 
- Make sure that the format you enter reads the same number of items from the file, and that they are in the same order. If this is not true, you may receive unexpected results.

- If you choose to add a prefix to ALL formats, you are given the prompt:

Enter Prefix for all FORMAT atatements $m$

- Enter a part of a FORMAT statement to be used as a prefix to all of the FORMAT statement (for example, "20X"). Do NOT use parentheses "()". This option would be useful if the file had descriptions preceding all entries.

6b. Repeat step $6 \mathrm{a}$ as necessary, then press 0 to exit.

Reading from Formatted Files. If the file you are reading from is a Formatted file and you are creating the specifications the first time (not reading them from a specifications file), you will get the following prompt for most files. (The Personnel file is broken into 4 records [see Special Notes on Importing Formatted Personnel Data], and you would receive four prompts similar to the following, one for each record.)

Example:

The FORMAT calculated to read this flle 18:

$(B N, I 6, A 8, A 8, A 32, A 40)$

Enter the FORMAT you would like to use, or <RETURN> to

accept. $\Rightarrow>$

6c. Enter the FORMAT statement that you wish to use or press RETURN to accept the one shown.

- Some features of the standard FORTRAN Format are not supported including: Repeat fields "2A10" and Nested formats "(2(1X,A5))").

- Numeric fields may need to be prefixed with (BN...) [see example in menu item \# 1 above] to correctly ignore spaces.

- Make sure that the format you enter reads the same number of items from the file and that they are in the same order. If this is not true, you may receive unexpected results. 


\section{Special Notes on Importing Formatted Personnel Data}

Since Personnel records contain more data than other types, they are broken down into four records. The format supported by IMPORT is as follows:

Record 1: Contains all the Names (First, Last, Middle Initial), Phone Numbers (Work, Home, Beeper, Emergency), and Title information

Record 2: Contains Address, City, State, Zip code, Agency Code and Available information.

Record 3: Contains the schedule information.

Record 4: Contains the Positions (and Status of each position) the person holds.

This Record format is the same as that used by the Personnel file in IBS (See PERSONNEL.DAT in the IBS Data Management Guide) so it will import any IBS Personnel files. 


\section{INSTALLON and INSTALLOFF}

The INSTALLON utility is a system management utility that installs CHGOSITE, IBS_SM, and XMAIL to the system. It requires CMKRNL privilege to run, and will most likely be run only at installation.

The INSTALLOFF utility is a system management utility that deinstalls CHGOSITE, IBS_SM, and XMAII from the system. It requires CMKRNL privilege to run, and will probably be run only during software upgrades.

\section{Installing IBS Images with INSTALLON}

To start this utility, enter INSTALLON at the system prompt. No further interaction is necessary.

\section{Deinstalling IBS Images with INSTALLOFF}

To start this utility, enter INSTALLOFF at the system prompt. No further interaction is necessary. 


\section{KERMIT}

The KERMIT utility is a general system utility that enables you to transfer sequential files over ordinary telecommunication lines between DIGITAL Equipment Corporation VAX computers operating under the VAX/VMS operating system. This utility supports the exchange of both binary and text files and allows for the exchange of multiple files.

KERMIT operates through the use of command keywords and associated parameters. KERMIT commands define the processing of sending, receiving, and other functions. The primary commands and control sequences for Kermit are the following:

\begin{tabular}{|c|c|}
\hline Command & Action / Result \\
\hline BYE & Close access to remote server. \\
\hline CONNECT & Connect to remote system. \\
\hline EXIT & Exit from KERMIT. \\
\hline FINISH & Shut down remote server. \\
\hline GET & Request a remote server to send specified file data. \\
\hline HELP & Help with KERMIT. \\
\hline LOCAL & Prefix used for local file management commands. \\
\hline LOG & Write user-specified information to a log file. \\
\hline LOGOUT & Logout of the remote server. \\
\hline PUSH & Access to VAX DCL command level. \\
\hline QUTT & Exit KERMIT Utility. \\
\hline RECEIVE & Receive files from the other system. \\
\hline REMOTE & Prefix used in remote file management commands. \\
\hline SEND & Send file(s) from VAX to other system. \\
\hline SERVER & Mode of remote operation. \\
\hline SET & Set various parameters. \\
\hline SHOW & Display various parameters. \\
\hline STATISTICS & Display statistics for the most recent file transfer. \\
\hline TAKE & Use KERMIT commands from a file. \\
\hline
\end{tabular}


Control Key

Sequence

CTRL-X

CTRL-A

CTRL-Z
Action / Result

Sends a cancellation request to the remote KERMIT. This key sequence is used to cancel receiving a file that you don't really want.

Displays a status report.

Interrupts the current file(s) transfer.

\section{Transferring Files with KERMIT}

To start the utility, enter KERMIT at the system prompt. You will then see the KERMIT prompt where you can enter specific commands.

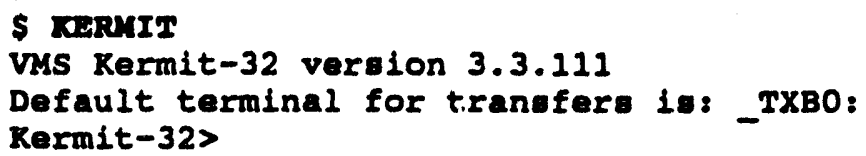

For more specific information about the proper syntax of each KERMIT command listed and about commands not specified here, refer to the documentation files KERMTT.DOC and KERMIT32.DOC contained in the IEMIS\$DOC: directory. 


\section{LIMITXX}

The LIMITXX utility is a database administration utility that creates a binary limit file containing all of the DMS (or TAC) files in the specified topography directory.

\section{What You Need to Know Before Using LIMITXX}

The LIMITXX utility requires a list of the names of the data files in the topography directory. Each topography directory generally includes such a list in a file named T.DAT.

The LIMITXX utility may create invalid limit files for any topographies which contain model output files (D2, EX, FI, VA, and WD), because all the DMS files with be put in the limit file, not just the one associated with the current model case.

See also the DOLIMITXX, CREATELIMITS, and SEELIMITS utilities. DOLIMITXX calls LIMITXX to do an entire database.

\section{Creating a Limit File for a Topography}

To start the utility, enter LIMITXX $x x$ at the system prompt, where $x x$ is the twocharacter code of the desired topography. If you do not specify the topography, the utility ends.

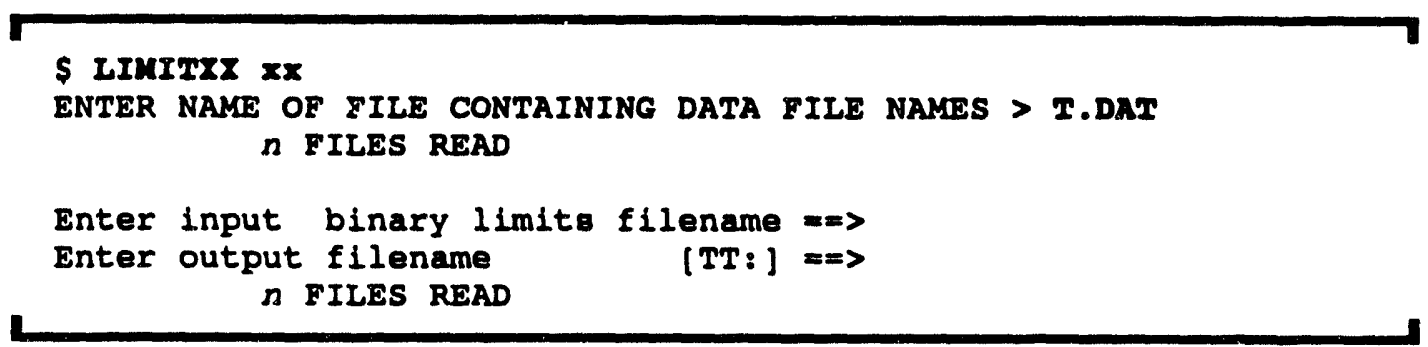




\section{LINEAR_JOIN}

A DMS file that has been built from other DMS files and/or from different data sources may contain discrete strings that should have exactly matching endpoints but which do not. The LINEAR JOIN utility reads a single DMS file and joins any vector strings that have closely matching end points and common attributes. The result is a new DMS file that contains a reduced number of strings. This reduces the number of "nodes" for network models that use the strings, such as the system evacuation model.

\section{Caution}

A joined string that results from jolning other strings may lose some attributes of the independent strings. Please read the following section carefully before using UNEAR_JOIN.

\section{What You Need to Know Before Using LINEAR_JOIN}

LINEAR JOIN first asks you to specify the names of the original DMS file and the new DMS file that will contain the joined strings. If the original DMS file references a supporting text file, then LINEAR_JOIN also asks you to specify the name of a new output.TXT file. The utility then enables you to control which vector strings will be joined by specifying criteria. The following sections describe criteria and strategies for joining strings with LINEAR_JOIN.

\section{Criteria for Joining Strings}

You can specify three criteria for joining strings within a DMS file:

attributes to INclude This refers to the attribute values (major, minor, and parameter values) that the strings to be joined must share in common. You may specify up to 15 sets of attributes to INclude. To be joined, two strings must share all of these attributes.

attributes to EXclude This refers to the attribute values that the strings to be joined must NOT share. Any string that contains these attributes is NOT joined, even if the string has all the "attributes to INclude." You may specify up to 15 sets of attributes to EXclude. To be joined, two strings must not contain any of these attributes. 
epsilon (in degrees) This refers to how close the endpoints of two strings must be to qualify for being joined. Two strings that have endpoints separated by no more than this number of degrees (of longitude and latitude) are considered to have a common endpoint.

A fourth criterion, which you cannot control, is that only two strings can be joined at a common endpoint: if more than two strings meet the first three criteria, then none of those strings are joined.

In other words, LINEAR_JOIN will join two strings within a DMS file if those strings do the following:

1) share all the "INclude" attributes,

2) do NOT share any "EXclude" attributes,

3) have endpoints that are within "epsilon" degrees of each other, and

4) are NOT within "epsilon" degrees of any other string endpoint.

\section{Caution}

A resulting joined string will have the display characteristics of the first string and only the specified "INclude" attributes. Any other attributes are discarded. Consider this as you plan how to use LNEAR_JOIN.

To specify attributes, you will need to know attributes values for the attributes you wish to specify. You can find a list of IBS attribute values in Appendix B.

\section{Strategles for Joining Strings}

If multiple attributes describe a classification of strings that you want to join, you may need to use LINEAR JOIN multiple times to get the best results. In general you may want to start at the highest resolution of attributes and work toward the lowest resolution. For example, class 1 roads is a general attribute for roads in IBS databases. Other attributes are used to further define class 1 roads as undivided, divided, one way, and so on. If you wanted to juin all of the "class 1 roads" in a DMS file together, you might want to start first by joining these subcategories of roads, executing LINEAR JOIN once for each of these attributes (combined with the general class 1 attribute). Each output file would then be used as the input file for the next use of LINEAR JOIN. After the subcategories of strings were joined, you could then join all the class 1 strings under the general class 1 attributes.

If more than two strings are estimated to have a common endpoint (within the 
epsilon distance), LINEAR JOIN does not attempt to join these multiple strings. To join these strings correctly, you will need to examine each string individually and join them manually: the GEDIT utility enables you to join specified strings.

\section{Reducing the Number of Strings in a DMS Flle with LINEAR_JOIN}

To start the utility, just enter LINEAR_JOIN at the system prompt.

The following simple example joins "Class 3 road" strings contained in a file named TOOELE.DMS. Two strings will be joined when the following criteria are met:

- Both strings have the attribute 5,140,0 (both strings are Class 3 roads).

- Neither string has the attribute $5,180,0$ (neither string is a bridge).

- The two strings have endpoints that match within 0.00001 degrees of both longitude and latitude (specified).

- No other strings meet at their common end point.

Example:

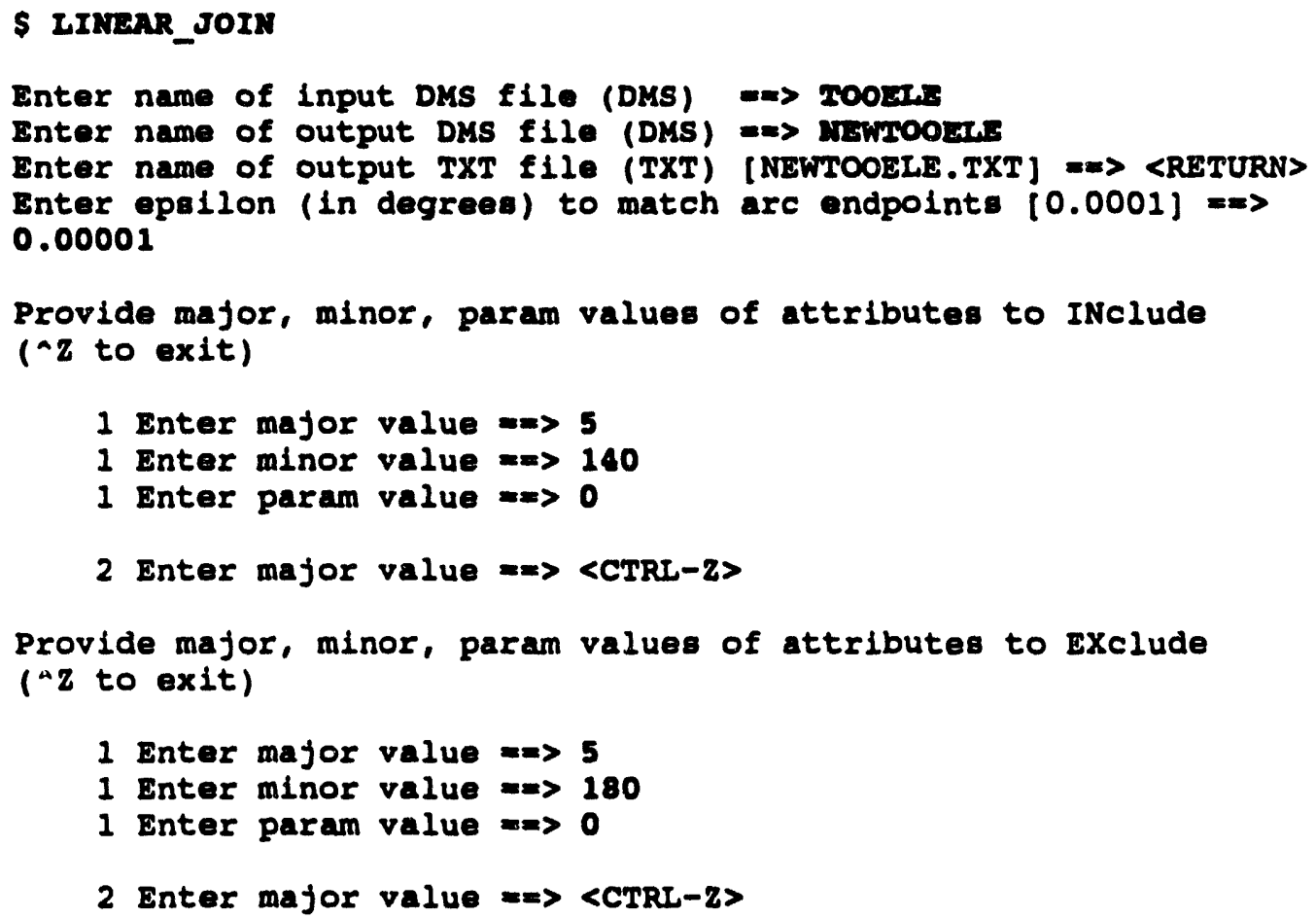

If you just press RETURN instead of entering an output TXT file name, the 
default name matching the DMS file name is used. You will see this prompt only if the original DMS file references a supporting .TXT file.

If you just press RETURN instead of entering an epsilon (endpoint separation) value, the default value of 0.0001 degrees is used. If this value is inappropriate for your data-if the results are not as they should be-you can enter a smaller (stricter) value to assure more exact matching, or you can enter a larger (less strict) value to join strings whose endpoints are further apart than 0.0001 degrees.

You can enter up to 15 attributes to INclude and 15 attributes to EXclude. To quit entering attribute values, press CTRL-Z at the "major value" prompt as indicated in the example.

\section{Results of Using LINEAR_JOIN}

LINEAR_JOIN searches through the DMS file's set of string objects in repetitive cycles, on each cycle 1) finding an string that meets the LINEAR JOIN criteria and 2) attempting to match and join it with a second qualifying string. A resulting joined string will have the display characteristics of the first string and only the specified "inclusion" attributes. Any other attributes are discarded.

When LINEAR JOIN is finished, it creates a new DMS file that contains the same data as the original DMS file except that the new DMS file should contain fewer strings (because some strings have been joined) and fewer string attributes (because some attributes may have been discarded).

As it runs, the program displays a series of status messages that indicate how many string objects have been processed and how many have been "saved" in the new file. If strings have been joined successfully, the number of objects saved should be fewer than the number of objects processed. In the following example, the status messages indicate that a total of 219 objects in the original DMS file were reduced to 187 objects in the new DMS file.

Example:

\begin{tabular}{|c|c|c|c|}
\hline $\begin{array}{l}\star \star \star \\
\star \star \star \\
\star \star \star \\
\star \star \star * \\
\star \star \star \\
\star \star \star \\
\star \star \star\end{array}$ & $\begin{array}{l}\text { MSG: } \\
\text { MSG: } \\
\text { MSG : } \\
\text { MSG: } \\
\text { MSG: } \\
\text { MSG: } \\
\text { MSG : }\end{array}$ & $\begin{array}{l}\text { Reading objects from } \\
\text { Processing object } \\
\text { Processing object } \\
\text { * of objects processec } \\
\text { Saving object : } \\
\text { * of objects saved: } \\
\text { Done }\end{array}$ & 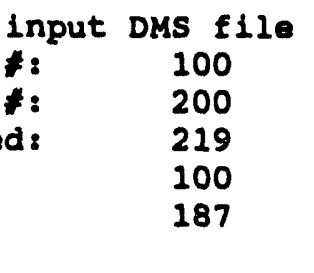 \\
\hline
\end{tabular}




\section{LISTCNX}

The LISTCNX utility is a diagnostic and reporting utility that lists all existing cases found in the Case Index file (CASEINDEX.DAT) of a user's current site. Each case found is listed and flagged as follows:

Flag Description of Case Type

(A) "Assigned"-This occurs only for Infomanager cases that have been checked out and assigned to someone else.

(M) "MCE" or Maximum Credible Event-This refers to key cases that are not to be modified.

(Y) "Yes" the case exists (and is flagged neither A nor $M$ ).

\section{Listing Model Cases Found in the Case Index File}

To start this utility, enter LISTCNX at the system prompt.

The output lists the model name, status of the individual case, and the case number. A sample of the output follows:

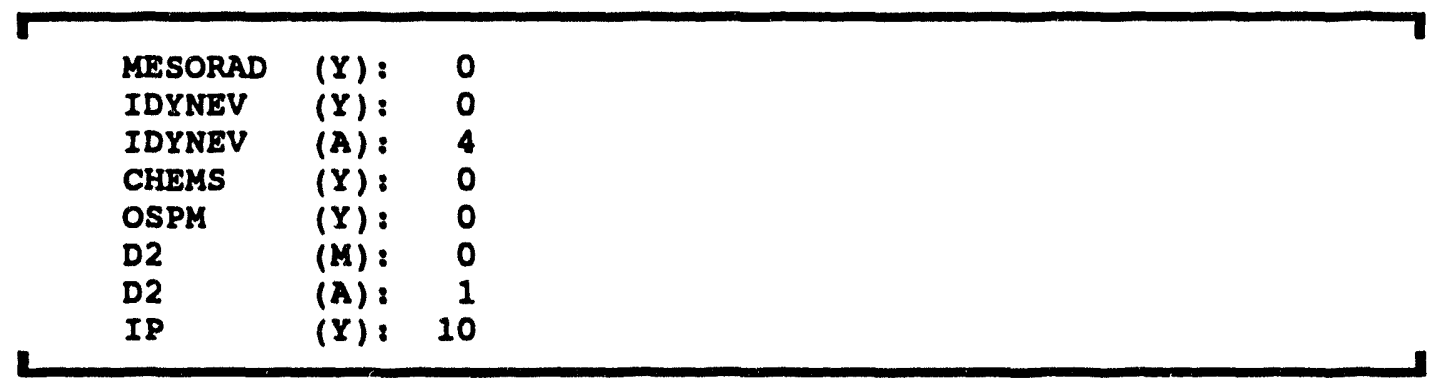

Note: If you are not in directory disk:[user.SITES site], you will be moved to SITESDIR. 


\section{LJ TIGER}

A DMS file that has been built from other DMS files and/or from different data sources may contain discrete strings that should have exactly matching endpoints but which do not. Both the LJ TIGER and LINEAR JOIN utilities read a single DMS file and join any vector strings that have closely matching endpoints and common attributes. The result is a new DMS file that contains a reduced number of strings. This can greatly reduce the disk space required for the file; it also reduces the number of "nodes" for network models that use the strings, such as the system evacuation model.

LJ TIGER has been optimized for use with DMS files that are based on TIGER data, and especially with DMS files that have been extracted from TIGER data. Unlike LINEAR JOIN, you do not specify attributes: all strings with the same attributes and the same text descriptions are joined if their endpoints are close enough together. You specify the criterion for closeness.

\section{What You Need to Know Before Using LJ_TIGER}

The utility prompts you to specify these items:

input DMS file This is the name of the original DMS file.

output DMS file This is the name of the new DMS file that will contain the joined strings.

epsilon

This is the criterion for how close the endpoints of two strings must be to qualify for being joined. Two strings that have endpoints separated by no more than this number of degrees (of longitude and latitude) are considered to have a common endpoint. If more than two strings have endpoints within this distance, the strings are NOT joined. The default epsilon value is 0.00001 degrees.

output TXT file This is the name of a new .TXT file that is associated with the new DMS file. You are prompted for a new .TXT file name only if the original DMS file has text data in a .TXT file. If you are prompted for a .TXT file name, the default name is the same as the output DMS file name.

Some text data is lost when TIGER data is joined: TIGER ID ("TID:

\#\#\#\#\#\#") and TIGER version ("TVER: \#\#\#\#\#") text fields are removed from any strings that are joined. 


\section{Joining Discrete Strings in a DMS File with LJ_TIGER}

To start the utility, just enter L TIGER at the system prompt. The following is an actual example that shows how LJ_TIGER reduced the number of strings in a DMS file.

Example:

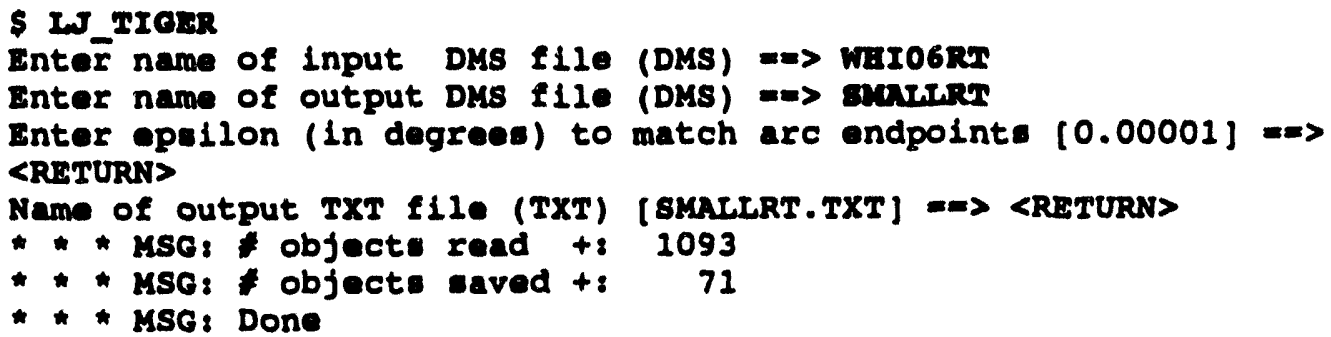

When LJ TIGER is finished it creates a new DMS file that contains the same data as the original DMS file except that the new DMS file should contain fewer strings (because some strings have been joined) and less text data (because "TID:

\#\#\#\#\#" and TVER: \#\#\#\#" have been discarded.)

The program displays status messages that indicate how many string objects have been read and then how many have been saved in the new file. In the example here, the status messages indicated that a total of 1093 objects in the original DMS file were reduced to 71 objects in the new DMS file. This also reduced the combined disk space required for both the DMS and TXT file from 272 blocks to 48 blocks. 


\section{LOOKTXT}

The LOOKTXT utility is a diagnostic and reporting utility that enables you to look at the contents of a DMS TXT file.

\section{What You Need to Know Before Using LOOKTXT}

In addition to an input text file name and output report file name, the LOOKTXT utility prompts for two items and two decisions:

TXT file name

output TXX file name

List everything?

Calculate EOF?
This is the file you want to look at.

This is the name of the report file. To display the information on the screen, just presis RETURN instead of entering an output file name.

For a short summary listing as shown in the following example, answer $\mathbf{N}$ to this prompt. For a longer numbered listing of each text item, just press RETURN or answer $Y$.

Some DMS TXT files do not follow standard conventions of listing the end-of-file record number in the header. In these cases, an end of file (EOF) must be calculated. Unless you are certain the TXT file follows standard conventions, it is best to answer $Y$ to calculate the EOF.

\section{Looking at the Contents of a DMS Text (.TXT) File}

To start this utility, enter LOOKTXT at the system prompt. The utility presents a series of prompts as shown in this example.

Example:

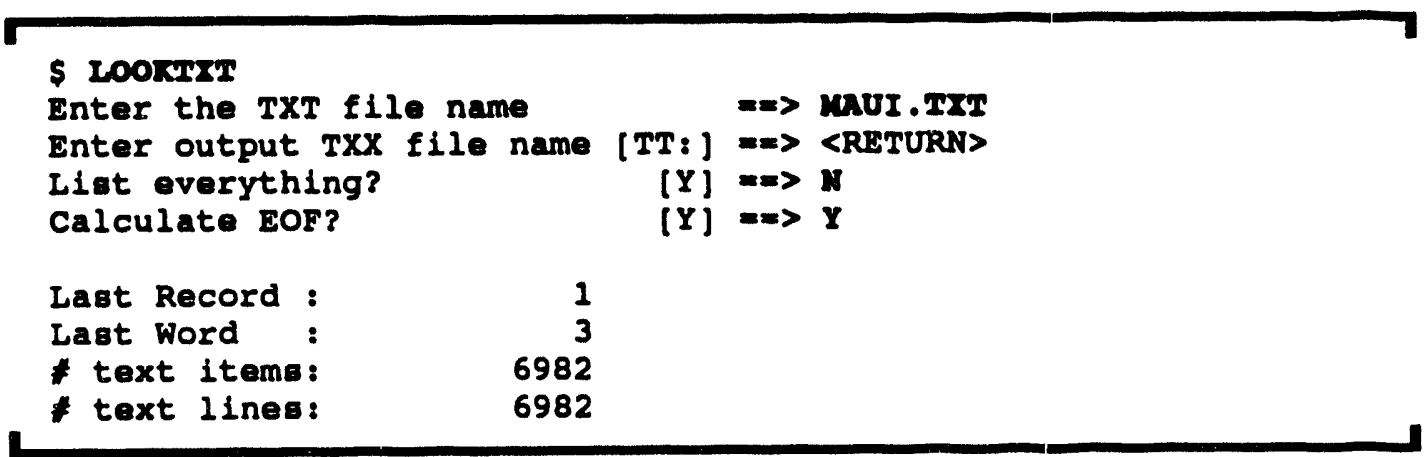




\section{MAIL C}

MAII_C is a background utility that checks the mail file of each IBS user once per minute to see if the user's mail status has changed. If a user's mail status has changed, MAIL C updates a mail status message (which may be no message when there is no mail) $\overline{-}$ at the bottom of the terminal screen.

MAIL_C is used in a background process that must be running at all times or else mail status messages will NOT change.

\section{What You Need to Know Before Using MAIL_C}

The MAIL_C utility runs in background (batch) mode on the VAX computer. Under usual conditions, this background process is already running and does not need to be started independently: the process is started by the batch command file IEMISSEXE:MAIL_C.BIS, which is executed when the system startup command file SYS\$MANAGER:SYSTARTUP_V5.COM is run at installation or whenever the computer is rebooted.

If this background process is stopped for some reason, IBS users will NOT be notified of any changes to their mail status. To restart this process, a user must have system privileges (SYSPRV).

\section{Restarting the Mail Checking Process}

To restart the background process that runs MAII_C, enter @IEMISSEXE:MAIL_C.BIS at the system prompt.

MAIL_C should run once a minute until someone stops the process.

\section{Results of Using MAIL_C}

MAII_C checks to see if the status of each user's mail has changed: if new mail has arrived, if unread mail has expired, or if all messages have been read. If the mail status has changed, the MAII_C utility notifies IBS, and IBS displays an appropriate message at the bottom of the user's screen:

Mail Status Change

New mail has arrived.

Existing mail has expired (not been read by a specified time)

\section{Message Displayed}

NEW MAIL

LATE MAIL 
Mail Status Change

All messages have been read.
Message Displayed

no message displayed

Note: The message displayed on the IBS screen does not change immediately when the mail status changes: The message changes only after MAII_C checks the mail status, once every minute.

When MAIL_C is running, the status of mail can also be checked by selecting SHOW USER ENVIRONMENT from the STATUS BOARD menu in the IBS program. If MAIL_C is not running, the SHOW USER ENVIRONMENT function may not report new or past due mail correctly. For further information, see the IBS User Guide. 


\section{MAIL LIST}

MAII_LIST is a general system program that enables you to use the electronic mail features of the system: sending and receiving messages; checking mail messages; and using mail distribution lists. MAIL_LIST is a stand-alone program that duplicates the mail-related functions of the MESSAGE BOARD menu in the IBS program, which is documented in the IBS User Guide.

\section{Using IBS Electronic Mail Features with MAIL_LIST}

To start the utility, enter MAIL_LIST at the system prompt.

\$ MAIL_LIST

MAII_LIST then displays the following menu.

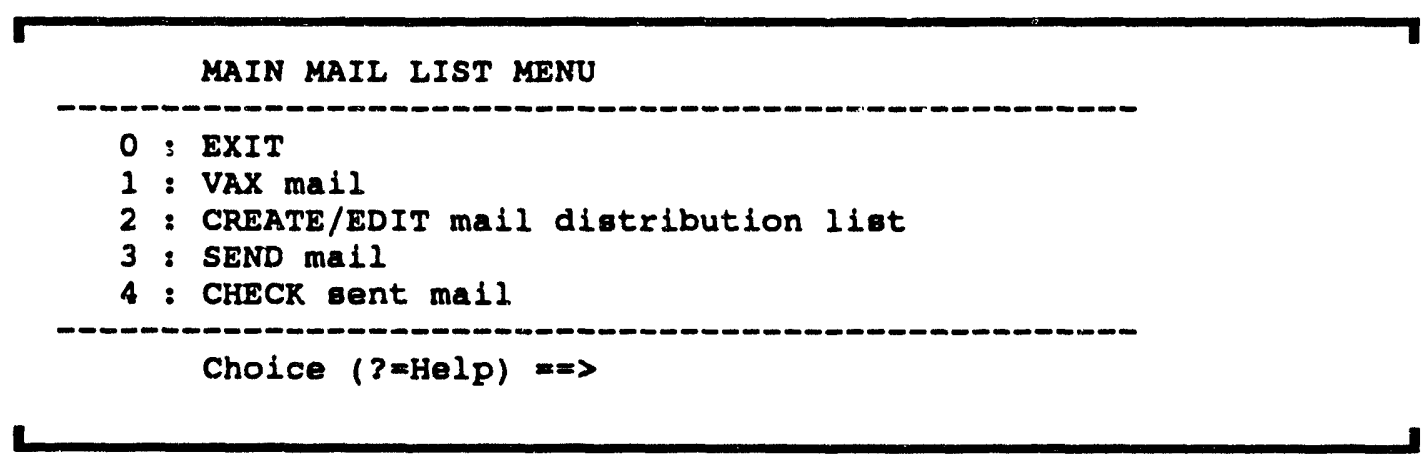

Pick the number of the option you wish to use and follow the prompts.

System Mailing Lists are those mailing lists in the Infomanager directory. To change one of the System Mailing Lists, the Infomanager user can edit that file by using option 2 above and either the Edit System Mailing List or Edit Personal Mailing List options on the resulting submenu.

For further information about each option, see the instructions for MESSAGE BOARD in the IBS User Guide. 


\section{MAKEDMS}

The MAKEDMS utility enables you to create DMS format files from sequential ASCII files (which you can create by using a text editor). You may include point or line structures in the ASCII input file.

\section{Creating DMS Files from ASCII Input Files with MAKEDMS}

To start the utility, enter MAKEDMS at the system prompt. You must supply the name of the ASCII input file and the name of the DMS file that you want to create.

Example:

SMATEDMS

Enter ASCII input filename > MYFIIs.DAT

Enter DMS output filename > MYFII. Dus

\section{Structure of the ASCII Input File}

Each entry within the input file contains two logical records. The first record describes the data type, display parameters, attributes, and number of longitude-latitude coordinates. The second record contains the longitude-latitude coordinates. Both logical records are entered in free field format (commas or spaces between parameters) and may span lines if necessary.

The format for the first record is:

PLOTFLAG, NPEN, of Attributes, of points, \{Attributes\}

where PLOTFLAG may be 0 or 1 ( 0 indicates a line data type, 1 indicates point) and NPEN is the pen number to be used when displaying the feature.

Each attribute has three subfields: Major code, Minor code, and parameter value. These three subfields are repeated for each attribute included with the feature. If the feature is a point for which an icon is to be displayed, the attribute describing the icon must be the first to appear in the attribute list.

The format of the second record is:

LONG1, LAT1, LONG2, LAT2, ...LONGn, LATn.

where longitude and latitude are described as decimal degrees. 
The following is an example of a file containing one point and one line element. The line will be displayed using pen number 6 and consists of 4 points. The point has one attribute associated with it, which may be presumed to signify the icon to be displayed.

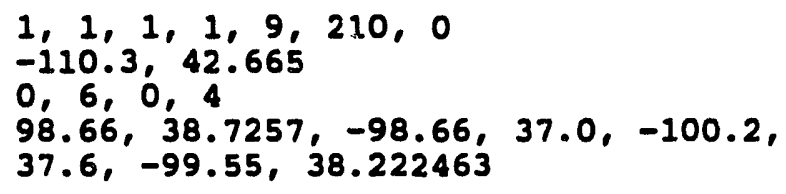

If this data were contained in MYFILE.DAT, then the example sequence would create the file MYFILE.DMS with the point and line data in DMS format. 


\section{MAKETAC}

The MAKETAC utility creates a Terrain Analysis Cell (TAC) data file with longitude-latitude extents and a uniform cell value that you specify. This capability in the IBS is intended only for supporting elevation data files and the sound impedance files used by the Outdoor Sound Propagation (OSPM) siren model.

\section{What You Need to Know Before Using MAKETAC}

The utility prompts you for the following items:

NAME OF TAC FULE

LONGITUDE, LATITUDE

NUMBER OF COLUMNS, NUMBER OF ROWS

CELL VALUE

SIZE OF DATA TYPE
This is the name of the TAC file to be created. If you do not specify a path name with the file name, the file is created in your default directory.

These are the extents of the new TAC file, the minimum and maximum longitude and latitude.

This is the grid size of the new TAC file: the columns and rows in the grid of cells.

This is the data value that will be stored for all cells of the file.

This is the number of bytes to be used for each cell data value. A value of 1 designates the data type as "byte," for example.

\section{Creating a New TAC File with MAKETAC}

To start the utility, enter MAKETAC at the system prompt. In the following example sequence, the utility creates a new file named TESTOSPM1.TAC (and the associated file TESTOSPM1.TAA) with a grid size of $500 \times 600$ cells and a 1 -byte value of zero in all cells. A message will confirm creation of the TAC file.

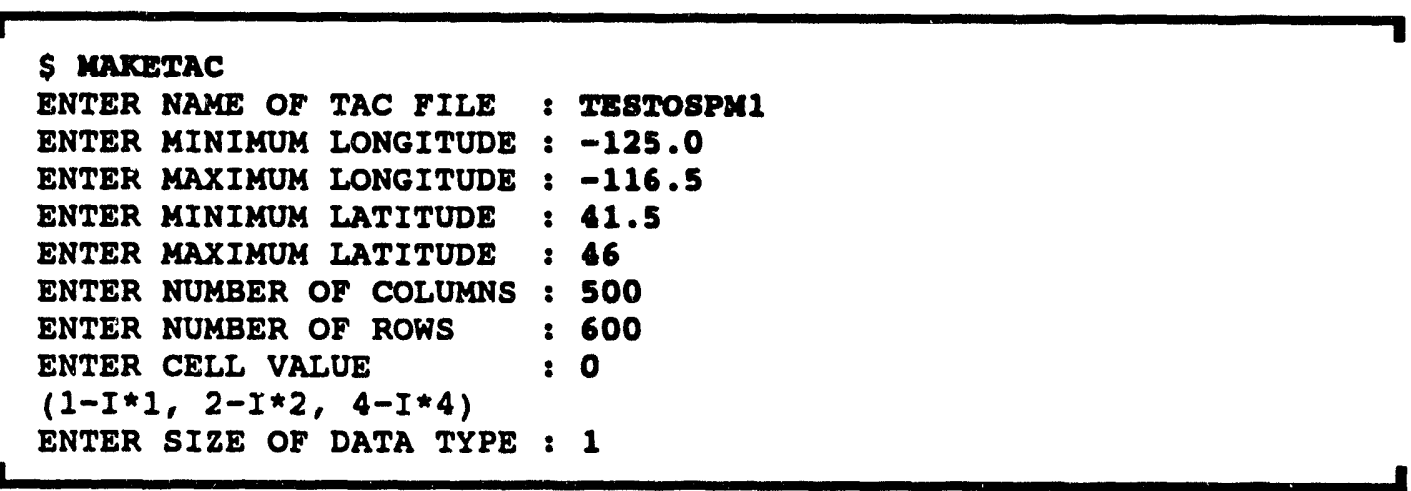




\section{MAPDBGEN}

The MAPDBGEN utility is used to extract a portion of a map database to create a separate "user-defined" map database. You may then modify the new map database to suit your needs.

This section covers the following topics:

- Why create a "user-defined" map database?

- What You Need to Know Before Using MAPDBGEN

- Creating a User-Defined Map Database with MAPDBGEN

- Results of Using MAPDBGEN.

\section{Why create a "user-defined" map database?}

A "user-defined" database is a separate subset of a larger DMS database. The difference between these databases, apart from size, is that you can edit and modify a "user-defined" map database without making system-wide changes. This allows you to tailor your DMS files for your own purposes.

\section{What You Need to Know Before Using MAPDBGEN}

Before using MAPDBGEN, there are certain pieces of information you need to know. If you are unsure about where to obtain this information, or if you need help, please contact your database administrator for more details.

MAPDBGEN asks you to specify the following information for the map database you are creating:

Top Level Directory The database top level directory is the actual path name of the directory where the new database will reside when it has been created.

Examples: Entering aaa would create the map database in a directory named aaa under your current directory.

Entering [aaa] would create the map database in the top level directory named [aas]. 
You will also need to know what geographic area you wish to extract from the existing map database to include in your new map database. This area can be defined in one of the three following ways:

Long/Lat Corners Requires two sets of longitude and latitude coordinates.

Center \& Radius (mi) Requires one set of longitude and latitude coordinates and a radius distance in miles.

Center \& Radius (km) Requires one set of longitude and latitude coordinates and a radius distance in kilometers.

Before you start creating the map database, make sure you have determined which longitude and latitude coordinates and which radius you plan to use (the following diagram clarifies those parameters). This kind of information can be obtained easily through the graphic mapping utilities in IBS (see MPDISPLAY, for example).

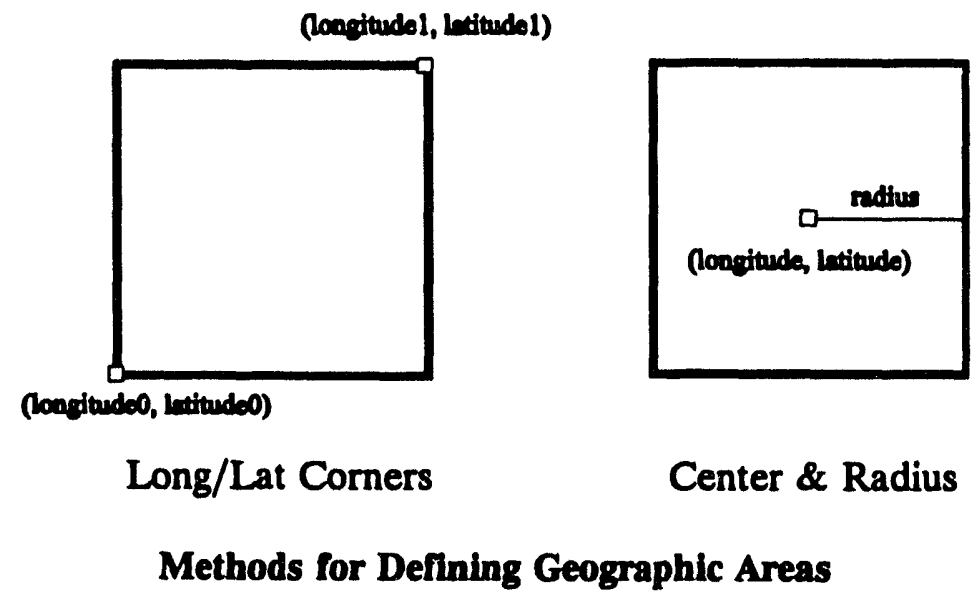

In addition to these parameters, you will also be asked if you wish to select cover maps. Cover maps (or overview maps) are displayed by the MPDISPLAY utility to show a database area from which you may select a region for display. Cover maps are usually low-detail maps that are represented by a single DMS file in the top directory of a map database.

If you do not use a cover map from the existing map database, you must furnish one before you can use the new database. This can be done by 1) placing one or more cover map DMS files in the top directory and 2) editing the topography control file, TOPOCTL.FI, to contain the name(s) of the cover map file(s). 


\section{Creating a User-Defined Map Database with MAPDBGEN}

The following is an example of how to create a "user-defined" map database using the MAPDBGEN utility.

Before beginning this process, you must know the system and topographic information explained in the previous section.

1. To run the utility, just enter MAPDBGEN at the system prompt. The utility starts by displaying the following prompt:

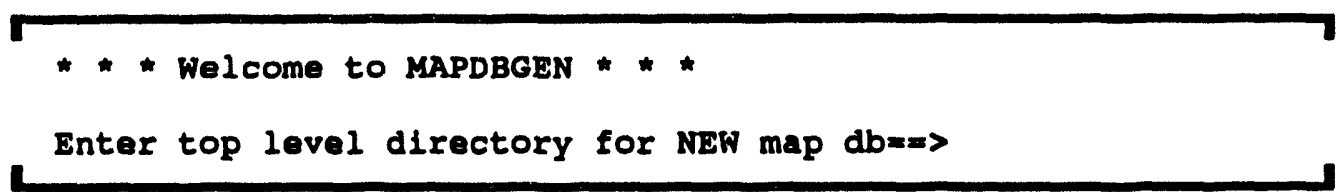

2. Type the name of the directory where you want your "user-defined" database to reside. When you are done, press RETURN. This tells the utility where to place any information you want to extract from the current map database.

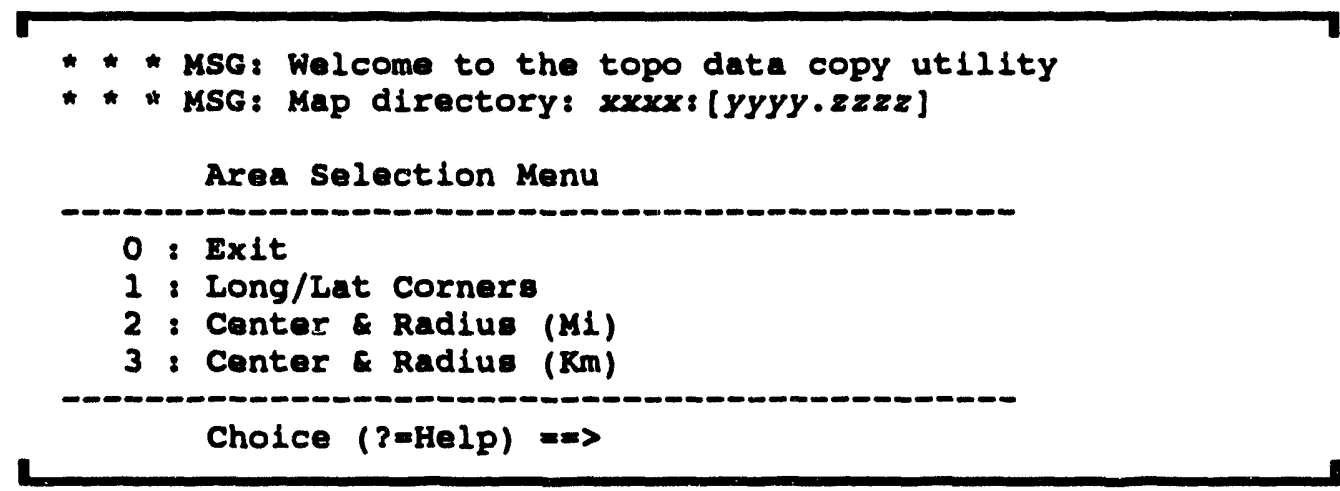

3. Select the option with which you want to define the area to be included in your "user-defined" map database.

If you need more information about the kind of input required by the area selection options, see What You Need to Know Before Using MAPDBGEN. Selecting Exit (0) in this menu returns you to the system prompt. Selecting one of the other options displays a screen asking you to enter the coordinates for the area.

4. Enter the coordinates that define the area of your map database. When you are done, another prompt asks you if you want to select cover maps: 
select from available cover mape? (N) $\Rightarrow$

5. To select cover map(s), enter $\mathbf{Y}$. Or to bypass cover map selection, enter $\mathbf{N}$ (or just press RETURN.

If you answer $\mathbf{Y}$ (yes), a menu similar to the following is displayed. If you answer $\mathbf{N}$ (no), skip ahead to the Available Topos shown in step 7.

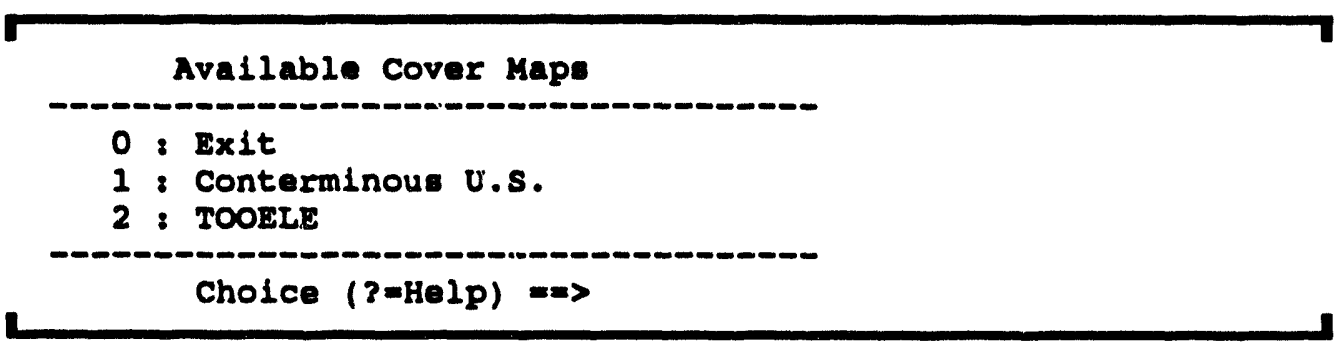

6. To select a cover map for your "user-defined" map database, type the number next to it and press RETURN. An asterisk (") then appears next to the selection, indicating that it will be included in the database. To deselect a map, enter the number again; the asterisk disappears, indicating that the map will not be copied.

7. When you have made all your cover map choices, select Exit (0).

The following menu then displays (note that different options may be in the menu, depending on your system setup):

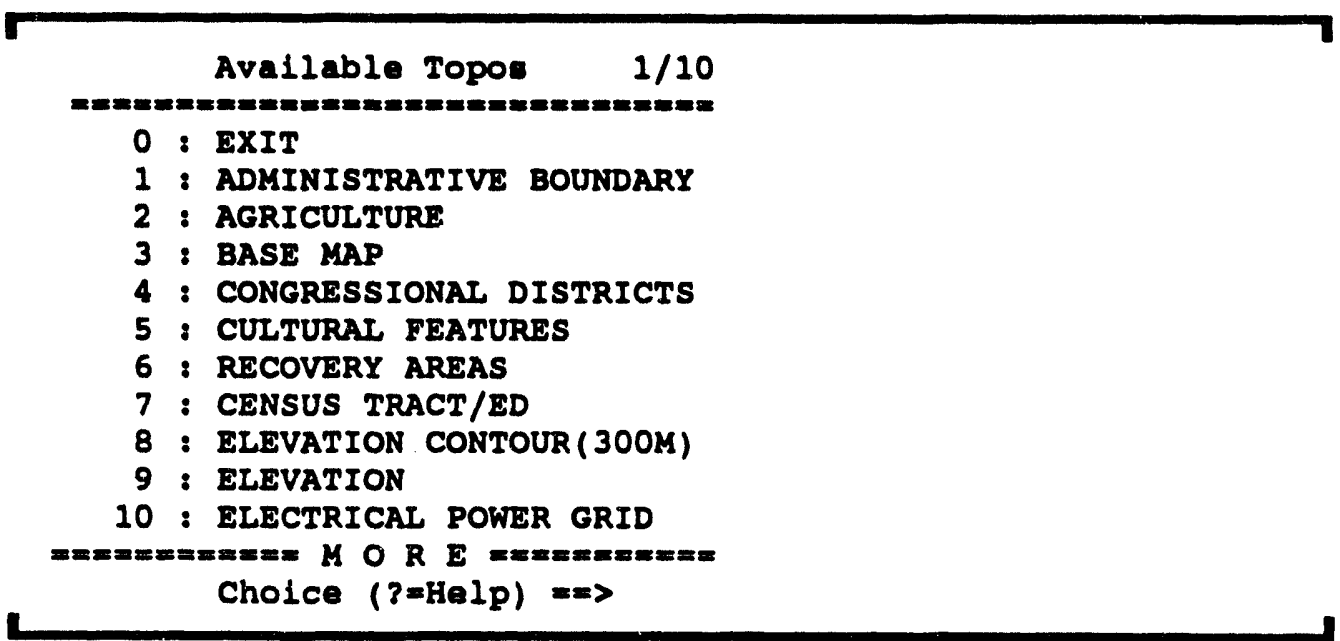


A few things should be noted about the Available Topos menu:

- The $1 / 10$ next to the title is an indication of the page currently being displayed, in this case, page 1 of 10 . To see different pages of the menu, type $U$ to move up a page or $D$ to move down a page.

- To select a topography (map layer), type the number after "Choice" and press RETURN. A text arrow $(=>)$ then appears next to the topography you wish to copy. If you type the same number again, the $=>$ disappears, indicating that the topography will NOT be copied.

- When you have selected all the topographies you want to copy to your current site, select Exit (0). This indicates to MAPDBGEN that all topographies have been selected and that you are ready to copy them to your new map database. If you wish to discontinue this operation, type $\mathbf{Q}$ to quit the menu.

8. Select topographies from the Available Topos menu.

\section{Caution}

Be careful not to overwitte topographles that already exist. If you select a topography that you have previously modffled in your current stte, you will overwrtte the files and lose your work.

9. When you have made all your topography choices, select Exit (0).

You will then see a sequence of messages similar to the following:

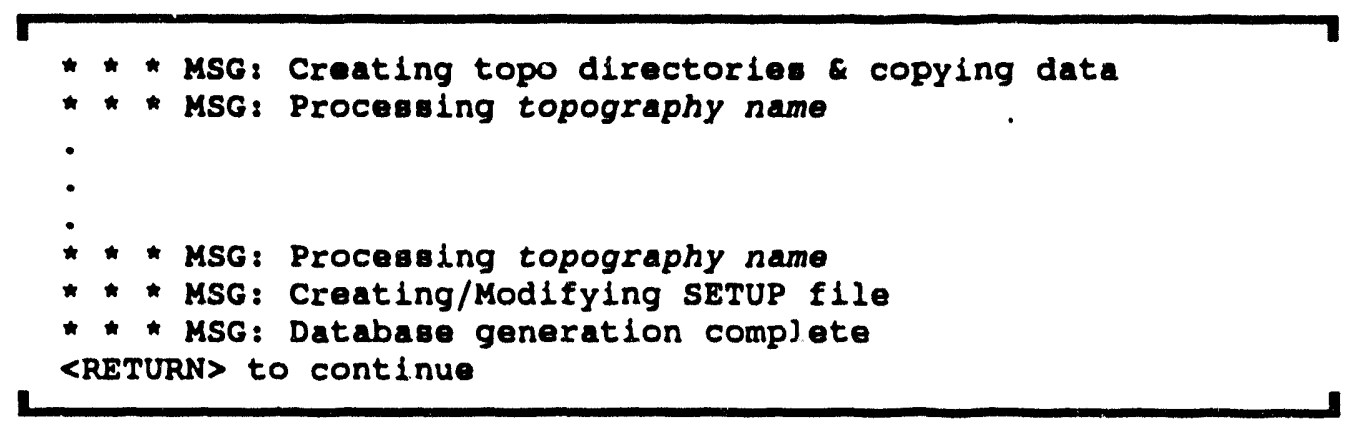

Once you press RETURN, the Area Selection menu in step 2 appears again. You have just completed creating a new map database. If you wish to add more topographies, you can do so by starting again at step 3 . 


\section{Results of Using MAPDBGEN}

For each topography you choose, MAPDBGEN examines the longitude-latitude extents of every DMS file corresponding to that topography in the existing map database. If the area of a topography file overlaps the area of the new map database, then the entire topography file is copied over into the new map database. The actual coverage of the new map database will be greater than the specified area because some of the copied DMS files will have areas that partially overlap the specified area and partially overlap a region outside the specified area. 


\section{MAPDBUPDT}

The MAPDBUPDT utility enables you to update a DMS database by adding new or modified files of topography data. Because this program has the potential to distort, lose, or otherwise damage necessary mapping information, it is important that you have a complete understanding of the update process before using MAPDBUPDT.

\section{Where Does the Update Data Come From?}

The simplest way to update topography data is to modify one of the topography files contained in a topography subdirectory of the database. In that case, the new version of the topography file is the update and MAPDBUPDT is not needed. $A$ more likely path is that you will do one of the following:

- Use the EXTRACT utility or MPDISPLAY's STORE REGION function to extract a region from the database, and then modify the topographies in that region.

- Create new data by digitizing geographic information from a map.

- Add or append new data converted to DMS format from an outside source of topographic data.

- Some combination of those methods.

For example, you might use the EXTRACT utility to copy a city region from the database, add detailed roads, and place name data, and then want to add the new data to the database. Whatever the method of obtaining the data, the result should be a topography file (or set of topography files) containing new data for a specific region.

\section{What Are the Requirements of Database Updating?}

To update a DMS database for a specific region, each altered topography of the region must be updated--one at a time. Each topography file containing new information is an Update File. Update Files have two requirements:

- Each Update File must be associated with a specific Update Region. This Update Region can be either

- the geographic extents (longitude-latitude limits) listed in the DMS file header, or 
- a region defined by a polygon file (a separate DMS file containing a polygon that describes the Update Region).

- Each Update File must represent all of the data for a particular topography within the Update Region.

The latter point is important: For the entire Update Region, MAPDBUPDT deletes old data for the topography and replaces it with the topography data in the Update File. Any "old" topography data that you want to save must be included in the Update File or that "old" data will disappear during the update. If the Update Region is larger than the area you really wish to change, a patch of "nothing" will appear along one or more edges of the area when the topography is displayed from the updated database. For this reason, it is crucial that the Update Region is exactly what you want it to be. Because the geographic extents of a DMS topography file can change during various storing, editing, appending, and manipulation operations...

Note: We strongly recommend that you create a polygon file that defines a polygon around the Update Region and then make sure that each topography you update contains the complete set of topography data for that region.

You can create a polygon file in several ways:

- You can use GEDIT to display topography files and draw a polygon defining the Update Region in a separate input file. (See Specifying Input Files in the description of GEDIT.) You can continue to display the polygon as you edit topography data in other input files.

- MPDISPLAY's STORE REGION option automatically saves a polygon of the stored region under the region name: For example, a stored region named MYAREA includes a polygon file named MYAREA.DMS.

- GEDIT's STORE REGION option automatically saves a polygon of the stored region under the name GEDIT.CLP.

You can check the correspondence between an Update Region polygon and the Update File by entering both DMS files as input files to PLOTDMS, a utility program that quickly plots DMS data. If there is a sharply defined empty space between the polygon and the topographic data, then there will likely be data lost if those files are used for an update. 


\section{What Happens During an Update?}

Background. Every topography in a DMS database has its own subdirectory. Each subdirectory contains a binary limit file named LIMITxx.BIN, where " $x x^{n}$ is the two-character code of the topography. This binary file specifies the names and geographic extents of all the topography files that make up the topography in that subdirectory. Each subdirectory also contains a reference file, T.DAT, which lists the names of the topography files.

Running MAPDBUPDT. MAPDBUPDT starts by asking you to specify a) the two-character abbreviation code of the topography you wish to update, b) the name of the Update File, and c) the Update Region.

The program then enters the corresponding topography subdirectory and reads the LIMIT file. For each database file specified in the LIMIT file, MAPDBUPDT determines whether the database file overlaps the Update Region.

- If no overlap occurs, the name and extents of the database file are placed in a new LIMIT file.

- If a complete overlap occurs, the name and extents of the database file are NOT included in the new LIMTT file.

- If a partial overlap occurs, the program creates a new version of the database file, deleting the graphics data in the overlapping area. The name and extents of the new database file are placed in the new LIMIT file.

After each database file specified in the original LIMIT file has been processed, a new version of the Update File is copied into the topography subdirectory, and the name of the new Update File is placed into the new LIMIT file. The new version of the Update File contains only the topographic data from within the Update Region. Each file name included in the LIMIT file is also placed in a new T.DAT file.

At the end of updating one topography, the topography subdirectory should contain:

- a new version of the Update File

- the rest of the data files that now constitute the database topography

- a new LIMIT file, LIMITxx.BIN, which specifies the names and extents of the new Update File and the rest of the files that now constitute the topography

- a new T.DAT file that lists the names of the same files 
- some old versions of files that are no longer part of the database data for that topography.

\section{A Procedure for Updating a DMS Database}

If an error occurs during the updating of a database, you must be able to reconstruct the original DMS database, determine what went wrong, and try again. The following is a methodology for using MAPDBUPDT to safely update a database.

1. Create one or more Update Files.

Remember, each Update File consists of all the data for a particular topography within a specific Update Region. It is preferable that the Update Region is specifically defined by a polygon in a separate DMS file.

2. Assign the logical names necessary for accessing the database by doing the following:

- If you are updating map data under a user account, set the default directory to disk:[user.SITES site] and then enter the following:

\section{\$ @SETUP disk user site associatedsite}

where site and associatedsite are the four-character codes for the site and its associated site (if there is one-see NEWSITE).

- If you are updating map data under a system map database, then either

- Set the default directory to the top-level directory of the map database and execute the SETUPDB.COM command file located there:

\section{\$@SETUPDB}

OR

- Enter the following command symbol, which does the same thing:

\section{S SYSmapDATABASE}

where map is the top-level directory name of the system map database.

3. Set the default directory to the database subdirectory of one topography that you wish to update. 
4. Check the topography subdirectory to become familiar with the topography files and to confirm that the LIMITxx.BIN and T.DAT files are in the subdirectory.

5. Copy the Update File for the particular topography into the subdirectory. (If you have a polygon file defining the Update Region, copy that file into the subdirectory also.)

6. If there is a separate text file (xcox.TXT) associated with the topography file, copy that file into the subdirectory also.

7. SAFETY FIRST: Create a complete copy of the topography subdirectory and its files where you can retrieve them and reconstruct the database if problems arise.

8. To start the update program, enter MAPDBUPDT.

You will see the following three (or four) prompts. The responses expected are explained as items $\mathrm{A}, \mathrm{B}, \mathrm{C}$, and D.

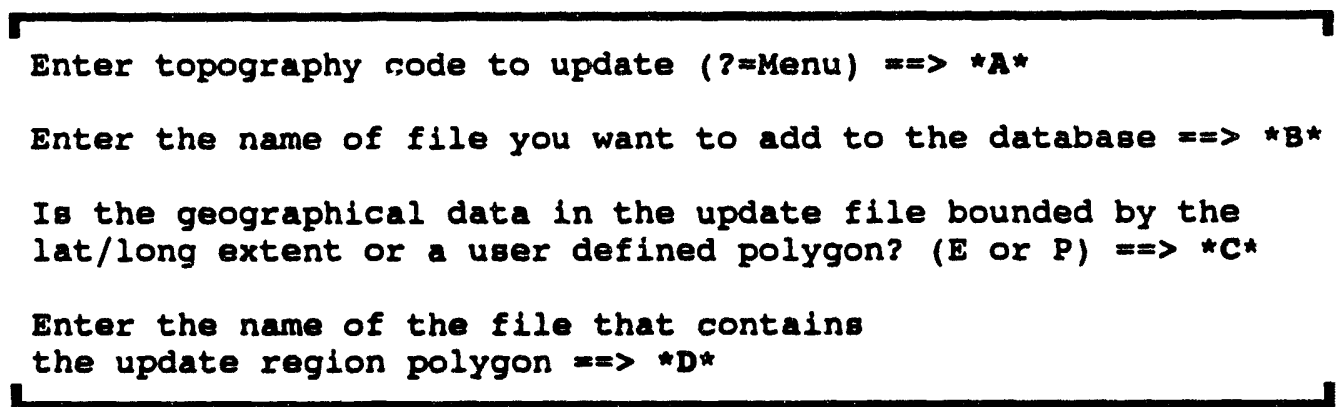

${ }^{*} A^{*} \quad$ Enter the correct 2-character code for the topography you are updating, or enter ? to see a menu list of topography codes.

*B* The Update File you specify must reside in the current default directory. If you specify a file name that points to a different directory, the program will respond with an error message and stop processing.

${ }^{*} C^{*}$ If you enter E, MAPDBUPDT uses the longitude-latitude extents of the Update File as the Update Region and does not prompt for an update region polygon. If you enter $\mathbf{P}$, you will see the prompt for the update region polygon.

${ }^{*} D^{*} \quad$ Enter the name of the polygon file to designate the Update Region. 
9. If an error message appears and the program does not run to completion:

a. Reload the original topography data, if necessary, and make the necessary corrections.

b. Try again, restarting the update process.

c. If the program terminated without a message or if you are completely stumped by what happened, reload the original database data and contact your System Manager.

10. If the program runs to completion without any error messages, check the update:

a. Check the directory to confirm that new versions of the LIMIT file and T.DAT have been created.

b. Check the contents of T.DAT to see that the name of the Update File has been added to the list of topography files.

11. Run MPDISPLAY to examine the Update Region and the area surrounding it.

12. If you see blank patches of missing data at the edges of the Update Region, reload the original database data. Then-
a. Determine a new, smaller Update Region, or
b. Modify the Update File to include the missing information.
c. Restart the update procedure.

13. If everything looks correct,

a. PURGE the topography subdirectory to delete old file versions.

b. Examine the T.DAT file to determine what files in the directory are still current.

Any remaining topography files that are not listed in the T.DAT file are for data that were totally overlapped by the Update File data.

c. Delete the topography files that were totally overlapped by the Update File data.

d. Delete the Update Region polygon file, if one was used. 
If you have successfully completed this procedure, then you have updated one database topography for a particular Update Region. Repeat the procedure for each topography you wish to update, restarting at step 3.

\section{Example of Updating a Database Topography}

Suppose that you have a user-defined Database that includes an AIRLINE ROUTES topography with a two-letter topographic code of "AR". Suppose that you wish to update the AIRLINE ROUTES data and have already completed steps 1 through 6 of the update procedure. The topography subdirectory [.AR] now includes 6 files:

- UPDATESAR.DMS;1: your Update File of new or modified AIRLINE ROUTES data

- ARREGION.DMS;1: a file containing a polygon that defines the Update Region

- LIMITAR.DMS;1: the binary LIMIT file for the AIRLINE ROUTES topography

- NORTHAR.DMS;1: a topography file that partially overlaps the data in UPDATESAR.DMS

- SOUTHAR.DMS;1: a topography file that completely overlaps the data in UPDATESAR.DMS

- T.DAT;1: a file that contains two file names, NORTHAR.DMS and SOUTHAR.DMS (the two current data files of the AIRLINE ROUTES topography)

Starting from the [AR] subdirectory, the interaction with MAPDBUPDT would look like this:

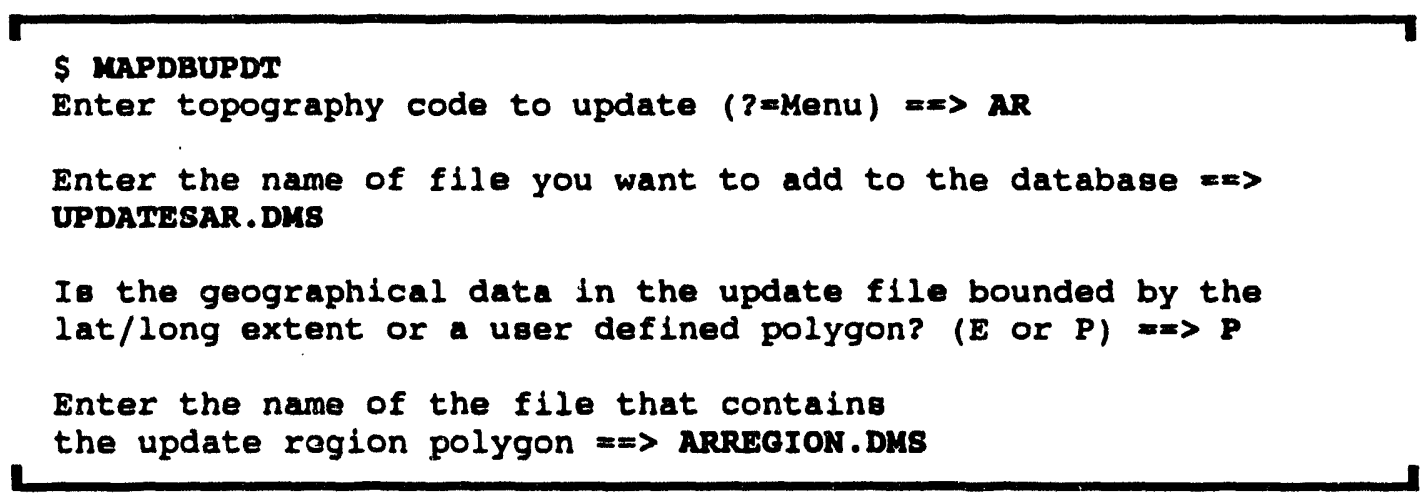


If the update was successful, the AIRLINE ROUTES subdirectory would then contain these 10 files:

- UPDATESAR.DMS;1 and UPDATESAR.DMS;2, a new file version that contains data only from within the Update Region

- ARREGION.DMS;1

- LIMITAR.DMS;1 and LIMITAR.DMS;2, a new version of the LIMIT file

- NORTHAR.DMS;1 and NORTHAR.DMS;2, a smaller, updated file that does not overlap the data in UPDATESAR.DMS;2

- SOUTHAR.DMS;1

- T.DAT;1 and T.DAT;2, a new file version that contains two file names, NORTHAR.DMS and UPDATESAR.DMS (the two current data files of the AIRLINE ROUTES topography)

Purging would remove the old file versions from the subdirectory. After ARREGION.DMS (the Update Region polygon) and SOUTHAR.DMS (the completely overlapped data) were deleted, the update for the AIRLINE ROUTES topography would be complete (for that Update Region). 


\section{MLMGR}

Options in the IBS program enable IBS users to send electronic mail to each other by picking names from a list (see the MESSAGE BOARD option in the IBS User Guide). The MLMGR utility enables a system manager (or someone else with appropriate IBS privileges) to update the system master mail list by adding or deleting names.

The MLMGR utility should be used whenever new IBS user accounts are added so that the system master mail list will be up to date. For example, NEWUSER puts in a partially complete record-you should fill it out completely using MLMGR. The DELUSER utility for deleting users from the system automatically removes the user's record from the mail list file, but you can also delete users with MLMGR.

\section{What You Need to Know Before Using MLMGR}

If you are adding people to the mail list or modifying list information, you need to supply the following information about each person:

\begin{tabular}{|c|c|}
\hline Name & $\begin{array}{l}\text { The name information includes the last name, first } \\
\text { name, and middle initial for each person. }\end{array}$ \\
\hline Agency & This is the code associated with the person's agency. \\
\hline Pasition & This is the position that will be listed for each person. \\
\hline $\begin{array}{l}\text { Electronic } \\
\text { Mail } \\
\text { Address }\end{array}$ & $\begin{array}{l}\text { If the person's user account is on a computer system } \\
\text { node that is networked to other nodes by DECNET, } \\
\text { then the address is just the name of the person's } \\
\text { account or home directory. }\end{array}$ \\
\hline
\end{tabular}

If the person is on a node that is NOT connected by DECNET (this includes users of the onpost system), then address refers to the entire mailing address. For example, if the user has an InterNet mailing address, you would enter that address.

If you are deleting people from the mail list, you must be able to pick their names or addresses from a listing.

Records that are added, modified, or deleted from the mail list file will automatically be changed in the mail list files of other systems on the network according to the contents of the IEMISSSYSF:MAII_SYSTEMS.DAT file (see the Data Management Guide for information about this file). The EVENT program must be running to route changes to the local mail list file to other networked 
systems. If EVENT is not running, these files will remain unprocessed until EVENT is started. When EVENT is restarted, it processes the waiting files, so changes made while EVENT is not running will NOT be lost.

\section{Updating the IBS Mail List}

To start this utility, enter MLMGR at the system prompt. The program starts by giving you a choice between adding, modifying, deleting, or updating user information on the IBS system mail list:

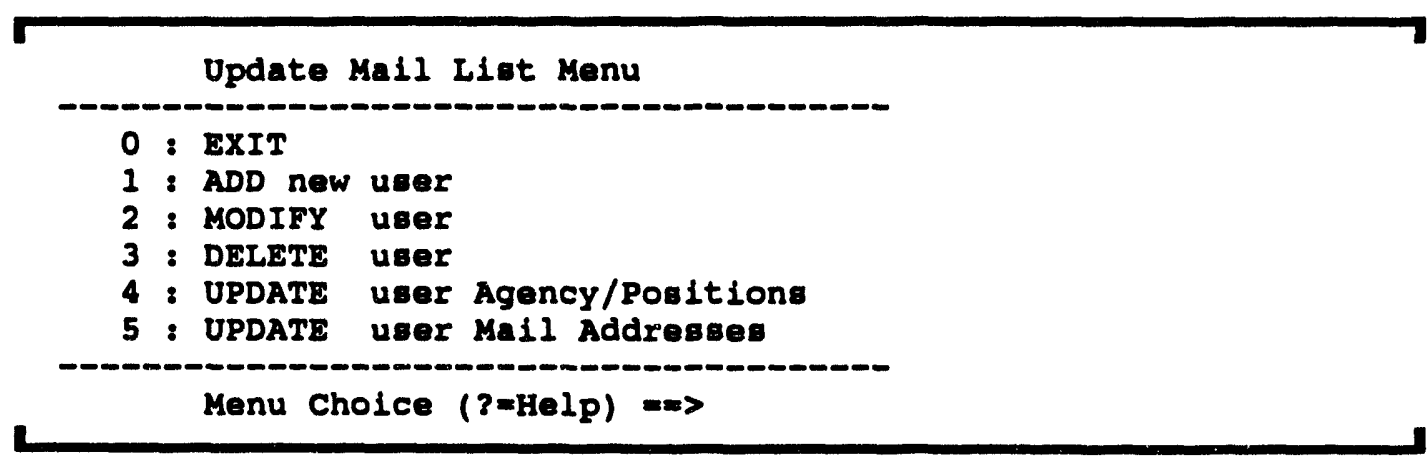

- To add new users to the list, enter 1.

- To modify information about a user, enter 2.

- To delete existing users from the list, enter 3.

- To update Agency/Position information on the list, enter 4.

- To update Mail Addresses by adding an address prefix or suffix, enter 5. 


\section{1 : Adding New Names to the Mall Lst}

The "ADD new user" option displays the following screen form for adding one name:

\begin{tabular}{|l|l|}
\hline Mall List Maintenance Form: Add & V2.00 \\
\hline Last Name: & Firet Name: \\
Agency: & Position: \\
& \\
Electronic Mail Address. & MI: \\
\hline
\end{tabular}

Enter the user's name, agency, position, and electronic mail address as described in the previous section. Then press GOLD $Z$ to exit the form and add the new user to the system mailing list. 


\section{2 : Modifying Information about Users on the Mall List}

The "MODIFY user" option displays this menu for listing users either by their mail addresses or by their real names:

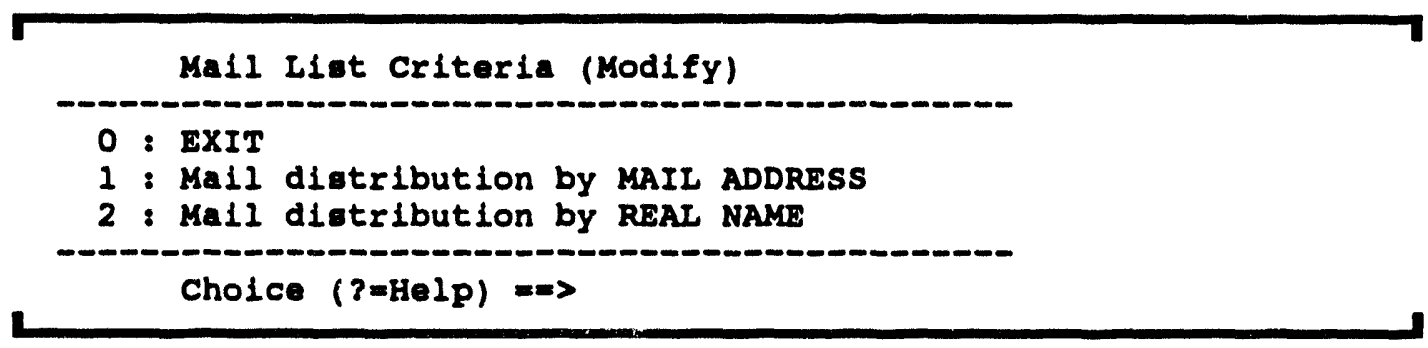

Each option presents a menu list of mail users for selecting which ones to delete. Each menu list is alphabetically sorted according to the indicated criterion.

Example: Menu List Sorted by Real Name (Option 2).

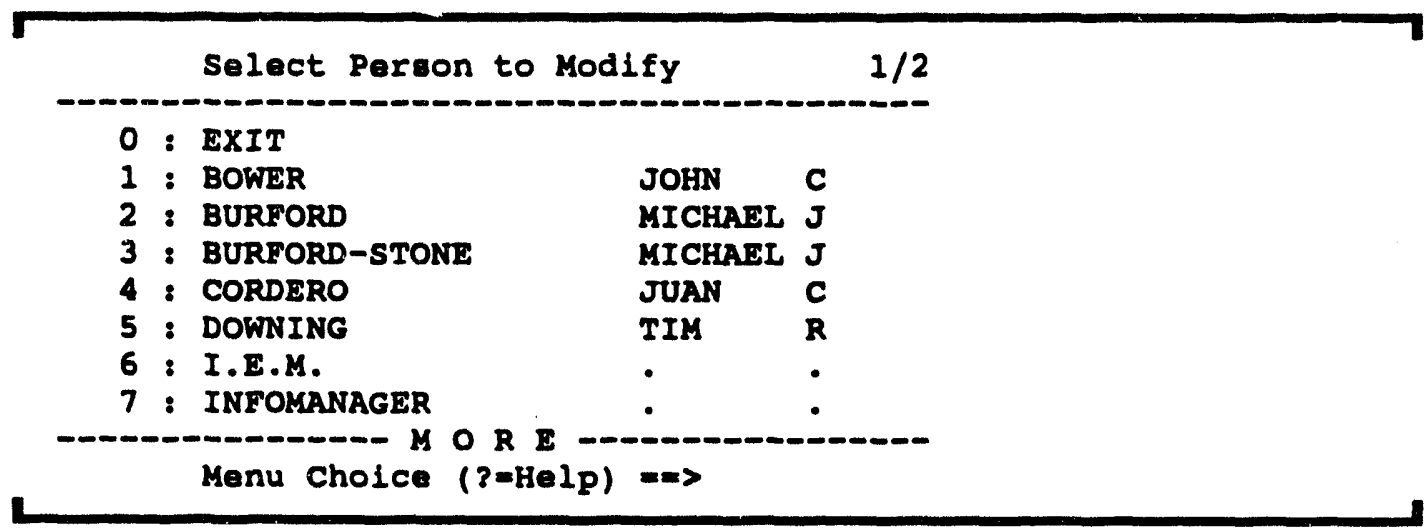

1. To choose the person you wish to modify, enter the corresponding number.

You are then presented with a form similar to the one described for adding a new user. The information for the chosen person is already filled in.

2. Modify any of the information items (name, agency, position, mail address).

3. To confirm the changes and save the modified information, press GOLD Z. To cancel the changes and exit without modifying the list, press GOLD Y.

4. Respond to: Update changes on all connected computers? $(Y / N)=>$ $Y(e s)$ updates the mailing information on all connected systems.

$\mathbf{N}(0)$ updates information only for the local system, which may have mail routing software that requires special addressing (see MLMGR option 5).

After either confirming or canceling, you will return to MLMGR's initial menu. 


\section{3 : Deleting Users from the Mall Lst}

The "DELETE user" option displays a menu for choosing how you want to list the users on the mail list:

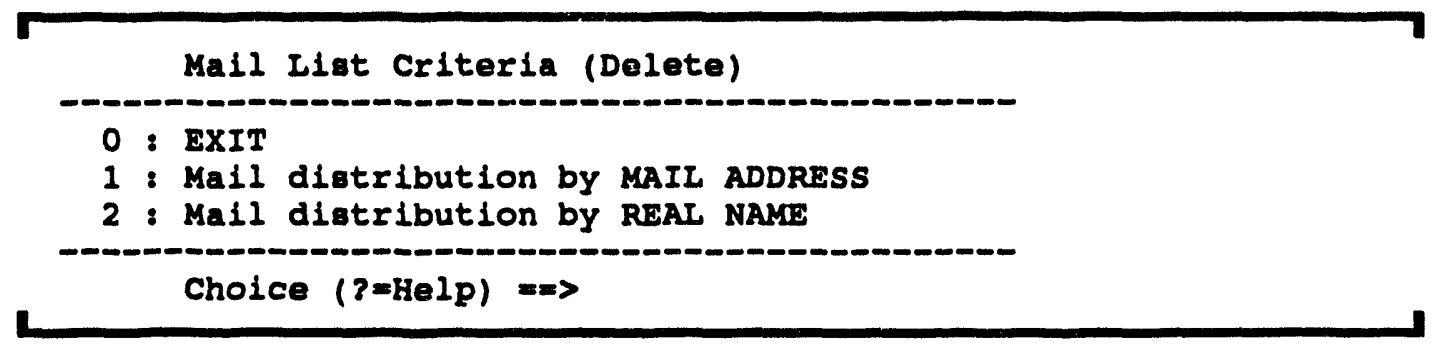

Each option presents a menu list of mail users for selecting which ones to modify. Each menu list is alphabetically sorted according to the indicated criterion:

- Option 1 lists the people by their mail addresses.

- Option 2 lists the people by their real names.

For example, the following menu list is sorted by electronic mail address (option 1).

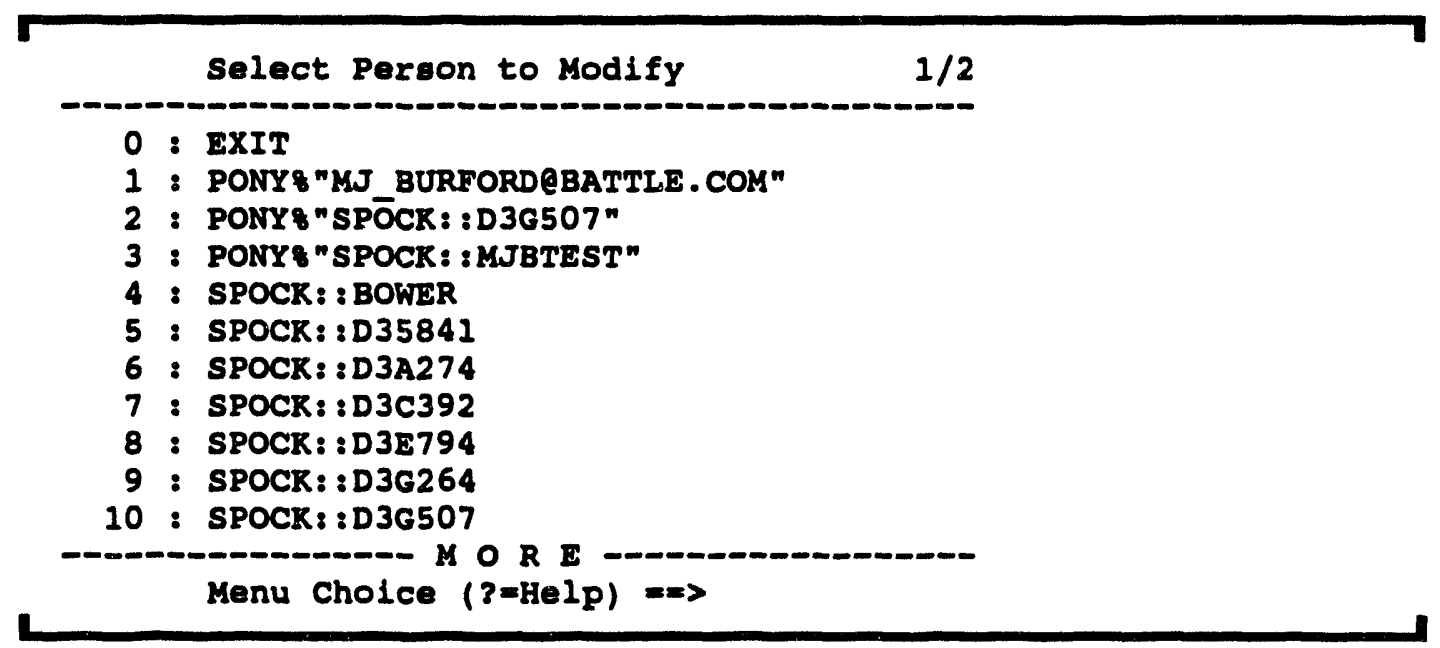

- To choose the person you wish to delete, enter the corresponding number. OR

To return to the MLMGR menu, choose 0 (EXIT). 


\section{4 : Updating Agency/Position Information on the Mall Lst}

The "UPDATE user Agency/Positions" option enables you to update all the positions in the mail list database with the correct position from the Personnel database. Only those people who have exactly the same names in the two databases will be updated. The program searches the entire Mail List and compares the user names to the names in the Personnel database. If the names are the same, the Agency/Position record from the Personnel database are propagated into the Mail List database. If the person's Agency/Position is different between the two databases, you will see the following prompt:

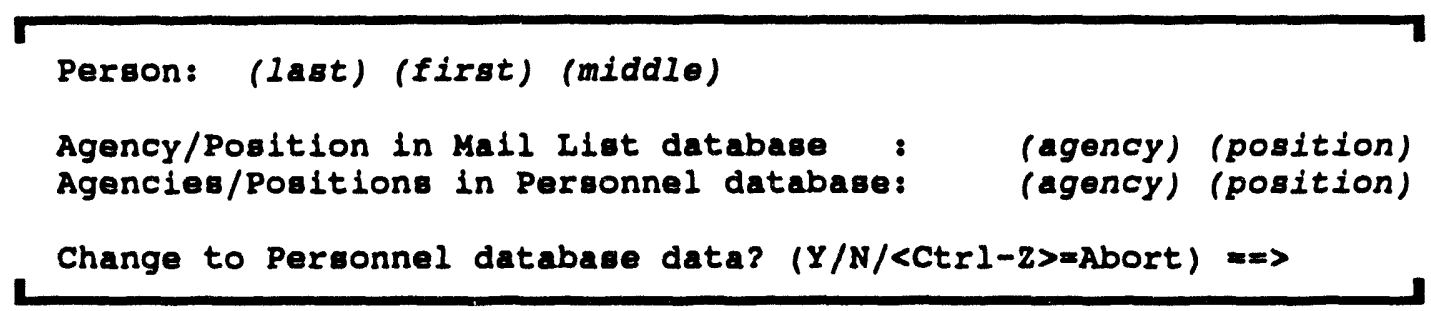

- To select which agency/position code to use, answer Y. You will then see a menu for selecting which agency and position codes to use.

OR

- To continue with the next name, answer $\mathbf{N}$.

OR

- To quit, press CTRL-Z. 


\section{5 : Updating Mall Address Information on the Mall Lst}

The "UPDATE user Mail Addresses" option enables you to add a prefix or suffix to selected mail addresses. This can be useful for adding information required by a mail router program. For example, the Pony Express program requires BONY\% as a prefix and ". as a suffix. The option displays a menu similar to the one below:

Example:

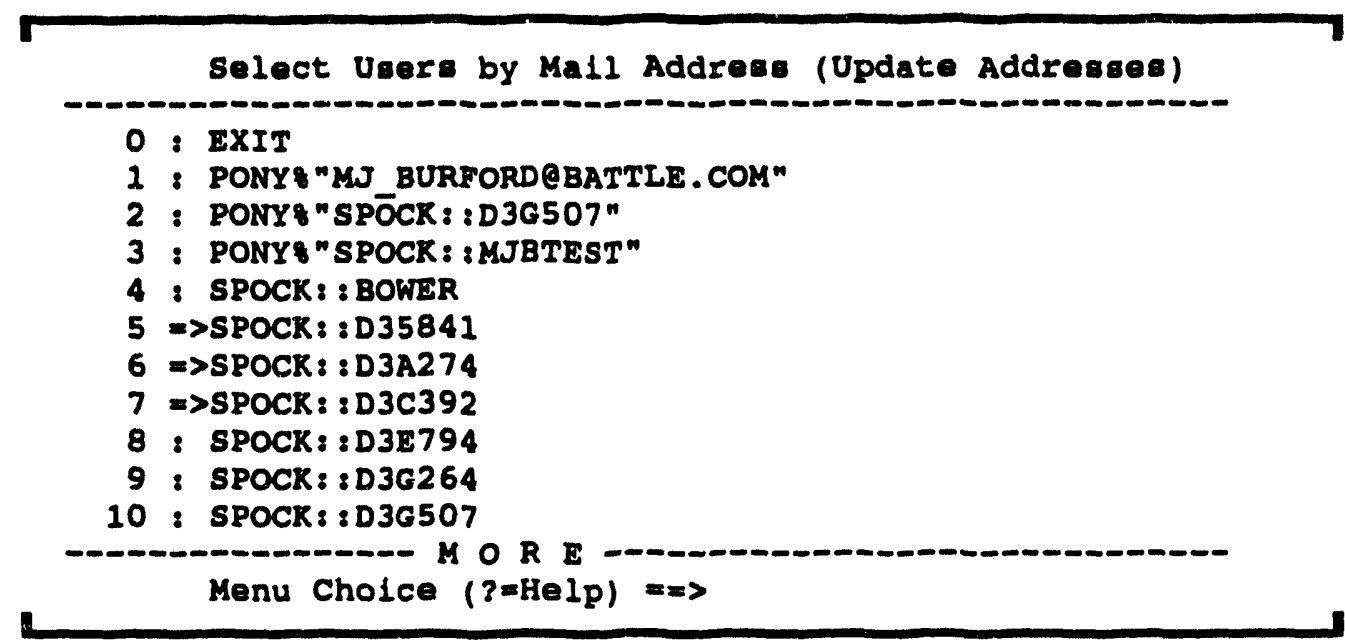

1. Select one or more mail addresses to modify by adding a prefix or suffix (or enter 0 (EXIT) to quit without updating addresses.

Each address you select is marked with $=>$, as illustrated for items 5-7 above.

2. Select 0 to exit the menu.

The program prompts for an address prefix and then a suffix:

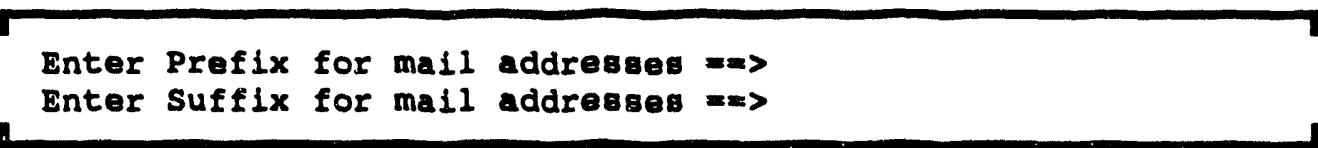

3. Enter each item, or press RETURN to skip an item.

A message shows the number of addresses updated.

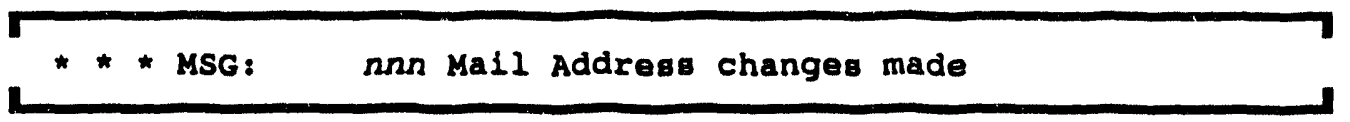

Press RETURN to continue and display the initial menu again (go to step 1). 


\section{MLROUTE}

The MLROUTE utility is intended for use by system managers (or someone else with appropriate privileges) to update not the local IBS master mail list file, but the IBS mail list files of remote computers that are connected to the local computer by a network. It does this by routing the contents of the system master mail list file to all networked computers according to the information in the

IEMISSSYSF:MAIL_SYSTEMS.DAT file (see the IBS Data Management Guide for information on this file.)

This program is normally executed only for one of the following two situations:

- when the system is being upgraded to IBS $2.0 x$

OR

- when new systems are added to IEMISSSYSF:MAIL_SYSTEMS.DAT

All normal changes to the system master mail list file (using MLMGR, NEWUSER, DELUSER, etc.) occur automatically and automatically update networked systems according to the contents of the IEMISSSYSF:MAII_SYSTEMS.DAT file.

\section{What You Need to Know Before Using MLROUTE}

MLROUTE starts by creating a series of files that the EVENT program will use to route the contents of the system master mail list. The EVENT program must be executing to route these files. If EVENT is not executing, these files remain unprocessed until EVENT is started. When EVENT is restarted, it processes the waiting files, so changes made while EVENT is not running are NOT lost. See EVENT in this document for information on starting the EVENT program.

\section{Updating the Mail Lists of Networked Systems with MLROUTE}

To start the utility, just enter MLROUTE at the system prompt. You should see status messages similar to the following if the program was successful.

Example:

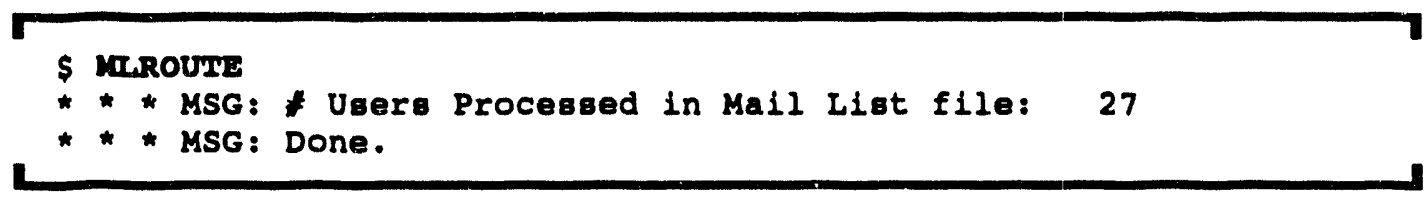




\section{MODATTDMS}

The MODATTDMS utility reads a DMS file and replaces all the DMS attributes of a certain major, minor code with a new major, minor.code that you specify.

\section{What You Need to Know Before Using MODATTDMS}

In addition to the name of the DMS file that you want to change, you must know the major, minor attribute code that you wish to replace and the new major, minor attribute code. For a listing of attributes in the IBS standard attribute dictionary, see Appendix B.

\section{Replacing Attribute Codes in a DMS File Using MODATTDMS}

To start this utility, enter MODATTDMS at the system prompt. The utility then presents the following sequence of prompts.

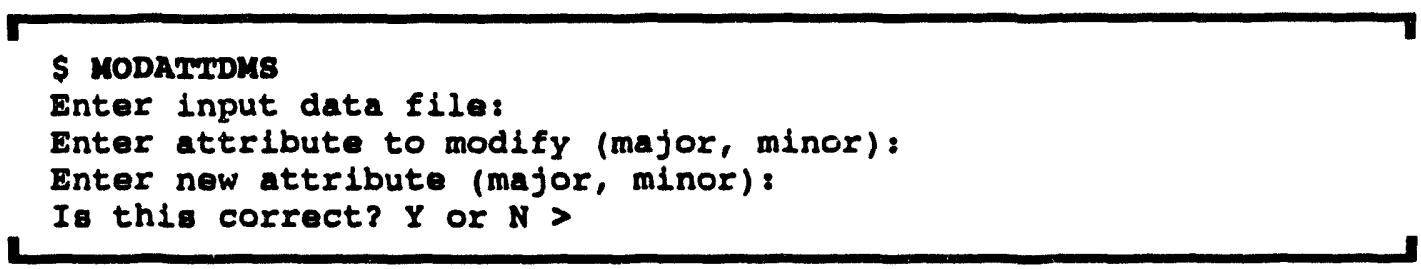

If you answer $Y$ to "Is this correct?", the old major, minor codes you specified will be replaced with new codes. If you answer $\mathbf{N}$, you will be reprompted for information.

Once you have completed the above steps, MODATTDMS begins another iteration of the same sequence. Continue through as many iterations as you wish. To exit, press CTRL-Z at the data file prompt. 


\section{MODTEXTNM}

Each DMS file containing graphically displayable text points has a text (.TXT) file associated with it. The name of this text file is included in the header section of the DMS file. If you rename a text file that is associated with a DMS file, you must also alter the contents of the DMS file header to refer to the correct text file.

The MODTEXTNM utility enables you to modify the name of the text file in the DMS file header.

\section{Changing the Text File Referenced in a DMS File Header}

To start the utility, just enter MODTEXTNM at the system prompt. The following is an example sequence that places a reference to the text file NEW.TXT in the header of a DMS file named MYFILE.DMS.

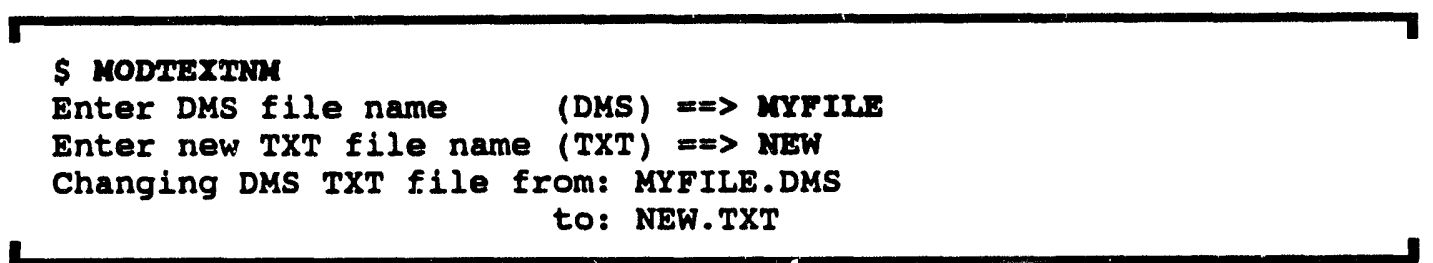

After you start MODTEXTNM, the utility asks you to :

following items:

DMS file name

new TXT file name

This is the name of the DMS you want to alter. If you do not specify an extension, the .DMS file extension is assumed. To cancel the program at this point, press CTRL-Z.

This is the new TXT file name to be included in the DMS file header. If you do not specify a file name extension, the .TXT extension is used. You can also do the following instead of entering the TXT file name:

To get this result:

To use the same name as the DMS file but with the .TXT extension:

To remove the text file reference and dissociate the DMS file from any text file:

To cancel the program:
Do this

Press RETURN.

Enter a blank space.

Press CTRL-Z. 


\section{Results of Using MODTEXTNM}

MODTEXTNM changes only the name of the text file referenced in the DMS file header. It does NOT rename the actual text file. To rename a text file, use the VMS RENAME command. 


\section{MPDISPLAY}

The MPDISPLAY utility enables you to selectively view and store IBS map information for different geographic areas. MPDISPLAY requires a graphic workstation of the type described in Section 1. This documentation covers the following topics:

- Description of the MPDISPLAY Software

- What You Need to Know Before Using MPDISPLAY

- Terminology for MPDISPLAY

- Starting an MPDISPLAY Session

- Using the MPDISPLAY Main Menu

Topic

- How to display topographic features

- How to "store" the displayed region for later use

- How to limit the display of data through attributes

- How to add and edit graphic data

- How to display elevation data

- How to specify which icons are available for display

- How to display icons, text, points, and filled polygons

- How to vary the display area

- How to display statistics associated with displayed features $\frac{\text { Menu Item/Function }}{\text { SELECT TOPOGRAPHY }} \frac{\text { Page }}{2.265}$

STORE REGION

ATTRIBUTE FUNCTIONS

EDIT FUNCTIONS

ELEVATION FUNCTIONS

ICON FUNCTIONS

ITEM DISPLAY FUNCTIONS

These principal functions of the MPDISPLAY main menu are explained in alphabetical order, starting on page 2.243 . 


\section{Description of the MPDISPLAY Software}

MPDISPLAY is a program that allows you to access the map databases on the IBS. You can interactively select regions and topographic features for display by using graphics input device (such as a mouse or joydisk) and menus. Standard topographic features, such as roads and streams, can be supplemented with userdefined data, such as specific buildings or populations that are linked to location. Individually tailored maps containing user-selected regions and features can then be clipped and stored for other applications and processing.

The map display software MPDISPLAY and the related map editing software GEDIT were created by the Pacific Northwest Laboratory. They contain a library of subprograms that are accessib: a by other programs for creating, storing, and displaying two-dimensional data. The map-related features of the system modeling functions are based on MPDISPLAY.

\section{What You Need to Know to Use the MPDISPLAY Software}

This documentation is intended as a guide for new users of the MPDISPLAY software. It contains information on the operation of MPDISPLAY and assumes that you will apply MPDISPLAY to the IBS map database or to another database that is constructed according to IBS standards for graphically represented geographic data. You should be familiar with using the graphics input device attached to your terminal-refer to the IBS User Guide.

\section{For the Typical User of MPDISPLAY}

This MPDISPLAY documentation covers all the concepts and interactions necessary to use the software for map display functions: choosing a region to display, choosing a type of map projection, choosing topographic features, using an edit file, and using the MPDISPLAY main menu. Graphics editing functions, some of which are accessible through MPDISPLAY, are described in the documentation for the GEDIT utility. Typical users need not be closely concerned with IBS geographic data standards and file structures.

\section{For the In-Depth User of MPDISPLAY}

In-depth users of MPDISPLAY can find map graphics standards described in the IBS Data Management Guide. 


\section{Terminology for MPDISPLAY}

- Digital Mapping Structure (DMS) -- Digital Mapping Structure is the data file format used to store graphic data for use by the IBS mapping functions (such as the MPDISPLAY map display program and the GEDIT graphic editing program). DMS files typically contain line or irregular point data depicting topographic features (such as boundaries, railroads, or hospitals) at particular longitude-latitude locations. DMS files have ".DMS" as the file name extension and are often part of a map database-a specially structured set of DMS data files that describe a geographic area. MPDISPLAY is used to display topographic features of regions within the database area.

- Edit file -- MPDISPLAY's EDIT FUNCTIONS allow you to use a subset of the graphic drawing and editing capabilities described for the GEDIT graphic editing utility in this guide. You can use these functions to modify one DMS file of your choice. This DMS file is referred to as the edit file.

- IBS Map Database -- The IBS map database for your site is a DMS database that contains site geographic information for use by all users. The database is generated by consolidating geographic information from various national, state, and local sources. Although most of the data is vector (line) data and text data that follow DMS conventions, it may also contain some 1:250,000scale cell (elevation) data for limited use. For detailed information on the IBS map database, please refer to the IBS Data Management Guide.

- MPDISPLAY - MPDISPLAY is the name of the program that starts when you enter the MPDISPLAY command at the system prompt.

- Stored region -- A "stored region" is a set of graphic data that has been copied from a region in the IBS map database. A stored region consists of 1) a DMS file containing a polygon that describes the boundaries of the stored region and 2) one DMS topography data file for each type of topography in the stored region. Stored regions are created by MPDISPLAY's STORE REGION option or by the EXTRACT utility program. The data files for a stored region are placed in your current default directory.

- Topography -- In general terms, a topography is a graphic description of a geographic region. In MPDISPLAY and the IBS, a topography (or map layer) is a set of graphic data that depicts a particular type of map feature. For example, "railroads," "streams," and "water bodies" are all different topographies used by the IBS map database. In MPDISPLAY you can choose which features are displayed by selecting them from a menu of topographies. 


\section{Starting an MPDISPLAY Session}

Start the MPDISPLAY software by entering MPDISPLAY at the system prompt. For example:

\section{\$ MPDIBPLAY <RETURN>}

The program starts by reading control files that specify the database areas, topographies, and actual data files within the database. This may take a few minutes if the system is heavily loaded or if the database contains a large number of files.

MPDISPLAY then presents a blank square outline (map grid) where the maps of geographic regions will be displayed. Next to the blank grid will be a graphics menu for selecting a geographic region. Your initial tasks are:

1. Choose a region to display.

2. Specify the type of geographic projection to be used.

3. Choose topographic features for display.

4. Enter the name of an "edit file."

These tasks are explained in the following subsections.

\section{Choosing a Region to Display}

MPDISPLAY's first menu lists options for choosing a region to display.

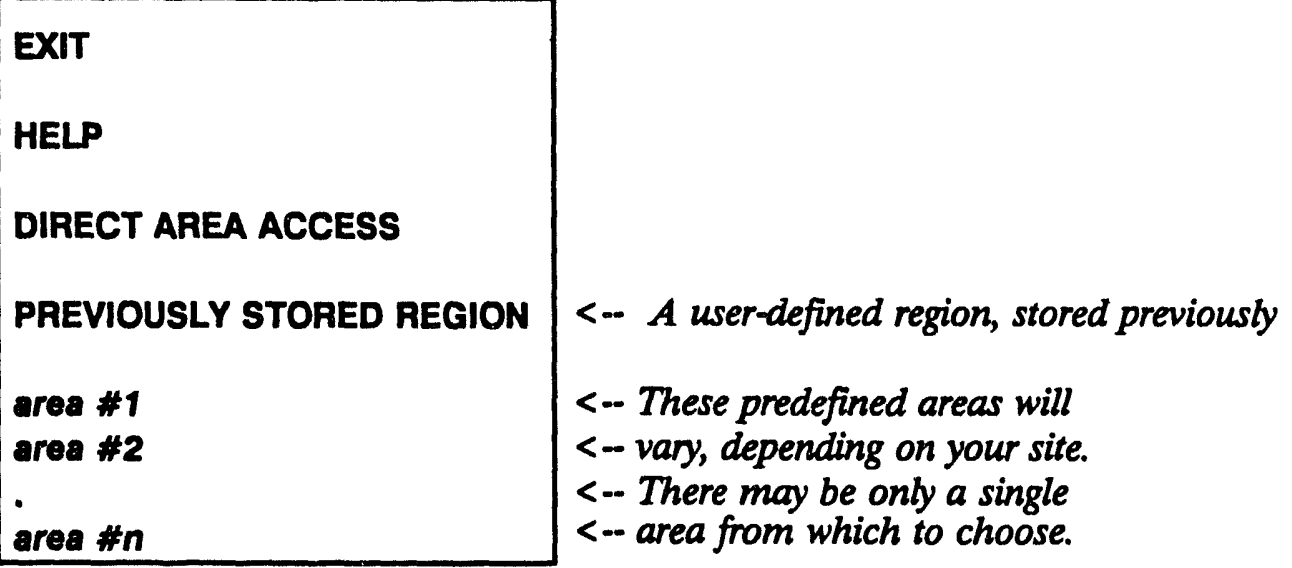

This menu allows you to access predefined areas (portions) of the database or regions that have already been stored with MPDISPLAY. The menu's principal options and their uses are described in the following list.

EXIT: If you select EXIT, MPDISPLAY will erase the current menu display and return you to the system prompt. 
DIRECT AREA ACCESS: If you select a DIRECT AREA ACCESS, MPDISPLAY will display a submenu:

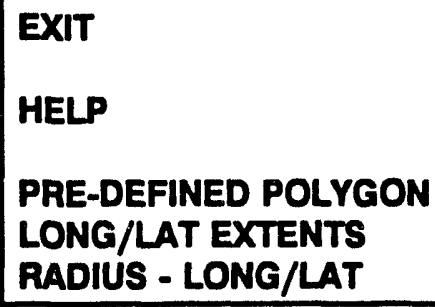

The principal options of this submenu are three different methods for directly specifying a display region from within either a previously stored region or a predefined area of the database. When you know the location and boundary of the region you want to display, direct area access is often the quickest way of displaying a region.

PRE-DEFINED POLYGON: Selecting this option allows you to enter the name of a polygon description file. A polygon description file is just a DMS file that contains a geographically defined polygon representing the boundary of the region you wish to display. You will see the prompt:

ENTER POLYGON DESCRIPTION FIIE NAME >

After you enter the name of the polygon description file, MPDISPLAY uses the polygon as the basis for extracting display information. For information on how to create a polygon description file, refer to the STORE REGION function (page 2.268).

- LONG/LAT EXTENTS: Selecting this option allows you to enter minimum and maximum longitude-latitude limits to define the boundary of the region you wish to display. You will see the prompt:

ENTER THE LONG/LAT EXTENTS OF THE AREA

( MIN-X, MAX-X, MIN-Y, MAX-Y ) >

Enter each extent (longitude values first), separating the values with commas or spaces. If the first longitude value is greater than the second (if MIN-X > MAX-X), then you will see an additional prompt that asks whether you want to cross the intemational dateline: enter NO. (See the following NOTE on crossing the international dateline.)

RADIUS - LONG/LAT: Selecting this option allows you to enter a longitude-latitude location and a radius around that location to define the boundary of the region you wish to display. You will see the prompt:

Enter RADIUS (in miles), Long, Lat > 
Enter the three values, separating the values with commas or spaces. If the defined region crosses the international dateline, you will see an additional message to that effect, and MPDISPLAY will attempt to display the defined region. Areas that contain no data will appear blank.

Note: MPDISPLAY will display a region that crosses the international dateline, as indicated above. The DIRECT AREA ACCESS of a region in the main database is the only way of accomplishing this. When displaying such a region, MPDISPLAY imposes some limitations:

- After the topographic features of the region are displayed initially, additional topographies cannot be added by the SELECT TOPOGRAPHY function (page 2.265).

- There will be no "edit file" for the region (page 2.240).

These limitations are consequences of the display method. A region that spans the international dateline can be stored by the STORE REGION function (page 2.268), but the longitude coordinates are shifted to allow storage within a normal DMS file. Although the latitudes and all other map relationships remain correct, the longitude coordinates are deliberately offset. For example, when the stored region is retrieved (using PREVIOUSLY STORED REGION), using RETURN COORDINATE (page 2.271) would yield faulty longitude values. The system database contains no data in the region of the international dateline, so such displays would be important only to those using a special DMS database.

After using one of these three methods to specify a display region, pick EXIT on the DIRECT AREA ACCESS menu. MPDISPLAY will redisplay the initial menu, allowing you to directly extract the display region from either a "previously stored region" or a predefined database area. Whichever of the three methods you use, the display region defined must exist entirely within the region from which the extraction is made. If this is not the case, blank areas will appear on the display where there is no data.

Pick either PREVIOUSLY STOAED REGION or one of the predefined database areas to identify which data you wish to directly access. If you pick a previously stored region, MPDISPLAY will prompt for the name of the stored region from which the display region data is to be extracted. This name would be the name that was entered when the STORE REGION command was used to store the region (page 2.268).

After you either enter the name of a nreviously stored region or pick a predefined database area, MPDISPLAY will ask you to choose the type of map projection (see page 2.237 ). 
PREVIOUSLY STORED REGION: If you want to retrieve data for an entire stored region, pick PREVIOUSLY STORED REGION instead of DIRECT AREA ACCESS. MPDISPLAY will prompt you for the name of the region. This name would be the name that was entered when the STORE REGION command was used to store the region (page 2.268). In conjunction with STORE REGION, this function provides a means of displaying data stored in local, user-owned files that are not associated with the main database.

After you enter the name of the previously stored region at your terminal keyboard, MPDISPLAY will present several options for choosing the type of map projection to display (see page 2.237 ).

- area \#1/area \#2/.../aree \#n (Predefined Database Areas): Some databases consist of areas so large that it is advisable to represent the database as several database areas (such as Alaska, Hawaii, and the conterminous United States). These predefined areas may be single sites or larger areas from which you would normally extract a smaller portion for display. It is possible that only your site map database will be listed.

If you pick one of the database areas (instead of DIRECT AREA ACCESS or PREVIOUSLY STORED REGION), MPDISPLAY will draw a low-detail overview map of the area and display a submenu with the following options:

EXIT
HELP
CONTINUE
DISPLAY COORDINATES
** SELECT REGION **

The principal options are described below:

DISPLAY COORDINATES: Picking DISPLAY COORDINATES and then a point on the map results in the display of

- longitude-latitude of the point

- the Great Circle distance from the point to the center of the display (shortest arc over the surface of the earth)

- the cumulative distance from the first point picked for a DISPLAY COORDINATES.

The DISPLAY COORDINATES function is a handy way of getting some 
quick coordinate information, which might be used for a DIRECT AREA ACCESS, for example. Picking subsequent points will continue to display coordinate information until you terminate the DISPLAY COORDINATES function by picking any menu item.

- SELECT REGION: This option allows you to select a region on the overview map by defining a square area that contains the desired region.

1. Use the graphics input device to "pick" a point on the map that is the center of the region that you want to capture for display:

Place the graphics cursor over a point on the map that is the center of the area that you want to capture, and press the SPACEBAR.

2. Place the cursor over a second point that will define a side of the captured area, and press the SPACEBAR.

Keep in mind that selecting a large region (such as an entire state) may mean that a large amount of data will have to be accessed to display the area, depending on what features are displayed. The time necessary to access the data and draw the map will increase with the number of topographies and the size of the area selected.

After you have chosen a region to display (by DIRECT ACCESS, by retrieving a PREVIOUSLY STORED REGION, or by extracting from a predefined database area), MPDISPIAY will present several options for the type of map projection to display.

\section{Choosing the Type of Map Projection}

MPDISPLAY then presents this menv of options for displaying map projections:

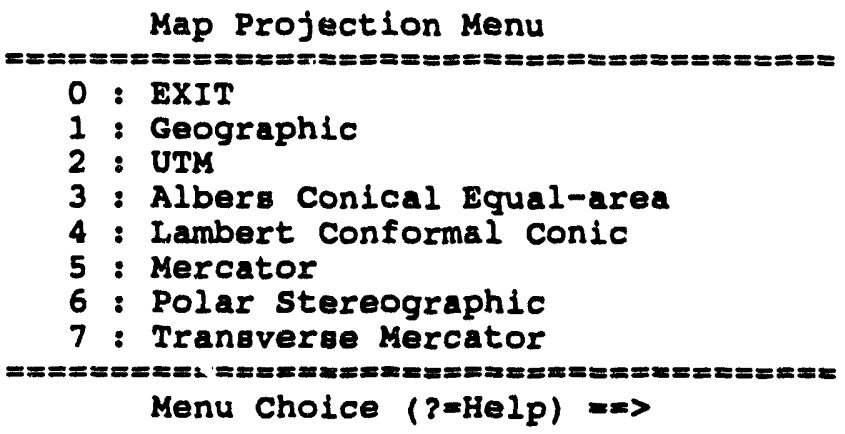

For most cases (up to 100-mile radius), enter 1 to select the "Geographic" projection. he Geographic projection is a straightforward projection onto a regular $X, Y$ plane If you desire a projection other than Geographic, enter a number (2 to 7) to choose one of the other projections. The following table is a simple summary of how the different map projections might be used. See Appendix D for background on the projection types. 
Map Projection Type

Geographic

Universal Transverse Mercator (UTM)

or Transverse Mercator

Albers Conical Equal-Area

or Lambert Conformal Conic

Polar Stereographic
Situations for Use

small areas (up to a 100-mile radius)

medium-sized areas (up to 1000-mile radius) or where the largest extent of the mapped area extends north-south rather than east-west

larger areas, or where the largest extent of the mapped area extends east-west

polar areas of the globe

After you select a projection type, MPDISPLAY will present a menu for choosing topographic features.

\section{Choosing Topographic Features}

The topography selection menu lists the topographies (topographic features or map layers) that are available for display, such as these standard IBS topographies:

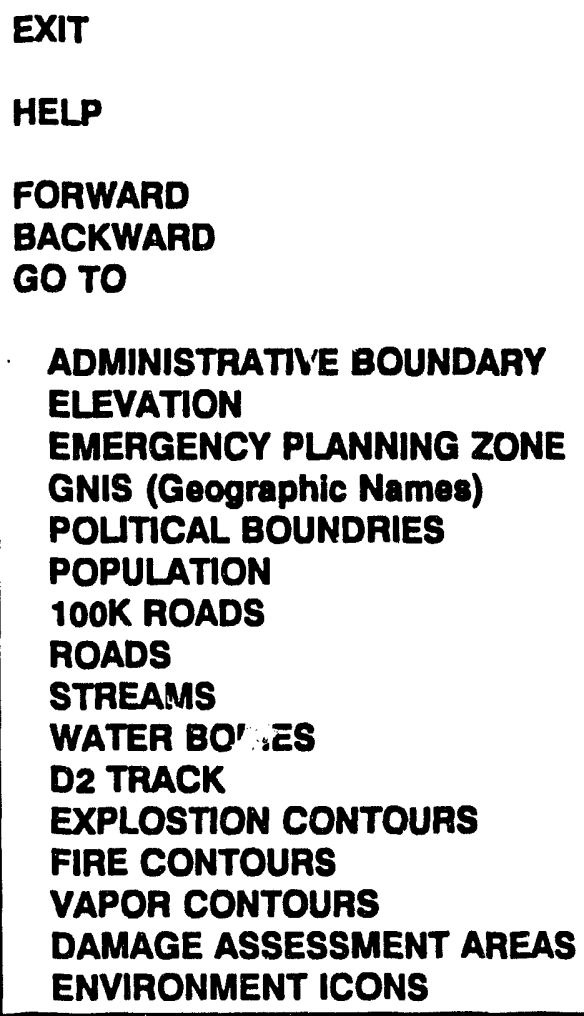

This list of topographies may vany If your database administrator has added or subtracted topographies.

Examples

clty boundaries

grióded elevation data

rivers, cemeteries, schools (USGS)

Use the graphics input device to pick topographic features for display: 
1. Pick each topographic feature (topography) that you want to display.

Each menu item picked will change color to indicate selection. If you change your mind about an item that you picked, pick it again. The menu item will return to its former color, indicating no selection.

If the menu area is not large enough to list all the topographies at one time, pick FORWARD to see the next part of the list. Pick BACKWARD to see a previous part of the list (after using FORWARD).

Do NOT pick a particular topography if you intend to edit that topography using the edit file (see Entering the Name of an Edit File, page 2.240).

2. When you have finished choosing topographies, pick the top menu item, EXIT.

The cursor will disappear momentarily, and the system will display a message: READING DATA. PLEASE WAIT.

What happens next depends on whether the display region is from a previously stored region or from a predefined database area:

Previously Stored Regions: For each topography that you choose, MPDISPLAY will read in the contents of one topographic data file containing the corresponding topographic information. (If you have used the DIRECT AREA ACCESS function to specify a portion of a stored region, then only a portion of each topographic data file may be read in.)

Extraction from a Database Area: For each topographic feature that you choose, MPDISPLAY will extract the corresponding topographic data from the current site map database for the area that you have defined. If you subsequently store the region, MPDISPLAY stores the data for each topographic feature in a separate data file. Refer to STORE REGION and SELECT TOPOGRAPHY.

The amount of time necessary to read (and subsequently display) the topographic data from the database is directly proportional to the size of the region selected and the number of topographies. After the system reads in the topographic data but before the topographies are displayed, you must specify the name of an edit file. 


\section{Entering the Name of an Edit File}

When MPDISPLAY prompts you for the name of an edit file:

Enter edit file name >

just type a file name and press RETURN.

Any graphic editing during the MPDISPLAY session will affect only this "edit file." (MPDISPLAY's EDIT FUNCTIONS option-page 2.251-enables you to use some of the editing functions of the GEDIT software.)

If you specify the name of an existing file, it must be a Digital Mapping Structure (DMS) file-such as a topographic data file, a previous "edit file," or a file created with the GEDIT software. Specifying a topographic data file from a previously stored region gives you the capability to edit the map data for that one file only. If you specify a topographic data file as the "edit file," do NOT also choose that topographic feature for display. Such a duplication could result in confusing displays that would not represent the available data.

If you specify a new file name, any text or graphics data that you add will be stored in that file, but the file will not be associated with the topographic data for the region.

After you specify an edit file name, MPDISPLAY will draw the map features on the map grid (as well as any graphic elements from the edit file).

The map area that is initially drawn within the map grid constitutes the base plot of the current MPDISPLAY session, the area for which topographic features are read in from the database. This base plot may be redefined later to expand or reduce the area for which topographic data may be input. When the map of the region is complete, MPDISPLAY will present its main menu of functions for manipulating the map. 


\section{Using the MPDISPLAY Main Menu}

After the initial startup tasks, MPDISPLAY presents the following main menu of map display functions:

$\left.\begin{array}{|l|}\hline \text { EXIT (SAVE EDIT FILE) } \\ \text { QUIT (DELETE EDIT FILE) } \\ \text { HELP } \\ \text { CANCEL } \\ \text { CONTINUE } \\ \text { SELECT TOPOGRAPHY } \\ \text { STORE REGION } \\ \text { SCREEN VIEW FUNCTIONS } \\ \text { ITEM DISPLAY FUNCTIONS } \\ \text { SUMMARY FUNCTIONS } \\ \text { ATTRIBUTE FUNCTIONS } \\ \text { ICON FUNCTIONS } \\ \text { ELEVATION FUNCTIONS } \\ \text { EDIT FUNCTIONS }\end{array}\right]$

These general functions are explained together on the following page.

These specific functions are explained in alphabetical order following the general functions.

Each item named FUNCTIONS results in a submenu of related options:

- SCREEN VIEW FUNCTIONS are options for changing the scale of the displayed area (ZOOM options) and for changing the boundaries of displayable topographic data.

- ITEM DISPLAY FUNCTIONS are options for enabling and disabling the display of specific data items (icons, text, attribute points, polygons) on the screen.

- SUMMARY FUNCTIONS are options for displaying numeric data values and statistics (coordinates, populations, attribute values, elevations, differences in elevation, areas, etc.) for data items and areas on the screen.

- ATTRIBUTE FUNCTIONS are options for displaying other "attributen information about map features and for selecting some features for display while eliminating others (based on attributes of the features).

- ICON FUNCTIONS are options 1or controlling which icons (graphic figures associated with point data) are displayed. 


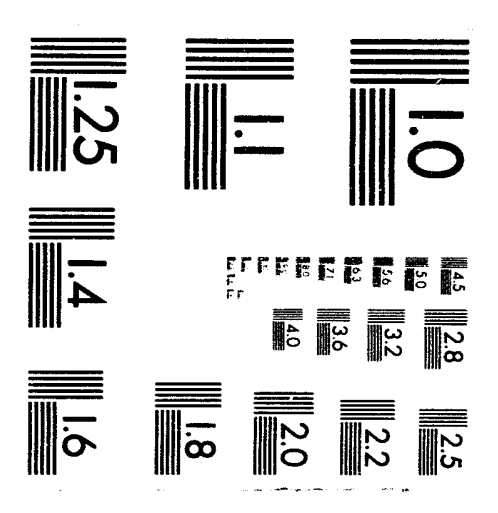



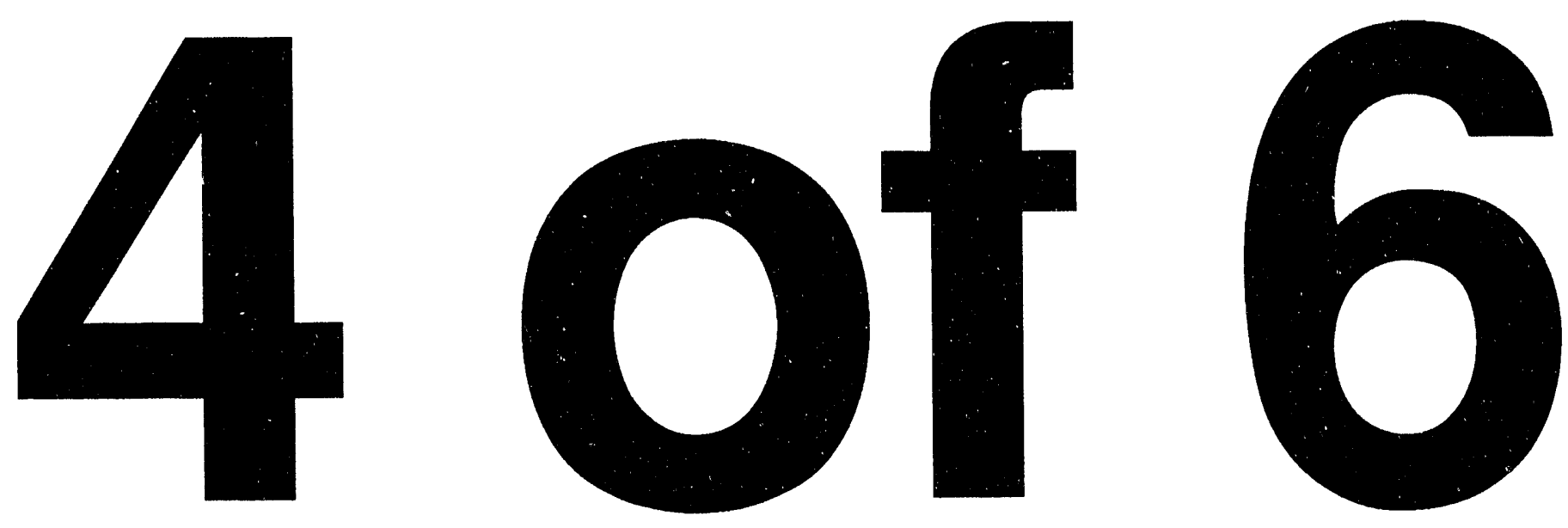
- ELEVATION FUNCTIONS are options for displaying elevation information (elevation, slope, summary statistics, elevation grid resolution, and contours) when elevation data are available for the displayed area.

- EDIT FUNCTIONS presents a submenu of graphic editing functions. These functions are a subset of the functions described for the GEDIT utility.

These "functions" menus and their options are described in alphabetical order after the following descriptions of the general functions on the MPDISPLAY menu.

\section{Exit (Save Edit File)}

Picking EXIT (SAVE EDIT FILE) saves the current edit file and returns control to MPDISPLAY's first menu (page 2.233) for selecting another region for display. On other menus, EXIT signals that you are through with the current menu and the operations on that menu. Operational control then returns to the previous or calling menu.

\section{Quit (Delete Edit File)}

Picking QUIT (DELETE EDIT FILE) cancels the current map display and returns control to MPDISPLAY's first menu (page 2.233) for selecting another region for display. Any graphic editing changes to the edit file are discarded.

Help

Picking HELP displays information about the current menu. When more than one screen of text is available, press RETURN to continue with the next screen.

\section{Cancel}

Picking CANCEL terminates the current function. Use this function when it becomes undesirable to complete a function that has been started. If the cursor disappears during the completion of a function, you will be unable to use CANCEL until the cursor reappears.

\section{Continue}

Picking CONTINUE signals the completion of an operation after you are satisfied that you have accomplished the correct action. For example, in an operation that requires you to choose a point on the screen, you might pick several points before finding the correct one and then selecting CONTINUE. 


\section{Attribute Functions}

You can use attribute functions to display text information about map features and to select some features for display while eliminating others. This is done by using attributes of the map features. Attributes are summarized here and explained in more detail in Appendix B and in the IBS Data Management Guide.

Attributes are numeric values used to describe data items or some characteristic of data items. Attributes consist of three numbers separated by semicolons:

- a Major attribute code (to indicate a major topographic group)

- a Minor attribute code (to indicate a feature within the group)

- a Parameter value (which may give data about the specific feature).

For instance, $1 ; 560 ; 300$ indicates

- a Major code of 1 (hydrography)

- a Minor code of 560 (permanent lake or pond)

- a Parameter value of $\mathbf{3 0 0}$ (length of the lake in decameters).

For each DMS database, a special file called an attribute dictionary defines the meaning of each Major;Minor pair of attribute codes. (The attribute dictionary for a standard IBS DMS database is listed in Appendix B.) Graphic data items may have up to 15 sets of different attributes. You can assign attributes to map data items of a DMS file by using the GEDIT utility (see ATTRIBUTE FUNCTIONS in GEDIT.)

The purpose of the attribute functions is that attributes can be used to control the display of graphic data. For example, because lakes are differentiated as permanent (Minor code $=560$ ) or intermittent (Minor code $=562$ ), you can specify the display of only permanent lakes, only intermittent lakes, both, or neither. ATTRIBUTE FUNCTIONS also includes some utility functions for finding attribute points in the display, displaying the attributes of data items, summarizing attribute point data, and looking up attributes in the attribute dictionary. 
Selecting ATTRIBUTE FUNCTIONS displays the following submenu:
EXIT
HELP
CANCEL
CONTINUE
ATTRIBUTE SELECTION
DISPLAY ATTRIBUTES
DICTIONARY LOOKUP
POINT DATA SUMMARY
ENABLE POINT DATA
DISABLE POINT DATA
REFRESH SCREEN

The principal options of this submenu are explained here.

ATTRIBUTE SELECTION: Allows you to selectively display only certain data items by specifying attribute codes.

After you pick ATTRIBUTE SELECTION on the menu, MPDISPLAY will prompt for a Boolean expression that indicates the criteria for selecting attributes:

ENTER SELECTION CRITERIA >

Your options are 1) press the RETURN key, which disables attribute selection, 2) enter a legal Boolean expression, or 3) press CTRL-Z, which aborts the function and returns control to the main menu.

The Boolean logical expression may be formed from the following list of operators and symbols:

$$
\begin{aligned}
& \text { V } \\
& \text { - logical AND } \\
& \text { - logical OR } \\
& \text { - }- \text { logical NOT } \\
& \text { - } \text { - comge specifier } \\
& \text { - }- \text { left parenthesis } \\
& \text { - right parenthesis }
\end{aligned}
$$


The attribute codes are combined with these symbols to create Boolean logical expressions.

Examples: 4;5:4;100 indicates all data items having an attribute with a Major code of 4 and a Minor code of 5 to 100 (inclusive)

$6 ; 13^{\wedge}(4 ; 5: 4 ; 100) \quad$ indicates all data items having an attribute with a Major code of 6 and a Minor code of 13 AND (as well as) having an attribute with a Major code of 4 and a Minor code of 5 to 100 (inclusive)

$5 ; 8 \mathrm{~V}\left(6 ; 13^{\wedge}(4 ; 5: 4 ; 100)\right) \quad$ indicates any data items having an attribute with EITHER a Major code of 5 and a Minor code of 8 OR the same code specifications as the previous example.

DISPLAY ATTRIBUTES: Displays the attributes associated with a particular data item.

\section{Pick DISPLAY ATTRIBUTES.}

2. Pick a point on or near the desired point or composite string.

The data item will blink, and if it has attributes associated with it, they will be displayed in a table. For example, this table displays information on two attributes:

$\begin{array}{ccccc}* & \text { MAJOR } & \text { MINOR } & \text { PARAM } & \text { DESCRIPTION } \\ 1 & 7 & 150 & 0 & \text { SEaplane ramp Or Landing area } \\ 2 & 8 & 7 & 6 & \text { NO ENTRY IN ATTRIBUTE DICTIONARY }\end{array}$

where \# is the index number and the following three columns list each attribute (the Major code, the Minor code, and the Parameter value) plus a text description of the data item (if a description exists in the attribute dictionary). Notice that the second set of attributes has "NO ENTRY IN ATTRIBUTE DICTIONARY": that is, the attributes 8;7;6 might have some meaning for the person who created the data item, but they are not in the master list of attributes normally used by GEDIT.

3. Repeat step 2 for any other data item.

As you continue to pick data items, old attribute information is retained on the text screen. To clear the screen and make it easier to read, use the dialogue erase key ( $<D$ ERASE $>$ ). 
4. Pick any other submenu item to end the DISPLAY ATTRIBUTES function.

- DICTIONARY LOOKUP: Allows you to look up an attribute in the attribute dictionary. You can reference an attribute by attribute codes or by the description in the attribute dictionary.

Picking DICTIONARY LOOKUP displays the following submenu:

\section{EXIT \\ HELP \\ MANOR/MINOR LOOKUP \\ DESCRIPTION LOOKUP}

The two principal options of this submenu are explained here.

- MANOR/MINOR LOOKUP: Allows you to display the attribute associated with a particular pairing of Major and Minor attribute codes in the attribute dictionary.

You will see the prompt

ENTER MAJOR MINOR ATTRIBUTE CODE >

Type a Major attribute code (up to two digits), a comma, and a Minor attribute code (up to four digits). Then press RETURN.

Example: 7,150

If the Major/Minor code pairing exists in the attribute dictionary, your entry will be displayed along with a parameter value (PARAM) and a description, as in this example:

$$
\begin{array}{crcl}
\text { MAJOR } & \text { MINOR } & \text { PARAM } & \text { DESCRIPTION } \\
7 & 150 & 0 & \text { SEaplane ramp or landing area }
\end{array}
$$

If no entry exists for the Major/Minor code pairing, the description note will read, "NO ENTRY IN ATTRIBUTE DICTIONARY."

Note: To see a list of all attributes that contain a particular Major code, enter a Minor code of zero. For example, the entry 5,0 will list every attribute thit hias a Major code of 5 (Roads and Trails) in the attribute dictionary. Be ready to press CTRL-S to "freeze" the listing as it prints on the screen: such listings are usually more than one screen long and can scroll by quickly. Press CTRL-Q to restart the listing. 
D DESCRIPTION LOOKUP: Allows you to display the attribute associated with a particular character string in the attribute dictionary descriptions.

You will see the prompt

ENTER DESCRIPTION TO LOOKUP >

Type a string of characters that you are seeking in the attribute dictionary descriptions (Example: car); then press RETURN.

You will see a form of your entry listed under DESCRIPTION, along with the corresponding major and minor codes and parameter value (PARAM) as in this example:

$\begin{array}{rrrl}\text { MAJOR } & \text { MINOR } & \text { PARAM } & \text { DESCRIPTION } \\ 1 & 280 & 0 & \text { Outline of a Carolina Bay } \\ 6 & 50 & 0 & \text { Carline } \\ 9 & 210 & 0 & \text { Carrying contour } \\ 9 & 560 & 0 & \text { Evacuee Reception/care points }\end{array}$

Note: Any DESCRIPTION that contains your character string in the dictionary will be displayed.

POINT DATA SUMMARY: Allows you to obtain a summary that tells how many points have a particular set of attribute codes in a specific area of the display.

Initially, you should select a mode for defining the area on which the attribute summary should be performed. The menu items CURRENT SCREEN, DRAW POLYGON, and SELECT POLYGON are the three means of defining an area. Only one of the above options, at most, is active at any given time. Selecting a second area input mode will disable the first. 
Picking POINT DATA SUMMARY displays the following submenu:

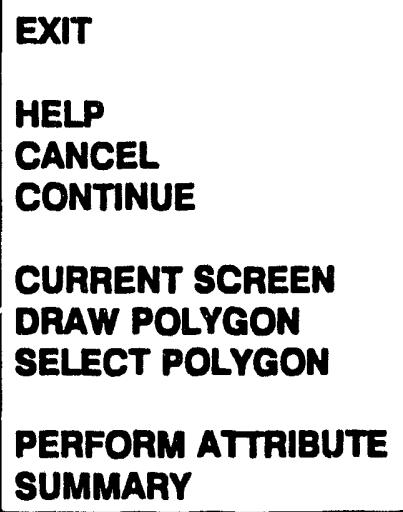

The principal options of this submenu are explained here.

- CONTINUE: Used in conjunction with DRAW POLYGON and SELECT POLYGON. Signals the completion of functions.

- CURRENT SCREEN: Sets the entire display screen as the area to which an attribute summary will apply. Subsequent attribute summaries will apply to this area until you define another area via DRAW POLYGON, or SELECT POLYGON.

D DRAW POLYGON: Allows you to pick points to define a selective area for performing an attribute summary. Subsequent attribute summaries will apply to this area until you define another area via CURRENT SCREEN, DRAW POLYGON, or SELECT POLYGON. When you select one of the above menu options to define a new area, the current polygon will disappear.

\section{Pick DRAW POLYGON.}

The menu item will blink in green to indicate that further input is required.

2. Pick points that define the sides of a polygon.

MPDISPLAY will draw line segments from point to point. 
3. Pick CONTINUE to complete the polygon.

The polygon will blink in green to indicate that a polygon has been properly selected. The menu option will stop blinking to indicate that no further input is required in selecting the polygon.

MPDISPLAY will draw the firal side between the first point and the last, closing the polygon area.

- SELECT POLYGON: Allows you to pick a predefined polygon on which to perform the attribute summary. Subsequent attribute summaries will apply to this area until you define another area via CURRENT SCREEN, DRAW POLYGON, or SELECT POLYGON. When you select one of the above menu options to define a new area, the current polygon will reappear in its original color.

\section{Pick SELECT POLYGON.}

The menu item will blink in green to indicate that further input is required.

2. Pick any point on a preexisting polygon.

The polygon will blink in white to indicate a pick. If the wrong polygon is highlighted, pick again on the desired polygon until it is properly selected.

3. Pick CONTINUE to confirm the polygon selection.

The polygon will blink in green to indicate that a polygon has been properly selected. The menu option will stop blinking to indicate that no further input is required in selecting the polygon.

D PERFORM ATTRIBUTE SUMMARY: Allows you to enter major and minor attribute codes to select attributes points to be summarized.

If used without selecting an "interactive area," this function can be used to search the entire screen.

You will see the prompt

ENTER MAJOR MINOR CODE >

Enter the major and minor code of an attribute point and press

RETURN. Example: 1, 2 
You will see summary table headings and the appropriate data, such as

$\begin{array}{cccccccc}\text { MAJOR } & \text { MINOR } & \text { COUNT } & \text { TOTAL } & \text { MINIMUM } & \text { MAXIMUM } & \text { AVG } \\ 1 & 2 & 13 & 906 & 3 & 333 & 69\end{array}$

ENABLE POINT DATA: Places an asterisk ${ }^{n * n}$ at the location of every point with attributes in each input file.

These points denote item points, icon seed points, text points, and color flood seed points. For example, if you are editing a single file that contains text, this feature will show how many text items there are and where the starting point of each item is located without taking the (possibly long) time to plot the text. ENABLE POINT DATA is particularly useful for locating text when the decluttering factor does not allow the text to be plotted on the screen.

DISABLE POINT DATA: Disables the display of points with attributes, allowing future plots to be created without showing the locations of attribute/point data.

DISABLE POINT DATA allows you to remove the attribute/point markers from the screen when used in conjunction with REFRESH SCREEN: Pick DISABLE POINT; then pick REFRESH SCREEN. The system will redraw the screen without the attribute/point markers.

- REFRESH SCREEN: Redraws the existing display image using the current ENABLE POINT and DISABLE POINT settings. 


\section{Edit Functions}

Picking the EDIT FUNCTIONS menu item will cause MPDISPLAY to present a submenu of functions for graphic editing. These functions (listed below) are a subset of the functions in the GEDIT utility.

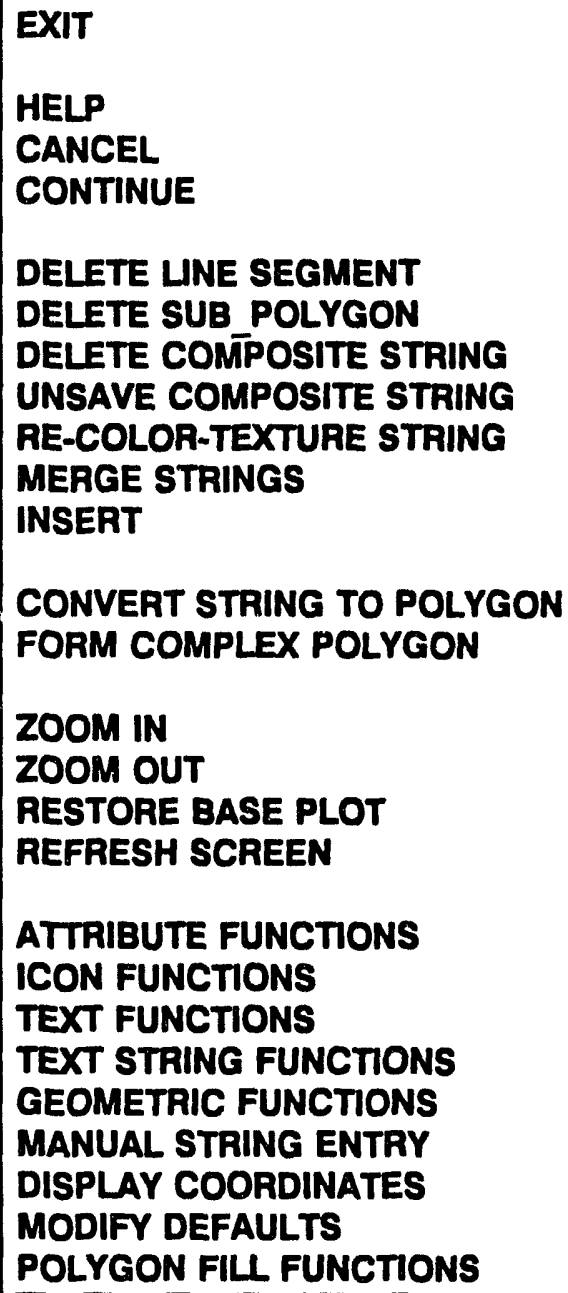

The selection of some options on this menu will result in the display of submenus from which further selections must be made. Please refer to the GEDIT utility for full descriptions of these functions.

These graphic editing functions may be used to modify the edit file, which you must specify at the beginning of a MPDISPLAY session (see Specifying the Name of an Edit File, page 2.240). This edit file is separate from the topographic data files that make up the rest of the displayed map, and any graphic editing during a MPDISPLAY seision affects only the edit file. 


\section{Elevation Functions}

This option is active only if the current geogaphic database is set up with an elevation topography and only if you have chosen to display the elevation topography (by selerting it when MPDISPLAY starts or by using the SELECT TOPOGRAPHY option). Otherwise you will see a message-NO ELEVATION TOPOGRAPHY EXISTS IN CURRENT DATABASE-and picking the option will have no effect. If an elevation topography is available, then picking the ELEVATION FUNCTIONS menu item displays the following submenu:

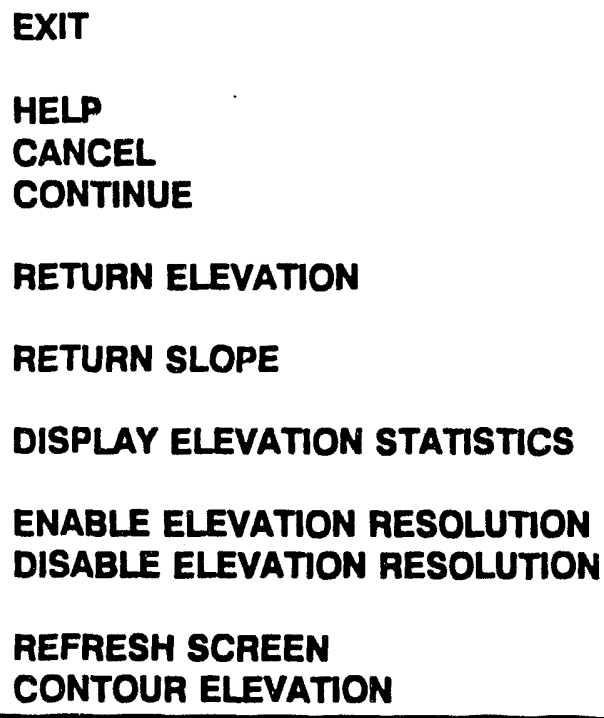

The principal options of this menu are explained here.

CONTINUE: Used in conjunction with RETURN ELEVATION and RETURN SLOPE.

RETURN ELEVATION: Displays the elevation at a selected point.

1. Pick Return Elevation.

2. Pick a point on the screen.

A yellow asterisk (*) will mark the selected location. Depending on the screen density of the elevation data, a box representing the borders of the elevation data cell may also appear.

The elevation of the selected point (center point of the cell) will be displayed. 
- RETURN SLOPE: Displays the absolute difference between the elevation values at two selected points.

1. Pick RETURn SLOPE.

2. Pick a point on the screen.

A yellow asterisk (*) will mark the selected location. Depending on the screen density of the elevation data, a box representing the borders of the elevation data cell may also appear.

3. Pick CONTINUE.

4. Pick a second point on the screen.

A second yellow asterisk will appear.

5. Pick CONTINUE again.

A line connecting the two points will be drawn. The absolute difference between the elevation values will be displayed on the terminal screen.

DISPLAY ELEVATION STATISTICS: Displays information about the elevation data with respect to 1) the base map and 2) the current view (screen display).

EXAMPLE: When the Base Map and the Current View are the Same

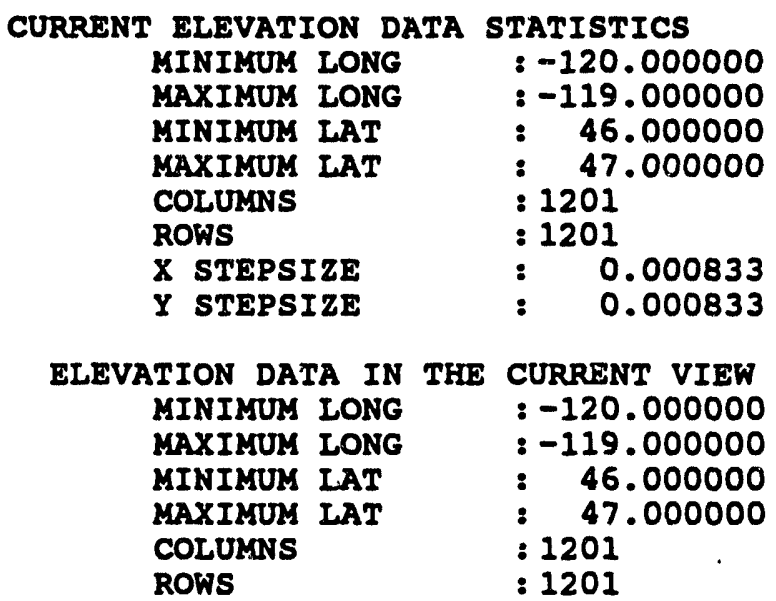


ENABLE ELEVATION RESOLUTION: Shows the resolution of the elevation data by drawing white grid lines around the elevation data cells.

To show the elevation data grid, just pick ENABLE ELEVATION RESOLUTION. If the current display screen requires more than 50 rows or columns of cells in the elevation data grid, you will see a warning message and a count of the number of rows and columns:

WARNING: Elevation data in current window is extremely dense.

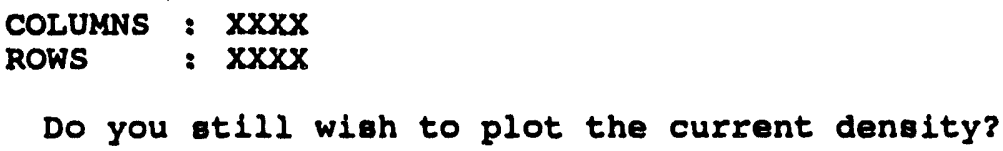

Because displaying a dense elevation data grid can effectively obliterate other displayed data, you should consider answering "No" and zooming in to a smaller area before displaying the elevation grid.

DISABLE ELEVATION RESOLUTION: Turns off the capability to display a line grid of elevation data cells.

This option does not erase currently marked elevation cells, but the markers will disappear the next time the screen is redrawn.

REFRESH SCREEN: Redraws the existing display image.

CONTOUR ELEVATION: Produces a display of vector contours that represent isopleths of the elevation data. You will see a display and prompt similar to the following:

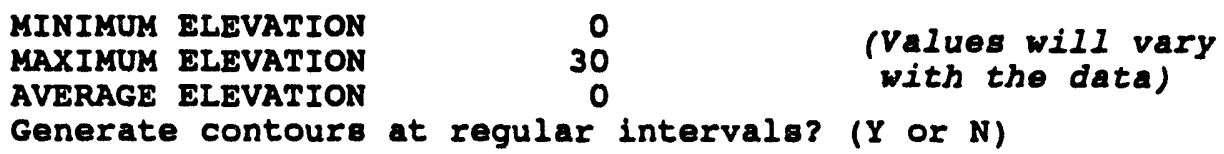

Note: If the map display is zoomed out and showing a large area (for example, if DISPLAY ELEVATION STATISTICS would report more than 100 or so rows and columns of elevation cells), then MPDISPLAY may take several minutes to check the elevation data before prompting you for information.

1. Enter $\mathbf{Y}$ (for regular intervals) or $\mathbf{N}$ (to specify intervals).

If you answer $\mathbf{N}$, the program will ask you to enter "contour levels" (elevations) one at a time. The program will keep prompting you until you press CTRL-Z to exit:

Enter contour level 1 <CTRL 2 to exits 
If you answer $\mathbf{Y}$ you will see the following prompt:

Enter start elevation, end elevation and spacing

2. Enter the contour values as indicated.

After you have specified the contour levels (either individually or by spacing) MPDISPLAY prompts you to choose the color(s) of each or all contours.

3. Enter your choice of contour color(s).

The following example shows how you can choose individual colors for each contour.

4. Enter the name of the DMS file that will contain the new contours.

The following example results in the creation of a DMS file, 400CONTOURS.DMS, which contains multicolored contour levels spaced 400 (feet) apart. User responses in the example are boldface and aligned to make them easier to distinguish.

Example of Using CONTOUR ELEVATION

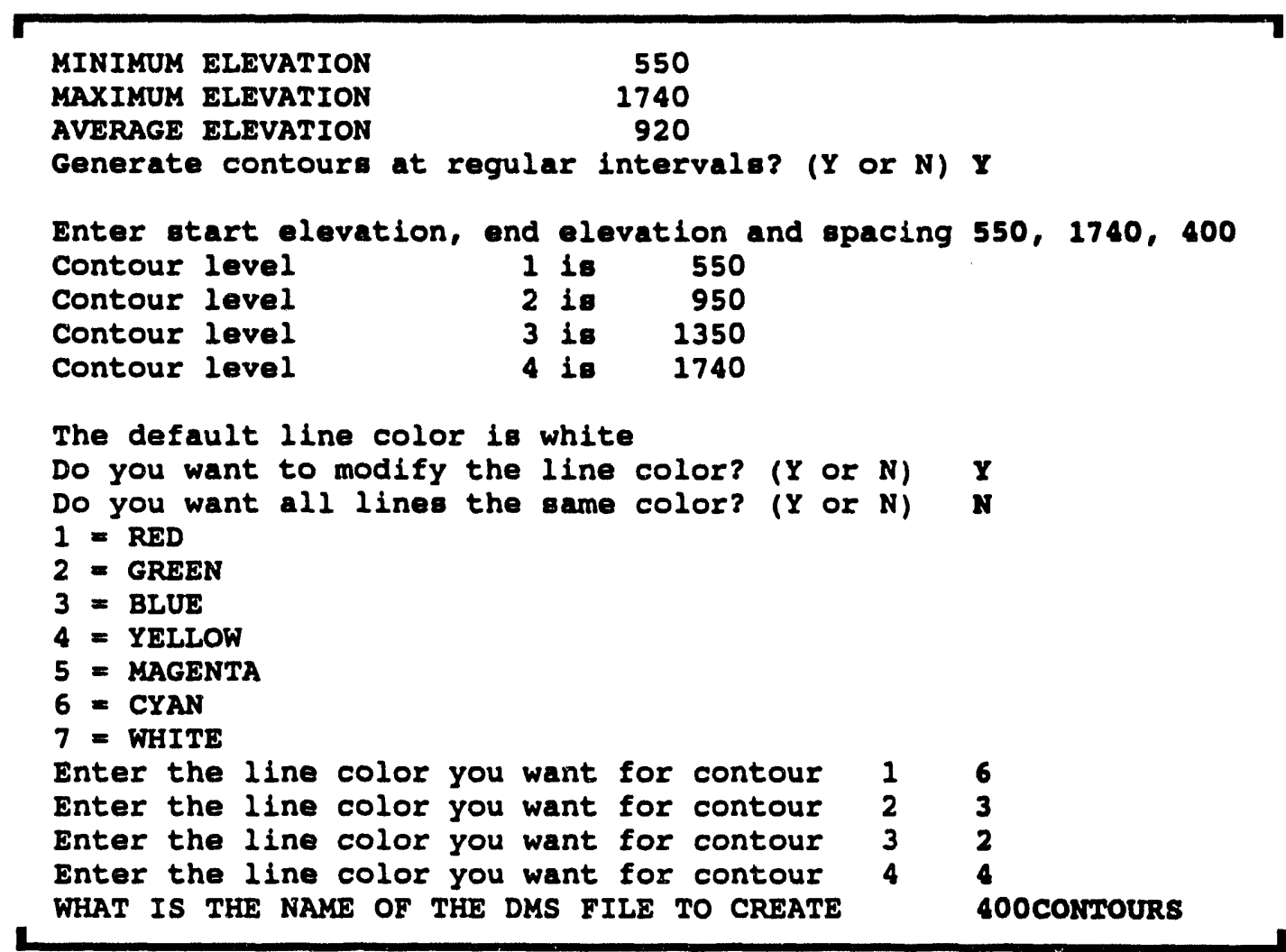




\section{Icon Functions}

An icon is a graphical figure that can be associated with a particular data point (location). Icons always appear at a constant viewing size, regardless of the map scale (which can be changed with the zoom functions). Figure 2.1 shows some examples of icons used by the IBS. The location of icons is governed by icon seed points, which indicate the type of icon to be drawn and where to center the icon.

The icons available for display are listed in the icon control file. The icon control file also contains the locations of the DMS files that are needed to plot each icon. A system default icon control file is used if you do not specify one (see Using an Icon Control File under the GEDIT utility). For examples of most of the standard icons supplied with the IBS, see the IBS User Guide.

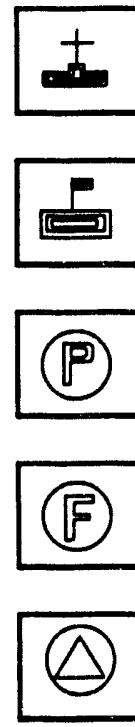

Church

School

Police Station

Fire Station

Emergency Operations Center (red)

Figure 2.1. Examples of Icons

Selecting ICON FUNCTIONS displays the following submenu:
EXIT
ICON DISPLAY LST
ENABLE ICON PLOTTING
DISABLE ICON PLOTTING
REFRESH SCREEN 
The principal menu options are explained below.

- ICON DISPLAY UST: Used to specify which of the available icons may be displayed. MPDISPLAY will present a submenu that lists the available icons:

\begin{tabular}{|l|}
\hline EXIT \\
FORWARD \\
BACKWARD \\
GO TO \\
Icon descriptor \#1 \\
Icon descriptor \#2 \\
icon descriptor \#n \\
\hline
\end{tabular}

For example, the default icon control file results in the following icon display list:

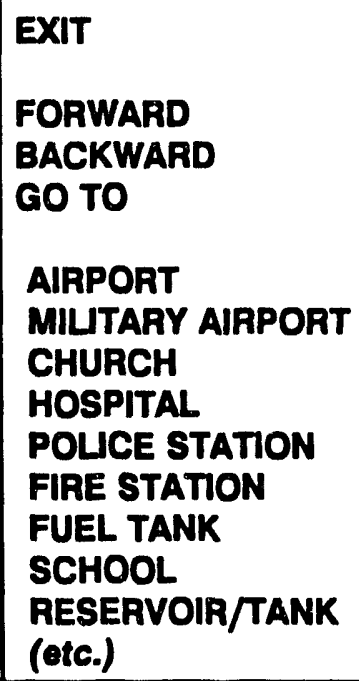

All available icons are in a paged menu with 16 icons per page. To scroll through the icon list, select the menu item (FORWARD, BACKWARD, GO TO) that describes which way you want to go:

FORWARD: Shows the next page of 16 icons. If you reach the end of the list, the system will return you to the first page of the icon listing.

D BACKWARD: Shows the previous page of 16 icons. If you reach the first page of the list, the system will return you to the last page of the icon listing. 
- GO TO: After selection of GO TO, the system will prompt you for the page number desired (ENTER PAGE \# OF ICONS DESIRED $>$ ). The system then shows you the icon page requested. If the page number requested is less than 1 , the system will display the first page of icons. If the page number requested is greater than the number of icon pages available, the system will send you to the last page of icons.

ㅁ icons: Select icon categories or types to be displayed by picking the appropriate descriptor in the menu. As a descriptor is selected, its color will toggle between the standard menu color (normally orange) and a secondary color (normally green) to indicate that the icon has been selected. By default all icons are set to be displayed.

To cancel a selection, simply pick that item again, and its color will toggle back to the standard color. The selected icons are eligible for display and will be plotted if ENABLE ICON PLOT has been picked and seed points exist for the selected area.

If you modify the icon display list, then the next time you use the function, the icon list will start at the page of the last icon selected for display.

ENABLE ICON PLOTTING: Used (along with DISABLE ICON PLOT) to specify whether the icons selected on the icon display list are actually displayed. When you pick ENABLE ICON PLOT, MPDISPLAY checks to see if any of the selected icon types occur in the display area and draws them at seed points on the display.

DISABLE ICON PLOTTING: Switches off the capability to display icons (without erasing any icons that are currently on the screen). Use the REFRESH SCREEN option to subsequently erase disabled icons.

REFRESH SCREEN: Redraws the current display. The REFRESH SCREEN function is included in the ICON FUNCTIONS menu for convenience in using the icon functions. Use the REFRESH SCREEN option to erase icons after disabling icons or after changing the Icon Display List. 


\section{Item Display Functions}

Picking ITEM DISPLAY FUNCTIONS displays the following submenu for displaying data items (icons, text, attribute points, polygons) on the screen.

EXIT
HELP
ENABLE ICONS
DISABLE ICONS
ENABLE TEXT
DISABLE TEXT
ENABLE POINT
DISABLE POINT
ENABLE POLYGON FILL
DISABLE POLYGON FILL
GRID OPTIONS
DECLUTTER ON/OFF
REFRESH SCREEN

The principal options of this menu are explained below.

ENABLE ICONS: Used (along with DISABLE ICON PLOT) to specify whether the icons selected on the icon display list are actually displayed. When you pick ENABLE ICON PLOT, MPDISPLAY checks to see if any of the selected icon types occur in the display area and draws them at seed points on the display.

- DISABLE ICONS: Switches off the capability to display icons (without erasing any icons that are currently on the screen). Use the REFRESH SCREEN option from the main menu to subsequently erase disabled icons.

- ENABLE TEXT: Displays any text associated with the DMS file topographic information displayed on the screen. (That is, the text must have already been created by the GEDIT software for the current region.) By default, text display is disabled upon entering MPDISPLAY.

Text will not be displayed if the currently displayed area is considerably larger than the scale at which the text was originally entered. If expected text is not displayed when you pick ENABLE TEXT DISPLAY, use a zoom function from the main menu to magnify the display to a scale that is close to that used 
when the text was entered. The text should then appear on the display.)

DISABLE TEXT: Switches off the text display capability. Any text on the screen will remain there until the screen is redrawn (for example, by picking REFRESH SCREEN).

- ENABLE POINT: Places an asterisk * at the location of every point with attributes in each input file.

These points denote item points, icon seed points, text points, and color flood seed points. For example, if you are displaying a single file that contains text, this feature will show how many text items there are and where the starting point of each item is located without taking the (possibly long) time to plot the text. ENABLE POINT is particularly useful for locating text when the decluttering factor does not allow the text to be plotted on the screen.

D DISABLE POINT: Disables the display of points with attributes, allowing future plots to be created without showing the locations of attribute/ point data.

DISABLE POINT allows you to remove the attribute/point markers from the screen when used in conjunction with REFRESH SCREEN: Pick DISABLE POINT; then pick REFRESH SCREEN. The system rill redraw the screen without the attribute/point data.

ENABLE POLYGON FIL: Draws and fills all polygon(s) that have an associated fill pattern or color.

This is a software function. A polygon to be filled must have already been associated with the fill color or pattern. This is usually accomplished through appropriate functions in the GEDIT utility.

DISABLE POLYGON FILL: Disables the automatic filling of polygons, negates the ENABLE POLYGON FIL command. To erase prior polygon fills, pick DISABLE POLYGON FIL; then pick REFRESH SCREEN.

GRID OPTIONS: Plots longitude-latitude lines on the map display area.

DECLUTTER ON/OFF: The DECLUTTER ON/OFF function can be used to declutter (reduce the detail and number of) features displayed on the map grid. When you pick the DECLUTTER ON/OFF, MPDISPLAY will prompt you for a decluttering factor $(0-30)$ that will be used in determining whether a feature will be displayed or removed from the screen:

ENTER DECLUTTERING LEVEL (CTRL Z TO TOGGLE) >

To enter a decluttering level, type a number from 0 to 30 (inclusive) and press RETURN. A higher number represents more decluttering. MPDISPLAY 
will indicate that decluttering has been turned on. When you set the decluttering level in this fashion, the decluttering level remains constant, even if you use ZOOM functions to change the display scale.

If you press CTRL-Z instead of entering a decluttering factor, MPDISPLAY will calculate a decluttering factor based on the size of the current plot. If you use any of the ZOOM functions, MPDISPLAY will recalculate the decluttering factor according to the display scale.

Subsequently selecting DECLUTTER ON/OFF and pressing CTRL-Z will turn off the decluttering function.

- Refresh SCREen: Picking Refresh SCREEN redraws the current display. 


\section{Screen View Functions}

Picking SCREEN VIEW FUNCTIONS results in a submenu for changing the size of the display and the area for which topographic data are available.

\begin{tabular}{|l|}
\hline EXIT \\
HELP \\
ZOOM IN \\
ZOOM OUT \\
MAP ZOOM \\
RESTORE BASE PLOT \\
RESET BASE AREA \\
REFRESH SCREEN \\
\hline
\end{tabular}

The principal options of this menu are explained below.

ZOOM IN: Pick ZOOM iN to enlarge a square portion of the displayed area.

1. Pick ZOOM IN.

2. Pick a point on the map at the center of the area that you want to enlarge.

3. Pick a second point that would lie on one side of the square area that you want to enlarge with the first point at the center.

After you define the area, MPDISPLAY will redraw the map, enlarging the defined area to fill the map grid.

If you are not satisfied with the area that you have enlarged, you can pick RESTORE BASE PLOT to redraw the area as it was first displayed (see RESTORE BASE PLOT).

ZOOM OUT: Pick ZOOM OUT to reduce the displayed area to a square within a larger area. Define the area to be reduced (scaled down) in the same manner as described for the ZOOM IN command:

1. Pick ZOOM OUT.

2. Pick a point on the map at the center of the area that you want to reduce (scale down). 
3. Pick a point on the map that would lie on the edge of a square with the first point at the center.

The currently displayed map area will be rescaled to the size of the square, and the new display will be centered at the center point of the square.

It is possible to zoom out too far, scaling the map down to be surrounded by a "blank" area within the map grid box. You can always use RESTORE BASE PLOT command to redraw the map at its original size.

MAP ZOOM: Pick MAP ZOOM to center the displayed area at a longitudelatitude (long-lat) that you provide. You can use the COORDINATE PICK function to find the longitude-latitude of a point on the display. Note that ongitudes in the western hemisphere are expressed as negative numbers.

\section{Pick MAP ZOOM.}

MPDISPLAY will query for the radius (in miles) and the center point (long-lat) of the area to be displayed. (If you have used the COORDINATE PICK function previously, the longitude-latitude of the last point picked may be on the text screen, if other functions have not used the screen for other purposes.)

2. Type the radius (in miles), the longitude, and then the latitude, separated by commas (or spaces).

(Pressing CTRL-Z will exit and abort the MAP ZOOM function.)

\section{Press RETURN.}

MPDISPLAY will redraw the displayed area with the specified point at the center.

RESTORE BASE PLOT: Initially the base plot is the area displayed when the main menu is first presented. The base area may be redefined later (see the next menu item, RESET BASE AREA). The concept of a base plot is extremely important because selection of topographic data is a function of the base plot area (see SELECT TOPOGRAPHY, page 2.265).

Picking RESTORE BASE PLOT causes the base plot to be redrawn in the display area of the screen. This function might be used after zooming, for instance, to restore the display to its original scale.

RESET BASE AREA: Picking RESET BASE AREA sets the base plot to the area currently being displayed. It enables you to restrict the amount of data being retrieved by the SELECT TOPOGRAPHY command. 
- Refresh SCREeN: Picking REFresh SCREEN redraws the current display.

This command can be useful for removing display elements that you no longer wish to display, such as text. 


\section{Displaying the Lst of Topographies}

All available topographies are listed in a paged menu with 19 topographies per page. To scroll through the topography list, select the menu item (FORWARD, BACKWARD, GO TO) that describes which way you want to go:

FORWARD: Shows the next page of topographies. If you reach the end of the list, the next FORWARD will return to the first page.

- BACKWARD: Shows the previous page of topographies. If you reach the first page of the list, the next BACKWARD will return to the last page.

- GO TO: Allows you to go directly to a specific page. After selection of GO TO, the system prompts, "ENTER PAGE \# OF topos DESIRED >". After your entry, the system shows the requested page of topographies. If the page number requested is less than 1 , the system displays the first page of topographies. If the page number requested is greater than the number of pages available, the system sends you to the last page.

If you select a topography, then the next time you use the function, the list will start at the page of the last topography selected.

\section{Picking Topographies from the Menu}

Use the graphics input device to select topographic features for display by picking the appropriate descriptor in the menu:

1. Pick each topographic feature that you want to display.

Each menu item picked will change color to indicate selection. If you change your mind about an item that you picked, pick it again. The menu item will return to the standard menu color, indicating no selection.

Any combination of topographies may be selected for simultaneous display.

2. When you have chosen all desired topographies, pick the top menu item, EXIT.

The cursor will disappear momentarily, and the system will display a message:

READING DATA. PLEASE WAIT.

This indicates that MPDISPLAY is reading in the data files for the selected topographic features. No topographic data is read in until you pick EXIT. After a period, MPDISPLAY will draw (or redraw) the map features in the map display area. The amount of data read is related to the number of topographies selected and to the area specified as the current base plot (see RESET BASE AREA under SCREEN VIEW FUNCTIONS). 
The amount of time necessary to read the topographic data from the database increases with the size of the region and the number of topographies selected.

If no topographies are selected when EXIT is picked, MPDISPLAY will erase the plot area and redisplay the main menu. This is a handy means of exiting the SELECT TOPOGRAPHY function without reading from the database.

\section{Topographic Codes}

Each topography is associated with a two-letter topographic code that is used in the topography subdirectory and data file names. For example, RT is the code for ROADS \& TRAILS. Generally you will have no reason to use these codes unless you individually access specific topography data files. (For a list of topographic codes, see Appendix A.) 


\section{Store Region}

Use the STORE REGION command to store areas of the map grid as a set of DMS data files for later use. STORE REGION is useful for combining DMS files and elevation data files (TAC files) across file boundaries. The resulting files of topographic data are stored in your current directory. STORE REGION does NOT store any graphics editing that you may have done in the "edit file." (For information on the edit file, see Specifying the Name of an Edit File, page 2.240.)

You may store the entire region displayed in the map grid or define a smaller area within the region (as shown in the following procedures).

To store the entire region in the current display:

1. Use the graphics cursor to pick STORE REGION.

2. Then pick CONTINUE.

3. Continue at step 4 of the following procedure.

To store a portion of the region within the current display:

\section{Pick STORE REGION.}

2. Use the graphics cursor to pick points on the map display that define a polygon that surrounds the desired region.

MPDISPLAY will draw lines between the points as they are entered, indicating the boundary of the desired region.

3. After picking the last point, pick CONTINUE to draw a line between the first and the last point, completing the polygon.

After you have specified the region to be stored (by either method), MPDISPLAY will present a STORE REGION submenu:

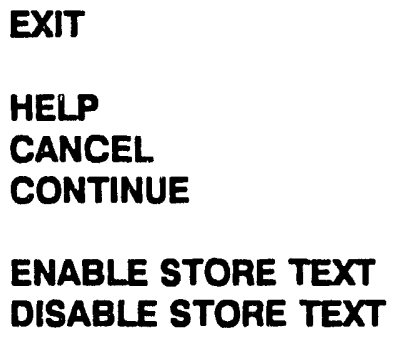


4. Choose whether to store text data associated with the specified region. (This step is optional):

- ENABle STORE TEXT: Chooses to store all text for all topographies in the region (regardless of whether the text is currently displayed).

D DISABLE STORE TEXT: Chooses to NOT store any text for the stored region (regardless of whether the text is currently displayed).

If you choose neither of these options, only text that is currently displayed will be saved with the stored region.

5. Pick EXIT on the STORE REGION submenu.

MPDISPLAY will then ask for an "output file designation" (name for the stored region) of up to seven characters:

$$
\text { ENTER OUTPUT FILE DESIGNATION ( } 7 \text { CHARACTERS) > }
$$

6. Type a name for the stored region and press RETURN.

MPDISPLAY will then query to determine if a direct access (polygon description) file is to be created:

DO YOU WANT A DIRECT AREA ACCESS FILE >

7. Enter $\mathbf{Y}$ for "yes" or $\mathbf{N}$ for "no."

If you answer yes, a DMS file will be created to contain a bounding polygon for selected region. No other data will be stored. This polygon description file is called a "direct access file" because you may use the file along with the DIRECT AREA ACCESS function to retrieve data for the bounded region without selecting from a database area (page 2.233).

If you answer no, MPDISPLAY will create a full set of data files describing the selected region, including the polygon description file. The contents and naming conventions for these data files are described after step 8 . If you have also selected ELEVATION data to be stored (and elevation data exists), then MPDISPLAY gives you the opportunity to modify the resolution of the elevat on data that will be saved:

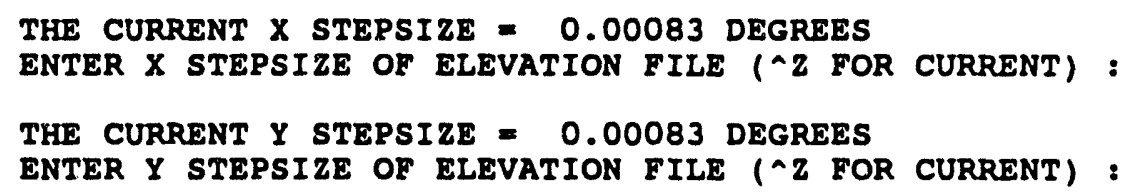

$\mathrm{X}$ refers to "horizontal" east-west increments; $\mathrm{Y}$ refers to "vertical" north-south increments. The resolution shown may vary. 
8. Enter modified step sizes, or just press CTRL-Z at each prompt to retain the resolution of the current elevation data.

\section{Results of using STORE REGION}

MPDISPLAY then takes a moment to save the data files. If you had entered an output file designation of "MYDATA," then when you exit, you would find the following files in your current directory:

- MYDATA.DMS

- MYDATAXx.DMS a polygon description file for the site area

one file for each selected vector topography, where "xx" is the two-character topographic code

If you included elevation data, these files would also be produced:
- MYDATAEL.TAC
the information "header file" for the TAC data set
- MYDATAEL.TAA
the cell data file for the TAC data set.

\section{Names of Map Display Data Files}

The "output file designation" (region specification) will be the major portion of the filenames for the files created by STORE REGION. For each topography in the current display, MPDISPLAY creates a file with the ".DMS" filename extension in the current default directory. A two-character topographic code is appended to the end of the region specification to identify each topography file.

For instance, suppose that streams and railroads are among the features in the current display. If the topographic code for streams is ST and the code for railroads is RR, then supplying "MYPLOT" as the region specification would result in the creation of the files MYPLOTST.DMS and MYPLOTRR.DMS. The codes for the standard IBS topographies are listed in Appendix A. (This list may vary if topographies are added or deleted by the database manager.)

The STORE REGION command in MPDISPLAY always creates a file that describes the bounding polygon for the clipped region. This polygon description file is provided for use with the DIRECT AREA ACCESS option (page 2.233) and the PASTE REGION command in the GEDIT software. The filename is derived by appending ".DMS" to the region specification-MYPLOT.DMS in the example above. 


\section{Summary Functions}

Picking SUMMARY FUNCTIONS displays the following submenu for displaying data values and statistics (coordinates, populations, attribute values, elevations, differences in elevation, areas, etc.) for items and areas on the screen.

EXIT
HELP
CANCEL
CONTINUE
RETURN COORDINATE
RETURN POPULATION
RETURN ATTRIBUTES
RETURN ELEVATION
RETURN SLOPE
DETERMINE AREA
POINT DATA SUMMARY
REFRESH SCREEN

The principal options of this menu are explained here.

RETURN COORDINATE: Picking RETURN COORDINATE and then a point on the map displays the

- longitude-latitude of the point

- the Great Circle distance from the point to the center of the display (shortest arc over the surface of the earth)

- the cumulative distance from the first point picked.

Picking subsequent points will display this information until you terminate the function by picking any menu item. 
RETURN POPULATION: RETURN POPULATION will display the population for an area that you define. Define the area of interest by specifying a polygon:

Initially, you should select a mode for defining the area on which the population gathering should be performed. The menu items DRAW POLYGON, and SELECT POLYGON are the two methods for defining the area. Only one of the above options, at most, is active at any given time. Selecting the alternative area input mode will disable the first.

Selecting the RETURN POPULATION option will bring up the following submenu:

\section{EXIT \\ HELP \\ CANCEL \\ CONTINUE \\ DRAW POLYGON \\ SELECT POLYGON}

The principal options of this submenu are explained here.

COMTINUE: Used in conjunction with DRAW POLYGON and SELECT

POLYGON. Signals the completion of functions.

DRAW POLYGON: Allows you to pick points to define a polygon on which to perform population gathering. When you select DRAW POLYGON or SELECT POLYGON to define a new area, the current polygon will disappear.

\section{Pick DRAW POLYGON.}

The menu item will blink in green to indicate that further input is required.

2. Pick points that define the sides of a polygon.

3. Pick CONTINUE to complete the polygon.

The polygon will blink in green to indicate that a polygon has been properly selected. The menu option will stop blinking to indicate that no further input is required in selecting the polygon. The polygon will be redrawn in white. The population of that bounded region will appear on the text screen. 
- SELECT POLYGON: Allows you to pick a predefined polygon on which to perform population gathering. When you select DAAW POLYGON or SELECT POLYGON to define a new area, the current polygon will reappear in its original color.

\section{Pick SELECT POLYGON.}

The menu item will blink in green to indicate that further input is required.

2. Pick any point on a preexisting polygon.

The polygon will blink in white to indicate pick. If the wrong polygon is highlighted, pick again on the desired polygon until it is properly selected.

3. Pick CONTINUE to confirm the polygon selection.

The polygon will be redrawn in its original color. The menu option will stop blinking to indicate that no further input is required in selecting the polygon. The population of that bounded region will appear on the text screen.

RETURN ATTRIBUTES: Displays the attributes associated with a particular data item.

\section{Pick Return Attributes.}

2. Pick a point on or near the desired point or composite string.

The data item will blink, and if it has attributes associated with it, they will be displayed in a table. For example, this table displays information on two attributes:

$\begin{array}{ccccc}* & \text { MAJOR } & \text { MINOR } & \text { PARAM } & \text { DESCRIPTION } \\ 1 & 7 & 150 & 0 & \text { Seaplane ramp or Landing area } \\ 2 & 8 & 7 & 6 & \text { NO ENTRY IN ATTRIBUTE DICTIONARY }\end{array}$

where \# is the index number and the following four columns list each attribute (the Major code, the Minor code, and the Parameter value) plus a text description of the data item (if a description exists in the attribute dictionary). Notice that the second set of attributes has "NO ENTRY IN ATTRIBUTE DICTIONARY": that is, the attributes 8;7;6 might have some meaning for the person who created the data item, but they are not in the master list of attributes normally used by the IBS. 
3. Repeat step 2 for any other data item.

As you continue to pick data items, old attribute information is retained on the text screen. To clear the screen and make it easier to read, press SHIFT and the dialogue erase key (<D ERASE >).

4. Pick any other submenu item to end the RETURN ATTRIBUTES function.

RETURN ELEVATION: Displays the elevation at a selected point. This option is active only if the current geographic database is set up with an elevation topography. Otherwise you will see a message-NO ELEVATION TOPOGRAPHY EXISTS IN CURRENT DATABASE-and the option will have no effect.

1. Pick Return Elevation.

2. Pick a point on the screen.

A yellow asterisk (*) will mark the selected location. Depending on the screen density of the elevation data, a box representing the borders of the elevation data cell may also appear.

3. Pick CONTINUE.

The elevation of the selected point (center point of the cell) will be displayed.

RETURN SLOPE: Displays the absolute difference between the elevation values at two selected points (if an elevation topography is used in the current geographic database).

\section{Pick RETURN SLOPE.}

2. Pick a point on the screen.

A yellow asterisk (*) will mark the selected location. Depending on the screen density of the elevation data, a box representing the borders of the elevation data cell may also appear.

3. Pick CONTINUE.

4. Pick a second point on the screen.

A second yellow asterisk will appear.

5. Pick CONTINUE again. 
A line connecting the two points will be drawn. The absolute difference between the elevation values will be displayed on the terminal screen.

DETERMINe AREA: Picking Determine AREA results in a submenu for determining the area of a region that you define graphically on the map. You will draw a polygon around the desired region.

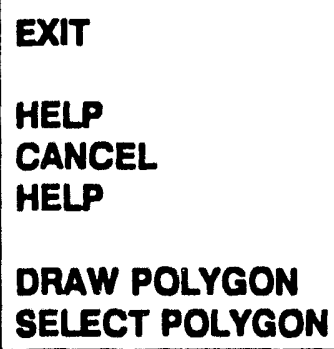

Initially, you should select a mode for defining the area on which the calculation should be performed. The menu items DRAW POLYGON, and SELECT POLYGON are the two methods for defining the area. Only one of the above options, at most, is active at any given time. Selecting the alternative area input mode will disable the first. The principal options of the submenu are explained here.

D CONTINUE: Used in conjunction with DRAW POLYGON and SELECT POLYGON. Signals the completion of functions.

D DRAW POLYGON: Allows you to pick points to define a polygon on which to perform area and circumference calculations. When you select this option to define a new area the current polygon will disappear.

1. Pick DRAW POLYGON.

The menu item will blink in green to indicate that further input is required.

2. Pick points that define the sides of a polygon.

3. Pick CONTINUE to complete the polygon.

The polygon will be redrawn in white. The menu option will stop blinking to indicate that no further input is required in selecting the polygon. The area (square miles) and circumference of the bounded region will appear on the text screen.

SELECT POLYGON: Allows you to pick a predefined polygon on which to perform area and circumference calculations. When you select this option to define a new area the current polygon will reappear in its 
original color.

1. Pick SELECT POLYGON.

The menu item will blink in green to indicate that further input is required.

2. Pick any point on a preexisting polygon.

The polygon will blink in white to indicate a pick. If the wrong polygon is highlighted pick again on the desired polygon until it is properly selected.

3. Pick CONTINUE to confirm the polygon selection.

The polygon will be redrawn in its original color. The menu option will stop blinking to indicate that no further input is required in selecting the polygon. The area (square miles) and circumference of the bounded region will appear on the text screen.

POINT DATA SUMMARY: Allows you to obtain a summary that tells how many points have a particular set of attribute codes in a specific area of the display.

Initially, you should select a mode for defining the area on which the attribute summary should be performed. The menu items CURRENT SCREEN, DRAW POLYGON, and SELECT POLYGON are the three means of defining an area. Only one of the above options, at most, is active at any given time. Selecting a second area input mode will disable the first.

Picking POINT DATA SUMMARY displays the following submenu:

\section{EXIT \\ HELP \\ CANCEL \\ CONTINUE \\ CURRENT SCREEN \\ DRAW POLYGON \\ SELECT POLYGON \\ PERFORM ATTRIBUTE SUMMARY}

The principal options of this submenu are explained here.

CONTINUE: Used in conjunction with DRAW POLYGON and SELECT

POLYGON. Signals the completion of functions. 
- CURRENT SCREEN: Sets the entire display screen as the area to which an attribute summary will apply. Subsequent attribute summaries will apply to this area until you define another area via DRAW POLYGON, or SELECT POLYGON.

D DRAW POLYGON: Allows you to pick points to define a selective area for performing an attribute summary. Subsequent attribute summaries will apply to this area until you define another area via CURRENT SCREEN, DRAW POLYGON, Or SELECT POLYGON. When you select one of the above menu options to define a new area, the current polygon will disappear.

\section{Pick DRAW POLYGON.}

The menu item will blink in green to indicate that further input is required.

2. Pick points that define the sides of a polygon.

MPDISPLAY will draw line segments from point to point.

3. Pick CONTINUE to complete the polygon.

The polygon will blink in green to indicate that a polygon has been properly selected. The menu option will stop blinking to indicate that no further input is required in selecting the polygon.

MPDISPLAY will draw the final side between the first point and the last, closing the polygon area.

- SELECT POLYGON: Allows you to pick a predefined polygon on which to perform the attribute summary. Subsequent attribute summaries will apply to this area until you define another area via CURRENT SCREEN, DRAW POLYGON, or SELECT POLYGON. When you select one of the above menu options to define a new area, the current polygon will reappear in its original color.

\section{Pick SELECT POLYGON.}

The menu item will blink in green to indicate that further input is required.

2. Pick any point on a preexisting polygon.

The polygon will blink in white to indicate a pick. If the wrong polygon is highlighted, pick again on the desired polygon until it is properly selected. 
3. Pick CONTINUE to confirm the polygon selection.

The polygon will blink in green to indicate that a polygon has been properly selected. The menu option will stop blinking to indicate that no further input is required in selecting the polygon.

- PERFORM ATTRIBUTE SUMMARY: Allows you to enter major and minor attribute codes to select attributes points to be summarized.

If used without selecting an "interactive area," this function can be used to search the entire screen.

You will see the prompt

ENTER MAJOR MINOR CODE >

Enter the major and minor code of an attribute point and press

RETURN. Example: 1, 2

You will see summary table headings and the appropriate data, such as

$\begin{array}{ccccccc}\text { MAJOR } & \text { MINOR } & \text { COUNT } & \text { TOTAL } & \text { MIN. } & \text { MAX. } & \text { AVG. } \\ 1 & 2 & 13 & 906 & 3 & 333 & 69\end{array}$




\section{MYTAPE}

The MYTAPE utility is a foreign tape processing program that simplifies tape file transfers between the VAX computer system and other systems. For example, the description of the USGS100KXDMS utility includes an (example of using MYTAPE to transfer USGS Digital Line Graph (DLG) data files from tape to disk before conversion to DMS format.

MYTAPE is added to the VAX Digital Command Language (DCL) command tables when the IBS is initialized at the time of system installation.

\section{Processing Foreign Tapes with MYTAPE}

To start MYTAPE, just enter MYTAPE at the system prompt. The program then displays its own command prompt for further tape processing commands:

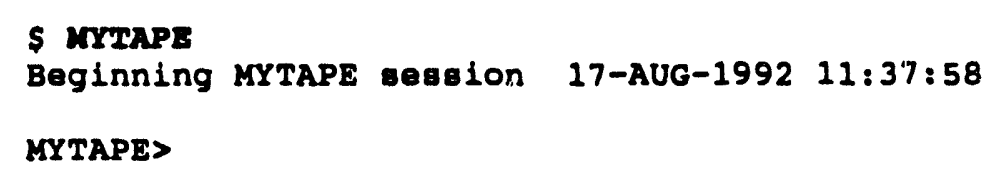

For detailed information on using the program and on MYTAPE commands, please refer to the complete documentation, which is available as IEMIS\$DOC:MYTAPE.DOC. To see online help messages, enter HELP at the MYTAPE > prompt. 


\section{NEWLOG}

The NEWLOG utility is a database administration utility that is designed to restart the IBS log journal files. The log journal files record various IBS reports exchanged by the offpost and onpost systems, messages, and other IBS activities. The IBS program uses these files to create "Message Board" reports.

If the $\log$ files are currently open, NEWLOG stops before doing anything. Otherwise it renames the current log files with the current date (filename_YYMMDD.LOG) and opens new ones.

\section{Caution}

When the logs are restarted, IBS users may no longer get reports based on the old logs.

\section{Starting New IBS Log Journals}

To start this utility, enter NEWLOG at the system prompt. No further interaction is necessary.

A series of messages should tell you which logs have been renamed, and what new log names are opened. 


\section{NEWSITE}

The NEWSITE utility is a database administration program that is used to assemble topographic, population, and other data that define a geographic site: an IBS site davabase. Once a site is created, it can be used as the "current site" for map display and computational modeling in the IBS.

This section covers the following topics:

- Why create a new site database?

- What You Need to Know Before Using NEWSITE

- Using NEWSITE to Define an IBS Site Database

- Results of Using NEWSITE.

\section{Why create a new site database?}

Emergency management analysts can set up different site databases for developing different scenarios and simulations using the various computational models available in IBS. The IBS site databases can be divided into the classes shown here:

- planning site databases

- operational site databases $-f \begin{aligned} & \text { real site databases } \\ & \text { exercise site databases }\end{aligned}$

Planning site databases are located under individual user accounts. Operational site databuses are located under the Information Manager (INFOMANAGER) account, and only one operational database is active at a time-the "current" operational site database. When the Information Manager and other IBS users use the current operational site database, they are said to be in operational mode. When the Information Manager and other IBS users are NOT using the current operational site database, they are said to be in planning mode.

The real difference between the current operational site database and all other site databases is that several users can use the current operational site database at once, in essence, sharing the data. Changes made to the data by one user are immediately reflected in the data available to the other operational-mode users. However, data in planning site databases or in non-current operational site databases is NOT shared: only one user at a time can use the data (the user under whose account the database is located). 
Planners require their own private site databases so that they can run model simulations and prepare Implementing Procedures (IPs) in the IBS program without affecting data in operational site databases or other users' site databases. Moreover, if a planner intends to modify topographic data, a private copy is necessary for the same reasons. After making changes to the data, the planner can, depending on privileges, copy the data to one of the operational site databases or copy the modified topographic data back into the map database.

There are two classes of operational site databases: real and exercise. Every exercise site must be associated with a real site, though not all real sites require an associated exercise site. Exercise site databases are used during exercises; real ones are not.

\section{What You Need to Know Before Using NEWSITE}

Before you create a site, you must know what type of site you are creating and where the site should be located on the system. If you are unsure about where to obtain this information, or need help, please contact your database administrator for more details.

The following information is used to specify the type of site and where it will be placed on the system:

Site Code This code must be four characters long. It will be used to identify the site that is to be created; for example, $A B C D$, TEST, TST2.

Site Name The site name can be up to forty characters long and is used as the full name of the site.

Disk Name The disk name is $\mathbf{1 5}$ characters long and is used to determine on which disk the site is to be created. This name can be either a device name or a logical name for a device. If no value is input, the default is your login disk.

Note: You can have sites on multiple disks as long as a top-level directory with your user name exists on each disk.

Site Type The site type is one character long and is used to define the kind of site to be created. Three different types exist (the default type is $\mathbf{P}$ ):

R Real site (only under the INFOMANAGER account)

E Exercise site (only under the INFOMANAGER account)

$P$ Planning site (not under the INFOMANAGER account) 
Assac Site This is the 4-character code of the "associated site." Only Exercise and Planning site require an associated site: any new Exercise or Planning site must be associated with a Real site. Real sites, on the other hand, do not necessarily have an associated site. If an Exercise site does or will exist for a Real site, use the name of that Exercise site as the associated site.

$\begin{array}{ll}\text { New Site Type } & \text { Assoc Site } \\ \text { Real } & =>\text { Exercise site code (possible) } \\ \text { Exercise } & \Rightarrow>\text { Real site code (required) } \\ \text { Planning } & =>\text { Real site code (required) }\end{array}$

Map Name This is the name of the map database from which topographic data will be used for the new site. If you are unsure about what map databases are available, consult the database administrator at your site.

ONSITE Code The "ONSITE code" is a 4-character code that identifies the onpost depot that corresponds to an operational (Real or Exercise) site being created under the INFOMANAGER account. Planning sites do not require an onsite code.

In addition to the parameters just defined, you must also know what geographic area you want to include in your site. For Planning and Exercise sites, the default is the same area as that used by the associated Real operational site, so you need not specify any area. If you want to change the area to something else, or if you are creating a Real operational site, four options are available for specifying what area to include in your site:

Longitude/Latitude Corners Requires two sets of longitude and latitude coordinates.

Center \& Radius (miles)

Requires one set of longitude and latitude coordinates and a radius distance in miles.

Center \& Radius (kilometers) Requires one set of longitude and latitude coordinates and a radius distance in kilometers.

Defaults: -112.34 40.30 37 Specifies defaults (site center and radius) that are set up specifically for each IBS installation and requires no further information. In this example, the longitude is -112.34 , the latitude is 40.30 , and the radius (in miles) is 37 .

If you plan to use one of the first three options to define the site area, then before you start creating the new site, make sure you have determined which longitude and latitude coordinates and which radius you will use. You can easily obtain this 
kind of information through the graphic mapping utilities in IBS. (See MPDISPLAY, for example.) The following diagram clarifies the methods for defining geographic areas in the first three options.

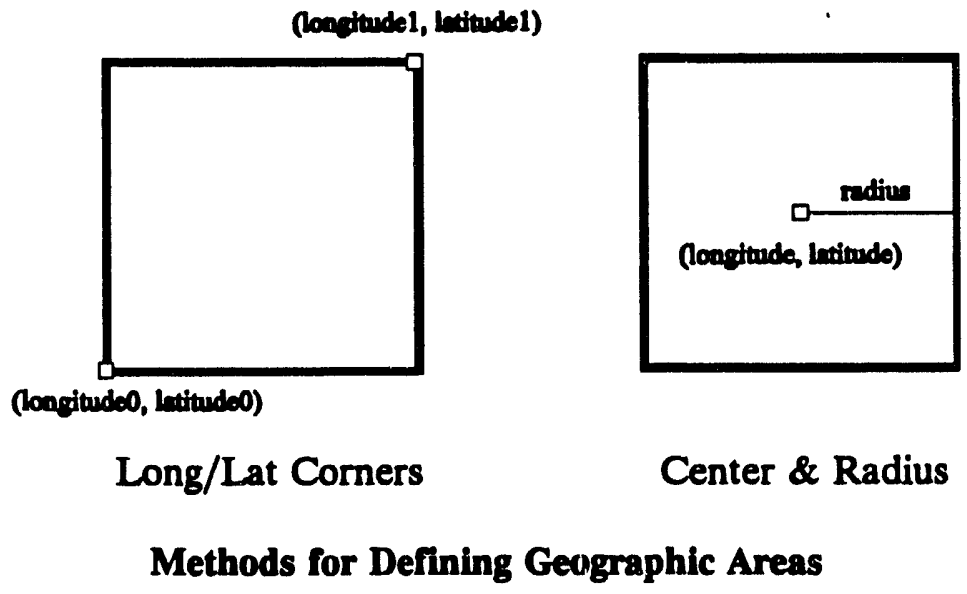

\section{Using NEWSITE to Define an IBS Site Database}

The following is an example of how to create a new site using the NEWSITE utility. This example steps through the process of creating new directories for a site and copying topographic data into the new directories.

Before beginning this process, you must know the system and topographic information explained in the previous section.

\section{Creating Directories for the New Slte}

1. To start the utility, just type NEWSITE at the system prompt. The utility begins by displaying the main menu for NEWSITE:

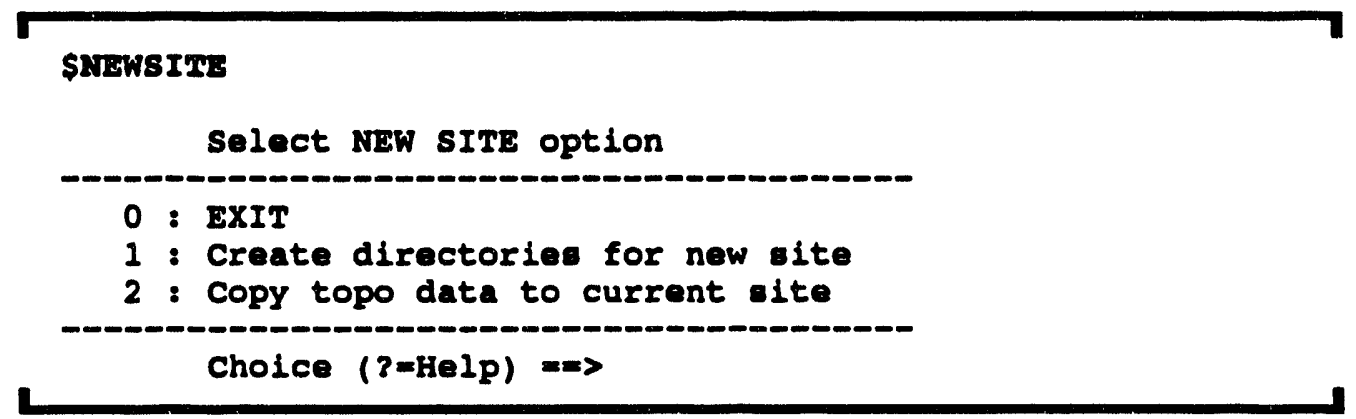

2. Select option 1 to define the new site system information.

This displays the following screen form, which indicates the system information you need to provide: 


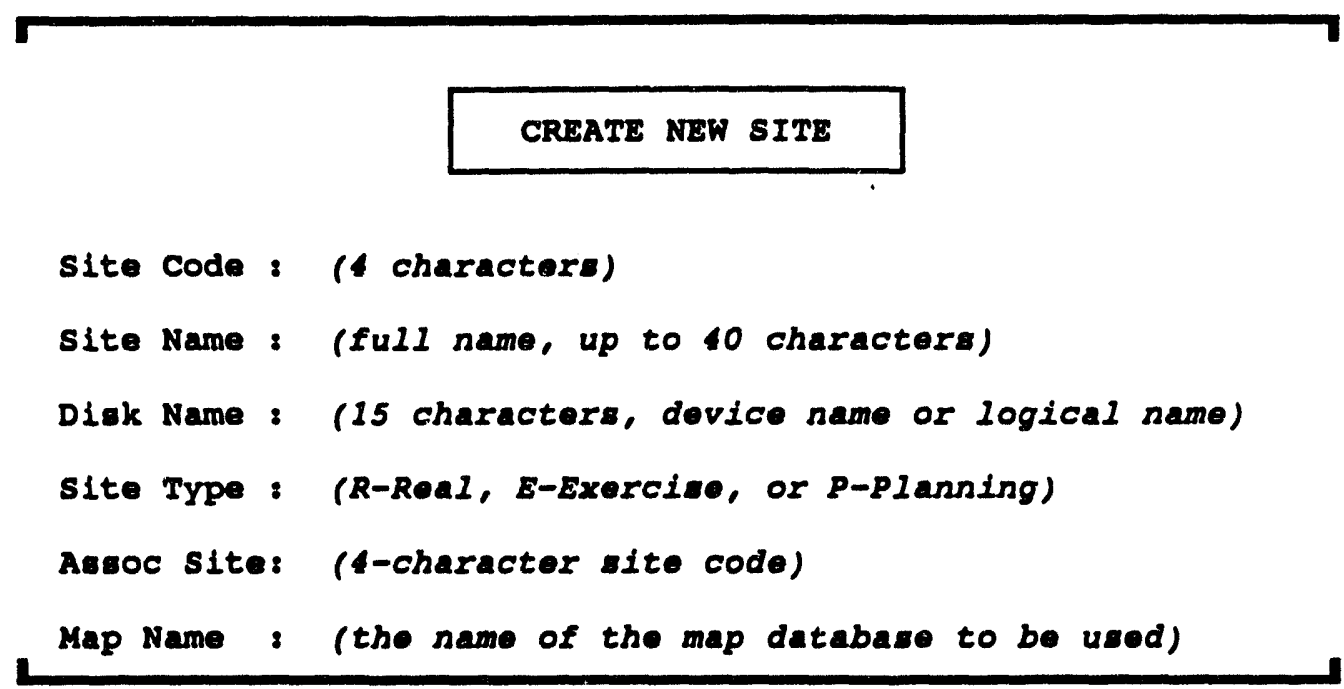

3. Enter the appropriate site code, site name, disk name, site type, associated site, and map name. Press GOLD $Z$ to finish and approve your entries (or press CTRL-W to abort the process).

Note: If you are creating a new operational (Real or Exercise) site under the INFOMANAGER account, then NEWSITE prompts you to enter a 4-character code that identifies the onpost depot that corresponds to the new OFFSITE directory:

Enter ONSITE code that corresponds to OFFSITE codes xexex $\Rightarrow=>$

After you approve your entries (or after you enter the ONSITE code), NEWSITE begins the process of creating the site directories and copying some standard files that serve as templates for supporting database files. After the site directories have been created and the necessary template files have been copied and possibly modified, NEWSITE returns you to the main menu.

You have just created a working site. The new site is now your current site, and you can use it in IBS applications with no further modifications or additions.

\section{Copying Topographic Data to the New Site}

Note: In general, most people do NOT need a private copy of any of the static topographic data-the standard map layer data that normally do not change, such as roadways. However, if you want to have a private copy of some of the static topographic data, then continue with the following procedure. 
1. To include copies of the normally static topographic data, select option 2 (Copy topo data to current site) from the NEWSITE menu. This displays summary information (similar to the following) and the Area Selection Menu:

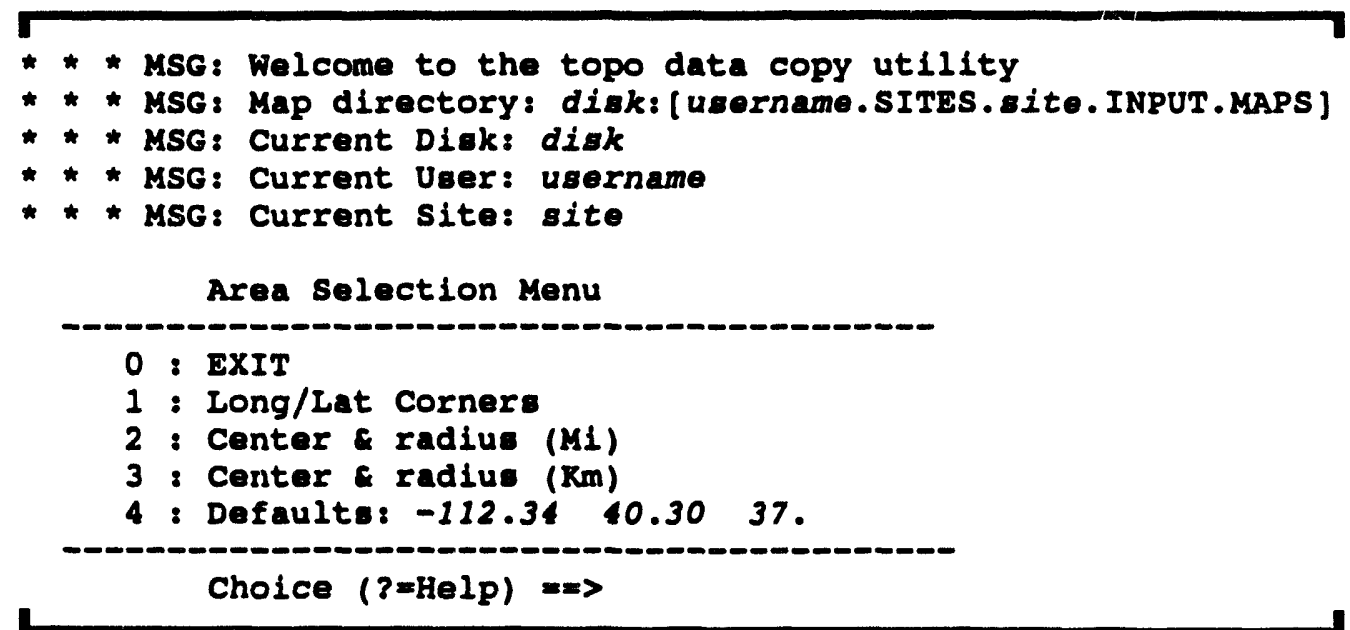

2. Select how you want to define the area for topographies to be included in your new site database:

- Option 1 displays a screen that asks you to enter the minimum and maximum longitude and latitude coordinates of the area.

- Options 2 and 3 display a screen that asks you to enter the site center coordinates and radius.

- Option 4 automatically sets the area to the default area (longitude, latitude, and radius in miles) shown in the menu.

- EXIT (0) returns you to the main menu.

If you need more information about the kind of input required by the area selection options, see What You Need to Know Before Using NEWSITE.

3. If you selected an option other than 4, enter the coordinates (and radius, if necessary) that define the area for which you want topographic information.

The following menu then appears. Different topographies (map layers) may be listed in the menu, depending on your system setup. 


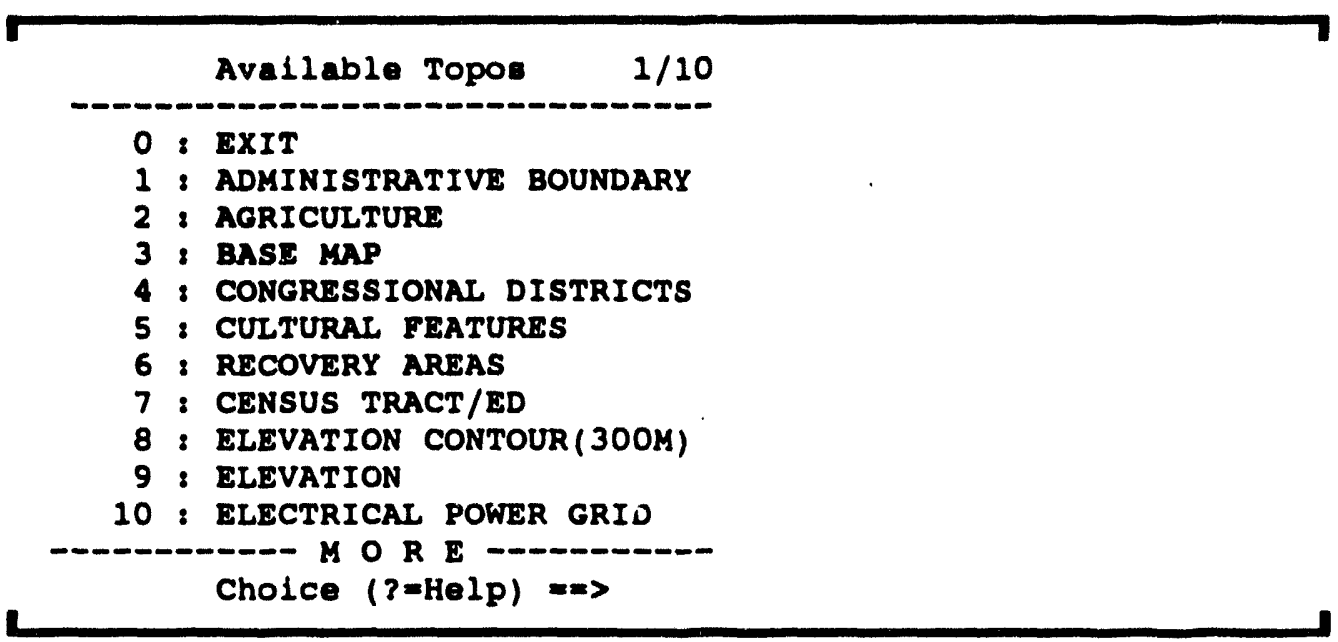

A few things should be noted about the Available Topos menu:

- The $1 / 10$ next to the title is an indication of the page currently being displayed, in this case, page 1 of 10 . To see different pages of the menu, type $U$ to move up a page or $D$ to move down a page.

- To select a topography (map layer), type the number after "Choice" and press RETURN. A text arrow $(=>)$ then appears next to the topography you wish to copy. If you type the same number again, the $=>$ disappears, indicating that the topography will NOT be copied to the current site.

- When you have selected all the topographies you want to copy to your current site, select EXIT (0). This indicates to NEWSITE that all topographies have been selected and that you are ready to copy them to your current site. If you want to discontinue this operation, type $\mathbf{Q}$ to quit the menu.

4. Select topographies from the Available Topos menu.

\section{Caution}

Be careful not to overwrtte topographies that already exist. If you select a topography that you have previously modified in your current site, you will overwrite the flles and lise your work. 
5. When you have made all your topography choices, select EXIT (0).

A sequence of messages reports the status of the process:

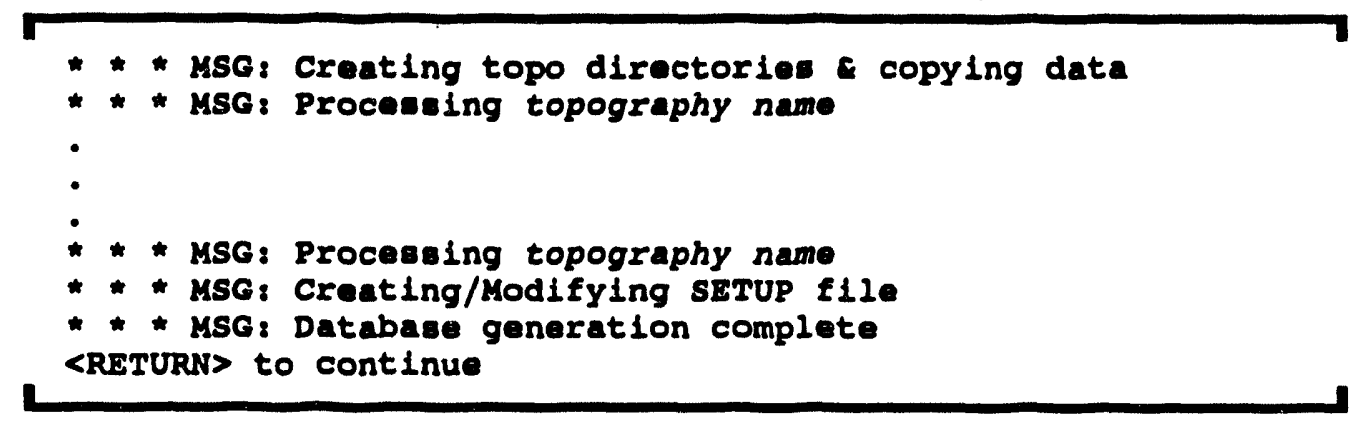

5a. If you elected to enter coordinates by selecting options 1,2 , or 3 in Step 2, the following prompt appears when you press RETURN:

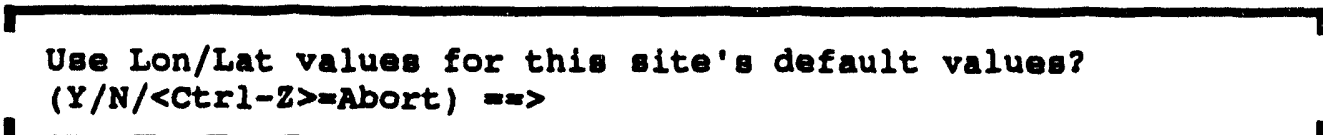

Enter $\mathbf{Y}$ or $\mathbf{N}$ to choose whether to use the new coordinate values as the default values for the IBS program.

- $\quad Y$ inserts these values as default values in your SITE.DAT file. When the IBS program starts, it checks SITE.DAT and displays the initial map using those coordinates.

- $\quad \mathbf{N}$ continues and does NOT change the SITE.DAT file.

Once you finish, the NEWSITE initial menu appears again. You have just completed adding topographies to the new site. If you want to add more topographies, you can do so by starting again at step 1 of the procedure.

\section{Results of Using NEWSITE}

NEWSITE creates a directory structure that contains site information. This directory structure can be used for simulations of the various models available in IBS. It is identical to the one described under the [INFOMANAGER][.SITES] tree in the IBS Data Management Guide.

NEWSITE also modifies the site database "SETUP" and control files so that your current site is set to whatever new site was created. 


\section{NEWUSER}

The NEWUSER utility is used by the system manager to create new user accounts for IBS users. Once cieated, a new user can login and access data on the system.

This section covers the following topies:

- Why create new user accounts?

- What You Need to Know Before Using NEWUSER

- Creating or Modifying a New User Account with NEWUSER

- Results of Using NEWUSER.

\section{Why create a new user account?}

It is necessary to create new user accounts to allow people to use IBS. By adding an account, you provide users with the ability to access IBS databases and perform whatever tasks they may be assigned to do. The NEWUSER utility provides a simple and direct means of adding user accounts and configuring them for use in IBS.

\section{What You Need to Know Before Using NEWUSER}

Before using NEWUSER, you must obtain the following necessary information:

username

real name

disk
The username is the name under which the user will login. It is the information which will entered by the new user at the system Ingin prompt.

The real name is the user's actual name. If specified in quotes, lowercase letters will be preserved in the name, if quotes are not used, the name will be converted to all caps.

$$
\text { Example: } \quad \begin{array}{cl}
\text { "John Doe" } & \rightarrow \text { John Doe } \\
\text { John Doe } & \rightarrow \text { JOHN DOE }
\end{array}
$$

This disk is the name of the disk on which the account is to be created.

Example: DISK1 
account

group number

member number

usertype
The account can be used to identify what type of account the new user will have access to. If you have a billing system for CPU time, this field can be used to specify who gets billed for use of the system. The account is specified as a string.

The group number is used to specify what group a user belongs to. Users working on the same projects should have identical group numbers. Because it is required by the operating system, the group number is specified as an octal (base eight) number instead of a decimal (base ten) number. So in octal sequence, group 7 is followed by group 10, group 17 by group 20, and group 77 by group 100 , and so on.

Note: It is strongly recommended that all IBS users have the same group number.

The member number should be a unique number for each user within a specific group. The member numbers are also specified as octal numbers.

The usertype indicates a set of IBS privileges that can be assigned to and shared by users. The default usertypes are ALL (all privileges), PLANNER (planning privileges), and NORMAL (operational data privileges). (These usertypes can be modified and other usertypes added by using PDMGR, the utility for managing user privileges and access to IBS programs and program functions.)

When a new account is created using NEWUSER, the password is automatically set to be the same as the username and to expire when the user logs in for the first time. NEWUSER also automatically adds the new user to the system mail list, but you should use MLMGR to verify that it is added correctly.

The NEWUSER utility automatically places a record for the new account in the mail list file, although the record may be incomplete. You should use the MLMGR program to verify that the newly created record is correct. The EVENT program must be running to update the mail list file. If EVENT is not running, these files remain unprocessed until EVENT is started. When EVENT is restarted, it processes the waiting files, so changes made while EVENT is not running will NOT be lost. The new record added to the mail list file is automatically routed to other systems on the network according to the contents of the IEMISSSYSF:MAII_SYSTEMS.DAT file (see the Data Management Guide for information about this file). 


\section{Creating or Modifying a User Account with NEWUSER}

The following is an example of how to create or modify a user account using the NEWUSER utility.

Before beginning this process you will have to know certain system and user information. Please refer to the previous section, What You Need to Know Before Using NEWUSER.

To start the utility, just type NEWUSER at the system prompt. The program then prompts you for the information outlined in the previous section. The series of prompts will look like the following (simulated answers are in bold):

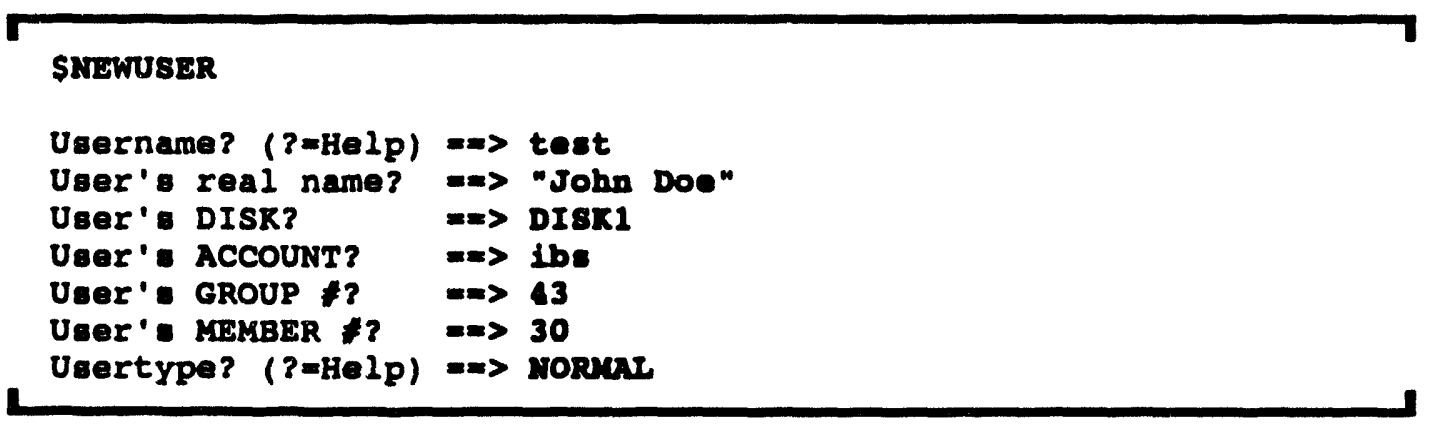

Once these fields have been entered, the utility will automatically create and configure the account for the new user. NEWUSER will generate messages that look something like the following:

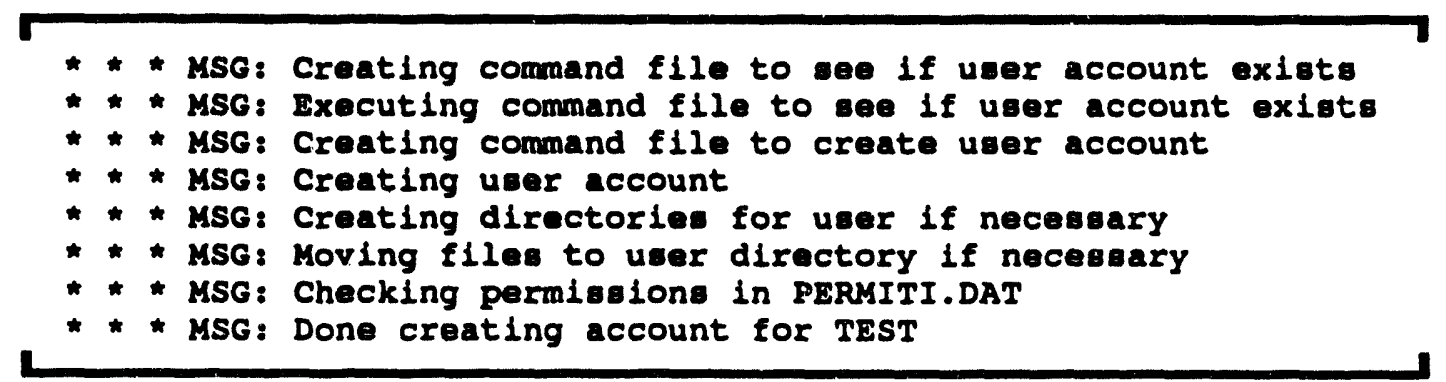

The password for the new user will automatically be the same as the username (so in the above example, the password would be TEST) and is set to expire upon the first login. 


\section{Results of Using NEWUSER}

NEWUSER creates a new account using the information which you provide it. It sets up the new user's password, and copies a base set of files into the new account. These files include JOB_ENV.DAT, LOGIN.COM and SITE.DAT. SITE.DAT contains no sites, just $\bar{a}$ couple of blank records. LOGIN.COM configures the user's environment so that the user can run IBS, and JOB_ENV.DAT contains IBS environment information. A SITES subdirectory [usermame.SITES] is also created, but it will be empty initially.

NEWUSER grants a new IBS user these VMS privileges: TMPMBX, NETMBX, EXQUOTA, SYSLCK, and GRPPRV. NEWUSER also modifies PERMITI.DAT so that the new user has the appropriate permissions to access the IBS databases.

If NEWUSER is used on an existing account, it will reset quota information to the IBS defaults. The quotas and their defaults are listed here:

\begin{tabular}{lr}
$\begin{array}{lr}\text { Process Quota } \\
\text { Parameter }\end{array}$ & Value \\
\hline ASTLM & 24 \\
BIOLM & 24 \\
BYTLM & 65536 \\
CPUTIME & 0 \\
DIOLM & 18 \\
ENOLM & 600 \\
FILLM & 70 \\
JTQUOTA & 8192 \\
MAXACCTJOBS & 0 \\
MAXDETACH & 0 \\
MAXJOBS & 0 \\
PBYTLM & 0 \\
PGFLOUOTA & 200000 \\
PRCLM & 4 \\
PRIORITY & 4 \\
PWDMINIMUM & 8 \\
OUEPRIO & 0 \\
SHRFILLM & 0 \\
TQELM & 10 \\
WSDEFAULT & 150 \\
WSEXTENT & 4096 \\
WSQUOTA & 1024
\end{tabular}




\section{PDMGR}

The PDMGR (Permiti.Dat Manager) utility is used by the system manager or database administrator to set IBS privileges for IBS users. The IBS privileges (specified in the IBS authorization file, PERMITI.DAT) control which programs and program functions an IBS user can use or see. For a list of IBS privileges and their correlation to IBS programs and program functions, refer to Appendix E.

\section{What You Need to Know Before Using PDMGR}

You can set privileges for a single user or of a group (usertype). Because it is expected that IBS users will fall into general categories (normal users, planners, data managers, and so on), PDMGR enables you to create and modify groups of privileges according to usertype. For example, planners should have more privileges than a normal user because they need access to the planning data. By creating a usertype with associated privileges, you can assign privileges to new users more easily and consistently: the usertype can be used to specify privileges when adding a new user (using the NEWUSER utility).

When IBS is installed, there are three default usertypes already defined, as shown in the following table. These usertypes contain the privileges thought to be necessary for those general roles. If these usertypes are not satisfactory, you can define new ones using PDMGR. Up to 100 usertypes can be defined for IBS.

\begin{tabular}{cl} 
Usertype & Privileges \\
\cline { 1 - 1 } ALL & All privileges within IBS \\
PLANNER & NORMAL privileges plus the privileges necessary to do planning \\
NORMAL & Privileges restricted to working; with the current operational data
\end{tabular}

\section{Privilege Required to Use PDMGR:}

- 29 PD_MANAGER

\section{Files Used:}

- IEMIS\$SYSF:PERMITI.DAT, the IBS authorization file

- IEMIS\$SYSF:PERMITI.DFT, file of default usertype permissions

- IEMIS\$HELP:PERMITI.DOC, the PDMGR help file

\section{Files Output:}

- IEMIS\$SYSF:PERMITI.DAT, the IBS authorization file

- IEMISSSYSF:PERMITI.DFT, file of default usertype permissions

\section{Interactive Input:}

- Menu and form interactions

- $\mathrm{Y}(\mathrm{es})$ or $\mathrm{N}(\mathrm{o})$ entries on forms to grant/revoke associated privileges 


\section{Modifying IBS Privileges with PDMGR}

To start this utility, enter PDMGR at the system prompt. PDMGR displays a menu similar to the following one. (The option to "Add a usertype" might be missing if there are already 100 usertypes.)

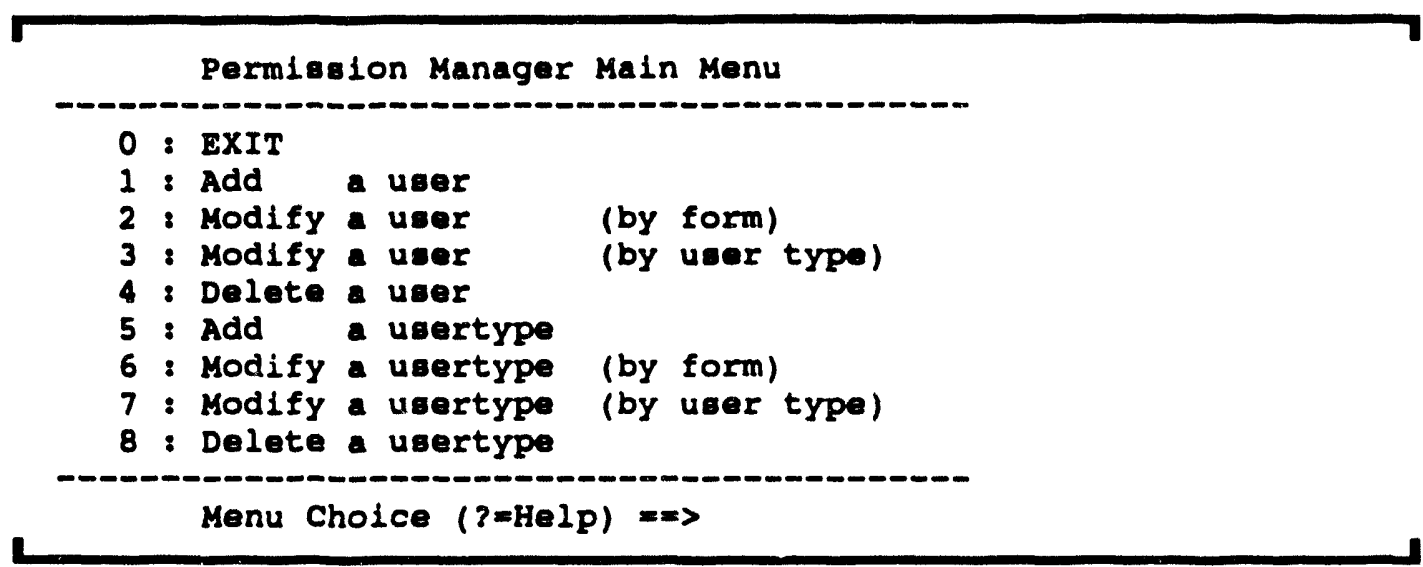

0 - EXIT: Exits PDMGR and saves the permissions changes that you have made. (See Accepting Changes to IBS Permissions, later in this discussion.)

1 - Add a user: 1) Prompts for a username and then 2) presents the following form of user privileges to set for that user.

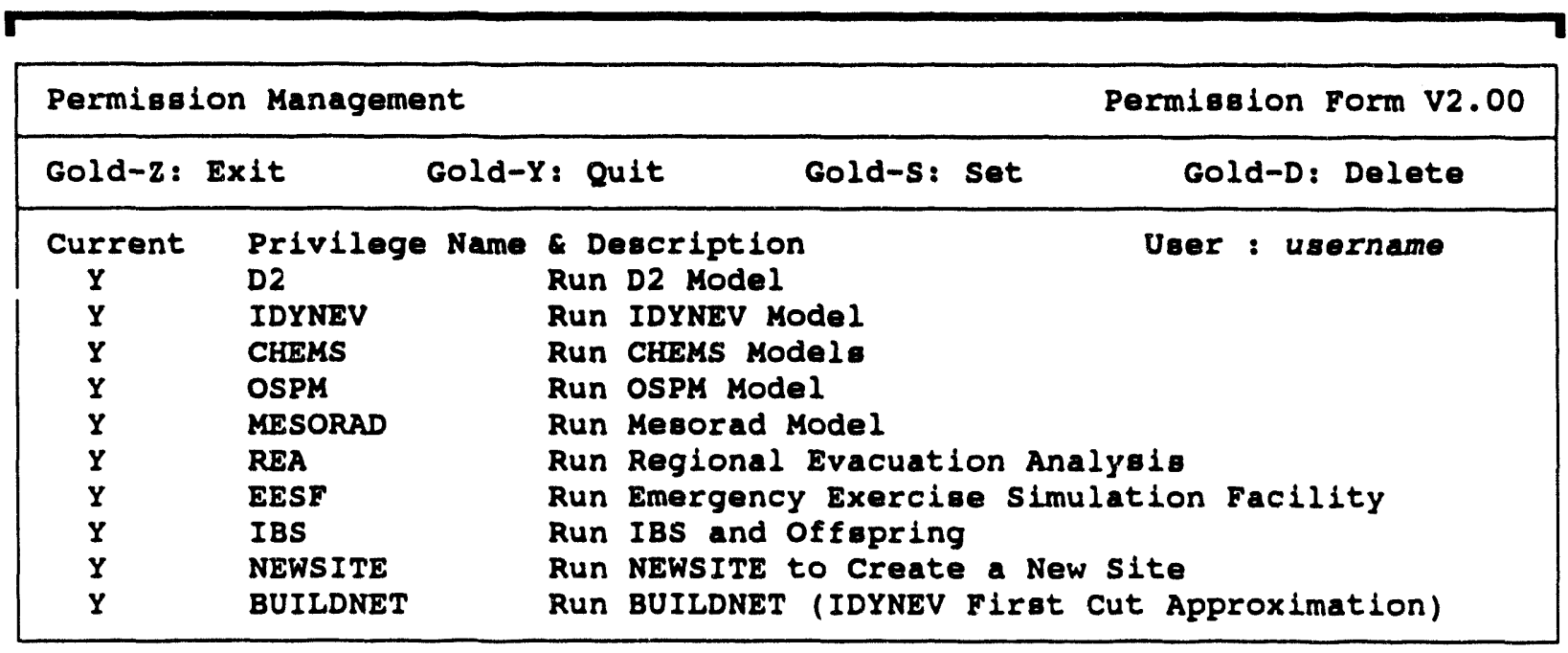


Enter $\mathbf{Y}$ or $\mathbf{N}$ beside each privilege that you want to grant or revoke.

Use the arrow keys to move between fields. Pressing the up/down arrow key when on the first/last line of the form will show the previous/next ten privileges, if there are any.

For a complete list of IBS Privileges and their correlation to IBS programs and program functions, see Appendix E.

2 - Modify a user (by form): Presents 1) a menu for selecting a specific user and then 2) the form for modifying the user's privileges (same form as for option 1).

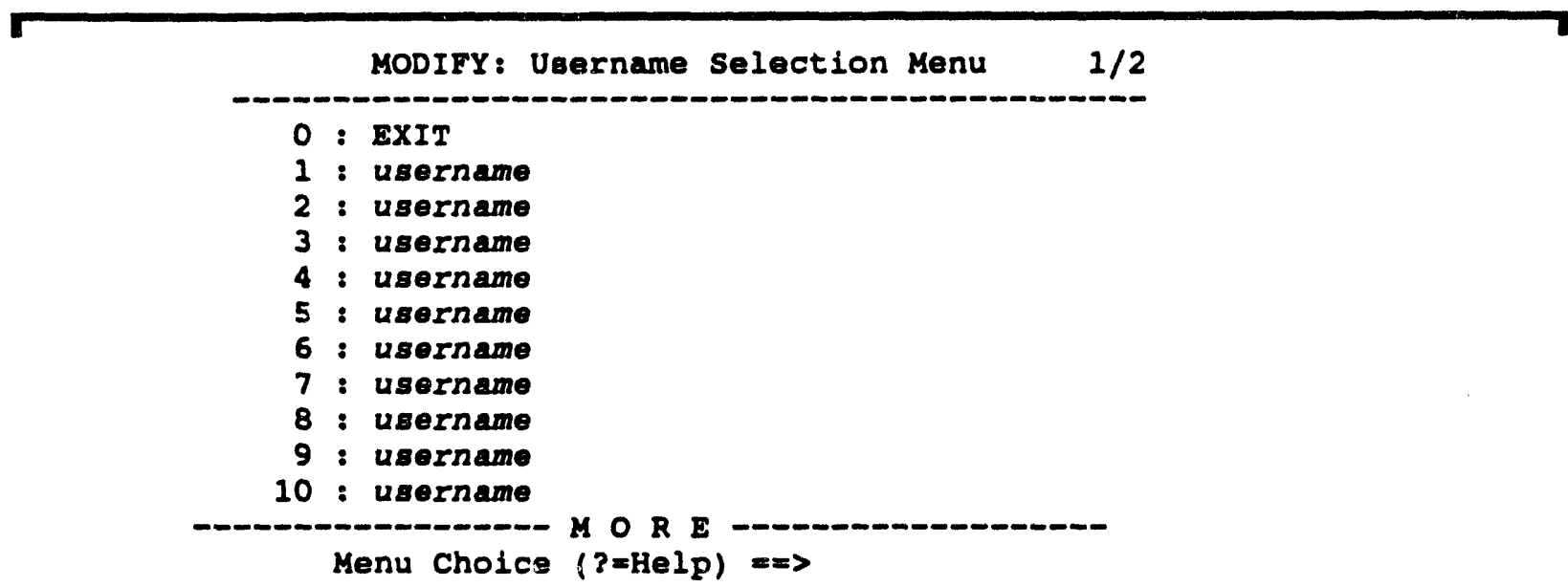

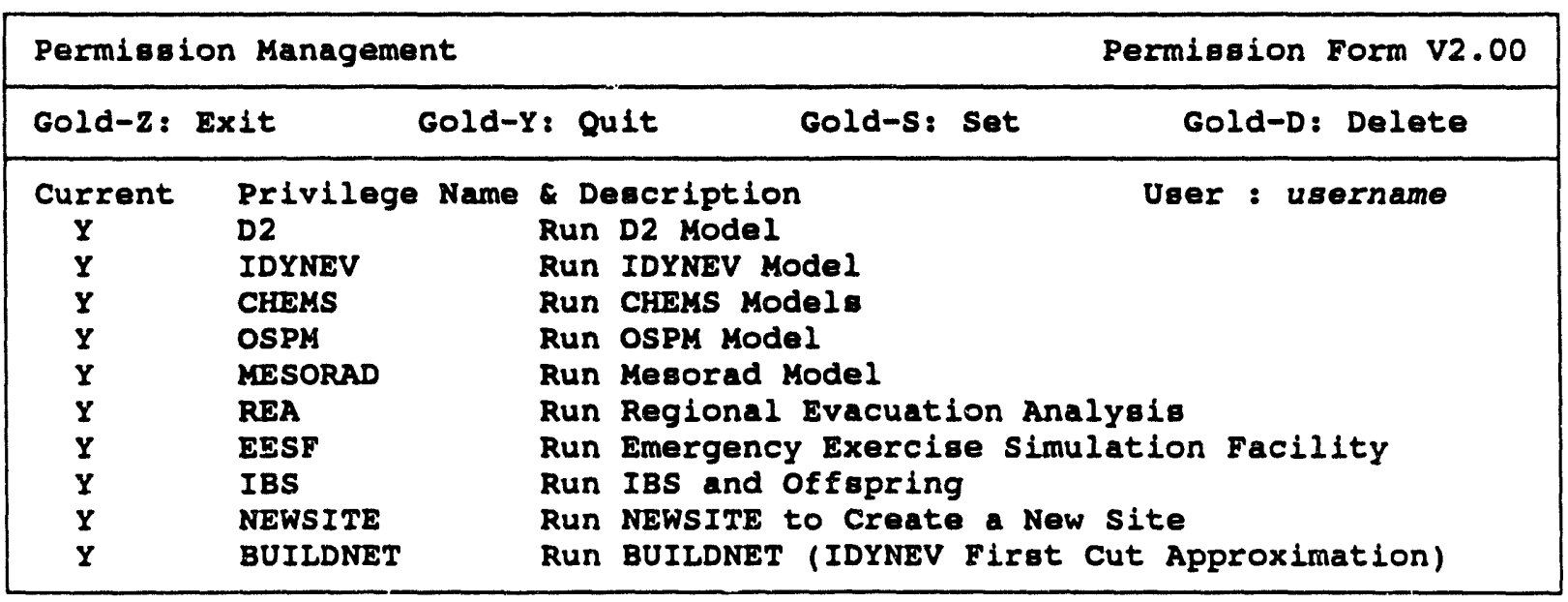


3 - Modify a user (by usertype): Presents 1) a similar menu for selecting a specific user and then 2) this second menu for selecting a usertype (predefined set of privileges) for that user.

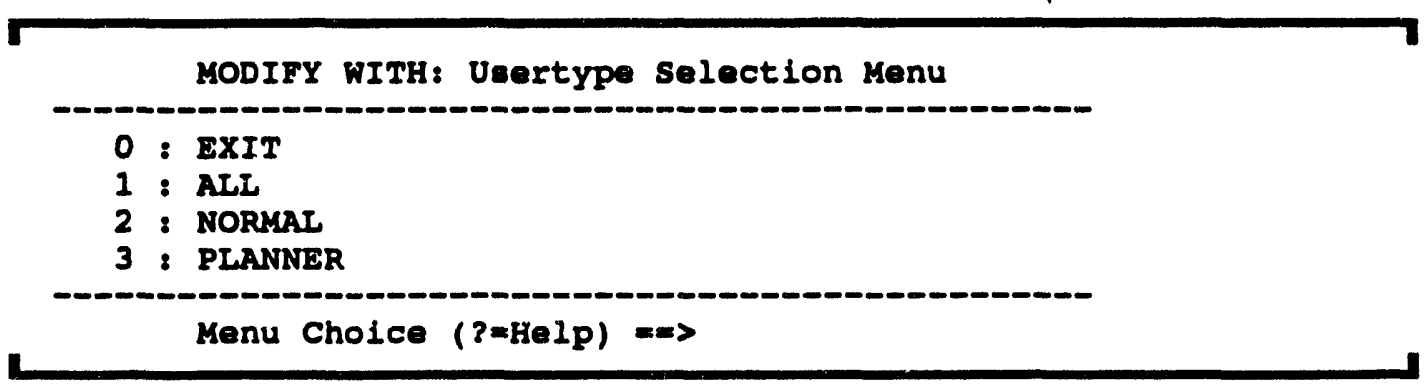

4 - Delete a user: Presents a menu for selecting a specific user to delete.

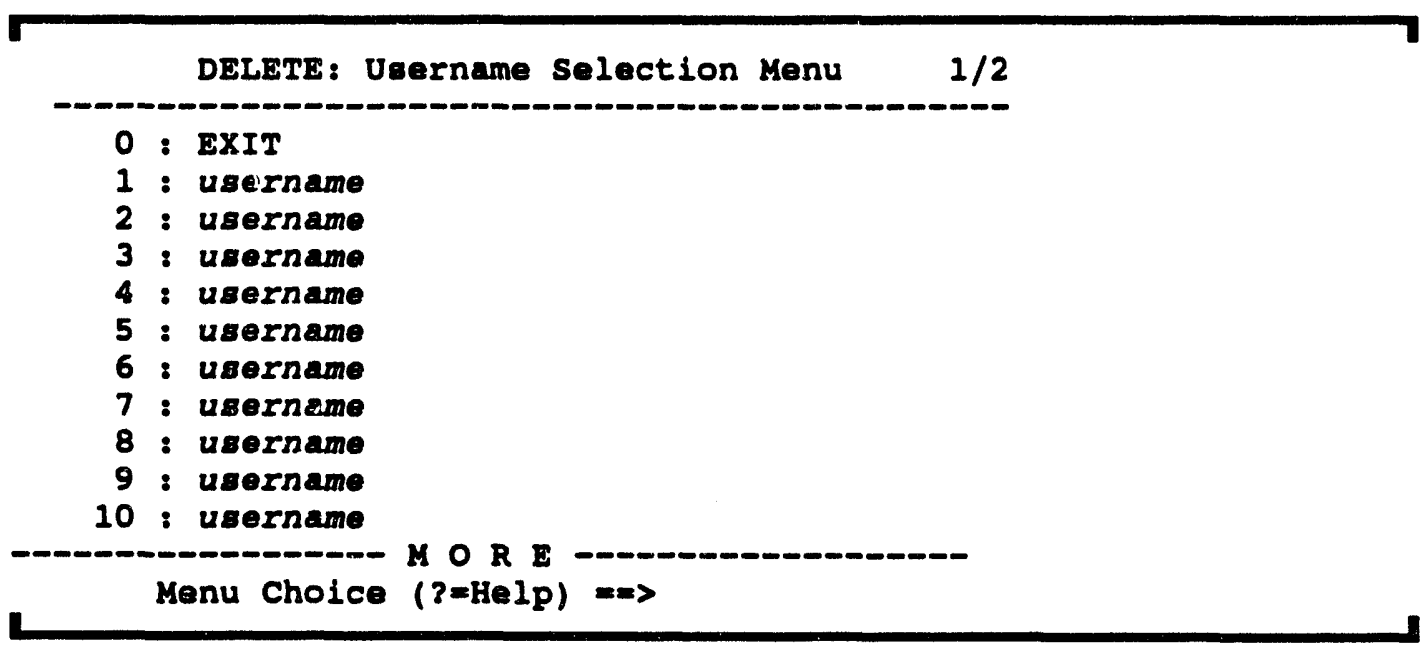

After you enter the user's index number, that user's privileges are canceled and the Username Selection Menu is redisplayed (without the deleted username).

5 - Add a usertype: 1) Prompts for the name of a new usertype and then 2) presents a form of user privileges for that new usertype, similar to the permission form for an individual user.

Enter $\mathbf{Y}$ or $\mathbf{N}$ beside each privilege that you want to grant or revoke.

Note: Only 100 total usertypes are permitted. If there are 100 usertypes, this option does NOT appear on the menu until another usertype is deleted. 
6 - Modify a usertype (by form): Presents 1) a menu of usertypes to choose from and then 2) a form of privileges to set for the selected usertype.

Example:

MODIFY: Usertype Selection Menu
$0:$ EXIT
$1:$ ALL
$2:$ NORMAL
$3:$ PLANNER
Menu Choice $(?=$ Help) $=0$

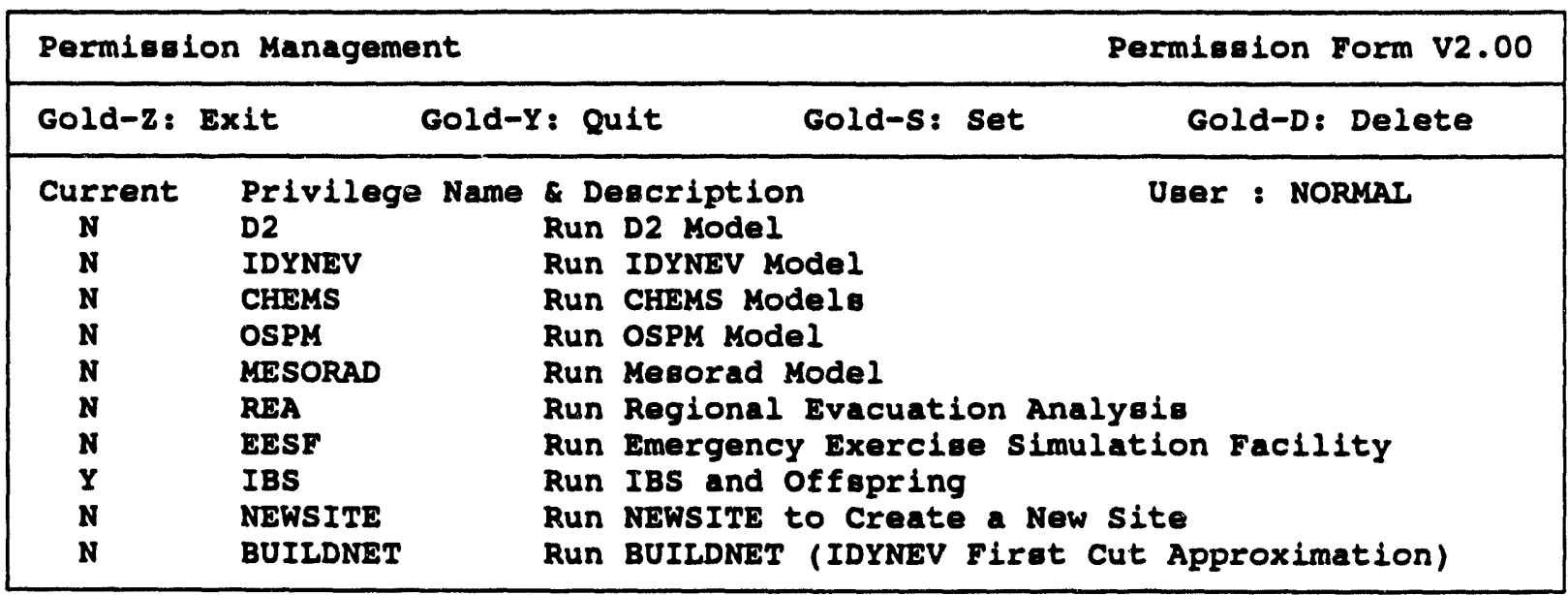

7 - Modify a usertype (by user type): Presents 1) the menu for selecting a usertype to modify and then 2) a second menu of usertypes for selecting one to use a basis for modifying the first usertype. That is, the permissions from the second usertype are copied over to the first usertype.

8 - Delete a usertype: Presents a menu for selecting a usertype to delete.

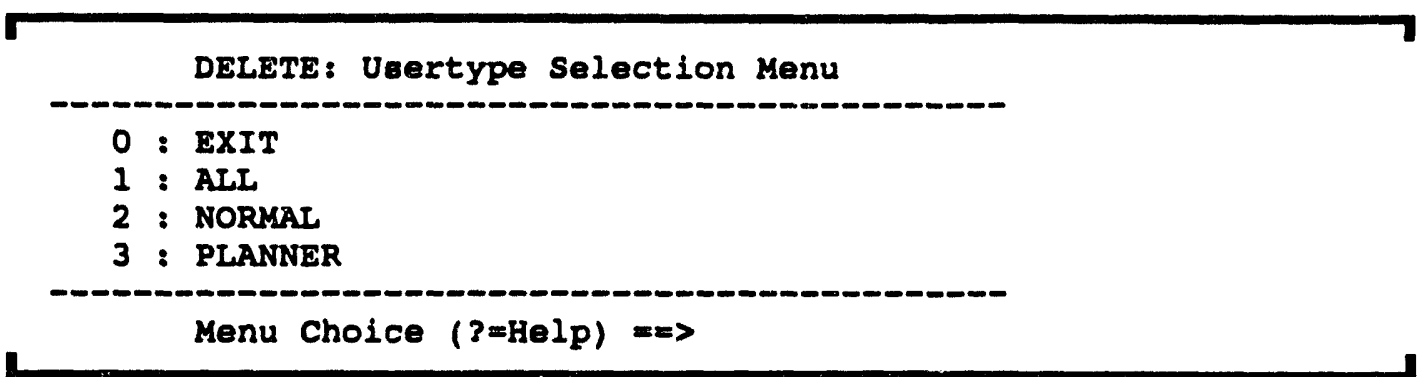


Accepting Changes to IBS Permissions. After you have made any changes to any IBS permissions, the PDMGR main menu adds two additional menu items:

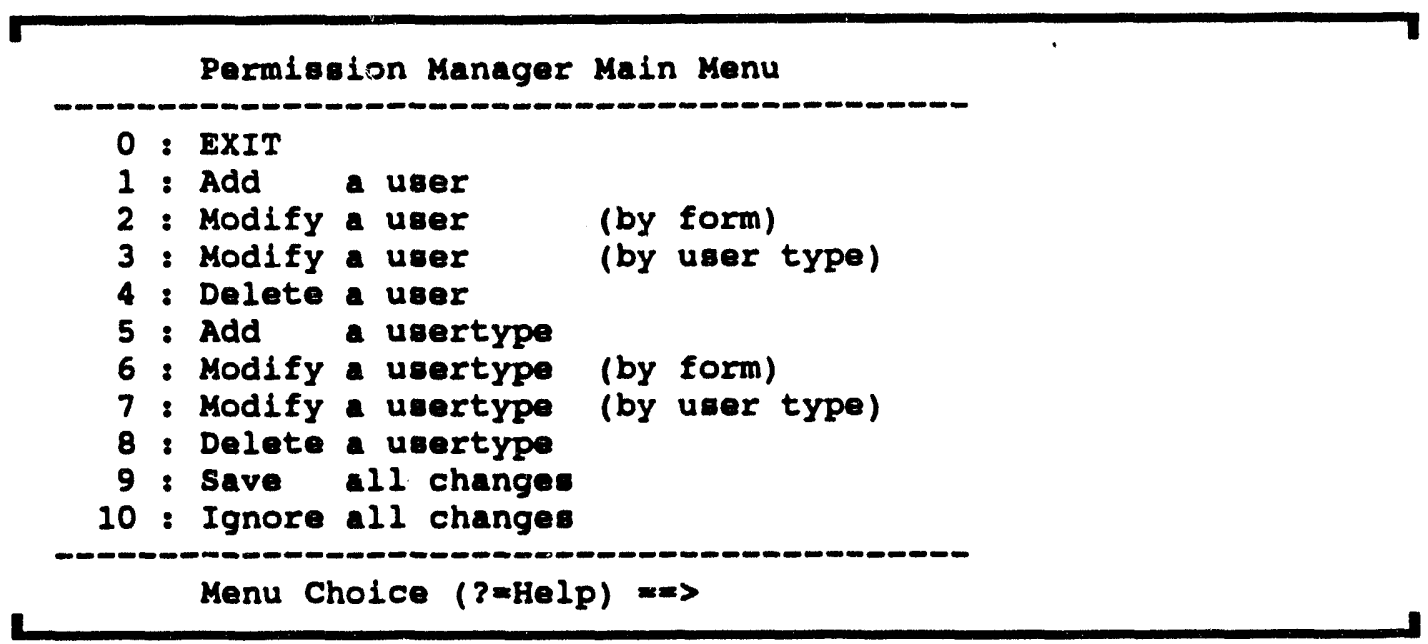

9 - Save all changes: Incorporates any changes in a new PERMITI.DAT file and then redisplays the main menu without the options to save or ignore all changes.

10 - Ignore all changes: Cancels all permissions changes made since the last time you used "Save all changes."

0 - EXIT: Exits PDMGR and saves any permissions changes made since you last used "Save all changes" or "Ignore all changes." 


\section{PLOTDMS}

The PLOTDMS utility displays one or more DMS files graphically on the terminal screen without border lines or menus.

You can use PLOTDMS as a simple way to visually verify the contents of a DMS file, perhaps after using data creation or conversion programs that create DMS files. A visual examination, coupled with numeric output from DMSXASCII and summary data from STATDMS, should verify whether or not the desired DMS file was created.

\section{What You Need to Know Before Using PLOTDMS}

PLOTDMS provides the capability to create a graphical plot display that represents the contents of a series of DMS files. The DMS files are plotted in the order they are entered. Therefore, if a particular file contains data you want overlaid on a base map, be sure to specify it last. Remember that the entire contents of each DMS file is plotted, so you may want to make sure the files are within the same area by using the DMSXASCII utility to check each file's extents.

\section{Plotting the Contents of a DMS File with PLOTDMS}

To start the utility, enter PLOTDMS at the system prompt. The utility then prompts you for the name of the DMS file(s) to be used. Enter the name of each .DMS file to be used, separating each file name by a carriage return: PLOTDMS repeats the prompt each time. To plot the specified DMS files, press the carriage return at the prompt instead of entering a file name. PLOTDMS then generates the graphical image.

Example:

\$ PLOTDMS

Enter DMS filename $(z=$ help, $q=E x i t,<C R>=P$ lot $==>$ NEWYORKST.DMs

Enter DMS filename $(z=$ hel $p, \wedge z=E x i t,\langle C R\rangle=P$ lot $=\Rightarrow$ NEWYORKWB.DMS

Enter DMS filename $(z=h e l p, \wedge z=E x i t,<C R>=P$ lot $=\Rightarrow>$ <RETURN $>$

(Display is generated and the prompt repeats)

Altering the Setup for a Plot. You also have the capability to alter criteria used to generate the image. Entering a forward slash (/) at the DMS filename prompt displays the Setup Menu. From this menu you can alter display parameters by choosing which parameter to alter and then entering the desired change. 
For example, if you wanted to display the streams and water bodies for New York using an Albers projection, with no rotation, at twice the scale, and with the display repositioned one inch higher, your responses might look like this:

Example:

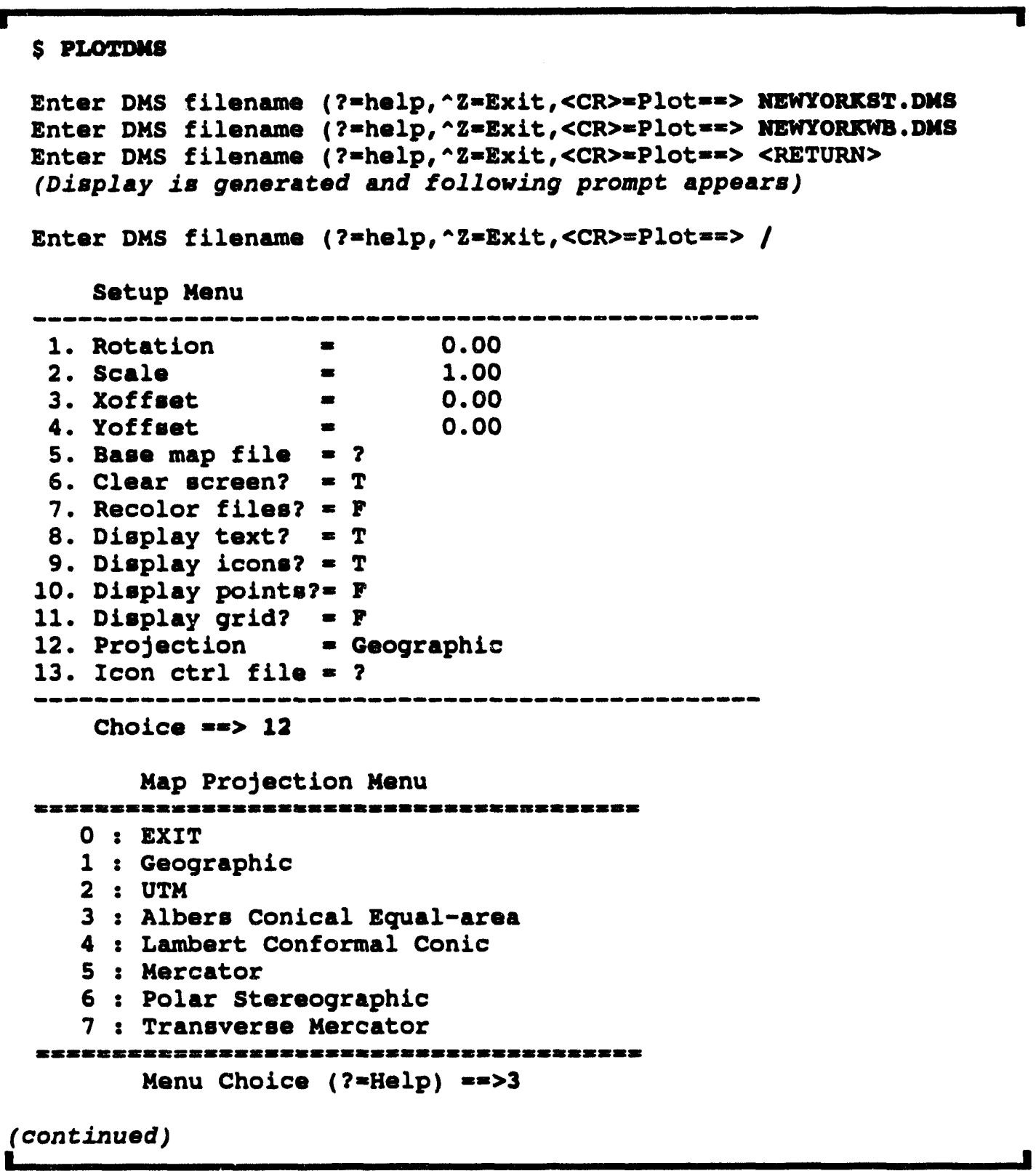


Example: (continued)

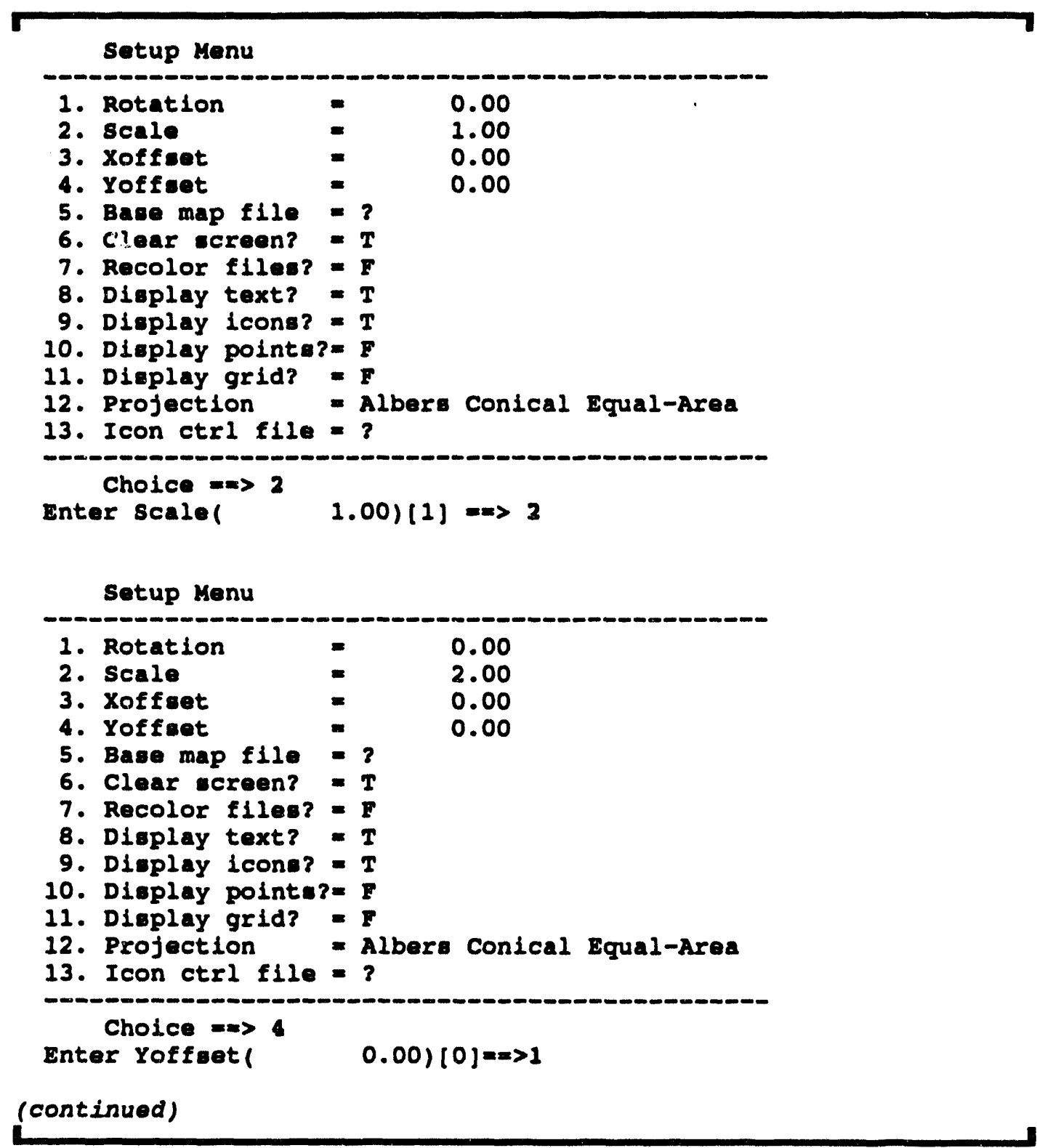


Example: (continued)

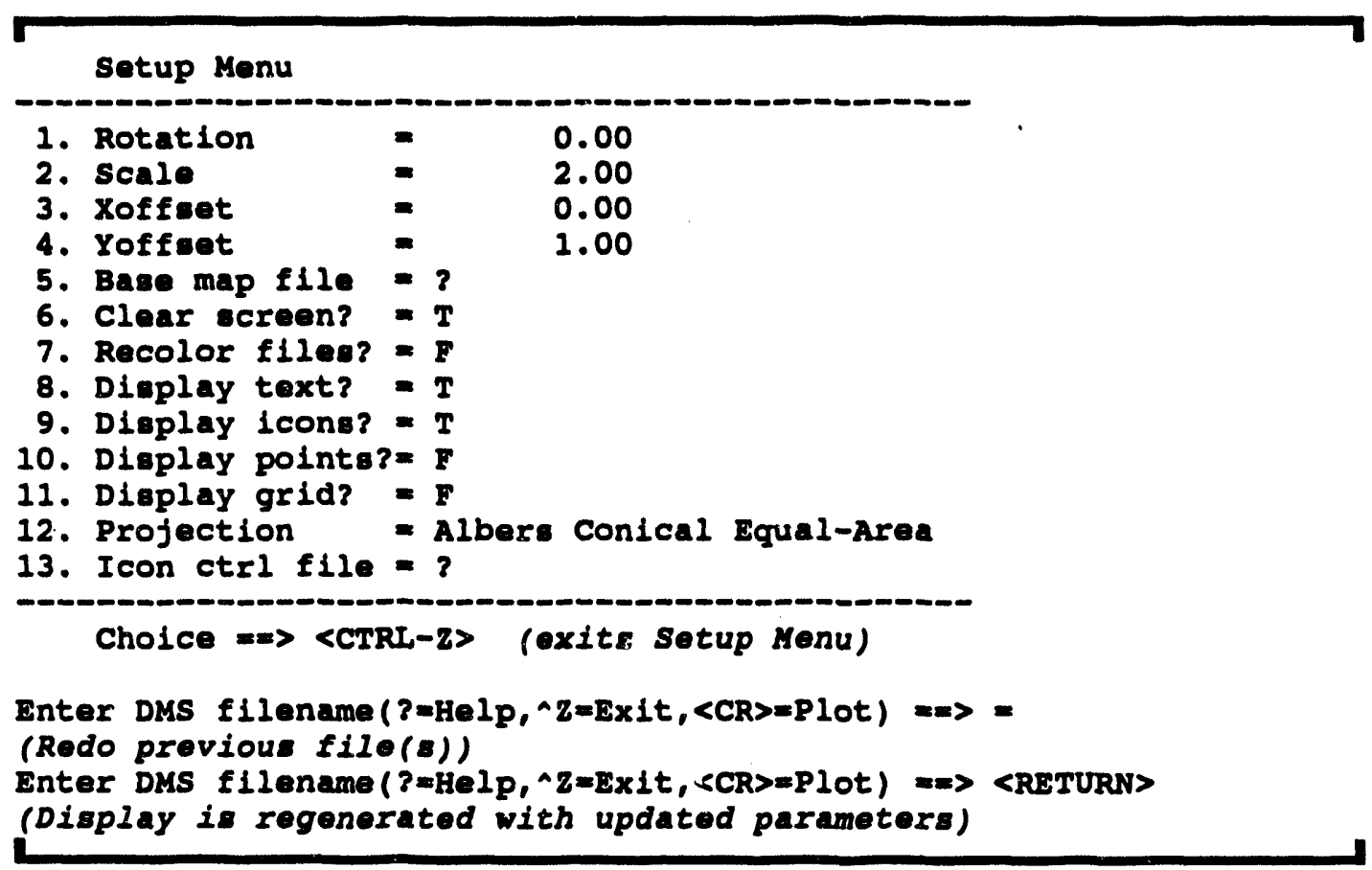

Entering Setup Menu Values. Parameters used in generating a plot can be changed by selecting the Setup Menu and entering the desired parameter values:

Setup Menu Option

1. Rotation

2. Scale

3. Xoffset

4. Yoffset

5. Base map file

6. Clear screen?

7. Recolor files?

8. Display text?

9. Display icons?

10. Display points?

11. Display grid?

12. Projection

13. Icon ctrl file $=$ ?
Parameter description or result

an angle (in degrees) through which the graphical plot is rotated a multiplication factor by which the base image is either increased or reduced in size

shift graphical image left/right on the display

shift graphical image up/down on the display

a file that overlays all other DMS files uses for the plot (You enter the file name.)

$Y$ clears the screen before the creation of a plot.

$Y$ reassigns colors used for each file in the plot.

$Y$ displays the respective items (text, icons, points, longitudelatitude grid lines) in the graphical plot.

$\mathbf{N}$ leaves them off the plot.

displays a menu that enables you to select a different projection type (see Choosing the Map Projection Type later)

allows you to read in the contents of the icon control file (icon dictionary), but this may be a lengthy process 
Getting Help with Using Other Features of PLOTDMS. If you enter a question mark at the DMS filename prompt, the utility displays a help screen of other possible responses to the prompt.

Example:

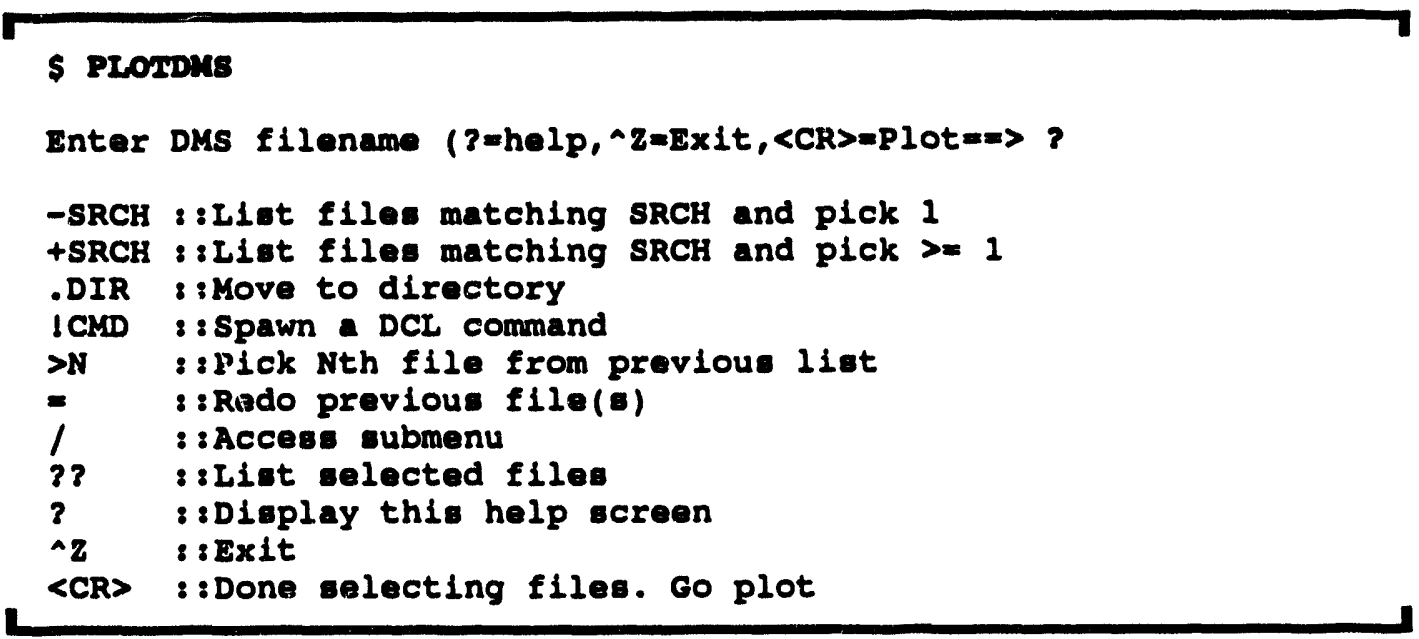

\begin{tabular}{|c|c|}
\hline Filename Prompt Response & Result \\
\hline -searchtext & $\begin{array}{l}\text { searches for DMS files whose file names match the search text } \\
\text { and then lists the files for you to choose one (see }>N \text { ) }\end{array}$ \\
\hline + searchteat & $\begin{array}{l}\text { searches for DMS files whose file names the search text and } \\
\text { lists the files for you to choose one or more (see }>N \text { ) }\end{array}$ \\
\hline DIR & $\begin{array}{l}\text { enables you to select a new directory used under PLOTDMS } \\
\text { while remaining on the same disk }\end{array}$ \\
\hline Icommand & $\begin{array}{l}\text { spawns the DCL command entered after the exclamation point } \\
\text { (such as !SHOW TIME) }\end{array}$ \\
\hline$>N$ & $\begin{array}{l}\text { picks the Nth DMS file from the previously shown list of DMS } \\
\text { files }\end{array}$ \\
\hline$=$ (equals sign) & replots previously selected files \\
\hline / (forward slash) & displays the Access Submenu \\
\hline ?? (double question mark) & displays a list of files selected for the previous plot. \\
\hline$\langle C T R L-Z\rangle$ & ends PLOTDMS \\
\hline$<$ RETURN > & plots the previously specified files \\
\hline
\end{tabular}


Choosing the Map Projection Type. For most cases (up to 100-mile radius), you will probably want to enter a 1 (or just press the RETURN key) to select the default "Geographic" projection. Enter a number (2 to 7) if you desire a projection other than Geographic. The following table is a simple summary of how the different map projections might be used. See Appendix D for background on the projection types.

Map Projection Type

Geographic

Universal Transverse Mercator (UTM) or Transverse Mercator

Albers Conical Equal-Area or Lambert Conformal Conic

Polar Stereographic
Situations for Use

small areas (up to a 100-mile radius)

medium-sized areas (up to 1000-mile radius) or where the largest extent of the mapped area extends north-south rather than east-west

larger areas, or where the largest extent of the mapped area extends east-west

polar areas of the globe 


\section{PLOTICON}

The PLOTICON utility is a diagnostic and reporting program that displays all icons specified in the current icon dictionary (icon control file).

Note: This utility requires a graphic terminal.

\section{What You Need to Know Before Using PLOTICON}

This utility asks you to specify several values that determine how the icons are displayed:

\begin{tabular}{llll} 
Value & Description & \multicolumn{2}{l}{$\begin{array}{l}\text { Recommended } \\
\text { Value }\end{array}$} \\
\cline { 1 - 1 } rows & \# of rows of icons & 5 \\
cols & \# of columns of icons & 5 \\
height & Height in inches of text & 0.25 \\
scale & Amount to scale icons (1=none) & 1 \\
color & Icon colors (-1 = DMS-specd) & -1 \\
text & Show icon \# \& description or not: & -1 \\
& $<0$ (show number; no description) & (number; \\
& 0 (show number; show description) & no description) \\
& $>0$ (no number; show description)
\end{tabular}

\section{Plotting the Available IBS Icons}

To start this utility, enter PLOTICON at the system prompt. The utility then prompts for multiple settings as shown.

$\$$ PLOTICOX

Enter rows, cols, height, scale, color, text $(z=$ Help) $=\Rightarrow$

Enter each value separated by commas. After all the values have been input, the icons in the current icon control file are displayed to the screen, page by page. To see the next page, press RETURN. To exit the screen display, press CTRL-Z. 


\section{POPXDMS}

The POPXDMS utility creates DMS data files from STF1B data files provided by the U.S. Department of Commerce, Bureau of the Census. The Bureau of the Census makes population data available at state, county, place, tract, group, and block levels. POPXDMS is designed to handle one county at a time.

Note: You will normally NOT use POPXDMS directly: for most purposes you will want to use the TIGERCVT utility program, which creates a special command file that runs POPXDMS for you. (See TIGERCVT.)

\section{What You Need to Know Before Using POPXDMS}

You will normally want to use the TIGERCVT utility rather than POPXDMS, as noted above. If you do use POPXDMS independently, you must specify at least some of the following items either by entering them or by choosing them from a menu:

Population Type This is the type of population data that will be converted for the county: state, county, county subdivision, place, census tract, block group, or block.

state name

county name

county/state

limits

combined state and county code

census directory and filename
For the state population data only: This is the name of the state for which you want state-level population data.

For population data other than state: This is the name of the county for which you want population data.

These are the longitude-latitude extents of the county (or state, for state data): the minimum and maximum longitude and the minimum and maximum latitude, in that order.

For data types other than state: This five-digit code consists of the two-digit FIPS state code followed by the three-digit FIPS county code.

This is the name of the directory where the census files are located and the name of the file. If you do not specify a directory name, the program looks for the file in the current default directory. 


\section{Creating DMS Population Files from Cenisus Bureau STF1B Files}

To start the utility, just enter POFXDMS at the system prompt. You will then see prompts for the information described in the previous section. The series of prompts will resemble the following two examples, which illustrate using POPXDMS for 1) data below the state level and for 2) data at the state level. (The state data is the same for all counties in a state; therefore, state data does not need to be converted more than once.) Simulated responses are in bold.

If subdirectories for each of the new map layers have not been created under the database top-level directory, POPXDMS will create the necessary subdirectories.

\section{Example of Converting Data Below the State Lievel}

\$ PORXDMS

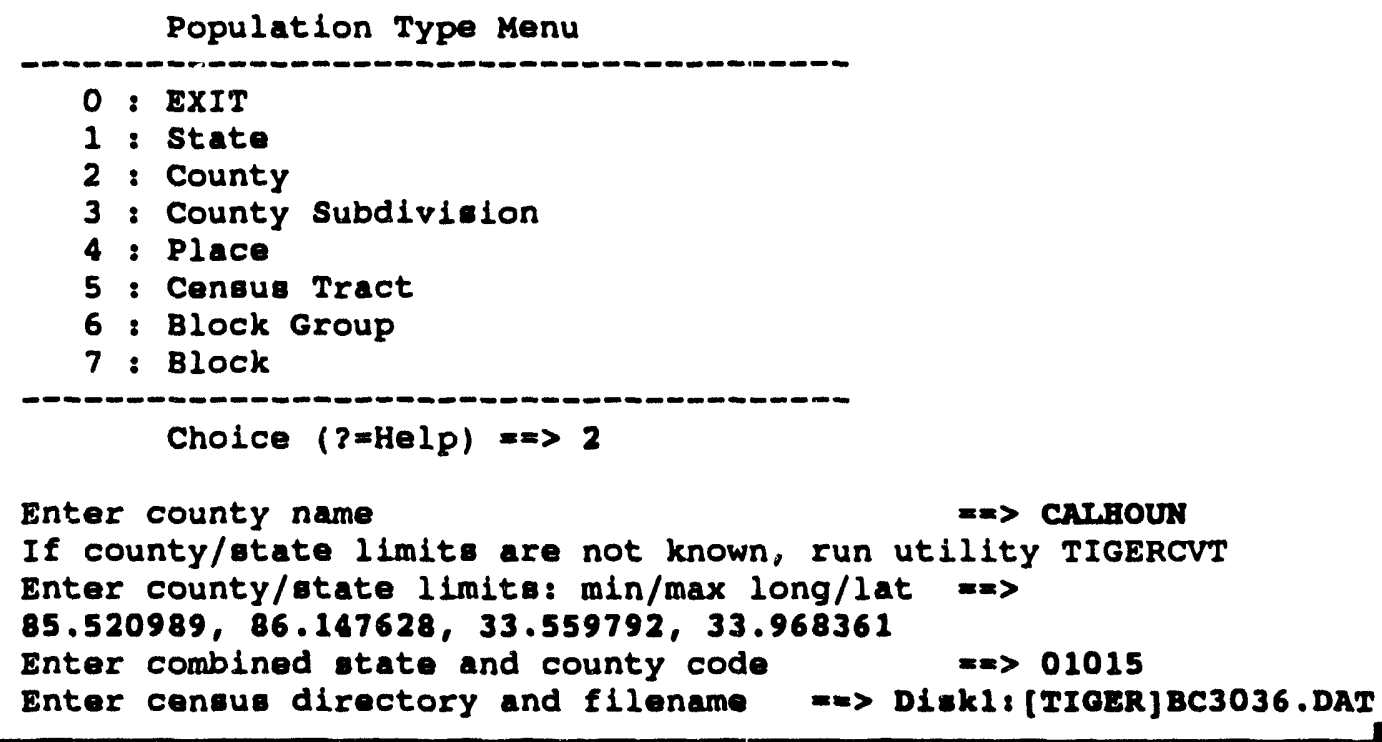

As POPXDMS processes the individual data records for conversion to DMS format, you will see a series of messages:

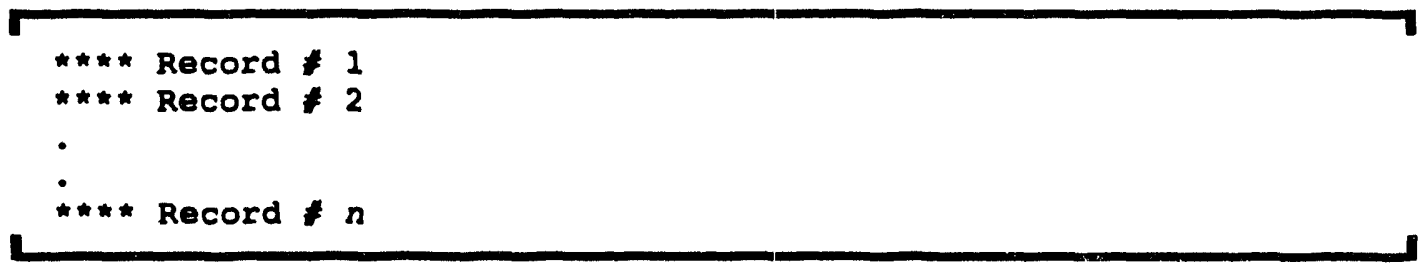


After all records have been read and the DMS files have been created, the program ends and the system prompt reappears.

\section{Example of Converting State-Level Data}

The state data is the same for all counties in a state; therefore, state data does not need to be converted more than once.

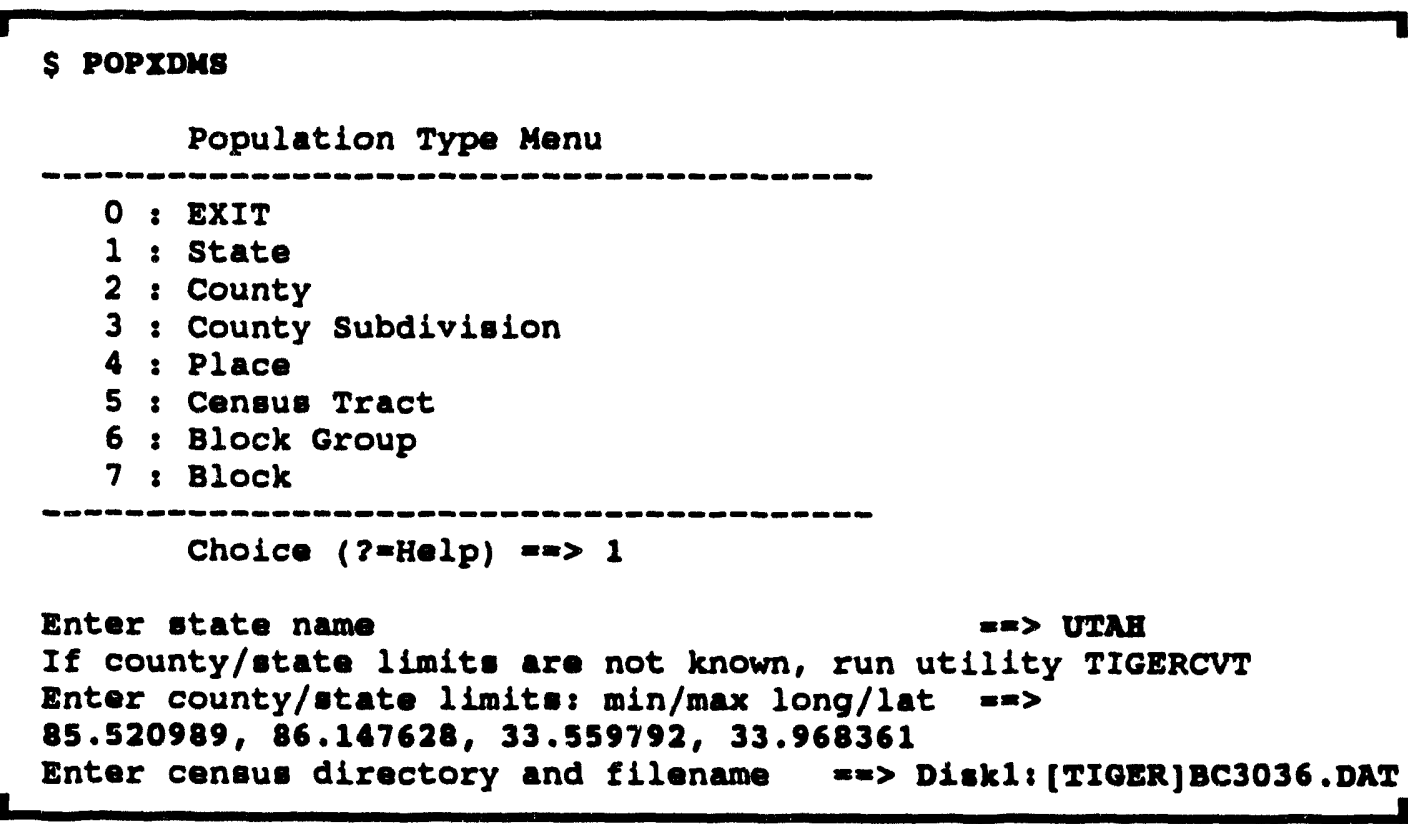

When you choose state data, the program prompts for the state name and the state extents. The extents should be set wide enough to include the entire state, as the exact location of the Census Bureau centroid for state data is not known. (See TIGERCVT). When you are converting state data, the prompt for a "combined state and county coden does not occur.

\section{POPXDMS Program Messages}

[directory] filename FILE COULD NOT BE OPENED

The data file did not exist in the directory specified. Check that the directory name is correct and that the data file is there.

*MSG* Problem with logical record numbers

The logical record numbers in the current TIGER record did not match. The program will ignore that record and proceed to the next. 
*MSG* Longitude/Latitude equals zero

Either the longitude or the latitude of the current TIGER record was given as zero. The program will ignore that record and proceed to the next.

*MSG* Out of object Iimits

The object specified in the current TIGER record did not fall within the long/lat limits specified for the current county. The program will ignore that record and proceed to the next.

*MSG* Invalid block suffix

The program encountered an invalid block suffix specification when converting TIGER block data. The program will ignore that record and proceed to the next.

\#MSG * COULD NOT OPEN FILE... nnn

The program encountered an error opening the DMS format files. Check that the logical names for the topography directories are correct, and that the directories exist. 


\section{RMCHK}

The RMCHK utility checks all Resource Management data for the current site and generates a report that includes the following:

- data that is referenced but does not exist

- data that contains blanks for important fields

- data that includes default items generated by the IMPORT utility, which should be updated with actual data

- the maximum size/count and the amount filled (current size/count) for all the main Resource Management data types.

Normally, RMCHK needs to be executed only after you have imported data with the IMPORT utility, to help determine which records should be updated.

\section{Checking Resource Management Data with RMCHK}

To start the utility, enter RMCHK at the system prompt. This utility is also available as a menu option from the RMMGR utility.

RMCHK starts by displaying the following menu:

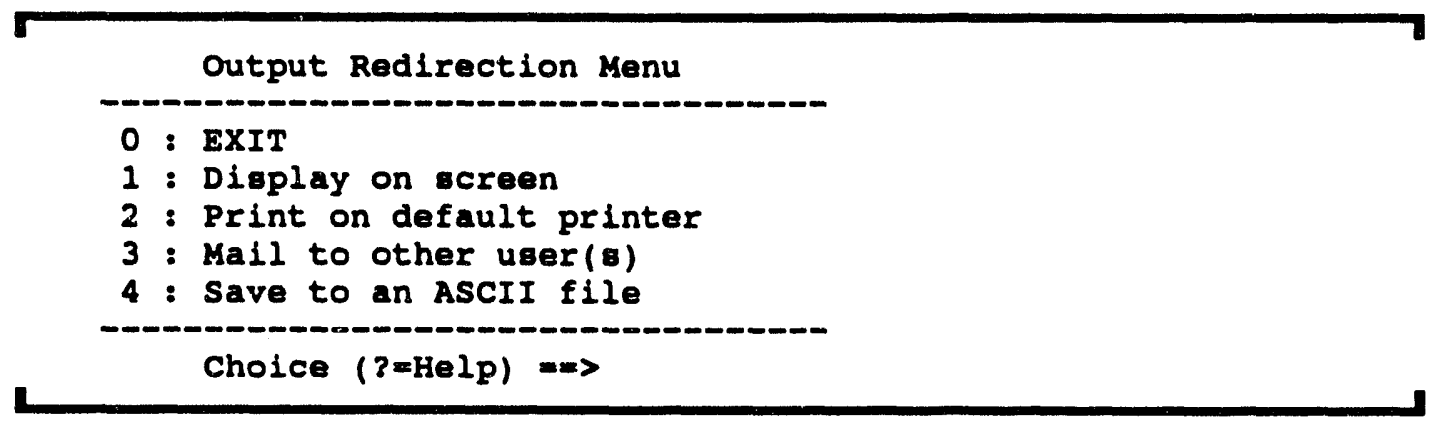

Choose a destination for the output report by entering the number shown next to the output method:

1 displays the report on the terminal screen

2 prints the report on the printer that is set up as the system default

3 displays a mail destination menu for choosing where to send the report by electronic mail (similar to MESSAGE BOARD-SEND MAIL option described in the IBS User Guide)

4 saves the report as an ASCII text file in your login directory under a name that you specify

After you choose the output method, the program sends the report to the destination that you chose. 
These abbreviations are used on the report form:
AG Agency
POS Positions
PER Personnel
FAC Facilities
FT Facility Tracks
KP Known Points
RES Resources 


\section{RMMGR}

The RMMGR (Resource Management ManaGeR) utility is a tool for managing information about the personnel, resources, and facilities that may be needed to handle an event. RMMGR displays site maps and graphic icons that show the locations of resources (materials, equipment, capabilities) and facilities (resource locations). RMMGR operates on data for the current site. A non-graphic version of the RMMGR program is available: the RMMGR_NOGR utility provides many of the same capabilities but does not display maps or require a graphics terminal.

Most of the capabilities of RMMGR are duplicated in the Resource Management, Personnel Management, and Map Analysis portions of the IBS program. Information managers and resource planners can use RMMGR for quick access to those assembled capabilities without running the IBS program. RMMGR also includes a few capabilities that are NOT found in the IBS program: these capabilities are of interest mostly to Information Managers.

Described here are the overall menus and operation of the RMMGR program as well as the capabilities that are unique in RMMGR. For background, terminology, and descriptions of the capabilities that overlap the IBS program, please refer to the IBS User Guide.

\section{What You Need to Know Before Using RMMGR}

You should be familiar with operating the IBS program as explained in Chapter 1 of the IBS User Guide. RMMGR operates with a graphic map display and a menu area similar to those used in the IBS program. The key operational difference between RMMGR and the IBS program is that you can choose functions from the principal RMMGR menus by typing a letter or number that corresponds to the item of your choice. For example, the top parts of the three principal menus in RMMGR are identical and provide direct access to each other by typing 2,3 , or 4 :

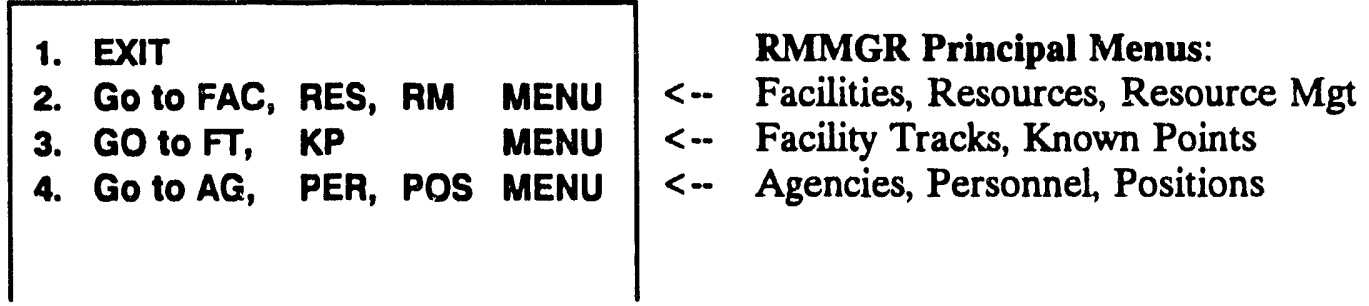

The Map Analysis menu and capabilities described in the IBS User Guide are directly accessible from all three of these menus. The RMMGR menus and associated capabilities are summarized on the following pages. As in the IBS program, an asterisk after a menu option indicates that special IBS privileges are required to use that capability. If you do not have privileges to use an option, or it is unavailable in your current site, then that option will not appear on the menu. 


\section{Using RMMGR}

To start the utility, just enter RMMGR at the system prompt. The screen map displays graphic icons that depicts the locations of facilities and resources used in IBS resource management and planning.

\section{Using the FAC, RES, RM Menu}

The Facilities, Resources, Resource Management Menu is the menu that displays when you first start RMMGR. This menu includes all the capabilities of the RESOURCE MANAGEMENT menus in the IBS program. (See Resource Management in the IBS User Guide.)

\begin{tabular}{|c|c|c|}
\hline $\begin{array}{l}\text { 1. EXIT } \\
\text { 2. Go to FAC, RES, RM MENU } \\
\text { 3. GO to FT, KP MENU } \\
\text { 4. Go to AG, PER, POS MENU } \\
\text { * * FAC, RES, RM * }\end{array}$ & & \\
\hline $\begin{array}{ll}\text { A. MAP ANALYSIS } \\
\text { B. CONTINUE } & \\
\text { C. ADD } & \text { FACIUTIES } \\
\text { D. MODIFY } & \text { FACIUTIES } \\
\text { E. DELETE } & \text { FACIUTIES } \\
\text { F. UST } & \text { FACIUTIES } \\
\text { G. DESCRIBE } & \text { FACIUTIES } \\
\text { H. HIGHUGHT } & \text { FACIUTIES } \\
\text { I. SUMMARIZE } & \text { FACILTIES } \\
\text { J. DEFINE } & \text { RESOURCES } \\
\text { K. ASSIGN } & \text { RESOURCES } \\
\text { L. MODIFY } & \text { RESOURCES } \\
\text { M. MOVE } & \text { RESOURCES } \\
\text { N. DELETE } & \text { RESOURCES } \\
\text { O. UST } & \text { RESOURCES } \\
\text { P. DESCRIBE } & \text { RESOURCES } \\
\text { Q. SUMMARIZE } & \text { RESOURCES } \\
\text { R. ENABLE } & \text { RESOURCES } \\
\text { S. DISABLE } & \text { RESOURCES } \\
\text { T. ZOOM IN } & \\
\text { U. ZOOM OUT } & \\
\text { V. REFRESH SCREEN }\end{array}$ & - & $\begin{array}{l}\text { See Map Analysis in the IBS User Guide. } \\
\text { Add a new facility to the database. } \\
\text { Modify facility information. } \\
\text { Delete a facility from the database. } \\
\text { Print a listing of current facilities. } \\
\text { Show facility information \& resources. } \\
\text { Show facilities that contain selected resources. } \\
\text { Show a summary of facilities. } \\
\text { Define a new resource. } \\
\text { Assign resources to a facility. } \\
\text { Modify resource information. } \\
\text { Move resources to another facility. } \\
\text { Delete a resource from the database. } \\
\text { Print a listing of current resources. } \\
\text { Show information about a resource. } \\
\text { List resources contained within a specific area } \\
\text { Turn on selected resource icons. } \\
\text { Remove selected resource icons from screen. } \\
\text { Enlarge a portion of the current screen. } \\
\text { Expand the view to show a larger area. } \\
\text { Redraw (and clean up) the current screen. }\end{array}$ \\
\hline
\end{tabular}




\section{Using the FT, KP Menu}

The Facility Tracks, Known Points Menu enables you to directly create or update database information about

1) facility tracks (facility names; facility status as active or inactive; numbers of people received at, remaining at, and released from facilities)

2) known points (point locations such as the locations of facilities).

\section{EXIT \\ 2. Go to FAC, RES, RM MENU \\ 3. GO to FT, KP MENU \\ 4. Go to AG, PER, POS MENU \\ * * FT, KP * * *}

A. MAP ANALYSIS

B. CONTINUE

C. ADD FACIUTY TRACKS

D. MODIFY FACIUTY TRACKS (F)

F, DELETE FACIUTY TRACKS

G. LIST FACIUTY TRACKS

I. ADD KNOWN POINTS

J. MODIFY KNOWN POINTS

K. DELETE KNOWN POINTS

L. LST KNOWN POINTS

M. GEO-LOCATE KNOWN POINTS

P. IMPORT DATA

Q. CHECK RM DATA

U. ZOOM IN

V. ZOOM OUT

W. REFRESH SCREEN
<-- See Map Analysis in the IBS User Guide.

- <-- Add tracking information for one facility.

- <-- Add information for a facility even if the facility does not actually exist at the moment.

- <- Remove information about one facility.

<-- List active facilities and facilities that have tracking information associated with them.

- -- Add new location to the points database.

- <-- Change the name/location of a known point.

* <- Delete known point location.

<- List all point names and long/lat coordinates.

<-- Register points to map locations.

<-- Import data like IMPORT utility

<-- Check Resource Management data like RMCHK utility

<-- Enlarge a portion of the current screen.

<- Expand the view to show a larger area.

<-- Redraw (and clean up) the current screen.

These capabilities are summarized on the following pages. 


\section{Modifying Facllity Tracks Information}

The facility tracks capabilities affect the contents of the facility tracking/loading data (contained in the FACILITY_TRACK.DAT file). This information includes the facility status (active or inactive) and people loading data for each facility: numbers of people received; current numbers of people; and numbers of people released.

\section{Caution}

Some of the facllty track capablities can corrupt data: see the specific capabllity descriptions here.

- ADD FACILTY TRACKS: This simulates the actions taken by the LOCATE program, enabling you to add facility track information for a specified existing facility. To specify which facility, you must enter the facility name.

- ADD FACIUTY TRACKS (F): This behaves exactly like ADD FACILITY TRACKS above, except that the information is added to the facility tracks file regardless of whether or not the facility you typed in really exists.

CAUTION: This function can easily corrupt your facility tracks database and should be used rarely (if ever).

D DELETE FACILITY TRACKS: This removes the facility tracks information from a facility. CAUTION: This function can easily corrupt your facility tracks database and should be used rarely (if ever).

aST FACIUTY TRACKS: This displays a list of facilities that are currently active and those that have a facility track associated with them.

\section{Modifying Known Points Information}

ADD KNOWN POINTS: This adds a new location to the database of Known Points. The following prompt appears first:

Enter new known Point name ==>

1. Enter any name (up to 40 characters) or press CTRL-Z to exit.

This location name also serves as the facility name for any facility that is added at that location.

If you enter a name that is already used for a location, the system prompts you to enter another name. After you enter a valid new location name, the following prompt appears: 
* * MSG: Select new Rnown Polnt location ueing mouse

2. Pick a location on the map screen.

If you pick a location that is already specified as a Known Point location, the system prompts you to select another point.

After you pick a valid location, that location is placed in the database of Known Points. The system then returns to step 1, where you can continue to add point locations until you quit by pressing CTRL-Z.

MODIFY KNOWN POINTS: This enables you to change either the name or location of a Known Point. First select a Known Point by using the cross hairs or one of the other methods on the Known Points Selection menu:

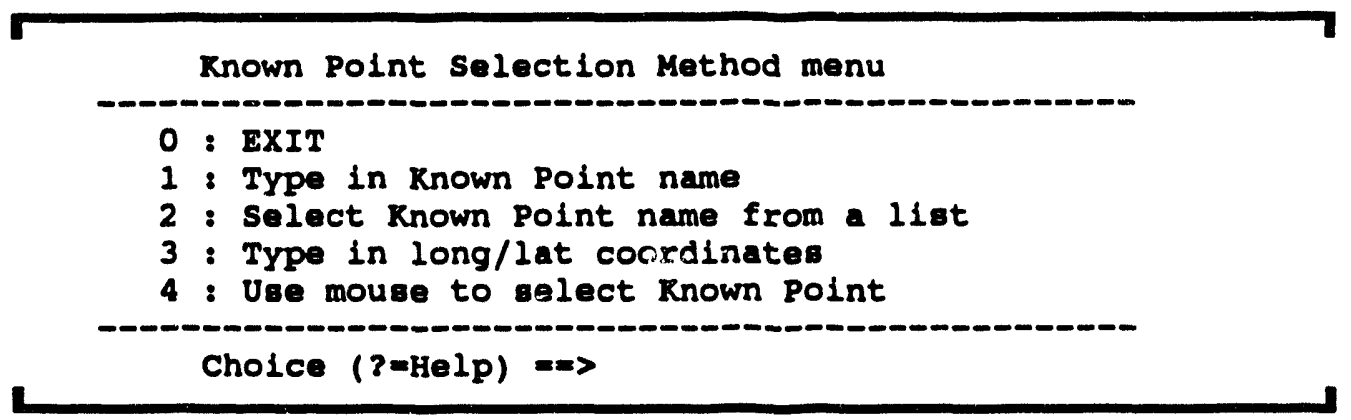

After you have selected a point, the program displays:

Modify the Rnown Point name? $[Y]=>$

- To change the name, enter $\mathbf{Y}$. The program displays:

Enter new known Point name $=2$

Enter any name (up to 40 characters) or press CTRL-Z to exit. If you enter a name that is already used for a location, the system prompts you to enter another name.

- To change the Known Point location (and its associated facility, if any), enter N. Select the new location for the Known Point with the cross hairs, and the Known Point location is changed.

DELETE KNOWN POINTS: This enables you to remove any locations from your database of Known Points. First select a Known Point. If there is no facility located at that point, the point is removed immediately. If there is a facility there, the program asks if you really want to delete this facility: To confirm the deletion and remove the facility, enter $\mathbf{Y}$. 
LST KNOWN POINTS: This lists all Known Points, giving the longitude and latitude of the point as well as the Known Point names.

- GeO-LOCATE KNOWN POINTS: This enables you to locate Known Points (and any associated facility) on the map display. This option is similar to Modify Known Points in RMMGR but has some changes to help deal with points that are not found in the current map area. (Using MODIFY FACILITY-MODIFY KNOWN POINT in the IBS program is another method to change the Known Point's location.)

You will initially receive the following menu (unless you have set defaults for selecting items using Setup - Job Environment options.)

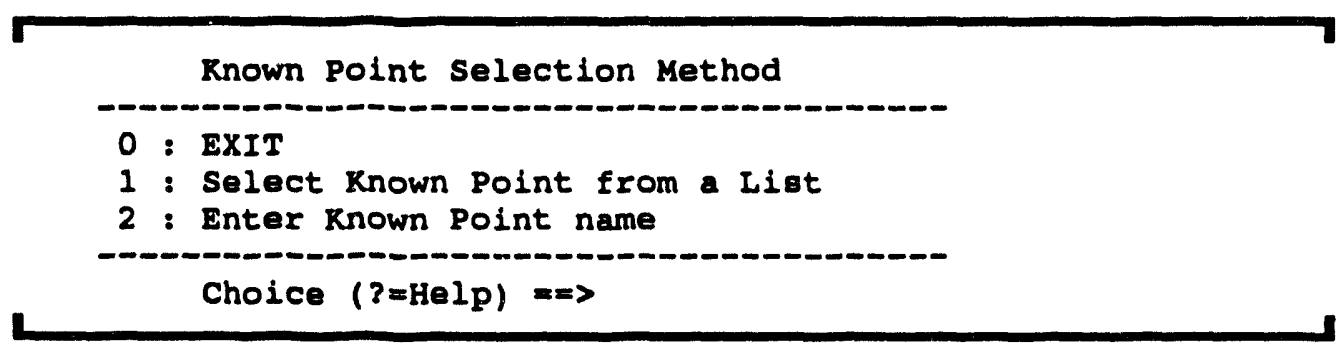

1. Select whether you wish to pick Known Points either from a list or by typing in the full name.

- If you selected to pick the Known Points from a list, you :vill see the following menu. Note that an arrow $<==$ to the right of an item indicates that it needs to be Located on the Map.

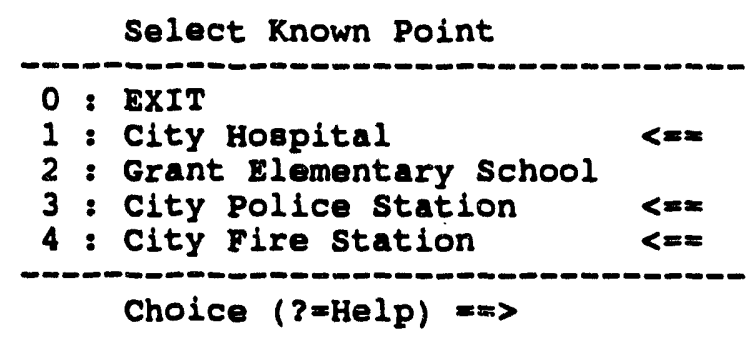

- If you selected typing in the Known Point name, you will receive the following prompt:

Enter known point name $==>$

You must enter the full name of the Point whose location you wish to change.

You will then receive the following menu (unless you have set defaults for selecting items using SETUP-JOB ENVIRONMENT options.) 


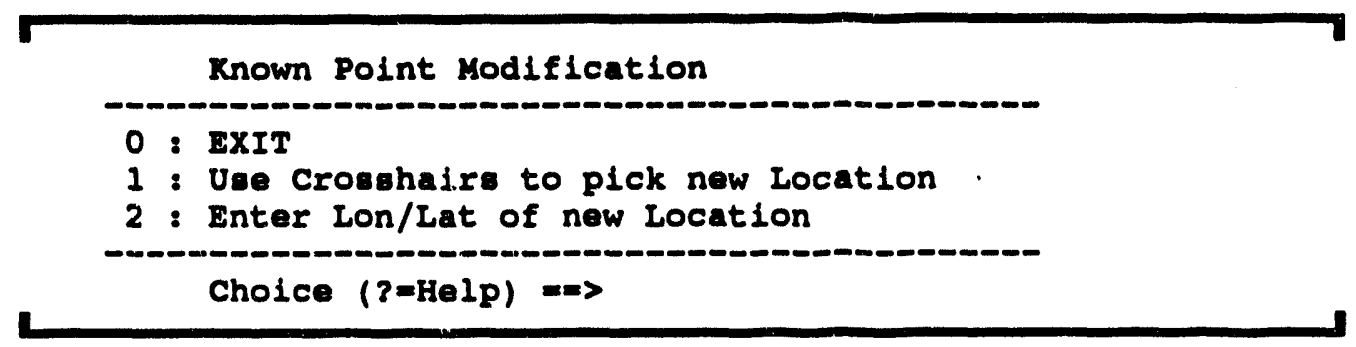

2. Select whether you want to pick the new location by either Pointing to the location on the current map display, or typing in the Longitude/Latitude coordinates of the point. You will receive the following prompt if you selected the first option:

* * * MSG: Select new Rnown Point location w/ mouse

3. Move the crosshairs over new location for the point and press a key.

Enter Longitude in either Decimal DDD.DDDD or Minute/Second DDD, MN, SS form. = $=>$

Enter Latitude in either Decimal DDD.DDDD or Minute/Second DDD, MA, SS form. $=\Rightarrow$

4. Type in the Longitude and Latitude coordinates for the new point.

IMPORT DATA: This function is the same as the IMPORT utility described elsewhere in this Utilities Guide.

CHECK RM DATA: This function is the same as the RMCHK utility described elsewhere in this Utilities Guide. 


\section{Using the AG, PER, POS Menu}

The Agency, Personnel, Position Menu duplicates many of the capabilities of the PERSONNel MANAGeMENT menu in the IBS program. (See Personnel Management in the IBS User Guide.) The descriptions of items that are unique to RMMGR are highlighted in bold below.

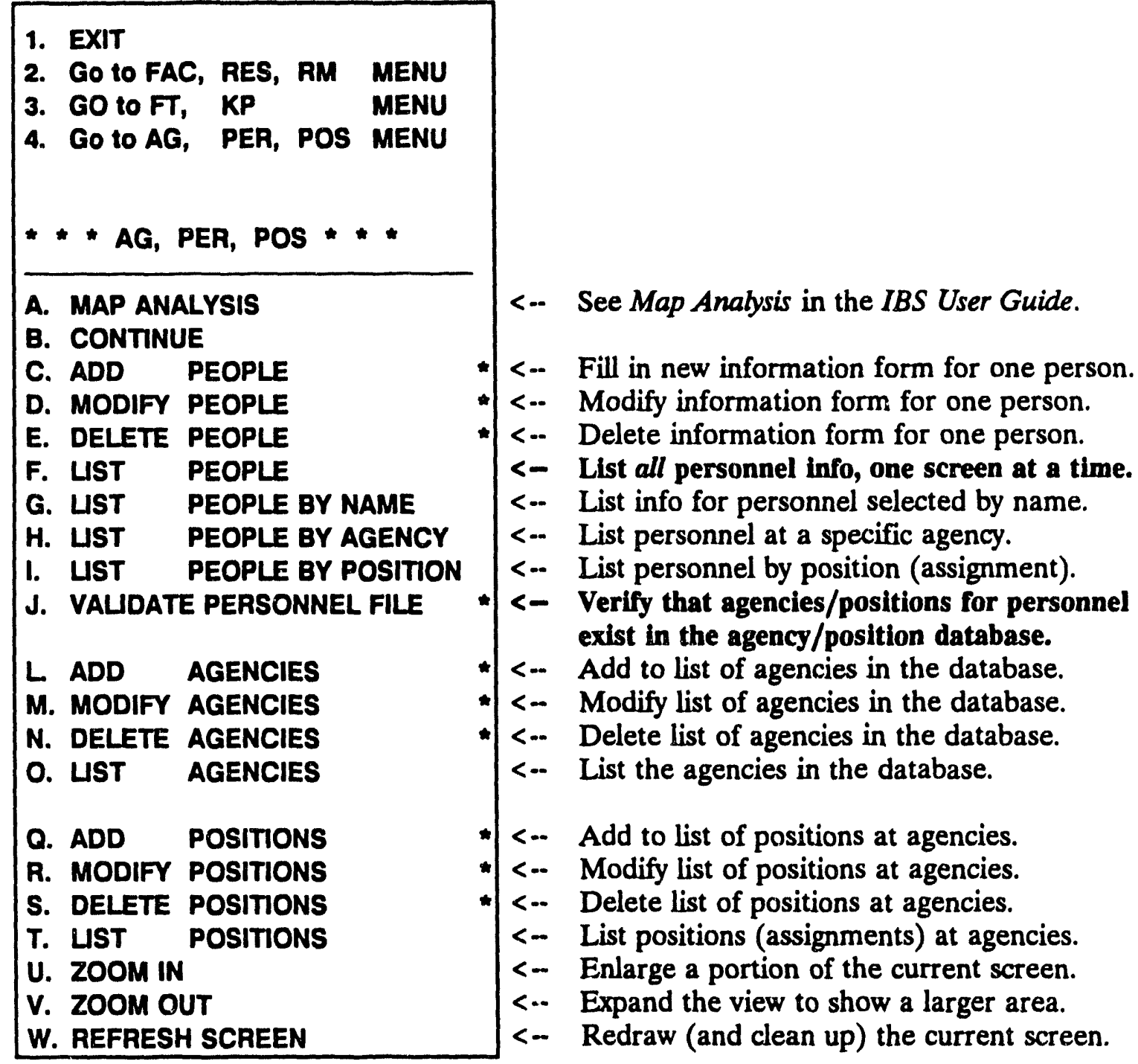

The two unique capabilities, LIST PEOPLE and VALDATE PERSONNEL FILE, are explained here. 
a UST PEOPLE: This lists all information in the personnel database, one screenful at a time.

- VAUDATE PERSONNel file: This verifies that all agencies and positions listed in the personnel database actually exist in the agency/position database.

If a discrepancy is found, the utility gives you the following options for handling the discrepancy:

$\mathbf{Y}$ : The data is correct; add it to the agency/position database.

$\mathbf{N}$ : The data in NOT correct; select an actual agency/position from a list.

C : The data is correct, but do the next Position for this person.

$\mathbf{X}$ : Done. Stop fixing the agency/position file and save changes.

Q : Done. Stop fixing the file, but do NOT save changes. 


\section{RMMGR_NOGR}

The RMMGR_NOGR (Resource Management ManaGeR, NOn-GRaphic) utility is a version of the RMMGR program that contains many of the same functions but which does not display maps or require a graphics terminal. Like RMMGR, the RMMGR_NOGR utility is a tool for managing information about the personnel, resources, and facilities that may be needed to handle an event.

With a few exceptions, the capabilities of RMMGR_NOGR are duplicated in RMMGR and in the Resource Management, Personnel Management, and Map Analysis portions of the IBS program. Described here are the overall menus and operation of the RMMGR_NOGR program. For background, terminology, and more specific operational descriptions, please refer to RMMGR and to the IBS User Guide.

Unique in RMMGR_NOGR (in the current version of IBS):

- the capability to "undefine" resources which expands on the capability to delete resources by enabling you not only to delete resources at facilities but also to delete the definition of a resource.

- independent capability to specify and list special populations, people who have special requirements in case of an event.

\section{What You Need to Know Before Using RMMGR_NOGR}

All operational menus in RMMGR_NOGR are text menus. You can choose the menu options only by typing a letter or number that corresponds to the item of your choice (rather than by selecting items with a mouse or joydisk). These menu options generally correspond to similar options in the RMMGR program.

As in RMMGR, any option that requires special privileges is followed 'y an asterisk *. If you do not have privileges to use an option, or it is unavailable in your current site, it will NOT appear in the menu of choices. The other menu options are then moved up on the menu accoridingly and leave no blank spaces. Therefore the actual screens you see may look somewhat different from the examples shown here.

\section{Managing Special IBS Data with RMMGR_NOGR}

To begin the program, enter RUN IEMIS\$EXE::RMMGR_NOGR at the system prompt. This displays the "Non-Graphic RMMGR Main Menu," which gives you access to the other principal menus for managing the eight kinds of data listed in the main menu. 


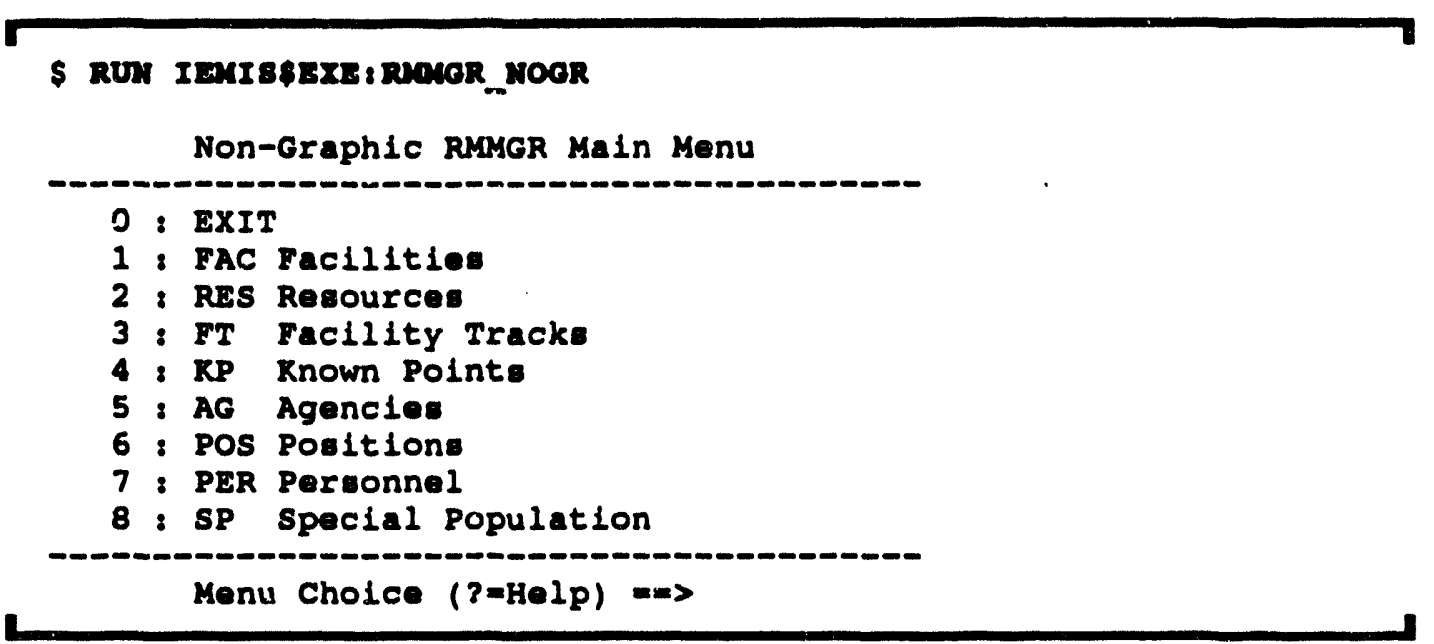

To continue, enter the index number associated with one kind of data.

The menus for each type of data are illustrated in the following sections.

\section{1 : Facilities}

The Facility Management menu includes options for working with facilities data:

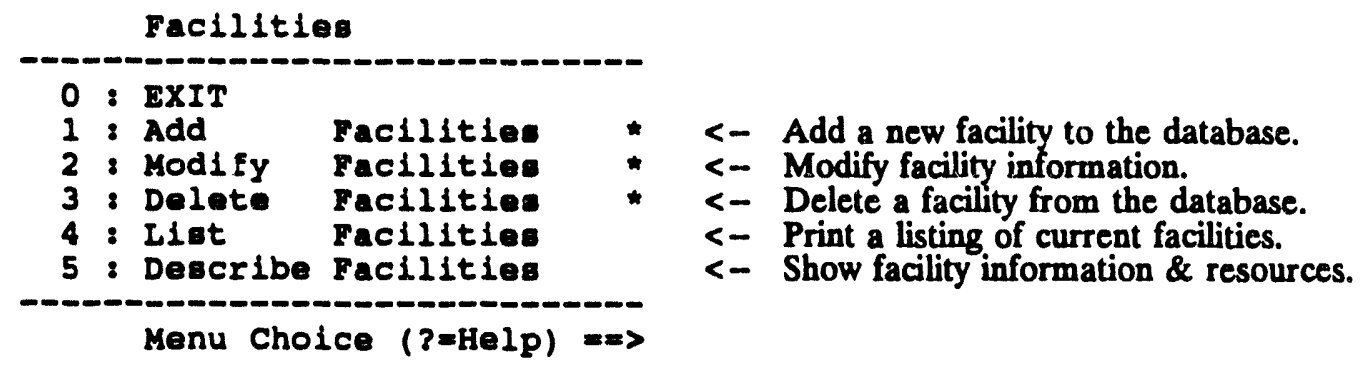

EXIT (0) on this menu returns you to the Non-Graphic RMMGR Main Menu.

The other options correspond to the same options on the RESOURCE

MANAGEMENT menus in the IBS program. For instructions on using these

capabilities, see Resource Management in the IBS User Guide. 


\section{2 : Resources}

The Resource Management menu includes options for working with resource data:

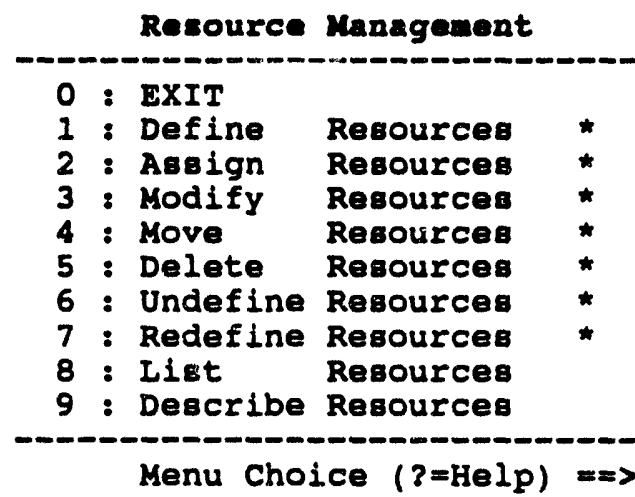

<- Define a new resource.

<- Assign resources to a facility.

<- Modify resource information.

<- Move resources to another facility.

<- Delete a resource from the database.

$<-$ Cancel a resource definition.

<- Edit a resource description and units.

<- Print a listing of current resources.

$<-$ Show information about a resource.

EXIT (0) on this menu returns you to the Non-Graphic RMMGR Main Menu. Options 1-5, 8, and 9 correspond to the same options on the RESOURCE MANAGEMENT menus in the IBS program. For instructions on using these capabilities, see Resource Management in the IBS User Guide. The additional options to Undefine Resources and Redefine Resources are explained here.

Selecting Resources. Both Undefine Resources and Redefine Resources require you to select a resource on which to operate. The general sequence for selecting a resource is similar for both options. The system prompts:

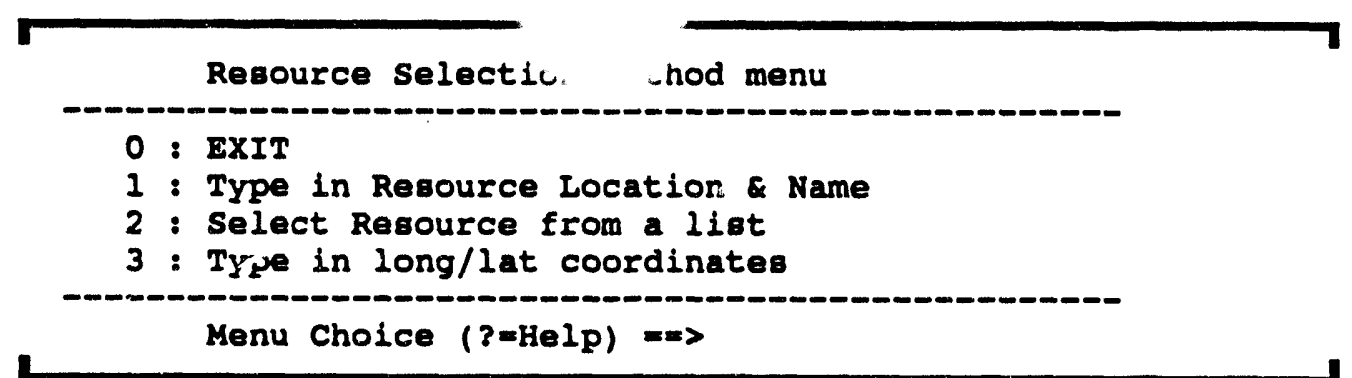

Use one of the following three methods to identify a resource:

1: Type in Resource Location \& Description: Prompts you to enter the name of an existing facility (location) and a resource description.

Enter Resource location $\Rightarrow=>$

Enter Resource description $=>$

2: Select Resource irom a list: Prompts you to choose one from a numbered list of resources at various facilities. For example: 


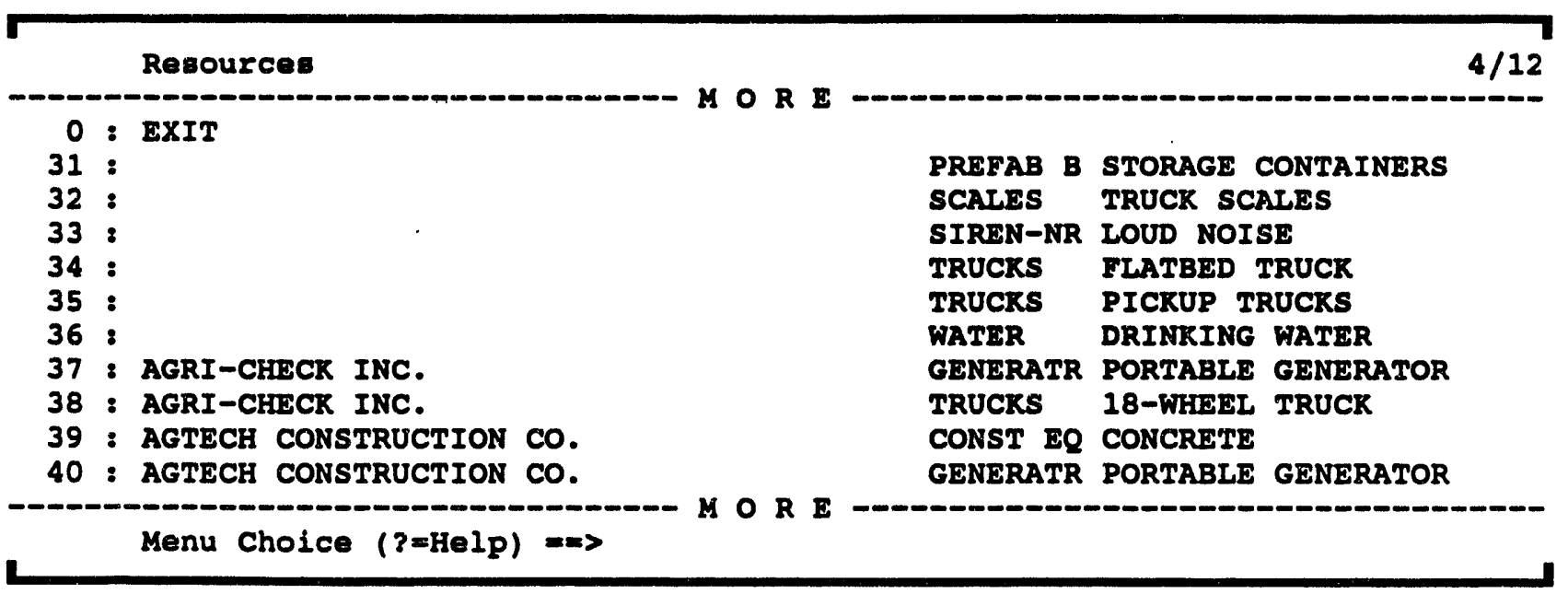

Facilities (locations) are listed alphabetically in the left column.

Resource types and specific resource descriptions are listed in the right column. If no specific instance of a defined resource is assigned to a facility, then no facility name is listed (as shown in the example for items 31 through 36).

Enter the number that corresponds to the desired resource, or enter 0 to exit.

3 : Type in long/lat coordinates: Prompts you for the longitude and then the latitude coordinates of the resource location.

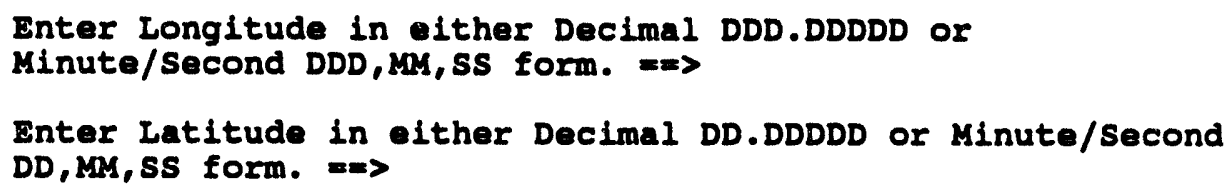

- Undefine Resources: This enables you to 1) delete a selected resource located at a specific facility location or 2) delete the definition of resource, disassociating a specific resource (such as "bandages") from its defined resource type (such as "1st Aid"). If you delete the definition of a resource, the resource can no longer be assigned to a specific facility/location.

Note: To protect the integrity of the data, RMMGR_NOGR prevents the deletion of a resource definition if some instance of that resource is currently assigned to a location. Before you can delete the definition of a resource, you must first delete any specific instances of that resource.

After you select a resource by way of the Resource Selection method menu, that resource (or resource definition) is deleted, and the program returns to 
the Resource Selection method menu.

To exit, select 0 (EXIT). If you have deleted assigned resources, this warning displays when you exit the Resource Selection method menu:

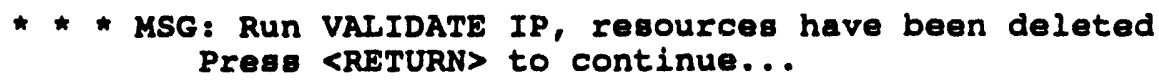

The VAUDATE IP option for validating Implementing Procedures (IPs) is available in the Status Board menu of the IBS program.

- Redefine Resources: This enables you to redefine a resource by changing the resource description and/or units of measure. All facilities that contain this resource will be updated in the resource database. Before showing the Resource Selection method menu, this option displays the following warning:

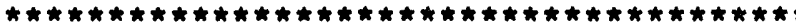

W A R N I N G I

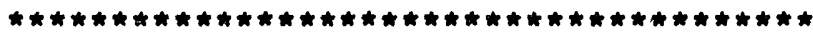

This function allows you to edit the resource Description and Units. Any IP that contains the resource WILL BE INVALIDATED!

Are you sure this is what you want to do? $(Y / N)=m$

Be sure you understand the consequences of changing the data before you answer yes $(Y)$ to the prompt. After you select a resource by way of the Resource Selection method menu, the information for that resource is displayed in a resource form similar to the following.

Example:

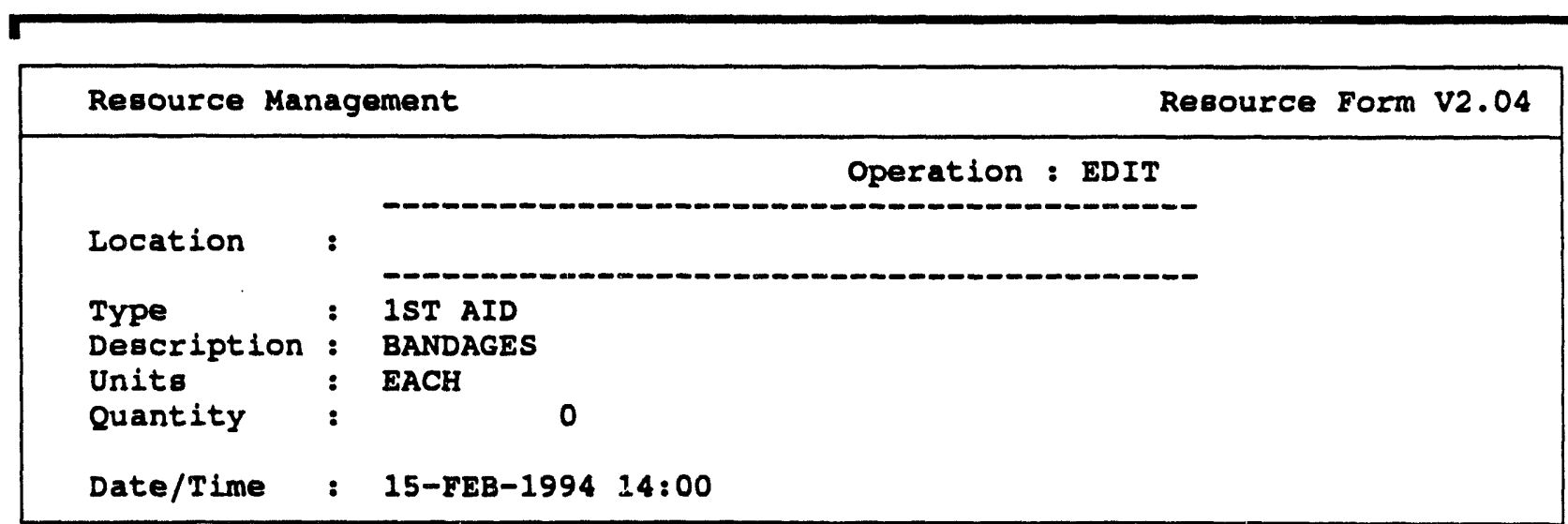

Only the resource description, units, and date/time can be changed on this form. The resource type and quantity cannot be changed. If you make a change, you will 
see a summary of the change and a final chance to change your mind.

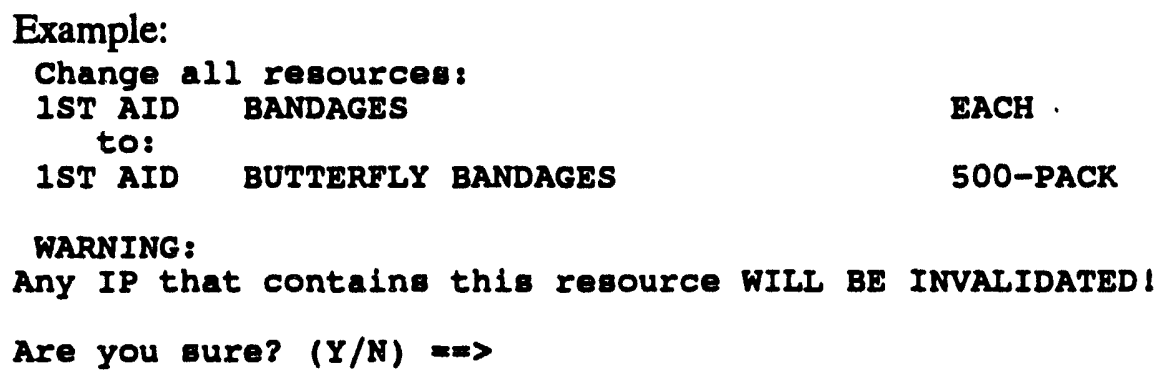

After you confirm (or cancel) the change, the Resource Selection method menu reappears for another selection.

\section{3 : Facility Tracks}

The facility tracks capabilities affect the contents of the facility tracking/loading data (contained in the FACILITY_TRACK.DAT file). This information includes the facility status (active or inactive) and people loading data for each facility: numbers of people received; current numbers of people; and numbers of people released. The Facility Tracks menu includes options for working with this data:

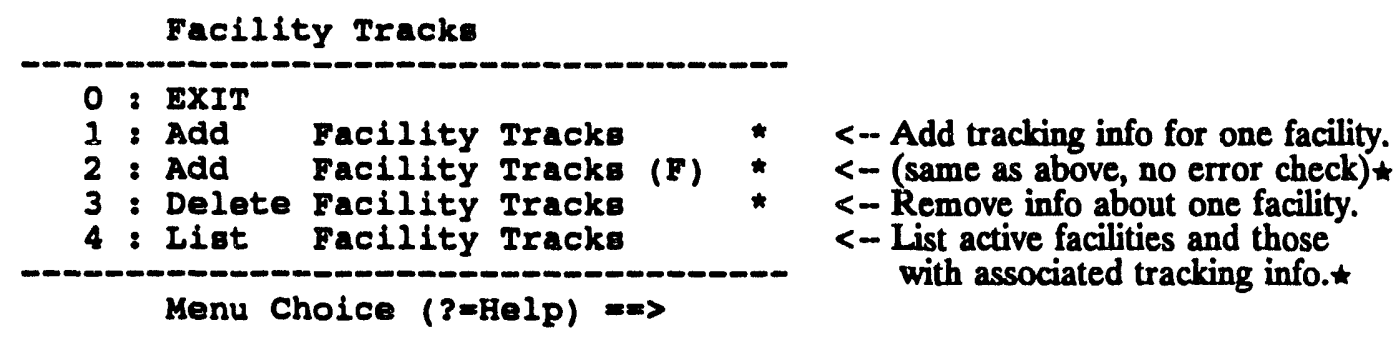

\section{Caution}

$\star$ Some of the facility tracks capabllities can corrupt data: 800 the specific capability descriptions for the FT, KP Menu in the RMMGR program.

Selecting EXIT (0) returns you to the RMMGR_NOGR main menu. The other options correspond to the menu choices of the same names in the FT, KP Menu in the RMMGR program. For instructions on using these capabilities, see Using the FT, KP Menu under RMMGR.

\section{4 : Known Points}

The Known Points menu enables you to directly add, delete, modify, or list database information about known points (point locations such as the locations of facilities). 


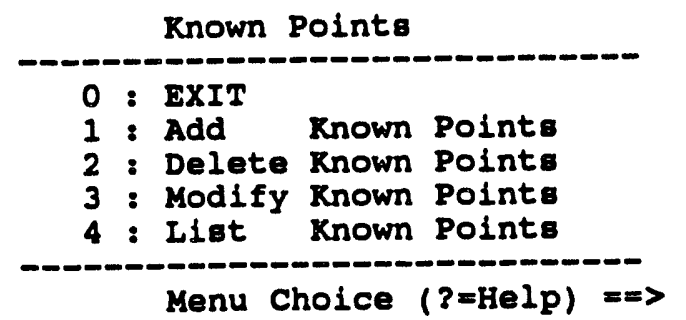

<- Add new location to the points database.

<- Delete known point location.

<- Change name/location of a known point.

$<-$ List all point names and long/lat coordinates.

Selecting EXIT (0) returns you to the RMMGR_NOGR main menu. The other options correspond to the menu choices of the same names in the FT, KP Menu in the RMMGR program. For instructions on using these capabilities, see Using the FT, KP Menu under RMMGR.

\section{5 : Agencies}

The Agencies menu enables you to add, modify, delete, or list information that pertains to agencies in the IBS personnel database.

\begin{aligned} & Agencies \\ \hline $0:$ ExIT & \\ $1:$ Add Agencies & $\star \\ 2:$ Modify Agencies & $* \\ 3:$ Delete Agencies & \\ $4:$ List Agencies & \end{aligned}

<- Add to list of agencies in the database. <- Modify list of agencies in the database. <- Delete list of agencies in the database.

Menu Choice $(z=$ Help) $\Rightarrow>$

Selecting EXIT (0) returns you to the RMMGR_NOGR main menu. The other options correspond to the menu choices of the same names the PERSONNEL MANAGEMENT menu in the IBS program. For instructions on using these capabilities, see Personnel Management in the IBS User Guide.

\section{6 : Positions}

The Positions menu enables you to add, modify, delete, or list information that pertains to job positions (assignments) in the IBS personnel database.

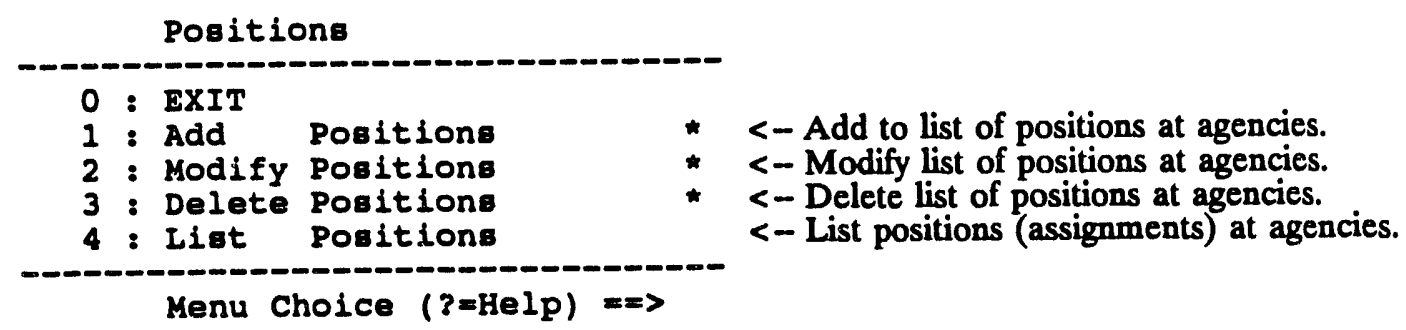

Selecting EXIT (0) returns you to the RMMGR_NOGR main menu. The other options correspond to the same options on the PERSONNEL MANAGEMENT menu 
in the IBS program. For instructions on using these capabilities, see Personnel Management in the IBS User Guide.

\section{7 : Personnel}

The Personnel menu enables you to add, modify, delete, or list information that pertains to people in the IBS personnel database.

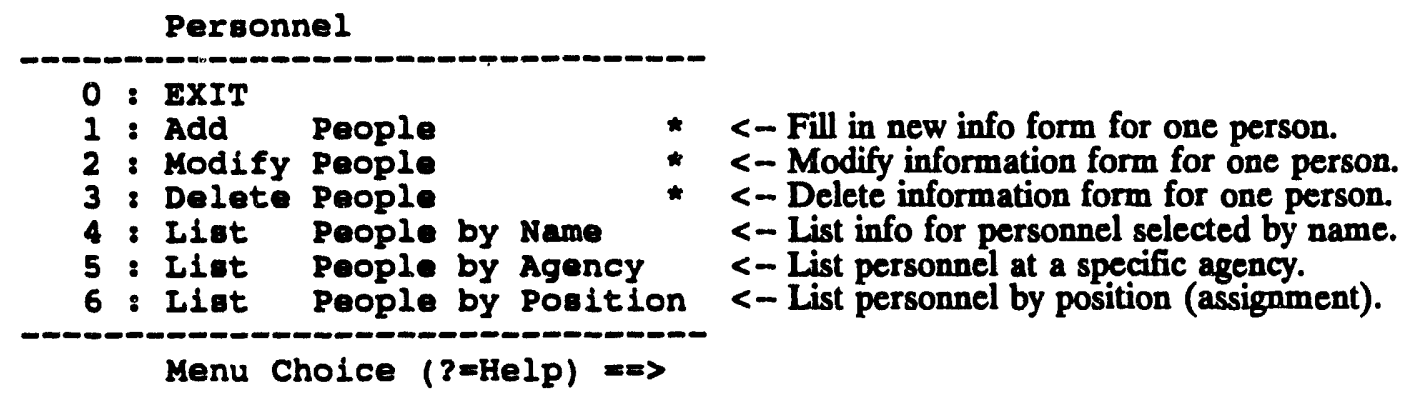

Selecting EXIT (0) returns you to the RMMGR_NOGR main menu. The other options correspond to the same options on the PERSONNEL MANAGEMENT menu in the IBS program. For instructions on using these capabilities, see Personnel Management in the IBS User Guide.

\section{8 : Special Population}

The Special Population menu enables you to add, update, delete, or list information that pertains to groups of people who have special requirements such as needs for notification or transportation.

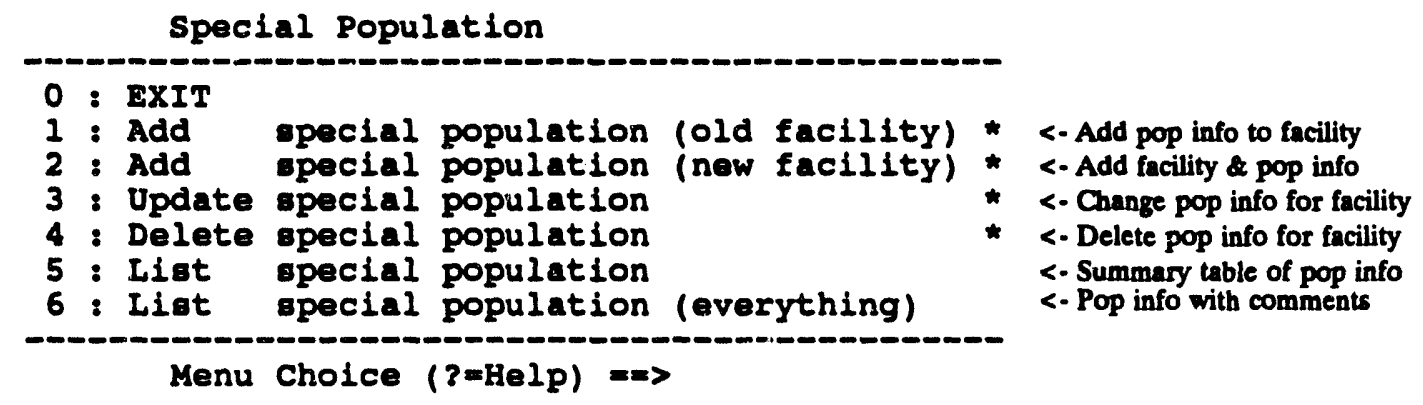

Selecting Facilities for Special Populations. The options on the Special Population menu first ask you to select (or add) the facility where the special population resides. The general sequence for selecting an existing facility begins with the Facility Selection method menu shown here. 


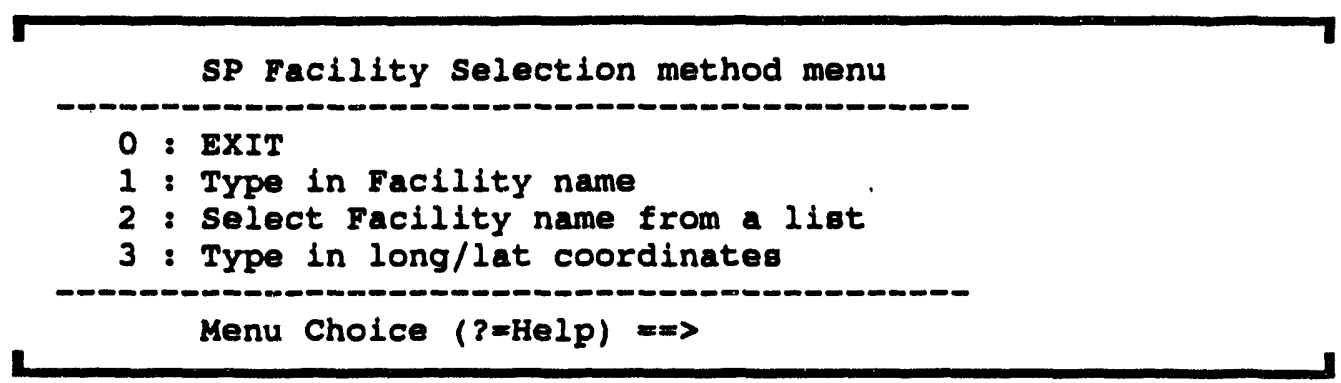

Use one of the three methods shown for selecting a facility: 1) type in the facility name, 2) select the facility name from a list, or 3) type in specific long/lat coordinates.

- Add special population (old facility): After you select a facility (using the SP Facility Selection method menu), RMMGR NOGR displays a form for entering special population information similar to the following example:

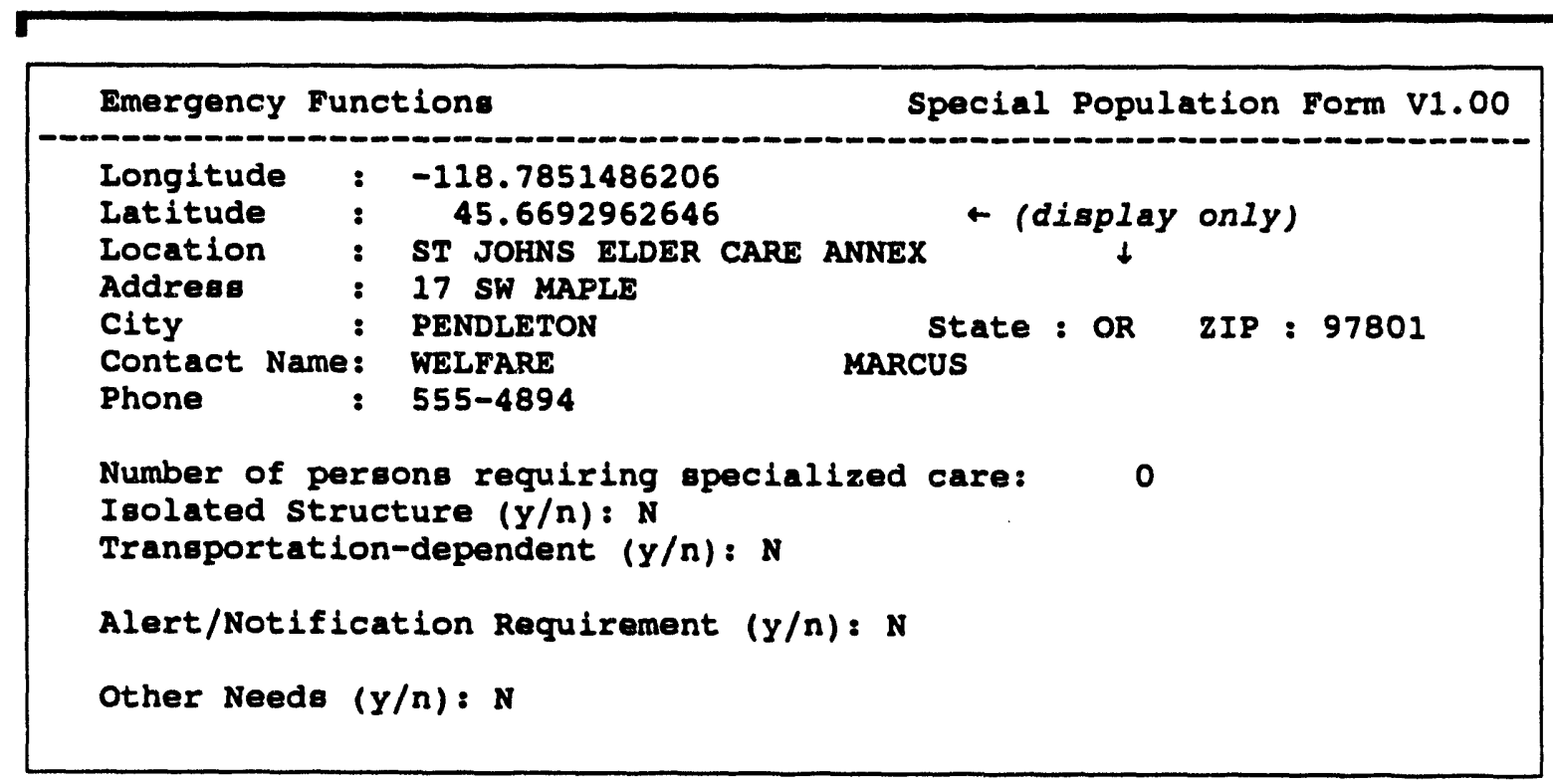

The upper part of the form is only for displaying facility information. The lower part of the form is for entering information about the special population. Enter the following information on the form.

Number of persons requiring specialized care: Enter the number of persons at the facility requiring specialized care (special population).

Isolated Structure $(\geqslant / \mathbf{n}):$ Y or $\mathbf{N}$-. Is the special population located at an isolated structure? 
Transportation-dependent $(\mathbf{y} / \mathbf{n}): \mathrm{Y}$ or $\mathrm{N}$-- Is the special population dependent on others for transportation? Use the following line on the form for adding comments.

Alert/Notification Requirement (y/n): $\mathrm{Y}$ or $\mathrm{N}$-- Is an alert/notification required for this special population? Use the following line on the form for adding comments.

Other Needs $(y / n): Y$ or $N$-- Are there other additional needs? Use the following line on the form for adding comments.

- Add special population (new facility): This option enables you to add a new facility before characterizing a special population at the new facility. For instructions on adding a facility, see ADD FACILTIES in the Resource

Management section of the IBS User Guide. After adding the facility, you then use the same Special Population form as in the previous option.

- Update special population: After you select a facility (using the SP Facility Selection method menu), RMMGR NOGR displays the Special Population form for modifying the special population data at the facility.

- Delete special population: After you select a facility (using the SP Facility Selection method menu), RMMGR_NOGR displays the following prompt to verify that you want to delete the special population data at this facility:

Delete special population: facilityname $(Y / N)[N]=m$ -

Enter ' $Y$ ' or <ENTER> to delete the special population data at the facility.

- List special population: RMMGR_NOGR displays a tabular listing of facilities that include special populations and a summary of the considerations specified for isolated structure, transportation dependency, alert/notification, and "other"-.Yes or No for each. (See the example on the next page.)

- List special population (everything): RMMGR_NOGR displays a similar tabular listing but also includes any comments made for each consideration of isolated structure, transportation dependency, alert/notification, and "other." (See the example on the next page.) 
Example: List special population

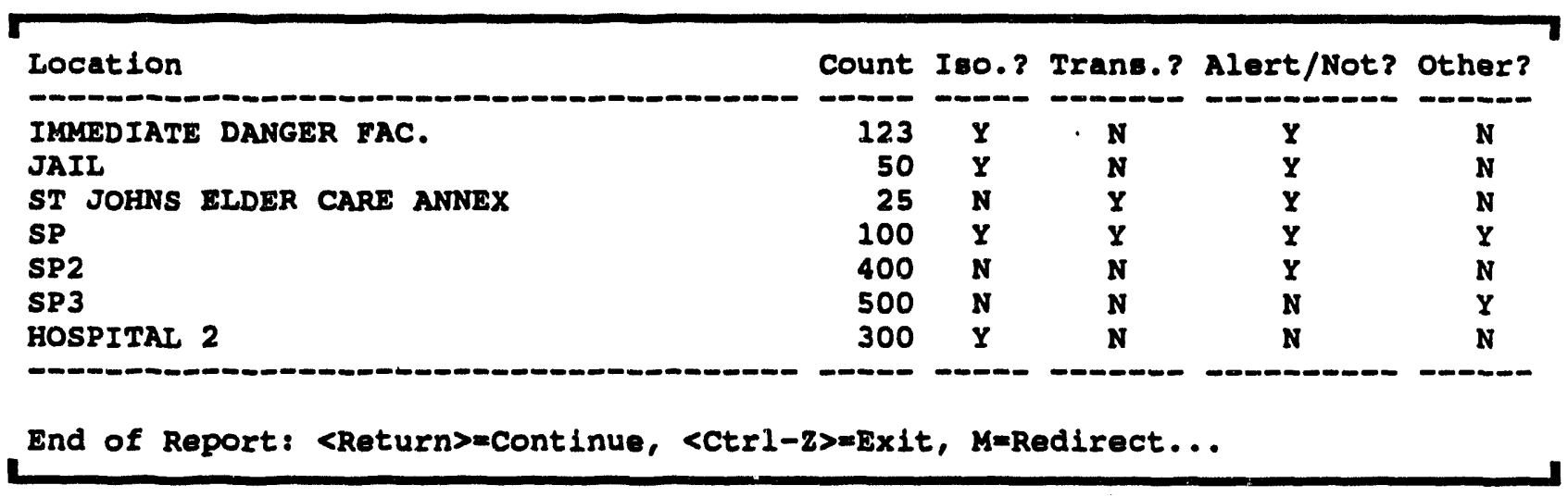

Example: List special population (everything)

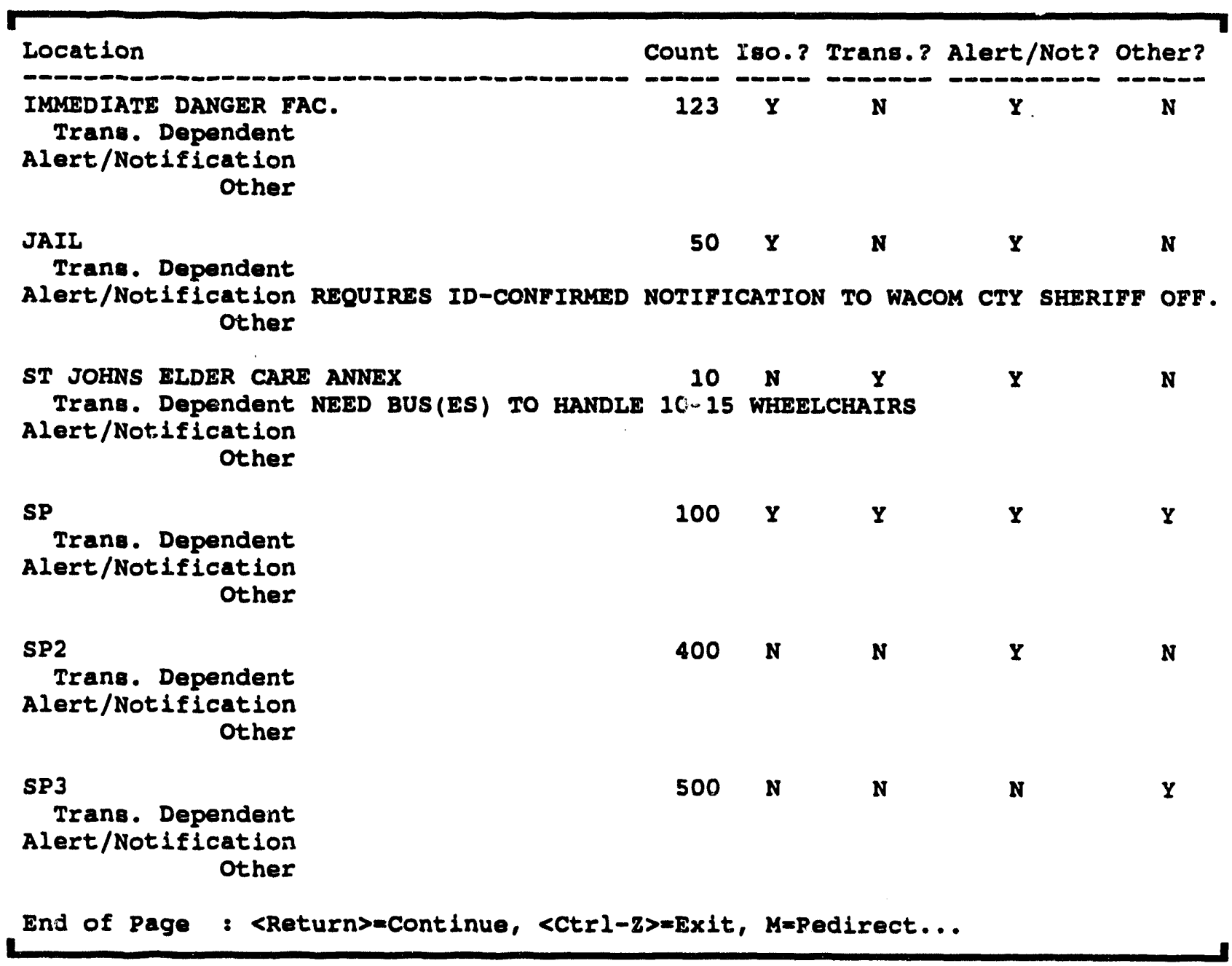




\section{SCALEDMS}

The SCALEDMS utility is a data file modification utility that reads a DMS file and creates a rescaled DMS file based on an $X, Y$ scale that you specify.

\section{What You Need to Know Before Using SCALEDMS}

In addition to the name of the input DMS file and a new output filename for the rescaled DMS file, SCALEDMS prompts for several items:

center point SCALEDMS enables you to change the center point of the DMS file. If you want to do this, you must know the longitude and latitude (in decimal degrees) of the new center point.

$X$ scale factor This is a horizontal scale factor (west-east).

$Y$ scale factor This is a vertical scale factor (north-south).

\section{Scaling a DMS File with SCALEDMS}

To start this utility, enter SCALEDMS at the system prompt. SCALEDMS prosnpts you for the information as shown in the sequence below..

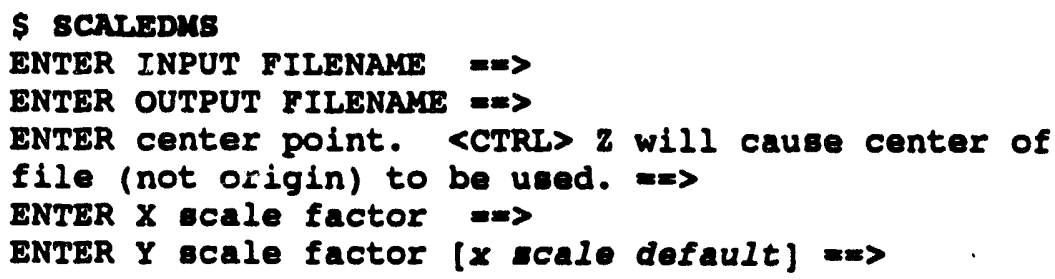

If you wish to specify a new center point for the new file, enter the longitude and latitude separated by a comma. If you wish to use the same center point as the input file, press CTRL-Z at the center point prompt; the center point will be echoed back to you:

CENTER POINT = [longitude, latitude]

Once you have input the $\mathrm{X}$ scale, the $\mathrm{Y}$ scale defaults to the same scale unless you override it by entering a different value.

After you supply all information, SCALEDMS creates the new rescaled DMS file. All objects in the file are rescaled according to the $\mathrm{X}, \mathrm{Y}$ scale factors. 


\section{SEELIMITS}

The SEELIMITS utility enables you to display the contents of a binary limit file or to save those contents in a readable ASCII text file.

\section{What You Need to Know Before Using SEELIMITS}

A binary limit file (LIMITxx.BIN) is a database support file located in each topography (map layer) subdirectory of a DMS geographic database. It specifies the names and geographic extents of all the topographic data files in the subdirectory (where " $2 x^{n}$ is the two-character code for that topography and also the subdirectory name). To create a binary limit file, you can use the CREATELIMITS utility.

The SEELIMITS utility asks you to specify two items:

input binary

limits filename

output filename
This is the name of the limit file that you want to display or to save in ASCII form. If the limit file is not in the current default directory, you should specify the path along with the filename.

This is the name of the ASCII file that you want to create.

\section{Displaying the Contents of a Limit file with SEELIMITS}

The following example shows how to list the binary limit file, LIMITWB.BIN, on the screen. Assumption for this example: You have set the default directory to be the MAPSDATASWB directory.

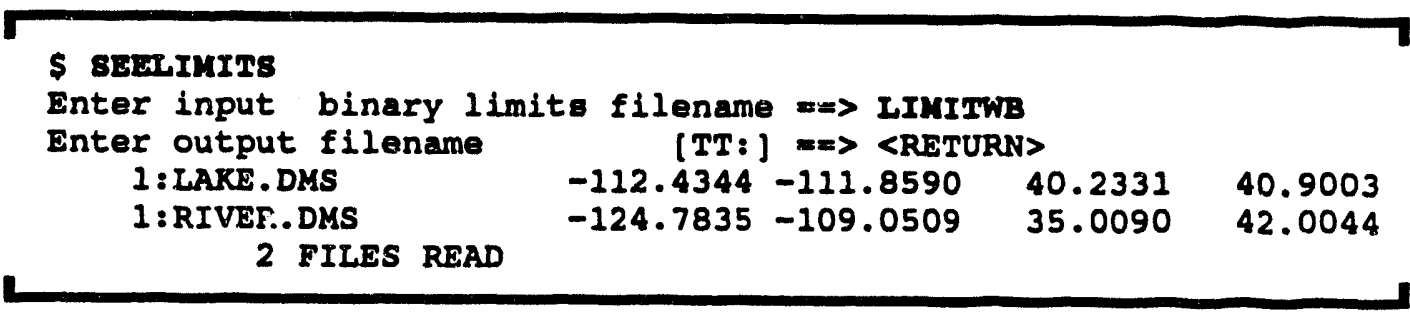

Result: The name and extents of each DMS topography file are displayed on the screen as shown. 


\section{Saving the Contents of a Limit file with SEELIMITS}

The following example shows how to save the same binary limit file to an ASCII file, LIMITWB.DAT. Assumption for this example: You have NOT set the default directory to be the MAPSDATASWB directory. Therefore the name of the path must be included with the input binary limit filename.

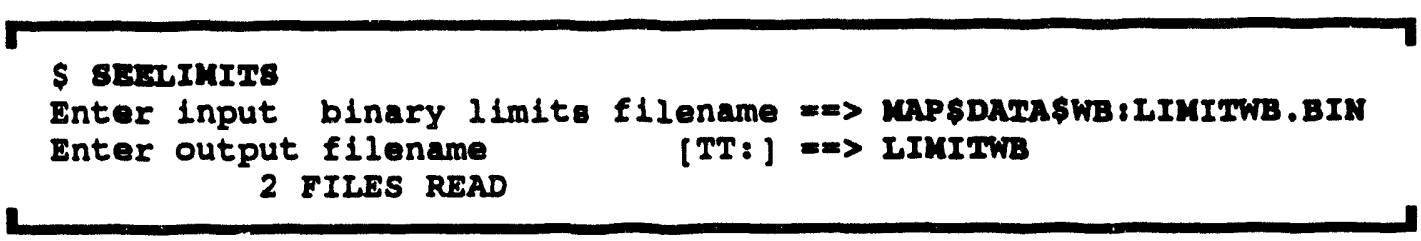

Result: The text file LIMITWB.DAT is created in your current default directory. 


\section{SEELIMITXX}

The SEELIMITXX utility is a database administration program that reads a specified binary limit file (LIMITxx.BIN) and creates an ASCII version (.DAT) listing the DMS (and TAC) files associated with the xx topography.

See also the DOSEELIMITXX utility, which runs SEELIMITXX for all of the topographies of a database.

\section{Creating an ASCII Version of a Binary Limit file}

To start this utility, enter

\section{SEELIMITXX [. $x x]$ LIMITxx.BIN [. .xx]LIMITJex.DAT}

at the system prompt, where $x x$ is the two-character topographic code, the first file name is the .BIN file to be read, and the second .DAT file name is the new ASCII file. If you do not specify the input limit file, you will be prompted. Although the output file can be any name you want, the format shown above is recommended for clarity.

Example:

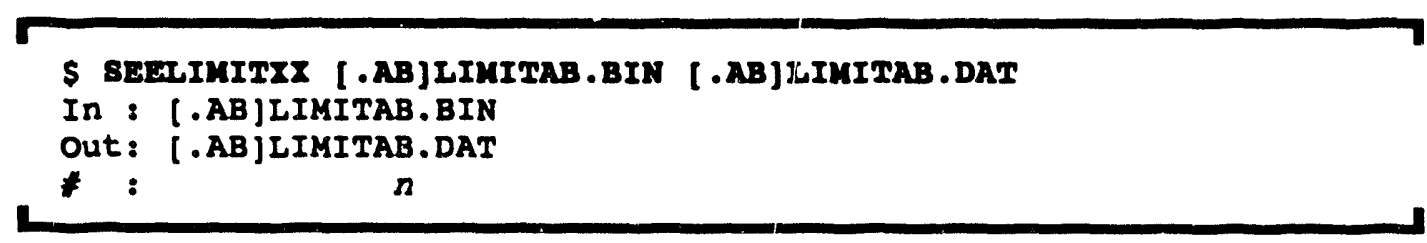

Example:

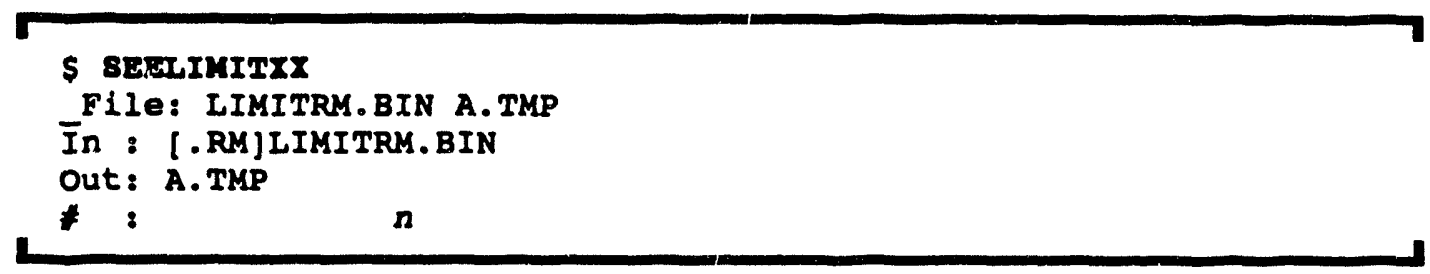




\section{SEETXTNM}

The SEETXTNM utility is a diagnostic and reporting prngram that displays the text (.TXT) file name associated with a specified DMS file.

\section{Seeing the Name of a DMS File's Text File with SEETXTNM}

To start this utility, enter SEETXTNM filename at the system prompt, where filename is the name of the DMS file for which you wish to find a .TXT name.

The output lists the DMS file name followed by the associated .TXT file name. 


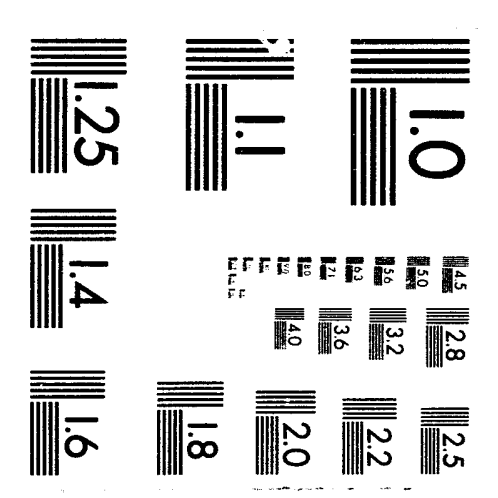



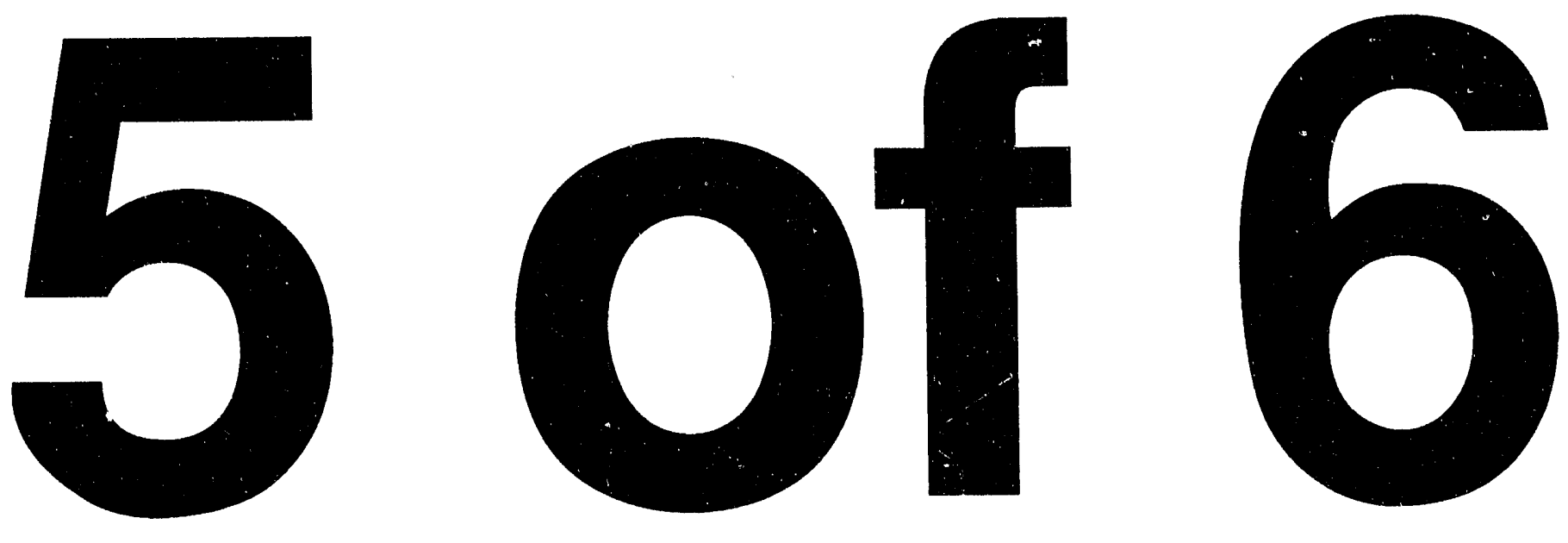


\section{SEQ2RND}

The SEQ2RND utility is a data file modification program that converts a sequential file to a random (direct) access file. This can be useful for fixing random access files that have been converted to sequential files by a file transfer process. The utility can also be of help in recovering data files that have been corrupted by ASCII editing, such as resource data files.

In addition to the name of the sequential file and a new name for the random access file, you must supply an appropriate record length for the file.

\section{Generating a Random Access File based on a Sequential File}

To start this utility, enter RUN IEMIS\$EXE:SEQ2RND at the system prompt. The program presents a series of prompts as shown.

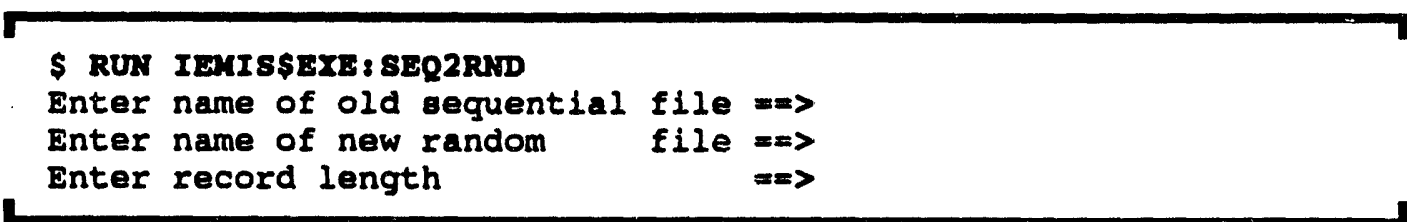

After you have entered a record length, SEQ2RND generates a new random access file based on the old sequential file. 


\section{SETUPTEK}

The SETUPTEK utility is a general system program that initializes a Tektronix 41 nn terminal for graphical and dialog use. You can use this to return the terminal to default characteristics.

See the TEKSETUP utility for setting customized terminal screen background and foreground colors.

\section{Returning a Tektronix Terminal to Default Settings with SETUPTEK}

To start this utility, enter SETUPTEK at the system prompt.

SETUPTEK then clears the screen and applies the default screen parameters. 


\section{SHOWATT}

The SHOWATT utility is a diagnostic and reporting program that enables you to specify an attribute value in one of three number bases (octal, hexadecimal, decimal) and convert the value to major/minor/parameter values. This is primarily a debugging tool for working with attribute values as they sometime appear in the software.

\section{Showing Equivalent Attribute Values in Octal/Hexadecimal/Decimal}

To start this utility, enter SHOWATT at the system prompt. SHOWATT displays a menu for converting among the number bases. In the following example, the decimal number 10 is shown as hexadecimal $0000000 \mathrm{~A}$ and octal 000000000012 . The major code is 0 ; the minor code is 10 ; and the parameter is 0 .

Example:

\$ 8BOWATT

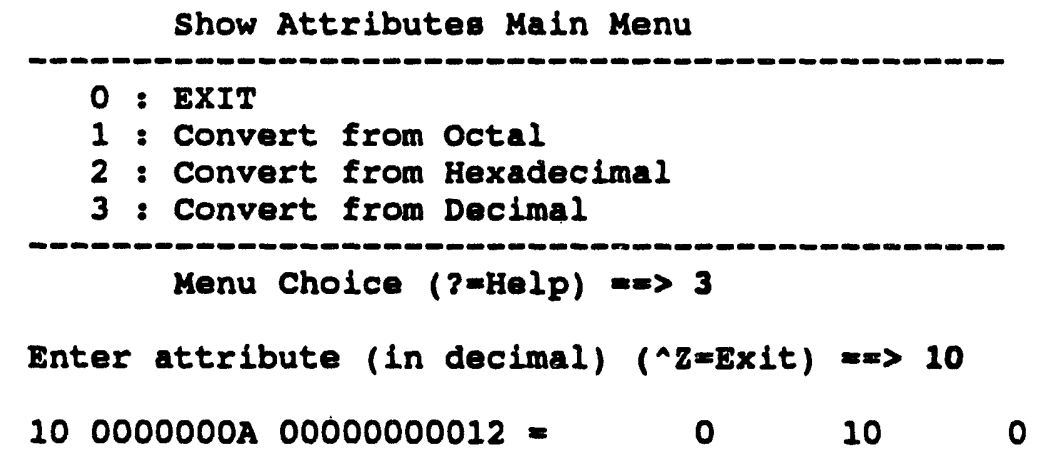

1. Select an option $(1,2$, or 3$)$. SHOWATT then prompts you to enter a value to convert (the numberbase is the base you just chose):

Enter attribute (in numberbase) ( $\mathrm{Z}=\mathrm{Exit}$ ) $=>$

2. Enter the number to be converted from the selected number base.

OR

Press CTRL-Z to exit the program.

OR

Press RETURN to return to the Show Attributes Main Menu.

If you enter a number to be converted, the number is redisplayed in decimal, hexadecimal, and octal (in that order). The major, minor, and parameter values are also displayed. 


\section{SHOWCNX}

The SHOWCNX utility is a diagnostic and reporting utility that shows the contents of the Case Index file (CASEINDEX.DAT) for a specified site.

\section{Showing the Model Case Index File for a Site}

Before starting SHOWCNX, first set the default directory to the site directory containing the caseindex of interest. Site directories are of the form disk:[user.SITES.site] where disk is the device name, user is the user name, and site is the four-character site code.

To start this utility, enter SHOWCNX at the system prompt. No further interaction is necessary.

The case numbers found in the CASEINDEX.DAT file will scroll across the screen as the file is read. 


\section{SHOWCOL}

The SHOWCOL utility is a handy diagnostic and reporting utility that shows the colors associated with Tektronix terminal pen numbers.

Note: This utility requires a graphic terminal.

\section{Showing Colors Associated with Terminal Pen Numbers}

To start this utility, enter SHOWCOL at the system prompt.

The pen numbers are displayed in their corresponding colors. 


\section{SHOWCOVER}

The SHOWCOVER utility is a database administration program that creates a DMS file showing the areas covered by all the DMS files listed in a topography (map layer) limit file. The resulting DMS file can be looked at directly through GEDIT or PLOTDMS.

\section{Creating an Area Coverage Map for a Topography (Map Layer)}

To start this utility, enter SHOWCOVER at the system prompt. SHOWCOVER presents the following series of prompts.

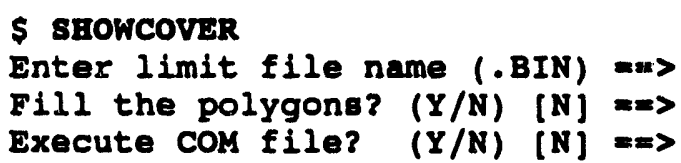

limit file name

This is the binary limit file (LIMITxx.BIN) containing all the graphic file extents for the map layer. Each map layer has its own directory named MAPSDATASxx, where $x x$ is the two-character topographic code.

Appendix A lists IBS map layers and their associated codes.

Fill the polygons? If you think you will want to turn on the polygon fill while editing this file sometime in the future, answer $\mathbf{Y}$. Otherwise answer $\mathbf{N}$.

Execute COM file? If you answer $\mathbf{N}$, two files are created in the MAPSDATASxx directory: LIMITxx.COM and LIMITxx.DMX. The LIMITxx.DMX file is an ASCII version of a DMS file outlining the data limits for the $x x$ map layer. The LIMITxx.COM is a command file to convert the LIMITxx.DMX to DMS format.

If you answer $\mathbf{N}$, only the area coverage map file MAPSDATASxx:LIMITxx.DMS will be created.

All output from the SHOWCOVER is placed in MAPSDATASxx. 


\section{SHOWENV}

The SHOWENV utility is a diagnostic and reporting utility that shows current environment characteristics for an IBS user (yourself or others) It generates the same report as STATUS BOARD-SHOW USER ENVIRONMENT in the IBS program. However, you have the option of ignoring shared memory (for models that don't know about shared memory).

\section{Showing User Environment Characteristics with SHOWENV}

To start the utility, type SHOWENV at the system prompt, followed by the disk name, user name, and site of an IBS user: SHOWENV disk user site. If you do not specify a disk-user-site combination on the command line, the utility shows information about your own environment.

Example: Show someone else's current environment'

S BEOWEN DUAO UEERY BATA

Use shared memory? $(Y / N)[Y]=Y Y$

Example: Show your current environment, but do not use shared memory'

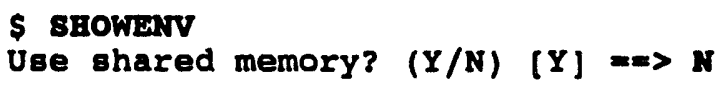

Example with output: Show your current environment.

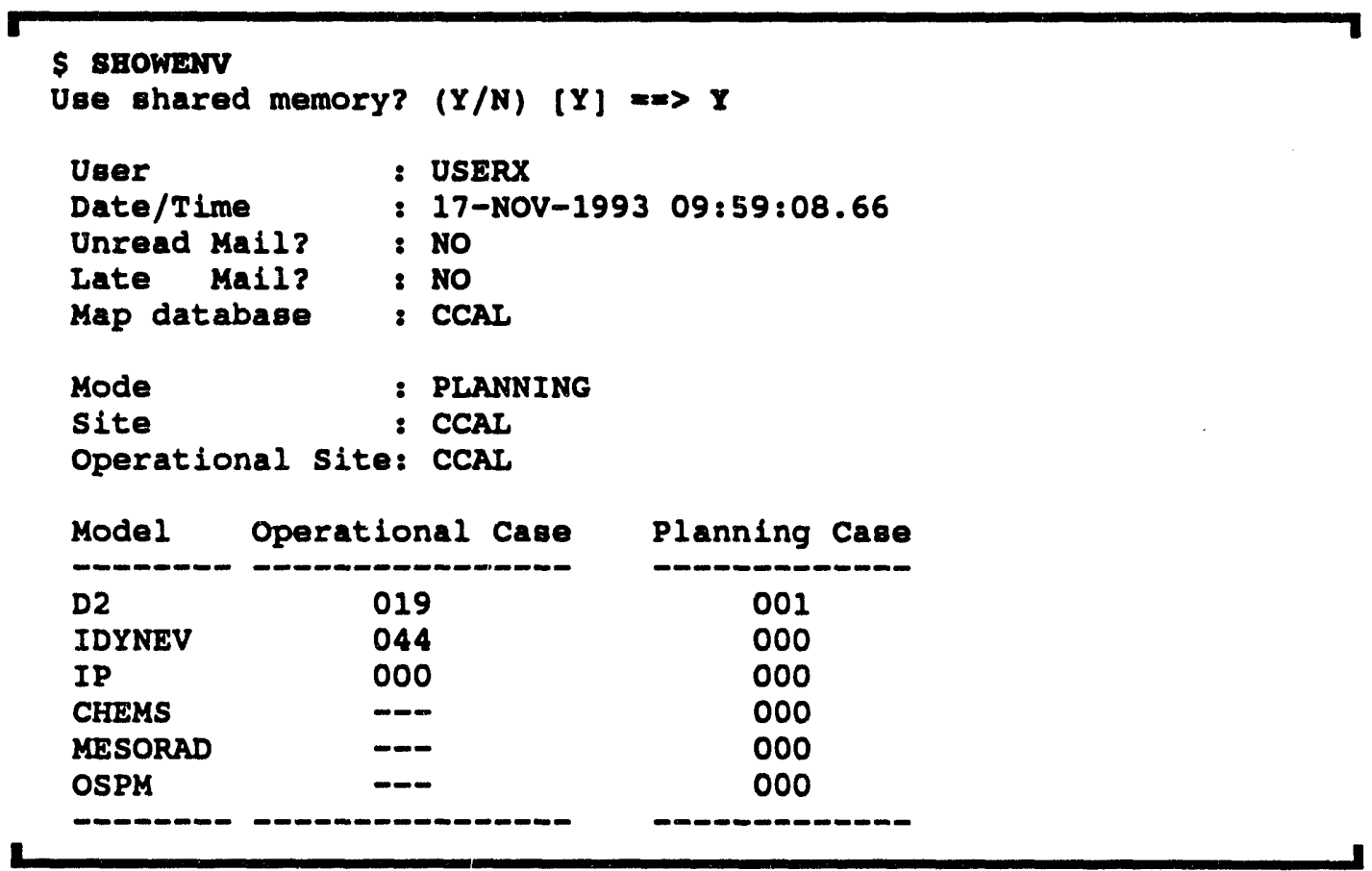




\section{SHOWTEK}

The SHOWTEK utility is a diagnostic and reporting program that displays current Tektronix TRPLOT settings.

Note: This utility requires a graphic terminal.

\section{Displaying Current Tektronix TRPLOT Settings with SHOWTEK}

To start this utility, enter SHOWTEK at the system prompt.

SHOWTEK fills the screen with TRPLOT device parameters. To exit the listing, press RETURN. 


\section{SITEBKP}

The SITEBKP utility is a database administration program that enables you to save and restore different types of site data, such as Resource Management data, or Model Case data.

\section{Backing Up or Restoring Selected IBS Site Data with SITEBKP}

To start the utility, enter SITEBKP at the system prompt. The following menu displays.

\begin{tabular}{l} 
Select Backup/Restore operation \\
\hline $0:$ ExIT \\
$1:$ Backup Selected Data \\
2 : Restore Selected Data \\
3 : List all Backup Seta \\
$4:$ Delete Backup set \\
Menu Choice $(\mathrm{P}=\mathrm{Help}) \mathrm{m}$
\end{tabular}

Select the menu option corresponding to your choice. The options are:

\section{1 : Backup Selected Data}

This option enables you to create a backup set containing selected types of IBS data. The following list will display.

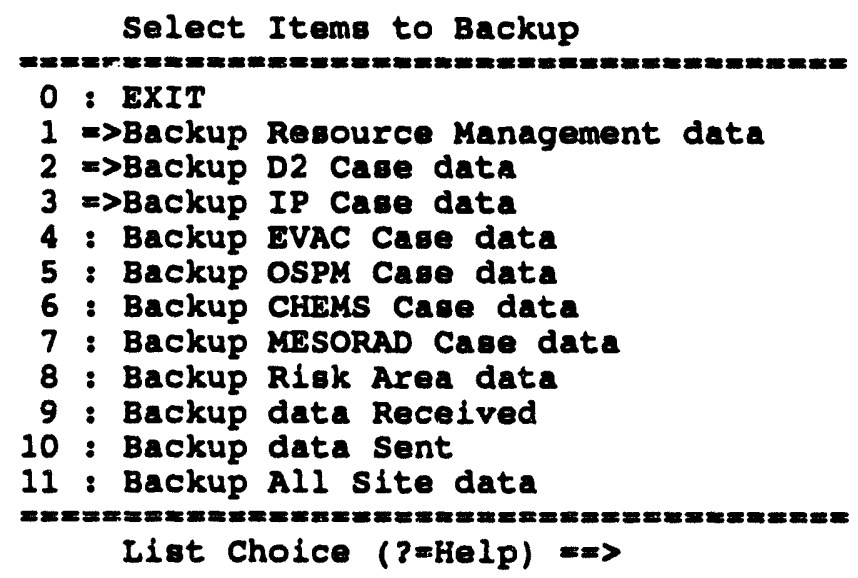

Select all the data types you want to include in this backup. An arrow $=>$ will display in front of any selected items (in the above example, items 1,2 , and 3 have been selected.) When you have selected all the items you want, select 0 to Exit. The following prompt will display:

View output of Backup? $(Y / N)=>$ 
Entering $Y$ will display a log of all files that are backed up on your screen. The backup then begins. Be patient: it may take over 10 minutes for the backup to complete, depending on how much data you selected to include.

Note: To get out of the backup at the View output prompt, press Ctrl-Z to return to the Select Backup/Restore operation menu.

You will then be prompted to enter a brief description of this backup.

Please enter a brief (one line) description of this backup. $\Rightarrow \quad 4 / 22 / 93$, KJB. Backup made before Exercise.

\section{2 : Restore Selected Data}

This option enables you to restore backed up data. This data will become the current data for a site.

Note: You must have write access to all directories when restoring data, and read access to all directories when backing up data.

The following menu will display. Select how to choose the Backup set.

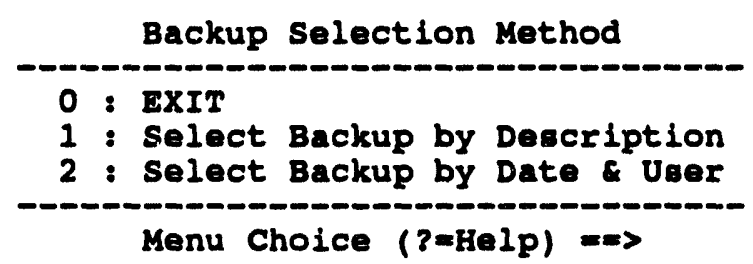

Depending on the method you select, menus similar to one of the following will display:

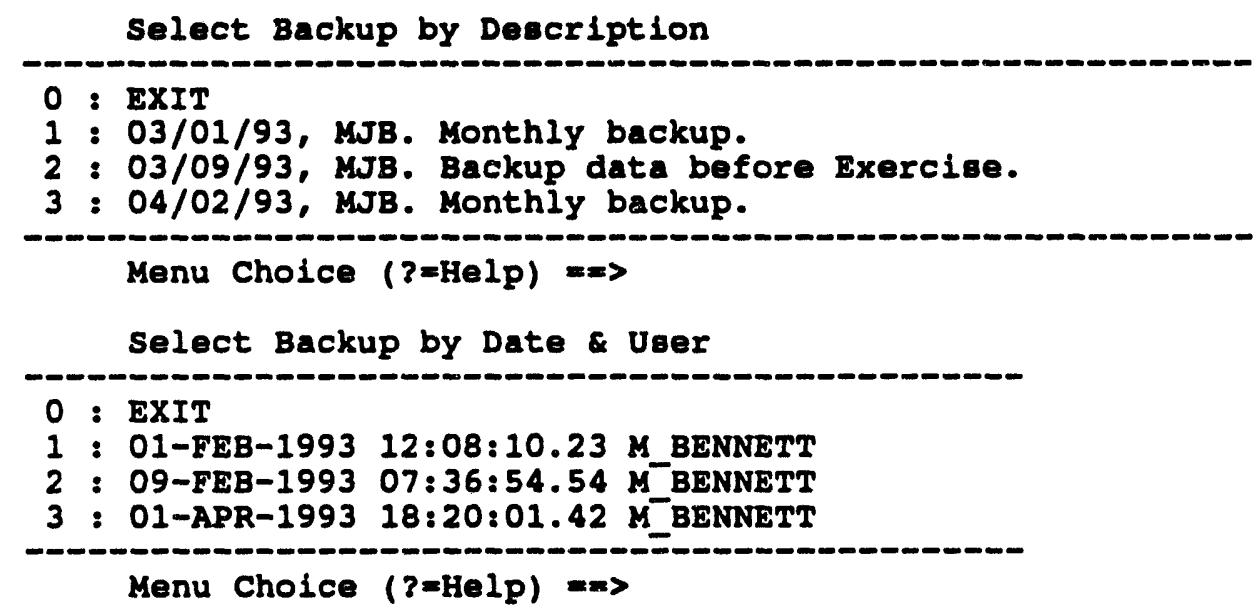

After selecting a backup set from the menu, a menu containing all the data types stored in the selected backup set will display. 


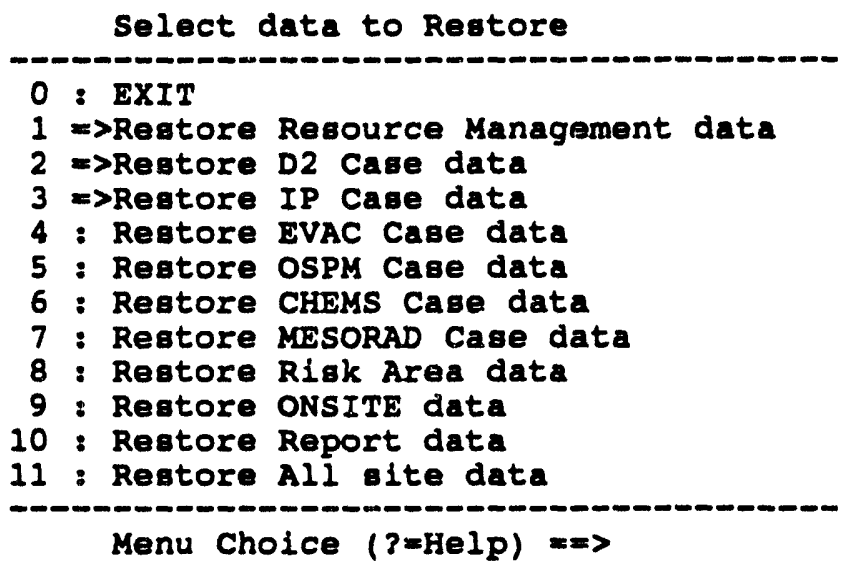

All data types are shown in the above menu, but only those types that are stored in the selected backup set will actually be shown. Select the data types you want to restore. An arrow $=>$ will display next to every selected item (in the above example, items 1, 2, and 3 are selected).

If some of the data you have selected to restore is Shared, such as the Resource Management data, the following prompt will display:

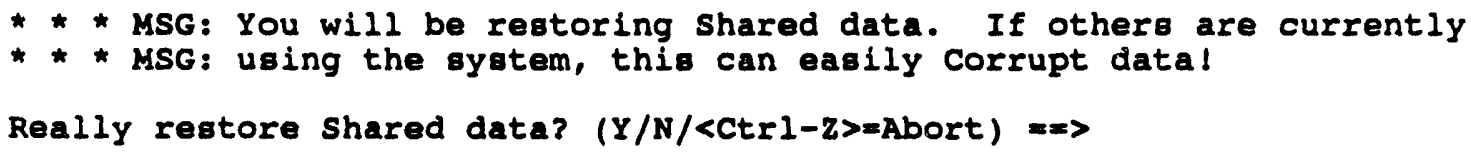

\section{Caution}

You should NEVER restore shared data while other users are using the system, it can easily cause data to be corrupted.

If you are not restoring shared data, or entered a $Y$ (Yes) at the above prompt, the following prompt will display:

* * MSG: Restoring All site data will cause ALL changes aince the Backup

* * * MSG: was made to be LOST!

Really restore All Site data? $(Y / N)=m$

If you type a $\mathrm{Y}$ (Yes) at the prompt, the following message will display.

View Output of Restore? $(Y / N)=>$

Typing $Y$ (Yes) will display a log of all files that are restored on your screen. The restore will then begin. It may take over 10 minutes for the restore to complete, depending on how much data you selected to include in the restore, so be patient. 


\section{3 : List all Backup Sets}

This option prints information about all the backup sets that have been made for the current site. The report will contain the following information:

- When the backup was made

- Who made it

- A brief description by the person who made the backup

- The data types included in the backup.

The report will be sent to your default report destination. If you have not specified a default output destination, the Output Redirection Menu will display and you can select a destination for the report.

The report will be similar to the following example:

BACKUP SET IIST

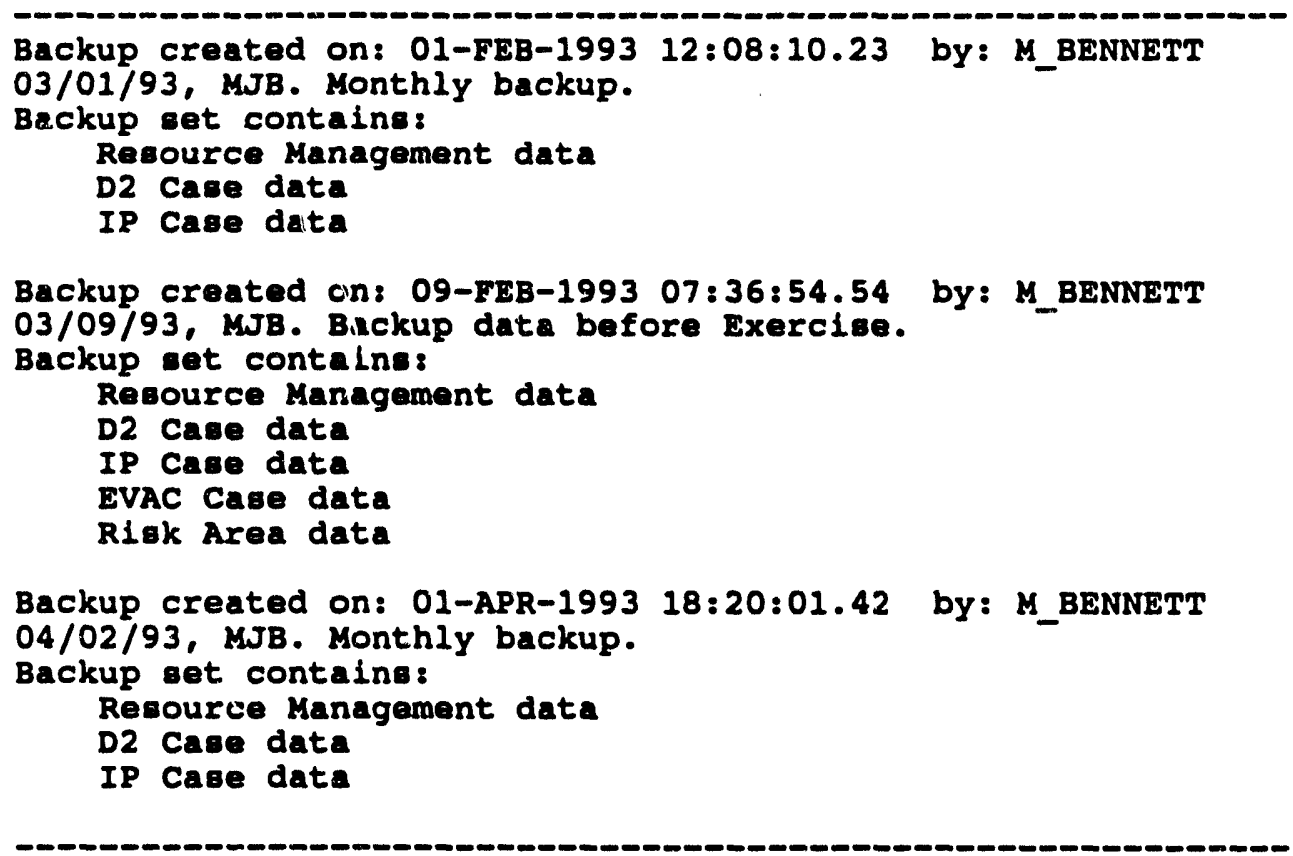




\section{4 : Delete Backup set}

Backed up data can use lots of disk space, especially if many backup sets are made. This option enables you to delete selected backup sets. This option displays a menu to select how to choose the Backup set to delete.

Backup Selection Method

\begin{tabular}{l}
$0:$ ExIT \\
$1:$ Select Backup by Description \\
2 : Select Backup by Date \& User \\
\hline Menu Choice $(?=$ Help $\Rightarrow=$
\end{tabular}

Depending on the method you select, menus similar to one of the following will display:

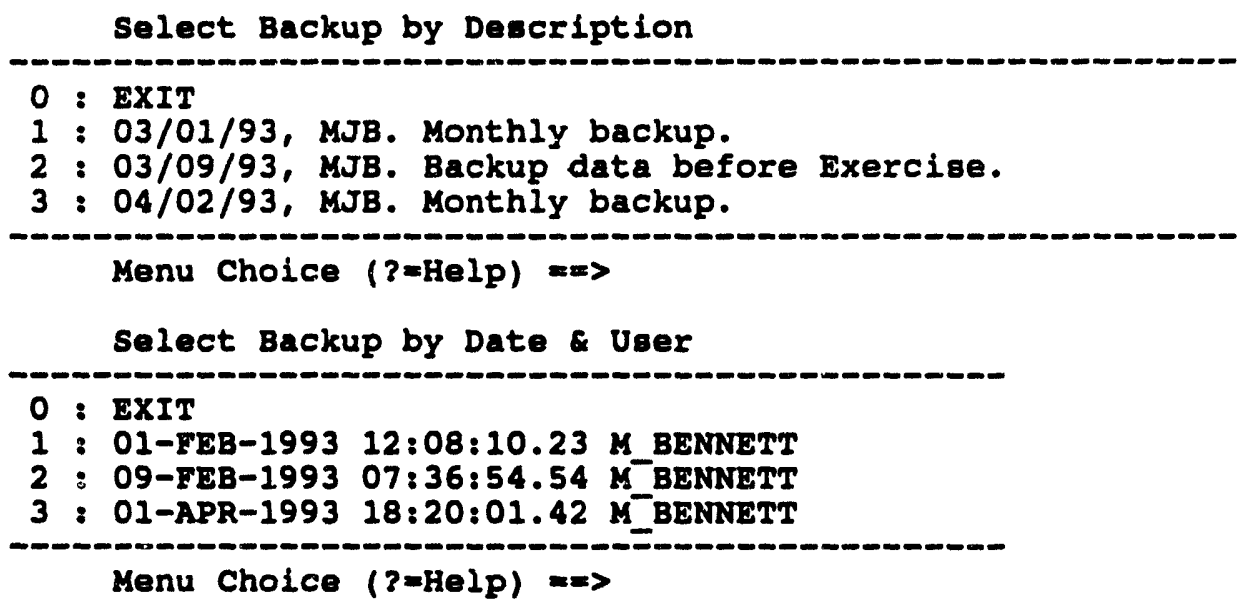

After selecting a backup set, the contents and information about that backup set will be displayed and you will be asked to verify that this is the backup set you wish to delete. The following is an example of a backup contents listing.

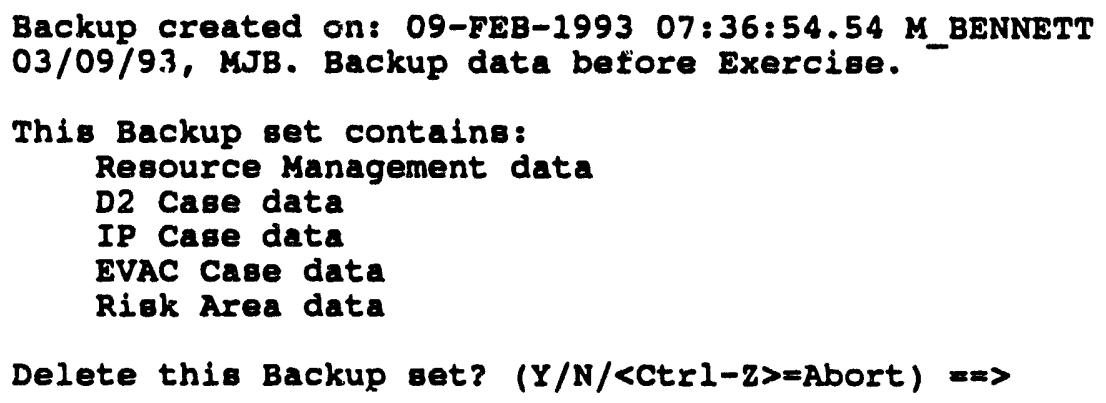

Type a Y (Yes) to delete the Backup. Unless the data has also been backed up to tape, the data will be lost. 


\section{SM_DUMP}

The SM_DUMP (Shared Memory DUMP) utility copies the current contents of the IBS shared memory block to a file or to the terminal screen.

\section{What You Need to Know Before Using SM_DUMP}

SM_DUMP is one of several related utilities that display or manipulate the shared memory block used by the IBS program. The IBS program uses this block of shared memory for storing shared variables that identify current operational data. SM_DUMP is primarily a tool for software developers or troubleshooters: you must have intimate knowledge of the IBS program to have any use for the information that SM_DUMP will produce.

\section{Copying Shared Memory to a File or to the Screen with SM_DUMP}

To start SM_DUMP, enter RUN IEMISSEXE:SM_DUMP at the system prompt. You will then be prompted for an output file name:

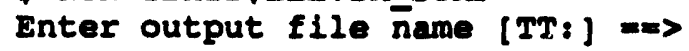

To save the contents of the shared memory block as a file, enter a file name.

Or to display the contents of the shared memory block on your screen, just press RETURN instead. 


\section{SM_INIT}

The SM_INIT utility initializes the variables stored in the shared memory block used by the IBS. This initialization of variables is necessary after the IBS system has been completely rebuilt from the program code.

\section{What You Need to Know Before Using SM_INIT}

\section{Caution}

SM INIT modfies the shared memory block used by the IBS program for storing shared varlables that identify current operational data. If SM INIT is used at the wrong time, it could overwrite this information. The SM INIT utility is intended for use only by an IBS system manager or database administrator.

Normally, SM_INIT would not be used independently: if the system is being completely rebuilt, the command file to completely rebuild the system should contain the command to run SM_INIT after the executable files have been rebuilt. The BUILDALL.BIS batch job file and BUILD*.COM command files for rebuilding the system do this already.

\section{Initializing Shared Memory Variables with SM_INIT}

To execute SM_INIT, just enter RUN IEMIS\$EXE:SM_INIT at the system prompt. No further interaction is necessary. 


\section{SM SET}

The SM_SET utility shows the current values of some of the shared memory variables and then enables you to change them.

\section{What You Need to Know Before Using SM SET}

\section{Caution}

SM_SET modifles the shared memory block used by the IBS program for storing shared yariables. If SM SET is used at the wrong time, It could overwrite this information. The SM_SET utility is intended for use only by an IBS system manager or database administrator. You must have intimate knowledge of the IBS program and your system before you can use this utility effectively.

\section{Setting Shared Memory Variables with SM_SET}

To start SM_SET, enter RUN IEMIS\$EXE:SM_SET at the system prompt. A series of prompts then asks you to enter values for the following specific variables:

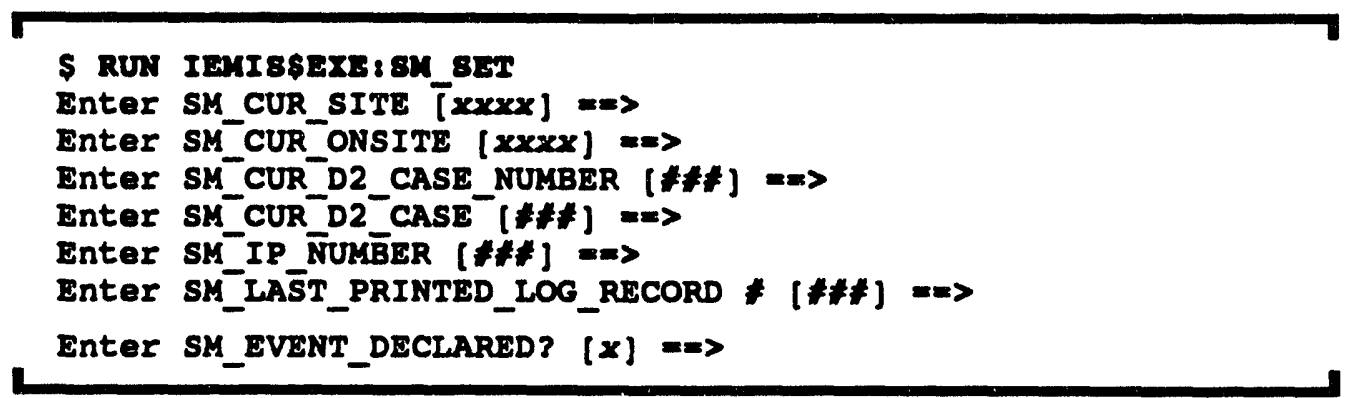

For each variable, enter a value or just press RETURN to accept the default values shown in brackets:

- For the current operational site (SM_CUR_SITE) and the associated onpost depot (SM_CUR_ONSITE), enter the four-character site codes.

- For the D2 case, IP, and printed log numbers, enter valid numeric values.

Note: SM_SET does NOT check to ensure that the case numbers you are selecting actually exist. You must know before you run this program which case numbers are valid and which are not.

- For whether an event has been declared (SM_EVENT_DECLARED), enter T or F (to stand for "True" or "False"). 


\section{SMOOTHER}

The SMOOTHER utility enables you to reduce the number of coordinate points that compose strings in a DMS file. SMOOTHER is very similar to the THINNER utility and would be used for many of the same reasons. The significant difference between the two is that they use a different method for removing points. THINNER determines which coordinates would appear as the same point on the screen. SMOOTHER calculates whether or not points form a straight (or nearly straight) line, if they do, some intermediate points may be removed. This is especially useful for extracted Tiger data that has been "linearjoined" using the LJTIGER utility.

\section{Reducing the Number of Points in a DMS File with SMOOTHER}

To start the utility, enter SMOOTHER at the system prompt. In the following example, SMDOThER reduces the number of points in the file TEST.DMS by nearly $30 \%$.

Example:

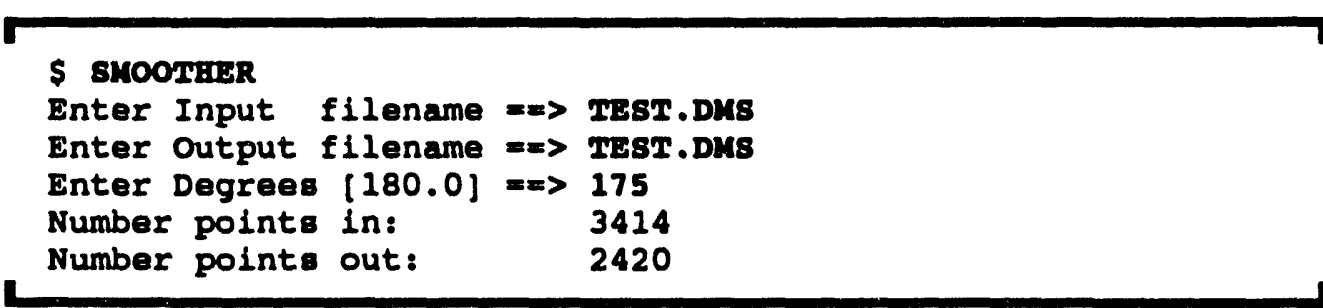

SMOOTHER first requests that you enter the original DMS file name and the name of the new DMS file that you want to create.

You can then specify the angle between points that qualifies as a straight line. If you press RETURN to choose 180 degrees (the default), then the points MUST form a straight line before intermediate points are removed. If you choose 180 degrees, there will be NO loss of visual data, although there should be fewer points to draw onscreen. Objects are therefore drawn faster and use less disk space.

If you specify an angle less than 180 degrees, then the removal of points between that angle and 180 degrees will cause some loss of data, but the number of points removed can be increased. If you use angles of less than about 170 degrees, you may remove so many points that the results get strange, but you may want to experiment with your particular data. 


\section{SPLITDMS}

The SPLITDMS utility is a data file modification program that reads a DMS file and splits it into two files: One file contains all records that have attribute values associated with them; the other file contains all records that do NOT have attribute values associated with them.

\section{Splitting Off DMS Objects that Have No Attributes with SPLITDMS}

To start this utility, enter SPLITDMS at the system prompt. SPLITDMS then displays the following series of prompts.

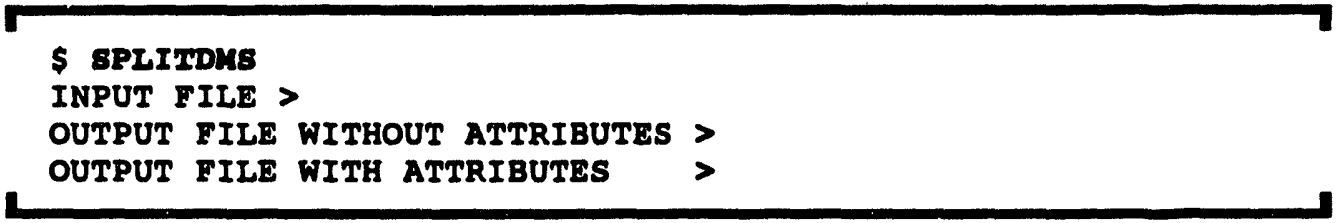

Enter each file name, including the .DMS extensions. After these inputs are complete, SPLITDMS creates two new DMS files with the names you specified. 


\section{STATDMS}

The STATDMS utility is a diagnostic and reporting program that computes and lists summary statistics about the attributes, lines, points, polygons, etc., contained in a DMS file. Normally STATDMS reads only one DMS file at a time; however, you do have the option of requesting summary information for a DMS database by supplying the name of the limit file associated with that database.

\section{What You Need to Know Before Using STATDMS}

If you are going to request statistics for (one topography of) an entire database, you must know the name of the database limit file.

\section{Producing DMS Data Statistics with STATDMS}

To start the utility, just enter STATDMS at the system prompt. STATDMS begins by asking if you wish to use a database limit file:

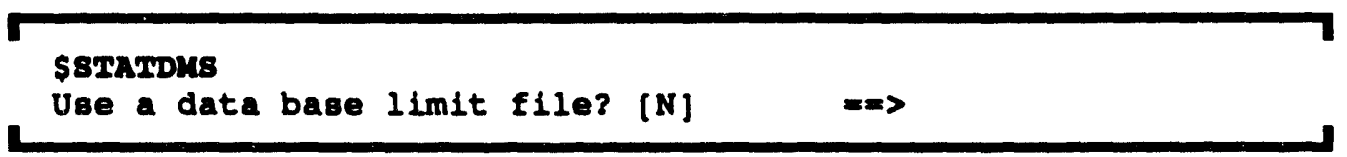

Your response to this request depends on what results you want:

To get this result:

To compute statistics for a single DMS file:

To compute statistics for an entire database (for one topography):

To cancel the program:
Do this:

Enter $\mathbf{N}$ or press RETURN.

Enter $\mathbf{Y}$.

Press CTRL-Z.

The following sections include examples of producing statistical reports for a single DMS file and for an entire database.

\section{Producing a Statistics Report for One DMS File}

The following example sequence shows how you can display summary statistics for a single DMS file (MYFILE.DMS) on your terminal screen: 


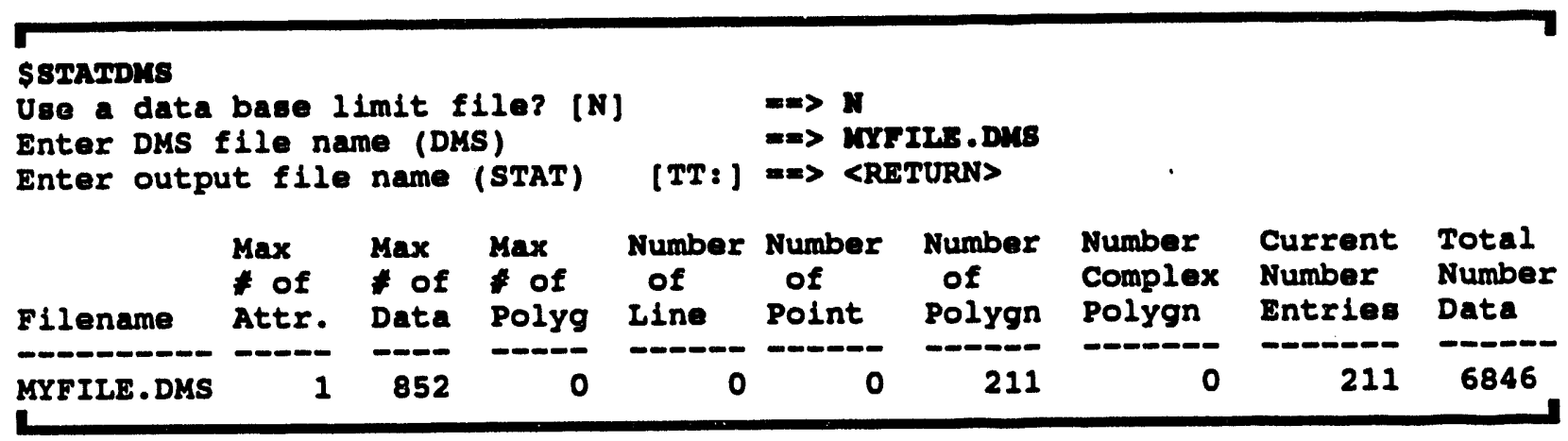

After you choose NOT to use a database limit file, the STATDMS utility asks you to specify the following items:

DMS file name

This is the name of the DMS file for which STATDMS will produce statistics. If you do not specify an extension, the .DMS file extension is assumed. To cancel this prompt and return to the first prompt, press CTRL-Z.

output file name This is a name for the statistical report file. If you do not specify a file name extension, the .STAT extension is used. You can also do the following instead of entering the output file name:

To get this result:

To use the same name as the DMS file but with the .STAT extension:

To display the results on the terminal screen (TT:):
Do this

Press CTRL-Z.

Press RETURN.

\section{Producing a Report of DMS File Statistles for a DMS Database}

The next example shows how you can calculate statistics for an entire database (one topography) and produce the statistics report in the file MYDBASE.STAT.

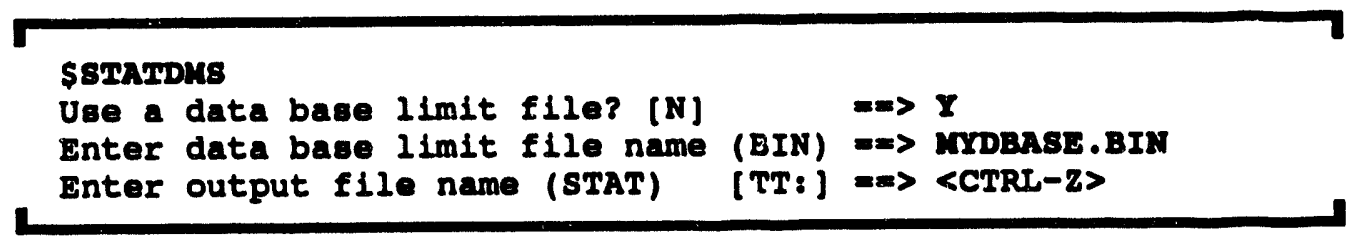

After you choose to use a database limit file, the STATDMS utility asks you to 
specify the following items:

data base limit file name

output file name
This is the name of the limit file, which lists several DMS files for STATDMS. If you do not specify an extension, the .BIN file extension is assumed. To cancel this prompt and return to the first prompt about the limit file, press CTRL-Z here.

This is a name for the statistical report file. If you do not specify a file name extension, the .STAT extension is used. You can also do the following instead of entering the output file name:

To get this result:

Do this

To use the same name as the Press CTRL-Z. limit file but with the .STAT extension:

To display the results on the terminal screen (TT:): 


\section{STOPEVENT}

The STOPEVENT utility stops the EVENT utility from running in the background. It requires system privileges (SYSPRV) to run. See EVENT in this document.

\section{Caution}

The EVENT utllity processes data from the onpost system and the onpost simulator. EVENT must be running in the background for the IBS to function correctly. If EVENT is not running, all data from the onpost system and the onpost simulator will be ignored.

\section{Stopping the EVENT Background Program with STOPEVENT}

To start this utility, enter STOPEVENT at the system prompt.

If the EVENT utility is running, it will be stopped and you will see the following message:

* * MSG: Deleting batch Job EVENT

To restart EVENT, enter EVENT at the system prompt. 


\section{STOPMAIL_C}

The STOPMAIL_C utility stops the MAIL_C utility from running in the background. It requires system privileges (SYSPRV) to run. See MAIL_C in this document.

\section{Caution}

The MAlL_C (mall checker) utility checks user accounts to see which users have new or expired mall. When new or expired mall is found, a message telling the IBS user is displayed on the screen. If the mall checker utility is not running, users running the IBS application will NOT be notified of new or explred mall.

\section{Stopping the MAIL_C Background Program with STOPMAIL_C}

To start this utility, enter STOPMAIL_C at the system prompt.

If the MAIL_C utility is running, it will be stopped and you will see the following message:

* * MSG: Deleting batch job MAIL_C

To restart the mail checker utility, enter MAIL_C at the system prompt. 


\section{TACFIXTAA}

The TACFIXTAA utility is a data file modification or reporting program that either changes or just displays the name of the TAA file reference contained in a Terrain Analysis Cell (TAC) file.

\section{Changing the TAA File Reference in a TAC File}

To start the utility, TACFIXTAA at the system prompt. To make the change, enter the TAC file name and a TAA file name. TACFIXTAA then displays the name of the TAC file that has been altered, the deleted $(-)$ TAA filename, and the added $(+)$ TAA file name, as in the following example.

Example:

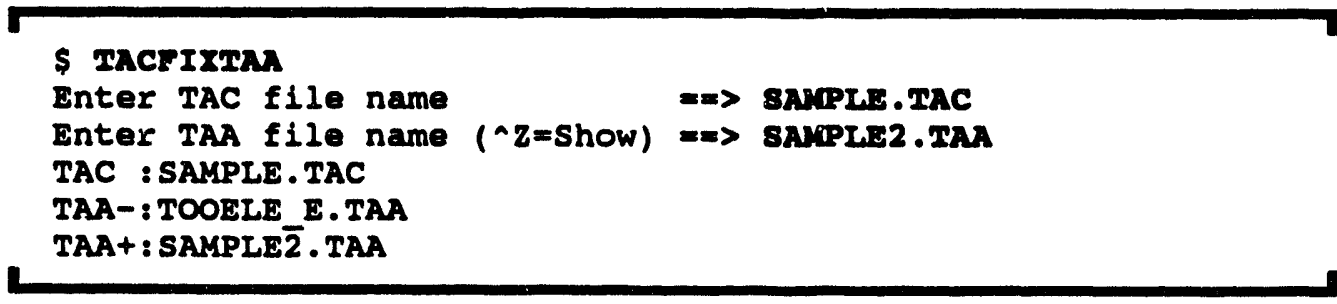

Displaying the Current TAA File Name. To display the name of the TAA file referenced in the TAC file without actually changing the TAC file, just press CTRL-Z at the TAA file prompt. TACFIXTAA displays the TAC file name followed by the included TAA file name.

Example:

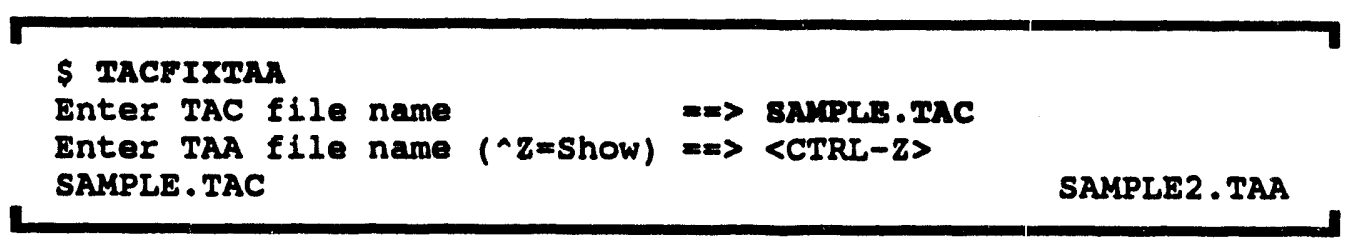




\section{TACLLXUTM}

The TACLLXUTM utility reads elevation data from an existing Terrain Analysis Cell (TAC) file (and its associated TAA file), and creates a new TAC file (and TAA file) with the X-Y coordinate data in a different coordinate system.

TACLLXUTM can convert TAC data between the longitude-latitude coordinate system and the Universal Transverse Mercator (UTM) coordinate system in both directions:

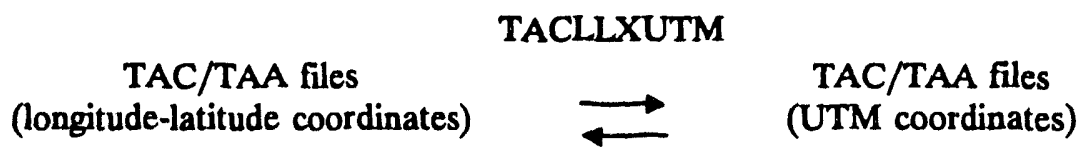

\section{What You Need to Know Before Using TACLLXUTM}

The TACLLXUTM utility prompts you for the following items:

Input File (.TAC) This is the name of the TAC file to be read.

Output File (.TAC) This is the name of the TAC file to be created.

Min and Max $X$ and $Y$ These are the minimum and maximum $X$ and $Y$ of the output map coordinate extents of the new map file (where $\mathrm{X}$ refers to the east-west values and $Y$ refers to the north-south value).

Pad Type

None

User supplied

Nearest Neigh
The pad value is a numeric value that will be used to fill in the output map cells at edge areas where the input map does not overlap the output map. This situation occurs when there are differences between the areas covered by the input and output map projections. The cells that contain these pad values are said to be "padded." The utility prompts you to choose one of the following pad types or methods for choosing pad values:

A pad type of "None" means that the background value (0) will be used.

A pad type of "User supplied" means that the utility will then prompt you to enter a pad value.

A pad type of "Nearest Neighbor" means that the pad values will be taken from the cells nearest to the cells to be padded. This is the default choice. 


\section{Converting TAC File Coordinate Data with TACLLXUTM}

To start the TACLLXUTM utility, just enter TACLLXUTM at the system prompt.

The following sequence is an example of creating a TAC file in the UTM coordinate system from a TAC file with longitude-latitude coordinates. The reverse sequence for creating a longitude-latitude TAC file from a UTM TAC file is identical except that a TAC file in UTM coordinates is used as the input file.

Example:

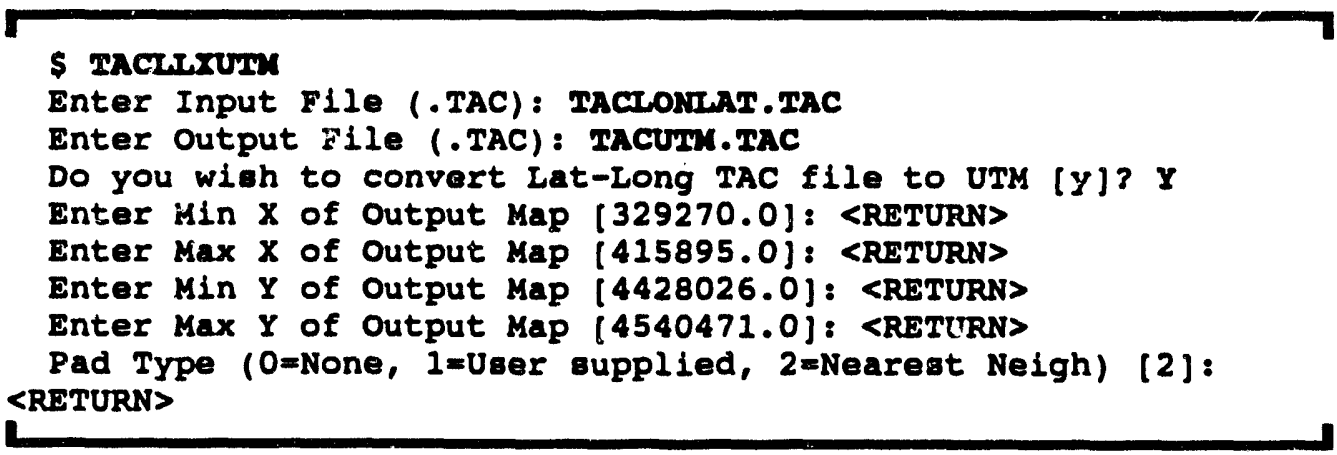

The utility provides default values for the output map extents; these are the extents of the input map. To accept the default output map extents, press RETURN at each prompt. Similarly, to accept the pad type default of "nearest neighbor," press RETURN.

\section{Results of Using TACLLXUTM}

After using TACLLXITM, you will have a new TAC data set: one TAC file (.TAC extension) and its associated TAA file (.TAA extension). In the example here, TACLLXUTM creates a TAC data set in the UTM coordinate system from a TAC data set in longitude/latitude. TACLLXUTM can also be used to create TAC data in longitude/latitude coordinates from TAC data in UTM coordinates. 


\section{TACXASCII}

The TACXASCII utility is a diagnostic and reporting program that displays the contents of the TAC header file. You can also display the data from the associated .TAA file at a selected resolution.

\section{Displaying the Contents of a TAC Header File with TACXASCII}

To start this utility, enter TA CXASCII at the system prompt.

The utility then prompts you for the name of the TAC header file (.TAC) and for whether you want to see just the header information (Y) or cell data as well (N). In the following example, just the header information is displayed.

Example: Header Only

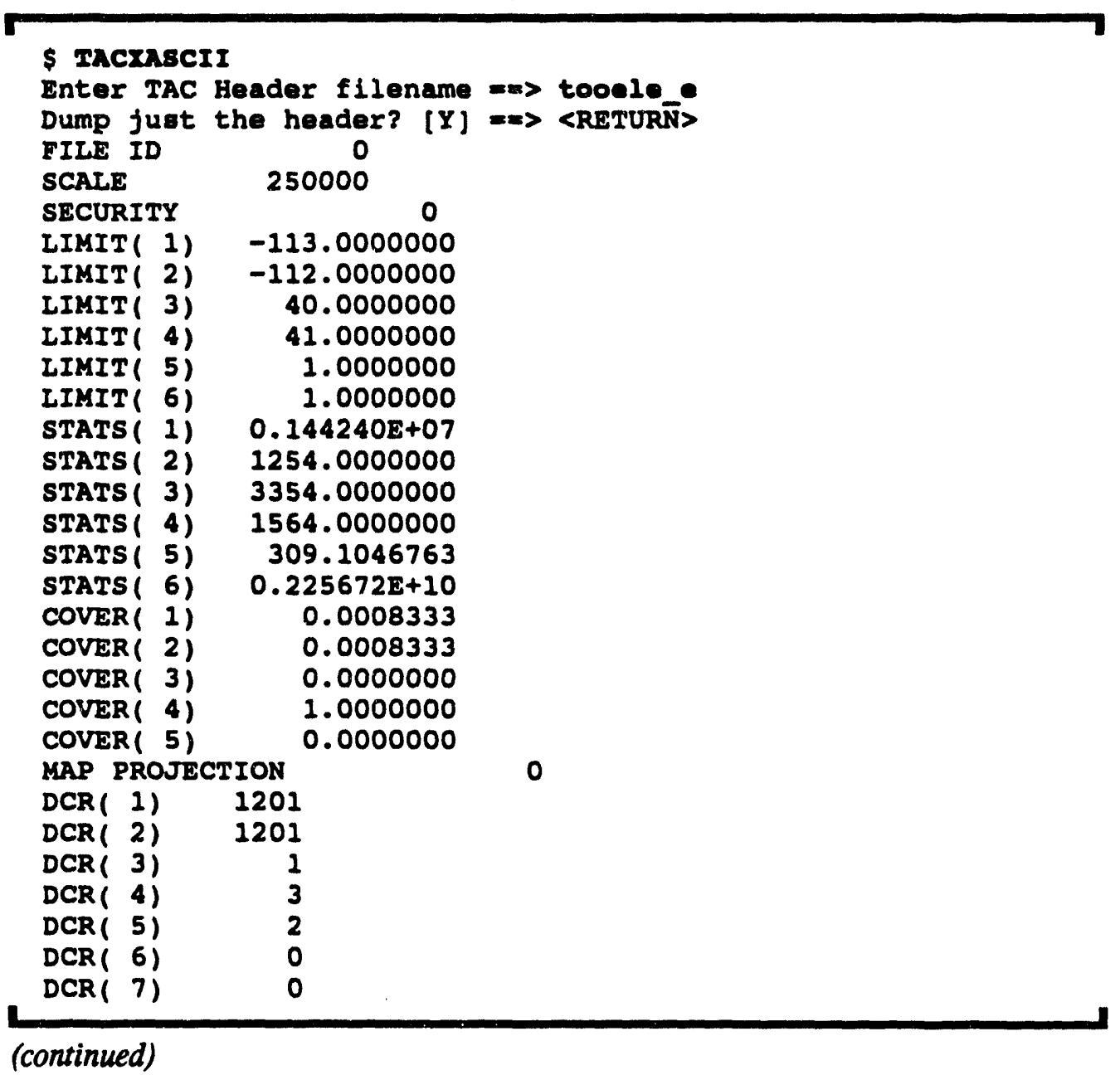


Example: Header Only (continued)

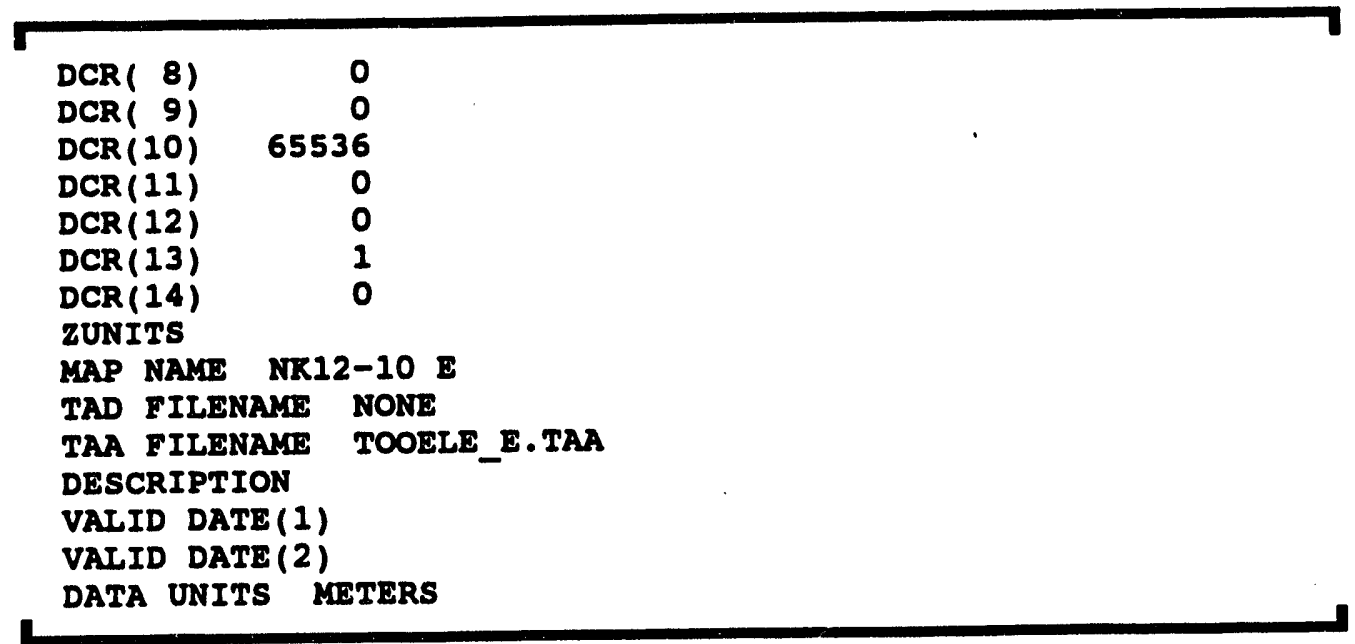

\section{Displaying TAC Data with TACXASCII}

If you answer $\mathbf{N}$ to "Dump just the header?", then the utility still shows the header information, pausing at each block of information. Press any key to continue or CTRL-Z to cancel the listing.

Following the header information, TACXASCII then displays a series of prompts similar to the following.

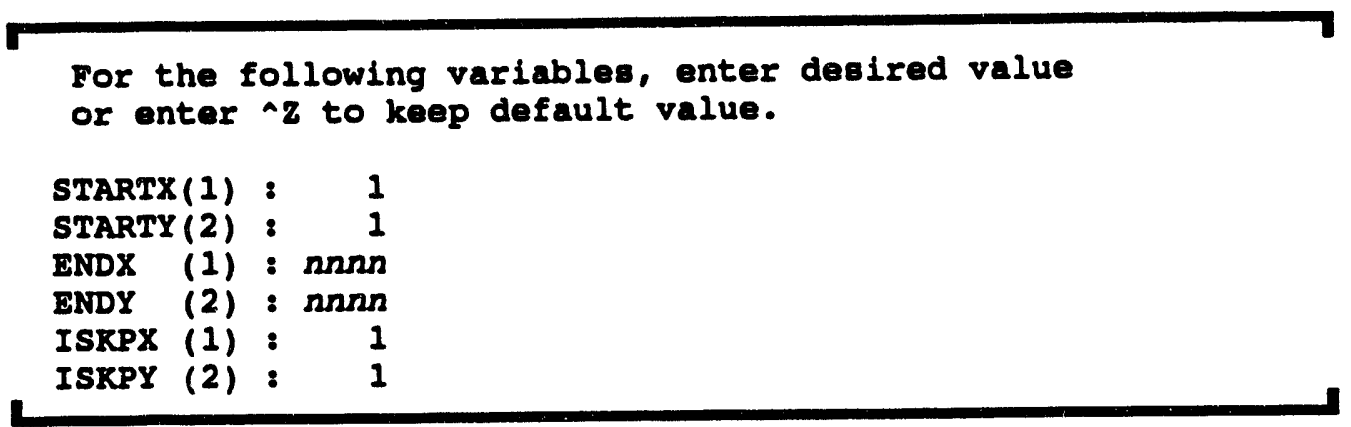

The prompts shows values for starting and ending $X, Y$ cell coordinates and a skip factor for the cell data. At each prompt you can either enter a different value or accept the default value by pressing CTRL-Z. After you specify each value, TACXASCII generates a table of cell data values based on that information.

In the following example the default values are for a 1201x1201 cell array. The new entered values divide the original area into a 10x10 cell array and then call for reporting a $5 \times 5$ representation of the array by using a skip factor of 2 (skipping every other cell value). 
Example: Displaying Cell Values

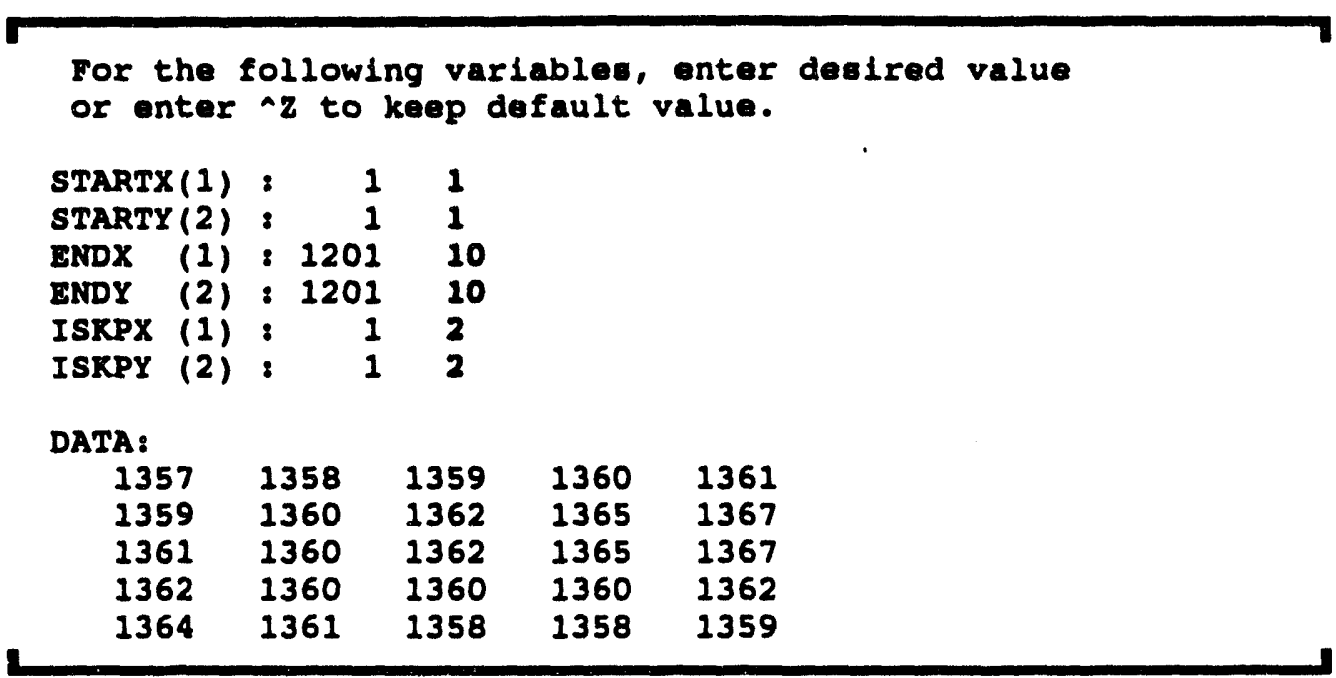

\section{Capturing the Cell Data in an ASCII File}

The cell data are written to the screen as shown. If you want to save the information to a ASCII file, you can do so by temporarily assigning system output to a file before using TACXASCII. The following example shows how you could make an ASCII file containing the same data as in the example above. Notice that you do NOT see any prompts. Be sure to deassign system output when finished.

Example: Capturing Cell Data in an ASCII File

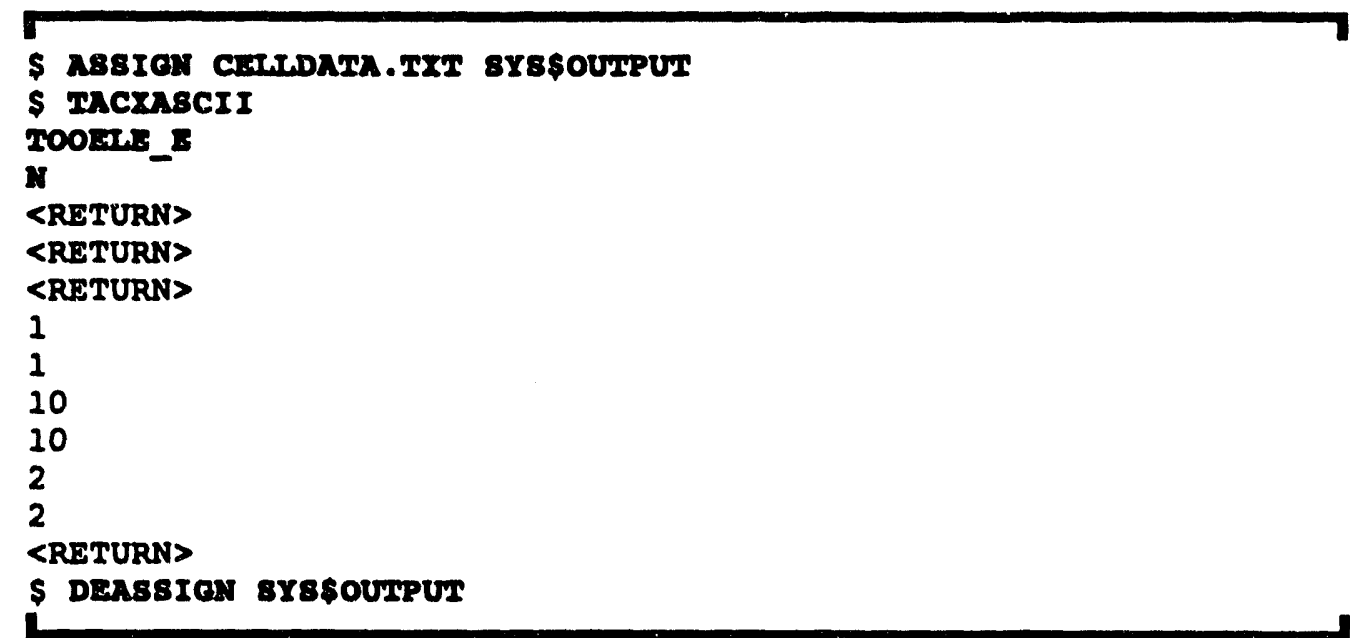

The resulting file captures all the output that would have been directed to the screen (SYSSOUTPUT) and places it a file named CELLDATA.TXT, which could then be edited. 


\section{TBXDMS}

The TBXDMS utility creates DMS boundary data files from TIGER/Line data files provided by the U.S. Department of Commerce, Bureau of the Census. The Bureau of the Census makes TIGER/Line data available for individual counties. TBXDMS is designed to handle one county at a time, converting boundary data from the Basic Data Record (Record Type 1) and Shape Coordinate Points (Record Type 2) files. Other data records of the TIGER/Line data files are ignored.

Note: You will normally NOT use TBXDMS directly: for most purposes you will want to use the TIGERCVT utility program, which creates a special command file that runs TBXDMS for you. (See TIGERCVT.)

\section{What You Need to Know Before Using TBXDMS}

You will normally want to use the TIGERCVT utility rather than TBXDMS, as noted above. If you do use TBXDMS independently, you must supply the following information:

county name

county limits

combined state and county code

drive:[directory] of TIGER data
This is the name of the county for which you want data.

These are the longitude-latitude extents of the county: the maximum and minimum longitude and the maximum and minimum latitude.

This five-digit code consists of the two-digit FIPS state code followed by the three-digit FIPS county code.

This is the name of the device and directory where the TIGER files are located.

\section{Creating DMS Boundary Data Files from TIGER/Line Data Files}

To start the utility, just enter TBXDMS at the system prompt. You will tl on see prompts for the information described in the previous section. The series of prompts will resemble the following example (simulated responses are in bold). 


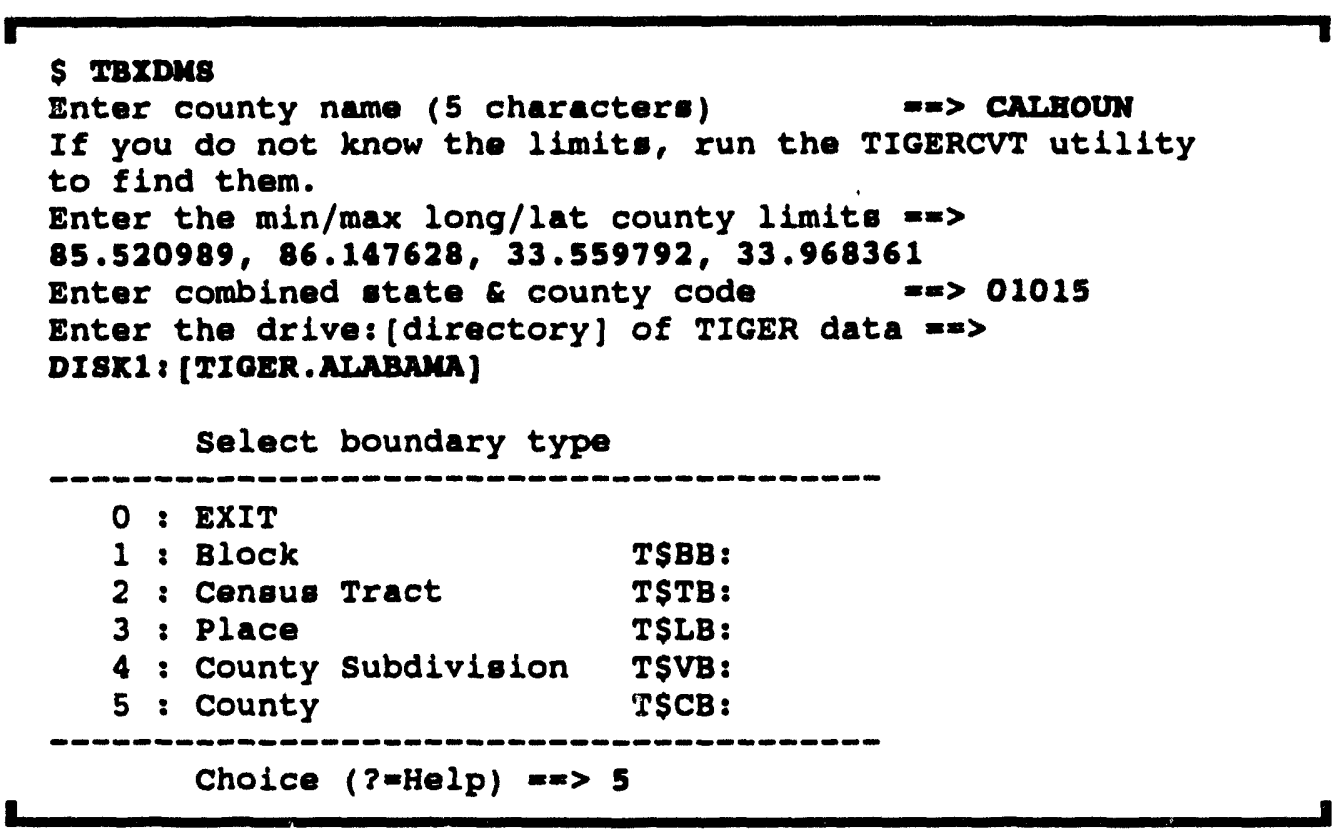

When TBXDMS begins reading the Record Type 1 file, you will see a series of messages:

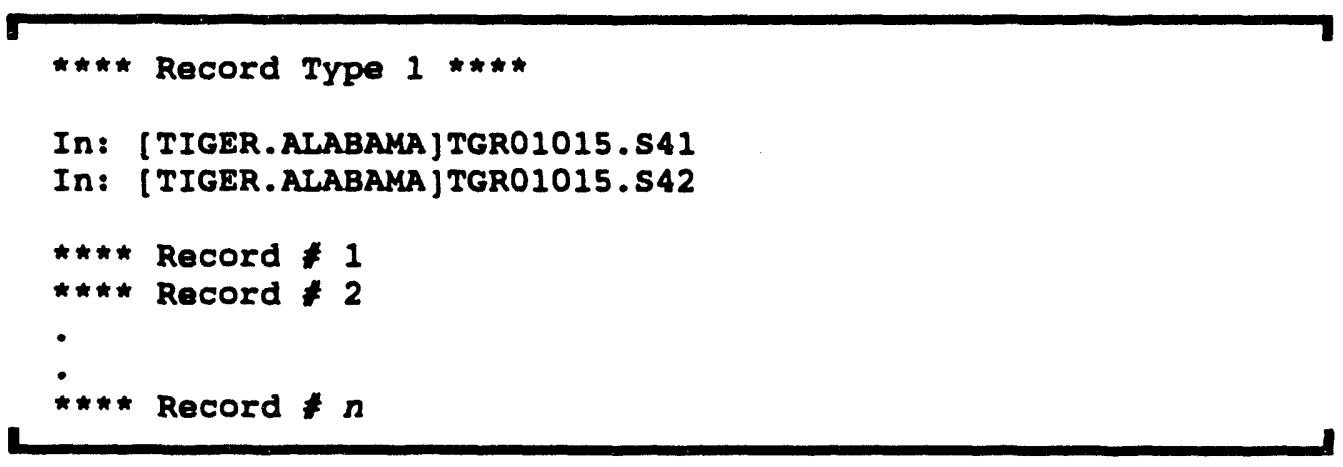

After all records have been read and the DMS files have been created, the program ends and the system prompt reappears.

Note: Some of the TIGER/Line boundary data is incomplete. After converting this data, you should view it with GEDIT, as editing may be necessary. 


\section{TBXDMS Program Messages}

*MSG* [directory] TGRnRnR .S41 FILE COULD NOT BE OPENED

*HSG* [directory]TGRnnnnn.S42 FILE COULD NOT BE OPENED

The program encountered an error while attempting to open the indicated

TIGER/Line file. The program will terminate. Check the combined state and county code and the location of the TIGER/Line files.

*MSG* Out of object limita

The program encountered an object that did not fall within the

longitude/latitude extents for the county. The program will ignore the object.

Check the longitude/latitude extents.

*MSG* Conflicting data for single-sided boundary

The program encountered a county boundary not listed as single-sided.

*MSG* COULD NOT OPEN FILE...1

The program encountered an error opening one of the DMS format files.

The program will create the remaining files. 


\section{TEKSETUP}

The TEKSETUP utility is a general system program that enables you to change the Tektronix screen color assignments for the screen background, for characters (foreground and background), and for the cursor (foreground and background).

See the SETUPTEK utility for restoring the Tektronix terminal screen to default characteristics.

\section{Changing Screen Color Assignments with TEKSETUP}

To start this utility, enter TEKSETUP at the system prompt. TEKSETUP displays a prompt listing the characteristics you can change:

Change what? (1=charb, $2=$ foreg, 3 =backg, $4=$ cursor $1,5=$ cursor 2 ) $=\equiv$

The prompt options correspond to the following changes:

1 - Change the character background color.

2 - Change the character foreground color.

3 - Change the background color.

4 - Change the cursor foreground color.

5 - Change the cursor background color.

After you select an option, TEKSETUP prompts you for a color choice:

select screencharacteristic colour $==>$

where screencharacteristic is the characteristic that you chose to change.

The valid color selections are :
-1 : current color
$4:$ Blue
0 : Black
$5:$ Cyan
1: White
6 : Magenta
2: Yellow
$7:$ Red
3 : Green
$>7:$ Red

You can continue to select a color for an option until satisfied; then use CTRL-Z to accept the color and continue. This causes the initial prompt to be redisplayed.

To exit TEKSETUP, press CTRL-Z at the initial prompt (or enter a value not listed in the initial prompt). 


\section{THINNER}

The THINNER utility enables you to reduce the number of coordinate points that compose strings in a DMS file. This is useful when the DMS file contains data to a greater precision than is needed. For example, if you extracted all the state boundaries from the $1: 2,000,000$ database with the intention of displaying regional maps (such as a 400-mile radius), many coordinates would map to identical pixels on the display device at this scale. THINNER can be used to remedy this situation and reduce the size of the DMS file.

\section{Reducing the Number of Points in a DMS File with THINNER}

To start the utility, enter THINNER at the system prompt. THINNER requests that you enter the original DMS file name and the name of the new DMS file that you want to create. You can also specify a plot radius parameter for controlling the data reduction or you can choose to have THINNER compute a default value that is based on the size of the area contained within the input file.

For example, the following interactions would reduce the data within the DMS file USAMAP.DMS, creating a second DMS file that contains only data that would be used in displaying a region of radius 500 miles or greater:

Example:

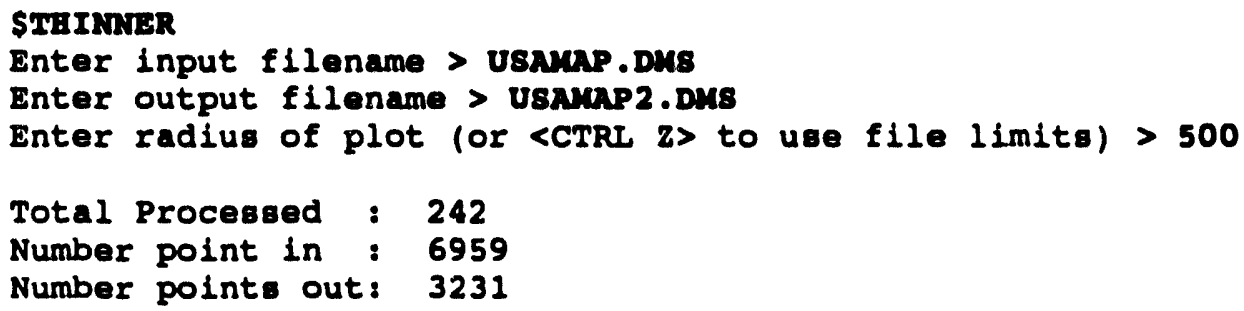




\section{TIGERCVT}

The TIGERCVT utility aids in creating DMS data files that are converted from TIGER/Line data files and STF1B population files provided by the U.S.

Department of Commerce, Bureau of the Census. TIGERCVT automatically reads the longitude-latitude extents of the TIGER/Line data and checks to see whether the data should be divided for separate, equal-area DMS files. TIGERCVT then creates command files that contain all the necessary inputs to the TIGERXDMS, POPXDMS, and TBXDMS data conversion utilities.

\section{What You Need to Know Before Using TIGERCVT}

Data files provided by the Census Bureau sometimes contain inaccurate data. Therefore it may be necessary to edit these Census Bureau data files with a text editor (such as EDT) before conversion with the TIGERXDMS, POPXDMS, and TBXDMS utilities is possible. After conversion, it may be necessary to edit the map graphics files with the GEDIT utility.

You should keep the following in mind when converting the Census Bureau Data:

- State population totals are placed at the state centroid specified in the Census Bureau STF1B files. Although this point is within the state for which the data is being stored, it may or may not lie within the site database radius.

- A census division (block, tract, etc.) may have more than one point at which data is stored.

The TIGERCVT utility asks you to specify several items, either by entering them or by choosing them from menus:

Population Type

state name

Boundary Type

county name

drive:[directory] of TIGER data
This refers to the type of population data to be converted to DMS format: state, county, county subdivision, place, census tract, block group, or block.

For the state population data only: This is the name of the state for which you want state-level population data. The state name must be at least 5 characters in length.

This refers to type of boundary data to be converted to DMS format: county, county subdivision, place, census tract, or block.

For population data other than state: This is the name of the county for which you want population data. The county name must be at least 5 characters in length.

This is the name of the disk device and directory where the TIGER census data files are located. 
basic record

filename

shape record

filename

population filename

database name

themes
This is the name of the file containing the TIGER/Line Basic Record Type (Record Type 1).

This is the name of the file containing the Shape Coordinate Points (Record Type 2).

This is the name of the Census Population (STF1B) file.

This is the database name or top-level directory under which the new DMS files will later be stored (in subdirectories).

The themes are the categories of topographic data that you wish to convert to DMS format. You can choose to convert some or all available themes.

The creation of DMS data files from TIGER/Line and STF1B population files is a two-part process:

I. Prepare for data conversion (create special command files) with TIGERCVT.

II. Create DMS files from Census Bureau data by using the special command files (which use the TIGERXDMS, POPXDMS, and TBXDMS utilities).

The following sections illustrate this process.

\section{Preparing for TIGER-to-DMS Data Conversion with TIGERCVT}

The following is an example of how you can use TIGERCVT to set up the creation of DMS data files from TIGER/Line and population data files. Simulated responses appear in bold.

1. To start the utility, just enter TIGERCVT at the system prompt.

The following menu prompts you to select the types of population data to be converted for the county that you will select.

Note: The state data is the same for all counties in a state; therefore, state data does not need to be converted more than once. 


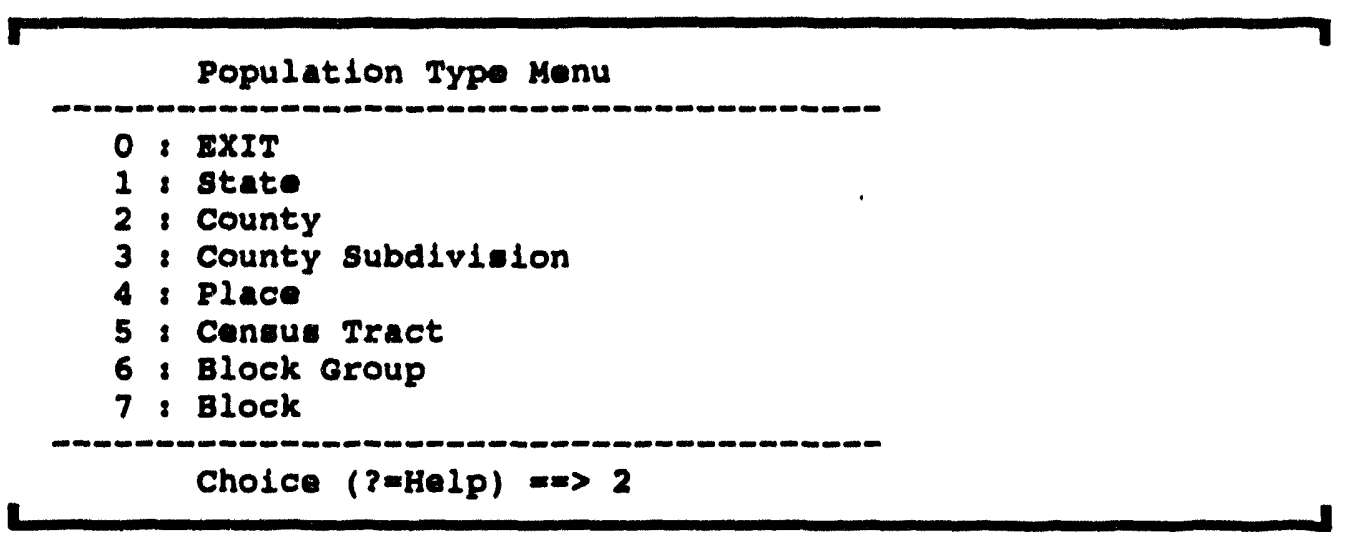

2. Choose the population type that you wish to convert from the Population Type Menu.

If you choose state data as the population type, the program prompts next for the state name:

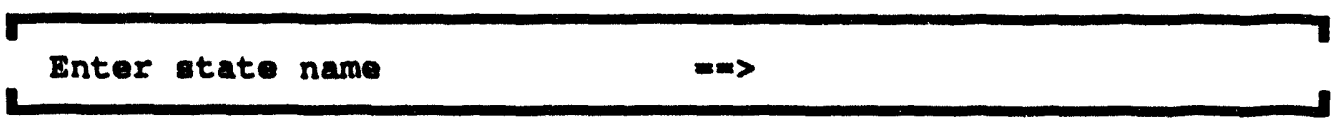

In that case, enter the state name before continuing. The state name must be at least 5 characters in length.

In all cases, the program then prompts for the type of boundary data to be converted:

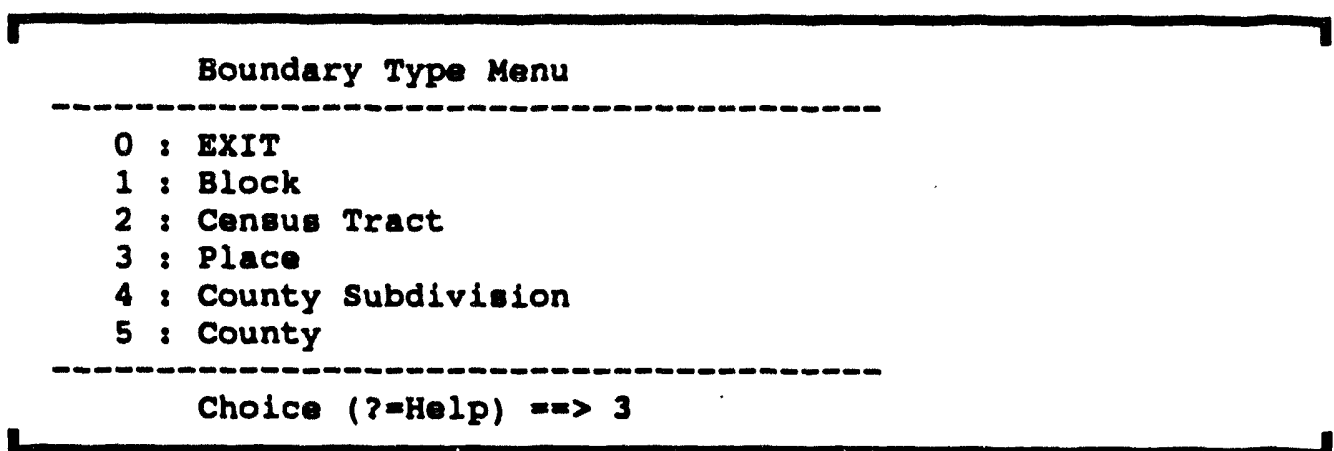

3. Choose one of the boundary types from the Boundary Type Menu.

Next the program prompts for the county name. 
Enter county name $m$

4. Enter a county name of 5 characters or longer.

The program then prompts for the location of the Census Bureau data files.

Enter drive: [directory] of TIGER data as DI8Kls [TIGER]

5. Specify both the disk drive and directory where the data files are located.

Next the program prompts for the names of the TIGER/Line Basic Record Type (Record Type 1), Shape Coordinate Points (Record Type 2), and Census Population (STF1B) files. If you do not wish to convert the population data file, press RETURN when prompted for the population filename.

\begin{tabular}{ll} 
Enter basic record filename & $\Rightarrow=$ TORO1015.F41 \\
Enter shape record filename & $\Rightarrow=>20 R 01015 . F 42$ \\
Enter population filename & $\Rightarrow=>$ BC3036.DAT \\
\hline
\end{tabular}

6. Enter the names of the Census Bureau data files.

The program then prompts you to choose which theme(s) to convert:

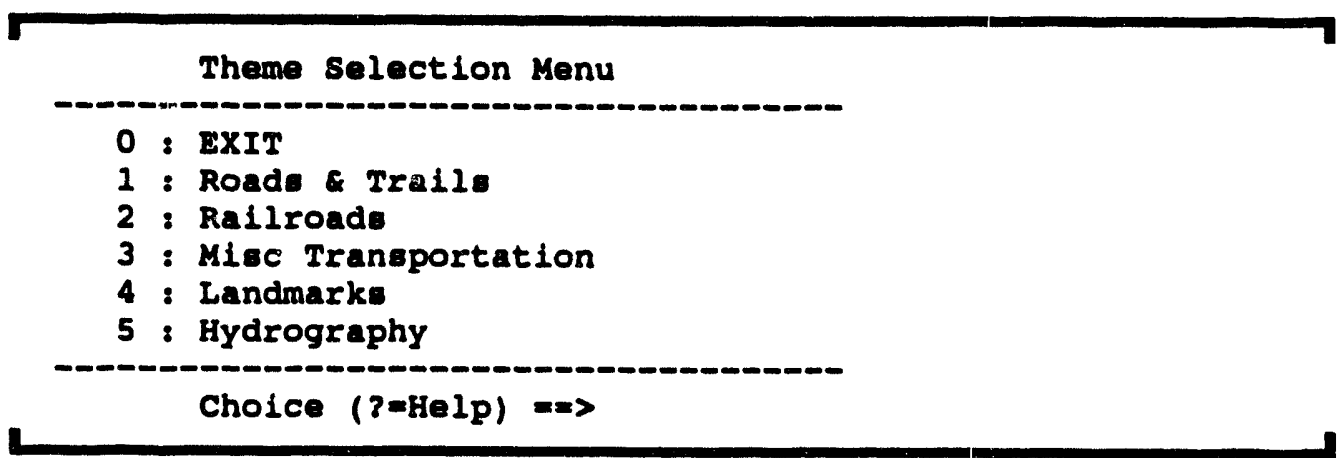

7. Enter the number of each theme that you wish to convert.

An asterisk appears to the left of each theme that you choose, indicating which themes will be converted.

8. When you are finished selecting themes, enter $\mathbf{0}$ to exit the Theme Selection Menu. 
Next the program prompts for the name of the database where the new DMS files will be stored. The currently selected database is shown in square brackets [ ].

For example:

Enter the database name [ANAD] $\Rightarrow$ ANAD

9. To specify the database, enter the database name.

OR

To use the current database [in brackets], just press RETURN.

The program then begins creating the command files.

\section{Results of Using TIGERCVT}

After you finish identifying the required information, TIGERCVT does the following:

- It reads the TIGER/Line Basic Data Record and Shape Coordinate Points files to determine the number of features of each theme and the longitude-latitude extents of the county. When this is complete, the following messages appear with statistical information filled in:

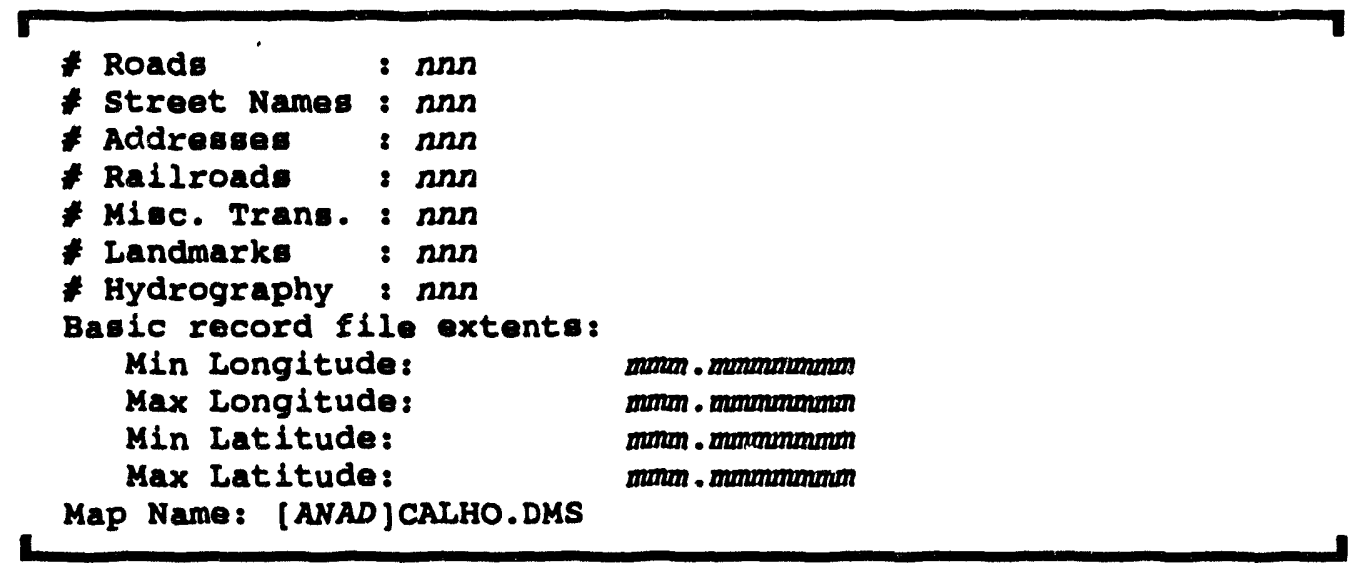

- The program then displays the following messages as it creates three command files in the current directory:

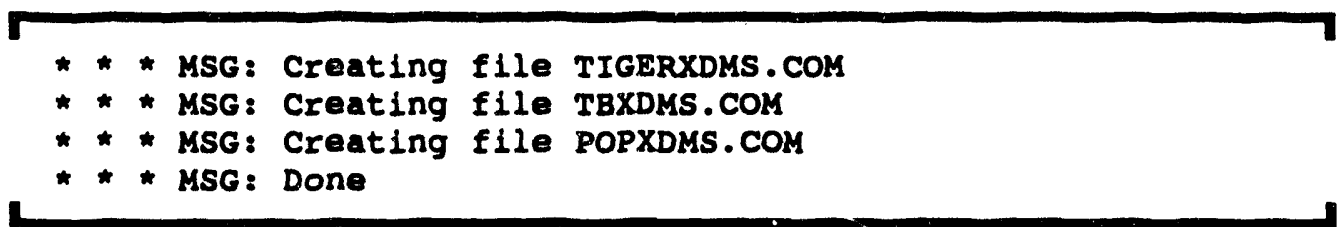


These command files-TIGERXDMS.COM, POPXDMS.COM, and TEXDMS.COM-can be used to execute the TIGERXDMS, POPXDMS, and TBXDMS utilities, respectively.

\section{Converting Census Bureau Data Using TIGERXDMS.COM, POPXDMS.COM, and TBXDMS.COM}

The following procedure describes the sequence for using the three command files to automatically run the TIGERXDMS, POPXDMS, and TBXDMS utilities. If subdirectories for each of the new map layers have not been created under the database top-level directory, the three utilities will create the necessary subdirectories.

1. Set the default directory to the directory where the command files TIGERXDMS.COM, POPXDMS.COM, and TBXDMS.COM are located. For example:

\section{S SET DEFAULT [ANAD]}

2. Then "is TIGERXDMS.COM to execute TIGERXDMS and convert TIGER/Line data to DMS formal:

\section{\$ OTIGERXDMS}

TIGERXDMS.COM automatically directs TIGERXDMS to sort the TIGER/Line data files and convert the entire county set of TIGER/Line data to DMS files. For more information and a list of TIGERXDMS operating messages, see TIGERXDMS.

If a selected topographic theme exceeds 5000 data objects, the theme data are split into a number of DMS files that represent equal-area sections of the county. The DMS file name for a theme that has been split into sections has three parts:

- the first three letters of the county name

- the number of the section

- the two-character code of the data theme.

For example, if Calhoun county contained 9,000 roads, the county would be split into two sections (01 and 02 ), and the $\mathrm{RD}$ subdirectory after conversion would contain the following roads-and-trails data files and associated text files:

\section{CALO1RD.DMS CAL02RD.DMS CAL01RD.TXT CAL02RD.TXT}

3. Use POPXDMS.COM to execute POPXDMS and convert Census Bureau population didid to DMS format: 


\section{$\$$ OPOPXDMS}

For more information and a list of POPXDMS operating messages, see POPXDMS.

4. Finally, use TBXDMS.COM to execute TBXDMS and convert TIGER/Line boundary data to DMS format:

\section{\$ @TBXDMS}

This executes TBXDMS.COM, which will run the TBXDMS program with the correct longitude-latitude extents and county name. For more information and a list of TBXDMS operating messages, see TBXDMS.

You can use the new DMS files and subdirectories as an independent DMS database. For example, to view the county data, you can enter GEDIT to start the IBS map editing utility.

Note: Some of the TIGER/Line boundary data is incomplete. After converting this data, you should view it with GEDIT: editing may be necessary.

\section{TIGERCVT Program Messages}

The following is a list of messages that you might see while using TIGERCVT. The list includes interpretations of each message and suggested actions.

* * ERra Bad directory

* * ERR: Creating directory [name] The directory name you specified was not a correct directory name. Check to make sure that the name contains the proper characters.

* * ERR: Bad theme. Try agaín

The theme number you selected was not in the proper range. Enter a new value. 
* * ERR: Inconsistent state and county codes

The five-digit combined state and county code you specified in the TIGER

filenames is not consistent. Check the names of the TIGER files.

* * ERR: nn Opening file filename

An error occurred when the program attempted to open the file specified. Check that the directory specification is correct.

* * ERR: nn Reading file filename

An error occurred when the program attempted to read a record from the file specified.

* * ERR: nn Writing file filename

An error occurred when the program attempted to write a record to the file specified.

* * * ERR: stopping

Due to an input/output error, the program is stopping execution. 


\section{TIGERXDMS}

The TIGERXDMS utility creates DMS data files from TIGER/Line data files provided by the U.S. Department of Commerce, Bureau of the Census. The Bureau of the Census makes TIGER/Line data available for individual counties. TIGERXDMS is designed to handle one county at a time, converting data from the following records:

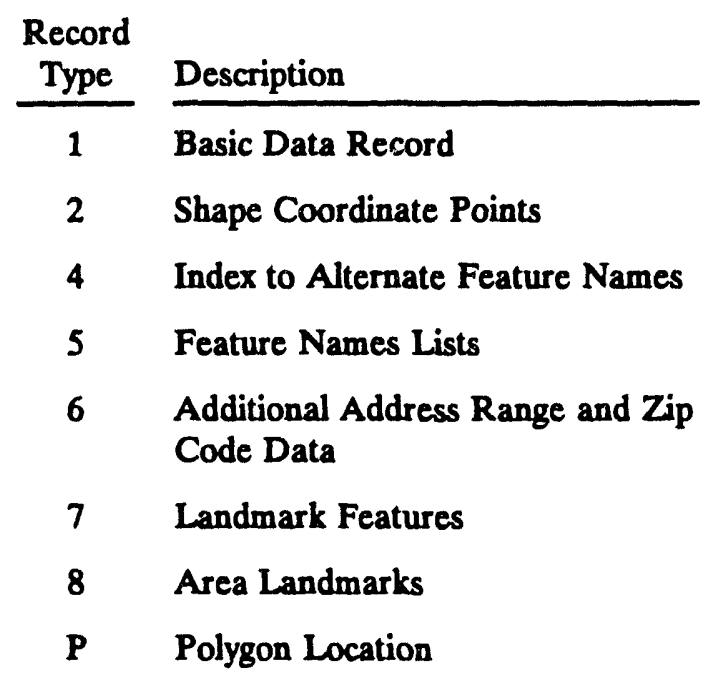

Other data records of the TIGER/Line data files are ignored.

Note: You will normally NOT use TIGERXDMS directly: for most purposes you will want to use the TIGERCVT utility program, which creates a special command file that runs TIGERXDMS for you. If a topographic theme (such as railroads) contains a large number of vector objects, the theme data may need to be split into more than one DMS file. TIGERCVT automatically reads the TIGER/Line data extents and (if necessary) manages the splitting of a county's data into separate DMS files. (See TIGERCVT.)

\section{What You Need to Know Before Using TIGERXDMS}

You will normally want to use the TIGERCVT utility rather than TIGERXDMS, as noted above. If you do use TIGERXDMS independently, you must supply the following information:

county name This is the name of the county for which you want data.

county limits These are the longitude-latitude extents of the county: the minimum and maximum longitude and the minimum and maximum latitude. 
combined state and county code

drive:[directory] of TIGER data

theme
This five-digit code consists of the two-digit FIPS state code followed by the three-digit FIPS county code.

This is the name of the disk device and directory where the TIGER files are located.

The theme is the category of topographic data that you wish to convert to DMS format. You can choose to convert one theme at a time. Your selection determines which records the program selects to convert to DMS format.

\section{Creating DMS Data Files from TIGER/Line Data Files}

To start the utility, just enter TIGERXDMS at the system prompt. You will then see prompts for the information described in the previous section. The series of prompts will resemble the following example (simulated responses are in bold).

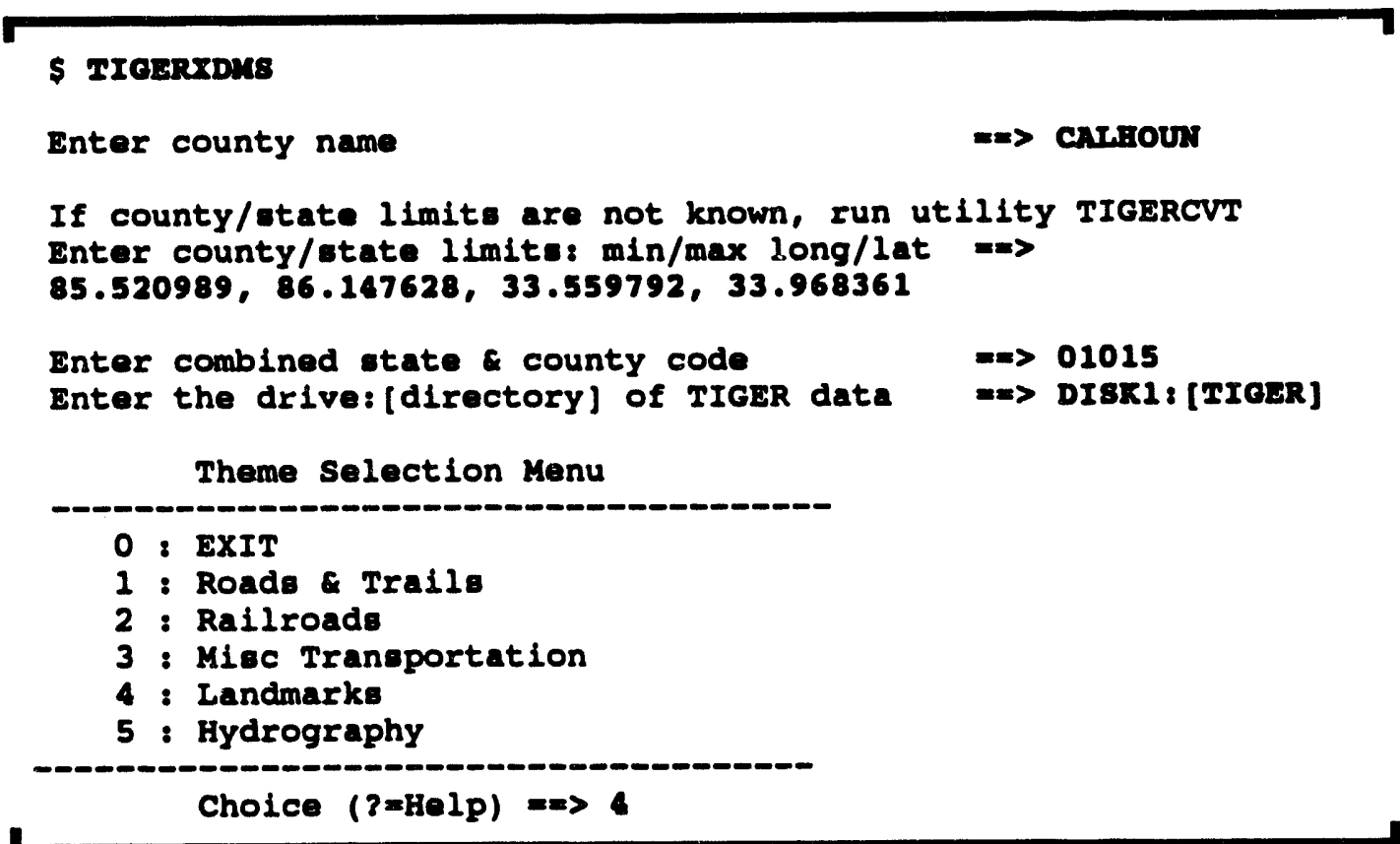

When TIGERXDMS begins reading the TIGER data for the data conversion, you will see a series of messages as the program reads each record: 


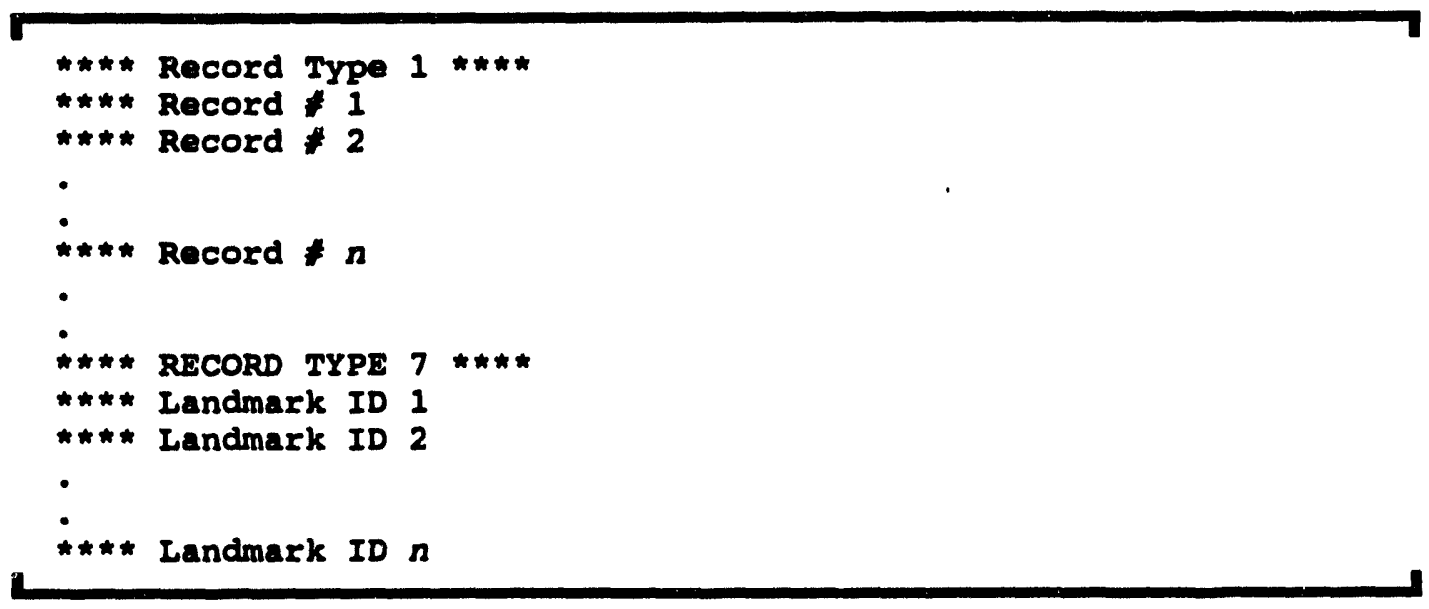

After all records have been read and the DMS files have been created, the program ends and the system prompt reappears.

\section{TIGERXDMS Program Messages}

*MSG* [directory] TGRnAnRn.Sam FILE COULD NOT BE OPENED. Here, the number nnnnn represents the five-digit state/county code that you have entered. One of the TIGER data files needed for the conversion could not be opened. If this happens, the program attempts to open the remaining files but will not perform a conversion. Check the directory and FIPS code, and make sure that all of the TIGER files needed are present.

BAD THEME NUMBER. TRY AGAIN

The theme number must be between one and five. Enter a new value.

*MSG* Out of object Iimits

The object specified in the current TIGER record did not fall within the longitudelatitude limits specified for the current county. The program will ignore that record and proceed to the next.

*MSG* Landmark not a single point

A TIGER landmark record that refers to more than one point was encountered.

*MSG* COULD NOT OPEN FILE... nnn

The program encountered an error opening the DMS format files. Check that the logical names for the topography directories are correct, and that the directories exist.

*MSG* Feature not found in Record Type 5 file

The name of a feature listed in another record was not found in the Feature Name List (Record Type 5) file. The feature will be stored without a name. 
*MSG* Addreev range on landmark feature

An address range was specified for a landmark feature.

«MSG* Unexpected CrCC

The program encountered a record in the Landmark Features file with a Census Feature Class Code (CFCC) specifying something other than Landmark Feature or Hydrography.

*MSG* nnnnnnnnnn Landmark ID not found in Record Type 8 File The program encountered a landmark record that referred to the Landmark Area (Record Type 8) file, which lists landmarks consisting of areas instead of points. However, no record was found in the Record Type 8 File.

*MSG* nnnnnnnnnn Polygon not found in Record Type P The program encountered a record referring to a polygon listed in the Polygon Location (Record Type P) file. However, no such record was found in the Type P file.

*MSG* Duplicate landmark point at mmmmmmmmm nnnnnnnnnn The program encountered a landmark record containing the given longitudelatitude coordinates, which were already used by an earlier record. The duplicate landmark will be ignored. 


\section{UPDATEATT}

The UPDATEATT utility is a database administration program that you can use to update the system attribute dictionary-the master listing of attribute codes that can be used to describe objects in a DMS file. The utility displays specified attributes on the terminal screen, enabling you to modify values associated with each attribute. The IBS attribute dictionary is MAPSATTRIB:ATTRIBDIC.BIN.

Note: An alternative to using UPDATEATT is to use the ATTXASC and ASCXATT utilities in combination. ATTXASC reads an attribute dictionary and creates an editable ASCII text version of the dictionary. After you carefully edit the ASCII file, ASCXATT can read the updated text file and create a new binary attribute dictionary file.

\section{What You Need to Know Before Using UPDATEATT}

To ensure consistency between IBS systems in the emergency management community, updates to the attribute dictionary should be managed through a central authority. Persons responsible for maintenance of the master file should take great care to preserve consistency when updating the attribute list. Whenever a new attribute code is a candidate for insertion into the list, criteria similar to the following should be used for evaluating the code's applicability:

- Is the code unique from others currently in the master attribute file?

- Which major category best describes the code?

- Could parameter values on current codes be used to describe this attribute?

- Because a limit of 16 major categories is presently imposed, no new major category should be added unless it is to contain a sizable number of attributes (at least 20). Also, the new category should be viewed as a substantial subdivision of geo-coded data.

If you want to change existing attributes, you must know the major and minor attribute codes for these attributes. For a listing of attributes in the IBS standard attribute dictionary, see Appendix B.

UPDATEATT enables you to display each attribute for updating on the terminal screen. The data record of one attribute that you specify is displayed in a form (data input screen) that contains fields (data entry spaces on the form). To update an attribute, you just change values of the data fields. 
Keyboard Commands for Using the Data Forms. The data input forms follow the conventions explained in Section 1 of the IBS User Guide. If you have not already done so, please read that section. In brief: Use the arrow keys to move from one field to another on a form. When you are finished making entries, press GOLD $Z$ to accept the entries-or press CTRL-W to quit and restore the original contents of the form. Pressing RETURN also advances from one field to the next. In the last field of a form, pressing RETURN (like GOLD Z) accepts the current form data and then exits.

Accepting Updates to the Attribute Dictionary. The utility stores all the changes and updates you make until you leave the utility. At that time, UPDATEATT gives you an opportunity to cancel all your updates (Quit) or to accept the updates and create a new attribute dictionary (Exi).

\section{Updating the IBS Attribute Dictionary with UPDATEATT}

To start the utility, just enter UPDATEATT at the system prompt. UPDATEATT then displays its menu prompt:

S UPDATEATY

DO YOU WANT TO (A)dd, (C)hange, (D)elete, (E)xit or (Q) uit >

To choose one of the options, just enter the appropriate letter:

A - Add a new attribute record.

C - Change any field on an existing attribute record.

D - Delete an attribute record.

E - Exit the program and update the attribute dictionary.

Q - Quit the program without updating the atribute dictionary.

The three options for updating individual attributes are explained in the following sections.

\section{A - Adding a New Attribute Record}

When you choose option A, the Attribute Description update form appears on the screen with only default data in all of the fields (see the following page). When a form is displayed, the first data field is highlighted to show that the field can be edited: you can change values in any of the fields. If a record already exists for the major and minor attribute codes entered, a new record will NOT be created. 


\section{Attribute Description Update Form}

ATTRIBUTE DESCRIPTION

MAJOR MINOR PARAMTERER

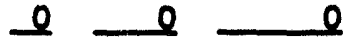

DESCRIPTION

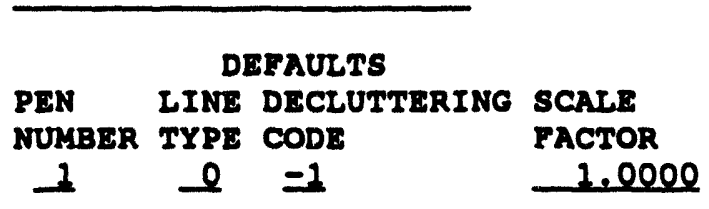

Prese <RETURN> or the GOLD <F5> key followed by a ' $Y$ '

to go back to Modify Selection Menu.

\section{C - Changing an Attribute Record}

When you choose option $\mathrm{C}$, the program will prompt for a major and minor attribute code:

\section{Example}

DO YOU WANT TO (A)dd, (C) hange, (D)elete, (E)xit or (Q) uit >C ENTER THE MAJOR AND MINOR ATTRIBUTE CODES $>5,20$

Enter the major and minor codes, separating them with a comma. For a listing of attributes in the IBS standard attribute dictionary, see Appendix B. After you enter these codes, the Attribute Description update form appears on the screen with the values for the specified attribute displayed in the form fields. You can change any field.

\section{D - Deleting an Attribute Record}

When you choose option $D$, the program prompts for a major and minor attribute code in the same way as for changing an attribute. After you enter these codes, you will return to the menu prompt. 


\section{USGS100KXDMS}

The USGS100KXDMS utility creates a DMS file from a USGS Digital Line Graph (DLG) data file that stores 1:100,000-scale data. For example, the following sequence will convert the data in a DLG file called BR201RD.DAT to DMS format in a file called BR201RDRT.DMS.

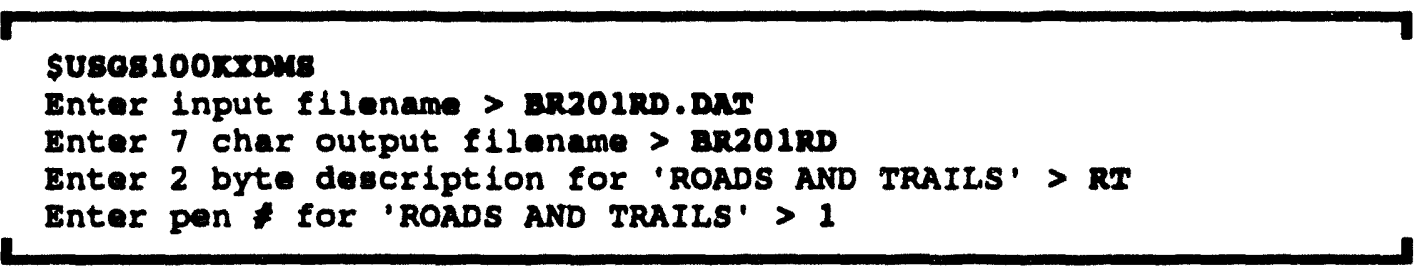

\section{Converting USGS Data Files for a Special-Purpose DMS Database}

The following procedure is designed to assist a System Manager or knowledgeable user in the process of extracting DLG data files from magnetic tape and converting them to DMS format. Your goal in converting files from DLG to DMS format may be to create a special-purpose DMS database or to add data for use with an existing DMS database. The conversion process is pertinent to the development of site-specific DMS databases as described in the IBS Data Management Guide.

Overview. You are starting with a set of magnetic tapes and a list of the DLG files on those tapes. You will follow three main steps:

1. Extract the DLG data files from magnetic tapes onto disk, using the MYTAPE utility.

2. Separate the files into topography subdirectories.

3. Convert each data file in each subdirectory to DMS format, using the USGS100KXDMS utility.

The final product is several subdirectories containing DMS formatted files. The conversion process is depicted by the following flow:

\begin{tabular}{|l|l|l|l|l|l|l|l|l|l}
\hline $\begin{array}{l}\text { magnetic } \\
\text { tapes }\end{array}$ & MYTAPB & $\begin{array}{l}\text { directory containing } \\
\text { USGS DLG data files }\end{array}$ & $\begin{array}{l}\text { Usos100KXDMS } \\
\text { subdirectory containing } \\
\text { DMS data files }\end{array}$
\end{tabular}

The transformations represent the state of the topographical data, and the descriptions beneath the arrows represent utilities that operate on the data.

We recommend that you convert the DLG files from one magnetic tape at a time, rather than all at once. The reason for this is that the USGS data files consume several times 
more disk space than the DMS files. You may need to delete USGS data files after DMS conversion to reserve enough disk space for extracting files from other tapes. Monitor disk space carefully throughout the conversion process.

\section{Requirements to Begin}

- data tape(s) containing the DLG files

- a list of the files on the tape(s)

- the number of characters for each logical record

- nine-track, 1600-bpi compatible tape drive

- MYTAPE utility (version 3.5 or later).

It is also expected that you are familiar with certain aspects of DMS topography files: the two-character "topographic codes" associated with DMS topographies and the "pen" numbers or colors used to draw topographic features on the screen. (Please refer to steps $2 \mathrm{~b}, 3 \mathrm{e}$, and $3 \mathrm{f}$ in the following procedure.)

\section{Procedure}

1. Extract the DLG data files from magnetic tapes onto disk: You will use the MYTAPE utility to get the DLG data files to disk. Complete MYTAPE documentation is available as PNLSDOCUMENT:MYTAPE.DOC.

a. Load the tape physically on the tape drive.

b. Set the default to the directory where the DMS files are to be located.

c. Enter MYTAPE

The MYTAPE prompt will appear as "MYTAPE > ". From this prompt, you may enter any MYTAPE command.

d. To mount the magnetic tape, enter a MOUNT command:

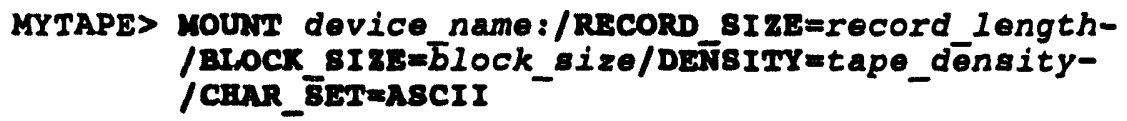

The parameter and /qualifier values that you must enter are:

device_name: physical name of tape drive used (MTA0:, MMA0:, MSA0:, etc.) or the logical name (TAPE0:, TAPE1:, etc.)

record_length number of bytes in a tape record 


$$
\begin{array}{ll}
\text { block_size } & \text { number of bytes in a tape block } \\
\text { tape_density } & \text { density of tape in bytes per inch (bpi). }
\end{array}
$$

The record length, block size, and tape density should appear on the label of the magnetic tape. The /CHAR_SET (character set) qualifier should always be "ASCII" for this use.

Example:

MYTAPED MOUAY TAPE1/RECORD_8I ZE=144/BLOCKSIZE=1440/DENSITY $=1600 /$ CEAR_8ET=ASCII

e. To retrieve each file from the magnetic tape, enter a COPY command:

$$
\begin{aligned}
& \text { MYTAPES COPY device name: file name }
\end{aligned}
$$

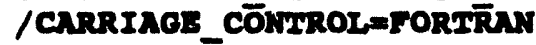

The parameters and /qualifier values that you must enter are:

$$
\begin{aligned}
& \text { device_name: } \begin{array}{l}
\text { physical name (or logical name) of the tape drive } \\
\text { used }
\end{array} \\
& \text { file_name } \\
& \text { the name of the USGS data file }
\end{aligned}
$$

The /CARRIAGE_CONTROL qualifier should always be "FORTRAN" to designate a FORTRAN carriage return as the end-of-line character.

To retrieve a set of files, you may place a number of filenames in a separate description file and then pass the name of the description file as a parameter to the MYTAPE COPY command:

$$
\begin{aligned}
& \text { MYTAPE> COPY device name: description_file/FIIE_IIsT- } \\
& \text { /CARRIAGE_cöTHROL-FORTRN }
\end{aligned}
$$

The FILE_LIST qualifier indicates that the input file is a list of filenames.

f. To dismount the magnetic tape drive, enter a DISMOUNT command:

$$
\text { MYTAPE> DIBMOUNT device_name: }
$$

g. Exit the MYTAPE utility:

MYTAPE> EXIT 
Example of MYTAPE execution:

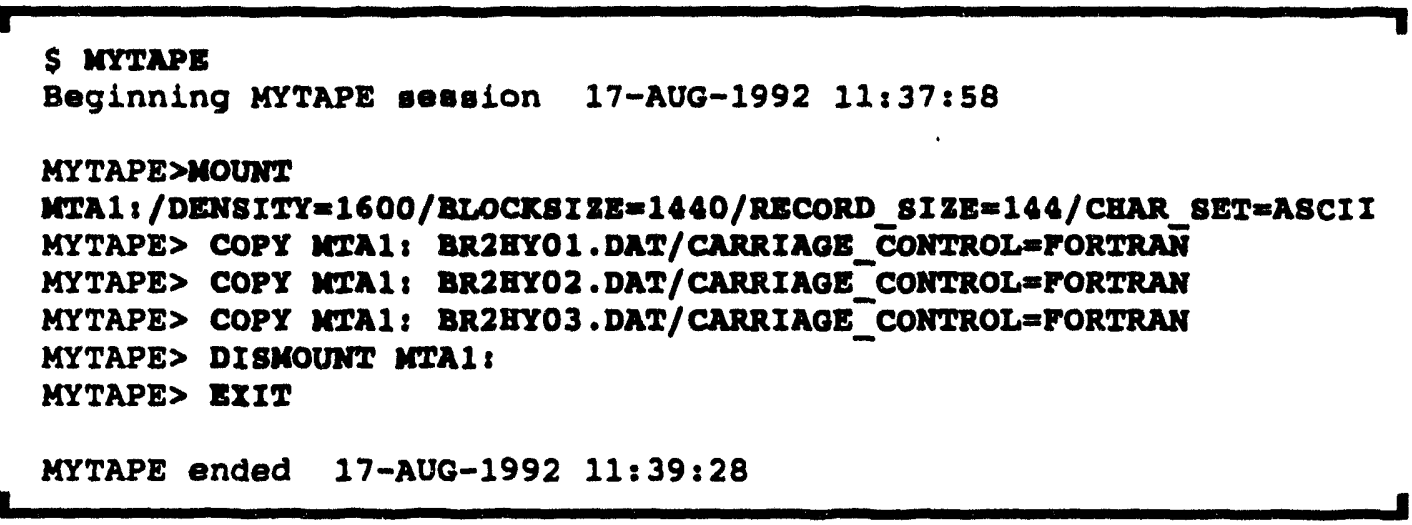

Output files created by this example:

$\begin{array}{ll}- & \text { BR2HY01.DAT } \\ \text { - } & \text { BR2HY02.DAT } \\ \text { - } & \text { BR2HY03.DAT }\end{array}$

Example of MYTAPE execution with a description list file:

For this example, the description file, BR2HY.LIS, contains the names of three DLG data files:
- BR2HY01.DAT
- BR2HY02.DAT
- BR2HY03.DAT

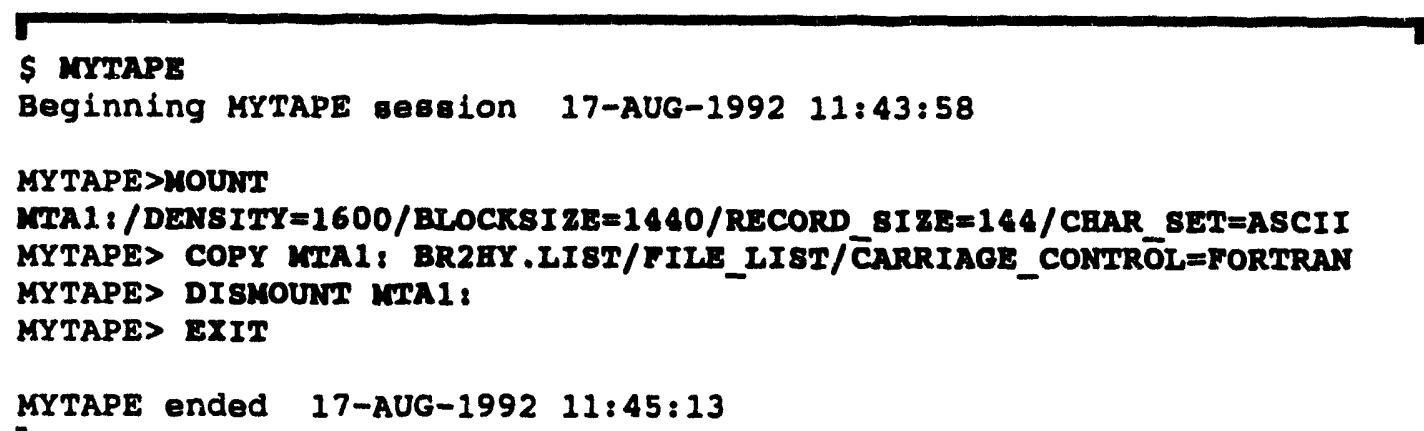


Output files created by this example:

- BR2HY01.DAT

- BR2HY02.DAT

- BR2HY03.DAT

2. Separate the data files into topographic subdirectories: Once a set of data files has been extracted from magnetic tape, the files should be sorted into topography subdirectories.

2. Check the output listing of the magnetic tape(s) to determine what topographic information is in the data file(s).

This listing should have been provided with the magnetic tapes. The two-letter topographic code will be embedded in the original data file filename. Typical codes are HY (hypsography), RD (roads and trails), RL (railroads), and MT (miscellaneous transportation).

b. For each topography file in the listing, locate the associated, extracted file and place that file in the appropriate DMS topography subdirectory.

An "appropriate DMS topography subdirectory" means 1) a subdirectory of a DMS database directory; 2) a subdirectory named with a two-character "topographic code" that corresponds to the type of data in the topography files. (Please refer to step $3 e$ of this procedure.)

3. Consert each data file in each subdirectory to DMS format: You will use the USGS100KXDMS utility.

a. SET DEFAULT to the topography subdirectory.

\section{b. Enter USGS100KXDMS}

This executes the conversion utility. (See also, Executing USGS100KXDMS in Batch Mode, after step 3g).

The utility will prompt you to enter an "input filename," an "output filename," a topography "description," and a "pen \#," as shown in steps 3c, $3 \mathrm{~d}$, and $3 \mathrm{e}$.

c. Enter the name of the DLG file to be converted.

EXample: ENTER INPUT FILENAME > BR201RD.DAT 
d. Enter a name for the new DMS file (up to 7 characters).

EXample: ENTER 7 CHAR OUTPUT FILENAME > BR201RD

(If the name of the DMS file is to be the same as the DLG filename, press the UP arrow or <scrollback > key; this will insert the input filename to the screen. Then delete the '.DAT' characters.)

USGS100KXDMS will check the DLG file to identify the topographic data.

e. Enter a 2-character topographic code for the DMS file.

EXample: ENTER 2 BYTE DESCRIPTION FOR 'ROADS AND TRAILS' $>\mathbf{R T}$

The utility will append the two characters to complete the name of the new DMS file.

The following list shows examples of topographies and topographic codes used in the IBS. Appendix A is a complete list of IBS topographies and their codes.

Topographic Descriptor

ADMINISTRATIVE BOUNDARY
AGRICULTURE
BASE MAP
CONGRESSIONAL DISTRICTS
CULTURAL FEATURES
RECOVERY AREAS
CENSUS TRACT/ED
ELEVATION CONTOUR(30OM)
ELEVATION
ELECTRICAL POWER GRID
EVACUATION ZONE
FAULT LINES
FEATURES (ICONS)
GNIS (GEOgraphic Names)
HYDROLOGY
1OOK HYDROGRAPHY
HYPSOGRAPHY
HAZMAT SITES
LIVESTOCK
LAND USES
1OOK MISC TRANSPORT

Two-Character Code

AB
BG
CD
CF
CN
CT
EC
EL
EP
EZ
FQ
FT
GN
HD
HG
HY
HZ
LS
LU
MT


f. Enter a pen number ("color" number) for the topographic data type.

Example: ENTER PEN * FOR 'ROADS \& TRAILS' > 1

A map database, for example, might use pen 1 for roads and trails, pen 4 for miscellaneous transportation, and pen 5 for railroads. When the topographic data are drawn with the system mapping functions, they will be drawn in the "color" corresponding to the pen number.

If you wish to follow the color conventions used in the IBS map databases, you will need to know the pen numbers associated with your topographies. You can examine an existing DMS topography file and identify the pen number by using the utility DMSXASCII.

USGS100KXDMS has completed when the \$ prompt returns.

g. Repeat $3 a-3 f$ for each DLG file in each topography subdirectory.

h. Delete the unconverted USGS files from each topography subdirectory.

\section{Executing USGS100KXDMS in Batch Mode}

USGS100KXDMS can be executed using a command file. If you execute the utility from a command file as part of a batch job, use the /LOG_FILE qualifier to generate a batch log. This will be helpful if an error occurs. The following example command file will convert three DLG input files (BR2D01.DAT, BR2D02.DAT, and BR2D03.DAT) to three DMS "roads and trails" files.

Command File Example:

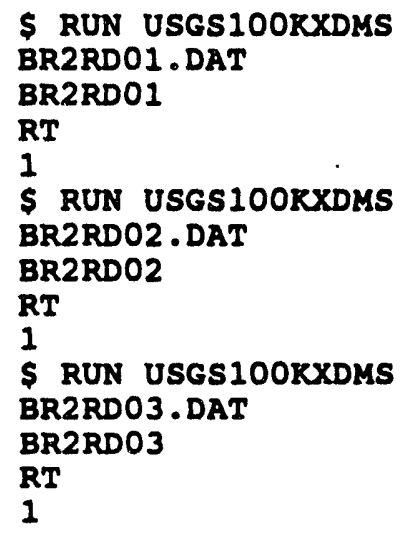


Output files created by this example command file:

- BR2RD01RT.DMS

- BR2RD02RT.DMS

- BR2RD03RT.DMS

\section{Errors Caused by Missing Attribute Codes}

To translate from DLG to DMS files, USGS100KXDMS contains a table of USGS attribute codes. If the DLG file contains new attribute codes that are not in the table, then USGS100KXDMS will stop and list the missing attributes by Major and Minor code.

Note: To fix this problem and complete the file conversion, system development personnel with access to the program source code must update the appropriate subroutine (called CNVT_ATTS) in the USGS100KXDMS utility, recompile and re-link the program code. Then the program could be re-executed for the DLG file that caused the error.

\section{Results of Using USGS100KXDMS}

At the end of this procedure you should have one or more topography subdirectories, each containing DMS files converted from the DLG data files.

If you are using the new DMS files to create a new DMS database, please refer to Generating a Site-Specific Map Database in the IBS Data Management Guide. That discussion explains the strategy for getting your data into the new database. 


\section{USGSXDMS}

The USGSXDMS utility reads data files in USGS format and creates new data files in DMS format. In general, the USGS files will be the output of processing a foreign tape using the MYTAPE utility.

\section{Converting USGS Format Data to DMS Format with USGSXDMS}

To start the utility, just enter USGSXDMS at the system prompt.

As USGSXDMS reads the data file header information, it requests that you supply file names, topographies, and pen (color) numbers to be used in the data conversion process.

The USGSXDMS utility does NOT convert DLG attribute codes to the corresponding DMS attribute codes. Rather, the major and minor code values are stored in the newly created DMS file. To translate DLG attributes information to DMS attribute codes, use the USGS100KXDMS utility.

To convert USGS Digital Line Graph (DLG) files to DMS format, use the DLGXDMS utility. 


\section{UTMXLL}

The UTMXIL utility is a general system program that converts in both directions between Universal Transverse Mercator (UTM) coordinates and longitude/latitude coordinates.

\section{Converting Between UTM and Longitude/Latitude with UTMXLL}

To start this utility, enter UTMXIL at the system prompt. The program then displays its initial prompt:

Convert from UTM? [Y]

Your answer to this prompt determines whether you will enter UTM coordinates for conversion to long/lat coordinates or long/lat coordinates for conversion to UTM coordinates. The following table illustrates these two paths through the program.

Convert from UTM? [Y]

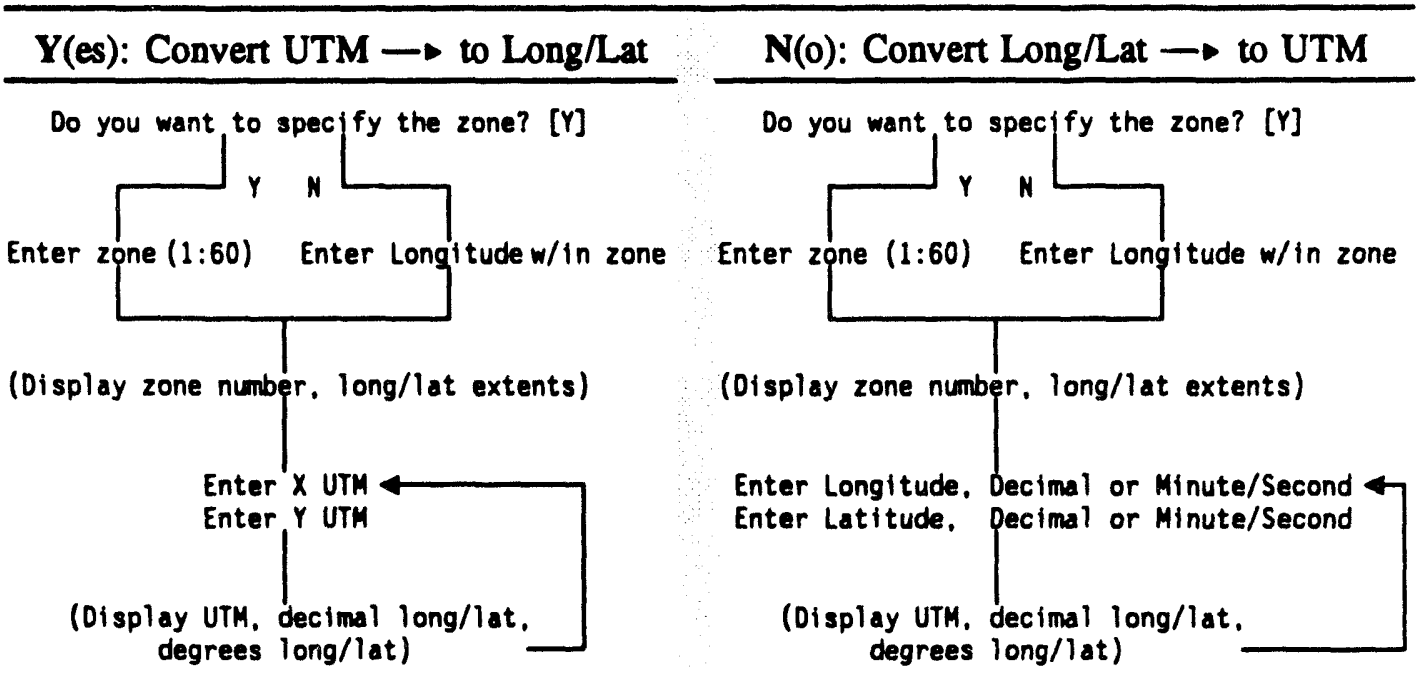

After you enter either kind of coordinates, UTMXIL displays UTM coordinate values as well as long/lat values in both decimals and degrees. The programs then continues to prompt you for more coordinates to convert. Convert as many as you like. If you want to return to an earlier prompt, press CTRL-Z until you reach the correct prompt. If you press CTRL- $Z$ at the initial prompt, the utility ends.

The following example shows first a conversion from UTM coordinates and then switches to show a conversion from decimal long/lat coordinates. 
Example:

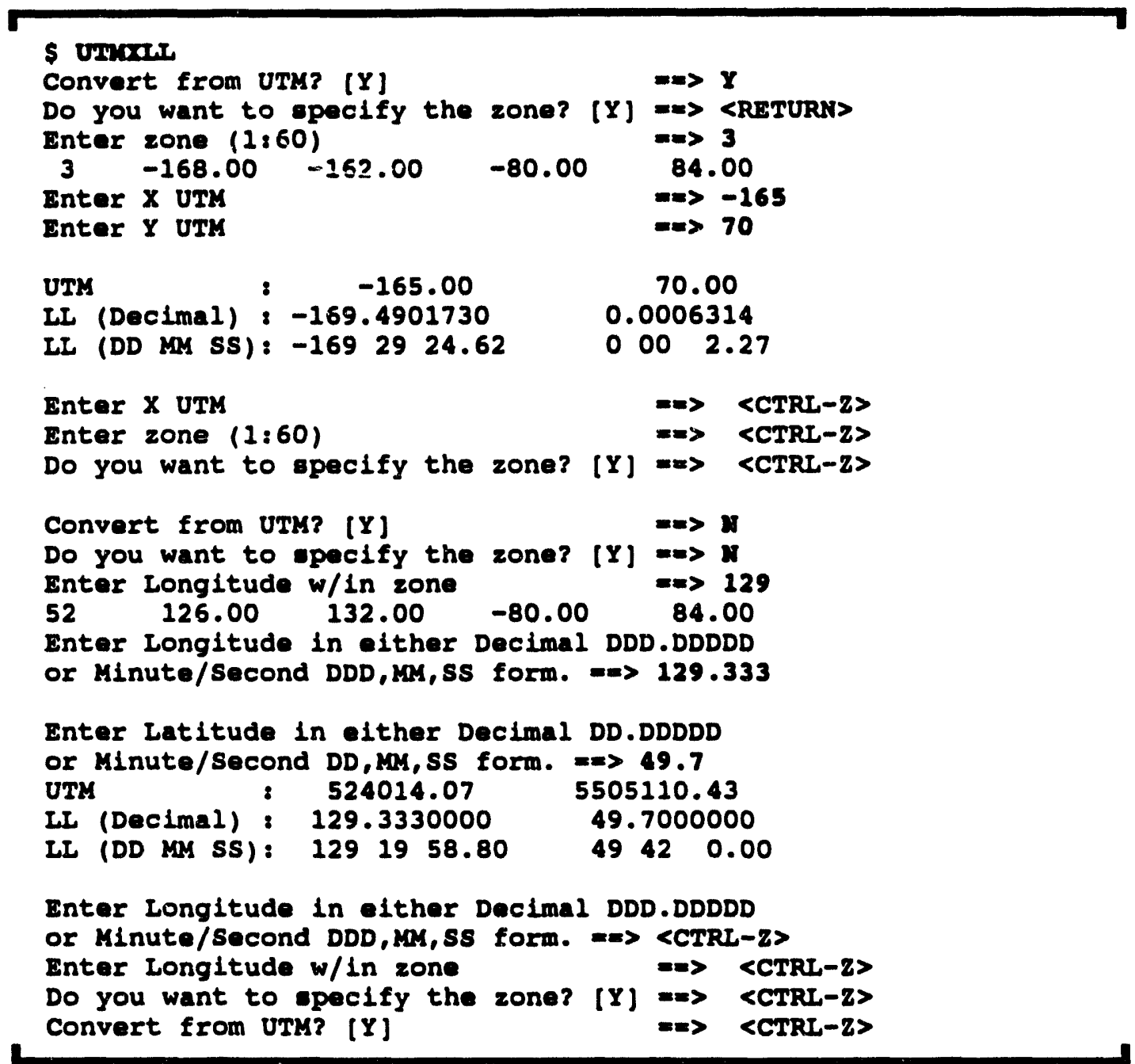




\section{XMAIL}

The XMAIL utility is a VAX MAIL enhancement program that is used as a basis for checking IBS user mailboxes for unread mail. XMAIL also has other useful electronic mail functions.

\section{Using Mail Enhancements in XMAIL}

To start this utility, enter XMAIL at the system prompt. The program then presents a command prompt for entering XMAIL commands.

$$
x-\operatorname{ma1} 1>
$$

The following is a list of a few XMAIL command functions and brief descriptions. For more information about a given function, please see the online help, which is available by entering HELP at the XMAIL command prompt.

\begin{tabular}{|c|c|}
\hline XMAIL Command & Description \\
\hline CHECK & $\begin{array}{l}\text { enables you to see if VMS mail messages that you sent } \\
\text { have been read }\end{array}$ \\
\hline CLEAN_VMSMAIL & $\begin{array}{l}\text { Deleting a username from SYSUAF.DAT does not } \\
\text { remove that username from the system mail file. } \\
\text { Subsequently, if a user is added with the same } \\
\text { username, an erroneous value for the number of new } \\
\text { mail messages may be displayed. This option deletes } \\
\text { records in the system mail file for which there is no } \\
\text { match in the SYSUAF.DAT file. }\end{array}$ \\
\hline EXIT & stops XMAIL and returns the user to DCL \\
\hline HELP & presents online help information, similar to VAX HELP \\
\hline NUMBER & $\begin{array}{l}\text { determines the number of mail messages that have not } \\
\text { been read by a given user or all users }\end{array}$ \\
\hline RESET_NEWMAIL & $\begin{array}{l}\text { resets the number of new mail messages that the system } \\
\text { thinks you have to the number that actually exist in your } \\
\text { VMS mail file }\end{array}$ \\
\hline SMAIL & $\begin{array}{l}\text { enables you to send a mail message to all users on the } \\
\text { system }\end{array}$ \\
\hline
\end{tabular}


This page is deliberately left BLANK. 


\section{Appendix A IBS Topographies (Map Layers)}

This appendix lists the names of the standard IBS topographies or map layers. In general terms, a topography is a graphic representation of the physical features of a geographic region. In IBS, a topography is a set of geographic data for depicting map features that have a common theme. Each topography has a name that describes that theme (for example: agriculture, roads, water bodies).

A topography consists of DMS files that contain data for mutually exclusive areas within a larger region (like tiles covering a floor).

- Static topographies contain DMS files that are not changed by anyone other than the IBS database administrator. These files are generated by the database administrator, placed in a topography within the map database, and made available to all users (who cannot modify them).

- Volatile topographies contain DMS files that can be modified by IBS users only through specific functions of the IBS application software. The volatile topographies usually represent features that change over time (for example: explosion contours, search/rescue areas). Before you can create or modify a volatile DMS file, the database administrator must authorize you to use that function of the IBS software.

The DMS files for a single static topography are stored in a common map database subdirectory, MAP\$DATASxx, where $x x$ is a different two-character topographic code for each topography. The DMS files for a single volatile topography are stored in a common subdirectory, VMAPSDATASsx. By convention, the names of the individual DMS files of one topography also include the topographic code: namexx.DMS.

For each topography of the standard IBS map database, this appendix includes the following:

- the topography name

- the topographic code

- an indication of whether the topography is static or volatile

- an expanded description (sometimes). 


\begin{tabular}{|c|c|c|c|}
\hline Toporraphy Name & Code & Type & Additional Description \\
\hline Administrative Boundary & $\mathbf{A B}$ & Static & -- \\
\hline Agriculture & AG & Static & -- \\
\hline Base Map & $\mathbf{B M}$ & Static & -- \\
\hline Congressional Districts & CD & Static & -- \\
\hline Cultural Features & $\mathbf{C F}$ & Static & -- \\
\hline Recovery Areas & CN & Static & -- \\
\hline Census Tract/ed & $\mathbf{C T}$ & Static & -- \\
\hline Elevation Contour $(300 \mathrm{~m})$ & EC & Static & -- \\
\hline Elevation & EL & Static & -- \\
\hline Electrical Power Grid & EP & Static & -- \\
\hline Evacuation Zone & $\mathbf{E Z}$ & Static & -- \\
\hline Fault Lines & FQ & Static & -- \\
\hline Features (Icons) & $\mathbf{F T}$ & Static & -- \\
\hline GNIS (Geographic Names) & GN & Static & -- \\
\hline Hydrology & HD & Static & -- \\
\hline 100K Hydrography & HG & Static & -- \\
\hline Hypsography & $\mathbf{H Y}$ & Static & -- \\
\hline Harmat Sites & $\mathbf{H Z}$ & Static & -- \\
\hline Livestock & LS & Static & -- \\
\hline Land Uses & $\mathbf{L U}$ & Static & -- \\
\hline 100K Misc Transport & MT & Static & -- \\
\hline NAPB Targets & NA & Static & -- \\
\hline New Features (Icons) & $\mathbf{N F}$ & Static & -- \\
\hline Pipe Lines & NG & Static & -- \\
\hline Nuc Power Plants & NP & Static & -- \\
\hline Political Boundries & PB & Static & -- \\
\hline Populated Place Names & PL & Static & -- \\
\hline Population & PO & Static & -- \\
\hline 500K Roads & $\mathbf{R 5}$ & Static & -- \\
\hline 100K Roads & $\mathbf{R D}$ & Static & -- \\
\hline 100K Railroads & $\mathbf{R L}$ & Static & -- \\
\hline Railroads & $\mathbf{R R}$ & Static & -- \\
\hline Roads & RT & Static & -- \\
\hline APA Sectors & SC & Static & -- \\
\hline Streams & ST & Static & -- \\
\hline Traffic Control & TC & Static & -- \\
\hline Tract Population & $\mathbf{T P}$ & Static & -- \\
\hline Water Bodies & WB & Static & -- \\
\hline Wells & WE & Static & -- \\
\hline Wildlife & WL & Static & -- \\
\hline Watersheds & WS & Static & -- \\
\hline D2 Track & D2 & Volatile & -- \\
\hline Explosion Contours & EX & Volatile & -- \\
\hline Fire Contours & $\mathbf{F I}$ & Volatile & -- \\
\hline Vapor Contours & VA & Volatile & -- \\
\hline Damage Assessment Areas & DA & Volatile & -- \\
\hline Environment Icons & EI & Volatile & -- \\
\hline
\end{tabular}




\begin{tabular}{|c|c|c|c|}
\hline Toporraphy Name & Code & Type & Additional Description \\
\hline Facilities \& Resources & $\mathbf{R M}$ & Volatile & -- \\
\hline Risk Areas & $\mathbf{R P}$ & Volatile & -- \\
\hline Search/rescue Areas & SR & Volatile & -- \\
\hline Onpost D2 Track & WD & Volatile & -- \\
\hline Weather Icons & $\mathbf{W I}$ & Volatile & -- \\
\hline Boundary - Block & BB & Static & -- \\
\hline Boundary - County & $\mathbf{C B}$ & Static & -- \\
\hline Boundary - Place & LB & Static & -- \\
\hline Boundary - Tract & TB & Static & -- \\
\hline Boundary - Subdivision & VB & Static & -- \\
\hline Street Names (TIGER) & SN & Static & -- \\
\hline Addresses (TIGER) & TA & Static & -- \\
\hline Landmarks & TF & Static & -- \\
\hline Misc Trans & TM & Static & -- \\
\hline Street Names (TIGER) & TN & Static & -- \\
\hline Railroads & TR & Static & -- \\
\hline (TIGER) & TS & Static & -- \\
\hline (TIGER) & TT & Static & -- \\
\hline Water Bodies (TIGER) & TW & Static & -- \\
\hline Water Body Names (TIGER) & WN & Static & -- \\
\hline Population - Block & B1 & Static & Population \\
\hline Demographic - Block & B2 & Static & Demographics \\
\hline House Size - Block & B3 & Static & Households \\
\hline Special Pop - Block & B4 & Static & Special Population \\
\hline Housing - Block & B5 & Static & Housing \\
\hline House Struct - Block & B6 & Static & Household Structures \\
\hline House Value - Block & B7 & Static & Household Values \\
\hline House Type - Block & $\mathbf{B 8}$ & Static & Household Types \\
\hline Population - County & C1 & Static & Population \\
\hline Demographic - County & $\mathbf{C 2}$ & Static & Demographics \\
\hline House Size - County & C3 & Static & Households \\
\hline Special Pop - County & $\mathrm{C4}$ & Static & Special Population \\
\hline Housing - County & C5 & Static & Housing \\
\hline House Struct - County & $\mathrm{C6}$ & Static & Household Structures \\
\hline House Value - County & $\mathrm{C7}$ & Static & Household Values \\
\hline House Type - County & $\mathbf{\infty}$ & Static & Household Types \\
\hline Population - Group & G1 & Static & Population \\
\hline Demographic - Group & G2 & Static & Demographics \\
\hline House Size - Group & G3 & Static & Households \\
\hline Special Pop - Group & G4 & Static & Special Population \\
\hline Housing - Group & G5 & Static & Housing \\
\hline House Struct - Group & G6 & Static & Household Structures \\
\hline House Value - Group & G7 & Static & Household Values \\
\hline House Type - Group & G8 & Static & Household Types \\
\hline Population - Place & $\mathbf{P 1}$ & Static & Population \\
\hline Demographic - Place & $\mathbf{P 2}$ & Static & Demographics \\
\hline House Size - Place & P3 & Static & Households \\
\hline
\end{tabular}


Toporraphy Name Special Pop - Place

Housing - Place

House Struet - Place

House Value - Place

House Type - Place

Population - State

Demographic - State

House Size - State

Special Pop - State

Housing - State

House Struct - State

House Value - State

House Type - State

Population - Tract

Demographic - Tract

House Size - Tract

Special Pop - Tract

Housing - Tract

House Struct - Tract

House Value - Tract

House Type - Tract

Population - Subdiv

Demographic - Subdiv

House Size - Subdiv

Special Pop - Subdiv

Housing - Subdiv

House Struct - Subdiv

House Value - Subdiv

House Type - Subdiv
Code Type

P4 Static

P5 Static

P6 Static

P7 Static

P8 Static

S1 Static

S2 Static

S3 Static

S4 Static

S5 Static

S6 Static

S7 Static

S8 Static

T1 Static

T2 Static

T3 Static

T4 Static

T5 Static

T6 Static

77 Static

T8 Static

V1 Static

V2 Static

V3 Static

V4 Static

V5 Static

V6 Static

V7 Static

V8 Static
Additional Description

Special ?'opulation

Housing

Household Structures

Household Values

Household Types

Population

Demographics

Households

Special Population

Housing;

Household Structures

Househiold Values

Household Types

Population

Demographics

Households

Special Population

Housing,

Household Structures

Household Values

Household Types

Population

Demographics

Housebolds

Special Population

Housing

Housebold Structures

Household Values

Housebold Types 


\section{Appendix B IBS Attribute Dictionary}

This appendix lists the contents of the IBS attribute dictionary-the current master set of attributes values that are used to assign physical or other descriptive features to spatial data objects in the Integrated Baseline System. Each spatial data object can be associated with one or more sets of attribute values that define and describe the object.

The attribute dictionary listing in this appendix contains the following fields:

- Major code: The major attribute code designates a major category of spatial data. The major code may range in value from 0 to 15 . The current major code values and their respective categories are as follows:

\begin{tabular}{|c|c|}
\hline $\begin{array}{l}\text { Major } \\
\text { Code }\end{array}$ & Major Category of Spatial Data \\
\hline$\overline{01}$ & Hydrography \\
\hline 02 & Surface Cover \\
\hline 03 & Boundaries \\
\hline 04 & Undisplayed Text(a) \\
\hline 05 & Roads and Trails \\
\hline 06 & Railroads \\
\hline 07 & $\begin{array}{l}\text { Pipelines, Trans. Lines, Miscellaneous } \\
\text { Transportation Features }\end{array}$ \\
\hline 08 & Other Significant Man-Made Features \\
\hline 09 & Miscellaneous \\
\hline 10 & Text Strings 1 (Displayed Text) $^{(b)}$ \\
\hline 11 & Place of Work Data \\
\hline 12 & Hazardous Material Sites \\
\hline 13 & Resources \\
\hline 14 & Land Use and Soil Type \\
\hline 15 & Property Values \\
\hline
\end{tabular}

(a) This text is NOT displayed on the screen map but is useful for associating additional descriptive text with certain topographic features (such as different portions of a road or different areas of a lake).

(b) This type of text is displayed on the map, often as the labels of point objects (the names of cities, for example). 
- Minor code: The minor code can be used to further define items within a category. For example, a minor code value of 560 designates a permanent lake in the hydrography category (major code $=5$ ).

Note: Some categories do not use the minor code construct in this way. For example, within the category of displayed text strings (major code $=10$ ), the minor code is used in combination with the parameter value to compose an index into a separate file that contains the text strings that are drawn on the map. The minor code may range in value from 0 to 4095 .

- Parameter value: The parameter value is a way of associating a specific value with a particular occurrence of an attribute. For example, if the major and minor codes define the attribute to be "number of hospital beds," then the parameter value could contain the actual number of beds at a particular hospital. The parameter value is not specified for most attribute codes. The parameter value may range from -32768 to 32767 . (See the description of the parameter-decluttering scale factor for more information on how the parameter value is used.)

Also included in this attribute dictionary listing are the default pen type, the default line type, and the decluttering code associated with each set of attribute values.

- Attribute description: The attribute description is a text description of the spatial data item specified by the attribute codes (up to 60 characters long).

- Default pen number: This field associates a pen number (color) with the attribute code. This can be used by map display software to provide a consistent display of DMS data based on attribute codes rather than on the DMS record pen number. The value of this field ranges from 0 to 127 .

- Default line type: This field associates a line type (texture, width, etc.) with an ctribute value. This can be used by graphics software to provide consistent display of DMS data based on attribute codes rather than on the DMS record line types. The value of this field ranges from 0 to 127 .

- Decluttering code: This field indicates the decluttering code number (from 0 to 100) for an attribute and is used by map display software to determine whether or not it is reasonable to display data items based on a specific window size (the size of an area viewed on the graphics display). That is, the map display software will "declutter" a graphics display, using the decluttering number as a basis for removing data items when the display is scaled such that some data items would become distracting clutter. For example, if you display a map that is 200 miles wide, detailed road data at the neighborhood level could be time-consuming to draw and unreadable afterward. A negative one $(-1)$ in the decluttering code field indicates that no decluttering is to occur for an attribute. 
If the attribute code has a value in the parameter value field, then the decluttering code field is used as the base index for calculating the actual decluttering number. See the following description of the parameter-decluttering scale factor for more information on how the decluttering code is used in combination with the parameter value.

- Parameter-decluttering scale factor: This field is used in conjunction with the decluttering code and parameter value to calculate a decluttering number. A default value of 1.00 is used for this field if no scaling is required and the decluttering code field contains a value other than -1 .

Note: The parameter-decluttering scale factors for all current attributes in the attribute dictionary are 1.00 . These are not included in this appendix listing of attribute dictionary values.

Decluttering numbers for attributes with parameter values are derived as follows:

$$
\text { decluttering \# = decluttering code }+\log \left(\mathrm{PV}^{\prime} / \mathrm{PV}\right) /[(\log 2) * \text { scale factor }]
$$

where PV' is the parameter value obtained from the decluttering attribute in the DMS data and PV is the parameter value of the attribute in the attribute dictionary. The decluttering number will always be clipped to $[0,30]$.

For example, if the attribute code was "river flow rate in cubic feet per second (CFS)", then the parameter field of any occurrence in a DMS file would contain the actual flow rate in CFS. If the attribute dictionary contained the following values:

$$
\begin{aligned}
& \text { Parameter Value }=16,000 \\
& \text { Decluttering Code }=28 \\
& \text { Parameter-Decluttering Scale Factor }=1
\end{aligned}
$$

then rivers could be assigned decluttering values based on their flow rates as follows:

$\begin{array}{ll}\frac{\text { Flow Rate }}{2,000} & \frac{\text { Decluttering \# }}{28+\log (.125) / \log (2)=25.00} \\ 7,000 & 28+\log (.4375) / \log (2)=26.81 \\ 10,000 & 28+\log (.625) / \log (2)=27.32 \\ 34,000 & 28+\log (2.125) / \log (2)=29.09\end{array}$

For examples about using decluttering values, see the DECLUTTER utility. 


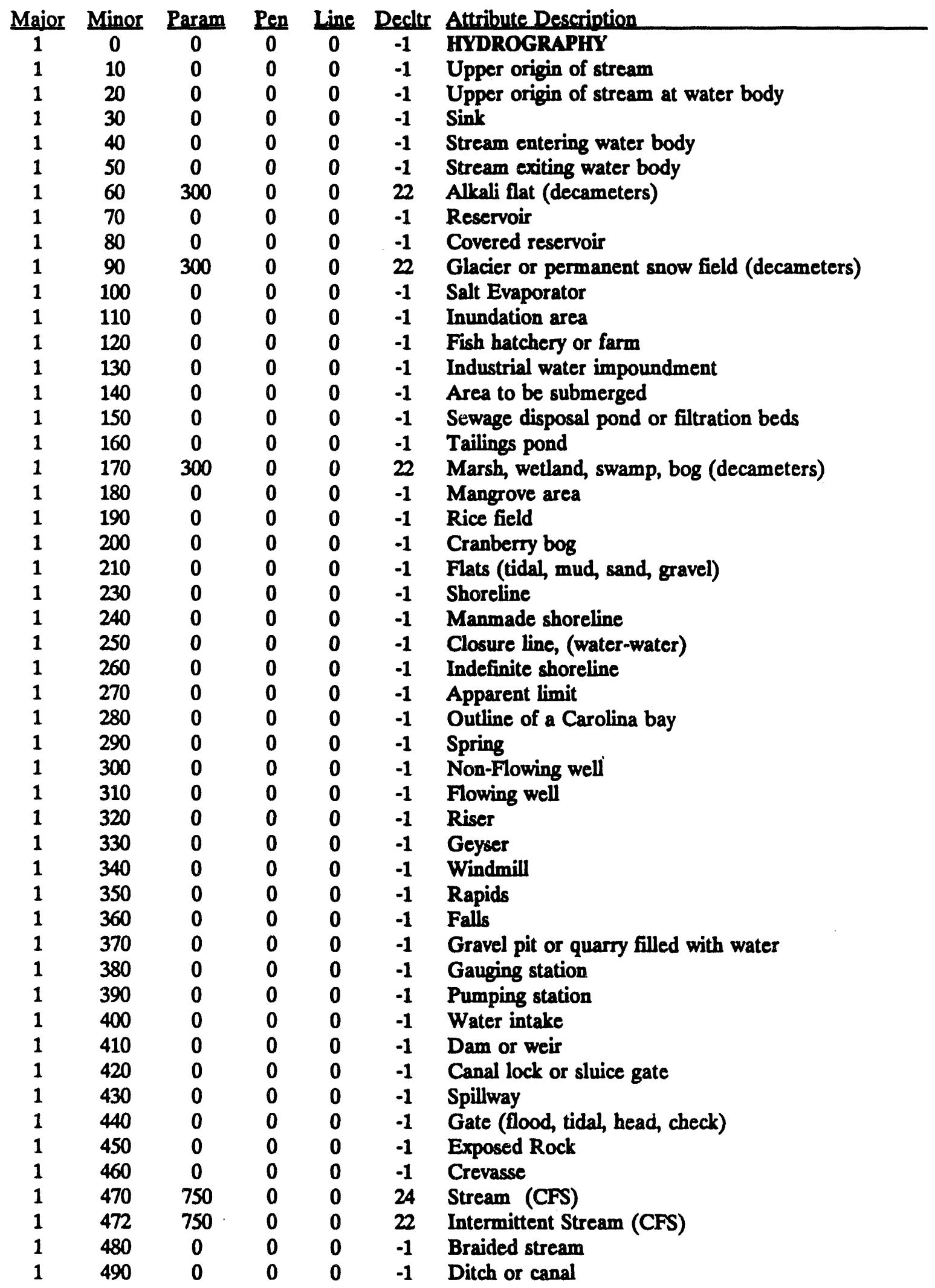




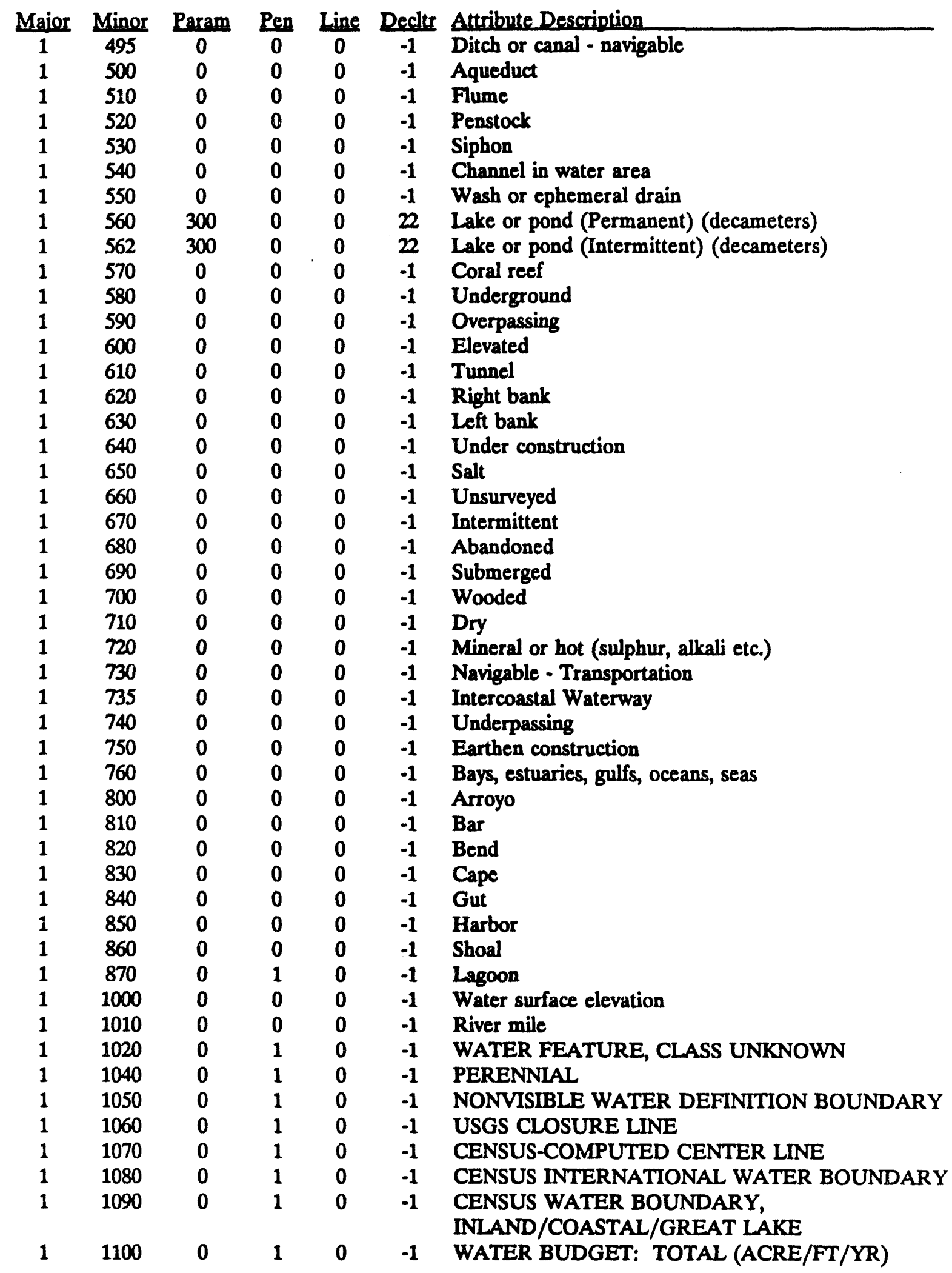




\begin{tabular}{|c|c|c|c|c|c|c|}
\hline Maior & Minor & Param & Pen & Line & Decitr & Attribute Description \\
\hline 1 & 1110 & $\mathbf{0}$ & 1 & $\mathbf{0}$ & -1 & $\begin{array}{l}\text { WATER BUDGET: M \& I DIVERSION } \\
\text { (ACRE/FT/YR) }\end{array}$ \\
\hline 1 & 1120 & $\mathbf{0}$ & 1 & $\mathbf{0}$ & -1 & $\begin{array}{l}\text { WATER BUDGET: IRRIGATION DIVERSION } \\
\text { (ACRE/FT/YR) }\end{array}$ \\
\hline 1 & 1130 & $\mathbf{0}$ & 1 & $\mathbf{0}$ & -1 & $\begin{array}{l}\text { WATER BUDGET: WILDLIFE REQUIREMENT } \\
\text { (ACRE/FT/YR) }\end{array}$ \\
\hline 1 & 1140 & 0 & 1 & 0 & -1 & $\begin{array}{l}\text { WATER BUDGET: LIVESTOCK REQUTREMENT } \\
\text { (ACRE/FT/YR) }\end{array}$ \\
\hline 1 & 1150 & 0 & 1 & 0 & -1 & $\begin{array}{l}\text { WATER BUDGET: EVAPORATION } \\
\text { (ACRE/FT/YR) }\end{array}$ \\
\hline 1 & 1160 & 0 & 1 & $\mathbf{0}$ & -1 & WATER BUDGET: BALANCE (ACRE/FT/YR) \\
\hline 1 & 2000 & 0 & 1 & $\mathbf{0}$ & -1 & 3-MILE LIMIT WATER BOUNDARY \\
\hline 1 & 2010 & 0 & 1 & $\mathbf{0}$ & -1 & SODA EVAPORATOR \\
\hline 1 & 2020 & $\mathbf{0}$ & 1 & 0 & -1 & DANGER CURVE \\
\hline 1 & 2030 & $\mathbf{0}$ & 1 & $\mathbf{0}$ & -1 & SAND IN OPEN WATER \\
\hline 1 & 2040 & $\mathbf{0}$ & 1 & $\mathbf{0}$ & -1 & SPOIL AREA \\
\hline 1 & 2050 & $\mathbf{0}$ & 1 & $\mathbf{0}$ & -1 & ANGLE OF CLOCKWISE ROTATION \\
\hline 1 & 2060 & $\mathbf{0}$ & 1 & $\mathbf{0}$ & -1 & BEST ESTIMATE OF CLASS OR POSITION \\
\hline 1 & 2070 & 0 & 1 & $\mathbf{0}$ & -1 & COINCIDENT FEATURE \\
\hline 1 & 2080 & 0 & 1 & $\mathbf{0}$ & -1 & PHOTOREVISED FEATURE \\
\hline 1 & 2090 & 0 & 1 & 0 & -1 & WELL ID \\
\hline 1 & 3000 & $\mathbf{0}$ & 1 & 0 & -1 & WELL NUMBER "A" \\
\hline 1 & 3010 & 0 & 1 & 0 & -1 & WELL NUMBER "B" \\
\hline 1 & 3020 & 0 & 1 & 0 & -1 & DIAMETER (INCHES) \\
\hline 1 & 3030 & 0 & 1 & $\mathbf{0}$ & -1 & DEPTH FROM (FEET) \\
\hline 1 & 3040 & $\mathbf{0}$ & 1 & $\mathbf{0}$ & -1 & DEPTH TO (FEET) \\
\hline 1 & 3070 & 0 & 1 & 0 & -1 & CFS X 1.0 \\
\hline 1 & 3071 & 0 & 1 & 0 & -1 & CFS $\times 0.1$ \\
\hline 1 & 3072 & 0 & 1 & 0 & -1 & CFS X 0.01 \\
\hline 1 & 3073 & 0 & 1 & 0 & -1 & CFS X 0.001 \\
\hline 1 & 3074 & 0 & 1 & $\mathbf{0}$ & -1 & CFS X 0.0001 \\
\hline 1 & 3075 & 0 & 1 & 0 & -1 & CFS X 0.00001 \\
\hline 1 & 3080 & 0 & 1 & 0 & -1 & ACRE FEET X 1 \\
\hline 1 & 3081 & 0 & 1 & $\mathbf{0}$ & -1 & ACRE FEET X 0.1 \\
\hline 1 & 3082 & 0 & 1 & $\mathbf{0}$ & -1 & ACRE FEET X 0.01 \\
\hline 1 & 3083 & $\mathbf{0}$ & 1 & 0 & -1 & ACRE FEET X 0.001 \\
\hline
\end{tabular}




\begin{tabular}{|c|c|c|c|c|c|c|}
\hline Maior & Minor & Param & Pen & Line & Decltr & Attribute Description \\
\hline 2 & $\mathbf{0}$ & 0 & $\mathbf{0}$ & $\mathbf{0}$ & -1 & SURFACE COVER \\
\hline 2 & 5 & $\mathbf{0}$ & 1 & $\mathbf{0}$ & -1 & TOPOGRAPHIC FEATURE \\
\hline 2 & 10 & 0 & 0 & 0 & -1 & Woods or brushwood \\
\hline 2 & 20 & $\mathbf{0}$ & 0 & $\mathbf{0}$ & -1 & Scrub \\
\hline 2 & 30 & 0 & $\mathbf{0}$ & $\mathbf{0}$ & -1 & Orchard or plantation \\
\hline 2 & 40 & 0 & 0 & $\mathbf{0}$ & -1 & Vineyard \\
\hline 2 & 50 & 0 & 0 & $\mathbf{0}$ & -1 & Scattered trees \\
\hline 2 & 60 & 0 & $\mathbf{0}$ & 0 & -1 & $\begin{array}{l}\text { Edge of surf. cov. at build up, house omission, urban } \\
\text { area }\end{array}$ \\
\hline 2 & 70 & $\mathbf{0}$ & $\mathbf{0}$ & $\mathbf{0}$ & -1 & Glacial moraine \\
\hline 2 & 80 & 0 & 0 & $\mathbf{0}$ & -1 & Beach \\
\hline 2 & 81 & 0 & $\mathbf{0}$ & $\mathbf{0}$ & -1 & Gravel beach \\
\hline 2 & 82 & 0 & 0 & $\mathbf{0}$ & -1 & Sand beach \\
\hline 2 & 90 & 0 & 0 & 0 & -1 & Shifting sand or dune area \\
\hline 2 & 100 & $\mathbf{0}$ & 0 & $\mathbf{0}$ & -1 & Lava \\
\hline 2 & 110 & 0 & 0 & $\mathbf{0}$ & -1 & Arch \\
\hline 2 & 120 & 0 & 0 & $\mathbf{0}$ & -1 & Area \\
\hline 2 & 130 & $\mathbf{0}$ & 0 & $\mathbf{0}$ & -1 & Basin \\
\hline 2 & 140 & 0 & 0 & $\mathbf{0}$ & -1 & Bench \\
\hline 2 & 150 & 0 & 0 & $\mathbf{0}$ & -1 & Cave \\
\hline 2 & 160 & 0 & 0 & $\mathbf{0}$ & -1 & Cliff \\
\hline 2 & 170 & 0 & 0 & 0 & -1 & Crater \\
\hline 2 & 180 & $\mathbf{0}$ & 0 & $\mathbf{0}$ & -1 & Flat \\
\hline 2 & 200 & $\mathbf{0}$ & 0 & $\mathbf{0}$ & -1 & Forest \\
\hline 2 & 210 & 0 & $\mathbf{0}$ & $\mathbf{0}$ & -1 & Gap \\
\hline 2 & 220 & 0 & $\mathbf{0}$ & $\mathbf{0}$ & -1 & Island \\
\hline 2 & 230 & 0 & $\mathbf{0}$ & $\mathbf{0}$ & -1 & Isthmus \\
\hline 2 & 340 & 0 & 0 & 0 & -1 & Pass \\
\hline 2 & 350 & 0 & 0 & $\mathbf{0}$ & -1 & Peak \\
\hline 2 & 360 & 0 & 0 & $\mathbf{0}$ & -1 & Pillar \\
\hline 2 & 370 & 0 & 0 & 0 & -1 & Plain \\
\hline 2 & 380 & 0 & $\mathbf{0}$ & $\mathbf{0}$ & -1 & Range \\
\hline 2 & 390 & 0 & $\mathbf{0}$ & 0 & -1 & Ridge \\
\hline 2 & 400 & 0 & 0 & $\mathbf{0}$ & -1 & Slope \\
\hline 2 & 410 & $\mathbf{0}$ & $\mathbf{0}$ & $\mathbf{0}$ & -1 & Summit \\
\hline 2 & 420 & 0 & $\mathbf{0}$ & $\mathbf{0}$ & -1 & Valley \\
\hline 2 & 430 & 0 & 1 & $\mathbf{0}$ & -1 & Mountain, Hill \\
\hline 2 & 440 & $\mathbf{0}$ & 1 & $\mathbf{0}$ & -1 & Rock \\
\hline 2 & 450 & 0 & 1 & $\mathbf{0}$ & -1 & Meadow \\
\hline 2 & 460 & 0 & 1 & $\mathbf{0}$ & -1 & Desert \\
\hline 2 & 470 & 0 & 1 & 0 & -1 & Plateau \\
\hline 2 & 480 & 0 & 1 & $\mathbf{0}$ & -1 & PHYSICAL FEATURE, CLASS UNKNOWN \\
\hline 2 & 490 & 0 & $\mathbf{0}$ & $\mathbf{0}$ & -1 & IMPROVED LANDS \\
\hline 2 & 500 & 0 & $\mathbf{0}$ & $\mathbf{0}$ & -1 & CROPLAND \\
\hline 2 & 510 & 0 & 0 & 0 & -1 & GRASSY/PHREATO./OPEN WATER AREAS \\
\hline 2 & 520 & 0 & 0 & 0 & -1 & RANGELAND \\
\hline 2 & 540 & 0 & 0 & 0 & -1 & NON-AGRICULTURAL USE \\
\hline 2 & 790 & 0 & 0 & 0 & -1 & HORTICULTURE \& SPECIALTY CROPS \\
\hline 2 & 800 & 0 & $\mathbf{0}$ & 0 & -1 & ROW AND CLOSE GROWN CROPS \\
\hline 2 & 810 & 0 & $\mathbf{0}$ & $\mathbf{0}$ & -1 & FRUIT \\
\hline
\end{tabular}




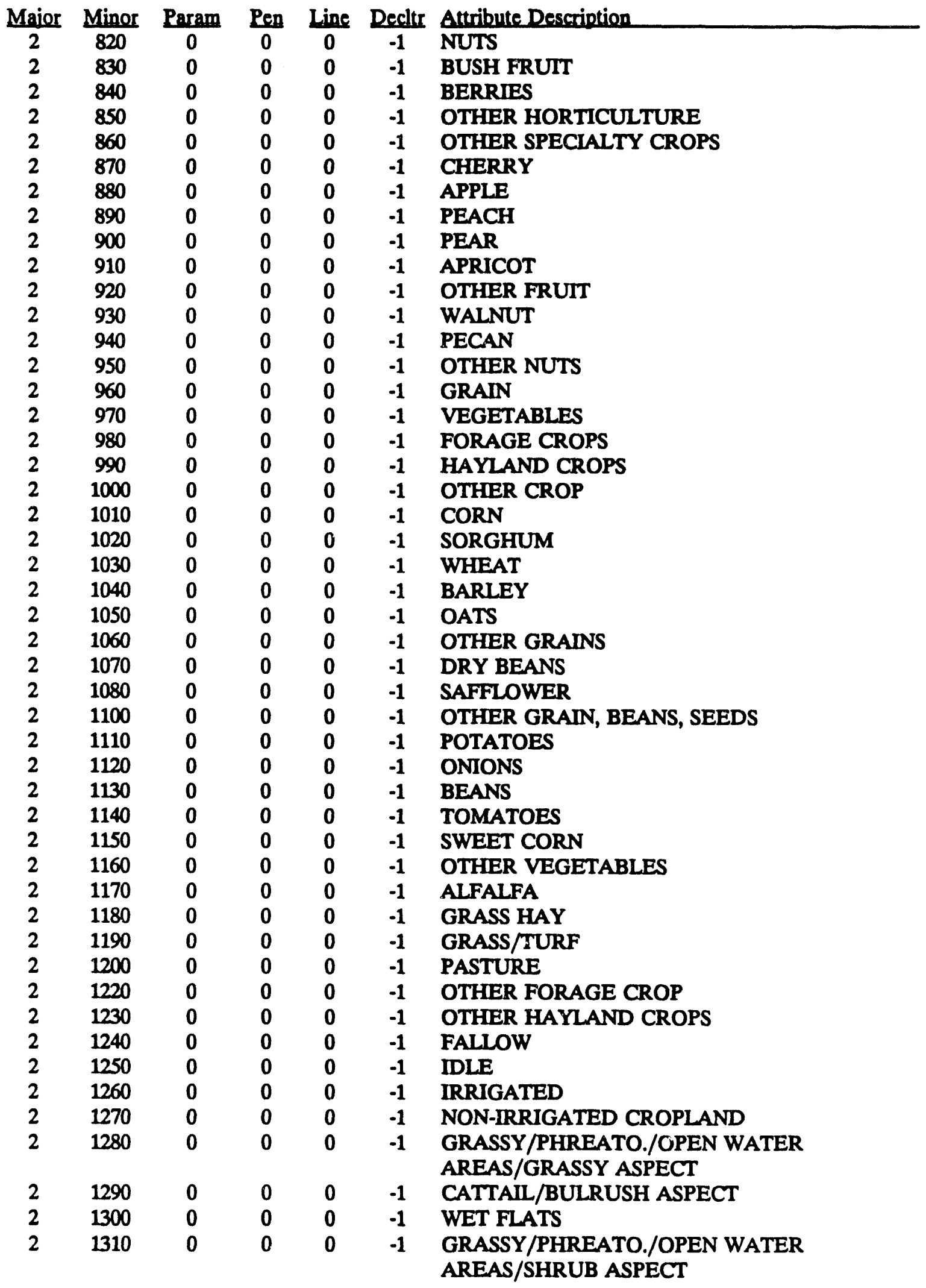




\begin{tabular}{|c|c|c|c|c|c|c|}
\hline Maior & Minor & Param & Pen & Line & Deciltr & Attribute Description \\
\hline 2 & 1320 & $\mathbf{0}$ & $\mathbf{0}$ & $\mathbf{0}$ & -1 & RIPARIAN \\
\hline 2 & 1330 & 0 & 0 & $\mathbf{0}$ & -1 & OPEN WATER \\
\hline 2 & 1350 & 0 & 0 & $\mathbf{0}$ & -1 & RIPARIAN FORESTED ASPECT \\
\hline 2 & 1360 & 0 & 0 & $\mathbf{0}$ & -1 & RIPARIAN SHRUB ASPECT \\
\hline 2 & 1370 & 0 & 0 & $\mathbf{0}$ & -1 & STREAMS \\
\hline 2 & 1380 & 0 & 0 & 0 & -1 & RESERVOIRS \\
\hline 2 & 1390 & $\mathbf{0}$ & $\mathbf{0}$ & $\mathbf{0}$ & -1 & PONDS \& LAKES \\
\hline 2 & 1400 & 0 & 0 & $\mathbf{0}$ & -1 & OTHER OPEN WATER \\
\hline 2 & 1410 & 0 & 0 & 0 & -1 & TEMPORARY FLOODED \\
\hline 2 & 1420 & $\mathbf{0}$ & 0 & $\mathbf{0}$ & -1 & SEWAGE LAGOON \\
\hline 2 & 1430 & $\mathbf{0}$ & $\mathbf{0}$ & $\mathbf{0}$ & -1 & EVAPORATION POND \\
\hline 2 & 1510 & 0 & $\mathbf{0}$ & $\mathbf{0}$ & -1 & ALPINE PLANT COMMUNTTIES \\
\hline 2 & 1520 & 0 & 0 & 0 & -1 & CONIFER \\
\hline 2 & 1530 & $\mathbf{0}$ & $\mathbf{0}$ & $\mathbf{0}$ & -1 & DECIDUOUS \\
\hline 2 & 1540 & $\mathbf{0}$ & $\mathbf{0}$ & $\mathbf{0}$ & -1 & RANGELAND \& FOREST/GRASSY ASPECT \\
\hline 2 & 1550 & $\mathbf{0}$ & 0 & 0 & -1 & RANGELAND \& FOREST/SHRUB ASPECT \\
\hline 2 & 1560 & 0 & 0 & 0 & -1 & DOUGLAS FIR - WHITE FIR \\
\hline 2 & 1570 & 0 & 0 & 0 & -1 & PONDEROSA PINE \\
\hline 2 & 1580 & 0 & 0 & 0 & -1 & FIR - SPRUCE \\
\hline 2 & 1590 & $\mathbf{0}$ & 0 & $\mathbf{0}$ & -1 & LODGEPOLE PINE \\
\hline 2 & 1600 & $\mathbf{0}$ & 0 & 0 & -1 & PINION PINE - JUNIPER \\
\hline 2 & 1610 & 0 & 0 & 0 & -1 & OTHER CONIFER \\
\hline 2 & 1620 & 0 & 0 & 0 & -1 & ASPEN \\
\hline 2 & 1630 & 0 & $\mathbf{0}$ & $\mathbf{0}$ & -1 & MOUNTAIN BRUSH \\
\hline 2 & 1640 & 0 & 0 & 0 & -1 & OTHER DECIDUOUS \\
\hline 2 & 1650 & $\mathbf{0}$ & $\mathbf{0}$ & $\mathbf{0}$ & -1 & DRY PASTURES - IMPROVED \\
\hline 2 & 1660 & $\mathbf{0}$ & $\mathbf{0}$ & $\mathbf{0}$ & -1 & NATTVE GRASSES \\
\hline 2 & 1670 & 0 & 0 & 0 & -1 & OTHER GRASS \\
\hline 2 & 1680 & $\mathbf{0}$ & 0 & 0 & -1 & NORTHERN DESERT SHRUBS \\
\hline 2 & 1690 & 0 & 0 & $\mathbf{0}$ & -1 & SOUTHERN DESERT SHRUBS \\
\hline 2 & 1700 & $\mathbf{0}$ & $\mathbf{0}$ & $\mathbf{0}$ & -1 & SALT DESERT SHRUBS \\
\hline 2 & 1710 & $\mathbf{0}$ & 0 & 0 & -1 & SAGEBRUSH \\
\hline 2 & 1720 & $\mathbf{0}$ & $\mathbf{0}$ & 0 & -1 & OTHER NORTHERN DESERT SHRUB \\
\hline 2 & 1730 & $\mathbf{0}$ & 0 & 0 & -1 & CREOSOTE BUSH \\
\hline 2 & 1740 & 0 & 0 & 0 & -1 & OTHER SOUTHERN DESERT SHRUB \\
\hline 2 & 1750 & 0 & $\mathbf{0}$ & 0 & -1 & SHADSCALE \\
\hline 2 & 1760 & 0 & 0 & 0 & -1 & GREASEWOOD \\
\hline 2 & 1770 & $\mathbf{0}$ & $\mathbf{0}$ & 0 & -1 & SALTBRUSH \\
\hline 2 & 1780 & 0 & $\mathbf{0}$ & 0 & -1 & DESERT MOLLEY \\
\hline 2 & 1790 & 0 & $\mathbf{0}$ & $\mathbf{0}$ & -1 & OTHER SALT DESERT SHRUB \\
\hline 2 & 1800 & $\mathbf{0}$ & 0 & $\mathbf{0}$ & -1 & BARE SOIL/SAND \\
\hline 2 & 1810 & 0 & $\mathbf{0}$ & $\mathbf{0}$ & -1 & ROCK OUTCROPS \\
\hline 2 & 1820 & 0 & 0 & 0 & -1 & EXCAVATED LANDS \\
\hline 2 & 1830 & 0 & 0 & 0 & -1 & OTHER BARREN LANDS \\
\hline 2 & 1840 & 0 & 0 & 0 & -1 & FARMSTEADS \\
\hline 2 & 1850 & 0 & $\mathbf{0}$ & 0 & -1 & RESIDENTIAL \\
\hline 2 & 1860 & 0 & 0 & 0 & -1 & COMMERCIAL/INDUSTRIAL \\
\hline 2 & 1870 & 0 & 0 & 0 & -1 & $\begin{array}{l}\text { TRANSPORTATION, COMMUNICATIONS, } \\
\text { UTILITIES }\end{array}$ \\
\hline 2 & 1880 & 0 & $\mathbf{0}$ & 0 & -1 & OTHER IMPROVED LANDS \\
\hline
\end{tabular}




\begin{tabular}{|c|c|c|c|c|c|c|}
\hline Maior & Minor & Param & Pen & Line & Decltr & Attribute Description \\
\hline 2 & 1990 & 0 & 1 & $\mathbf{0}$ & -1 & OPEN SPACE \\
\hline 2 & 2000 & 0 & $\mathbf{0}$ & $\mathbf{0}$ & -1 & FARMSTEAD BUILDINGS/HOMES \\
\hline 2 & 2010 & 0 & $\mathbf{0}$ & $\mathbf{0}$ & -1 & FARMSTEAD OPEN SPACES \\
\hline 2 & 2020 & 0 & $\mathbf{0}$ & $\mathbf{0}$ & -1 & $\begin{array}{l}\text { HIGH DENSITY RESIDENTIAL } \\
\text { BUILDINGS/HOMES }\end{array}$ \\
\hline 2 & 2030 & 0 & $\mathbf{0}$ & 0 & -1 & $\begin{array}{l}\text { LOW DENSITY RESIDENTIAL } \\
\text { BUILDINGS/HOMES }\end{array}$ \\
\hline 2 & 2040 & 0 & $\mathbf{0}$ & $\mathbf{0}$ & -1 & RESIDENTIAL OPEN SPACES \\
\hline 2 & 2050 & $\mathbf{0}$ & 0 & 0 & -1 & RESIDENTIAL IDLE SPACES \\
\hline 2 & 2060 & 0 & 0 & $\mathbf{0}$ & -1 & COMMERCIAL \\
\hline 2 & 2070 & $\mathbf{0}$ & $\mathbf{0}$ & $\mathbf{0}$ & -1 & INDUSTRIAL \\
\hline 2 & 2080 & 0 & 0 & 0 & -1 & COMMERCIAL/INDUSTRIAL OPEN SPACES \\
\hline 2 & 2090 & 0 & 0 & 0 & -1 & DRY SALT FLATS \\
\hline 2 & 2100 & 0 & 0 & 0 & -1 & NOT CLASSIFIED \\
\hline 2 & 2110 & 0 & 0 & 0 & -1 & OTHER BARE SOIL/SAND \\
\hline
\end{tabular}




\begin{tabular}{|c|c|c|c|c|c|c|}
\hline Maior & Minor & Param & Pen & Line & Decltr & Attribute Description \\
\hline 3 & 0 & 0 & 0 & 0 & -1 & BOUNDARIES \\
\hline 3 & 10 & 0 & 0 & 0 & -1 & Monumented point on a boundary \\
\hline 3 & 15 & 0 & $\mathbf{0}$ & 0 & 29 & State boundary \\
\hline 3 & 20 & 0 & 0 & 0 & -1 & Civil township, district, precinct, or barrio \\
\hline 3 & 30 & 0 & 0 & 0 & -1 & Large incorporated city \\
\hline 3 & 40 & 0 & 0 & $\mathbf{0}$ & -1 & $\begin{array}{l}\text { Nat. park,monument, lakeshore, prkwy, battlefld, rec. } \\
\text { area }\end{array}$ \\
\hline 3 & 45 & 0 & $\mathbf{0}$ & 0 & -1 & Large city, county or private park \\
\hline 3 & 47 & 0 & 0 & 0 & -1 & Small city, county, or private park \\
\hline 3 & 50 & 0 & 0 & 0 & -1 & National forest or grassland \\
\hline 3 & 60 & 0 & $\mathbf{0}$ & $\mathbf{0}$ & -1 & $\begin{array}{l}\text { National wildlife refuge, game preserve, or fish } \\
\text { hatchery }\end{array}$ \\
\hline 3 & 70 & 0 & 0 & 0 & -1 & National scenic waterway or wilderness area \\
\hline 3 & 80 & 0 & 0 & 0 & -1 & Indian reservation \\
\hline 3 & 90 & 0 & 0 & 0 & -1 & Military reservation (active) \\
\hline 3 & 100 & 0 & 0 & 0 & -1 & Non-military government reservation \\
\hline 3 & 110 & 0 & 0 & 0 & -1 & Federal prison \\
\hline 3 & 120 & 0 & 0 & 0 & -1 & State park, recreation area, arboretum, or lake \\
\hline 3 & 130 & 0 & 0 & $\mathbf{0}$ & -1 & State wildlife refuge, game preserve, or fish hatchery \\
\hline 3 & 131 & 0 & 1 & 0 & -1 & $\begin{array}{l}\text { CRITICAL WILDLIFE HABITAT BOUNDARY: } \\
\text { FISHERIES }\end{array}$ \\
\hline 3 & 132 & 0 & 1 & $\mathbf{0}$ & -1 & $\begin{array}{l}\text { CRITICAL WILDLIFE HABITAT BOUNDARY: } \\
\text { BIG GAME }\end{array}$ \\
\hline 3 & 133 & 0 & 1 & 0 & -1 & $\begin{array}{l}\text { CRITICAL WILDLIFE HABITAT BOUNDARY: } \\
\text { UPLAND GAME BIRD }\end{array}$ \\
\hline 3 & 135 & 0 & 1 & 0 & -1 & OTHER FEDERAL LAND \\
\hline 3 & 140 & 0 & 0 & 0 & -1 & State forest or grassland \\
\hline 3 & 150 & 0 & 0 & 0 & -1 & State prison \\
\hline 3 & 160 & 0 & 0 & 0 & -1 & County game preserve \\
\hline 3 & 170 & 0 & 0 & 0 & 25 & County Boundary \\
\hline 3 & 180 & 0 & 0 & 0 & 24 & Small city, village, town, borough, or hamlet \\
\hline 3 & 185 & 0 & 0 & 0 & -1 & International treaty line \\
\hline 3 & 190 & 0 & 0 & 0 & -1 & USSR \\
\hline 3 & 200 & 0 & 0 & 0 & -1 & Canada \\
\hline 3 & 210 & 0 & $\mathbf{0}$ & 0 & -1 & Mexico \\
\hline 3 & 220 & 0 & 0 & 0 & -1 & $\begin{array}{l}\text { Non-sovereign area outside of United States } \\
\text { boundaries }\end{array}$ \\
\hline 3 & 225 & 0 & 0 & 0 & -1 & National boundary (land) \\
\hline 3 & 226 & 0 & 0 & 0 & -1 & National boundary (water) \\
\hline 3 & 227 & 0 & 0 & 0 & -1 & Continental Divide \\
\hline 3 & 230 & 0 & 0 & 0 & -1 & Approximate boundary \\
\hline 3 & 240 & 0 & 0 & 0 & -1 & Indefinite boundary \\
\hline 3 & 250 & 0 & 0 & 0 & -1 & Disputed boundary \\
\hline 3 & 260 & 0 & 0 & 0 & -1 & Historical line \\
\hline 3 & 270 & 0 & 0 & 0 & -1 & Boundary closure line \\
\hline 3 & 280 & 0 & 0 & 0 & -1 & $\begin{array}{l}\text { Reference monuments for monumented point on } \\
\text { boundary }\end{array}$ \\
\hline 3 & 290 & 0 & 0 & 0 & -1 & Civil \\
\hline 3 & 300 & 0 & 0 & 0 & -1 & Populated Place (ppl) \\
\hline 3 & 310 & $\mathbf{0}$ & 0 & 0 & -1 & Park \\
\hline
\end{tabular}




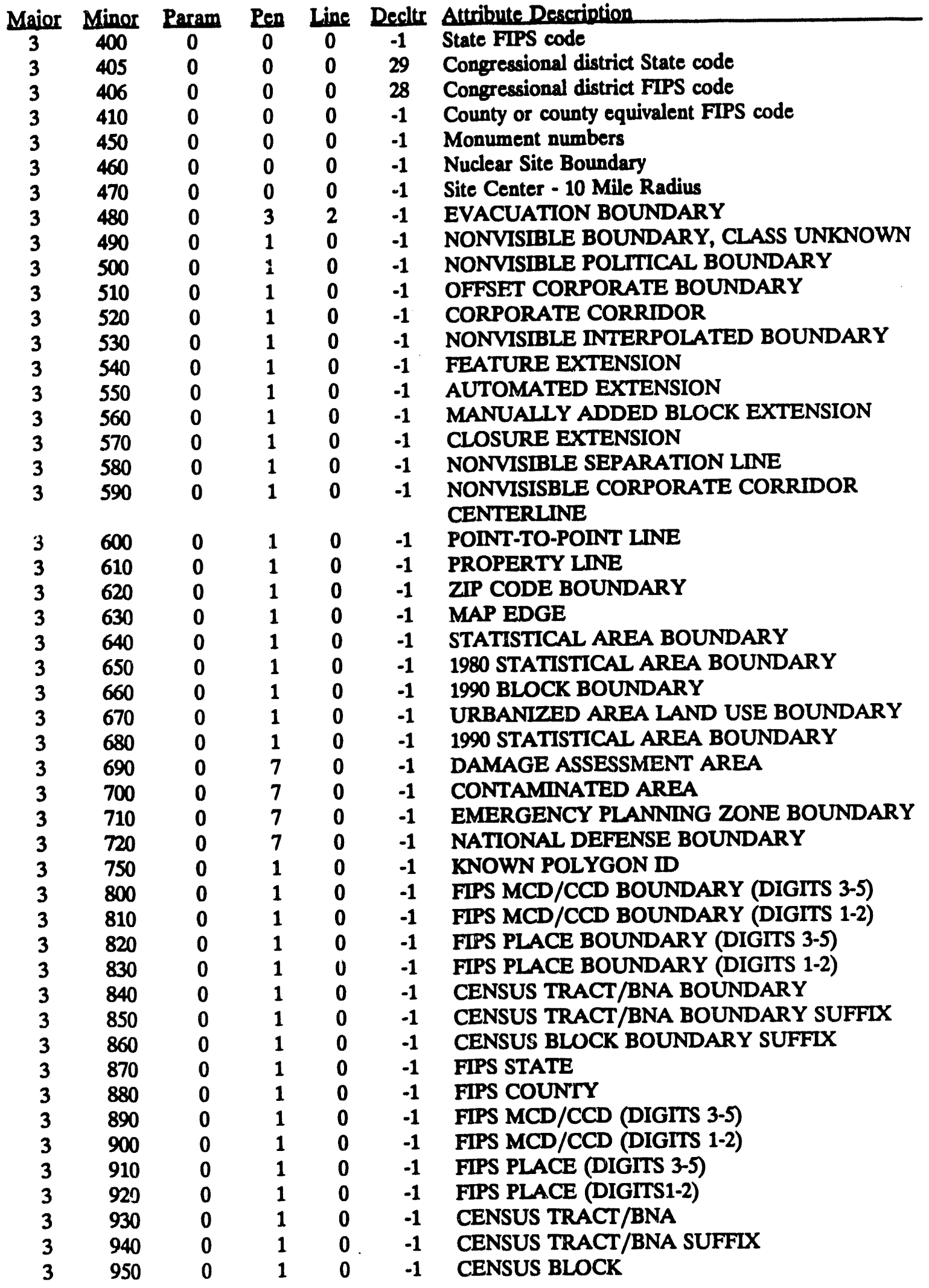


Maior Minor Param Pen Line Decltr Attribute Desarintion

$\begin{array}{lllllll}3 & 960 & 0 & 1 & 0 & -1 & \text { CENSUS BLOCK SUFFIX }\end{array}$

$\begin{array}{lllllll}3 & 970 & 0 & 1 & 0 & -1 & \text { BLOCK GROUP }\end{array}$ 


\begin{tabular}{|c|c|c|c|c|c|c|}
\hline Major & Minor & Param & Pen & Line & Declit & Attribute Description \\
\hline 5 & $\mathbf{0}$ & $\mathbf{0}$ & 0 & 0 & -1 & ROADS AND TRAILS \\
\hline 5 & 10 & $\mathbf{0}$ & $\mathbf{0}$ & 0 & -1 & Bridge abutment \\
\hline 5 & 20 & 0 & 0 & 0 & -1 & Tunnel portal \\
\hline 5 & 30 & 0 & 0 & 0 & -1 & Gate \\
\hline 5 & 40 & $\mathbf{0}$ & $\mathbf{0}$ & $\mathbf{0}$ & -1 & Cul-de-sac \\
\hline 5 & 50 & $\mathbf{0}$ & 0 & 0 & -1 & Dead end \\
\hline 5 & 51 & 0 & 0 & 0 & -1 & Interstate (Proposed) \\
\hline 5 & 52 & 0 & 0 & 0 & 30 & Interstate \\
\hline 5 & 53 & $\mathbf{0}$ & 0 & 0 & -1 & Interstate (Under Construction) \\
\hline 5 & 54 & 0 & 0 & 0 & 22 & U.S. Highway \\
\hline 5 & 55 & 0 & 0 & 0 & -1 & U.S. Highway (Under Construction) \\
\hline 5 & 56 & $\mathbf{0}$ & 0 & 0 & 15 & State Highway \\
\hline 5 & 57 & 0 & $\mathbf{0}$ & 0 & -1 & State Highway (Under Construction) \\
\hline 5 & 58 & $\mathbf{0}$ & 0 & 0 & 7 & Secondary state and counity \\
\hline 5 & 59 & 0 & 0 & 0 & -1 & Secondary state and county (Under Construction) \\
\hline 5 & 60 & $\mathbf{0}$ & $\mathbf{0}$ & $\mathbf{0}$ & -1 & Primary route, class 1 , symbol undivided \\
\hline 5 & 70 & 0 & 0 & 0 & -1 & Primary route, class 1 , symbol divided by centerline \\
\hline 5 & 80 & 0 & 0 & 0 & -1 & Primary route, class 1 , divided, lanes separated \\
\hline 5 & 90 & 0 & 0 & 0 & -1 & $\begin{array}{l}\text { Primary route, class } 1 \text {, one way, other than divided } \\
\text { highway }\end{array}$ \\
\hline 5 & 91 & 0 & 1 & 0 & -1 & PRIMARY ROUTE, CLASS 1 \\
\hline 5 & 100 & $\mathbf{0}$ & 0 & $\mathbf{0}$ & -1 & Secondary route, class 2 , symbol undivided \\
\hline 5 & 110 & $\mathbf{0}$ & 0 & 0 & -1 & Secondary route, class 2 , symbol divided by centerline \\
\hline 5 & 120 & $\mathbf{0}$ & 0 & $\mathbf{0}$ & -1 & $\begin{array}{l}\text { Secondary route, class } 2 \text {, symbol divided, lanes } \\
\text { separated }\end{array}$ \\
\hline 5 & 130 & 0 & 0 & 0 & -1 & $\begin{array}{l}\text { Secondary route, class } 2 \text {, one way, other than divided } \\
\text { highwy }\end{array}$ \\
\hline 5 & 131 & 0 & 1 & $\mathbf{0}$ & -1 & SECONDARY ROUTE, CLASS 2 \\
\hline 5 & 140 & 0 & 0 & 0 & -1 & Road or street, class 3 \\
\hline 5 & 141 & 0 & 1 & 0 & -1 & ROAD, CLASS 3, DIVIIJED \\
\hline 5 & 142 & $\mathbf{0}$ & 1 & 0 & -1 & ROAD, CLASS 3, UNDIVIDED \\
\hline 5 & 150 & $\mathbf{0}$ & $\mathbf{0}$ & 0 & -1 & Road or street class 4 \\
\hline 5 & 151 & $\mathbf{0}$ & 1 & $\mathbf{0}$ & -1 & ROAD OR STREET, CLLASS 4, DIVIDED \\
\hline 5 & 152 & 0 & 1 & 0 & -1 & ROAD OR STREET, CLASS 4, UNDIVIDED \\
\hline 5 & 160 & 0 & 0 & 0 & -1 & Trail, class 5 , other than four wheel drive vehicle \\
\hline 5 & 170 & 0 & 0 & 0 & -1 & Trail, class 5 , four wheel drive vehicle \\
\hline 5 & 171 & 0 & 1 & 0 & -1 & TRAII, CLASS 5, UNDIVIDED \\
\hline 5 & 172 & $\mathbf{0}$ & 1 & 0 & -1 & ROAD, CLASSIFICATION UNKNOWN \\
\hline 5 & 173 & 0 & 1 & 0 & -1 & ROAD, UNDIVIDED \\
\hline 5 & 174 & 0 & 1 & 0 & -1 & ROAD DIVIDED \\
\hline 5 & 175 & 0 & 0 & 0 & -1 & Interstate Connector \\
\hline 5 & 179 & 0 & 1 & 0 & -1 & $\begin{array}{l}\text { ROAD-RELATED ATIRIBUTE ADDED BY JRW } \\
\text { 'CUZ FILE REFD IT }\end{array}$ \\
\hline 5 & 180 & 0 & 0 & 0 & -1 & Bridge \\
\hline 5 & 181 & 0 & 0 & 0 & -1 & Footbridge \\
\hline 5 & 190 & 0 & 0 & 0 & -1 & Ferry crossing \\
\hline 5 & 200 & 0 & 0 & 0 & -1 & Perimeter of parking area \\
\hline 5 & 210 & 0 & 0 & 0 & -1 & Arbitrary extension of line (join or closure) \\
\hline 5 & 220 & 0 & 0 & 0 & -1 & Road or street, class 3 , symbol divided by centerline \\
\hline 5 & 230 & 0 & 0 & 0 & -1 & Road or street, class 3, divided, lanes separated \\
\hline
\end{tabular}




\begin{tabular}{|c|c|c|c|c|c|c|}
\hline Major & Minor & Param & Pen & Line & Decltr & Attribute Descriotion \\
\hline 5 & 240 & 0 & 0 & 0 & -1 & Traffic circle \\
\hline 5 & 250 & $\mathbf{0}$ & $\mathbf{0}$ & $\mathbf{0}$ & -1 & Cloverleaf or interchange \\
\hline 5 & 260 & 0 & $\mathbf{0}$ & $\mathbf{0}$ & -1 & Toll gate, toll plaza or perimeter of toll plaza \\
\hline 5 & 270 & 0 & $\mathbf{0}$ & $\mathbf{0}$ & -1 & Historical \\
\hline 5 & 280 & 0 & 0 & $\mathbf{0}$ & -1 & In tunnel \\
\hline 5 & 290 & $\mathbf{0}$ & $\mathbf{0}$ & 0 & -1 & Overpassing on bridge \\
\hline 5 & 300 & 0 & 0 & 0 & -1 & Under construction, classification known \\
\hline 5 & 310 & 0 & 0 & 0 & -1 & Under construction, classification unknown \\
\hline 5 & 320 & 0 & 0 & 0 & -1 & Labeled "old railroad grade" \\
\hline 5 & 330 & 0 & $\mathbf{0}$ & $\mathbf{0}$ & -1 & Submerged or in ford \\
\hline 5 & 340 & 0 & $\mathbf{0}$ & $\mathbf{0}$ & -1 & Underpassing \\
\hline 5 & 350 & 0 & 0 & 0 & -1 & Limited access \\
\hline 5 & 360 & $\mathbf{0}$ & $\mathbf{0}$ & 0 & -1 & Toll Road \\
\hline 5 & 365 & $\mathbf{0}$ & $\mathbf{0}$ & $\mathbf{0}$ & -1 & Toll Connector \\
\hline 5 & 370 & $\mathbf{0}$ & $\mathbf{0}$ & 0 & -1 & Privately operated or controlled public access \\
\hline 5 & 380 & 0 & 0 & 0 & -1 & Proposed \\
\hline 5 & 390 & 0 & $\mathbf{0}$ & 0 & -1 & Double-decked \\
\hline 5 & 400 & 0 & 0 & 0 & -1 & In service facility or rest area \\
\hline 5 & 410 & 0 & 0 & 0 & -1 & Elevated \\
\hline 5 & 420 & 0 & $\mathbf{0}$ & 0 & -1 & Bypass route \\
\hline 5 & 430 & $\mathbf{0}$ & $\mathbf{0}$ & 0 & -1 & Alternate route \\
\hline 5 & 440 & $\mathbf{0}$ & $\mathbf{0}$ & 0 & -1 & Business route \\
\hline 5 & 450 & 0 & $\mathbf{0}$ & 0 & -1 & On drawbridge \\
\hline 5 & 453 & 0 & 1 & 0 & -1 & $\begin{array}{l}\text { ROAD WIDTH 16-25 FEET, .010 INCHES AT } \\
1: 24,000\end{array}$ \\
\hline 5 & 454 & 0 & 1 & 0 & -1 & $\begin{array}{l}\text { ROAD WIDTH } 26-35 \text { FEET, } .015 \text { INCHES AT } \\
\text { 24:000 }\end{array}$ \\
\hline 5 & 455 & 0 & 1 & 0 & -1 & $\begin{array}{l}\text { ROAD WIDTH } 36-45 \text { FEET, .020 INCHES AT } \\
1: 24,000\end{array}$ \\
\hline 5 & 460 & 0 & 0 & 0 & -1 & Road width $46-55$ feet, .025 inches at $1: 24,000$ \\
\hline 5 & 470 & 0 & $\mathbf{0}$ & $\mathbf{0}$ & -1 & Road width $46-65$ feet, .030 inches at $1: 24,000$ \\
\hline 5 & 480 & 0 & $\mathbf{0}$ & $\mathbf{0}$ & -1 & Road width $66-75$ feet, .035 inches at $1: 24,000$ \\
\hline 5 & 490 & 0 & 0 & 0 & -1 & Road width $76-85$ feet, .040 inches at $1: 24,000$ \\
\hline 5 & 500 & 0 & $\mathbf{0}$ & $\mathbf{0}$ & -1 & Road width $86-95$ feet, .045 inches at $1: 24,000$ \\
\hline 5 & 510 & 0 & 0 & $\mathbf{0}$ & -1 & Road width $96-105$ feet, .055 inches at $1: 24,000$ \\
\hline 5 & 520 & 0 & 0 & 0 & -1 & Road width $106-115$ feet, .055 inches at $1: 24,000$ \\
\hline 5 & 530 & $\mathbf{0}$ & $\mathbf{0}$ & 0 & -1 & Road width $116-125$ feet, .060 inches at $1: 24,000$ \\
\hline 5 & 540 & $\mathbf{0}$ & $\mathbf{0}$ & $\mathbf{0}$ & -1 & Road width $126-135$ feet, .065 inches at $1: 24,000$ \\
\hline 5 & 550 & 0 & $\mathbf{0}$ & $\mathbf{0}$ & -1 & Road width $136-145$ feet, .070 inches at $1: 24,000$ \\
\hline 5 & 600 & 0 & $\mathbf{0}$ & $\mathbf{0}$ & -1 & Number of lanes \\
\hline 5 & 650 & $\mathbf{0}$ & $\mathbf{0}$ & $\mathbf{0}$ & -1 & Interstate route number \\
\hline 5 & 700 & 0 & $\mathbf{0}$ & 0 & -1 & U.S. route number \\
\hline 5 & 750 & 0 & 0 & $\mathbf{0}$ & -1 & State route number \\
\hline 5 & 800 & 0 & $\mathbf{0}$ & 0 & -1 & Reservation, park, or military route number \\
\hline 5 & 850 & 0 & 0 & 0 & -1 & County route \\
\hline 5 & 860 & 0 & 1 & 0 & -1 & RAIL LINE IN CENTER \\
\hline 5 & 888 & 500 & 1 & 0 & 21 & DECLUTTERING ATTRIBUTE \\
\hline 5 & 930 & $\mathbf{0}$ & 1 & 0 & -1 & SERVICE DRIVE \\
\hline 5 & 940 & $\mathbf{0}$ & 1 & 0 & -1 & SPECIAL ROAD FEATURE \\
\hline 5 & 950 & 0 & 1 & 0 & -1 & OTHER THOROUGHFARE \\
\hline
\end{tabular}




\begin{tabular}{|c|c|c|c|c|c|c|}
\hline Major & Minor & Param & Pen & ine & Decltr & Attribute Description \\
\hline 5 & 960 & $\mathbf{0}$ & 1 & 0 & -1 & WALKWAY \\
\hline 5 & 970 & $\mathbf{0}$ & 1 & 0 & -1 & STAIRWAY \\
\hline 5 & 980 & 0 & 1 & $\mathbf{0}$ & -1 & ALLEY \\
\hline 5 & 990 & $\mathbf{0}$ & 1 & $\mathbf{0}$ & -1 & WEIGH STATION \\
\hline 5 & 1000 & 0 & 1 & 0 & -1 & SPUR \\
\hline 5 & 1010 & $\mathbf{0}$ & 1 & $\mathbf{0}$ & -1 & $\begin{array}{l}\text { XXYY ALPHABETIC PORTION OF ROUTE } \\
\text { NUMBER }\end{array}$ \\
\hline 5 & 1020 & 0 & 1 & 0 & -1 & COINCIDENT FEATURE \\
\hline 5 & 1030 & $\mathbf{0}$ & 1 & $\mathbf{0}$ & -1 & BEST ESTIMATE OF CLASS OR POSITION \\
\hline 5 & 1040 & 0 & 1 & 0 & -1 & PHOTOREVISED FEATURE \\
\hline 5 & 1100 & 0 & 1 & $\mathbf{0}$ & -1 & TRAFFIC CONTROL \\
\hline 5 & 1101 & 0 & 1 & 0 & -1 & TRAFFIC CONTROL (INACT) \\
\hline
\end{tabular}




\begin{tabular}{ccccccl} 
Major & Minor & Param & $\frac{\text { Pen }}{\text { Line }}$ & Decltr & Attribute Description \\
\hline 6 & 0 & 0 & 0 & 0 & -1 & RAILROADS \\
6 & 10 & 0 & 0 & 0 & -1 & Bridge abutment \\
6 & 20 & 0 & 0 & 0 & -1 & Tunnel portal \\
6 & 30 & 0 & 0 & 0 & -1 & Railroad \\
6 & 40 & 0 & 0 & 0 & -1 & Railroad in street or road \\
6 & 50 & 0 & 0 & 0 & -1 & Carline \\
6 & 60 & 0 & 0 & 0 & -1 & Cog railroad, incline railway, logging tram \\
6 & 70 & 0 & 0 & 0 & -1 & Ferry crossing \\
6 & 80 & 0 & 0 & 0 & -1 & Railroad siding \\
6 & 90 & 0 & 0 & 0 & -1 & Perimeter or limit of yard \\
6 & 100 & 0 & 0 & 0 & -1 & Arbitrary line extension (join, closure) \\
6 & 110 & 0 & 0 & 0 & -1 & Railroad station, perimeter of station \\
6 & 120 & 0 & 0 & 0 & -1 & Turntable \\
6 & 130 & 0 & 0 & 0 & -1 & Roundhouse \\
6 & 140 & 0 & 0 & 0 & -1 & Historical \\
6 & 150 & 0 & 0 & 0 & -1 & In tunnel \\
6 & 160 & 0 & 0 & 0 & -1 & Overpassing, on bridge \\
6 & 170 & 0 & 0 & 0 & -1 & Abandoned \\
6 & 180 & 0 & 0 & 0 & -1 & Dismantled \\
6 & 190 & 0 & 0 & 0 & -1 & Underpassing \\
6 & 200 & 0 & 0 & 0 & -1 & Narrow gauge \\
6 & 210 & 0 & 0 & 0 & -1 & In snowshed or under structure \\
6 & 220 & 0 & 0 & 0 & -1 & Under construction \\
6 & 230 & 0 & 0 & 0 & -1 & Elevated \\
6 & 240 & 0 & 0 & 0 & -1 & Rapid transit \\
6 & 250 & 0 & 0 & 0 & -1 & On drawbridge \\
6 & 300 & 0 & 0 & 0 & -1 & Number of tracks \\
6 & 310 & 0 & 1 & 0 & -1 & RAIlROAD MAIN TRACK \\
6 & 320 & 0 & 1 & 0 & -1 & RAIlROAD SPUR TRACK \\
6 & 330 & 0 & 1 & 0 & -1 & RAILROAD YARD \\
6 & 340 & 0 & 1 & 0 & -1 & OTHER RAIl FEATURE \\
6 & 350 & 0 & 1 & 0 & -1 & PRIVATE \\
6 & 360 & 0 & 1 & 0 & -1 & U.S. GOVERNMENT \\
6 & 370 & 0 & 1 & 0 & -1 & JUXTAPOSITION \\
6 & 380 & 0 & 1 & 0 & -1 & PHOTOREVISED FEATURE \\
6 & 390 & 0 & 1 & 0 & -1 & NUMBER OF TRACKS \\
6 & 400 & 0 & 1 & 0 & -1 & BEST ESTIMATE OF CLASS OR POSITION \\
6 & 410 & 0 & 1 & 0 & -1 & COINCIDENT FEATURE \\
6 & 3646 & 0 & 1 & 0 & -1 & NUMBER OF UNITS IN HOUSING UNIT \\
& & & & & & STRUCTURE - 10 TO 19 \\
& & & & & &
\end{tabular}




\begin{tabular}{|c|c|c|c|c|c|c|}
\hline Maior & Minor & Param & Pen & Line & Decltr & Attribute Description. \\
\hline 7 & 0 & $\mathbf{0}$ & 0 & 0 & -1 & $\begin{array}{l}\text { PIPELINES, TRANS. LINES, MISC. } \\
\text { TRANSPORTATION FEATURES }\end{array}$ \\
\hline 7 & 10 & 0 & 0 & 0 & -1 & End of trans. line at power sta., substa., hydro. plant \\
\hline 7 & 20 & 0 & 0 & 0 & -1 & End of pipeline at oil or gas field \\
\hline 7 & 30 & 0 & $\mathbf{0}$ & $\mathbf{0}$ & -1 & End of pipeline at refinery, depot, or tank farm \\
\hline 7 & 40 & 0 & $\mathbf{0}$ & $\mathbf{0}$ & -1 & Pipeline \\
\hline 7 & 50 & 0 & 0 & $\mathbf{0}$ & -1 & Power transmission line \\
\hline 7 & 60 & $\mathbf{0}$ & $\mathbf{0}$ & $\mathbf{0}$ & -1 & Telephone or telegraph line \\
\hline 7 & 70 & $\mathbf{0}$ & 0 & $\mathbf{0}$ & -1 & Aerial tramway, monorail, ski lift \\
\hline 7 & 80 & 0 & 0 & $\mathbf{0}$ & -1 & Power Station \\
\hline 7 & 90 & $\mathbf{0}$ & 0 & $\mathbf{0}$ & -1 & Substation \\
\hline 7 & 100 & 0 & 0 & $\mathbf{0}$ & -1 & Hydroelectric Plant \\
\hline 7 & 110 & 0 & 0 & $\mathbf{0}$ & -1 & Landing strip, airport, perimeter of airport \\
\hline 7 & 111 & $\mathbf{0}$ & 0 & $\mathbf{0}$ & -1 & AIRPORT \\
\hline 7 & 112 & 0 & 6 & $\mathbf{0}$ & -1 & CIVILIAN AIR HUB \\
\hline 7 & 115 & $\mathbf{0}$ & 0 & $\mathbf{0}$ & -1 & Military Landing strip, airport, perimeter of airport \\
\hline 7 & 120 & 0 & 0 & $\mathbf{0}$ & -1 & Heliport, perimeter of heliport \\
\hline 7 & 130 & $\mathbf{0}$ & $\mathbf{0}$ & $\mathbf{0}$ & -1 & Launch complex, perimeter of launch complex \\
\hline 7 & 140 & $\mathbf{0}$ & $\mathbf{0}$ & $\mathbf{0}$ & -1 & Pumping station (other than water) \\
\hline 7 & 150 & $\mathbf{0}$ & 0 & $\mathbf{0}$ & -1 & Seaplane ramp or landing area \\
\hline 7 & 160 & 0 & $\mathbf{0}$ & $\mathbf{0}$ & -1 & Underground \\
\hline 7 & 170 & 0 & 0 & 0 & -1 & Under construction \\
\hline 7 & 180 & 0 & 0 & $\mathbf{0}$ & -1 & Abandoned \\
\hline 7 & 190 & 0 & 0 & $\mathbf{0}$ & -1 & Above ground \\
\hline 7 & 200 & 0 & 0 & $\mathbf{0}$ & -1 & Labeled "closed" \\
\hline 7 & 210 & 0 & 0 & $\mathbf{0}$ & -1 & Unimproved, loose surface \\
\hline 7 & 220 & 0 & 1 & $\mathbf{0}$ & -1 & $\begin{array}{l}\text { SPECIAL TRANSPORTATION FEATURE, CLASS } \\
\text { UNKNOWN }\end{array}$ \\
\hline 7 & 230 & 0 & 1 & $\mathbf{0}$ & -1 & OTHER SPECIAL TRANSPORTAION FEATURE \\
\hline 7 & 240 & 0 & 1 & $\mathbf{0}$ & -1 & FEATURE NOT ELSEWHERE CLASSIFIED \\
\hline 7 & 250 & $\mathbf{0}$ & 1 & $\mathbf{0}$ & -1 & TRANSPORTAION TERMINAL \\
\hline 7 & 260 & $\mathbf{0}$ & 1 & $\mathbf{0}$ & -1 & ARBITRARY EXTENSION CLOSURE LINE \\
\hline 7 & 270 & $\mathbf{0}$ & 1 & $\mathbf{0}$ & -1 & SUBMERGED \\
\hline 7 & 280 & 0 & 1 & $\mathbf{0}$ & -1 & NUCLEAR \\
\hline 7 & 290 & 0 & 1 & 0 & -1 & PHOTOREVISED FEATURE \\
\hline 7 & 300 & 0 & 1 & $\mathbf{0}$ & -1 & ANGLE OF CLOCKWISE ROTATION \\
\hline 7 & 310 & 0 & 1 & $\mathbf{0}$ & -1 & BEST ESTIMATE OF CLASS OR POSITION \\
\hline 7 & 320 & 0 & 1 & $\mathbf{0}$ & -1 & COINCIDENT FEATURE \\
\hline 7 & 330 & 0 & 1 & $\mathbf{0}$ & -1 & SEAPLANE ANCHORAGE \\
\hline 7 & 340 & $\mathbf{0}$ & 1 & $\mathbf{0}$ & -1 & MARINE TERMINAL \\
\hline
\end{tabular}




\begin{tabular}{|c|c|c|c|c|c|c|}
\hline Major & Minor & Param & Pen & Line & Decltr & Attribute Description \\
\hline 8 & $\mathbf{0}$ & $\mathbf{0}$ & $\mathbf{0}$ & $\mathbf{0}$ & -1 & OTHER SIGNIFICANT MAN-MADE STRUCTURES \\
\hline 8 & 1 & $\mathbf{0}$ & $\mathbf{0}$ & $\mathbf{0}$ & -1 & End point of linear man-made feature \\
\hline 8 & 2 & 0 & $\mathbf{0}$ & $\mathbf{0}$ & -1 & Point on linear man-made feature \\
\hline 8 & 10 & 0 & $\mathbf{0}$ & $\mathbf{0}$ & -1 & Church complex \\
\hline 8 & 20 & $\mathbf{0}$ & $\mathbf{0}$ & $\mathbf{0}$ & -1 & School campus \\
\hline 8 & 30 & 0 & 0 & $\mathbf{0}$ & -1 & Hospital complex \\
\hline 8 & 31 & 0 & 1 & 0 & -1 & $\begin{array}{l}\text { VETERANS ADMINISTRATION HOSPITAL } \\
\text { COMPLEX }\end{array}$ \\
\hline 8 & 32 & 0 & 1 & 0 & -1 & HOSPITAL (INACTIVE) \\
\hline 8 & 35 & 0 & 1 & 0 & -1 & NURSING HOME \\
\hline 8 & 40 & 0 & 0 & 0 & -1 & Orphanage \\
\hline 8 & 45 & $\mathbf{0}$ & 1 & $\mathbf{0}$ & -1 & CÚSTODIAL FACILITY \\
\hline 8 & 46 & 0 & 1 & 0 & -1 & COUNTY HOME OR POOR FARM \\
\hline 8 & 50 & 0 & 0 & 0 & -1 & Prison compound \\
\hline 8 & 55 & $\mathbf{0}$ & 1 & $\mathbf{0}$ & -1 & HALFWAY HOUSE \\
\hline 8 & 60 & 0 & 0 & 0 & -1 & Trailer park \\
\hline 8 & 70 & $\mathbf{0}$ & 0 & 0 & -1 & Ski area \\
\hline 8 & 80 & 0 & 0 & 0 & -1 & Stadium \\
\hline 8 & 90 & 0 & 0 & 0 & -1 & Athletic fields \\
\hline 8 & 100 & 0 & 0 & 0 & -1 & Golf course \\
\hline 8 & 110 & 0 & 0 & $\mathbf{0}$ & -1 & Shopping center \\
\hline 8 & 120 & 0 & 0 & 0 & -1 & Zoo \\
\hline 8 & 130 & 0 & 0 & 0 & -1 & Drive-in theater \\
\hline 8 & 140 & 0 & 0 & 0 & -1 & Race track \\
\hline 8 & 150 & $\mathbf{0}$ & $\mathbf{0}$ & $\mathbf{0}$ & -1 & Raceway, drag strip \\
\hline 8 & 160 & $\mathbf{0}$ & 0 & $\mathbf{0}$ & -1 & Marina \\
\hline 8 & 170 & 0 & 0 & 0 & -1 & Cable area \\
\hline 8 & 175 & $\mathbf{0}$ & 1 & $\mathbf{0}$ & -1 & BUS TERMINAL \\
\hline 8 & 180 & 0 & 0 & 0 & -1 & Urban area, house omission area \\
\hline 8 & 181 & 0 & $\mathbf{0}$ & 0 & -1 & PUBLIC UTILITY \\
\hline 8 & 185 & 0 & 0 & 0 & -1 & TRANSPORTATION FACILITY \\
\hline 8 & 190 & 0 & 0 & 0 & -1 & Industrial park \\
\hline 8 & 195 & 0 & $\mathbf{0}$ & 0 & -1 & PRIVATE BUSINESS \\
\hline 8 & 200 & 0 & 0 & $\mathbf{0}$ & -1 & Materials storage area \\
\hline 8 & 205 & 0 & 4 & $\mathbf{0}$ & -1 & NUCLEAR PLANT \\
\hline 8 & 210 & 0 & 0 & 0 & -1 & Refinery or plant \\
\hline 8 & 215 & 0 & 1 & 0 & -1 & EMPLOYMENT CENTER \\
\hline 8 & 216 & 0 & 1 & 0 & -1 & OTHER EMPLOYMENT CENTER \\
\hline 8 & 220 & 0 & 0 & 0 & -1 & Tailings \\
\hline 8 & 230 & 0 & $\mathbf{0}$ & $\mathbf{0}$ & -1 & Intricate surface area \\
\hline 8 & 240 & 0 & $\mathbf{0}$ & $\mathbf{0}$ & -1 & Oil sump or sludge pit \\
\hline 8 & 250 & 0 & 0 & $\mathbf{0}$ & -1 & Slurry disposal area \\
\hline 8 & 260 & 0 & $\mathbf{0}$ & 0 & -1 & Tank farm \\
\hline 8 & 270 & $\mathbf{0}$ & 0 & 0 & -1 & Feedlot \\
\hline 8 & 280 & 0 & $\mathbf{0}$ & $\mathbf{0}$ & -1 & Experimental farm \\
\hline 8 & 290 & 0 & 0 & 0 & -1 & Proving grounds \\
\hline 8 & 300 & 0 & 0 & 0 & -1 & Firing range \\
\hline 8 & 310 & 0 & 0 & $\mathbf{0}$ & -1 & Conveyor \\
\hline 8 & 320 & 0 & 0 & 0 & -1 & Boardwalk \\
\hline 8 & 330 & 0 & 0 & 0 & -1 & Wall \\
\hline
\end{tabular}




\begin{tabular}{|c|c|c|c|c|c|c|}
\hline Maior & Minor & Param & Pen & Line & Decltr & Attribute Description \\
\hline 8 & 340 & $\mathbf{0}$ & 0 & $\mathbf{0}$ & -1 & Sea wall \\
\hline 8 & 350 & $\mathbf{0}$ & 0 & $\mathbf{0}$ & -1 & Levee \\
\hline 8 & 360 & 0 & 0 & 0 & -1 & Fence Line \\
\hline 8 & 370 & 0 & $\mathbf{0}$ & 0 & -1 & Cross \\
\hline 8 & 380 & 0 & $\mathbf{0}$ & 0 & -1 & Flag \\
\hline 8 & 390 & 0 & 0 & $\mathbf{0}$ & -1 & Sewer line \\
\hline 8 & 400 & 0 & $\mathbf{0}$ & $\mathbf{0}$ & -1 & Spoil bank \\
\hline 8 & 410 & 0 & $\mathbf{0}$ & 0 & -1 & Coke ovens \\
\hline 8 & 420 & 0 & $\mathbf{0}$ & 0 & -1 & Arbitrary closure line \\
\hline 8 & 430 & 0 & 0 & 0 & -1 & Grave site \\
\hline 8 & 440 & 0 & 0 & 0 & -1 & Historical marker \\
\hline 8 & 441 & 0 & 1 & $\mathbf{0}$ & -1 & SPECIAL PURPOSE LANDMARK \\
\hline 8 & 445 & 0 & 1 & 0 & -1 & $\begin{array}{l}\text { LANDMARK FEATURE, CLASSIFICATION } \\
\text { UNKNOWN OR NOT ELSEWHERE }\end{array}$ \\
\hline 8 & 450 & 0 & 0 & 0 & -1 & Mine funnel entrance or cave \\
\hline 8 & 460 & $\mathbf{0}$ & 0 & 0 & -1 & Mine shaft \\
\hline 8 & 470 & 0 & 0 & 0 & -1 & Prospect \\
\hline 8 & 480 & $\mathbf{0}$ & 0 & $\mathbf{0}$ & -1 & Tower \\
\hline 8 & 490 & $\mathbf{0}$ & 0 & $\mathbf{0}$ & -1 & Burner/stack \\
\hline 8 & 500 & $\mathbf{0}$ & $\mathbf{0}$ & $\mathbf{0}$ & -1 & Drilled well \\
\hline 8 & 510 & $\mathbf{0}$ & 0 & $\mathbf{0}$ & -1 & Cliff dwelling \\
\hline 8 & 520 & 0 & 0 & 0 & -1 & Light \\
\hline 8 & 530 & $\mathbf{0}$ & 5 & $\mathbf{0}$ & -1 & SHELTER \\
\hline 8 & 536 & $\mathbf{0}$ & 1 & $\mathbf{0}$ & -1 & SHELTER (INACTIVE) \\
\hline 8 & 540 & 0 & $\mathbf{0}$ & $\mathbf{0}$ & -1 & Class 1 building \\
\hline 8 & 550 & 0 & $\mathbf{0}$ & 0 & -1 & Class 2 building \\
\hline 8 & 560 & $\mathbf{0}$ & $\mathbf{0}$ & $\mathbf{0}$ & -1 & Police Station. \\
\hline 8 & 561 & $\mathbf{0}$ & 1 & $\mathbf{0}$ & -1 & POLICE STATION (INACTIVE) \\
\hline 8 & 570 & $\mathbf{0}$ & $\mathbf{0}$ & $\mathbf{0}$ & -1 & Fire Station \\
\hline 8 & 571 & $\mathbf{0}$ & 1 & $\mathbf{0}$ & -1 & FIRE STATION (INACTIVE) \\
\hline 8 & 575 & $\mathbf{0}$ & 10 & $\mathbf{0}$ & -1 & RESCUE SQUAD \\
\hline 8 & 576 & $\mathbf{0}$ & 1 & $\mathbf{0}$ & -1 & RESCUE SQUAD (INACTIVE) \\
\hline 8 & 580 & $\mathbf{0}$ & $\mathbf{0}$ & $\mathbf{0}$ & -1 & Municipal building \\
\hline 8 & 585 & $\mathbf{0}$ & $\mathbf{0}$ & 0 & -1 & GOVERNMENT BUILDING \\
\hline 8 & 590 & $\mathbf{0}$ & 0 & 0 & -1 & Court house \\
\hline 8 & 600 & 0 & 0 & 0 & -1 & Post office \\
\hline 8 & 610 & 0 & 0 & 0 . & -1 & City or town hall \\
\hline 8 & 620 & 0 & 1 & 0 & -1 & EMERGENCY OPERATING CENTER \\
\hline 8 & 621 & 0 & 1 & $\mathbf{0}$ & -1 & $\begin{array}{l}\text { EMERGENCY OPERATIONS CENTER } \\
\text { (INACTIVE) }\end{array}$ \\
\hline 8 & 630 & $\mathbf{0}$ & 1 & $\mathbf{0}$ & -1 & HOTEL \\
\hline 8 & 635 & $\mathbf{0}$ & 1 & $\mathbf{0}$ & -1 & ROOMING OR BOARDING HOUSE \\
\hline 8 & 636 & $\mathbf{0}$ & 1 & $\mathbf{0}$ & -1 & SORORITY OR FRATERNITY \\
\hline 8 & 637 & $\mathbf{0}$ & 1 & $\mathbf{0}$ & -1 & HOUSING FACILITY FOR WORKERS \\
\hline 8 & 640 & 0 & $\mathbf{0}$ & $\mathbf{0}$ & -1 & $\begin{array}{l}\text { Town, village, settlement, locality, unincorporated } \\
\text { village }\end{array}$ \\
\hline 8 & 660 & 0 & 0 & $\mathbf{0}$ & -1 & Cemetery \\
\hline 8 & 670 & $\mathbf{0}$ & $\mathbf{0}$ & $\mathbf{0}$ & -1 & Sewage disposal plant \\
\hline 8 & 680 & 0 & 0 & 0 & -1 & Waterworks \\
\hline 8 & 690 & 0 & 0 & 0 & -1 & Oil reservoir \\
\hline
\end{tabular}




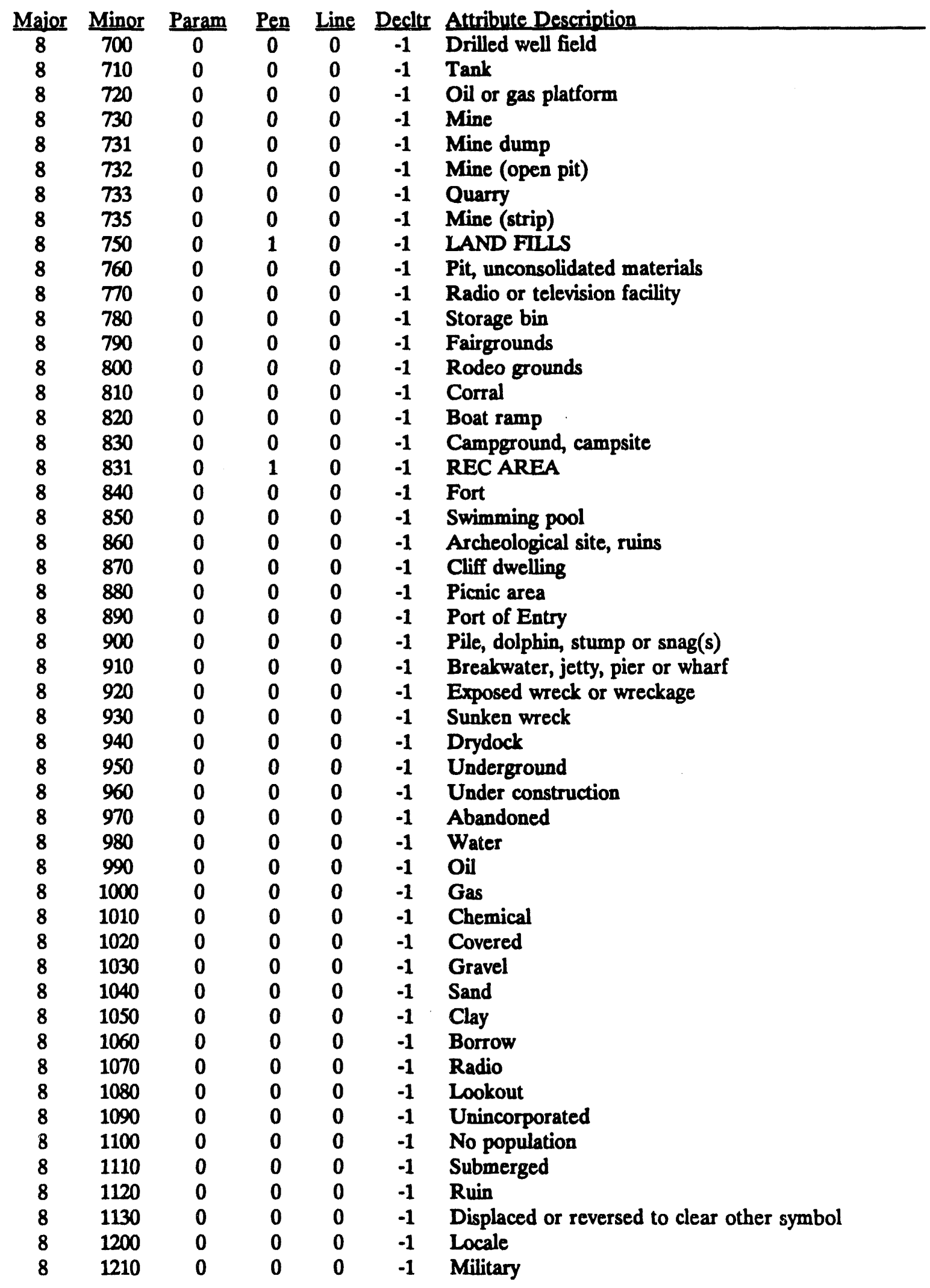


Major Minor Param Pen Line Decltr Attribute Description.

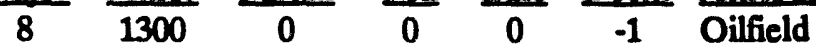

$\begin{array}{lllllll}8 & 1400 & 0 & 1 & 0 & -1 & \text { SIREN - ROTATING }\end{array}$

$\begin{array}{lllllll}8 & 1401 & 0 & 1 & 0 & -1 & \text { SIREN - NON-ROTATING }\end{array}$

$\begin{array}{lllllll}8 & 1402 & 0 & 1 & 0 & -1 & \text { SIREN }\end{array}$ 


\begin{tabular}{|c|c|c|c|c|c|c|}
\hline Maior & Minor & Param & Pen & Line & Decltr & Attribute Description \\
\hline 9 & 0 & 0 & 0 & $\mathbf{0}$ & -1 & MISCELLANEOUS \\
\hline 9 & 10 & 0 & 0 & $\mathbf{0}$ & -1 & Population number \\
\hline 9 & 20 & 0 & $\mathbf{0}$ & $\mathbf{0}$ & -1 & Population number in 10 's \\
\hline 9 & 21 & 0 & 1 & $\mathbf{0}$ & -1 & POPULATION 0-5 YRS IN 10'S \\
\hline 9 & 22 & 0 & 1 & $\mathbf{0}$ & -1 & POPULATION 6-19 YRS IN 10'S \\
\hline 9 & 23 & 0 & 1 & 0 & -1 & POPULATION 20-64 YRS IN 10'S \\
\hline 9 & 24 & 0 & 1 & 0 & -1 & POPULATION 65 YRS OR OLDER IN 10'S \\
\hline 9 & 30 & $\mathbf{0}$ & $\mathbf{0}$ & $\mathbf{0}$ & -1 & Population number in 100 's \\
\hline 9 & 31 & 0 & 1 & 0 & -1 & POPUULATION 0-5 YRS IN 100'S \\
\hline 9 & 32 & $\mathbf{0}$ & 1 & $\mathbf{0}$ & -1 & POPULATION 6-19 YRS IN 100'S \\
\hline 9 & 33 & 0 & 1 & $\mathbf{0}$ & -1 & POPULATION 20-64 YRS IN 100'S \\
\hline 9 & 34 & 0 & 1 & 0 & -1 & POPULATION 65 YRS OR OLDER IN 100'S \\
\hline 9 & 40 & 0 & 1 & $\mathbf{0}$ & -1 & Population number in 1000 's \\
\hline 9 & 41 & $\mathbf{0}$ & 1 & $\mathbf{0}$ & -1 & POPULATION 0-5 YRS IN 1000'S \\
\hline 9 & 42 & 0 & 1 & $\mathbf{0}$ & -1 & POPULATION 6-19 YRS IN 1000'S \\
\hline 9 & 43 & 0 & 1 & 0 & -1 & POPULATION 20-64 YRS IN 1000'S \\
\hline 9 & 44 & 0 & 1 & 0 & -1 & POPULATION 65 YRS OR OLDER IN 1000'S \\
\hline 9 & 50 & 0 & 1 & 0 & -1 & SPECIAL POPULATION \\
\hline 9 & 51 & 0 & 1 & 0 & -1 & $\begin{array}{l}\text { INSTITUTIONAL, TRANSIT-DEPENDENT } \\
\text { POPULATION }\end{array}$ \\
\hline 9 & 52 & $\mathbf{0}$ & 1 & $\mathbf{0}$ & -1 & $\begin{array}{l}\text { NON-INSTITUTIONAL, TRANSIT-DEPENDENT } \\
\text { POPULATION }\end{array}$ \\
\hline 9 & 53 & 0 & 1 & 0 & -1 & CREW OF VESSEL \\
\hline 9 & 99 & $\mathbf{0}$ & $\mathbf{0}$ & 0 & -1 & Polygon fill descriptor (graphics: spacing, pen, fill) \\
\hline 9 & 100 & 0 & 0 & 0 & -1 & Sensors \\
\hline 9 & 200 & 0 & 0 & 0 & -1 & Basic contour (METERS) \\
\hline 9 & 210 & $\mathbf{0}$ & $\mathbf{0}$ & $\mathbf{0}$ & -1 & Carrying contour \\
\hline 9 & 220 & 0 & 0 & 0 & -1 & Supplementary contour \\
\hline 9 & 230 & $\mathbf{0}$ & 0 & $\mathbf{0}$ & -1 & Continuation contour \\
\hline 9 & 240 & 0 & $\mathbf{0}$ & 0 & -1 & Auxiliary contour \\
\hline 9 & 250 & 0 & 0 & 0 & -1 & Bathymetric contour \\
\hline 9 & 260 & $\mathbf{0}$ & 0 & 0 & -1 & Depth curve \\
\hline 9 & 270 & $\mathbf{0}$ & 0 & 0 & -1 & Spot elevation, less than third order \\
\hline 9 & 280 & $\mathbf{0}$ & 0 & 0 & -1 & Decimal fractions of feet or meters \\
\hline 9 & 290 & $\mathbf{0}$ & $\mathbf{0}$ & 0 & -1 & Approximate \\
\hline 9 & 300 & 0 & 0 & 0 & -1 & Depression \\
\hline 9 & 310 & 0 & 0 & 0 & -1 & Glacier or snow field \\
\hline 9 & 320 & 0 & 0 & 0 & -1 & Underwater \\
\hline 9 & 330 & 0 & 0 & 0 & -1 & Cut \\
\hline 9 & 340 & 0 & 0 & $\mathbf{0}$ & -1 & Fill \\
\hline 9 & 350 & 0 & 0 & $\mathbf{0}$ & -1 & Levee \\
\hline 9 & 360 & 0 & 0 & 0 & -1 & Elevation in whole feet or meters, right-justified \\
\hline 9 & 370 & 0 & 0 & $\mathbf{0}$ & -1 & $\begin{array}{l}\text { Horiz. control station - 3rd order or better, permanent } \\
\text { mark }\end{array}$ \\
\hline 9 & 375 & 0 & $\mathbf{0}$ & 0 & -1 & Horizontal control station - 3rd order or better \\
\hline 9 & 380 & 0 & $\mathbf{0}$ & 0 & -1 & Horizontal control station - vertical angle, bench mark \\
\hline 9 & 385 & $\mathbf{0}$ & $\mathbf{0}$ & 0 & -1 & Horizontal control station - checked spot elevation \\
\hline 9 & 390 & 0 & $\mathbf{0}$ & 0 & -1 & Vertical control station - 3rd order or better - tablet \\
\hline 9 & 395 & 0 & 0 & 0 & -1 & $\begin{array}{l}\text { Vert. cont. sta. -recov. mark-3rd ord. or better - no } \\
\text { tablet }\end{array}$ \\
\hline
\end{tabular}




\begin{tabular}{|c|c|c|c|c|c|c|}
\hline Major & Minor & Param & Pen & Line & Decltr & Attribute Description \\
\hline 9 & 400 & 0 & 0 & 0 & -1 & Boundary monument - 3rd order or better - tablet \\
\hline 9 & 405 & $\mathbf{0}$ & $\mathbf{0}$ & $\mathbf{0}$ & -1 & Boundary monument - 3rd order or better - no tablet \\
\hline 9 & 410 & $\mathbf{0}$ & $\mathbf{0}$ & $\mathbf{0}$ & -1 & Reference monument \\
\hline 9 & 420 & $\mathbf{0}$ & 0 & $\mathbf{0}$ & -1 & U.S. Mineral or location monument \\
\hline 9 & 430 & 0 & $\mathbf{0}$ & $\mathbf{0}$ & -1 & Other control point \\
\hline 9 & 500 & $\mathbf{0}$ & $\mathbf{0}$ & $\mathbf{0}$ & -1 & State FIPS code \\
\hline 9 & 510 & 0 & 0 & $\mathbf{0}$ & -1 & County FIPS code \\
\hline 9 & 520 & $\mathbf{0}$ & 0 & $\mathbf{0}$ & -1 & Elevation to nearest foot \\
\hline 9 & 530 & $\mathbf{0}$ & $\mathbf{0}$ & $\mathbf{0}$ & -1 & Elevation to nearest meter \\
\hline 9 & 550 & $\mathbf{0}$ & $\mathbf{0}$ & $\mathbf{0}$ & -1 & Evacuee Origination Points \\
\hline 9 & 560 & $\mathbf{0}$ & $\mathbf{0}$ & $\mathbf{0}$ & -1 & Evacuee Reception/Care Points \\
\hline 9 & 561 & $\mathbf{0}$ & 1 & $\mathbf{0}$ & -1 & RECEPTION CENTER \\
\hline 9 & 562 & 0 & 1 & $\mathbf{0}$ & -1 & RECEPTION CENTER CAPACITY \\
\hline 9 & 563 & $\mathbf{0}$ & 1 & $\mathbf{0}$ & -1 & RECEPTION CENTER LOAD \\
\hline 9 & 565 & $\mathbf{0}$ & 1 & $\mathbf{0}$ & -1 & RECEPTION CENTER (INACTIVE) \\
\hline 9 & 566 & $\mathbf{0}$ & 1 & $\mathbf{0}$ & -1 & DECONTAMINATION CENTER \\
\hline 9 & 567 & $\mathbf{0}$ & 1 & $\mathbf{0}$ & -1 & DECONTAMINATION CENTER (INACTIVE) \\
\hline 9 & 570 & $\mathbf{0}$ & 1 & 0 & -1 & MORGUE \\
\hline 9 & 571 & $\mathbf{0}$ & 1 & $\mathbf{0}$ & -1 & MORGUE (INACTIVE) \\
\hline 9 & 600 & 0 & $\mathbf{0}$ & $\mathbf{0}$ & -1 & Time (year/mo) \\
\hline 9 & 601 & $\mathbf{0}$ & $\mathbf{0}$ & $\mathbf{0}$ & -1 & Time (day/hr/min) \\
\hline 9 & 602 & $\mathbf{0}$ & $\mathbf{0}$ & $\mathbf{0}$ & -1 & Time (milliseconds) \\
\hline 9 & 603 & $\mathbf{0}$ & 1 & $\mathbf{0}$ & -1 & TIME STEP \\
\hline 9 & 610 & $\mathbf{0}$ & 1 & $\mathbf{0}$ & -1 & SET CODE \\
\hline 9 & 611 & 0 & 1 & $\mathbf{0}$ & -1 & SET ELEMENT PONNTER \\
\hline 9 & 620 & $\mathbf{0}$ & 1 & 0 & -1 & CONCENTRATION PARTS PER MILLION (PPM) \\
\hline 9 & 621 & $\mathbf{0}$ & 1 & $\mathbf{0}$ & -1 & CONCENTRATION PARTS PER BILLION (PPB) \\
\hline 9 & 622 & $\mathbf{0}$ & 1 & $\mathbf{0}$ & -1 & HEAT FLUX WATTS/M**2 \\
\hline 9 & 623 & $\mathbf{0}$ & 1 & $\mathbf{0}$ & -1 & HEAT FLUX K WATTS/M**2 \\
\hline 9 & 624 & $\mathbf{0}$ & 1 & $\mathbf{0}$ & -1 & POOL FIRE POOL BOUNDARY \\
\hline 9 & 630 & $\mathbf{0}$ & 1 & $\mathbf{0}$ & -1 & EXPLOSION DAMAGE CONTOURR \\
\hline 9 & 631 & $\mathbf{0}$ & 1 & 0 & -1 & THRESHOLD LUNG COLLAPSE \\
\hline 9 & 632 & $\mathbf{0}$ & 1 & $\mathbf{0}$ & -1 & THRESHOLD EAR RUPTURE \\
\hline 9 & 633 & $\mathbf{0}$ & 1 & $\mathbf{0}$ & -1 & THRESHOLD SKULL DAMAGE \\
\hline 9 & 634 & 0 & 1 & $\mathbf{0}$ & -1 & THRESHOLD FATALITIES \\
\hline 9 & 635 & $\mathbf{0}$ & 1 & $\mathbf{0}$ & -1 & THRESHOLD GLASS BREAKAGE \\
\hline 9 & 636 & $\mathbf{0}$ & 1 & $\mathbf{0}$ & -1 & THRESHOLD BUILDING DAMAGE \\
\hline 9 & 637 & 0 & 1 & $\mathbf{0}$ & -1 & DISTANCE IN THOUSANDS OF METERS \\
\hline 9 & 638 & 0 & 1 & $\mathbf{0}$ & -1 & DISTANCE IN HUNDREDS OF METERS \\
\hline 9 & 639 & $\mathbf{0}$ & 1 & $\mathbf{0}$ & -1 & DISTANCE IN TENS OF METERS \\
\hline 9 & 640 & 0 & 1 & $\mathbf{0}$ & -1 & DISTANCE IN METERS \\
\hline 9 & 641 & 0 & 1 & 0 & -1 & $\begin{array}{l}\text { UNUSED: MAXIMUM DOSAGE IN MG- } \\
\text { MIN/METERS CUBED / 10M }\end{array}$ \\
\hline 9 & 642 & 0 & 1 & $\mathbf{0}$ & -1 & $\begin{array}{l}\text { UNUSED: MAXIMUM DOSAGE IN MG- } \\
\text { MIN/METERS CUBED / } \mathbf{1 k}\end{array}$ \\
\hline 9 & 643 & 0 & 1 & 0 & -1 & $\begin{array}{l}\text { TIME IN TENTHS OF MINUTES OF TIP OF } \\
\text { PIUME }\end{array}$ \\
\hline 9 & 644 & 0 & 1 & $\mathbf{0}$ & -1 & $\begin{array}{l}\text { TOTAL DOSAGE IN MG-MIN/METERS CUBED } \\
/ \text { 10M }\end{array}$ \\
\hline 9 & 645 & $\mathbf{0}$ & 1 & $\mathbf{0}$ & -1 & DOSAGE IN MG-MIN/METERS CUBED / 1K \\
\hline
\end{tabular}




\begin{tabular}{|c|c|c|c|c|c|c|}
\hline Maior & Minor & Param & Pen & Line & Decltr & Attribute Description \\
\hline 9 & 646 & 0 & 1 & 0 & -1 & $\begin{array}{l}\text { TIME IN TENTHS OF MINUTES FOR TAIL OF } \\
\text { PLUME }\end{array}$ \\
\hline 9 & 647 & 0 & 1 & $\mathbf{0}$ & -1 & $\begin{array}{l}\text { UNUSED: MAXIMUM DOSAGE IN } \\
\text { MG-MIN/METERS CUBED }\end{array}$ \\
\hline 9 & 648 & 0 & 1 & 0 & -1 & TOTAL DOSAGE IN MG-MIN/METERS CUBED \\
\hline 9 & 649 & 0 & 1 & 0 & -1 & $\begin{array}{l}\text { UNUSED: LOW PORTION OF DOSAGE IN } \\
\text { TENTHS OF \% }\end{array}$ \\
\hline 9 & 650 & $\mathbf{0}$ & 1 & $\mathbf{0}$ & -1 & $\begin{array}{l}\text { UNUSED: MEDIUM PORTION OF DOSAGE IN } \\
\text { TENTHS OF \% }\end{array}$ \\
\hline 9 & 651 & 0 & 1 & 0 & -1 & $\begin{array}{l}\text { UNUSED: HIGH PORTION OF DOSAGE IN } \\
\text { TENTHS OF } \%\end{array}$ \\
\hline 9 & 652 & 0 & 1 & 0 & -1 & $\begin{array}{l}\text { UNUSED: TIME FOR DOSAGE TO EQUAL LOW } \\
\text { DOSAGE IN TENTHS OF MINUTES }\end{array}$ \\
\hline 9 & 653 & 0 & 1 & 0 & -1 & $\begin{array}{l}\text { UNUSED: TIME FOR DOSAGE TO EQUAL } \\
\text { MEDIUM DOSAGE IN TENTHS OF MINUTES }\end{array}$ \\
\hline 9 & 654 & 0 & 1 & 0 & -1 & $\begin{array}{l}\text { UNUSED: TIME FOR DOSAGE TO EQUAL } \\
\text { HIGH DOSAGE IN TENTHS OF MINUTES }\end{array}$ \\
\hline 9 & 655 & 0 & 1 & 0 & -1 & $\begin{array}{l}\text { UNUSED: LOW DOSAGE PLUME IN TENTHS } \\
\text { (MG-MIN/M**3) }\end{array}$ \\
\hline 9 & 656 & 0 & 1 & 0 & -1 & $\begin{array}{l}\text { UNUSED: MEDIUM DOSAGE PLUME IN } \\
\text { TENTHS (MG-MIN/M**3) }\end{array}$ \\
\hline 9 & 657 & 0 & 1 & 0 & -1 & $\begin{array}{l}\text { UNUSED: HIGH DOSAGE PLUME IN TENTHS } \\
\text { (MG-MIN/M**3) }\end{array}$ \\
\hline 9 & 658 & 0 & 1 & 0 & -1 & $\begin{array}{l}\text { UNUSED: LOW CONCENTRATION IN TENTHS } \\
\text { (MG/M**3) PLUME }\end{array}$ \\
\hline 9 & 659 & 0 & 1 & 0 & -1 & $\begin{array}{l}\text { UNUSED: MEDIUM CONCENTRATION IN } \\
\text { TENTHS (MG/M**3) PLLIME }\end{array}$ \\
\hline 9 & 660 & 0 & 1 & 0 & -1 & $\begin{array}{l}\text { UNUSED: HIGH CONCENTRATION IN TENTHS } \\
\left(M G / M^{* *} 3\right) \text { PLUME }\end{array}$ \\
\hline 9 & 661 & 0 & 1 & 0 & -1 & $\begin{array}{l}\text { UNUSED: LOW CONCENTRATION IN TENTHS } \\
\text { (PPM) PLUME }\end{array}$ \\
\hline 9 & 662 & 0 & 1 & 0 & -1 & $\begin{array}{l}\text { UNUSED: MEDIUM CONCENTRATION IN } \\
\text { TENTHS (PPM) PLUME }\end{array}$ \\
\hline 9 & 663 & 0 & 1 & 0 & -1 & $\begin{array}{l}\text { UNUSED: HIGH CONCENTRATION IN TENTHS } \\
\text { (PPM) PLUME }\end{array}$ \\
\hline 9 & 664 & 0 & 1 & $\mathbf{0}$ & -1 & $\begin{array}{l}\text { UNUSED: LOW FUMIGATION } \\
\text { CONCENTRATION IN TENTHS PLUME }\end{array}$ \\
\hline 9 & 665 & 0 & 1 & 0 & -1 & $\begin{array}{l}\text { UNUSED: MEDIUM FUMIGATION } \\
\text { CONCENTRATION IN TENTHS PLUME }\end{array}$ \\
\hline 9 & 666 & $\mathbf{0}$ & 1 & 0 & -1 & $\begin{array}{l}\text { UNUSED: HIGH FUMIGATION } \\
\text { CONCENTRATION IN TENTHS PLUME }\end{array}$ \\
\hline 9 & 667 & 0 & 1 & 0 & -1 & WIND DIRECTION (FROM) \\
\hline 9 & 670 & 0 & 7 & $\mathbf{0}$ & -1 & RISK POLYGON \\
\hline 9 & 671 & 0 & 7 & 0 & -1 & USER DEFINED RISK POLYGON \\
\hline 9 & $67 ?$ & 0 & 1 & 0 & -1 & $\begin{array}{l}\text { DOSAGE LEVEL IN HUNDREDTHS } \\
\text { (MG-MIN/M**3) }\end{array}$ \\
\hline 9 & 473 & 0 & 1 & 0 & -1 & $\begin{array}{l}\text { PORTION OF DOSAGE LEVEL IN TENTHS OF } \\
\%\end{array}$ \\
\hline 9 & 674 & 0 & 1 & 0 & -1 & $\begin{array}{l}\text { TIME FOR DOSAGE TO EQUAL DOSAGE } \\
\text { LEVEL IN TENTHS OF MINUTES }\end{array}$ \\
\hline
\end{tabular}




\begin{tabular}{|c|c|c|c|c|c|c|}
\hline Maior & Minor & Param & Pen & Line & Decltr & Attribute Description \\
\hline 9 & 700 & $\mathbf{0}$ & 5 & $\mathbf{0}$ & -1 & EARTHQUAKE FAULT \\
\hline 9 & 701 & $\mathbf{0}$ & 5 . & 0 & -1 & TROPICAL STORM \\
\hline 9 & 702 & 0 & 1 & 0 & -1 & HURRICANE \\
\hline 9 & 703 & 0 & 1 & $\mathbf{0}$ & -1 & EARTHQUAKE (ICON) \\
\hline 9 & 704 & 0 & 1 & 0 & -1 & FIRE ICON \\
\hline 9 & 705 & 0 & 1 & 0 & -1 & BLIZZARD ICON \\
\hline 9 & 706 & 0 & 1 & $\mathbf{0}$ & -1 & TORNADO ICON \\
\hline 9 & 707 & $\mathbf{0}$ & 1 & $\mathbf{0}$ & -1 & FLOOD ICON \\
\hline 9 & 708 & $\mathbf{0}$ & 1 & $\mathbf{0}$ & -1 & CITY ICON \\
\hline 9 & 709 & 0 & 1 & 0 & -1 & $\begin{array}{llllll}9 & 710 & 0 & 1 & 0 & -1\end{array}$ \\
\hline 9 & 711 & $\mathbf{0}$ & 1 & 0 & -1 & FIRE STATION \\
\hline 9 & 712 & $\mathbf{0}$ & 1 & $\mathbf{0}$ & -1 & MOBILE MEDICAL UNIT \\
\hline 9 & 713 & 0 & 1 & $\mathbf{0}$ & -1 & HOSPITAL RESOURCE \\
\hline 9 & 714 & $\mathbf{0}$ & 1 & $\mathbf{0}$ & -1 & EQUIPMENT \\
\hline 9 & 715 & $\mathbf{0}$ & 1 & 0 & $-?$ & PRIVATE RESIDENCE \\
\hline 9 & 730 & 0 & 7 & 0 & -1 & SEARCH \& RESCUE AREA \\
\hline 9 & 740 & $\mathbf{0}$ & 1 & $\mathbf{0}$ & -1 & KNOWN POINT ID \\
\hline 9 & 760 & $\mathbf{0}$ & 1 & 0 & -1 & KNOWN ROUTE ID \\
\hline 9 & 812 & 0 & 1 & 0 & -1 & ENVIRONMENT REPORT \\
\hline 9 & 813 & $\mathbf{0}$ & 1 & $\mathbf{0}$ & -1 & WEATHER REPORT \\
\hline 9 & 900 & $\mathbf{0}$ & 1 & 0 & -1 & NA.P.B. TARGET - KILOTONNAGE \\
\hline 9 & 901 & $\mathbf{0}$ & 1 & 0 & -1 & CANADA \\
\hline 9 & 902 & 0 & 1 & $\mathbf{0}$ & -1 & TARGET - MILTAARY INSTALLATION \\
\hline 9 & 903 & $\mathbf{0}$ & 1 & $\mathbf{0}$ & -1 & TARGET - OTMIL \\
\hline 9 & 904 & $\mathbf{0}$ & 1 & $\mathbf{0}$ & -1 & TARGET - PORT \\
\hline 9 & 905 & $\mathbf{0}$ & 1 & $\mathbf{0}$ & -1 & HAZARD \\
\hline 9 & 906 & 0 & 1 & 0 & -1 & HAZMAT SITE \\
\hline 9 & 907 & $\mathbf{0}$ & 1 & $\mathbf{0}$ & -1 & TARGET - POLITICAL \\
\hline 9 & 908 & $\mathbf{0}$ & 1 & 0 & -1 & TARGET - POWER \\
\hline 9 & 909 & 0 & 1 & 0 & -1 & TARGET - COFCE \\
\hline 9 & 1000 & 0 & 1 & 0 & -1 & TARGET - CHEMICAL \\
\hline 9 & 1001 & $\mathbf{0}$ & 1 & 0 & $-i$ & TARGET - MREFIN \\
\hline 9 & 1002 & 0 & 1 & 0 & -1 & ZIP CODE \\
\hline 9 & 1003 & 0 & 1 & $\mathbf{0}$ & -1 & EOC SQUARE FOOTAGE \\
\hline 9 & 1004 & $\mathbf{0}$ & 1 & $\mathbf{0}$ & -1 & PERSONNEL STAFFING - EMERGENCY \\
\hline 9 & 1005 & 0 & 1 & 0 & -1 & PERSONNEL STAFFING - NORMAL \\
\hline 9 & 1006 & 0 & 1 & $\mathbf{0}$ & -1 & REFINERY \\
\hline 9 & 1007 & 0 & $\mathbf{I}$ & $\mathbf{0}$ & -1 & COAL MINE \\
\hline 9 & 1008 & $\mathbf{0}$ & 1 & $\mathbf{0}$ & -1 & COKE PRODUCTION \\
\hline 9 & 1009 & 0 & 1 & 0 & -1 & NATURAL GAS PRESSURE PLANT \\
\hline 9 & 1010 & $\mathbf{0}$ & 1 & $\mathbf{0}$ & -1 & NATURAL GAS UNDERGROUND STORAGE \\
\hline 9 & 1011 & 0 & 1 & 0 & -1 & PETROLEUM IMPORT FACILITY \\
\hline 9 & 1012 & 0 & 1 & 0 & -1 & PORT FACILITY \\
\hline 9 & 1013 & $\mathbf{0}$ & 1 & $\mathbf{0}$ & -1 & GRAIN STORAGE FACILITY \\
\hline 9 & 1014 & 0 & 1 & $\mathbf{0}$ & -1 & FLOUR MILLS \\
\hline 9 & 1015 & 0 & 1 & $\mathbf{0}$ & -1 & FOOD DISTRIBUTION FACILITY \\
\hline 9 & 1016 & 0 & 1 & $\mathbf{0}$ & -1 & POULTRY PRODUCTION \\
\hline 9 & 1017 & 0 & 1 & 0 & -1 & AGRICULTURE EMERGENCY OPS CENTER \\
\hline 9 & 1018 & $\mathbf{0}$ & 1 & $\mathbf{0}$ & -1 & EGG PRODUCERS \\
\hline 9 & 1019 & $\mathbf{0}$ & 1 & 0 & -1 & FOOD PRODUCTION FACILITY \\
\hline
\end{tabular}




\begin{tabular}{|c|c|c|c|c|c|c|}
\hline Maior & Minor & Param & Pen & Line & Decltr & Attribute Description \\
\hline 9 & 1020 & 0 & $\mathbf{0}$ & 0 & -1 & NUMBER OF DAIRY COWS \\
\hline 9 & 1021 & 0 & 1 & $\mathbf{0}$ & -1 & PLANT CAPACITY \\
\hline 9 & 2001 & $\mathbf{0}$ & 1 & $\mathbf{0}$ & -1 & ALLENS CREEK NPP \\
\hline 9 & 2002 & 0 & 1 & 0 & -1 & ARKANSAS NPP \\
\hline 9 & 2003 & 0 & 1 & $\mathbf{0}$ & -1 & DUANE ARNOLD NPP \\
\hline 9 & 2004 & 0 & 1 & 0 & -1 & BEAVER VALLEY NPP \\
\hline 9 & 2005 & 0 & 1 & 0 & -1 & BELLEFONTE NPP \\
\hline 9 & 2006 & $\mathbf{0}$ & 1 & $\mathbf{0}$ & -1 & BIG ROCK POINT NPP \\
\hline 9 & 2007 & $\mathbf{0}$ & 1 & $\mathbf{0}$ & -1 & BRAIDWOOD NPP \\
\hline 9 & 2008 & 0 & 1 & $\mathbf{0}$ & -1 & BROWNS FERRY NPP \\
\hline 9 & 2009 & 0 & 1 & 0 & -1 & BRUNSWICK NPP \\
\hline 9 & 2010 & $\mathbf{0}$ & 1 & 0 & -1 & BYRON NPP \\
\hline 9 & 2011 & $\mathbf{0}$ & 1 & $\mathbf{0}$ & -1 & CALLAWAY NPP \\
\hline 9 & 2012 & 0 & 1 & $\mathbf{0}$ & -1 & CALVERT CLIFFS NPP \\
\hline 9 & 2013 & 0 & 1 & $\mathbf{0}$ & -1 & CATAWBA NPP \\
\hline 9 & 2014 & 0 & 1 & 0 & -1 & CLINTON NPP \\
\hline 9 & 2015 & 0 & 1 & 0 & -1 & COMANCHE PEAK \\
\hline 9 & 2016 & 0 & 1 & 0 & -1 & COOK NPP \\
\hline 9 & 2017 & 0 & 1 & 0 & -1 & COOPER NPP \\
\hline 9 & 2018 & 0 & 1 & 0 & -1 & CRYSTAL RIVER NPP \\
\hline 9 & 2019 & 0 & 1 & 0 & -1 & DAVIS-BESSIE NPP \\
\hline 9 & 2020 & 0 & 1 & 0 & -1 & DIABLO CANYON NPP \\
\hline 9 & 2021 & 0 & 1 & 0 & -1 & DRESDEN NPP \\
\hline 9 & 2022 & 0 & 1 & 0 & -1 & FARLEY NPP \\
\hline 9 & 2023 & 0 & 1 & 0 & -1 & FERMI NPP \\
\hline 9 & 2024 & 0 & 1 & 0 & -1 & FITZPATRICK NPP \\
\hline 9 & 2025 & 0 & 1 & 0 & -1 & FORT CALHOUN NPP \\
\hline 9 & 2026 & 0 & 1 & $\mathbf{0}$ & -1 & FORT ST. VRAIN NPP \\
\hline 9 & 2027 & 0 & 1 & 0 & -1 & GINNA (BROOKWOOD) NPP \\
\hline 9 & 2028 & 0 & 1 & 0 & -1 & GRAND GULF NPP \\
\hline 9 & 2029 & 0 & 1 & 0 & -1 & HADDAM NECK (CONN YANKEE) NPP \\
\hline 9 & 2030 & 0 & 1 & $\mathbf{0}$ & -1 & HATCH NPP \\
\hline 9 & 2031 & 0 & 1 & 0 & -1 & HOPE CREEK NPP \\
\hline 9 & 2032 & 0 & 1 & 0 & -1 & HUMBOLDT BAY NPP \\
\hline 9 & 2033 & $\mathbf{0}$ & 1 & 0 & -1 & INDIAN POINT NPP \\
\hline 9 & 2034 & 0 & 1 & $\mathbf{0}$ & -1 & KEWAUNEE NPP \\
\hline 9 & 2035 & $\mathbf{0}$ & 1 & 0 & -1 & LA CROSSE (GENOA) NPP \\
\hline 9 & 2036 & 0 & 1 & 0 & -1 & LASALLE NPP \\
\hline 9 & 2037 & 0 & 1 & 0 & -1 & LIMERICK NPP \\
\hline 9 & 2038 & $\mathbf{0}$ & 1 & $\mathbf{0}$ & -1 & MAINE YANKEE NPP \\
\hline 9 & 2039 & 0 & 1 & 0 & -1 & MCGUIRE NPP \\
\hline 9 & 2040 & 0 & 1 & 0 & -1 & MILLSTONE NPP \\
\hline 9 & 2041 & 0 & 1 & 0 & -1 & MONTICELLO NPP \\
\hline 9 & 2042 & 0 & 1 & 0 & -1 & NINE MILE POINT NPP \\
\hline 9 & 2043 & 0 & 1 & 0 & -1 & NORTH ANNA NPP \\
\hline 9 & 2044 & 0 & 1 & 0 & -1 & OCONEE NPP \\
\hline 9 & 2045 & 0 & 1 & $\mathbf{0}$ & -1 & OYSTER CREEK NPP \\
\hline 9 & 2046 & 0 & 1 & 0 & -1 & PALISADES NPP \\
\hline 9 & 2047 & 0 & 1 & 0 & -1 & PALO VERDE NPP \\
\hline 9 & 2048 & 0 & 1 & 0 & -1 & PEACH BOTTOM NPF \\
\hline
\end{tabular}




\begin{tabular}{|c|c|c|c|c|c|c|}
\hline Maior & Minor & Param & Pen & Line & Decltr & Attribute Description \\
\hline 9 & 2049 & 0 & 1 & 0 & -1 & PERRY NPP \\
\hline 9 & 2050 & 0 & 1 & 0 & -1 & PILGRIM (ROCKY POINT) NPP \\
\hline 9 & 2051 & $\mathbf{0}$ & 1 & $\mathbf{0}$ & -1 & POINT BEACH NPP \\
\hline 9 & 2052 & 0 & 1 & 0 & -1 & PRAIRIE ISLAND NPP \\
\hline 9 & 2053 & $\mathbf{0}$ & 1 & 0 & -1 & QUAD CITIES NPP \\
\hline 9 & 2054 & $\mathbf{0}$ & 1 & $\mathbf{0}$ & -1 & RANCHO SECO NPP \\
\hline 9 & 2055 & $\mathbf{0}$ & 1 & $\mathbf{0}$ & -1 & RIVER BEND NPP \\
\hline 9 & 2056 & $\mathbf{0}$ & 1 & 0 & -1 & ROBINSON NPP \\
\hline 9 & 2057 & $\mathbf{0}$ & 1 & $\mathbf{0}$ & -1 & ST. LUCIE NPP \\
\hline 9 & 2058 & $\mathbf{0}$ & 1 & 0 & -1 & SALEM NPP \\
\hline 9 & 2059 & 0 & 1 & $\mathbf{0}$ & -1 & SAN ONOFRE NPP \\
\hline 9 & 2060 & $\mathbf{0}$ & 1 & $\mathbf{0}$ & -1 & SEABROOK NPP \\
\hline 9 & 2061 & $\mathbf{0}$ & 1 & 0 & -1 & SEQUOYAH NPP \\
\hline 9 & 2062 & $\mathbf{0}$ & 1 & $\mathbf{0}$ & -1 & SHEARON HARRIS \\
\hline 9 & 2063 & $\mathbf{0}$ & 1 & $\mathbf{0}$ & -1 & SHOREHAM NPP \\
\hline 9 & 2064 & 0 & 1 & 0 & -1 & SOUTH TEXAS NPP \\
\hline 9 & 2065 & 0 & 1 & 0 & -1 & SUMMER NPP \\
\hline 9 & 2066 & $\mathbf{0}$ & 1 & 0 & -1 & SURRY NPP \\
\hline 9 & 2067 & 0 & 1 & $\mathbf{0}$ & -1 & SUSQUEHANNA NPP \\
\hline 9 & 2068 & $\mathbf{0}$ & 1 & $\mathbf{0}$ & -1 & THREE MILE ISLAND) NPP \\
\hline 9 & 2069 & 0 & 1 & $\mathbf{0}$ & -1 & TROJAN IIPP \\
\hline 9 & 2070 & 0 & 1 & $\mathbf{0}$ & -1 & TURKEY POINT NPP \\
\hline 9 & 2071 & 0 & 1 & 0 & -1 & VERMONT YANKEE NPP \\
\hline 9 & 2072 & $\mathbf{0}$ & 1 & 0 & -1 & VOGTLE NPP \\
\hline 9 & 2073 & $\mathbf{0}$ & 1 & $\mathbf{0}$ & -1 & WPPSS STEAM/N-REACTOR \\
\hline 9 & 2074 & $\mathbf{0}$ & 1 & $\mathbf{0}$ & -1 & WPPSS 2 NPP \\
\hline 9 & 2075 & 0 & 1 & 0 & -1 & WATERFORD NPP \\
\hline 9 & 2076 & 0 & 1 & $\mathbf{0}$ & -1 & WOLF CREEK NPP \\
\hline 9 & 2077 & $\mathbf{0}$ & 1 & $\mathbf{0}$ & -1 & YANKEE NPP \\
\hline 9 & 2078 & $\mathbf{0}$ & 1 & $\mathbf{0}$ & -1 & ZION NPP \\
\hline 9 & 2201 & $\mathbf{0}$ & 1 & $\mathbf{0}$ & -1 & SITE NAME: WALI,IS, TEXAS \\
\hline 9 & 2202 & $\mathbf{0}$ & 1 & $\mathbf{0}$ & -1 & SITE NAME: RUSSELLVILLE, ARKANSAS \\
\hline 9 & 2203 & 0 & 1 & 0 & -1 & SITE NAME: PALC, IOWA \\
\hline 9 & 2204 & $\mathbf{0}$ & 1 & $\mathbf{0}$ & -1 & SITE NAME: SHIPPINGPORT, PENNSYLVANIA \\
\hline 9 & 2205 & 0 & 1 & $\mathbf{0}$ & -1 & SITE NAME: SCO'TTSBORO, ALABAMA \\
\hline 9 & 2206 & $\mathbf{0}$ & 1 & $\mathbf{0}$ & -1 & SITE NAME: BIG ROCK POINT, MICHIGAN \\
\hline 9 & 2207 & $\mathbf{0}$ & 1 & $\mathbf{0}$ & -1 & SITE NAME: BRAIDWOOD, ILLINOIS \\
\hline 9 & 2208 & $\mathbf{0}$ & 1 & 0 & -1 & SITE NAME: DECATUR, ALABAMA \\
\hline 9 & 2209 & $\mathbf{0}$ & 1 & $\mathbf{0}$ & -1 & SITE NAME: SOI.THPORT, NORTH CAROLINA \\
\hline 9 & 2210 & $\mathbf{0}$ & 1 & $\mathbf{0}$ & -1 & SITE NAME: BYRON, ILLINOIS \\
\hline 9 & 2211 & $\mathbf{0}$ & 1 & 0 & -1 & SITE NAME: FULTON, MISSOURI \\
\hline 9 & 2212 & $\mathbf{0}$ & 1 & 0 & -1 & SITE NAME: LUSBY, MARYLAND \\
\hline 9 & 2213 & $\mathbf{0}$ & 1 & $\mathbf{0}$ & -1 & SITE NAME: LAKE WYLIE, SOUTH CAROLINA \\
\hline 9 & 2214 & $\mathbf{0}$ & 1 & 0 & -1 & SITE NAME: CLINTON, ILLINOIS \\
\hline 9 & 2215 & $\mathbf{0}$ & 1 & $\mathbf{0}$ & -1 & SITE NAME: GLEN ROSE, TEXAS \\
\hline 9 & 2216 & 0 & 1 & $\mathbf{0}$ & -1 & SITE NAME: EIRIDGMAN, MICHIGAN \\
\hline 9 & 2217 & 0 & 1 & 0 & -1 & SITE NAME: IROWNVILLE, NEBRASKA \\
\hline 9 & 2218 & 0 & 1 & $\mathbf{0}$ & -1 & SITE NAME: IRED LEVEL, FLORIDA \\
\hline 9 & 2219 & 0 & 1 & $\mathbf{0}$ & -1 & SITE NAME: OAK HARBOR, OHIO \\
\hline 9 & 2220 & 0 & 1 & 0 & -1 & SITE NAME: DIABLO CANYON, CAL \\
\hline
\end{tabular}




\begin{tabular}{|c|c|c|c|c|c|c|}
\hline Maior & Minor & Param & Pen & Line & Deciltr & Attribute Descriotion \\
\hline 9 & 2221 & 0 & 1 & $\mathbf{0}$ & -1 & SITE NAME: MORRIS, ILLINOIS \\
\hline 9 & 22222 & $\mathbf{0}$ & 1 & 0 & -1 & SITE NAME: DOTHAN, ALABAMA \\
\hline 9 & 2223 & $\mathbf{0}$ & 1 & 0 & -1 & SITE NAME: NEWPORT, MICHIGAN \\
\hline 9 & 2224 & $\mathbf{0}$ & 1 & $\mathbf{0}$ & -1 & SITE NAME: SCRIBA, NEW YORK \\
\hline 9 & 2225 & $\mathbf{0}$ & 1 & $\mathbf{0}$ & -1 & SITE NAME: FORT CALHOUN, NEBRASKA \\
\hline 9 & 2226 & $\mathbf{0}$ & 1 & $\mathbf{0}$ & -1 & SITE NAME: PLATTVILLE, COLORADO \\
\hline 9 & 2227 & 0 & 1 & 0 & -1 & SITE NAME: ONTARIO, NEW YORK \\
\hline 9 & 2228 & 0 & 1 & 0 & -1 & SITE NAME: PORT GIBSON, MISSISSIPPI \\
\hline 9 & 2229 & $\mathbf{0}$ & 1 & $\mathbf{0}$ & -1 & SITE NAME: HADDAM NECK, CONNECTICUT \\
\hline 9 & 2230 & $\mathbf{0}$ & 1 & 0 & -1 & SITE NAME: BAXILEY, GEORGIA \\
\hline 9 & 2231 & $\mathbf{0}$ & 1 & 0 & -1 & SITE NAME: SALEM, NEW JERSEY \\
\hline 9 & 2232 & 0 & 1 & $\mathbf{0}$ & -1 & SITE NAME: EUREKA, CALIFORNIA \\
\hline 9 & 2233 & 0 & 1 & $\mathbf{0}$ & -1 & SITE NAME: BUCHANAN, NEW YORK \\
\hline 9 & 2234 & 0 & 1 & $\mathbf{0}$ & -1 & SITE NAME: CARLTON, WISCONSIN \\
\hline 9 & 2235 & $\mathbf{0}$ & 1 & $\mathbf{0}$ & -1 & SITE NAME: LA CROSSE, WISCONSIN \\
\hline 9 & 2236 & $\mathbf{0}$ & 1 & $\mathbf{0}$ & -1 & SITE NAME: SENECA, ILLINOIS \\
\hline 9 & 2237 & $\mathbf{0}$ & 1 & $\mathbf{0}$ & -1 & SITE NAME: POTTSTOWN, PENNSYLVANIA \\
\hline 9 & 2238 & $\mathbf{0}$ & 1 & $\mathbf{0}$ & -1 & SITE NAME: WISCASSET, MAIN \\
\hline 9 & 2239 & 0 & 1 & $\mathbf{0}$ & -1 & $\begin{array}{l}\text { SITE NAME: COWANSFORD DAM, NORTH } \\
\text { CAROLINA }\end{array}$ \\
\hline 9 & 2240 & 0 & 1 & $\mathbf{0}$ & -1 & SITE NAME: WATERFORD, CONNECTICUT \\
\hline 9 & 2241 & $\mathbf{0}$ & 1 & $\mathbf{0}$ & -1 & SITE NAME: MONTICELLO, MINNESOTA \\
\hline 9 & 2242 & 0 & 1 & $\mathbf{0}$ & -1 & SITE NAME: SCRIBA, NEW YORK \\
\hline 9 & 2243 & $\mathbf{0}$ & 1 & $\mathbf{0}$ & -1 & SITE NAME: MINERAL, VIRGINIA \\
\hline 9 & 2244 & 0 & 1 & $\mathbf{0}$ & -1 & SITE NAME: SENECA, SOUTH CAROLINA \\
\hline 9 & 2245 & $\mathbf{0}$ & 1 & $\mathbf{0}$ & -1 & SITE NAME: TOMA RIVER, NEW JERSEY \\
\hline 9 & 2246 & $\mathbf{0}$ & 1 & $\mathbf{0}$ & -1 & SITE NAME: SOUTH HAVEN, MICHIGAN \\
\hline 9 & 2247 & $\mathbf{0}$ & 1 & $\mathbf{0}$ & -1 & SITE NAME: WINTERSBURG, ARIZONA \\
\hline 9 & 2248 & 0 & 1 & 0 & -1 & SITE NAME: PEACH BOTTOM, PENNSYLVANIA \\
\hline 9 & 2249 & $\mathbf{0}$ & 1 & $\mathbf{0}$ & -1 & SITE NAME: PERRY, OHIO \\
\hline 9 & 2250 & 0 & 1 & $\mathbf{0}$ & -1 & SITE NAME: PLYMOUTH, MASSACHUSETTS \\
\hline 9 & 2251 & 0 & 1 & 0 & -1 & SITE NAME: TWO CREEKS, WISCONSIN \\
\hline 9 & 2252 & $\mathbf{0}$ & 1 & 0 & -1 & SITE NAME: RED WING, MINNESOTA \\
\hline 9 & 2253 & $\mathbf{0}$ & 1 & $\mathbf{0}$ & -1 & SITE NAME: CORDOVA, ILLINOIS \\
\hline 9 & 2254 & $\mathbf{0}$ & 1 & $\mathbf{0}$ & -1 & SITE NAME: CLAYSTATION, CALIFORNIA \\
\hline 9 & 2255 & $\mathbf{0}$ & 1 & $\mathbf{0}$ & -1 & SITE NAME: ST. FRANCISVILLE, LOUISIANA \\
\hline 9 & 2256 & $\mathbf{0}$ & 1 & $\mathbf{0}$ & -1 & SITE NAME: HARTSVILLE, SOUTH CAROLINA \\
\hline 9 & 2257 & 0 & 1 & $\mathbf{0}$ & -1 & SITE NAME: FT. PIERCE, FLORIDA \\
\hline 9 & 2258 & 0 & 1 & $\mathbf{0}$ & -1 & SITE NAME: SALEM, NEW JERSEY \\
\hline 9 & 2259 & $\mathbf{0}$ & 1 & 0 & -1 & SITE NAME: SAN CLEMENTE, CALIFORNIA \\
\hline 9 & 2260 & $\mathbf{0}$ & 1 & 0 & -1 & SITE NAME: SEABROOK, NEW HAMPSHIRE \\
\hline 9 & 2261 & $\mathbf{0}$ & 1 & 0 & -1 & SITE NAME: DAISY, TENNESSEE \\
\hline 9 & 2262 & $\mathbf{0}$ & 1 & $\mathbf{0}$ & -1 & SITE NAME: BONSAL, NORTH CAROLINA \\
\hline 9 & 2263 & $\mathbf{0}$ & 1 & $\mathbf{0}$ & -1 & SITE NAME: BROOKHAVEN, NEW YORK \\
\hline 9 & 2264 & 0 & 1 & $\mathbf{0}$ & -1 & SITE NAME: MATAGORDA COUNTY, TEXAS \\
\hline 9 & 2265 & 0 & 1 & $\mathbf{0}$ & -1 & $\begin{array}{l}\text { SITE NAME: BROAD RIVER, SOUTH } \\
\text { CAROLINA }\end{array}$ \\
\hline 9 & 2266 & 0 & 1 & $\mathbf{0}$ & -1 & SITE NAME: GRAVEL NECK, VIRGINIA \\
\hline 9 & 2267 & 0 & 1 & 0 & -1 & SITE NAME: BERWICK, PENNSYLVANIA \\
\hline 9 & 2268 & 0 & 1 & 0 & -1 & A \\
\hline
\end{tabular}




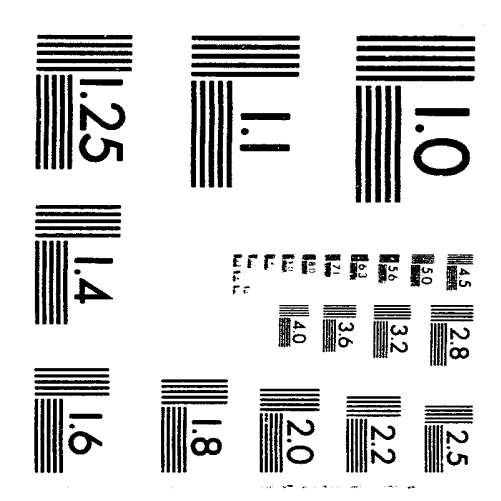



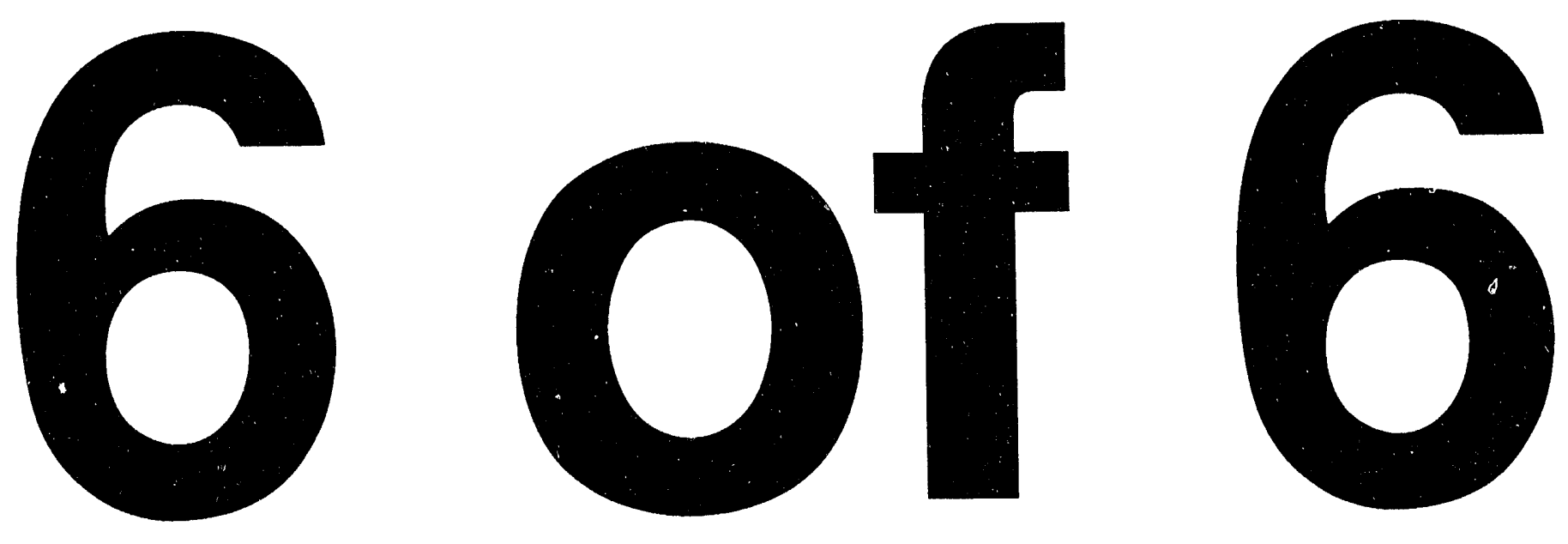


\begin{tabular}{|c|c|c|c|c|c|c|}
\hline Maior & Minor & Param & Pen & Line & Decltr & Attribute Description \\
\hline 9 & 2269 & $\mathbf{0}$ & 1 & 0 & -1 & SITE NAME: PRESCOTT, OREGON \\
\hline 9 & 2270 & $\mathbf{0}$ & 1 & $\mathbf{0}$ & -1 & SITE NAME: FLORIDA CITY, FLORIDA \\
\hline 9 & 2271 & 0 & 1 & 0 & -1 & SITE NAME: VERNON, VERMONT \\
\hline 9 & 2272 & $\mathbf{0}$ & 1 & $\mathbf{0}$ & -1 & SITE NAME: WAYNESBORO, GEORGIA \\
\hline 9 & 2273 & $\mathbf{0}$ & 1 & $\mathbf{0}$ & -1 & SITE NAME: RICHIAND, WASHINGTON \\
\hline 9 & 2274 & $\mathbf{0}$ & 1 & 0 & -1 & SITE NAME: RICHLAND, WASHINGTON \\
\hline 9 & 2275 & 0 & 1 & $\mathbf{0}$ & -1 & SITE NAME: TAFT, LOUISIANA \\
\hline 9 & 2276 & $\mathbf{0}$ & 1 & $\mathbf{0}$ & -1 & SITE NAME: BURLNNGTON, KANSAS \\
\hline 9 & 2277 & $\mathbf{0}$ & 1 & $\mathbf{0}$ & -1 & SITE NAME: ROWE, MASSACHUSETTS \\
\hline 9 & 2278 & $\mathbf{0}$ & 1 & $\mathbf{0}$ & -1 & SITE NAME: ZION, ILLINOIS \\
\hline 9 & 2401 & 0 & 1 & $\mathbf{0}$ & -1 & COUNTY NAME: AUSTIN \\
\hline 9 & 2402 & 0 & 1 & 0 & -1 & COUNTY NAME: POPE \\
\hline 9 & 2403 & 0 & 1 & $\mathbf{0}$ & -1 & COUNTY NAME: LINN \\
\hline 9 & 2404 & $\mathbf{0}$ & 1 & 0 & -1 & COUNTY NAME: BEAVER \\
\hline 9 & 2405 & 0 & 1 & $\mathbf{0}$ & -1 & COUNTY NAME: JACKSON \\
\hline 9 & 2406 & $\mathbf{0}$ & 1 & 0 & -1 & COUNTY NAME: CHARLEVODX \\
\hline 9 & 2407 & 0 & 1 & 0 & -1 & COUNTY NAME: WILL \\
\hline 9 & 2408 & 0 & 1 & $\mathbf{0}$ & -1 & COUNTY NAME: MORGAN \\
\hline 9 & 2409 & 0 & 1 & $\mathbf{0}$ & -1 & COUNTY NAME: BRUNSWICK \\
\hline 9 & 2410 & $\mathbf{0}$ & 1 & 0 & -1 & COUNTY NAME: MACON \\
\hline 9 & 2411 & 0 & 1 & 0 & -1 & COUNTY NAME: CALLAWAY \\
\hline 9 & 2412 & 0 & 1 & 0 & -1 & COUNTY NAME: CALVERT \\
\hline 9 & 2413 & 0 & 1 & 0 & -1 & COUNTY NAME: YORK \\
\hline 9 & 2414 & 0 & 1 & 0 & -1 & COUNTY NAME: DE WITT \\
\hline 9 & 2415 & 0 & 1 & 0 & -1 & COUNTY NAME: SOMERVELL \\
\hline 9 & 2416 & $\mathbf{0}$ & 1 & $\mathbf{0}$ & -1 & COUNTY NAME: BERRIEN \\
\hline 9 & 2417 & $\mathbf{0}$ & 1 & 0 & -1 & COUNTY NAME: NEMAHA \\
\hline 9 & 2418 & 0 & 1 & $\mathbf{0}$ & -1 & COUNTY NAME: CITRUS \\
\hline 9 & 2419 & $\mathbf{0}$ & 1 & 0 & -1 & COUNTY NAME: OTTAWA \\
\hline 9 & 2420 & 0 & 1 & $\mathbf{0}$ & -1 & COUNTY NAME: SAN LUIS OBISPO \\
\hline 9 & 2421 & $\mathbf{0}$ & 1 & $\mathbf{0}$ & -1 & COUNTY NAME: GRUNDY \\
\hline 9 & 2422 & 0 & 1 & $\mathbf{0}$ & -1 & COUNTY NAME: HOUSTON \\
\hline 9 & 2423 & $\mathbf{0}$ & 1 & $\mathbf{0}$ & -1 & COUNTY NAME: MONROE \\
\hline 9 & 2424 & $\mathbf{0}$ & 1 & $\mathbf{0}$ & -1 & COUNTY NAME: OSWEGO \\
\hline 9 & 2425 & 0 & 1 & 0 & -1 & COUNTY NAME: WASHINGTON \\
\hline 9 & 2426 & $\mathbf{0}$ & 1 & 0 & -1 & COUNTY NAME: WELD \\
\hline 9 & 2427 & 0 & 1 & 0 & -1 & COUNTY NAME: WAYNE \\
\hline 9 & 2428 & 0 & 1 & 0 & -1 & COUNTY NAME: CLAIBORNE \\
\hline 9 & 2429 & 0 & 1 & 0 & -1 & COUNIY NAME: MIDDLESEX \\
\hline 9 & 2430 & 0 & 1 & 0 & -1 & COUNTY NAME: APPLING \\
\hline 9 & 2431 & $\mathbf{0}$ & 1 & 0 & -1 & COUNTY NAME: SALEM \\
\hline 9 & 2432 & 0 & 1 & 0 & -1 & COUNTY NAME: HUMBOLDT \\
\hline 9 & 2433 & 0 & 1 & 0 & -1 & COUNTY NAME: WESTCHESTER \\
\hline 9 & 2434 & 0 & 1 & 0 & -1 & COUNTY NAME: KEWAUNEE \\
\hline 9 & 2435 & $\mathbf{0}$ & 1 & 0 & -1 & COUNTY NAME: LA CROSSE \\
\hline 9 & 2436 & 0 & 1 & 0 & -1 & COUNTY NAME: LA SALLE \\
\hline 9 & 2437 & 0 & 1 & 0 & -1 & COUNTY NAME: BERKS \\
\hline 9 & 2438 & 0 & 1 & 0 & -1 & COUNTY NAME: LINCOLN \\
\hline 9 & 2439 & $\mathbf{0}$ & 1 & 0 & -1 & COUNTY NAME: LINCOLN \\
\hline 9 & 2440 & 0 & 1 & 0 & -1 & COUNTY NAME: NEW LONDON \\
\hline
\end{tabular}




\begin{tabular}{|c|c|c|c|c|c|c|}
\hline Maior & Minor & Param & Pen & Line & Decltr & Attribute Description \\
\hline 9 & 2441 & 0 & 1 & 0 & -1 & COUNTY NAME: WRIGHT \\
\hline 9 & 2442 & 0 & 1 & 0 & -1 & COUNTY NAME: OSWEGO \\
\hline 9 & 2443 & 0 & 1 & 0 & -1 & COUNTY NAME: LOUISA \\
\hline 9 & 2444 & 0 & 1 & 0 & -1 & COUNTY NAME: OCONEE \\
\hline 9 & 2445 & 0 & 1 & 0 & -1 & COUNTY NAME: OCEAN \\
\hline 9 & 2446 & 0 & 1 & 0 & -1 & COUNTY NAME: VAN BUREN \\
\hline 9 & 2447 & 0 & 1 & 0 & -1 & COUNTY NAME: MARICOPA \\
\hline 9 & 2448 & 0 & 1 & 0 & -1 & COUNTY NAME: LANCASTER \\
\hline 9 & 2449 & 0 & 1 & 0 & -1 & COUNTY NAME: LAKE \\
\hline 9 & 2450 & 0 & 1 & 0 & -1 & COUNTY NAME: PLYMOUTH \\
\hline 9 & 2451 & 0 & 1 & 0 & -1 & COUNTY NAME: MANITOWOC \\
\hline 9 & 2452 & 0 & 1 & 0 & -1 & COUNTY NAME: GOODHUE \\
\hline 9 & 2453 & 0 & 1 & 0 & -1 & COUNTY NAME: ROCK ISLAND \\
\hline 9 & 2454 & 0 & 1 & 0 & -1 & COUNTY NAME: AMADOR \\
\hline 9 & 2455 & 0 & 1 & 0 & -1 & COUNTY NAME: POINTE COUPEE \\
\hline 9 & 2456 & 0 & 1 & 0 & -1 & COUNTY NAME: DARIINGTON \\
\hline 9 & 2457 & 0 & 1 & 0 & -1 & COUNTY NAME: ST. LUCIE \\
\hline 9 & 2458 & 0 & 1 & 0 & -1 & COUNTY NAME: SALEM \\
\hline 9 & 2459 & 0 & 1 & 0 & -1 & COUNTY NAME: ORANGE \\
\hline 9 & 2460 & 0 & 1 & 0 & -1 & COUNTY NAME: ROCKINGHAM \\
\hline 9 & 2461 & 0 & 1 & 0 & -1 & COUNTY NAME: HAMILTON \\
\hline 9 & 2462 & 0 & 1 & 0 & -1 & COUNTY NAME: WAKE \\
\hline 9 & 2463 & $\mathbf{0}$ & 1 & 0 & -1 & COUNTY NAME: SUFFOLK \\
\hline 9 & 2464 & 0 & 1 & 0 & -1 & COUNTY NAME: MATAGORDA \\
\hline 9 & 2465 & 0 & 1 & 0 & -1 & COUNTY NAME: FAIRFIELD \\
\hline 9 & 2466 & 0 & 1 & 0 & -1 & COUNTY NAME: SURRY \\
\hline 9 & 2467 & $\mathbf{0}$ & 1 & 0 & -1 & COUNTY NAME: COLUMBIA \\
\hline 9 & 2468 & 0 & 1 & 0 & -1 & COUNTY NAME: DAUPHIN \\
\hline 9 & 2469 & 0 & 1 & 0 & -1 & COUNTY NAME: COLUMBIA \\
\hline 9 & 2470 & 0 & 1 & 0 & -1 & COUNTY NAME: DADE \\
\hline 9 & 2471 & 0 & 1 & 0 & -1 & COUNTY NAME: WINDHAM \\
\hline 9 & 2472 & 0 & 1 & 0 & -1 & COUNTY NAME: BURKE \\
\hline 9 & 2473 & 0 & 1 & 0 & -1 & COUNTY NAME: BENTON \\
\hline 9 & 2474 & 0 & 1 & 0 & -1 & COUNTY NAME: BENTON \\
\hline 9 & 2475 & 0 & 1 & 0 & -1 & COUNTY NAME: ST. JOHN THE BAPTIST \\
\hline 9 & 2476 & 0 & 1 & 0 & -1 & COUNTY NAME: COFFEE \\
\hline 9 & 2477 & 0 & 1 & 0 & -1 & COUNTY NAME: FRANKIIN \\
\hline 9 & 2478 & $\mathbf{0}$ & 1 & $\mathbf{0}$ & -1 & COUNTY NAME: LAKE \\
\hline 9 & 2601 & $\mathbf{0}$ & 1 & 0 & -1 & FIPS CODE: 48015 \\
\hline 9 & 2602 & 0 & 1 & $\mathbf{0}$ & -1 & FIPS CODE: 05115 \\
\hline 9 & 2603 & 0 & 1 & $\mathbf{0}$ & -1 & FIPS CODE: 19113 \\
\hline 9 & 2604 & $\mathbf{0}$ & 1 & 0 & -1 & FIPS CODE: 42007 \\
\hline 9 & 2605 & 0 & 1 & $\mathbf{0}$ & -1 & FIPS CODE: 01071 \\
\hline 9 & 2606 & $\mathbf{0}$ & 1 & $\mathbf{0}$ & -1 & FIPS CODE: 26029 \\
\hline 9 & 2607 & $\mathbf{0}$ & 1 & $\mathbf{0}$ & -1 & FIPS CODE: 17197 \\
\hline 9 & 2608 & $\mathbf{0}$ & 1 & $\mathbf{0}$ & -1 & FIPS CODE: 01103 \\
\hline 9 & 2609 & 0 & 1 & $\mathbf{0}$ & -1 & FIPS CODE: 37019 \\
\hline 9 & 2610 & 0 & 1 & 0 & -1 & FIPS CODE: 17141 \\
\hline 9 & 2611 & 0 & 1 & $\mathbf{0}$ & -1 & FIPS CODE: 29027 \\
\hline 9 & 2612 & 0 & 1 & 0 & -1 & FIPS CODE: 24009 \\
\hline
\end{tabular}




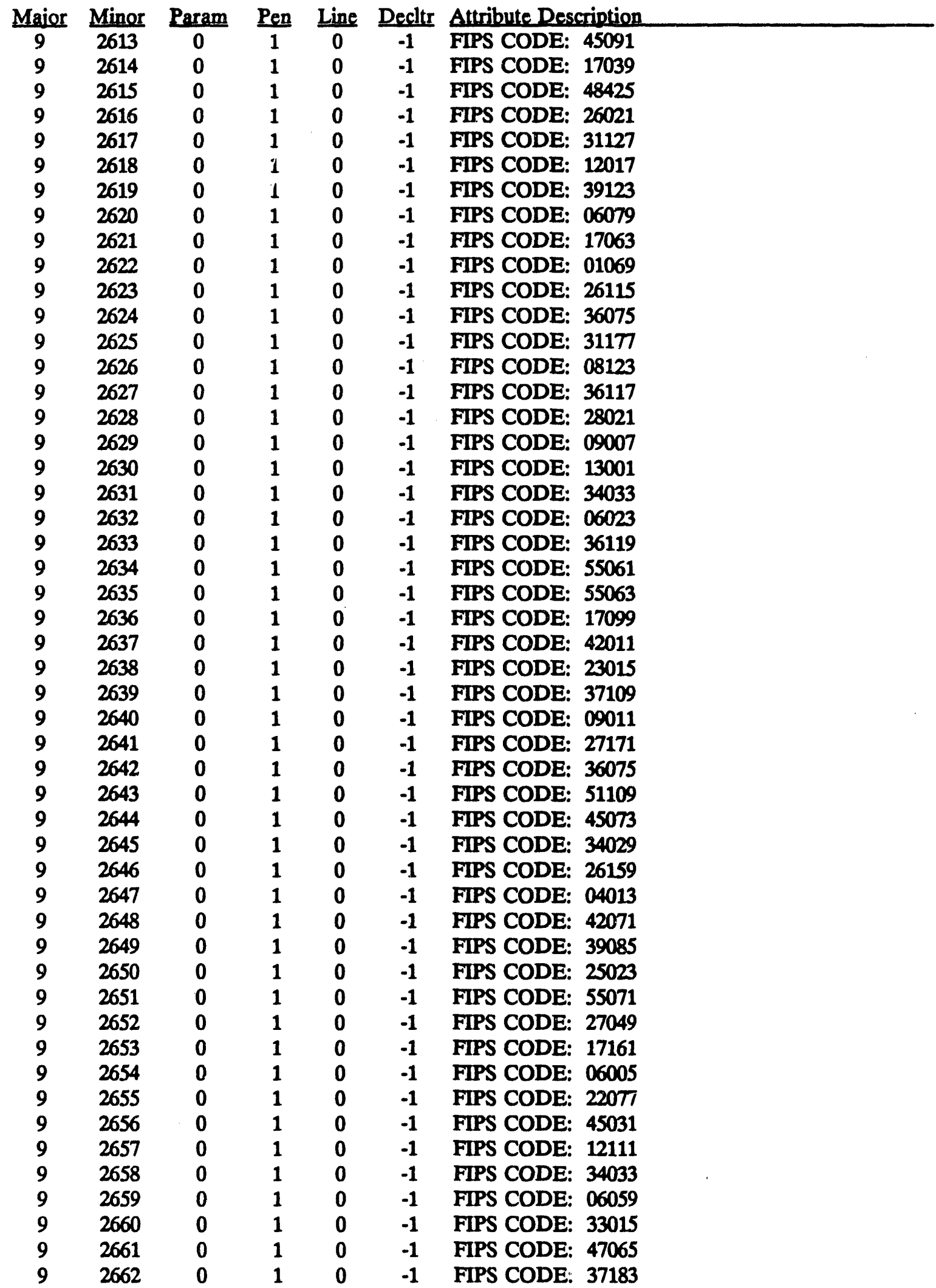




\begin{tabular}{|c|c|c|c|c|c|c|}
\hline Maior & Minor & Param & Pen & Line & Decitr & Attribute Descriotion \\
\hline 9 & 2663 & 0 & 1 & 0 & -1 & FIPS CODE: 36103 \\
\hline 9 & 2664 & $\mathbf{0}$ & 1 & 0 & -1 & FIPS CODE: 48321 \\
\hline 9 & 2665 & 0 & 1 & 0 & -1 & FIPS CODE: 45039 \\
\hline 9 & 2666 & 0 & 1 & $\mathbf{0}$ & -1 & FIPS CODE: 51181 \\
\hline 9 & 2667 & 0 & 1 & $\mathbf{0}$ & -1 & FIPS CODE: 42037 \\
\hline 9 & 2668 & 0 & 1 & 0 & -1 & FIPS CODE: 42043 \\
\hline 9 & 2669 & $\mathbf{0}$ & 1 & 0 & -1 & FIPS CODE: 41009 \\
\hline 9 & 2670 & 0 & 1 & 0 & -1 & FIPS CODE: 12025 \\
\hline 9 & 2671 & $\mathbf{0}$ & 1 & $\mathbf{0}$ & -1 & FIPS CODE: 50025 \\
\hline 9 & 2672 & 0 & 1 & $\mathbf{0}$ & -1 & FIPS CODE: 13033 \\
\hline 9 & 2673 & $\mathbf{0}$ & 1 & 0 & -1 & FIPS CODE: 53005 \\
\hline 9 & 2674 & 0 & 1 & $\mathbf{0}$ & -1 & FIPS CODE: 53005 \\
\hline 9 & 2675 & $\mathbf{0}$ & 1 & 0 & -1 & FIPS CODE: 22095 \\
\hline 9 & 2676 & $\mathbf{0}$ & 1 & 0 & -1 & FIPS CODE: 20031 \\
\hline 9 & 2677 & 0 & 1 & 0 & -1 & FIPS CODE: 25011 \\
\hline 9 & 2678 & 0 & 1 & $\mathbf{0}$ & -1 & FIPS CODE: 17098 \\
\hline 9 & 2801 & $\mathbf{0}$ & 1 & 0 & -1 & UTILITY: HOUSTON LIGHTING \& POWER CO. \\
\hline 9 & 2802 & 0 & 1 & $\mathbf{0}$ & -1 & UTILITY: ARKANSAS POWER \& LIGHT CO. \\
\hline 9 & 2803 & 0 & 1 & 0 & -1 & $\begin{array}{l}\text { UTILITY: IOWA ELECTRIC LIGHT \& POWER } \\
\text { CO. }\end{array}$ \\
\hline 9 & 2804 & 0 & 1 & 0 & -1 & $\begin{array}{l}\text { UTILITY: DUQUESNE LIGHT CO. -OHIO } \\
\text { EDISON }\end{array}$ \\
\hline 9 & 2805 & 0 & 1 & 0 & -1 & UTILITY: TENNESSEE VALLEY AUTHORITY \\
\hline 9 & 2806 & $\mathbf{0}$ & 1 & 0 & -1 & UTILITY: CONSUMERS POWER CO. \\
\hline 9 & 2807 & $\mathbf{0}$ & 1 & 0 & -1 & UTHLTY: COMMONWEALTH EDISON CO. \\
\hline 9 & 2808 & $\mathbf{0}$ & 1 & $\mathbf{0}$ & -1 & UTILITY: TENNESSEE VALLEY AUTHORITY \\
\hline 9 & 2809 & $\mathbf{0}$ & 1 & $\mathbf{0}$ & -1 & UTILITY: CAROLINA POWER \& LIGHT CO. \\
\hline 9 & 2810 & $\mathbf{0}$ & 1 & 0 & -1 & UTILITY: COMMONWEALTH EDISON CO. \\
\hline 9 & 2811 & 0 & 1 & 0 & -1 & UTILITY: UNION ELECTRIC CO. \\
\hline 9 & 2812 & $\mathbf{0}$ & 1 & $\mathbf{0}$ & -1 & UTHLITY: BALTIMORE GAS \& ELECTRIC CO. \\
\hline 9 & 2813 & 0 & 1 & $\mathbf{0}$ & -1 & UTILITY: DUKE POWER CO. \\
\hline 9 & 2814 & $\mathbf{0}$ & 1 & $\mathbf{0}$ & -1 & UTILITY: ILLINOIS POWER CO. \\
\hline 9 & 2815 & 0 & 1 & 0 & -1 & UTILITY: TEXAS UTILITIES SVC., INC. \\
\hline 9 & 2816 & 0 & 1 & $\mathbf{0}$ & -1 & $\begin{array}{l}\text { UTILITY: INDIANA \& MICHIGAN ELECTRIC } \\
\text { CO. }\end{array}$ \\
\hline 9 & 2817 & 0 & 1 & $\mathbf{0}$ & -1 & $\begin{array}{l}\text { UTILTTY: NEBRASKA PUBLIC POWER DIST \& } \\
\text { IOWA P \& L CO. }\end{array}$ \\
\hline 9 & 2818 & 0 & 1 & $\mathbf{0}$ & -1 & UTILITY: FLORIDA POWER CORP. \\
\hline 9 & 2819 & 0 & 1 & 0 & -1 & $\begin{array}{l}\text { UTILITY: TOLEDO EDISON-CLEVELAND EL. } \\
\text { ILLUM CO. }\end{array}$ \\
\hline 9 & 2820 & $\mathbf{0}$ & 1 & $\mathbf{0}$ & -1 & UTILITY: PACIFIC GAS \& ELECTRIC CO. \\
\hline 9 & 2821 & 0 & 1 & $\mathbf{0}$ & -1 & UTHLITY: COMMONWEALTH EDISON CO. \\
\hline 9 & 2822 & 0 & 1 & $\mathbf{0}$ & -1 & UTILTY: ALABAMA POWER CO. \\
\hline 9 & 2823 & 0 & 1 & $\mathbf{0}$ & -1 & UTTLITY: DETROIT EDISON CO. \\
\hline 9 & 2824 & 0 & 1 & 0 & -1 & $\begin{array}{l}\text { UTILITY: POWER AUTHORITY OF THE STATE } \\
\text { OF NEW YORK }\end{array}$ \\
\hline 9 & 2825 & 0 & 1 & $\mathbf{0}$ & -1 & UTHITY: OMAHA PUBLIC POWER DISTRICT \\
\hline 9 & 2826 & $\mathbf{0}$ & 1 & $\mathbf{0}$ & -1 & $\begin{array}{l}\text { UTILITY: PUBLIC SERVICE CO. OF } \\
\text { COLORADO }\end{array}$ \\
\hline 9 & 2827 & $\mathbf{0}$ & 1 & $\mathbf{0}$ & -1 & UTILITY: ROCHESTER GAS \& ELEC. CORP. \\
\hline
\end{tabular}




\begin{tabular}{|c|c|c|c|c|c|c|}
\hline Maior & Minor & Param & Pen & Line & Decltr & Attribute Description \\
\hline 9 & 2828 & 0 & 1 & $\mathbf{0}$ & -1 & UTILITY: MISSISSIPPI POWER \& LIGHT CO. \\
\hline 9 & 2829 & 0 & 1 & 0 & -1 & $\begin{array}{l}\text { UTILITY: CONN. YANKEE ATOMIC POWER } \\
\text { CO. }\end{array}$ \\
\hline 9 & 2830 & $\mathbf{0}$ & 1 & 0 & -1 & UTILITY: GEORGIA POWER CO. \\
\hline 9 & 2831 & $\mathbf{0}$ & 1 & 0 & -1 & $\begin{array}{l}\text { UTILITY: PUBLIC SERVICE ELECTRIC \& GAS, } \\
\text { NEW JERSEY }\end{array}$ \\
\hline 9 & 2832 & $\mathbf{0}$ & 1 & $\mathbf{0}$ & -1 & UTILITY: PACIFIC GAS \& ELECTRIC CO. \\
\hline 9 & 2833 & $\mathbf{0}$ & 1 & $\mathbf{0}$ & -1 & UTILITY: CONSOLIDATED EDISON \\
\hline 9 & 2834 & $\mathbf{0}$ & 1 & $\mathbf{0}$ & -1 & UTILITY: WISCONSIN PUBLIC SVC. CORP. \\
\hline 9 & 2835 & 0 & 1 & $\mathbf{0}$ & -1 & UTILITY: DAIRYLAND POWER COOPERATIVE \\
\hline 9 & 2836 & $\mathbf{0}$ & 1 & $\mathbf{0}$ & -1 & UTILITY: COMMONWEALTH EDISON CO. \\
\hline 9 & 2837 & $\mathbf{0}$ & 1 & $\mathbf{0}$ & -1 & UTILITY: PHILADELPHIA ELECTRIC CO. \\
\hline 9 & 2838 & $\mathbf{0}$ & 1 & 0 & -1 & $\begin{array}{l}\text { UTILITY: MAINE YANKEE ATOMIC POWER } \\
\text { CO. }\end{array}$ \\
\hline 9 & 2839 & $\mathbf{0}$ & 1 & $\mathbf{0}$ & -1 & UTILITY: DUKE POWER CO. \\
\hline 9 & 2840 & $\mathbf{0}$ & 1 & $\mathbf{0}$ & -1 & $\begin{array}{l}\text { UTILITY: NORTHEAST NUCLEAR ENERGY } \\
\text { CO. }\end{array}$ \\
\hline 9 & 2841 & 0 & 1 & $\mathbf{0}$ & -1 & UTILTTY: NORTHERN STATES POWER CO. \\
\hline 9 & 2842 & $\mathbf{0}$ & 1 & 0 & -1 & UTILTY: NIAGARA MOHAWK POWER CORP. \\
\hline 9 & 2843 & $\mathbf{0}$ & 1 & $\mathbf{0}$ & -1 & UTILITY: VIRGINIA ELEC. \& POWER CORP. \\
\hline 9 & 2844 & $\mathbf{0}$ & 1 & $\mathbf{0}$ & -1 & UTILTY: DUKE POWER CO. \\
\hline 9 & 2845 & $\mathbf{0}$ & 1 & $\mathbf{0}$ & -1 & $\begin{array}{l}\text { UTILTYY: JERSEY CENTRAL POWER \& LIGHT } \\
\text { CO. }\end{array}$ \\
\hline 9 & 2846 & $\mathbf{0}$ & 1 & 0 & -1 & UTHITY: CONSUMERS POWER CO. \\
\hline 9 & 2847 & $\mathbf{0}$ & 1 & $\mathbf{0}$ & -1 & UTILITY: ARIZONA PUBLIC SERVICE \\
\hline 9 & 2848 & $\mathbf{0}$ & 1 & $\mathbf{0}$ & -1 & UTILITY: PHILADELPHIA ELECTRIC CO. \\
\hline 9 & 2849 & $\mathbf{0}$ & 1 & $\mathbf{0}$ & -1 & UTILITY: CLEVELAND ELEC. ILLUM. CO. \\
\hline 9 & 2850 & $\mathbf{0}$ & 1 & $\mathbf{0}$ & -1 & UTILTYY: BOSTON EDISON CO. \\
\hline 9 & 2851 & $\mathbf{0}$ & 1 & $\mathbf{0}$ & -1 & UTILITY: WISCONSIN-MICHIGAN POWER CO. \\
\hline 9 & 2852 & 0 & 1 & 0 & -1 & UTILTY: NORTHERN STATES POWER CO. \\
\hline 9 & 2853 & $\mathbf{0}$ & 1 & 0 & -1 & $\begin{array}{l}\text { UTILITY: COMM. ED. CO.-IA. -ILL. GAS \& } \\
\text { ELEC. CO. }\end{array}$ \\
\hline 9 & 2854 & 0 & 1 & $\mathbf{0}$ & -1 & $\begin{array}{l}\text { UTILITY: SACRAMENTO MUNICIPAL UTILITY } \\
\text { DISTRICT }\end{array}$ \\
\hline 9 & 2855 & $\mathbf{0}$ & 1 & 0 & -1 & UTILTTY: GULF STATES UTILITIES CO. \\
\hline 9 & 2856 & $\mathbf{0}$ & 1 & $\mathbf{0}$ & -1 & UTILTYY: CAROLINA POWER \& LIGHT CO. \\
\hline 9 & 2857 & $\mathbf{0}$ & 1 & $\mathbf{0}$ & -1 & UTILITY: FLORIDA POWER \& LIGHT CO. \\
\hline 9 & 2858 & 0 & 1 & $\mathbf{0}$ & -1 & $\begin{array}{l}\text { UTILITY: PUBLIC SVC. ELECTRIC \& GAS, NEW } \\
\text { JERSEY }\end{array}$ \\
\hline 9 & 2859 & 0 & 1 & $\mathbf{0}$ & -1 & $\begin{array}{l}\text { UTILITY: SO. CALIF. ED. \& SAN DIEGO GAS \& } \\
\text { ELECT CO. }\end{array}$ \\
\hline 9 & 2860 & $\mathbf{0}$ & 1 & 0 & -1 & $\begin{array}{l}\text { UTILITY: PUBLIC SERVICE OF NEW } \\
\text { HAMPSHIRE }\end{array}$ \\
\hline 9 & 2861 & $\mathbf{0}$ & 1 & $\mathbf{0}$ & -1 & UTILITY: TENNESSEE VALLEY AUTHORITY \\
\hline 9 & 2862 & $\mathbf{0}$ & 1 & $\mathbf{0}$ & -1 & UTILITY: CAROLINA POWER \& LIGHT CO. \\
\hline 9 & 2863 & $\mathbf{0}$ & 1 & 0 & -1 & UTILITY: LONG ISLAND LIGHTING CO. \\
\hline 9 & 2864 & $\mathbf{0}$ & 1 & 0 & -1 & $\begin{array}{l}\text { UTILITY: CENTRAL POWR \& LT. -HOUSTON } \\
\text { LT. \& POWER }\end{array}$ \\
\hline 9 & 2865 & 0 & 1 & 0 & -1 & UTILITY: SOUTH CAROLINA ELEC. \& GAS CO. \\
\hline 9 & 2866 & $\mathbf{0}$ & 1 & 0 & -1 & UTILITY: VIRGINIA ELE \\
\hline
\end{tabular}




\begin{tabular}{|c|c|c|c|c|c|c|}
\hline Maior & Minor & Param & Pen & Line & Decltr & Attribute Description \\
\hline 9 & 2867 & 0 & 1 & $\mathbf{0}$ & -1 & UTILITY: PENNSYLVANIA POWER \& LIGHT \\
\hline 9 & 2868 & 0 & 1 & $\mathbf{0}$ & -1 & UTHITY: METROPOLITAN EDISON CO. \\
\hline 9 & 2869 & 0 & 1 & 0 & -1 & $\begin{array}{l}\text { UTILITY: PORTLAND GENERAL ELECTRIC } \\
\text { CO. }\end{array}$ \\
\hline 9 & 2870 & 0 & 1 & 0 & -1 & UTIUTTY: FLORIDA POWER \& LIGHT CO. \\
\hline 9 & 2871 & 0 & 1 & 0 & -1 & $\begin{array}{l}\text { UTULTY: VERMONT YANKEE NUCLEAR } \\
\text { POWER CORP. }\end{array}$ \\
\hline 9 & 2872 & 0 & 1 & 0 & -1 & UTILITY: GEORGIA POWER CO. \\
\hline 9 & 2873 & 0 & 1 & 0 & -1 & UTILITY: DEPARTMENT OF ENERGY \\
\hline 9 & 2874 & 0 & 1 & 0 & -1 & $\begin{array}{l}\text { UTHLITY: WASH. PUBLIC. POWER SUPPLY } \\
\text { SYSTEMS }\end{array}$ \\
\hline 9 & 2875 & 0 & 1 & $\mathbf{0}$ & -1 & UTILITY: LOUISIANA POWER \& LIGHT CO. \\
\hline 9 & 2876 & $\mathbf{0}$ & 1 & $\mathbf{0}$ & -1 & UTILITY: KANSAS GAS \& ELEC. -KC P\&L \\
\hline 9 & 2877 & 0 & 1 & 0 & -1 & UTILITY: YANKEE ATOMIC ELECTRIC CO. \\
\hline 9 & 2878 & 0 & 1 & $\mathbf{0}$ & -1 & UTILTTY: COMMONWEALTH EDISON CO. \\
\hline 9 & 3001 & 0 & 1 & 0 & -1 & WEEKDAY POPULATION: FEMALE \\
\hline 9 & 3002 & 0 & 1 & 0 & -1 & WEEKDAY POPULATION: MALE \\
\hline 9 & 3003 & 0 & 1 & 0 & -1 & EVENING POPULATION: FEMALE \\
\hline 9 & 3004 & 0 & 1 & 0 & -1 & EVENING POPULATION: MALE \\
\hline 9 & 3005 & 0 & 1 & $\mathbf{0}$ & -1 & NIGHT POPULATION: FEMALE \\
\hline 9 & 3006 & $\mathbf{0}$ & 1 & $\mathbf{0}$ & -1 & NIGHT POPULATION: MALE \\
\hline 9 & 3010 & $\mathbf{0}$ & 1 & $\mathbf{0}$ & -1 & POPULATION 0.5 YRS: TOTAL \\
\hline 9 & 3011 & 0 & 1 & $\mathbf{0}$ & -1 & POPULATION 0-5 YRS: TOTAL FEMALE \\
\hline 9 & 3012 & $\mathbf{0}$ & 1 & $\mathbf{0}$ & -1 & POPULATION 0.5 YRS: CAUCASIAN FEMALE \\
\hline 9 & 3013 & 0 & 1 & $\mathbf{0}$ & -1 & $\begin{array}{l}\text { POPULATION 0-5 YRS: NON-CAUCASIAN } \\
\text { FEMALE }\end{array}$ \\
\hline 9 & 3021 & $\mathbf{0}$ & 1 & $\mathbf{0}$ & -1 & POPULATION 0-5 YRS: TOTAL MALE \\
\hline 9 & 3022 & 0 & 1 & $\mathbf{0}$ & -1 & POPULATION 0.5 YRS: CAUCASIAN MALE \\
\hline 9 & 3023 & $\mathbf{0}$ & 1 & $\mathbf{0}$ & -1 & $\begin{array}{l}\text { POPULATION 0.5 YRS: NON-CAUCASIAN } \\
\text { MALE }\end{array}$ \\
\hline 9 & 3030 & $\mathbf{0}$ & 1 & $\mathbf{0}$ & -1 & POPULATION 6-19 YRS: TOTAL \\
\hline 9 & 3031 & 0 & 1 & $\mathbf{0}$ & -1 & POPULATION 6-19 YRS: TOTAL FEMALE \\
\hline 9 & 3032 & $\mathbf{0}$ & 1 & 0 & -1 & POPULATION 6-19 YRS: CAUCASIAN FEMALE \\
\hline 9 & 3033 & $\mathbf{0}$ & 1 & 0 & -1 & $\begin{array}{l}\text { POPULATION 6-19 YRS: NON-CAUCASIAN } \\
\text { FEMALE }\end{array}$ \\
\hline 9 & 3041 & 0 & 1 & $\mathbf{0}$ & -1 & POPULATION 6-19 YRS: TOTAL MALE \\
\hline 9 & 3042 & 0 & 1 & $\mathbf{0}$ & -1 & POPULATION 6-19 YRS: CAUCASIAN MALE \\
\hline 9 & 3043 & 0 & 1 & 0 & -1 & $\begin{array}{l}\text { POPULATION 6-19 YRS: NON-CAUCASIAN } \\
\text { MALE }\end{array}$ \\
\hline 9 & 3050 & 0 & 1 & 0 & -1 & POPULATION 20-64 YRS: TOTAL \\
\hline 9 & 3051 & $\mathbf{0}$ & 1 & $\mathbf{0}$ & -1 & POPULATION 20-64 YRS: TOTAL FEMALE \\
\hline 9 & 3052 & 0 & 1 & 0 & -1 & POPULATION 20-64 YRS: CAUCASIAN FEMALE \\
\hline 9 & 3053 & 0 & 1 & $\mathbf{0}$ & -1 & $\begin{array}{l}\text { POPULATION 20-64 YRS: NON-CAUCASION } \\
\text { FEMALE }\end{array}$ \\
\hline 9 & 3061 & $\mathbf{0}$ & 1 & $\mathbf{0}$ & -1 & POPULATION $20-64$ YRS: TOTAL MALE \\
\hline 9 & 3062 & 0 & 1 & $\mathbf{0}$ & -1 & POPULATION 20-64 YRS: CAUCASIAN MALE \\
\hline 9 & 3063 & 0 & 1 & 0 & -1 & $\begin{array}{l}\text { POPULATION } 20-64 \text { YRS: NON-CAUCASIAN } \\
\text { MALE }\end{array}$ \\
\hline 9 & 3070 & 0 & $\mathbf{1}$ & $\mathbf{0}$ & -1 & POPULATION 65 YRS OR OLDER: TOTAL \\
\hline
\end{tabular}




\begin{tabular}{|c|c|c|c|c|c|c|}
\hline Maior & Minor & Param & Pen & Line & Decltr & Attribute Description \\
\hline 9 & 3071 & 0 & 1 & 0 & -1 & $\begin{array}{l}\text { POPULATION } 65 \text { YRS OR OLDER: TOTAL } \\
\text { FEMALE }\end{array}$ \\
\hline 9 & 3072 & 0 & 1 & 0 & -1 & $\begin{array}{l}\text { POPULATION } 65 \text { YRS OR OLDER: CAUCASIAN } \\
\text { FEMALE }\end{array}$ \\
\hline 9 & 3073 & 0 & 1 & 0 & -1 & $\begin{array}{l}\text { POPULATION } 65 \text { YRS OR OLDER: } \\
\text { NON-CAUCASIAN FEMALE }\end{array}$ \\
\hline 9 & 3081 & $\mathbf{0}$ & 1 & 0 & -1 & $\begin{array}{l}\text { POPULATION } 65 \text { YRS OR OLDER: TOTAL } \\
\text { MALE }\end{array}$ \\
\hline 9 & 3082 & 0 & 1 & 0 & -1 & $\begin{array}{l}\text { POPULATION } 65 \text { YRS OR OLDER: CAUCASIAN } \\
\text { MALE }\end{array}$ \\
\hline 9 & 3083 & 0 & 1 & $\mathbf{0}$ & -1 & $\begin{array}{l}\text { POPULATION } 65 \text { YRS OR OLDER: } \\
\text { NON-CAUCASIAN MALE }\end{array}$ \\
\hline 9 & 3101 & 0 & 1 & 0 & -1 & AGE: UNDER 1 YEAR \\
\hline 9 & 3102 & 0 & 1 & 0 & -1 & AGE: 1 AND 2 YEARS \\
\hline 9 & 3103 & $\mathbf{0}$ & 1 & $\mathbf{0}$ & -1 & AGE: 3 AND 4 YEARS \\
\hline 9 & 3104 & 0 & 1 & $\mathbf{0}$ & -1 & AGE: 5 YEARS \\
\hline 9 & 3105 & 0 & 1 & 0 & -1 & AGE: 6 YEARS \\
\hline 9 & 3106 & $\mathbf{0}$ & 1 & $\mathbf{0}$ & -1 & AGE: 7 TO 9 YEARS \\
\hline 9 & 3107 & $\mathbf{0}$ & 1 & $\mathbf{0}$ & -1 & 10 AND 11 YEARS \\
\hline 9 & 3108 & $\mathbf{0}$ & 1 & $\mathbf{0}$ & -1 & AGE: 12 AND 13 YEARS \\
\hline 9 & 3109 & $\mathbf{0}$ & 1 & $\mathbf{0}$ & -1 & AGE: 14 YEARS \\
\hline 9 & 3110 & 0 & 1 & $\mathbf{0}$ & -1 & AGE: 15 YEARS \\
\hline 9 & 3111 & 0 & 1 & 0 & -1 & AGE: 16 YEARS \\
\hline 9 & 3112 & 0 & 1 & $\mathbf{0}$ & -1 & AGE: 17 YEARS \\
\hline 9 & 3113 & 0 & 1 & $\mathbf{0}$ & -1 & AGE: 18 YEARS \\
\hline 9 & 3114 & 0 & 1 & $\mathbf{0}$ & -1 & AGE: 19 YEARS \\
\hline 9 & 3115 & $\mathbf{0}$ & 1 & $\mathbf{0}$ & -1 & AGE: 20 YEARS \\
\hline 9 & 3116 & 0 & 1 & $\mathbf{0}$ & -1 & AGE: 21 YEARS \\
\hline 9 & 3117 & 0 & 1 & 0 & -1 & AGE: 22 TO 24 YEARS \\
\hline 9 & 3118 & 0 & 1 & $\mathbf{0}$ & -1 & AGE: 25 TO 29 YEARS \\
\hline 9 & 3119 & $\mathbf{0}$ & 1 & $\mathbf{0}$ & -1 & AGE: 30 TO 34 YEARS \\
\hline 9 & 3120 & $\mathbf{0}$ & 1 & $\mathbf{0}$ & -1 & AGE: 35 TO 39 YEARS \\
\hline 9 & 3121 & 0 & 1 & 0 & -1 & AGE: 31 TO 44 YEARS \\
\hline 9 & 3122 & $\mathbf{0}$ & 1 & 0 & -1 & AGE: 45 TO 49 YEARS \\
\hline 9 & 3123 & 0 & 1 & 0 & -1 & AGE: 50 TO 54 YEARS \\
\hline 9 & 3124 & 0 & 1 & $\mathbf{0}$ & -1 & $\begin{array}{l}\text { AGE: } 55 \text { TO } 59 \text { YEARS } \\
\text { AGE: } 60 \text { AND } 61 \text { YEARS }\end{array}$ \\
\hline 9 & 3125 & 0 & 1 & 0 & -1 & $\begin{array}{l}\text { AGE: } 60 \text { AND } 61 \text { YEARS } \\
\text { AGE: } 62 \text { T0 } 64 \text { YEARS }\end{array}$ \\
\hline 9 & 3126 & 0 & 1 & 0 & -1 & $\begin{array}{l}\text { AGE: } 62 \text { T0 } 64 \text { YEARS } \\
\text { AGE: } 65 \text { TO } 69 \text { YEARS }\end{array}$ \\
\hline $\begin{array}{l}9 \\
0\end{array}$ & $\begin{array}{l}3127 \\
3128\end{array}$ & $\begin{array}{l}0 \\
0\end{array}$ & 1 & 0 & $\begin{array}{l}-1 \\
-1\end{array}$ & $\begin{array}{l}\text { AGE: } 651069 \text { YEARS } \\
\text { AGE: } 70 \text { TO } 74 \text { YEARS }\end{array}$ \\
\hline $\begin{array}{l}9 \\
9\end{array}$ & 3129 & 0 & 1 & 0 & -1 & AGE: 75 TO 79 YEARS \\
\hline 9 & 3130 & 0 & 1 & 0 & -1 & AGE: 80 TO 84 YEARS \\
\hline 9 & 3131 & $\mathbf{0}$ & 1 & $\mathbf{0}$ & -1 & AGE: 85 YEARS AND OVER \\
\hline 9 & 3141 & 0 & 1 & 0 & -1 & $\begin{array}{l}\text { AGE OF HOUSEHOLD MEMBERS, } 1 \text { OR MORE } \\
\text { OVER 65: } 1 \text { PERSON }\end{array}$ \\
\hline 9 & 3142 & 0 & 1 & $\mathbf{0}$ & -1 & $\begin{array}{l}\text { AGE OF HOUSEHOLD MEMBERS, } 65 \text { OR OVER: } \\
\text { >2, FAMILY HOUSEHOLD }\end{array}$ \\
\hline 9 & 3143 & $\mathbf{0}$ & 1 & 0 & -1 & $\begin{array}{l}\text { AGE OF MEMBERS, } 65 \text { OR OVER: }>2 \text { NON } \\
\text { FAMILY HOUSEHOLDS }\end{array}$ \\
\hline
\end{tabular}




\begin{tabular}{|c|c|c|c|c|c|c|}
\hline Maior & Minor & Param & Pen & Line & Decltr & Attribute Description \\
\hline 9 & $\overline{3144}$ & 0 & 1 & $\mathbf{0}$ & -1 & $\begin{array}{l}\text { AGE OF MEMBERS, NONE } 65 \text { OR OVER: } 1 \\
\text { PERSON }\end{array}$ \\
\hline 9 & 3145 & 0 & 1 & 0 & -1 & $\begin{array}{l}\text { AGE OF MEMBERS, NONE 65: >1, FAMILY } \\
\text { HOUSEHOLD }\end{array}$ \\
\hline 9 & 3146 & 0 & 1 & $\mathbf{0}$ & -1 & $\begin{array}{l}\text { AGE OF MEMBERS, NONE } 65:>1 \text {, NONFAMILY } \\
\text { HOUSEHOLD }\end{array}$ \\
\hline 9 & 3151 & 0 & 1 & 0 & -1 & PERSONS BY RACE: WHITE \\
\hline 9 & 3152 & 0 & 1 & $\mathbf{0}$ & -1 & PERSONS BY RACE: BLACK \\
\hline 9 & 3153 & 0 & 1 & 0 & -1 & $\begin{array}{l}\text { PERSONS BY RACE: AMERICAN INDIAN, } \\
\text { ESKIMO, OR ALEUT }\end{array}$ \\
\hline 9 & 3154 & 0 & 1 & 0 & -1 & $\begin{array}{l}\text { PERSONS BY RACE: ASIAN OR PACIFIC } \\
\text { ISLANDER }\end{array}$ \\
\hline 9 & 3155 & 0 & 1 & 0 & -1 & PERSONS BY RACE: OTHER \\
\hline 9 & 3156 & 0 & 1 & 0 & -1 & PERSONS OF HISPANIC ORIGIN \\
\hline 9 & 3160 & 0 & 1 & 0 & -1 & PERSONS BY SEX: MALE \\
\hline 9 & 3170 & 0 & 1 & 0 & -1 & PERSONS BY SEX: FEMALE \\
\hline 9 & 3200 & $\mathbf{0}$ & 1 & 0 & -1 & TOTAL FAMILIES \\
\hline 9 & 3201 & 0 & 1 & $\mathbf{0}$ & -1 & HOUSEHOLD TYPE AND SIZE - FAMILY,2 \\
\hline 9 & 3202 & 0 & 1 & 0 & -1 & HOUSEHOLD TYPE AND SIZE - FAMILY,3 \\
\hline 9 & 3203 & 0 & 1 & 0 & -1 & HOUSEHOLD TYPE AND SIZE - FAMILY,4 \\
\hline 9 & 3204 & 0 & 1 & 0 & -1 & HOUSEHOLD TYPE AND SIZE - FAMILY,5 \\
\hline 9 & 3205 & 0 & 1 & 0 & -1 & HOUSEHOLD TYPE AND SIZE - FAMILY,6 \\
\hline 9 & 3206 & 0 & 1 & 0 & -1 & $\begin{array}{l}\text { HOUSEHOLD TYPE AND SIZE - FAMILY,7 OR } \\
\text { MORE }\end{array}$ \\
\hline 9 & 3210 & 0 & 1 & $\mathbf{0}$ & -1 & TOTAL HOUSEHOLDS \\
\hline 9 & 3211 & $\mathbf{0}$ & 1 & $\mathbf{0}$ & -1 & HOUSEHOLD TYPE AND SIZE - NONFAMILY,1 \\
\hline 9 & 3212 & $\mathbf{0}$ & 1 & 0 & -1 & HOUSEHOLD TYPE AND SIZE - NONFAMILY,2 \\
\hline 9 & 3213 & 0 & 1 & $\mathbf{0}$ & -1 & HOUSEHOLD TYPE AND SIZE - NONFAMILY,3 \\
\hline 9 & 3214 & 0 & 1 & 0 & -1 & HOUSEHOLD TYPE AND SIZE - NONFAMILY,4 \\
\hline 9 & 3215 & 0 & 1 & $\mathbf{0}$ & -1 & HOUSEHOLD TYPE AND SIZE - NONFAMILY,5 \\
\hline 9 & 3216 & $\mathbf{0}$ & 1 & 0 & -1 & HOUSEHOLD TYPE AND SIZE - NONFAMILY,6 \\
\hline 9 & 3217 & 0 & 1 & 0 & -1 & $\begin{array}{l}\text { HOUSEHOLD TYPE AND SIZE - NONFAMILY,7 } \\
\text { OR MORE }\end{array}$ \\
\hline 9 & 3221 & 0 & 1 & 0 & -1 & PERSONS IN HOUSEHOLD: HOUSEHOLDER \\
\hline 9 & 3222 & $\mathbf{0}$ & 1 & 0 & -1 & PERSONS IN HOUSEHOLD: SPOUSE \\
\hline 9 & 3223 & $\mathbf{0}$ & 1 & $\mathbf{0}$ & -1 & $\begin{array}{l}\text { PERSONS IN HOUSEHOLD: CHILD, NATURAL } \\
\text { BORN OR ADOPTED }\end{array}$ \\
\hline 9 & 3224 & 0 & 1 & $\mathbf{0}$ & -1 & PERSONS IN HOUSEHOLD: CHILD, STEP \\
\hline 9 & 3225 & $\mathbf{0}$ & 1 & $\mathbf{0}$ & -1 & $\begin{array}{l}\text { PERSONS IN HOUSEHOLD: CHILD, } \\
\text { GRANDCHILD }\end{array}$ \\
\hline 9 & 3226 & 0 & 1 & 0 & -1 & $\begin{array}{l}\text { PERSONS IN HOUSEHOLD: CHILD, OTHER } \\
\text { RELATIVE }\end{array}$ \\
\hline 9 & 3227 & 0 & 1 & 0 & -1 & $\begin{array}{l}\text { PERSONS IN HOUSEHOLD: CHILD, } \\
\text { NONRELATIVES }\end{array}$ \\
\hline 9 & 3231 & 0 & 1 & $\mathbf{0}$ & -1 & $\begin{array}{l}\text { NON FAMILY HOUSEHOLD: HOUSEHOLDER } \\
\text { LIVING ALONE }\end{array}$ \\
\hline 9 & 3232 & 0 & 1 & $\mathbf{0}$ & -1 & $\begin{array}{l}\text { NON FAMILY HOUSEHOLD: HOUSEHOLDER } \\
\text { NOT LIVING ALONE }\end{array}$ \\
\hline 9 & 3233 & 0 & 1 & $\mathbf{0}$ & -1 & NON FAMILY HOUSEHOLD: NONRELATIVES \\
\hline
\end{tabular}




\begin{tabular}{|c|c|c|c|c|c|c|}
\hline Maior & Minor & Param & Pen & Line & Decltr & Attribute Description \\
\hline 9 & 3300 & $\mathbf{0}$ & 1 & 0 & -1 & $\begin{array}{l}\text { PERSONS IN HOUSEHOLD, GROUP } \\
\text { QUARTERS: INSTITUTIONALIZED }\end{array}$ \\
\hline 9 & 3301 & 0 & 1 & 0 & -1 & $\begin{array}{l}\text { GROUP } \\
\text { QUARTERS;INSTITUTIONS,CORRECTIONAL } \\
\text { INSTITUTIONS }\end{array}$ \\
\hline 9 & 3302 & $\mathbf{0}$ & 1 & 0 & -1 & $\begin{array}{l}\text { GROUP QUARTERS; INSTITUTIONS, NURSING } \\
\text { HOMES }\end{array}$ \\
\hline 9 & 3303 & 0 & 1 & $\mathbf{0}$ & -1 & $\begin{array}{l}\text { GROUP QUARTERS; INSTITUTIONS, MENTAL } \\
\text { HOSPITALS }\end{array}$ \\
\hline 9 & 3304 & $\mathbf{0}$ & 1 & $\mathbf{0}$ & -1 & GROUP QUARTERS; DISTITUTIONS, JUVENILE \\
\hline 9 & 3305 & 0 & 1 & 0 & -1 & GROUP QUARTERS; INSTITUTIONS, OTHERS \\
\hline 9 & 3310 & $\mathbf{0}$ & 1 & 0 & -1 & $\begin{array}{l}\text { PERSONS IN HOUSEHOLD, GROUP } \\
\text { QUARTERS: OTHERS }\end{array}$ \\
\hline 9 & 3311 & 0 & 1 & 0 & -1 & $\begin{array}{l}\text { GROUP QUARTERS; OTHER, COLLEGE } \\
\text { DORMTORIES }\end{array}$ \\
\hline 9 & 3312 & $\mathbf{0}$ & 1 & 0 & -1 & $\begin{array}{l}\text { GROUP QUARTERS; OTHER, MILITARY } \\
\text { QUARTERS }\end{array}$ \\
\hline 9 & 3313 & 0 & 1 & 0 & -1 & $\begin{array}{l}\text { GROUP QUARTERS; OTHER, EMERGENCY } \\
\text { SHELTERS FOR HOMELESS }\end{array}$ \\
\hline 9 & 3314 & $\mathbf{0}$ & 1 & 0 & -1 & $\begin{array}{l}\text { GROUP QUARTERS; OTHER, VISIBLE IN } \\
\text { STREET LOCATIONS }\end{array}$ \\
\hline 9 & 3315 & $\mathbf{0}$ & 1 & 0 & -1 & $\begin{array}{l}\text { GROUP QUARTERS; OTHER, OTHER } \\
\text { NONINSTITUTIONAL }\end{array}$ \\
\hline 9 & 3600 & $\mathbf{0}$ & 1 & $\mathbf{0}$ & -1 & TOTAL HOUSING UNITS \\
\hline 9 & 3611 & 0 & 1 & 0 & -1 & HOUSING OCCUPANCY STATUS; OCCUPIED \\
\hline 9 & 3612 & $\mathbf{0}$ & 1 & $\mathbf{0}$ & -1 & HOUSING OCCUPANCY STATUS; VACANT \\
\hline 9 & 3615 & $\mathbf{0}$ & 1 & $\mathbf{0}$ & -1 & OCCUPIED HOUSING TENURE; OWNER \\
\hline 9 & 3616 & $\mathbf{0}$ & 1 & $\mathbf{0}$ & -1 & OCCUPIED HOUSING TENURE; RENTER \\
\hline 9 & 3621 & $\mathbf{0}$ & 1 & 0 & -1 & $\begin{array}{l}\text { NUMBER OF HOUSING UNITS INSIDE } \\
\text { URBANIZED AREA }\end{array}$ \\
\hline 9 & 3622 & $\mathbf{0}$ & 1 & $\mathbf{0}$ & -1 & $\begin{array}{l}\text { NUMBER OF HOUSING UNITS OUTSIDE } \\
\text { URBANIZED AREA }\end{array}$ \\
\hline 9 & 3623 & $\mathbf{0}$ & 1 & $\mathbf{0}$ & -1 & NUMBER OF RURAL HOUSING UNITS \\
\hline 9 & 3624 & $\mathbf{0}$ & 1 & $\mathbf{0}$ & -1 & $\begin{array}{l}\text { NUMBER OF HOUSING UNITS NOT DEFINED } \\
\text { IN URBAN OR RURAL AREAS }\end{array}$ \\
\hline 9 & 3631 & $\mathbf{0}$ & 1 & $\mathbf{0}$ & -1 & VACANT HOUSING UNITS FOR RENT \\
\hline 9 & 3632 & $\mathbf{0}$ & 1 & $\mathbf{0}$ & -1 & VACANT HOUSING UNITS FOR SALE \\
\hline 9 & 3633 & 0 & 1 & $\mathbf{0}$ & -1 & $\begin{array}{l}\text { VACANT HOUSING UNITS RENTED OR SOLD, } \\
\text { NOT OCCUPIED }\end{array}$ \\
\hline 9 & 3634 & $\mathbf{0}$ & 1 & $\mathbf{0}$ & -1 & $\begin{array}{l}\text { VACANT HOUSING UNITS FOR } \\
\text { SEASONAL,RECR., OR OCCASIONAL USE }\end{array}$ \\
\hline 9 & 3635 & 0 & 1 & $\mathbf{0}$ & -1 & VACANT HOUSING FOR MIGRANT WORKERS \\
\hline 9 & 3636 & $\mathbf{0}$ & 1 & $\mathbf{0}$ & -1 & VACANT HOUSING UNITS, OTHER VACANT \\
\hline 9 & 3641 & 0 & 1 & $\mathbf{0}$ & -1 & $\begin{array}{l}\text { NUMBER OF UNITS IN HOUSING UNIT } \\
\text { STRUCTURE - 1,DETACHED }\end{array}$ \\
\hline 9 & 3642 & 0 & 1 & 0 & -1 & $\begin{array}{l}\text { NUMBER OF UNITS IN HOUSING UNIT } \\
\text { STRUCTURE - } 1 \text { ATTACHED }\end{array}$ \\
\hline 9 & 3643 & 0 & 1 & 0 & -1 & $\begin{array}{l}\text { NUMBER OF UNITS IN HOUSING UNIT } \\
\text { STRUCTURE - } 2\end{array}$ \\
\hline
\end{tabular}




\begin{tabular}{|c|c|c|c|c|c|c|}
\hline Maior & Minor & Param & Pen & Line & Declit & Attribute Description \\
\hline 9 & 3644 & 0 & 1 & $\mathbf{0}$ & -1 & $\begin{array}{l}\text { NUMBER OF UNITS IN HOUSING STRUCTURE } \\
\text { UNIT - } 3 \text { OR } 4\end{array}$ \\
\hline 9 & 3645 & 0 & 1 & 0 & -1 & $\begin{array}{l}\text { NUMBER OF UNITS IN HOUSING UNIT } \\
\text { STRUCTURE - } 5 \text { TO } 9\end{array}$ \\
\hline 9 & 3646 & 0 & 1 & 0 & -1 & $\begin{array}{l}\text { NUMBER OF UNITS IN HOUSING UNIT } \\
\text { STRUCTURE - } 10 \text { TO } 19\end{array}$ \\
\hline 9 & 3647 & 0 & 1 & 0 & -1 & $\begin{array}{l}\text { NUMBER OF UNITS IN HOUSING UNIT } \\
\text { STRUCTURE - } 20 \text { TO } 49\end{array}$ \\
\hline 9 & 3648 & 0 & 1 & 0 & -1 & $\begin{array}{l}\text { NUMBER OF UNITS IN HOUSING UNIT } \\
\text { STRUCTURE - } 50 \text { OR MORE }\end{array}$ \\
\hline 9 & 3649 & 0 & 1 & 0 & -1 & $\begin{array}{l}\text { NUMBER OF UNITS IN HOUSING UNIT } \\
\text { STRUCTURE - MOBILE HOME }\end{array}$ \\
\hline 9 & 3650 & 0 & 1 & $\mathbf{0}$ & -1 & $\begin{array}{l}\text { NUMBER OF UNITS IN HOUSING UNIT } \\
\text { STRUCTURE - OTHER }\end{array}$ \\
\hline 9 & 3660 & $\mathbf{0}$ & 1 & $\mathbf{0}$ & -1 & $\begin{array}{l}\text { NUMBER OF AGGREGATE ROOMS IN EACH } \\
\text { HOUSING UNIT; TOT } \$ \perp \perp\end{array}$ \\
\hline 9 & 3671 & 0 & 1 & $\mathbf{0}$ & -1 & BOARDED UP VACANT HOUSING UNITS \\
\hline 9 & 3672 & $\mathbf{0}$ & 1 & $\mathbf{0}$ & -1 & VACANT HOUSING UNITS NOT BOARDED UP \\
\hline 9 & 3700 & $\mathbf{0}$ & 1 & $\mathbf{0}$ & -1 & $\begin{array}{l}\text { AGGREGATE VALUE OF OWNER OCCUPIED } \\
\text { HOUSING UNITS; TOTAL }\end{array}$ \\
\hline 9 & 3701 & 0 & 1 & $\mathbf{0}$ & -1 & $\begin{array}{l}\text { OWNER OCCUPIED HOUSING VALUE, LESS } \\
\text { THAN } \$ 15,000\end{array}$ \\
\hline 9 & 3702 & 0 & 1 & $\mathbf{0}$ & -1 & $\begin{array}{l}\text { OWNER OCCUPIED HOUSING VALUE (\$) } 15,000 \\
\text { TO } 19,999\end{array}$ \\
\hline 9 & 3703 & $\mathbf{0}$ & 1 & $\mathbf{0}$ & -1 & $\begin{array}{l}\text { OWNER OCCUPIED HOUSING VALUE (\$) } 20,000 \\
\text { TO } 24,999\end{array}$ \\
\hline 9 & 3704 & $\mathbf{0}$ & 1 & $\mathbf{0}$ & -1 & $\begin{array}{l}\text { OWNER OCCUPIED HOUSING VALUE (\$) } 25,000 \\
\text { TO } 29,999\end{array}$ \\
\hline 9 & 3705 & 0 & 1 & 0 & -1 & $\begin{array}{l}\text { OWNER OCCUPIED HOUSING VALUE (\$) } 30,000 \\
\text { TO } 34,999\end{array}$ \\
\hline 9 & 3706 & 0 & 1 & 0 & -1 & $\begin{array}{l}\text { OWNER OCCUPIED HOUSING VALUE (\$) } 35,000 \\
\text { TO } 39,999\end{array}$ \\
\hline 9 & 3707 & 0 & 1 & 0 & -1 & $\begin{array}{l}\text { OWNER OCCUPIED HOUSING VALUE (\$) } 40,000 \\
\text { TO } 44,999\end{array}$ \\
\hline 9 & 3708 & 0 & 1 & 0 & -1 & $\begin{array}{l}\text { OWNER OCCUPIED HOUSING VALUE ( } \$ \text { ) } 45,000 \\
\text { TO } 49,999\end{array}$ \\
\hline 9 & 3709 & 0 & 1 & 0 & -1 & $\begin{array}{l}\text { OWNER OCCUPIED HOUSING VALUE (\$) } 50,000 \\
\text { TO } 59,999\end{array}$ \\
\hline 9 & 3710 & 0 & 1 & 0 & -1 & $\begin{array}{l}\text { OWNER OCCUPIED HOUSING VALUE ( } \$ 60,000 \\
\text { TO } 74,999\end{array}$ \\
\hline 9 & 3711 & 0 & 1 & 0 & -1 & $\begin{array}{l}\text { OWNER OCCUPIED HOUSING VALUE (\$) } 57,000 \\
\text { TO } 99,999\end{array}$ \\
\hline 9 & 3712 & 0 & 1 & 0 & -1 & $\begin{array}{l}\text { OWNER OCCUPIED HOUSING VALUE (\$) } \\
100,000 \text { TO } 124,999\end{array}$ \\
\hline 9 & 3713 & 0 & 1 & 0 & -1 & $\begin{array}{l}\text { OWNER OCCUPIED HOUSING VALUE (\$) } \\
125,000 \text { TO } 149,999\end{array}$ \\
\hline 9 & 3714 & 0 & 1 & 0 & -1 & $\begin{array}{l}\text { OWNER OCCUPIED HOUSING VALUE (\$) } \\
150,000 \text { TO } 174,999\end{array}$ \\
\hline 9 & 3715 & 0 & 1 & 0 & -1 & $\begin{array}{l}\text { OWNER OCCUPIED HOUSING VALUE (\$) } \\
175,000 \text { TO } 199,999\end{array}$ \\
\hline
\end{tabular}




\begin{tabular}{|c|c|c|c|c|c|}
\hline Minor & Param & Pen & Line & Decltr & Attribute Description \\
\hline 3716 & 0 & $\overline{1}$ & 0 & -1 & $\begin{array}{l}\text { OWNER OCCUPIED HOUSING VALUE (\$) } \\
200,000 \text { TO } 249,999\end{array}$ \\
\hline 3717 & $\mathbf{0}$ & 1 & 0 & -1 & $\begin{array}{l}\text { OWNER OCCUPIED HOUSING VALUE (\$) } \\
250,000 \text { TO } 299,999\end{array}$ \\
\hline 3718 & 0 & 1 & 0 & -1 & $\begin{array}{l}\text { OWNER OCCUPIED HOUSING VALUE (\$) } \\
300,000 \text { TO } 399,999\end{array}$ \\
\hline 3719 & 0 & 1 & 0 & -1 & $\begin{array}{l}\text { OWNER OCCUPIED HOUSING VALUE (\$) } \\
400,000 \text { TO } 499,999\end{array}$ \\
\hline 3720 & 0 & 1 & 0 & -1 & $\begin{array}{l}\text { OWNER OCCUPIED HOUSING VALUE (\$) } \\
500,000 \text { OR MORE }\end{array}$ \\
\hline 3730 & 0 & 1 & $\mathbf{0}$ & -1 & $\begin{array}{l}\text { AGGREGATE CONTRACT RENT FOR RENTER } \\
\text { OCCUPIED HOUSING UNTTS }\end{array}$ \\
\hline 3750 & 0 & 1 & 0 & -1 & $\begin{array}{l}\text { AGGREGA'TE PRICE ASKED FOR } \\
\text { VACANT-FOR-SALE-ONLY HOUSING UNITS }\end{array}$ \\
\hline 3760 & 0 & 1 & 0 & -1 & $\begin{array}{l}\text { AGGREGATE RENT ASKED IN } \\
\text { VACANT-FOR-RENT HOUSING UNITS; TOTAL }\end{array}$ \\
\hline 3800 & $\mathbf{0}$ & 1 & 0 & -1 & D2 RELEASE TYPE (REL) \\
\hline 3801 & 0 & 1 & 0 & -1 & D2 METHOD OF ASSESSMENT (IMA) \\
\hline 3802 & 0 & 1 & 0 & -1 & D2 NUMBER OF TRACKS FOR CASE \\
\hline 3803 & 0 & 1 & 0 & -1 & $\begin{array}{l}\text { D2 DOSAGE OR CONCENTRATION OF } \\
\text { INTEREST }\end{array}$ \\
\hline 3805 & 0 & 1 & $\mathbf{0}$ & -1 & D2 AGENT CODE (AGN) \\
\hline 3810 & 0 & 1 & 0 & -1 & D2 SEASON (SEA) \\
\hline 3815 & $\mathbf{0}$ & 1 & $\mathbf{0}$ & -1 & D2 STABLITY (STB) \\
\hline 3820 & 0 & 1 & $\mathbf{0}$ & -1 & D2 MUNITION (MÚN) \\
\hline 3825 & $\mathbf{0}$ & 1 & 0 & -1 & D2 NUMBER OF MUNITIONS (NMU) \\
\hline 3830 & 0 & 1 & $\mathbf{0}$ & -1 & D2 WIND DIRECTION \\
\hline 3835 & 0 & 1 & 0 & -1 & D2 WIND SPEED (WND) \\
\hline 3840 & 0 & 1 & $\mathbf{0}$ & -1 & D2 TEMPERATURE (TMP) \\
\hline 3841 & 0 & 1 & 0 & -1 & D2 ONPOST OR OFFPOST \\
\hline 3842 & 0 & 1 & 0 & -1 & D2 TOTAL DIST DIV 10000 \\
\hline 3843 & 0 & 1 & 0 & -1 & D2 TOTAL DIST \\
\hline 3844 & 0 & 1 & $\mathbf{0}$ & -1 & D2 CURRENT TRACK DIST DIV 10000 \\
\hline 3845 & 0 & 1 & 0 & -1 & D2 CURRENT TRACK DIST \\
\hline
\end{tabular}




\begin{tabular}{|c|c|c|c|c|c|c|}
\hline Major & Minor & Param & Pen & Line & Decitr & Attribute Description \\
\hline 10 & 0 & 0 & $\overline{0}$ & 0 & -1 & TEXT STRINGS 1 \\
\hline 11 & $\mathbf{0}$ & 0 & 1 & $\mathbf{0}$ & -1 & PLACE OF WORK DATA \\
\hline 11 & 10 & 0 & $\mathbf{0}$ & 0 & -1 & AGRICULTURAL PRODUCTION, CROPS \\
\hline 11 & 11 & 0 & 0 & 0 & -1 & AGRICULTURAL PRODUCTION, LIVESTOCK \\
\hline 11 & 20 & $\mathbf{0}$ & 0 & 0 & -1 & $\begin{array}{l}\text { AGRICULTURAL SERVICES, EXCEPT } \\
\text { HORTICULTURAL }\end{array}$ \\
\hline 11 & 21 & 0 & $\mathbf{0}$ & 0 & -1 & HORTICULTURAL SERVICES \\
\hline 11 & 30 & $\mathbf{0}$ & $\mathbf{0}$ & 0 & -1 & FORESTRY \\
\hline 11 & 31 & 0 & $\mathbf{0}$ & 0 & -1 & FISHING, HUNTING, \& TRAPPING \\
\hline 11 & 40 & 0 & 0 & 0 & -1 & METAL MINING \\
\hline 11 & 41 & $\mathbf{0}$ & $\mathbf{0}$ & $\mathbf{0}$ & -1 & COAL MINING \\
\hline 11 & 42 & 0 & $\mathbf{0}$ & 0 & -1 & $\begin{array}{l}\text { CRUDE PETROLEUM \& NATURAL GAS } \\
\text { EXTRACTION }\end{array}$ \\
\hline 11 & 50 & 0 & 0 & 0 & -1 & $\begin{array}{l}\text { NONMETALLIC MINING \& QUARRYING, } \\
\text { EXCEPT FUEL }\end{array}$ \\
\hline 11 & 60 & $\mathbf{0}$ & 0 & 0 & -1 & CONSTRUCTION \\
\hline 11 & 100 & $\mathbf{0}$ & 0 & 0 & -1 & MEAT PRODUCTS \\
\hline 11 & 101 & $\mathbf{0}$ & $\mathbf{0}$ & 0 & -1 & DAIRY PRODUCTS \\
\hline 11 & 102 & 0 & 0 & 0 & -1 & $\begin{array}{l}\text { CANNED \& PRESERVED FRUITS \& } \\
\text { VEGETABLES }\end{array}$ \\
\hline 11 & 110 & 0 & $\mathbf{0}$ & $\mathbf{0}$ & -1 & GRAIN MILL PRODUCTS \\
\hline 11 & 111 & 0 & 0 & 0 & -1 & BAKERY PRODUCTS \\
\hline 11 & 112 & $\mathbf{0}$ & 0 & 0 & -1 & SUGAR \& CONFECTIONERY PRODUCTS \\
\hline 11 & 120 & $\mathbf{0}$ & $\mathbf{0}$ & $\mathbf{0}$ & -1 & BEVERAGE INDUSTRIES \\
\hline 11 & 121 & $\mathbf{0}$ & $\mathbf{0}$ & 0 & -1 & $\begin{array}{l}\text { MISCELLANEOUS FOOD PREPARATIONS \& } \\
\text { KINDRED PRODUCTS }\end{array}$ \\
\hline 11 & 122 & $\mathbf{0}$ & 0 & $\mathbf{0}$ & -1 & NOT SPECIFIED FOOD INDUSTRIES \\
\hline 11 & 130 & $\mathbf{0}$ & $\mathbf{0}$ & $\mathbf{0}$ & -1 & TOBACCO MANUFACTURES \\
\hline 11 & 132 & $\mathbf{0}$ & 0 & 0 & -1 & KNITTING MILLS \\
\hline 11 & 140 & 0 & 0 & 0 & -1 & $\begin{array}{l}\text { DYEING \& FINISHING TEXTILES, EXCEPT } \\
\text { WOOL \& KNIT GOODS }\end{array}$ \\
\hline 11 & 141 & $\mathbf{0}$ & $\mathbf{0}$ & $\mathbf{0}$ & -1 & FLOOR COVERINGS, EXCEPT HARD SURFACE \\
\hline 11 & 142 & $\mathbf{0}$ & $\mathbf{0}$ & $\mathbf{0}$ & -1 & YARN, THREAD, \& FABRIC MILLS \\
\hline 11 & 150 & $\mathbf{0}$ & $\mathbf{0}$ & $\mathbf{0}$ & -1 & MISCELLANEOUS TEXTILE MILL PRODUCTS \\
\hline 11 & 151 & $\mathbf{0}$ & $\mathbf{0}$ & $\mathbf{0}$ & -1 & APPAREL \& ACCESSORIES, EXCEPT KNIT \\
\hline 11 & 152 & 0 & $\mathbf{0}$ & 0 & -1 & $\begin{array}{l}\text { MISCELLANEOUS FABRICATED TEXTILE } \\
\text { PRODUCTS }\end{array}$ \\
\hline 11 & 160 & 0 & $\mathbf{0}$ & $\mathbf{0}$ & -1 & PULP, PAPER, \& PAPERBOARD MILLS \\
\hline 11 & 161 & 0 & $\mathbf{0}$ & 0 & -1 & MISCELLANEOUS PAPER \& PULP PRODUCTS \\
\hline 11 & 162 & $\mathbf{0}$ & $\mathbf{0}$ & 0 & -1 & PAPERBOARD CONTAINERS \& BOXES \\
\hline 11 & 171 & 0 & 0 & $\mathbf{0}$ & -1 & NEWSPAPER PIURLISHING \& PRINTING \\
\hline 11 & 172 & 0 & 0 & 0 & -1 & $\begin{array}{l}\text { PRINTING, PUBLISHING, \& ALLIED } \\
\text { INDUSTRIES, EXCEPT NEWSPAPERS }\end{array}$ \\
\hline 11 & 180 & 0 & $\mathbf{0}$ & $\mathbf{0}$ & -1 & PLASTICS, SYNTHETICS, \& RESINS \\
\hline 11 & 181 & 0 & $\mathbf{0}$ & 0 & -1 & DRUGS \\
\hline 11 & 182 & 0 & $\mathbf{0}$ & $\mathbf{0}$ & -1 & SOAPS \& COSMETICS \\
\hline 11 & 190 & 0 & 0 & $\mathbf{0}$ & -1 & PAINTS, VARNISHES, \& RELATED PRODUCTS \\
\hline 11 & 191 & 0 & $\mathbf{0}$ & 0 & -1 & AGRICULTURAL CHEMICALS \\
\hline 11 & 192 & 0 & $\mathbf{0}$ & 0 & -1 & INDUSTRIAL \& MISCELLANEOUS CHEMICALS \\
\hline
\end{tabular}




\begin{tabular}{|c|c|c|c|c|c|c|}
\hline Major & Minor & Param & Pen & Line & Decltr & Attribute Description \\
\hline 11 & $\overline{200}$ & 0 & $\overline{0}$ & $\mathbf{0}$ & -1 & PETROLEUM REFINING \\
\hline 11 & 201 & $\mathbf{0}$ & $\mathbf{0}$ & $\mathbf{0}$ & -1 & $\begin{array}{l}\text { MISCELLANEOUS PETROLEUM \& COAL } \\
\text { PRODUCTS }\end{array}$ \\
\hline 11 & 210 & $\mathbf{0}$ & 0 & 0 & -1 & TIRES \& INNER TUBES \\
\hline 11 & 211 & 0 & 0 & $\mathbf{0}$ & -1 & $\begin{array}{l}\text { OTHER RUBBER PRODUCTS, \& PLASTICS } \\
\text { FOOTWEAR \& BELTING }\end{array}$ \\
\hline 11 & 212 & 0 & $\mathbf{0}$ & $\mathbf{0}$ & -1 & MISCELLANEOUS PLASTICS PRODUCTS \\
\hline 11 & 220 & 0 & 0 & $\mathbf{0}$ & -1 & LEATHER TANNING \& FINISHING \\
\hline 11 & 221 & $\mathbf{0}$ & $\mathbf{0}$ & $\mathbf{0}$ & -1 & FOOTWEAR, EXCEPT RUBBER \& PLASTIC \\
\hline 11 & 222 & $\mathbf{0}$ & 0 & 0 & -1 & LEATHER PRODUCTS, EXCEPT FOOTWEAR \\
\hline 11 & 230 & $\mathbf{0}$ & $\mathbf{0}$ & 0 & -1 & LOGGING \\
\hline 11 & 231 & $\mathbf{0}$ & $\mathbf{0}$ & $\mathbf{0}$ & -1 & SAWMILLS, PLANING MILLS, \& MILLWORK \\
\hline 11 & 232 & $\mathbf{0}$ & $\mathbf{0}$ & $\mathbf{0}$ & -1 & WOOD BUILDINGS \& MOBILE HOMES \\
\hline 11 & 241 & $\mathbf{0}$ & $\mathbf{0}$ & 0 & -1 & MISCELLANEOUS WOOD PRODUCTS \\
\hline 11 & 242 & $\mathbf{0}$ & $\mathbf{0}$ & $\mathbf{0}$ & -1 & FURNITURE \& FIXTURES \\
\hline 11 & 250 & $\mathbf{0}$ & $\mathbf{0}$ & 0 & -1 & GLASS \& GLASS PRODUCTS \\
\hline 11 & 251 & $\mathbf{0}$ & $\mathbf{0}$ & $\mathbf{0}$ & -1 & $\begin{array}{l}\text { CEMENT, CONCRETE, GYPSUM, \& PLASTER } \\
\text { PRODUCTS }\end{array}$ \\
\hline 11 & 252 & 0 & $\mathbf{0}$ & $\mathbf{0}$ & -1 & STRUCTURAL CLAY PRODUCTS \\
\hline 11 & 261 & $\mathbf{0}$ & $\mathbf{0}$ & $\mathbf{0}$ & -1 & POTTERY \& RELATED PRODUCTS \\
\hline 11 & 262 & $\mathbf{0}$ & 0 & $\mathbf{0}$ & -1 & $\begin{array}{l}\text { MISCELLANEOUS NONMETALLIC MINERAL \& } \\
\text { STONE PRODUCTS }\end{array}$ \\
\hline 11 & 270 & $\mathbf{0}$ & $\mathbf{0}$ & $\mathbf{0}$ & -1 & $\begin{array}{l}\text { BLAST FURNACES, STEELWORKS, ROLLING \& } \\
\text { FINISHING MILLS }\end{array}$ \\
\hline 11 & 271 & 0 & $\mathbf{0}$ & $\mathbf{0}$ & -1 & IRON \& STEEL FOUNDRIES \\
\hline 11 & 272 & 0 & 0 & $\mathbf{0}$ & -1 & PRIMARY ALUMINUM INDUSTRIES \\
\hline 11 & 280 & 0 & 0 & 0 & -1 & OTHER PRIMARY METAL INDUSTRIES \\
\hline 11 & 281 & 0 & $\mathbf{0}$ & 0 & -1 & $\begin{array}{l}\text { CUTLERY, HAND TOOLS, \& OTHER } \\
\text { HARDWARE }\end{array}$ \\
\hline 11 & 282 & 0 & $\mathbf{0}$ & 0 & -1 & $\begin{array}{l}\text { FABRICATED STRUCTURAL METAL } \\
\text { PRODUCTS }\end{array}$ \\
\hline 11 & 290 & $\mathbf{0}$ & $\mathbf{0}$ & 0 & -1 & SCREW MACHINE PRODUCTS \\
\hline 11 & 291 & $\mathbf{0}$ & $\mathbf{0}$ & $\mathbf{0}$ & -1 & METAL FORGINGS \& STAMPINGS \\
\hline 11 & 292 & $\mathbf{0}$ & $\mathbf{0}$ & $\mathbf{0}$ & -1 & ORDNANCE \\
\hline 11 & 300 & $\mathbf{0}$ & 0 & $\mathbf{0}$ & -1 & $\begin{array}{l}\text { MISCELLANEOUS FABRICATED METAL } \\
\text { PRODUCTS }\end{array}$ \\
\hline 11 & 301 & $\mathbf{0}$ & $\mathbf{0}$ & $\mathbf{0}$ & -1 & NOT SPECIFIED METAL INDUSTRIES \\
\hline 11 & 310 & $\mathbf{0}$ & 0 & 0 & -1 & ENGINES \& TURBINES \\
\hline 11 & 311 & 0 & 0 & $\mathbf{0}$ & -1 & FARM MACHINERY \& EQUIPMENT \\
\hline 11 & 312 & $\mathbf{0}$ & 0 & 0 & -1 & $\begin{array}{l}\text { CONSTRUCTION \& MATERIAL HANDLING } \\
\text { MACHINES }\end{array}$ \\
\hline 11 & 320 & 0 & $\mathbf{0}$ & 0 & -1 & METALWORKING MACHINERY \\
\hline 11 & 321 & $\mathbf{0}$ & $\mathbf{0}$ & $\mathbf{0}$ & -1 & OFFICE \& ACCOUNTING MACHINES \\
\hline 11 & 322 & $\mathbf{0}$ & $\mathbf{0}$ & $\mathbf{0}$ & -1 & ELECTRONIC COMPUTING EQUIPMENT \\
\hline 11 & 331 & 0 & 0 & $\mathbf{0}$ & -1 & MACHINERY, EXCEPT ELECTRICAL, N.E.C. \\
\hline 11 & 332 & $\mathbf{0}$ & $\mathbf{0}$ & $\mathbf{0}$ & -1 & NOT SPECIFIED MACHINERY \\
\hline 11 & 340 & 0 & 0 & $\mathbf{0}$ & -1 & HOUSEHOLD APPLIANCES \\
\hline 11 & 341 & $\mathbf{0}$ & 0 & $\mathbf{0}$ & -1 & RADIO, TV, \& COMMUNICATION EQUIPMENT \\
\hline 11 & 342 & $\mathbf{0}$ & 0 & 0 & -1 & $\begin{array}{l}\text { ELECTRICAL MACHINERY, EQUIPMENT, \& } \\
\text { SUPPLIES, N.E.C. }\end{array}$ \\
\hline
\end{tabular}




\begin{tabular}{|c|c|c|c|c|c|c|}
\hline Maior & Minor & Param & Pen & Line & Decltr & Attribute Description \\
\hline 11 & 350 & 0 & 0 & 0 & -1 & $\begin{array}{l}\text { NOT SPECIFIED ELECTRICAL MACHINERY, } \\
\text { EQUIPMENT, \& SUPPLIES }\end{array}$ \\
\hline 11 & 351 & 0 & $\mathbf{0}$ & $\mathbf{0}$ & -1 & $\begin{array}{l}\text { MOTOR VEHICLES \& MOTOR VEHICLE } \\
\text { EQUIPMENT }\end{array}$ \\
\hline 11 & 352 & $\mathbf{0}$ & $\mathbf{0}$ & $\mathbf{0}$ & -1 & AIRCRAFT \& PARTS \\
\hline 11 & 360 & 0 & 0 & $\mathbf{0}$ & -1 & SHIP \& BOAT BUILDING \& REPAIRING \\
\hline 11 & 361 & 0 & $\mathbf{0}$ & 0 & -1 & RAILROAD LOCOMOTIVES \& EQUIPMENT \\
\hline 11 & 362 & 0 & $\mathbf{0}$ & $\mathbf{0}$ & -1 & GUIDED MISSILES, SPACE VEHICLES \& PARTS \\
\hline 11 & 370 & 0 & 0 & $\mathbf{0}$ & -1 & $\begin{array}{l}\text { CYCLES \& MISCELLANEOUS } \\
\text { TRANSPORTATION EQUIPMENT }\end{array}$ \\
\hline 11 & 371 & 0 & 0 & 0 & -1 & SCIENTIFIC \& CONTROLLING INSTRUMENTS \\
\hline 11 & 372 & 0 & 0 & $\mathbf{0}$ & -1 & OPTICAL \& HEALTH SERVICES SUPPLIES \\
\hline 11 & 380 & 0 & $\mathbf{0}$ & 0 & -1 & PHOTOGRAPHIC EQUIPMENT \& SUPPLIES \\
\hline 11 & 381 & 0 & $\mathbf{0}$ & $\mathbf{0}$ & -1 & $\begin{array}{l}\text { WATCHES, CLOCKS, \& CLOCKWORK } \\
\text { OPERATED DEVICES }\end{array}$ \\
\hline 11 & 382 & 0 & $\mathbf{0}$ & 0 & -1 & NOT SPECIFIED PROFESSIONAL EQUIPMENT \\
\hline 11 & 390 & 0 & $\mathbf{0}$ & $\mathbf{0}$ & -1 & TOYS, AMUSEMENT, \& SPORTING GOODS \\
\hline 11 & 391 & 0 & $\mathbf{0}$ & 0 & -1 & $\begin{array}{l}\text { MISCELLANEOUS MANUFACTURING } \\
\text { INDUSTRIES }\end{array}$ \\
\hline 11 & 392 & 0 & 0 & $\mathbf{0}$ & -1 & $\begin{array}{l}\text { NOT SPECIFIED MANUFACTURING } \\
\text { INDUSTRIES }\end{array}$ \\
\hline 11 & 400 & 0 & $\mathbf{0}$ & $\mathbf{0}$ & -1 & RAILROADS \\
\hline 11 & 401 & 0 & 0 & 0 & -1 & BUS SERVICE \& URBAN TRANSIT \\
\hline 11 & 402 & $\mathbf{0}$ & $\mathbf{0}$ & $\mathbf{0}$ & -1 & TAXICAB SERVICE \\
\hline 11 & 410 & 0 & $\mathbf{0}$ & $\mathbf{0}$ & -1 & TRUCKING SERVICE \\
\hline 11 & 411 & $\mathbf{0}$ & $\mathbf{0}$ & 0 & -1 & WAREHOUSING \& STORAGE \\
\hline 11 & 412 & 0 & $\mathbf{0}$ & 0 & -1 & U.S. POSTAL SERVICE \\
\hline 11 & 420 & $\mathbf{0}$ & $\mathbf{0}$ & $\mathbf{0}$ & -1 & WATER TRANSPORTATION \\
\hline 11 & 421 & $\mathbf{0}$ & 0 & $\mathbf{0}$ & -1 & AIR TRANSPORTATION \\
\hline 11 & 422 & $\mathbf{0}$ & $\mathbf{0}$ & 0 & -1 & PIPE LINES, EXCEPT NATURAL GAS \\
\hline 11 & 432 & $\mathbf{0}$ & $\mathbf{0}$ & $\mathbf{0}$ & -1 & SERVICES INCIDENTAL TO TRANSPORTATION \\
\hline 11 & 440 & 0 & 0 & 0 & -1 & RADIO \& TELEVISION BROADCASTING \\
\hline 11 & 441 & 0 & $\mathbf{0}$ & 0 & -1 & TELEPHONE (WIRE \& RADIO) \\
\hline 11 & 442 & 0 & 0 & 0 & -1 & $\begin{array}{l}\text { TELEGRAPH \& MISCELLANEOUS } \\
\text { COMMUNICATION SERVICES }\end{array}$ \\
\hline 11 & 460 & $\mathbf{0}$ & $\mathbf{0}$ & 0 & -1 & ELECTRIC LIGHT \& POWER \\
\hline 11 & 461 & $\mathbf{0}$ & $\mathbf{0}$ & $\mathbf{0}$ & -1 & GAS \& STEAM SUPPLY SYSTEMS \\
\hline 11 & 462 & $\mathbf{0}$ & $\mathbf{0}$ & $\mathbf{0}$ & -1 & ELECTRIC \& GAS, \& OTHER COMBINATIONS \\
\hline 11 & 470 & 0 & $\mathbf{0}$ & $\mathbf{0}$ & -1 & WATER SUPPLY \& IRRIGATION \\
\hline 11 & 471 & $\mathbf{0}$ & $\mathbf{0}$ & 0 & -1 & SANITARY SERVICES \\
\hline 11 & 472 & 0 & 0 & $\mathbf{0}$ & -1 & NOT SPECIFIED UTHLTIES \\
\hline 11 & 500 & 0 & 0 & 0 & -1 & MOTOR VEHICLES \& EQUIPMENT \\
\hline 11 & 501 & $\mathbf{0}$ & $\mathbf{0}$ & $\mathbf{0}$ & -1 & FURNITURE \& HOME FURNISHINGS \\
\hline 11 & 502 & 0 & 0 & 0 & -1 & LUMBER \& CONSTRUCTION MATERIALS \\
\hline 11 & 510 & 0 & 0 & 0 & -1 & SPORTING GOODS, TOYS, \& HOBBY GOODS \\
\hline 11 & 511 & 0 & 0 & 0 & -1 & METALS \& MINERALS, EXCEPT PETROLEUM \\
\hline 11 & 512 & 0 & $\mathbf{0}$ & $\mathbf{0}$ & -1 & ELECTRICAL GOODS \\
\hline 11 & 521 & $\mathbf{0}$ & $\mathbf{0}$ & 0 & -1 & $\begin{array}{l}\text { HARDWARE, PLUMBING \& HEATING } \\
\text { SUPPLIES }\end{array}$ \\
\hline
\end{tabular}




\begin{tabular}{|c|c|c|c|c|c|c|}
\hline Major & Minor & Param & Pen & Line & Decltr & Attribute Description \\
\hline 11 & 522 & $\mathbf{0}$ & 0 & $\mathbf{0}$ & -1 & $\begin{array}{l}\text { NOT SPECIFIED ELECTRICAL \& HARDWARE } \\
\text { PRODUCTS }\end{array}$ \\
\hline 11 & 530 & $\mathbf{0}$ & $\mathbf{0}$ & $\mathbf{0}$ & -1 & MACHINERY, EQUIPMENT, \& SUPPLIES \\
\hline 11 & 531 & $\mathbf{0}$ & 0 & $\mathbf{0}$ & -1 & SCRAP \& WASTE MATERIALS \\
\hline 11 & 532. & $\mathbf{0}$ & 0 & 0 & -1 & $\begin{array}{l}\text { MISCELLANEOUS WHOLESALE, DURABLE } \\
\text { GOODS }\end{array}$ \\
\hline 11 & 540 & $\mathbf{0}$ & 0 & $\mathbf{0}$ & -1 & PAPER \& PAPER PRODUCTS \\
\hline 11 & 541 & $\mathbf{0}$ & 0 & $\mathbf{0}$ & -1 & DRUGS, CHEMICALS, \& ALLIED PRODUCTS \\
\hline 11 & 542 & $\mathbf{0}$ & 0 & $\mathbf{0}$ & -1 & APPAREL, FABRICS, \& NOTIONS \\
\hline 11 & 550 & $\mathbf{0}$ & 0 & $\mathbf{0}$ & -1 & GROCERIES \& RELATED PRODUCTS \\
\hline 11 & 551 & $\mathbf{0}$ & $\mathbf{0}$ & $\mathbf{0}$ & -1 & FARM PRODUCTS-RAW MATERIALS \\
\hline 11 & 552 & $\mathbf{0}$ & $\mathbf{0}$ & $\mathbf{0}$ & -1 & PETROLEUM PRODUCTS \\
\hline 11 & 560 & $\mathbf{0}$ & 0 & $\mathbf{0}$ & -1 & ALCOHOLIC BEVERAGES \\
\hline 11 & 561 & $\mathbf{0}$ & $\mathbf{0}$ & $\mathbf{0}$ & -1 & FARM SUPPLIES \\
\hline 11 & 562 & 0 & $\mathbf{0}$ & $\mathbf{0}$ & -1 & $\begin{array}{l}\text { MISCELLANEOUS WHOLESALE, } \\
\text { NONDURABLE GOODS }\end{array}$ \\
\hline 11 & 571 & $\mathbf{0}$ & $\mathbf{0}$ & $\mathbf{0}$ & -1 & NOT SPECIFIED WHOLESALE TRADE \\
\hline 11 & 580 & $\mathbf{0}$ & 0 & $\mathbf{0}$ & -1 & LUMBER \& BUILDING MATERIAL RETAILING \\
\hline 11 & 581 & $\mathbf{0}$ & $\mathbf{0}$ & $\mathbf{0}$ & -1 & HARDWARE STORES \\
\hline 11 & 582 & 0 & $\mathbf{0}$ & $\mathbf{0}$ & -1 & RETAII NURSERIES \& GARDEN STORES \\
\hline 11 & 590 & $\mathbf{0}$ & 0 & $\mathbf{0}$ & -1 & MOBILE HOME DEALERS \\
\hline 11 & 591 & $\mathbf{0}$ & $\mathbf{0}$ & $\mathbf{0}$ & -1 & DEPARTMENT STORES \\
\hline 11 & 592 & $\mathbf{0}$ & 0 & 0 & -1 & VARIETY STORES \\
\hline 11 & 600 & 0 & 0 & $\mathbf{0}$ & -1 & $\begin{array}{l}\text { MISCELLANEOUS GENERAL MERCHANDISE } \\
\text { STORES }\end{array}$ \\
\hline 11 & 601 & $\mathbf{0}$ & 0 & $\mathbf{0}$ & -1 & GROCERY STORES \\
\hline 11 & 602 & 0 & $\mathbf{0}$ & $\mathbf{0}$ & -1 & DAIRY PRODUCTS STORES \\
\hline 11 & 610 & 0 & $\mathbf{0}$ & 0 & -1 & RETAIL BAKERIES \\
\hline 11 & 611 & $\mathbf{0}$ & $\mathbf{0}$ & $\mathbf{0}$ & -1 & FOOD STORES, N.E.C. \\
\hline 11 & 612 & $\mathbf{0}$ & $\mathbf{0}$ & $\mathbf{0}$ & -1 & MOTOR VEHICLE DEALERS \\
\hline 11 & 620 & $\mathbf{0}$ & $\mathbf{0}$ & $\mathbf{0}$ & -1 & AUTO \& HOME SUPPLY STORES \\
\hline 11 & 621 & $\mathbf{0}$ & $\mathbf{0}$ & $\mathbf{0}$ & -1 & GASOLINE SERVICE STATIONS \\
\hline 11 & 622 & 0 & $\mathbf{0}$ & $\mathbf{0}$ & -1 & MISCELLANEOUS VEHICLE DEALERS \\
\hline 11 & 630 & $\mathbf{0}$ & $\mathbf{0}$ & $\mathbf{0}$ & -1 & $\begin{array}{l}\text { APPAREL \& ACCESSORY STORES, EXCEPT } \\
\text { SHOE }\end{array}$ \\
\hline 11 & 631 & $\mathbf{0}$ & $\mathbf{0}$ & $\mathbf{0}$ & -1 & SHOE STORES \\
\hline 11 & 632 & $\mathbf{0}$ & $\mathbf{0}$ & $\mathbf{0}$ & -1 & FURNITURE \& HOME FURNISHINGS STORES \\
\hline 11 & 640 & $\mathbf{0}$ & $\mathbf{0}$ & $\mathbf{0}$ & -1 & $\begin{array}{l}\text { HOUSEHOLD APPLIANCES, TV, \& RADIO } \\
\text { STORES }\end{array}$ \\
\hline 11 & 641 & $\mathbf{0}$ & $\mathbf{0}$ & $\mathbf{0}$ & -1 & EATING \& DRINKING PLACES \\
\hline 11 & 642 & $\mathbf{0}$ & $\mathbf{0}$ & $\mathbf{0}$ & -1 & DRUG STORES \\
\hline 11 & 650 & 0 & 0 & $\mathbf{0}$ & -1 & LIQUOR STORES \\
\hline 11 & 651 & $\mathbf{0}$ & $\mathbf{0}$ & $\mathbf{0}$ & -1 & $\begin{array}{l}\text { SPORTING GOODS, BICYCLES, \& HOBBY } \\
\text { STORES }\end{array}$ \\
\hline 11 & 652 & $\mathbf{0}$ & 0 & $\mathbf{0}$ & -1 & BOOK \& STATIONERY STORES \\
\hline 11 & 660 & 0 & 0 & $\mathbf{0}$ & -1 & JEWELRY STORES \\
\hline 11 & 661 & 0 & $\mathbf{0}$ & 0 & -1 & $\begin{array}{l}\text { SEWING, NEEDLEWORK, \& PIECE GOODS } \\
\text { STORES }\end{array}$ \\
\hline 11 & 662 & $\mathbf{0}$ & $\mathbf{0}$ & $\mathbf{0}$ & -1 & MAIL ORDER HOUSES \\
\hline 11 & 670 & 0 & 0 & $\mathbf{0}$ & -1 & VENDING MACHINE OPERATORS \\
\hline
\end{tabular}




\begin{tabular}{|c|c|c|c|c|c|c|}
\hline Maior & Minor & Param & Pen & Line & Decltr & Attribute Description \\
\hline 11 & 671 & 0 & 0 & 0 & -1 & DIRECT SELLING ESTABLISHMENTS \\
\hline 11 & 672 & 0 & 0 & 0 & -1 & FUEL \& ICE DEALERS \\
\hline 11 & 681 & 0 & 0 & $\mathbf{0}$ & -1 & RETAII FLORISTS \\
\hline 11 & 682 & $\mathbf{0}$ & $\mathbf{0}$ & 0 & -1 & MISCELLANEOUS RETAIL STORES \\
\hline 11 & 691 & 0 & $\mathbf{0}$ & 0 & -1 & NOT SPECIFIED RETAIL TRADE \\
\hline 11 & 700 & $\mathbf{0}$ & 0 & 0 & -1 & BANKING \\
\hline 11 & 701 & 0 & 0 & 0 & -1 & SAVINGS \& LOAN ASSOCIATIONS \\
\hline 11 & 702 & 0 & 0 & 0 & -1 & CREDIT AGENCIES, N.E.C. \\
\hline 11 & 710 & 0 & 0 & 0 & -1 & $\begin{array}{l}\text { SECURITY, COMMODITY BROKERAGE, \& } \\
\text { INVESTMENT COMPANIES }\end{array}$ \\
\hline 11 & 711 & 0 & 0 & 0 & -1 & INSURANCE \\
\hline 11 & 712 & 0 & 0 & $\mathbf{0}$ & -1 & $\begin{array}{l}\text { REAL ESTATE, INCLUDING REAL } \\
\text { ESTATE-INSURANCE-LAW OFFICES }\end{array}$ \\
\hline 11 & 721 & 0 & 0 & 0 & -1 & ADVERTISING \\
\hline 11 & 722 & 0 & 0 & 0 & -1 & $\begin{array}{l}\text { SERVICES TO DWELLINGS \& OTHER } \\
\text { BUILDINGS }\end{array}$ \\
\hline 11 & 730 & 0 & 0 & 0 & -1 & $\begin{array}{l}\text { COMMERCIAL RESEARCH, DEVELOPMENT, \& } \\
\text { TESTING LABS }\end{array}$ \\
\hline 11 & 731 & 0 & 0 & $\mathbf{0}$ & -1 & PERSONNEL SUPPLY SERVICES \\
\hline 11 & 732 & 0 & 0 & 0 & -1 & $\begin{array}{l}\text { BUSINESS MANAGEMENT \& CONSULTING } \\
\text { SERVICES }\end{array}$ \\
\hline 11 & 740 & 0 & 0 & 0 & -1 & COMPUTER \& DATA PROCESSING SERVICES \\
\hline 11 & 741 & 0 & 0 & 0 & -1 & DETECTIVE \& PROTECTIVE SERVICES \\
\hline 11 & 742 & 0 & $\mathbf{0}$ & 0 & -1 & BUSINESS SERVICES, N.E.C. \\
\hline 11 & 750 & 0 & $\mathbf{0}$ & 0 & -1 & AUTOMOTIVE SERVICES, EXCEPT REPAIR \\
\hline 11 & 751 & $\mathbf{0}$ & $\mathbf{0}$ & $\mathbf{0}$ & -1 & AUTOMOTIVE REPAIR SHOPS \\
\hline 11 & 752 & $\mathbf{0}$ & 0 & $\mathbf{0}$ & -1 & ELECTRICAL REPAIR SHOPS \\
\hline 11 & 760 & 0 & 0 & 0 & -1 & MISCELLANEOUS REPAIR SERVICES \\
\hline 11 & 761 & $\mathbf{0}$ & $\mathbf{0}$ & 0 & -1 & PRIVATE HOUSEHOLDS \\
\hline 11 & 762 & 0 & $\mathbf{0}$ & 0 & -1 & HOTELS \& MOTELS \\
\hline 11 & 770 & 0 & $\mathbf{0}$ & 0 & -1 & $\begin{array}{l}\text { LODGING PLACES, EXCEPT HOTELS \& } \\
\text { MOTELS }\end{array}$ \\
\hline 11 & 771 & $\mathbf{0}$ & $\mathbf{0}$ & 0 & -1 & $\begin{array}{l}\text { LAUNDRY, CLEANING, \& GARMENT } \\
\text { SERVICES }\end{array}$ \\
\hline 11 & 772 & 0 & $\mathbf{0}$ & 0 & -1 & BEAUTY SHOPS \\
\hline 11 & 780 & 0 & $\mathbf{0}$ & 0 & -1 & BARBER SHOPS \\
\hline 11 & 781 & $\mathbf{0}$ & $\mathbf{0}$ & 0 & -1 & FUNERAL SERVICE \& CREMATORIES \\
\hline 11 & 782 & 0 & $\mathbf{0}$ & 0 & -1 & SHOE REPAIR SHOPS \\
\hline 11 & 790 & 0 & $\mathbf{0}$ & 0 & -1 & DRESSMAKING SHOPS \\
\hline 11 & 791 & 0 & $\mathbf{0}$ & $\mathbf{0}$ & -1 & MISCELLANEOUS PERSONAL SERVICES \\
\hline 11 & 800 & $\mathbf{0}$ & 0 & 0 & -1 & THEATERS \& MOTION PICTURES \\
\hline 11 & 801 & 0 & $\mathbf{0}$ & $\mathbf{0}$ & -1 & $\begin{array}{l}\text { BOWLING ALLEYS, BILLIARD \& POOL } \\
\text { PARLORS }\end{array}$ \\
\hline 11 & 802 & 0 & C & 0 & -1 & $\begin{array}{l}\text { MISCELLANEOUS ENTERTAINMENT \& } \\
\text { RECREATION SERVICES }\end{array}$ \\
\hline 11 & 812 & 0 & 0 & $\mathbf{0}$ & -1 & OFFICES OF PHYSICIANS \\
\hline 11 & 820 & 0 & 0 & 0 & -1 & OFFICES OF DENTISTS \\
\hline 11 & 821 & 0 & 0 & 0 & -1 & OFFICES OF CHIROPRACTORS \\
\hline 11 & 822 & 0 & 0 & 0 & -1 & OFFICES OF OPTOMETRISTS \\
\hline 11 & 830 & 0 & $\mathbf{0}$ & $\mathbf{0}$ & -1 & OFFICES OF HEALTH PRACTTTIONERS, N.E.C. \\
\hline
\end{tabular}




\begin{tabular}{|c|c|c|c|c|c|c|}
\hline Major & Minor & Param & Pen & $\underline{\text { Line }}$ & Decltr & Attribute Description \\
\hline 11 & 831 & $\mathbf{0}$ & 0 & $\mathbf{0}$ & -1 & HOSPITALS \\
\hline 11 & 832 & 0 & $\mathbf{0}$ & $\mathbf{0}$ & -1 & NURSING \& PERSONAL CARE FACILITIES \\
\hline 11. & 840 & 0 & 0 & $\mathbf{0}$ & -1 & HEALTH SERVICES, N.E.C. \\
\hline 11 & 841 & 0 & $\mathbf{0}$ & $\mathbf{0}$ & -1 & LEGAL SERVICES \\
\hline 11 & 842 & $\mathbf{0}$ & $\mathbf{0}$ & 0 & -1 & ELEMENTARY \& SECONDARY SCHOOLS \\
\hline 11 & 850 & $\mathbf{0}$ & $\mathbf{0}$ & $\mathbf{0}$ & -1 & COLLEGES \& UNIVERSITIES \\
\hline 11 & 851 & $\mathbf{0}$ & $\mathbf{0}$ & $\mathbf{0}$ & -1 & BUSINESS, TRADE, \& VOCATIONAL SCHOOLS \\
\hline 11 & 852 & $\mathbf{0}$ & $\mathbf{0}$ & 0 & -1 & LIBRARIES \\
\hline 11 & 860 & 0 & 0 & $\mathbf{0}$ & -1 & EDUCATIONAL SERVICES, N.E.C. \\
\hline 11 & 861 & 0 & 0 & 0 & -1 & $\begin{array}{l}\text { JOB TRAINING \& VOCATIONAL } \\
\text { REHABILITATION SERVICES }\end{array}$ \\
\hline 11 & 862 & 0 & 0 & $\mathbf{0}$ & -1 & CHILD DAY CARE SERVICES \\
\hline 11 & 870 & 0 & 0 & 0 & -1 & $\begin{array}{l}\text { RESIDENTIAL CARE FACILITIES, WITHOUT } \\
\text { NURSING }\end{array}$ \\
\hline 11 & 871 & 0 & $\mathbf{0}$ & $\mathbf{0}$ & -1 & SOCIAL SERVICES, N.E.C. \\
\hline 11 & 872 & 0 & $\mathbf{0}$ & 0 & -1 & MUSEUMS, ART GALLERIES, \& ZOOS \\
\hline 11 & 880 & $\mathbf{0}$ & 0 & 5 & -1 & RELIGIOUS ORGANIZATIONS \\
\hline 11 & 881 & $\mathbf{0}$ & $\mathbf{0}$ & $\mathbf{0}$ & -1 & MEMBERSHIP ORGANIZATIONS \\
\hline 11 & 882 & $\mathbf{0}$ & $\mathbf{0}$ & $\mathbf{0}$ & -1 & $\begin{array}{l}\text { ENGINEERING, ARCHITECTURAL, \& } \\
\text { SURVEYING SERVICES }\end{array}$ \\
\hline 11 & 890 & 0 & $\mathbf{0}$ & $\mathbf{0}$ & -1 & $\begin{array}{l}\text { ACCOUNTING, AUDITING, \& BOOKKEEPING } \\
\text { SERVICES }\end{array}$ \\
\hline 11 & 891 & $\mathbf{0}$ & $\mathbf{0}$ & $\mathbf{0}$ & -1 & $\begin{array}{l}\text { NONCOMMERCIAL EDUCATIONAL \& } \\
\text { SCIENTIFIC RESEARCH }\end{array}$ \\
\hline 11 & 892 & 0 & $\mathbf{0}$ & $\mathbf{0}$ & -1 & $\begin{array}{l}\text { MISCELLANEOUS PROFESSIONAL \& RELATED } \\
\text { SERVICES }\end{array}$ \\
\hline 11 & 900 & $\mathbf{0}$ & $\mathbf{0}$ & $\mathbf{0}$ & -1 & EXECUTIVE \& LEGISLATIVE OFFICES \\
\hline 11 & 901 & $\mathbf{0}$ & $\mathbf{0}$ & $\mathbf{0}$ & -1 & GENERAL GOVERNMENT, N.E.C. \\
\hline 11 & 910 & $\mathbf{0}$ & $\mathbf{0}$ & $\mathbf{0}$ & -1 & JUSTICE, PUBLIC ORDER, \& SAFETY \\
\hline 11 & 921 & 0 & $\mathbf{0}$ & $\mathbf{0}$ & -1 & $\begin{array}{l}\text { PUBLIC FINANCE, TAXATION, \& MONETARY } \\
\text { POLICY }\end{array}$ \\
\hline 11 & 922 & 0 & 0 & $\mathbf{0}$ & -1 & $\begin{array}{l}\text { ADMINISTRATION OF HUMAN RESOURCES } \\
\text { PROGRAMS }\end{array}$ \\
\hline 11 & 930 & $\mathbf{0}$ & $\mathbf{0}$ & 0 & -1 & $\begin{array}{l}\text { ADMINISTRATION OF ENVIRONMENTAL } \\
\text { QUALITY \& HOUSING PROGRAMS }\end{array}$ \\
\hline 11 & 931 & $\mathbf{0}$ & $\mathbf{0}$ & $\mathbf{0}$ & -1 & ADMINISTRATION OF ECONOMIC PROGRAMS \\
\hline 11 & 932 & $\mathbf{0}$ & $\mathbf{0}$ & $\mathbf{0}$ & -1 & $\begin{array}{l}\text { NATIONAL SECURITY \& INTERNATIONAL } \\
\text { AFFAIRS }\end{array}$ \\
\hline 11 & 1003 & $\mathbf{0}$ & $\mathbf{0}$ & $\mathbf{0}$ & -1 & LEGISLATORS \\
\hline 11 & 1004 & $\mathbf{0}$ & $\mathbf{0}$ & $\mathbf{0}$ & -1 & $\begin{array}{l}\text { CHIEF EXECUTIVES \& OFFICIALS, PUBLIC } \\
\text { ADMINISTRATION }\end{array}$ \\
\hline 11 & 1005 & $\mathbf{0}$ & $\mathbf{0}$ & $\mathbf{0}$ & -1 & $\begin{array}{l}\text { ADMINISTRATORS \& OFFICIALS, PUBLIC } \\
\text { ADMINISTRATION }\end{array}$ \\
\hline 11 & 1006 & $\mathbf{0}$ & $\mathbf{0}$ & $\mathbf{0}$ & -1 & ADMINISTRATORS, PROTECTIVE SERVICES \\
\hline 11 & 1007 & $\mathbf{0}$ & $\mathbf{0}$ & $\mathbf{0}$ & -1 & FINANCIAL MANAGERS \\
\hline 11 & 1008 & $\mathbf{0}$ & 0 & $\mathbf{0}$ & -1 & $\begin{array}{l}\text { PERSONNEL \& LABOR RELATIONS } \\
\text { MANAGERS }\end{array}$ \\
\hline 11 & 1009 & 0 & $\mathbf{0}$ & $\mathbf{0}$ & -1 & PURCHASING MANAGERS \\
\hline 11 & 1013 & $\mathbf{0}$ & $\mathbf{0}$ & $\mathbf{0}$ & -1 & $\begin{array}{l}\text { MANAGERS, MARKETING, ADVERTISING \& } \\
\text { PUBLIC RELATIONS }\end{array}$ \\
\hline
\end{tabular}




\begin{tabular}{|c|c|c|c|c|c|c|}
\hline Maior & Minor & Param & Pen & Line & Decltr & Attribute Description \\
\hline 11 & 1014 & 0 & 0 & 0 & -1 & $\begin{array}{l}\text { ADMINISTRATORS, EDUCATION \& RELATED } \\
\text { FIELDS }\end{array}$ \\
\hline 11 & 1015 & 0 & $\mathbf{0}$ & $\mathbf{0}$ & -1 & MANAGERS, MEDICINE \& HEALTH \\
\hline 11 & 1016 & 0 & 0 & $\mathbf{0}$ & -1 & MANAGERS, PROPERTIES \& REAL ESTATE \\
\hline 11 & 1017 & 0 & $\mathbf{0}$ & $\mathbf{0}$ & -1 & POSTMASTERS \& MAIl SUPERINTENDENTS \\
\hline 11 & 1018 & 0 & $\mathbf{0}$ & $\mathbf{0}$ & -1 & FUNERAL DIRECTORS \\
\hline 11 & 1019 & 0 & $\mathbf{0}$ & $\mathbf{0}$ & -1 & MANAGERS \& ADMINISTRATORS, N.E.C. \\
\hline 11 & 1023 & 0 & $\mathbf{0}$ & $\mathbf{0}$ & -1 & ACCOUNTANTS \& AUDITORS \\
\hline 11 & 1024 & 0 & $\mathbf{0}$ & $\mathbf{0}$ & -1 & UNDERWRTIERS \\
\hline 11 & 1025 & $\mathbf{0}$ & $\mathbf{0}$ & $\mathbf{0}$ & -1 & OTHER FINANCIAL OFFICERS \\
\hline 11 & 1026 & 0 & $\mathbf{0}$ & $\mathbf{0}$ & -1 & MANAGEMENT ANALYSTS \\
\hline 11 & 1027 & 0 & $\mathbf{0}$ & $\mathbf{0}$ & -1 & $\begin{array}{l}\text { PERSONNEL, TRAINING, \& LABOR RELATIONS } \\
\text { SPECIALISTS }\end{array}$ \\
\hline 11 & 1028 & 0 & 0 & 0 & -1 & $\begin{array}{l}\text { PURCHASING AGENTS \& BUYERS, FARM } \\
\text { PRODUCTS }\end{array}$ \\
\hline 11 & 1029 & 0 & 0 & 0 & -1 & $\begin{array}{l}\text { BUYERS, WHOLESALE \& RETAIL, EXCEPT } \\
\text { FARM PRODUCTS }\end{array}$ \\
\hline 11 & 1033 & 0 & $\mathbf{0}$ & $\mathbf{0}$ & -1 & PURCHASING AGENTS \& BUYERS, N.E.C. \\
\hline 11 & 1034 & 0 & $\mathbf{0}$ & 0 & -1 & BUSINESS \& PROMOTION AGENTS \\
\hline 11 & 1035 & 0 & $\mathbf{0}$ & $\mathbf{0}$ & -1 & CONSTRUCTION INSPECTORS \\
\hline 11 & 1036 & 0 & 0 & 0 & -1 & $\begin{array}{l}\text { INSPECTORS \& COMPLIANCE OFFICERS, } \\
\text { EXCEPT CONSTRUCTION }\end{array}$ \\
\hline 11 & 1037 & 0 & 0 & 0 & -1 & $\begin{array}{l}\text { MANAGEMENT RELATED OCCUPATIONS, } \\
\text { N.E.C. }\end{array}$ \\
\hline 11 & 1043 & 0 & $\mathbf{0}$ & $\mathbf{0}$ & -1 & ARCHITECTS \\
\hline 11 & 1044 & 0 & $\mathbf{0}$ & $\mathbf{0}$ & -1 & AEROSPACE ENGINEERS \\
\hline 11 & 1045 & 0 & $\mathbf{0}$ & $\mathbf{0}$ & -1 & METALLURGICAL \& MATERIALS ENGINEERS \\
\hline 11 & 1046 & 0 & $\mathbf{0}$ & 0 & -1 & MINING ENGINEERS \\
\hline 11 & 1047 & 0 & 0 & $\mathbf{0}$ & -1 & PETROLEUM ENGINEERS \\
\hline 11 & 1048 & 0 & $\mathbf{0}$ & $\mathbf{0}$ & -1 & CHEMICAL ENGINEERS \\
\hline 11 & 1049 & 0 & $\mathbf{0}$ & $\mathbf{0}$ & -1 & NUCLEAR ENGINEERS \\
\hline 11 & 1053 & 0 & $\mathbf{0}$ & $\mathbf{0}$ & -1 & CIVIL ENGINEERS \\
\hline 11 & 1054 & 0 & $\mathbf{0}$ & $\mathbf{0}$ & -1 & AGRICULTURAL ENGINEERS \\
\hline 11 & 1055 & 0 & $\mathbf{0}$ & $\mathbf{0}$ & -1 & ELECTRICAL \& ELECTRONIC ENGINEERS \\
\hline 11 & 1056 & 0 & $\mathbf{0}$ & $\mathbf{0}$ & -1 & INDUSTRIAL ENGINEERS \\
\hline 11 & 1057 & 0 & $\mathbf{0}$ & $\mathbf{0}$ & -1 & MECHANICAL ENGINEERS \\
\hline 11 & 1058 & 0 & $\mathbf{0}$ & $\mathbf{0}$ & -1 & MARINE ENGINEERS \& NAVAL ARCHITECTS \\
\hline 11 & 1059 & 0 & 0 & $\mathbf{0}$ & -1 & ENGINEERS, N.E.C. \\
\hline 11 & 1063 & 0 & $\mathbf{0}$ & $\mathbf{0}$ & -1 & SURVEYORS \& MAPPING SCIENTISTS \\
\hline 11 & 1064 & 0 & 0 & 0 & -1 & $\begin{array}{l}\text { COMPUTER SYSTEMS ANALYSTS \& } \\
\text { SCIENTISTS }\end{array}$ \\
\hline 11 & 1065 & 0 & 0 & 0 & -1 & $\begin{array}{l}\text { OPERATIONS \& SYSTEMS RESEARCHERS \& } \\
\text { ANALYSTS }\end{array}$ \\
\hline 11 & 1066 & 0 & 0 & 0 & -1 & ACTUARIES \\
\hline 11 & 1067 & $\mathbf{0}$ & $\mathbf{0}$ & $\mathbf{0}$ & -1 & STATISTICIANS \\
\hline 11 & 1068 & 0 & 0 & $\mathbf{0}$ & -1 & MATHEMATICAL SCIENTISTS, N.E.C. \\
\hline 11 & 1069 & 0 & 0 & 0 & -1 & PHYSICISTS \& ASTRONOMERS \\
\hline 11 & 1073 & 0 & 0 & 0 & -1 & CHEMISTS, EXCEPT BIOCHEMISTS \\
\hline 11 & 1074 & $\mathbf{0}$ & 0 & $\mathbf{0}$ & -1 & ATMOSPHERIC \& SPACE SCIENTISTS \\
\hline 11 & 1075 & $\mathbf{0}$ & $\mathbf{0}$ & $\mathbf{0}$ & -1 & GEOLOGISTS \& GEODESISTS \\
\hline
\end{tabular}




\begin{tabular}{|c|c|c|c|c|c|c|}
\hline Maior & Minor & Param & Pen & Line & Decitr & Attribute Descriotion \\
\hline 11 & 1076 & $\mathbf{0}$ & 0 & $\mathbf{0}$ & -1 & PHYSICAI SCIENTISTS, N.E.C. \\
\hline 11 & 1077 & $\mathbf{0}$ & $\mathbf{0}$ & $\mathbf{0}$ & -1 & AGRICULTURAL \& FOOD SCIENTISTS \\
\hline 11 & 1078 & $\mathbf{0}$ & $\mathbf{0}$ & $\mathbf{0}$ & -1 & BIOLOGICAL \& LIFE SCIENTISTS \\
\hline 11 & 1079 & $\mathbf{0}$ & $\mathbf{0}$ & $\mathbf{0}$ & -1 & FORESTRY \& CONSERVATION SCIENTISTS \\
\hline 11 & 1083 & $\mathbf{0}$ & $\mathbf{0}$ & $\mathbf{0}$ & -1 & MEDICAL SCIENTISTS \\
\hline 11 & 1084 & $\mathbf{0}$ & $\mathbf{0}$ & $\mathbf{0}$ & -1 & PHYSICIANS \\
\hline 11 & 1085 & 0 & 0 & $\mathbf{0}$ & -1 & DENTISTS \\
\hline 11 & 1086 & $\mathbf{0}$ & $\mathbf{0}$ & $\mathbf{0}$ & -1 & VETERINARIANS \\
\hline 11 & 1087 & $\mathbf{0}$ & 0 & $\mathbf{0}$ & -1 & OPTOMETRISTS \\
\hline 11 & 1088 & $\mathbf{0}$ & $\mathbf{0}$ & $\mathbf{0}$ & -1 & PODIATRISTS \\
\hline 11 & 1089 & $\mathbf{0}$ & 0 & $\mathbf{0}$ & -1 & HEALTH DIAGNOSING PRACTITIONERS, N.E.C \\
\hline 11 & 1095 & $\mathbf{0}$ & $\mathbf{0}$ & $\mathbf{0}$ & -1 & REGISTERED NURSES \\
\hline 11 & 1096 & 0 & $\mathbf{0}$ & $\mathbf{0}$ & -1 & PHARMACISTS \\
\hline 11 & 1097 & 0 & $\mathbf{0}$ & $\mathbf{0}$ & -1 & DIETITIANS \\
\hline 11 & 1098 & $\mathbf{0}$ & $\mathbf{0}$ & $\mathbf{0}$ & -1 & INHALATION THERAPISTS \\
\hline 11 & 1099 & $\mathbf{0}$ & $\mathbf{0}$ & $\mathbf{0}$ & -1 & OCCUPATIONAL THERAPISTS \\
\hline 11 & 1103 & $\mathbf{0}$ & 0 & $\mathbf{0}$ & -1 & PHYSICAL THERAPISTS \\
\hline 11 & 1104 & 0 & $\mathbf{0}$ & $\mathbf{0}$ & -1 & SPEECH THERAPISTS \\
\hline 11 & 1105 & 0 & 0 & $\mathbf{0}$ & -1 & THERAPISTS, N.E.C. \\
\hline 11 & 1106 & 0 & $\mathbf{0}$ & $\mathbf{0}$ & -1 & PHYSICIANS' ASSISTANTS \\
\hline 11 & 1113 & $\mathbf{0}$ & $\mathbf{0}$ & $\mathbf{0}$ & -1 & $\begin{array}{l}\text { EARTH, ENVIRONMENTAL, \& MARINE } \\
\text { SCIENCE TEACHERS }\end{array}$ \\
\hline 11 & 1114 & 0 & 0 & $\mathbf{0}$ & -1 & BIOLOGICAL SCIENCE TEACHERS \\
\hline 11 & 1115 & $\mathbf{0}$ & $\mathbf{0}$ & $\mathbf{0}$ & -1 & CHEMISTRY TEACHERS \\
\hline 11 & 1116 & $\mathbf{0}$ & $\mathbf{0}$ & $\mathbf{0}$ & -1 & PHYSICS TEACHERS \\
\hline 11 & 1117 & $\mathbf{0}$ & $\mathbf{0}$ & $\mathbf{0}$ & -1 & NATURAL SCIENCE TEACHERS, N.E.C. \\
\hline 11 & 1118 & $\mathbf{0}$ & 0 & $\mathbf{0}$ & -1 & PSYCHOLOGY TEACHERS \\
\hline 11 & 1119 & $\mathbf{0}$ & $\mathbf{0}$ & 0 & -1 & ECONOMICS TEACHERS \\
\hline 11 & 1123 & $\mathbf{0}$ & $\mathbf{0}$ & $\mathbf{0}$ & -1 & HISTORY TEACHERS \\
\hline 11 & 1124 & $\mathbf{0}$ & $\mathbf{0}$ & $\mathbf{0}$ & -1 & POLTICAL SCIENCE TEACHERS \\
\hline 11 & 1125 & $\mathbf{0}$ & $\mathbf{0}$ & $\mathbf{0}$ & -1 & SOCIOLOGY TEACHERS \\
\hline 11 & 1126 & $\mathbf{0}$ & $\mathbf{0}$ & 0 & -1 & SOCIAL SCIENCE TEACHERS, N.E.C. \\
\hline 11 & 1127 & 0 & $\mathbf{0}$ & $\mathbf{0}$ & -1 & ENGINEERING TEACHERS \\
\hline 11 & 1128 & 0 & $\mathbf{0}$ & $\mathbf{0}$ & -1 & MATHEMATICAL SCIENCE TEACHERS \\
\hline 11 & 1129 & $\mathbf{0}$ & 0 & 0 & -1 & COMPUTER SCIENCE TEACHERS \\
\hline 11 & 1133 & $\mathbf{0}$ & $\mathbf{0}$ & 0 & -1 & MEDICAL SCIENCE TEACHERS \\
\hline 11 & 1134 & 0 & $\mathbf{0}$ & $\mathbf{0}$ & -1 & HEALTH SPECIALTIES TEACHERS \\
\hline 11 & 1135 & 0 & $\mathbf{0}$ & $\mathbf{0}$ & -1 & $\begin{array}{l}\text { BUSINESS, COMMERCE, \& MARKETING } \\
\text { TEACHERS }\end{array}$ \\
\hline 11 & 1136 & 0 & $\mathbf{0}$ & $\mathbf{0}$ & -1 & AGRICULTURE \& FORESTRY TEACHERS \\
\hline 11 & 1137 & 0 & 0 & 0 & -1 & ART, DRAMA, \& MUSIC TEACHERS \\
\hline 11 & 1138 & $\mathbf{0}$ & 0 & $\mathbf{0}$ & -1 & PHYSICAL EDUCATION TEACHERS \\
\hline 11 & 1139 & 0 & $\mathbf{0}$ & 0 & -1 & EDUCATION TEACHERS \\
\hline 11 & 1143 & $\mathbf{0}$ & $\mathbf{0}$ & 0 & -1 & ENGLISH TEACHERS \\
\hline 11 & 1144 & 0 & $\mathbf{0}$ & 0 & -1 & FOREIGN LANGUAGE TEACHERS \\
\hline 11 & 1145 & 0 & $\mathbf{0}$ & $\mathbf{0}$ & -1 & LAW TEACHERS \\
\hline 11 & 1146 & $\mathbf{0}$ & 0 & 0 & -1 & SOCIAL WORK TEACHERS \\
\hline 11 & 1147 & $\mathbf{0}$ & $\mathbf{0}$ & $\mathbf{0}$ & -1 & THEOLOGY TEACHERS \\
\hline 11 & 1148 & $\mathbf{0}$ & $\mathbf{0}$ & $\mathbf{0}$ & -1 & TRADE \& INLUSTRIAL TEACHERS \\
\hline 11 & 1149 & 0 & 0 & $\mathbf{0}$ & -1 & HOME ECONOMICS TEACHERS \\
\hline
\end{tabular}




\begin{tabular}{|c|c|c|c|c|c|c|}
\hline Maior & Minor & Param & Pen & Line & Decltr & Attribute Description \\
\hline 11 & 1153 & 0 & 0 & 0 & -1 & TEACHERS, POSTSECONDARY, N.E.C. \\
\hline 11 & 1154 & 0 & 0 & $\mathbf{0}$ & -1 & $\begin{array}{l}\text { POSTSECONDARY TEACHERS, SUBJECT NOT } \\
\text { SPECIFIED }\end{array}$ \\
\hline 11 & 1155 & 0 & 0 & $\mathbf{0}$ & -1 & $\begin{array}{l}\text { TEACHERS, PREKINDERGARTEN \& } \\
\text { KINDERGARTEN }\end{array}$ \\
\hline 11 & 1156 & 0 & 0 & 0 & -1 & TEACHERS, ELEMENTARY SCHOOL \\
\hline 11 & 1157 & 0 & $\mathbf{0}$ & $\mathbf{0}$ & -1 & TEACHERS, SECONDARY SCHOOL \\
\hline 11 & 1158 & 0 & $\mathbf{0}$ & $\mathbf{0}$ & -1 & TEACHERS, SPECIAL EDUCATION \\
\hline 11 & 1159 & $\mathbf{0}$ & $\mathbf{0}$ & 0 & -1 & TEACHERS, N.E.C. \\
\hline 11 & 1163 & 0 & 0 & $\mathbf{0}$ & -1 & $\begin{array}{l}\text { COUNSELORS, EDUCATIONAL \& } \\
\text { VOCATIONAL }\end{array}$ \\
\hline 11 & 1164 & $\mathbf{0}$ & $\mathbf{0}$ & $\mathbf{0}$ & -1 & LIBRARIANS \\
\hline 11 & 1165 & $\mathbf{0}$ & $\mathbf{0}$ & $\mathbf{0}$ & -1 & ARCHIVISTS \& CURATORS \\
\hline 11 & 1166 & 0 & $\mathbf{0}$ & $\mathbf{0}$ & -1 & ECONOMISTS \\
\hline 11 & 1167 & $\mathbf{0}$ & 0 & $\mathbf{0}$ & -1 & PSYCHOLOGISTS \\
\hline 11 & 1168 & 0 & 0 & $\mathbf{0}$ & -1 & SOCIOLOGISTS \\
\hline 11 & 1169 & 0 & $\mathbf{0}$ & $\mathbf{0}$ & -1 & SOCIAL SCIENTISTS, N.E.C. \\
\hline 11 & 1173 & 0 & 0 & $\mathbf{0}$ & -1 & URBAN PLANNERS \\
\hline 11 & 1174 & 0 & 0 & $\mathbf{0}$ & -1 & SOCIAL WORKERS \\
\hline 11 & 1175 & $\mathbf{0}$ & 0 & $\mathbf{0}$ & -1 & RECREATION WORKERS \\
\hline 11 & 1176 & $\mathbf{0}$ & 0 & $\mathbf{0}$ & -1 & CLERCY \\
\hline 11 & 1177 & $\mathbf{0}$ & 0 & $\mathbf{0}$ & -1 & RELIGIOUS WORKERS, N.E.C. \\
\hline 11 & 1178 & $\mathbf{0}$ & 0 & $\mathbf{0}$ & -1 & LAWYERS \\
\hline 11 & 1179 & $\mathbf{0}$ & 0 & $\mathbf{0}$ & -1 & JUDGES \\
\hline 11 & 1183 & $\mathbf{0}$ & $\mathbf{0}$ & $\mathbf{0}$ & -1 & AUTHORS \\
\hline 11 & 1184 & 0 & $\mathbf{0}$ & $\mathbf{0}$ & -1 & TECHNICAL WRITERS \\
\hline 11 & 1185 & $\mathbf{0}$ & $\mathbf{0}$ & $\mathbf{0}$ & -1 & DESIGNERS \\
\hline 11 & 1186 & 0 & $\mathbf{0}$ & $\mathbf{0}$ & -1 & MUSICIANS \& COMPOSERS \\
\hline 11 & 1187 & 0 & $\mathbf{0}$ & $\mathbf{0}$ & -1 & ACTORS \& DIRECTORS \\
\hline 11 & 1188 & 0 & $\mathbf{0}$ & $\mathbf{0}$ & -1 & $\begin{array}{l}\text { PAINTERS, SCULPTORS, CRAFT-ARTISTS, \& } \\
\text { ARTIST PRINTMAKERS }\end{array}$ \\
\hline 11 & 1189 & 0 & $\mathbf{0}$ & $\mathbf{0}$ & -1 & PHOTOGRAPHERS \\
\hline 11 & 1193 & $\mathbf{0}$ & 0 & 0 & -1 & DANCERS \\
\hline 11 & 1194 & 0 & $\mathbf{0}$ & 0 & -1 & $\begin{array}{l}\text { ARTISTS, PERFORMERS, \& RELATED } \\
\text { WORKERS, N.E.C. }\end{array}$ \\
\hline 11 & 1195 & 0 & $\mathbf{0}$ & $\mathbf{0}$ & -1 & EDITORS \& REPORTERS \\
\hline 11 & 1197 & $\mathbf{0}$ & $\mathbf{0}$ & 0 & -1 & PUBLIC RELATIONS SPECIALISTS \\
\hline 11 & 1198 & 0 & $\mathbf{0}$ & 0 & -1 & ANNOUNCERS \\
\hline 11 & 1199 & 0 & $\mathbf{0}$ & 0 & -1 & ATHLETES \\
\hline 11 & 1203 & 0 & $\mathbf{0}$ & $\mathbf{0}$ & -1 & Health Technologists \& Technicians \\
\hline 11 & 1204 & $\mathbf{0}$ & $\mathbf{0}$ & $\mathbf{0}$ & -1 & DENTAL HYGIENISTS \\
\hline 11 & 1205 & 0 & 0 & 0 & -1 & $\begin{array}{l}\text { HEALTH RECORD TECHNOLOGISTS \& } \\
\text { TECHNICIANS }\end{array}$ \\
\hline 11 & 1206 & 0 & $\mathbf{0}$ & $\mathbf{0}$ & -1 & RADIOLOGIC TECHNICIANS \\
\hline 11 & 1207 & $\mathbf{0}$ & $\mathbf{0}$ & $\mathbf{0}$ & -1 & LICENSED PRACTICAL NURSES \\
\hline 11 & 1208 & $\mathbf{0}$ & 0 & 0 & -1 & $\begin{array}{l}\text { HEALTH TECHNOLOGISTS \& TECHNICIANS, } \\
\text { N.E.C. }\end{array}$ \\
\hline 11 & 1213 & $\mathbf{0}$ & $\mathbf{0}$ & $\mathbf{0}$ & -1 & ELECTRICAL \& ELECTRONIC TECHNICIANS \\
\hline 11 & 1214 & $\mathbf{0}$ & $\mathbf{0}$ & $\mathbf{0}$ & -1 & INDUSTRIAL ENGINEERING TECHNICIANS \\
\hline 11 & 1215 & $\mathbf{0}$ & 0 & $\mathbf{0}$ & -1 & MECHANICAL ENGINEERING TECHNICIANS \\
\hline
\end{tabular}




\begin{tabular}{|c|c|c|c|c|c|c|}
\hline Major & Minor & Param & Ren & Line & Decitr & Attribute Description \\
\hline 11 & 1216 & 0 & $\mathbf{0}$ & $\mathbf{0}$ & -1 & ENGINEERING TECHNICIANS, N.E.C. \\
\hline 11 & 1217 & $\mathbf{0}$ & 0 & $\mathbf{0}$ & -1 & DRAFIING OCCUPATIONS \\
\hline 11 & 1218 & $\mathbf{0}$ & 0 & $\mathbf{0}$ & -1 & SURVEYING \& MAPPING TECHNICIANS \\
\hline 11 & 1223 & $\mathbf{0}$ & 0 & $\mathbf{0}$ & -1 & BIOLOGICAL TECHNICIANS \\
\hline 11 & 1224 & $\mathbf{0}$ & 0 & $\mathbf{0}$ & -1 & CHEMICAL TECFINICIANS \\
\hline 11 & 1225 & $\mathbf{0}$ & $\mathbf{0}$ & $\mathbf{0}$ & -1 & SCIENCE TECHNICIANS, N.E.C. \\
\hline 11 & 1226 & $\mathbf{0}$ & $\mathbf{0}$ & $\mathbf{0}$ & -1 & AIRPLANE PILOTS \& NAVIGATORS \\
\hline 11 & 1227 & $\mathbf{0}$ & $\mathbf{0}$ & $\mathbf{0}$ & -1 & AIR TRAFFIC CONTROLLERS \\
\hline 11 & 1228 & $\mathbf{0}$ & $\mathbf{0}$ & $\mathbf{0}$ & -1 & BROADCAST EQUIPMENT OPERATORS \\
\hline 11 & 1229 & $\mathbf{0}$ & 0 & $\mathbf{0}$ & -1 & COMPUTER PROGRAMMERS \\
\hline 11 & 1233 & $\mathbf{0}$ & 0 & 0 & -1 & $\begin{array}{l}\text { TOOL PROGRAMMERS, NUMERICAL } \\
\text { CONTROL }\end{array}$ \\
\hline 11 & 1234 & $\mathbf{0}$ & $\mathbf{0}$ & $\mathbf{0}$ & -1 & LEGAL ASSISTANTS \\
\hline 11 & 1235 & $\mathbf{0}$ & $\mathbf{0}$ & $\mathbf{0}$ & -1 & TECHNICIANS, N.E.C. \\
\hline 11 & 1243 & $\mathbf{0}$ & 0 & 0 & -1 & $\begin{array}{l}\text { SUPERVISORS \& PROPRIETORS, SALES } \\
\text { OCCUPATIONS }\end{array}$ \\
\hline 11 & 1253 & $\mathbf{0}$ & $\mathbf{0}$ & $\mathbf{0}$ & -1 & INSURANCE SALES OCCUPATIONS \\
\hline 11 & 1254 & $\mathbf{0}$ & $\mathbf{0}$ & $\mathbf{0}$ & -1 & REAL ESTATE SALES OCCUPATIONS \\
\hline 11 & 1255 & $\mathbf{0}$ & $\mathbf{0}$ & $\mathbf{0}$ & -1 & $\begin{array}{l}\text { SECURITIES \& FINANCIAL SERVICES SALES } \\
\text { OCCUPATIONS }\end{array}$ \\
\hline 11 & 1256 & $\mathbf{0}$ & $\mathbf{0}$ & $\mathbf{0}$ & -1 & $\begin{array}{l}\text { ADVERTISING \& RELATED SALES } \\
\text { OCCUPATIONS }\end{array}$ \\
\hline 11 & 1257 & $\mathbf{0}$ & $\mathbf{0}$ & $\mathbf{0}$ & -1 & $\begin{array}{l}\text { SALES OCCUPATIONS, OTHER BUSINESS } \\
\text { SERVICES }\end{array}$ \\
\hline 11 & 1258 & $\mathbf{0}$ & $\mathbf{0}$ & $\mathbf{0}$ & -1 & SALES ENGINEERS \\
\hline 11 & 1259 & $\mathbf{0}$ & $\mathbf{0}$ & $\mathbf{0}$ & -1 & SALES REPRESENTATTVES \\
\hline 11 & 1263 & $\mathbf{0}$ & $\mathbf{0}$ & $\mathbf{0}$ & -1 & SALES WORKERS, MOTOR VEHICLES \& BOATS \\
\hline 11 & 1264 & $\mathbf{0}$ & $\mathbf{0}$ & $\mathbf{0}$ & -1 & SALES WORKERS, APPAREL \\
\hline 11 & 1265 & $\mathbf{0}$ & $\mathbf{0}$ & $\mathbf{0}$ & -1 & SALES WORKERS, SHOES \\
\hline 11 & 1266 & $\mathbf{0}$ & 0 & 0 & -1 & $\begin{array}{l}\text { SALES WORKERS, FURNITURE \& HOME } \\
\text { FURNISHINGS }\end{array}$ \\
\hline 11 & 1267 & $\mathbf{0}$ & $\mathbf{0}$ & $\mathbf{0}$ & -1 & $\begin{array}{l}\text { SALES WORKERS; RADIO, TELEVISION, HI-FI, } \\
\text { \& APPLIANCES }\end{array}$ \\
\hline 11 & 1268 & 0 & $\mathbf{0}$ & $\mathbf{0}$ & -1 & $\begin{array}{l}\text { SALES WORKERS, HARDWARE \& BUILDING } \\
\text { SUPPLIES }\end{array}$ \\
\hline 11 & 1269 & 0 & $\mathbf{0}$ & $\mathbf{0}$ & -1 & SALES WORKERS, PARTS \\
\hline 11 & 1274 & $\mathbf{0}$ & $\mathbf{0}$ & $\mathbf{0}$ & -1 & SALES WORKERS, OTHER COMMOD. \\
\hline 11 & 1275 & $\mathbf{0}$ & $\mathbf{0}$ & $\mathbf{0}$ & -1 & SALES COUNTER CLERKS \\
\hline 11 & 1276 & $\mathbf{0}$ & $\mathbf{0}$ & $\mathbf{0}$ & -1 & CASHIERS \\
\hline 11 & 1277 & $\mathbf{0}$ & $\mathbf{0}$ & $\mathbf{0}$ & -1 & STREET \& DOOR-TO-DOOR SALES WORKERS \\
\hline 11 & 1278 & $\mathbf{0}$ & $\mathbf{0}$ & $\mathbf{0}$ & -1 & NEWS VENDORS \\
\hline 11 & 1283 & $\mathbf{0}$ & $\mathbf{0}$ & $\mathbf{0}$ & -1 & $\begin{array}{l}\text { DEMONSTRATORS, PROMOTERS \& MODELS, } \\
\text { SALES }\end{array}$ \\
\hline 11 & 1284 & $\mathbf{0}$ & $\mathbf{0}$ & $\mathbf{0}$ & -1 & AUCTIONEERS \\
\hline 11 & 1285 & $\mathbf{0}$ & $\mathbf{0}$ & $\mathbf{0}$ & -1 & SALES SUPPORT OCCUPATIONS, N.E.C. \\
\hline 11 & 1303 & $\mathbf{0}$ & $\mathbf{0}$ & $\mathbf{0}$ & -1 & SUPERVISORS, GENERAL OFFICE \\
\hline 11 & 1304 & $\mathbf{0}$ & $\mathbf{0}$ & $\mathbf{0}$ & -1 & $\begin{array}{l}\text { SUPERVISORS, COMPUTER EQUIPMENT } \\
\text { OPERATORS }\end{array}$ \\
\hline 11 & 1305 & $\mathbf{0}$ & $\mathbf{0}$ & $\mathbf{0}$ & -1 & $\begin{array}{l}\text { SUPERVISORS, FINANCIAL RECORDS } \\
\text { PROCESSING }\end{array}$ \\
\hline
\end{tabular}




\begin{tabular}{|c|c|c|c|c|c|c|}
\hline Maior & Minor & Param & Pen & Line & Decitr & Attribute Description \\
\hline 11 & 1306 & $\mathbf{0}$ & 0 & $\mathbf{0}$ & -1 & CHIEF COMMUNICATIONS OPERATORS \\
\hline 11 & 1307 & 0 & $\mathbf{0}$ & $\mathbf{0}$ & -1 & $\begin{array}{l}\text { SUPERVISORS; DISTRIBUTION, SCHEDULING, } \\
\text { \& ADJUSTING CLERKS }\end{array}$ \\
\hline 11 & 1308 & $\mathbf{0}$ & 0 & 0 & -1 & COMPUTER OPERATORS \\
\hline 11 & 1309 & $\mathbf{0}$ & $\mathbf{0}$ & $\mathbf{0}$ & -1 & PERIPHERAL EQUIPMENT OPERATORS \\
\hline 11 & 1313 & $\mathbf{0}$ & $\mathbf{0}$ & 0 & -1 & SECRETARIES \\
\hline 11 & 1314 & 0 & $\mathbf{0}$ & 0 & -1 & STENOGRAPHERS \\
\hline 11 & 1315 & $\mathbf{0}$ & 0 & $\mathbf{0}$ & -1 & TYPISTS \\
\hline 11 & 1316 & 0 & $\mathbf{0}$ & 0 & -1 & INTERVIEWERS \\
\hline 11 & 1317 & 0 & $\mathbf{0}$ & $\mathbf{0}$ & -1 & HOTEL CLERKS \\
\hline 11 & 1318 & 0 & $\mathbf{0}$ & $\mathbf{0}$ & -1 & $\begin{array}{l}\text { TRANSPORTATION TICKET \& RESERVATION } \\
\text { AGENTS }\end{array}$ \\
\hline 11 & 1319 & $\mathbf{0}$ & $\mathbf{0}$ & 0 & -1 & RECEPTIONISTS \\
\hline 11 & 1323 & $\mathbf{0}$ & 0 & 0 & -1 & INFORMATION CLERKS, N.E.C. \\
\hline 11 & 1325 & $\mathbf{0}$ & $\mathbf{0}$ & 0 & -1 & CLASSIFIED-AD CLERKS \\
\hline 11 & 1326 & $\mathbf{0}$ & $\mathbf{0}$ & 0 & -1 & CORRESPONDENCE CLERKS \\
\hline 11 & 1327 & 0 & $\mathbf{0}$ & 0 & -1 & ORDER CLERKS \\
\hline 11 & 1328 & 0 & $\mathbf{0}$ & 0 & -1 & $\begin{array}{l}\text { PERSONNEL CLERKS, EXCEPT PAYROLL \& } \\
\text { TIMEKEEPING }\end{array}$ \\
\hline 11 & 1329 & $\mathbf{0}$ & $\mathbf{0}$ & $\mathbf{0}$ & -1 & LIBRARY CLERKS \\
\hline 11 & 1335 & $\mathbf{0}$ & $\mathbf{0}$ & $\mathbf{0}$ & -1 & FILE CLERKS \\
\hline 11. & 1336 & $\mathbf{0}$ & $\mathbf{0}$ & $\mathbf{0}$ & -1 & RECORDS CLERKS \\
\hline 11 & 1337 & $\mathbf{0}$ & $\mathbf{0}$ & $\mathbf{0}$ & -1 & $\begin{array}{l}\text { BOOKKEEPERS, ACCOUNTING, \& AUDITING } \\
\text { CLERKS }\end{array}$ \\
\hline 11 & 1338 & 0 & 0 & $\mathbf{0}$ & -1 & PAYROLL \& TIMEKEEPING CLERKS \\
\hline 11 & 1339 & 0 & $\mathbf{0}$ & $\mathbf{0}$ & -1 & BILLING CLERKS \\
\hline 11 & 1343 & 0 & 0 & $\mathbf{0}$ & -1 & COST \& RATE CLERKS \\
\hline 11 & 1344 & 0 & $\mathbf{0}$ & $\mathbf{0}$ & -1 & $\begin{array}{l}\text { BILLING, POSTING, \& CALCULATING } \\
\text { MACHINE OPERATORS }\end{array}$ \\
\hline 11 & 1345 & 0 & $\mathbf{0}$ & $\mathbf{0}$ & -1 & DUPLICATING MACHINE OPERATORS \\
\hline 11 & 1346 & $\mathbf{0}$ & 0 & 0 & -1 & $\begin{array}{l}\text { MAIL PREPARING \& PAPER HANDLING } \\
\text { MACHINE OPERATORS }\end{array}$ \\
\hline 11 & 1347 & $\mathbf{0}$ & $\mathbf{0}$ & $\mathbf{0}$ & -1 & OFFICE MACHINE OPERATORS, N.E.C. \\
\hline 11 & 1348 & 0 & $\mathbf{0}$ & 0 & -1 & TELEPHONE OPERATORS \\
\hline 11 & 1349 & 0 & $\mathbf{0}$ & 0 & -1 & TELEGRAPHERS \\
\hline 11 & 1353 & $\mathbf{0}$ & 0 & $\mathbf{0}$ & -1 & $\begin{array}{l}\text { COMMUNICATIONS EQUIPMENT OPERATORS, } \\
\text { N.E.C. }\end{array}$ \\
\hline 11 & 1354 & 0 & $\mathbf{0}$ & $\mathbf{0}$ & -1 & POSTAL CLERKS, EXCEPT MAIL CARRIERS \\
\hline 11 & 1355 & 0 & $\mathbf{0}$ & $\mathbf{0}$ & -1 & MAIL CARRIERS, POSTAL SERVICE \\
\hline 11 & 1356 & $\mathbf{0}$ & 0 & 0 & -1 & MAIL CLERKS, EXCEPT POSTAL SERVICE \\
\hline 11 & 1357 & $\mathbf{0}$ & $\mathbf{0}$ & $\mathbf{0}$ & -1 & MESSENGERS \\
\hline 11 & 1359 & $\mathbf{0}$ & $\mathbf{0}$ & $\mathbf{0}$ & -1 & DISPATCHERS \\
\hline 11 & 1363 & $\mathbf{0}$ & $\mathbf{0}$ & $\mathbf{0}$ & -1 & PRODUCTION COORDINATORS \\
\hline 11. & 1364 & $\mathbf{0}$ & $\mathbf{0}$ & $\mathbf{0}$ & -1 & TRAFFIC, SHIPPING, \& RECEIVING CLERKS \\
\hline 11 & 1365 & $\mathbf{0}$ & $\mathbf{0}$ & $\mathbf{0}$ & -1 & STOCK \& INVENTORY CLERKS \\
\hline 11 & 1366 & $\mathbf{0}$ & $\mathbf{0}$ & $\mathbf{0}$ & -1 & METER READERS \\
\hline 11 & 1368 & 0 & 0 & 0 & -1 & WEIGHERS, MEASURERS, \& CHECKERS \\
\hline 11 & 1369 & $\mathbf{0}$ & $\mathbf{0}$ & $\mathbf{0}$ & -1 & SAMPLERS \\
\hline 11 & 1373 & 0 & $\mathbf{0}$ & $\mathbf{0}$ & -1 & EXPEDITERS \\
\hline
\end{tabular}




\begin{tabular}{|c|c|c|c|c|c|c|}
\hline Maior & Minor & Param & Pen & Line & Decltr & Attribute Description \\
\hline 11 & 1374 & $\mathbf{0}$ & $\mathbf{0}$ & $\mathbf{0}$ & -1 & $\begin{array}{l}\text { MATERIAL RECORDING, SCHEDULING, \& } \\
\text { DISTRIB. CLERKS, N.E.C. }\end{array}$ \\
\hline 11 & 1375 & $\mathbf{0}$ & $\mathbf{0}$ & 0 & -1 & $\begin{array}{l}\text { INSURANCE ADJUSTERS, EXAMINERS, \& } \\
\text { INVESTIGATORS }\end{array}$ \\
\hline 11 & 1376 & 0 & $\mathbf{0}$ & 0 & -1 & $\begin{array}{l}\text { INVESTIGATORS \& ADJUSTERS, EXCEPT } \\
\text { INSURANCE }\end{array}$ \\
\hline 11 & 1377 & $\mathbf{0}$ & 0 & $\mathbf{0}$ & -1 & ELIGIBILITY CLERKS, SOCIAL WELFARE \\
\hline 11 & 1378 & $\mathbf{0}$ & $\mathbf{0}$ & $\mathbf{0}$ & -1 & BILL \& ACCOUNT COLLECTORS \\
\hline 11 & 1379 & $\mathbf{0}$ & $\mathbf{0}$ & $\mathbf{0}$ & -1 & GENERAL OFFICE CLERKS \\
\hline 11 & 1383 & $\mathbf{0}$ & 0 & $\mathbf{0}$ & -1 & BANK TELLERS \\
\hline 11 & 1384 & $\mathbf{0}$ & 0 & 0 & -1 & PROOFREADERS \\
\hline 11 & 1385 & $\mathbf{0}$ & $\mathbf{0}$ & $\mathbf{0}$ & -1 & DATA-ENTRY KEYERS \\
\hline 11 & 1386 & $\mathbf{0}$ & $\mathbf{0}$ & $\mathbf{0}$ & -1 & STATISTICAL CLERKS \\
\hline 11 & 1387 & $\mathbf{0}$ & $\mathbf{0}$ & 0 & -1 & TEACHERS' AIDES \\
\hline 11 & 1389 & 0 & 0 & 0 & -1 & $\begin{array}{l}\text { ADMINISTRATIVE SUPPORT OCCUPATION, } \\
\text { N.E.C. }\end{array}$ \\
\hline 11 & 1403 & $\mathbf{0}$ & 0 & $\mathbf{0}$ & -1 & LAUNDERERS \& IRONERS \\
\hline 11 & 1404 & $\mathbf{0}$ & $\mathbf{0}$ & 0 & -1 & COOKS, PRIVATE HOUSEHOLD \\
\hline 11 & 1405 & $\mathbf{0}$ & $\mathbf{0}$ & $\mathbf{0}$ & -1 & HOUSEKEEPERS \& BUTLERS \\
\hline 11 & 1406 & $\mathbf{0}$ & $\mathbf{0}$ & $\mathbf{0}$ & -1 & $\begin{array}{l}\text { CHILD CARE WORKERS, PRIVATE } \\
\text { HOUSEHOLD }\end{array}$ \\
\hline 11 & 1407 & 0 & 0 & 0 & -1 & $\begin{array}{l}\text { PRIVATE HOUSEHOLD CLEANERS \& } \\
\text { SERVANTS }\end{array}$ \\
\hline 11 & 1413 & 0 & $\mathbf{0}$ & 0 & -1 & $\begin{array}{l}\text { SUPERVISORS, FIREFIGHTING \& FIRE } \\
\text { PREVENTION OCCUPATIONS }\end{array}$ \\
\hline 11 & 1414 & $\mathbf{0}$ & $\mathbf{0}$ & $\mathbf{0}$ & -1 & SUPERVISORS, POLICE \& DETECTIVES \\
\hline 11 & 1415 & $\mathbf{0}$ & $\mathbf{0}$ & $\mathbf{0}$ & -1 & SUPERVISORS, GUARDS \\
\hline 11 & 1416 & $\mathbf{0}$ & $\mathbf{0}$ & $\mathbf{0}$ & -1 & $\begin{array}{l}\text { FIRE INSPECTION \& FIRE PREVENTION } \\
\text { OCCUPATIONS }\end{array}$ \\
\hline 11 & 1417 & $\mathbf{0}$ & $\mathbf{0}$ & 0 & -1 & FIREFIGHTING OCCUPATIONS \\
\hline 11 & 1418 & $\mathbf{0}$ & $\mathbf{0}$ & $\mathbf{0}$ & -1 & POLICE \& DETECTIVES, PUBLIC SERVICE \\
\hline 11 & 1423 & $\mathbf{0}$ & 0 & $\mathbf{0}$ & -1 & $\begin{array}{l}\text { SHERIFFS, BAILIFFS, \& OTHER LAW } \\
\text { ENFORCEMENT OFFICERS }\end{array}$ \\
\hline 11 & 1424 & $\mathbf{0}$ & $\mathbf{0}$ & $\mathbf{0}$ & -1 & CORRECTIONAL INSTITUTION OFFICERS \\
\hline 11 & 1425 & $\mathbf{0}$ & 0 & $\mathbf{0}$ & -1 & CROSSING GUARDS \\
\hline 11 & 1426 & $\mathbf{0}$ & $\mathbf{0}$ & $\mathbf{0}$ & -1 & GUARDS \& POLICE, EXCEPT PUBLIC SERVICE \\
\hline 11 & 1427 & $\mathbf{0}$ & 0 & 0 & -1 & PROTECTIVE SERVICE OCCUPATIONS, N.E.C. \\
\hline 11 & 1433 & $\mathbf{0}$ & 0 & $\mathbf{0}$ & -1 & $\begin{array}{l}\text { SUPERVISORS, FOOD PREPARATION \& } \\
\text { SERVICE OCCUPATIONS }\end{array}$ \\
\hline 11 & 1434 & $\mathbf{0}$ & $\mathbf{0}$ & $\mathbf{0}$ & -1 & BARTENDERS \\
\hline 11 & 1435 & $\mathbf{0}$ & 0 & $\mathbf{0}$ & -1 & WAITERS \& WAITRESSES \\
\hline 11 & 1436 & $\mathbf{0}$ & 0 & $\mathbf{0}$ & -1 & COOKS, EXCEPT SHORT ORDER \\
\hline 11 & 1437 & $\mathbf{0}$ & $\mathbf{0}$ & $\mathbf{0}$ & -1 & SHORT-ORDER COOKS \\
\hline 11 & 1438 & $\mathbf{0}$ & $\mathbf{0}$ & $\mathbf{0}$ & -1 & $\begin{array}{l}\text { FOOD COUNTER, FOUNTAIN \& RELATED } \\
\text { OCCUPATIONS }\end{array}$ \\
\hline 11 & 1439 & $\mathbf{0}$ & $\mathbf{0}$ & 0 & -1 & KITCHEN WORKERS, FOOD PREPARATION \\
\hline 11 & 1443 & $\mathbf{0}$ & 0 & 0 & -1 & WAITERS' \& WAITRESSES' ASSISTANTS \\
\hline 11 & 1444 & $\mathbf{0}$ & 0 & 0 & -1 & $\begin{array}{l}\text { MISCELLANEOUS FOOD P PEPARATION } \\
\text { OCCUPATIONS }\end{array}$ \\
\hline 11 & 1445 & $\mathbf{0}$ & 0 & 0 & -1 & DENTAL ASSISTANTS \\
\hline
\end{tabular}




\begin{tabular}{|c|c|c|c|c|c|c|}
\hline Major & Minor & Param & Ben & Line & Decltr & Attribute Description \\
\hline 11 & 1446 & 0 & $\mathbf{0}$ & $\mathbf{0}$ & -1 & HEALTH AIDES, EXCEPT NURSING \\
\hline 11 & 1447 & $\mathbf{0}$ & $\mathbf{0}$ & 0 & -1 & NURSING AIDES, ORDERLIES, \& ATTENDANTS \\
\hline 11 & 1448 & 0 & 0 & $\mathbf{0}$ & -1 & $\begin{array}{l}\text { SUPERVISORS, CLEANING \& BUILDING } \\
\text { SERVICE WORKERS }\end{array}$ \\
\hline 11 & 1449 & 0 & 0 & 0 & -1 & MAIDS \& HOUSEMEN \\
\hline 11 & 1453 & 0 & $\mathbf{0}$ & 0 & -1 & JANITORS \& CLEANERS \\
\hline 11 & 1454 & $\mathbf{0}$ & $\mathbf{0}$ & $\mathbf{0}$ & -1 & ELEVATOR OPERATORS \\
\hline 11 & 1455 & $\mathbf{0}$ & $\mathbf{0}$ & 0 & -1 & PEST CONTROL OCCUPATIONS \\
\hline 11 & 1456 & 0 & s & 0 & -1 & $\begin{array}{l}\text { SUPERVISORS, PERSONAL SERVICE } \\
\text { OCCUPATIONS }\end{array}$ \\
\hline 11 & 1457 & 0 & 0 & $\mathbf{0}$ & -1 & BARBERS \\
\hline 11 & 1458 & 0 & $\mathbf{0}$ & $\mathbf{0}$ & -1 & HAIRDRESSERS \& COSMETOLOGISTS \\
\hline 11 & 1459 & 0 & $\mathbf{0}$ & $\mathbf{0}$ & -1 & $\begin{array}{l}\text { ATTENDANTS, AMUSEMENT \& RECREATION } \\
\text { FACILITIES }\end{array}$ \\
\hline 11 & 1463 & $\mathbf{0}$ & $\mathbf{0}$ & $\mathbf{0}$ & -1 & GUIDES \\
\hline 11 & 1464 & 0 & $\mathbf{0}$ & $\mathbf{0}$ & -1 & USHERS \\
\hline 11 & 1465 & $\mathbf{0}$ & $\mathbf{0}$ & 0 & -1 & PUBLIC TRANSPORTATION ATTENDANTS \\
\hline 11 & 1466 & 0 & $\mathbf{0}$ & $\mathbf{0}$ & -1 & BAGGAGE PORTERS \& BELLHOPS \\
\hline 11 & 1467 & $\mathbf{0}$ & $\mathbf{0}$ & $\mathbf{0}$ & -1 & WELFARE SERVICE AIDES \\
\hline 11 & 1468 & 0 & $\mathbf{0}$ & $\mathbf{0}$ & -1 & $\begin{array}{l}\text { CHILD CARE WORKERS, EXCEPT PRIVATE } \\
\text { HOUSEHOLD }\end{array}$ \\
\hline 11 & 1469 & $\mathbf{0}$ & 0 & $\mathbf{0}$ & -1 & PERSONAL SERVICE OCCUPATIONS, N.E.C. \\
\hline 11 & 1473 & $\mathbf{0}$ & 0 & 0 & -1 & FARMERS, EXCEPT HORTICULTURAL \\
\hline 11 & 1474 & 0 & $\mathbf{0}$ & 0 & -1 & HORTICULTURAL SPECIALTY FARMERS \\
\hline 11 & 1475 & 0 & 0 & $\mathbf{0}$ & -1 & $\begin{array}{l}\text { MANAGERS, FARMS, EXCEPT } \\
\text { HORTICULTURAL }\end{array}$ \\
\hline 11 & 1476 & 0 & $\mathbf{0}$ & $\mathbf{0}$ & -1 & $\begin{array}{l}\text { MANAGERS, HORTICULTURAL SPECIALTY } \\
\text { FARMS }\end{array}$ \\
\hline 11 & 1477 & $\mathbf{0}$ & $\mathbf{0}$ & $\mathbf{0}$ & -1 & SUPERVISORS, FARM WORKERS \\
\hline 11 & 1479 & $\mathbf{0}$ & $\mathbf{0}$ & $\mathbf{0}$ & -1 & $\begin{array}{l}\text { FARM WORKERS, AGRICULTURAL } \\
\text { PRODUCIION, CROPS }\end{array}$ \\
\hline 11 & 1483 & $\mathbf{0}$ & $\mathbf{0}$ & $\mathbf{0}$ & -1 & MARINE LIFE CULTIVATION WORKERS \\
\hline 11 & 1484 & 0 & 0 & 0 & -1 & NURSERY WORKERS \\
\hline 11 & 1485 & 0 & 0 & 0 & -1 & $\begin{array}{l}\text { SUPERVISORS, RELATED AGRICULTURAL } \\
\text { OCCUPATIONS }\end{array}$ \\
\hline 11 & 1486 & $\mathbf{0}$ & 0 & 0 & -1 & $\begin{array}{l}\text { GROUNDSKEEPERS \& GARDENERS, EXCEPT } \\
\text { FARM }\end{array}$ \\
\hline 11 & 1487 & 0 & 0 & 0 & -1 & ANIMAL CARETAKERS, EXCEPT FARM \\
\hline 11 & 1488 & $\mathbf{0}$ & $\mathbf{0}$ & 0 & -1 & $\begin{array}{l}\text { GRADERS \& SORTERS, AGRICULTURAL } \\
\text { PRODUCTS }\end{array}$ \\
\hline 11 & 1489 & $\mathbf{0}$ & $\mathbf{0}$ & $\mathbf{0}$ & -1 & INSPECTORS, AGRICULTURAL PRODUCTS \\
\hline 11 & 1494 & $\mathbf{0}$ & $\mathbf{0}$ & 0 & -1 & $\begin{array}{l}\text { SUPERVISORS, FOIRESTRY \& LOGGING } \\
\text { WORKERS }\end{array}$ \\
\hline 11 & 1495 & 0 & 0 & $\mathbf{0}$ & -1 & FORESTRY WORKERS, EXCEPT LOGGING \\
\hline 11 & 1496 & 0 & $\mathbf{0}$ & $\mathbf{0}$ & -1 & TIMBER CUTTING \& LOGGING OCCUPATIONS \\
\hline 11 & 1497 & 0 & $\mathbf{0}$ & $\mathbf{0}$ & -1 & $\begin{array}{l}\text { CAPTAINS \& OTHER OFFICERS, FISHING } \\
\text { VESSELS }\end{array}$ \\
\hline 11 & 1498 & $\mathbf{0}$ & 0 & 0 & -1 & FISHERS \\
\hline 11 & 1499 & 0 & 0 & 0 & -1 & HUNTERS \& TRAPPERS \\
\hline 11 & 1503 & $\mathbf{0}$ & 0 & 0 & -1 & SUPERVISORS, MECHANICS \& REPAIRERS \\
\hline
\end{tabular}




\begin{tabular}{|c|c|c|c|c|c|c|}
\hline Maior & Minor & Param & Pen & Line & Decltr & Attribute Description \\
\hline 11 & 1505 & 0 & 0 & $\mathbf{0}$ & -1 & $\begin{array}{l}\text { AUTOMOBILE MECHANICS, EXCEPT } \\
\text { APPRENTICES }\end{array}$ \\
\hline 11 & 1506 & $\mathbf{0}$ & 0 & $\mathbf{0}$ & -1 & AUTOMOBILE MECHANIC APPRENTICES \\
\hline 11 & 1507 & 0 & 0 & 0 & -1 & $\begin{array}{l}\text { BUS, TRUCK, \& STATIONARY ENGINE } \\
\text { MECHANICS }\end{array}$ \\
\hline 11 & 1508 & 0 & 0 & $\mathbf{0}$ & -1 & AIRCRAFT ENGINE MECHANICS \\
\hline 11 & 1509 & $\mathbf{0}$ & $\mathbf{0}$ & $\mathbf{0}$ & -1 & SMALL ENGINE REPAIRERS \\
\hline 11 & 1514 & $\mathbf{0}$ & $\mathbf{0}$ & $\mathbf{0}$ & -1 & AUTOMOBILE BODY \& RELATED REPAIRERS \\
\hline 11 & 1515 & $\mathbf{0}$ & 0 & $\mathbf{0}$ & -1 & AIRCRAFT MECHANICE, EXCEPT ENGINE \\
\hline 11 & 1516 & $\mathbf{0}$ & 0 & $\mathbf{0}$ & -1 & HEAVY EQUIPMENT MECHANICS \\
\hline 11 & 1517 & $\mathbf{0}$ & $\mathbf{0}$ & $\mathbf{0}$ & -1 & FARM EQUIPMENT MECHANICS \\
\hline 11 & 1518 & $\mathbf{0}$ & $\mathbf{0}$ & $\mathbf{0}$ & -1 & INDUSTRIAL MACHINERY REPAIRERS \\
\hline 11 & 1519 & $\mathbf{0}$ & $\mathbf{0}$ & $\mathbf{0}$ & -1 & MACHINERY MAINTENANCE OCCUPATIONS \\
\hline 11 & 1523 & $\mathbf{0}$ & 0 & $\mathbf{0}$ & -1 & $\begin{array}{l}\text { ELECTRONIC REPAIRERS, COMMUNICATIONS } \\
\text { \& INDUSTRIAL EOUIPMENT }\end{array}$ \\
\hline 11 & 1525 & $\mathbf{0}$ & $\mathbf{0}$ & $\mathbf{0}$ & -1 & DATA PROCESSING EQUIPMENT REPAIRERS \\
\hline 11 & 1526 & 0 & $\mathbf{0}$ & 0 & -1 & $\begin{array}{l}\text { HOUSEHOLD APPLIANCE \& POWER TOOL } \\
\text { REPAIRERS }\end{array}$ \\
\hline 11 & 1527 & $\mathbf{0}$ & $\mathbf{0}$ & $\mathbf{0}$ & -1 & TELEPHONE LINE INSTALLERS \& REPAIRERS \\
\hline 11 & 1529 & $\mathbf{0}$ & $\mathbf{0}$ & $\mathbf{0}$ & -1 & TELEPHONE INSTALLERS \& REPAIRERS \\
\hline 11 & 1533 & $\mathbf{0}$ & $\mathbf{0}$ & $\mathbf{0}$ & -1 & $\begin{array}{l}\text { MISCELLANEOUS ELECTRICAL \& } \\
\text { ELECTRONIC EQUIPMENT REPAIRERS }\end{array}$ \\
\hline 11 & 1534 & 0 & $\mathbf{0}$ & 0 & -1 & $\begin{array}{l}\text { HEATING, AIR CONDITIONING, \& } \\
\text { REFRIGERATION MECHANICS }\end{array}$ \\
\hline 11 & 1535 & 0 & $\mathbf{0}$ & 0 & -1 & $\begin{array}{l}\text { CAMERA, WATCH, \& MUSICAL INSTRUMENT } \\
\text { REPAIRERS }\end{array}$ \\
\hline 11 & 1536 & $\mathbf{0}$ & $\mathbf{0}$ & $\mathbf{0}$ & -1 & LOCKSMITHS \& SAFE REPAIRERS \\
\hline 11 & 1538 & 0 & $\mathbf{0}$ & $\mathbf{0}$ & -1 & OFFICE MACHINE REPAIRERS \\
\hline 11 & 1539 & $\mathbf{0}$ & 0 & $\mathbf{0}$ & -1 & $\begin{array}{l}\text { MECHANICAL CONTROLS \& VALVE } \\
\text { REPAIRERS }\end{array}$ \\
\hline 11 & 1543 & 0 & $\mathbf{0}$ & $\mathbf{0}$ & -1 & ELEVATOR INSTALLERS \& REPAIRERS \\
\hline 11 & 1544 & $\mathbf{0}$ & $\mathbf{0}$ & $\mathbf{0}$ & -1 & MILLWRIGHTS \\
\hline 11 & 1547 & 0 & 0 & 0 & -1 & SPECIFIED MECHANICS \& REPAIRERS, N.E.C. \\
\hline 11 & 1549 & 0 & 0 & $\mathbf{0}$ & -1 & NOT SPECIFIED MECHANICS \& REPAIRERS \\
\hline 11 & 1553 & 0 & $\mathbf{0}$ & $\mathbf{0}$ & -1 & $\begin{array}{l}\text { SUPERVISORS; BRICKMASONS, } \\
\text { STONEMASONS, \& TILE SETTERS }\end{array}$ \\
\hline 11 & 1554 & 0 & $\mathbf{0}$ & $\mathbf{0}$ & -1 & $\begin{array}{l}\text { SUPERVISORS, CARPENTERS \& RELATED } \\
\text { WORKERS }\end{array}$ \\
\hline 11 & 1555 & 0 & 0 & $\mathbf{0}$ & -1 & $\begin{array}{l}\text { SUPERVISORS, ELECTRICIANS \& POWER } \\
\text { TRANSMISSION INSTALLERS }\end{array}$ \\
\hline 11 & 1556 & 0 & 0 & 0 & -1 & $\begin{array}{l}\text { SUPERVISORS; PAINTERS, PAPERHANGERS, \& } \\
\text { PLASTERERS }\end{array}$ \\
\hline 11 & 1557 & 0 & 0 & $\mathbf{0}$ & -1 & $\begin{array}{l}\text { SUPERVISORS; PLUMBERS, PIPEFITTERS, \& } \\
\text { STEAMFITTERS }\end{array}$ \\
\hline 11 & 1558 & 0 & 0 & $\mathbf{0}$ & -1 & $\begin{array}{l}\text { CONSTRUCTION TRADES SUPERVISORS, } \\
\text { N.E.C. }\end{array}$ \\
\hline 11 & 1563 & 0 & 0 & 0 & -1 & $\begin{array}{l}\text { BRICKMASONS \& STONEMASONS, EXCEPT } \\
\text { APPRENTICES }\end{array}$ \\
\hline 11 & 156 & 0 & 0 & 0 & -1 & BRICKMASON \& STONEMASON APPRENTICES \\
\hline 11 & 1565 & 0 & 0 & 0 & -1 & TILE SETTERS, HARD \& SOFT \\
\hline
\end{tabular}




\begin{tabular}{|c|c|c|c|c|c|c|}
\hline Maior & Minor & Param & Pen & Line & Decltr & Attribute Description \\
\hline 11 & 1566 & 0 & 0 & $\mathbf{0}$ & -1 & CARPET INSTALLERS \\
\hline 11 & 1567 & $\mathbf{0}$ & 0 & 0 & -1 & CARPENTERS, EXCEPT APPRENTICES \\
\hline 11 & 1569 & 0 & 0 & 0 & -1 & CARPENTER APPRENTICES \\
\hline 11 & 1573 & 0 & 0 & 0 & -1 & DRYWALL INSTALLERS \\
\hline 11 & 1575 & 0 & 0 & 0 & -1 & ELECTRICIANS, EXCEPT APPRENTICES \\
\hline 11 & 1576 & $\mathbf{0}$ & 0 & 0 & -1 & ELECTRICIAN APPRENTICES \\
\hline 11 & 1577 & 0 & 0 & 0 & -1 & $\begin{array}{l}\text { ELECTRICAL POWER INSTALLERS \& } \\
\text { REPAIRERS }\end{array}$ \\
\hline 11 & 1579 & 0 & 0 & $\mathbf{0}$ & -1 & PAINTERS, CONSTRUCTION \& MAINTENANCE \\
\hline 11 & 1583 & 0 & 0 & 0 & -1 & PAPERHANGERS \\
\hline 11 & 1584 & $\mathbf{0}$ & $\mathbf{0}$ & $\mathbf{0}$ & -1 & PLASTERERS \\
\hline 11 & 1585 & 0 & 0 & $\mathbf{0}$ & -1 & $\begin{array}{l}\text { PLUMBERS, PIPEFITTERS, \& STEAMFITTERS, } \\
\text { EXCEPT APPRENTICES }\end{array}$ \\
\hline 11 & 1587 & 0 & $\mathbf{0}$ & 0 & -1 & $\begin{array}{l}\text { PLUMBER, PIPEFITTER, \& STEAMFITTER } \\
\text { APPRENTICES }\end{array}$ \\
\hline 11 & 1588 & 0 & 0 & 0 & -1 & CONCRETE \& TERRAZZO FINISHERS \\
\hline 11 & 1589 & $\mathbf{0}$ & 0 & $\mathbf{0}$ & -1 & GLAZIERS \\
\hline 11 & 1593 & 0 & $\mathbf{0}$ & $\mathbf{0}$ & -1 & INSULATION WORKERS \\
\hline 11 & 1594 & 0 & 0 & $\mathbf{0}$ & -1 & $\begin{array}{l}\text { PAVING, SURFACING, \& TAMPING } \\
\text { EQUIPMENT OPERATORS }\end{array}$ \\
\hline 11 & 1595 & $\mathbf{0}$ & $\mathbf{0}$ & 0 & -1 & ROOFERS \\
\hline 11 & 1596 & $\mathbf{0}$ & $\mathbf{0}$ & $\mathbf{0}$ & -1 & SHEETMETAL DUCT INSTALLERS \\
\hline 11 & 1597 & $\mathbf{0}$ & $\mathbf{0}$ & $\mathbf{0}$ & -1 & STRUCTURAL METAL WORKERS \\
\hline 11 & 1598 & 0 & $\mathbf{0}$ & 0 & -1 & DRILLERS, EARTH \\
\hline 11 & 1599 & $\mathbf{0}$ & $\mathbf{0}$ & $\mathbf{0}$ & -1 & CONSTRUCTION TRADES, N.E.C. \\
\hline 11 & 1613 & $\mathbf{0}$ & $\mathbf{0}$ & $\mathbf{0}$ & -1 & SUPERVISORS, EXTRACTIVE OCCUPATIONS \\
\hline 11 & 1614 & $\mathbf{0}$ & $\mathbf{0}$ & $\mathbf{0}$ & -1 & DRILLERS, OIL WELL \\
\hline 11 & 1615 & 0 & $\mathbf{0}$ & $\mathbf{0}$ & -1 & EXPLOSIVES WORKERS \\
\hline 11 & 1616 & $\mathbf{0}$ & $\mathbf{0}$ & $\mathbf{0}$ & -1 & MINING MACHINE OPERATORS \\
\hline 11 & 1617 & $\mathbf{0}$ & $\mathbf{0}$ & 0 & -1 & MINING OCCUPATIONS, N.E.C. \\
\hline 11 & 1633 & $\mathbf{0}$ & $\mathbf{0}$ & $\mathbf{0}$ & -1 & SUPERVISORS, PRODUCTION OCCUPATIONS \\
\hline 11 & 1634 & $\mathbf{0}$ & $\mathbf{0}$ & $\mathbf{0}$ & -1 & TOOL \& DIE MAKERS, EXCEPT APPRENTICES \\
\hline 11 & 1635 & $\mathbf{0}$ & $\mathbf{0}$ & $\mathbf{0}$ & -1 & TOOL \& DIE MAKER APPRENTICES \\
\hline 11 & 1636 & $\mathbf{0}$ & $\mathbf{0}$ & $\mathbf{0}$ & -1 & PRECISION ASSEMBLERS, METAL \\
\hline 11 & 1637 & 0 & $\mathbf{0}$ & $\mathbf{0}$ & -1 & MACHINISTS, EXCEPT APPRENTICES \\
\hline 11 & 1639 & $\mathbf{0}$ & $\mathbf{0}$ & $\mathbf{0}$ & -1 & MACHINIST APPRENTICES \\
\hline 11 & 1643 & $\mathbf{0}$ & $\mathbf{0}$ & $\mathbf{0}$ & -1 & BOILERMAKERS \\
\hline 11 & 1644 & $\mathbf{0}$ & 0 & 0 & -1 & $\begin{array}{l}\text { PRECISION GRINDERS, FILERS, \& TOOL } \\
\text { SHARPENERS }\end{array}$ \\
\hline 11 & 1645 & $\mathbf{0}$ & $\mathbf{0}$ & 0 & -1 & PATTERNMAKERS \& MODEL MAKERS, META \\
\hline 11 & 1646 & $\mathbf{0}$ & 0 & $\mathbf{0}$ & -1 & LAY-OUT WORKERS \\
\hline 11 & 1647 & $\mathbf{0}$ & $\mathbf{0}$ & 0 & -1 & $\begin{array}{l}\text { PRECIOUS STONES \& METALS WORKERS } \\
\text { (JEWELERS) }\end{array}$ \\
\hline 11 & 1649 & $\mathbf{0}$ & $\mathbf{0}$ & 0 & -1 & ENGRAVERS, METAL \\
\hline 11 & 1653 & $\mathbf{0}$ & $\mathbf{0}$ & 0 & -1 & $\begin{array}{l}\text { SHEET METAI WORKERS, EXCEPT } \\
\text { APPRENTICES }\end{array}$ \\
\hline 11 & 1654 & 0 & 0 & 0 & -1 & SHEET METAL WORKER APPRENTICES \\
\hline 11 & 1655 & 0 & 0 & 9 & -1 & $\begin{array}{l}\text { MISCELLANEOUS PRECISION METAL } \\
\text { WORKERS }\end{array}$ \\
\hline 11 & 1656 & $\mathbf{0}$ & $\mathbf{0}$ & $\mathbf{0}$ & -1 & PATTERNMAKERS \& MODEL MAKERS, WOOD \\
\hline
\end{tabular}




\begin{tabular}{|c|c|c|c|c|c|c|}
\hline Major & Minor & Baram & Pen & Line & Decltr & Attribute Description \\
\hline 11 & 1657 & $\mathbf{0}$ & 0 & $\mathbf{0}$ & -1 & CABINET MAKERS \& BENCH CARPENTERS \\
\hline 11 & 1658 & $\mathbf{0}$ & $\mathbf{0}$ & $\mathbf{0}$ & -1 & FURNITURE \& WOOD FINISHERS \\
\hline 11 & 1659 & 0 & 0 & $\mathbf{0}$ & -1 & MISCELLANEOUS PRECISION WOODWORKERS \\
\hline 11 & 1666 & $\mathbf{0}$ & 0 & $\mathbf{0}$ & -1 & DRESSMAKERS \\
\hline 11 & 1667 & $\mathbf{0}$ & 0 & 0 & -1 & TAILORS \\
\hline 11 & 1668 & $\mathbf{0}$ & 0 & 0 & -1 & UPHOLSTERERS \\
\hline 11 & 1669 & $\mathbf{0}$ & $\mathbf{0}$ & 0 & -1 & SHOE REPAIRERS \\
\hline 11 & 1673 & $\mathbf{0}$ & 0 & $\mathbf{0}$ & -1 & APPAREL \& FABRIC PATTERNMAKERS \\
\hline 11 & 1674 & $\mathbf{0}$ & 0 & 0 & -1 & $\begin{array}{l}\text { MISCELLANEOUS PRECISION APPAREL \& } \\
\text { FABRIC WORKERS }\end{array}$ \\
\hline 11 & 1675 & $\mathbf{0}$ & 0 & 0 & -1 & $\begin{array}{l}\text { HAND MOLDERS \& SHAPERS, EXCEPT } \\
\text { JEWELERS }\end{array}$ \\
\hline 11 & 1676 & $\mathbf{0}$ & 0 & $\mathbf{0}$ & -1 & $\begin{array}{l}\text { PATTERNMAKERS, LAY-OUT WORKERS, \& } \\
\text { CUTTERS }\end{array}$ \\
\hline 11 & 1677 & 0 & 0 & 0 & -1 & OPTICAL GOODS WORKERS \\
\hline 11 & 1678 & $\mathbf{0}$ & 0 & 0 & -1 & $\begin{array}{l}\text { DENTAL LABORATORY \& MEDICAL } \\
\text { APPLIANCE TECHNICIANS }\end{array}$ \\
\hline 11 & 1679 & 0 & 0 & $\mathbf{0}$ & -1 & BOOKBINDERS \\
\hline 11 & 1683 & $\mathbf{0}$ & $\mathbf{0}$ & $\mathbf{0}$ & -1 & $\begin{array}{l}\text { ELECTRICAL \& ELECTRONIC EQUIPMENT } \\
\text { ASSEMBLERS }\end{array}$ \\
\hline 11 & 1684 & $\mathbf{0}$ & $\mathbf{0}$ & $\mathbf{0}$ & -1 & $\begin{array}{l}\text { MISCELLANEOUS PRECISION WORKERS, } \\
\text { N.E.C. }\end{array}$ \\
\hline 11 & 1686 & $\mathbf{0}$ & $\mathbf{0}$ & $\mathbf{0}$ & -1 & BUTCHERS \& MEAT CUTTERS \\
\hline 11 & 1687 & $\mathbf{0}$ & $\mathbf{0}$ & $\mathbf{0}$ & -1 & BAKERS \\
\hline 11 & 1688 & $\mathbf{0}$ & 0 & $\mathbf{0}$ & -1 & FOOD BATCHMAKERS \\
\hline 11 & 1689 & 0 & $\mathbf{0}$ & $\mathbf{0}$ & -1 & INSPECTORS, TESTERS, \& GRADERS \\
\hline 11 & 1693 & 0 & $\mathbf{0}$ & $\mathbf{0}$ & -1 & ADJUSTERS \& CALIBRATORS \\
\hline 11 & 1694 & $\mathbf{0}$ & 0 & $\mathbf{0}$ & -1 & $\begin{array}{l}\text { WATER \& SEWAGE TREATMENT PLANT } \\
\text { OPERATORS }\end{array}$ \\
\hline 11 & 1695 & 0 & 0 & $\mathbf{0}$ & -1 & POWER PLANT OPERATORS \\
\hline 11 & 1696 & 0 & $\mathbf{0}$ & $\mathbf{0}$ & -1 & STATIONARY ENGINEERS \\
\hline 11 & 1699 & 0 & $\mathbf{0}$ & $\mathbf{0}$ & -1 & $\begin{array}{l}\text { MISCELLANEOUS PLANT \& SYSTEM } \\
\text { OPERATORS }\end{array}$ \\
\hline 11 & 1703 & $\mathbf{0}$ & $\mathbf{0}$ & $\mathbf{0}$ & -1 & $\begin{array}{l}\text { LATHE \& TURNING MACHINE SET-UP } \\
\text { OPERATORS }\end{array}$ \\
\hline 11 & 1704 & 0 & $\mathbf{0}$ & $\mathbf{0}$ & -1 & LATHE \& TURNING MACHINE OPERATORS \\
\hline 11 & 1705 & $\mathbf{0}$ & $\mathbf{0}$ & $\mathbf{0}$ & -1 & MILLING \& PLANING MACHINE OPERATORS \\
\hline 11 & 1706 & 0 & $\mathbf{0}$ & 0 & -1 & $\begin{array}{l}\text { PUNCHING \& STAMPING PRESS MACHINE } \\
\text { OPERATORS }\end{array}$ \\
\hline 11 & 1707 & $\mathbf{0}$ & $\mathbf{0}$ & $\mathbf{0}$ & -1 & ROLLING MACHINE OPERATORS \\
\hline 11 & 1708 & 0 & $\mathbf{0}$ & $\mathbf{0}$ & -1 & DRILLING \& BORING MACHINE OPERATORS \\
\hline 11 & 1709 & 0 & 0 & $\mathbf{0}$ & -1 & $\begin{array}{l}\text { GRINDING, ABRADING, BUFFING, \& } \\
\text { POLISHING MACHINE OPERATORS }\end{array}$ \\
\hline 11 & 1713 & $\mathbf{0}$ & $\mathbf{0}$ & $\mathbf{0}$ & -1 & FORGING MACHINE OPERATORS \\
\hline 11 & 1714 & $\mathbf{0}$ & $\mathbf{0}$ & 0 & -1 & $\begin{array}{l}\text { NUMERICAL CONTROL MACHINE } \\
\text { OPERATORS }\end{array}$ \\
\hline 11 & 1715 & $\mathbf{0}$ & 0 & $\mathbf{0}$ & -1 & $\begin{array}{l}\text { MISC. METAL, PLASTIC, STONE, \& GLASS } \\
\text { WORKING MACHINE OPS. }\end{array}$ \\
\hline 11 & 1717 & 0 & 0 & 0 & -1 & FABRICATING MACHINE OPERATORS, N.E.C. \\
\hline 11 & 1719 & 0 & $\mathbf{0}$ & 0 & -1 & MOLDING \& CASTING MACHINE \\
\hline
\end{tabular}




\begin{tabular}{|c|c|c|c|c|c|c|}
\hline Major & Minor & Param & Pen & Line & Decitr & Attribute Description \\
\hline 11 & 1723 & 0 & 0 & $\mathbf{0}$ & -1 & METAL PLATING MACHINE OPERATORS \\
\hline 11 & 1724 & $\mathbf{0}$ & $\mathbf{0}$ & $\mathbf{0}$ & -1 & HEAT TREATING EQUIPMENT OPERATORS \\
\hline 11 & 1725 & 0 & 0 & 0 & -1 & $\begin{array}{l}\text { MISCELLANEOUS METAL \& PLASTIC } \\
\text { PROCESSING MACHINE OPERATORS }\end{array}$ \\
\hline 11 & 1726 & 0 & $\mathbf{0}$ & 0 & -1 & $\begin{array}{l}\text { WOOD LATHE, ROUTING, \& PLANING } \\
\text { MACHINE OPERATORS }\end{array}$ \\
\hline 11 & 1727 & $\mathbf{0}$ & $\mathbf{0}$ & $\mathbf{0}$ & -1 & SAWING MACHINE OPERATORS \\
\hline 11 & 1728 & $\mathbf{0}$ & $\mathbf{0}$ & $\mathbf{0}$ & -1 & SHAPING \& JOINING MACHINE OPERATORS \\
\hline 11 & 1729 & $\mathbf{0}$ & $\mathbf{0}$ & 0 & -1 & NAILING \& TACKING MACHINE OPERATORS \\
\hline 11 & 1733 & 0 & 0 & $\mathbf{0}$ & -1 & $\begin{array}{l}\text { MISCELLANEOUS WOODWORKING MACHINE } \\
\text { OPERATORS }\end{array}$ \\
\hline 11 & 1734 & $\mathbf{0}$ & 0 & $\mathbf{0}$ & -1 & PRINTING MACHINE OPERATORS \\
\hline 11 & 1735 & $\mathbf{0}$ & $\mathbf{0}$ & $\mathbf{0}$ & -1 & PHOTOENGRAVERS \& LITHOGRAPHERS \\
\hline 11 & 1736 & $\mathbf{0}$ & $\mathbf{0}$ & $\mathbf{0}$ & -1 & TYPESETTERS \& COMPOSITORS \\
\hline 11 & 1737 & 0 & 0 & 0 & -1 & $\begin{array}{l}\text { MISCELLANEOUS PRINTING MACHINE } \\
\text { OPERATORS }\end{array}$ \\
\hline 11 & 1738 & $\mathbf{0}$ & $\mathbf{0}$ & $\mathbf{0}$ & -1 & WINDING \& TWISTING MACHINE OPERATORS \\
\hline 11 & 1739 & $\mathbf{0}$ & $\mathbf{0}$ & $\mathbf{0}$ & -1 & $\begin{array}{l}\text { KNITTING, LOOPING, TAPING, \& WEAVING } \\
\text { MACHINE OPERATORS }\end{array}$ \\
\hline 11 & 1743 & $\mathbf{0}$ & $\mathbf{0}$ & $\mathbf{0}$ & -1 & TEXTILE CUTTING MACHINE OPERATORS \\
\hline 11 & 1744 & 0 & $\mathbf{0}$ & 0 & -1 & TEXTILE SEWING MACHINE OPERATORS \\
\hline 11 & 1745 & 0 & $\mathbf{0}$ & 0 & -1 & SHOE MACHINE OPERATORS \\
\hline 11 & 1747 & $\mathbf{0}$ & 0 & $\mathbf{0}$ & -1 & PRESSING MACHINE OPERATORS \\
\hline 11 & 1748 & 0 & $\mathbf{0}$ & $\mathbf{0}$ & -1 & $\begin{array}{l}\text { LAUNDERING \& DRY CLEANING MACHINE } \\
\text { OPERATORS }\end{array}$ \\
\hline 11 & 1749 & $\mathbf{0}$ & $\mathbf{0}$ & $\mathbf{0}$ & -1 & $\begin{array}{l}\text { MISCELLANEOUS TEXTILE MACHINE } \\
\text { OPERATORS }\end{array}$ \\
\hline 11 & 1753 & 0 & $\mathbf{0}$ & 0 & -1 & $\begin{array}{l}\text { CEMENTING \& GLUING MACHINE } \\
\text { OPERATORS }\end{array}$ \\
\hline 11 & 1754 & 0 & 0 & $\mathbf{0}$ & -1 & $\begin{array}{l}\text { PACKAGING \& FILLING MACHINE } \\
\text { OPERATORS }\end{array}$ \\
\hline 11 & 1755 & 0 & $\mathbf{0}$ & 0 & -1 & $\begin{array}{l}\text { EXTRUDING \& FORMING MACHINE } \\
\text { OPERATORS }\end{array}$ \\
\hline 11 & 1756 & 0 & $\mathbf{0}$ & $\mathbf{0}$ & -1 & MIXING \& BLENDING MACHINE OPERATORS \\
\hline 11 & 1757 & $\mathbf{0}$ & 0 & $\mathbf{0}$ & -1 & $\begin{array}{l}\text { SEPARATING, FILTERING, \& CLARIFYING } \\
\text { MACHINE OPERATORS }\end{array}$ \\
\hline 11 & 1758 & 0 & 0 & $\mathbf{0}$ & -1 & $\begin{array}{l}\text { COMPRESSING \& COMPACTING MACHINE } \\
\text { OPERATORS }\end{array}$ \\
\hline 11 & 1759 & $\mathbf{0}$ & 0 & $\mathbf{0}$ & -1 & $\begin{array}{l}\text { PAINTING \& PAINT SPRAYING MACHINE } \\
\text { OPERATORS }\end{array}$ \\
\hline 11 & 1763 & 0 & 0 & 0 & -1 & $\begin{array}{l}\text { ROASTING \& BAKING MACHINE OPERATORS, } \\
\text { FOOD }\end{array}$ \\
\hline 11 & 1764 & 0 & 0 & 0 & -1 & $\begin{array}{l}\text { WASHING, CLEANING, \& PICKLING MACHINE } \\
\text { OPERATORS }\end{array}$ \\
\hline 11 & 1765 & 0 & $\mathbf{0}$ & 0 & -1 & FOLDING MACHINE OPERATORS \\
\hline 11 & 1766 & 0 & 0 & 0 & -1 & $\begin{array}{l}\text { FURNACE, KILN, \& OVEN OPERATORS, } \\
\text { EXCEPT FOOD }\end{array}$ \\
\hline 11 & 1768 & 0 & $\mathbf{0}$ & $\mathbf{0}$ & -1 & $\begin{array}{l}\text { CRUSHING \& GRINDING MACHINE } \\
\text { OPERATORS }\end{array}$ \\
\hline 11 & 1769 & 0 & 0 & 0 & -1 & CING \& CUTTING MACHINE OPERATORS \\
\hline
\end{tabular}




\begin{tabular}{|c|c|c|c|c|c|c|}
\hline Major & Minor & Param & Pen & Line & Decltr & Attribute Description \\
\hline 11 & 1773 & $\mathbf{0}$ & $\mathbf{0}$ & $\mathbf{0}$ & -1 & MOTION PICTURE PROJECTIONISTS \\
\hline 11 & 1774 & $\mathbf{0}$ & $\mathbf{0}$ & $\mathbf{0}$ & -1 & $\begin{array}{l}\text { PHOTOGRAPHIC PROCESS MACHINE } \\
\text { OPERATORS }\end{array}$ \\
\hline 11 & 1777 & 0 & 0 & 0 & -1 & $\begin{array}{l}\text { MISCELLANEOUS MACHINE OPERATORS, } \\
\text { N.E.C. }\end{array}$ \\
\hline 11 & 1779 & $\mathbf{0}$ & $\mathbf{0}$ & $\mathbf{0}$ & -1 & MACHINE OPERATORS, NOT SPECIFIED \\
\hline 11 & 1783 & 0 & 0 & 0 & -1 & WELDERS \& CUTTERS \\
\hline 11 & 1784 & 0 & $\mathbf{0}$ & $\mathbf{0}$ & -1 & SOLDERERS \& BRAZERS \\
\hline 11 & 1785 & $\mathbf{0}$ & $\mathbf{0}$ & $\mathbf{0}$ & -1 & ASSEMBLERS \\
\hline 11 & 1786 & 0 & 0 & $\mathbf{0}$ & -1 & HAND CUTTING \& TRIMMING OCCUPATIONS \\
\hline 11 & 1787 & 0 & 0 & 0 & -1 & $\begin{array}{l}\text { HAND MOLDING, CASTING, \& FORMING } \\
\text { OCCUPATIONS }\end{array}$ \\
\hline 11 & 1789 & 0 & 0 & 0 & -1 & $\begin{array}{l}\text { HAND PAINTING, COATING, \& DECORATING } \\
\text { OCCUPATIONS }\end{array}$ \\
\hline 11 & 1793 & 0 & 0 & 0 & -1 & $\begin{array}{l}\text { HAND ENGRAVING \& PRINTING } \\
\text { OCCUPATIONS }\end{array}$ \\
\hline 11 & 1794 & 0 & 0 & 0 & -1 & $\begin{array}{l}\text { HAND GRINDING \& POLISHING } \\
\text { OCCUPATIONS }\end{array}$ \\
\hline 11 & 1795 & 0 & 0 & $\mathbf{0}$ & -1 & $\begin{array}{l}\text { MISCELLANEOUS HAND WORKING } \\
\text { OCCUPATIONS }\end{array}$ \\
\hline 11 & 1796 & $\mathbf{0}$ & 0 & 0 & -1 & $\begin{array}{l}\text { PRODUCTION INSPECTORS, CHECKERS, \& } \\
\text { EXAMINERS }\end{array}$ \\
\hline 11 & 1797 & $\mathbf{0}$ & 0 & $\mathbf{0}$ & -1 & PRODUCTION TESTERS \\
\hline 11 & 1798 & 0 & 0 & 0 & -1 & PRODUCTION SAMPLERS \& WEIGHERS \\
\hline 11 & 1799 & 0 & $\mathbf{0}$ & $\mathbf{0}$ & -1 & $\begin{array}{l}\text { GRADERS \& SORTERS, EXCEPT } \\
\text { AGRICULTURAL }\end{array}$ \\
\hline 11 & 1803 & $\mathbf{0}$ & $\mathbf{0}$ & $\mathbf{0}$ & -1 & SUPERVISORS, MOTOR VEHICLE OPERATORS \\
\hline 11 & 1804 & $\mathbf{0}$ & $\mathbf{0}$ & $\mathbf{0}$ & -1 & TRUCK DRIVERS, HEAVY \\
\hline 11 & 1805 & $\mathbf{0}$ & $\mathbf{0}$ & 0 & -1 & TRUCK DRIVERS, LIGHT \\
\hline 11 & 1806 & 0 & $\mathbf{0}$ & $\mathbf{0}$ & -1 & DRIVER-SALES WORKERS \\
\hline 11 & 1808 & 0 & 0 & 0 & -1 & BUS DRIVERS \\
\hline 11 & 1809 & 0 & 0 & 0 & -1 & TAXICAB DRIVERS \& CHAUFFEURS \\
\hline 11 & 1813 & $\mathbf{0}$ & $\mathbf{0}$ & $\mathbf{0}$ & -1 & PARKING LOT ATTENDANTS \\
\hline 11 & 1814 & 0 & 0 & $\mathbf{0}$ & -1 & $\begin{array}{l}\text { MOTOR TRANSPORTATION OCCUPATIONS, } \\
\text { N.E.C. }\end{array}$ \\
\hline 11 & 1823 & 0 & 0 & $\mathbf{0}$ & -1 & RAILROAD CONDUCTORS \& YARDMASTERS \\
\hline 11 & 1824 & 0 & 0 & $\mathbf{0}$ & -1 & LOCOMOTIVE OPERATING OCCUPATIONS \\
\hline 11 & 1825 & 0 & 0 & $\mathbf{0}$ & -1 & $\begin{array}{l}\text { RAILROAD BRAKE, SIGNAL, \& SWITCH } \\
\text { OPERATORS }\end{array}$ \\
\hline 11 & 1826 & $\mathbf{0}$ & 0 & $\mathbf{0}$ & -1 & RAIL VEHICLE OPERATORS, N.E.C. \\
\hline 11 & 1828 & 0 & 0 & $\mathbf{0}$ & -1 & $\begin{array}{l}\text { SHIP CAPTAINS \& MATES, EXCEPT FISHING } \\
\text { BOATS }\end{array}$ \\
\hline 11 & 1829 & 0 & 0 & $\mathbf{0}$ & -1 & SAILORS \& DECKHANDS \\
\hline 11 & 1833 & 0 & $\mathbf{0}$ & $\mathbf{0}$ & -1 & MARINE ENGINEERS \\
\hline 11 & 1834 & 0 & 0 & $\mathbf{0}$ & -1 & BRIDGE, LOCK, \& LIGHTHOUSE TENDERS \\
\hline 11 & 1843 & 0 & 0 & 0 & -1 & $\begin{array}{l}\text { SUPERVISORS, MATERIAL MOVING } \\
\text { EQUIPMENT OPERATORS }\end{array}$ \\
\hline 11 & 1844 & $\mathbf{0}$ & 0 & 0 & -1 & OPERATING ENGINEERS \\
\hline 11 & 1845 & 0 & 0 & 0 & -1 & LONGSHORE EQUIPMENT OPERATORS \\
\hline 11 & 1848 & 0 & 0 & 0 & -1 & HOIST \& WINCH OPERATORS \\
\hline
\end{tabular}




\begin{tabular}{|c|c|c|c|c|c|c|}
\hline Maior & Minor & Param & Pen & Line & Decltr & Attribute Description \\
\hline 11 & 1849 & 0 & $\mathbf{0}$ & 0 & -1 & CRANE \& TOWER OPERATORS \\
\hline 11 & 1853 & $\mathbf{0}$ & 0 & 0 & -1 & $\begin{array}{l}\text { EXCAVATING \& LOADING MACHINE } \\
\text { OPERATORS }\end{array}$ \\
\hline 11 & 1855 & $\mathbf{0}$ & $\mathbf{0}$ & $\mathbf{0}$ & -1 & GRADER, DOZER, \& SCRAPER OPERATORS \\
\hline 11 & 1856 & 0 & 0 & 0 & -1 & $\begin{array}{l}\text { INDUSTRIAI TRUCK \& TRACTOR EQUIPMENT } \\
\text { OPERATORS }\end{array}$ \\
\hline 11 & 1859 & 0 & 0 & $\mathbf{0}$ & -1 & $\begin{array}{l}\text { MISCELLANEOUS MATERIAL MOVING } \\
\text { EOUIPMENT OPERATORS }\end{array}$ \\
\hline 11 & 1863 & 0 & 0 & 0 & -1 & $\begin{array}{l}\text { SUPERVISORS; HANDLERS, EQUIP. CLEANERS } \\
\text { \& LABORERS, N.E.C. }\end{array}$ \\
\hline 11 & 1864 & $\mathbf{0}$ & $\mathbf{0}$ & $\mathbf{0}$ & -1 & HELPERS, MECHANICS \& REPAIRERS \\
\hline 11 & 1865 & 0 & $\mathbf{0}$ & $\mathbf{0}$ & -1 & HELPERS, CONSTRUCTION TRADES \\
\hline 11 & 1866 & 0 & 0 & $\mathbf{0}$ & -1 & HELPERS, SURVEYOR \\
\hline 11 & 1867 & $\mathbf{0}$ & 0 & $\mathbf{0}$ &.-1 & HELPERS, EXTRACTIVE OCCUPATIONS \\
\hline 11 & 1869 & 0 & 0 & $\mathbf{0}$ & -1 & CONSTRUCTION LABORERS \\
\hline 11 & 1873 & 0 & 0 & 0 & -1 & PRODUCTION HELPERS \\
\hline 11 & 1875 & 0 & 0 & 0 & -1 & GARBAGE COLLECTORS \\
\hline 11 & 1876 & 0 & 0 & 0 & -1 & STEVEDORES \\
\hline 11 & 1877 & 0 & 0 & $\mathbf{0}$ & -1 & STOCK HANDLERS \& BAGGERS \\
\hline 11 & 1878 & $\mathbf{0}$ & $\mathbf{0}$ & 0 & -1 & MACHINE FEEDERS \& OFFBEARERS \\
\hline 11 & 1883 & 0 & $\mathbf{0}$ & 0 & -1 & $\begin{array}{l}\text { FREIGHT, STOCK, \& MATERIAL HANDLERS, } \\
\text { N.E.C. }\end{array}$ \\
\hline 11 & 1885 & 0 & $\mathbf{0}$ & 0 & -1 & $\begin{array}{l}\text { GARAGE \& SERVICE STATION RELATED } \\
\text { OCCUPATIONS }\end{array}$ \\
\hline 11 & 1887 & 0 & $\mathbf{0}$ & $\mathbf{0}$ & -1 & $\begin{array}{l}\text { VEHICLE WASHERS \& EQUIPMENT } \\
\text { CLEANERS }\end{array}$ \\
\hline 11 & 1888 & $\mathbf{0}$ & $\mathbf{0}$ & $\mathbf{0}$ & -1 & HAND PACKERS \& PACKAGERS \\
\hline 11 & 1889 & 0 & $\mathbf{0}$ & $\mathbf{0}$ & -1 & LABORERS, EXCEPT CONSTRUCTION \\
\hline 11 & 2000 & 0 & 1 & 0 & -1 & RESTRICTED BY HANDICAP \\
\hline 11 & 2001 & 0 & 1 & 0 & -1 & * OF HOURS WORKED PER WEEK \\
\hline 11 & 2002 & $\mathbf{0}$ & 1 & $\mathbf{0}$ & -1 & CLASS OF WORKER \\
\hline 11 & 2003 & $\mathbf{0}$ & 1 & $\mathbf{0}$ & -1 & TIME \\
\hline 11 & 2004 & $\mathbf{0}$ & 1 & $\mathbf{0}$ & -1 & SOCIAL SECURTY INCOME (THOUSANDS) \\
\hline 11 & 2005 & $\mathbf{0}$ & 1 & 0 & -1 & WAGE INCOME (THOUSANDS) \\
\hline 11 & 2006 & $\mathbf{0}$ & 1 & $\mathbf{0}$ & -1 & PUBLIC ASSISTANCE INCOME (THOUSANDS) \\
\hline 11 & 2007 & 0 & 1 & $\mathbf{0}$ & -1 & NON FARM SECTOR INCOME (THOUSANDS) \\
\hline 11 & 2008 & 0 & 1 & $\mathbf{0}$ & -1 & OTHER INCOME (THOUSANDS) \\
\hline 11 & 2009 & 0 & 1 & $\mathbf{0}$ & -1 & $\begin{array}{l}\text { FARM SECTOR EMPLOYMENT INCOME } \\
\text { (THOUSANDS) }\end{array}$ \\
\hline 11 & 2010 & 0 & 1 & $\mathbf{0}$ & -1 & PROPERTY INCOME (THOI SANDS) \\
\hline 11 & 2011 & 0 & 1 & 0 & -1 & TOTAL INCOME (THOUSANDS) \\
\hline 11 & 3200 & $\mathbf{0}$ & 0 & 0 & -1 & NUMBER OF FARMS (1 - 9 ACRES) \\
\hline 11 & 3210 & $\mathbf{0}$ & 0 & 0 & -1 & NUMBER OF FARMS (10 - 49 ACRES) \\
\hline 11 & 3220 & 0 & 0 & $\mathbf{0}$ & -1 & NUMBER OF FARMS (50 - 179 ACRES) \\
\hline 11 & 3230 & $\mathbf{0}$ & $\mathbf{0}$ & $\mathbf{0}$ & -1 & NUMBER OF FARMS (180 - 499 ACRES) \\
\hline 11 & 3240 & $\mathbf{0}$ & $\mathbf{0}$ & $\mathbf{0}$ & -1 & NUMBER OF FARMS (500 - 999 ACRES) \\
\hline 11 & 3250 & $\mathbf{0}$ & $\mathbf{0}$ & $\mathbf{0}$ & -1 & NUMBER OF FARMS (1000 - 1999 ACRES) \\
\hline 11 & 3260 & 0 & $\mathbf{0}$ & $\mathbf{0}$ & -1 & NUMBER OF FARMS (2000 OR MORE ACRES) \\
\hline 11 & 3280 & $\mathbf{0}$ & 0 & $\mathbf{0}$ & -1 & NUMBER OF POULTRY FARMS \\
\hline
\end{tabular}




\begin{tabular}{|c|c|c|c|c|c|c|}
\hline Maior & Minor & Param & Pen & Line & Decltr & Attribute Description \\
\hline 12 & 0 & 0 & 1 & 0 & -1 & HAZARDOUS MATERIAL SITES \\
\hline 13 & 1 & 0 & 1 & $\mathbf{0}$ & -1 & 1ST AID \\
\hline 13 & 2 & 0 & 1 & 0 & -1 & BARRICAD \\
\hline 13 & 3 & $\mathbf{0}$ & 1 & $\mathbf{0}$ & -1 & CARS \\
\hline 13 & 4 & 0 & 1 & $\mathbf{0}$ & -1 & COMM EO \\
\hline 13 & 5 & $\mathbf{0}$ & 1 & $\mathbf{0}$ & -1 & CONST EQ \\
\hline 13 & 6 & 0 & 1 & 0 & -1 & DRY GOOD \\
\hline 13 & 7 & $\mathbf{0}$ & 1 & $\mathbf{0}$ & -1 & EMERG EQ \\
\hline 13 & 8 & $\mathbf{0}$ & 1 & $\mathbf{0}$ & -1 & FOOD \\
\hline 13 & 9 & $\mathbf{0}$ & 1 & 0 & -1 & GENERATR \\
\hline 13 & 10 & $\mathbf{0}$ & 1 & $\mathbf{0}$ & -1 & HAND TL \\
\hline 13 & 11 & 0 & 1 & $\mathbf{0}$ & -1 & HEAVY EQ \\
\hline 13 & 12 & $\mathbf{0}$ & 1 & $\mathbf{0}$ & -1 & LIGHTS \\
\hline 13 & 13 & 0 & 1 & 0 & -1 & MEDIC EQ \\
\hline 13 & 14 & 0 & 1 & $\mathbf{0}$ & -1 & OFFICE $M$ \\
\hline 13 & 15 & 0 & 1 & $\mathbf{0}$ & -1 & PAVE EQ \\
\hline 13 & 16 & $\mathbf{0}$ & 1 & $\mathbf{0}$ & -1 & PEOPLE \\
\hline 13 & 17 & 0 & 1 & 0 & -1 & POWER TL \\
\hline 13 & 18 & 0 & 1 & 0 & -1 & PREFAB B \\
\hline 13 & 19 & 0 & $\mathbf{1}$ & 0 & -1 & RESCUE \\
\hline 13 & 20 & 0 & 1 & $\mathbf{0}$ & -1 & SCALES \\
\hline 13 & 21 & 0 & 1 & 0 & -1 & SIREN-NR \\
\hline 13 & 22 & 0 & 1 & $\mathbf{0}$ & -1 & SIREN-R \\
\hline 13 & 23 & 0 & 1 & 0 & -1 & TRANSPRT \\
\hline$\overline{13}$ & 24 & 0 & 1 & 0 & -1 & TRUCKS \\
\hline 13 & 25 & 0 & 1 & 0 & -1 & WATER \\
\hline 13 & 26 & 0 & 1 & 0 & -1 & WEAPONS \\
\hline 13 & 27 & 0 & 1 & $\mathbf{0}$ & -1 & FUEL \\
\hline 13 & 3071 & 0 & 1 & 0 & -1 & PROPANE \\
\hline 13 & 3072 & $\mathbf{0}$ & 1 & 0 & -1 & DIESEL \\
\hline 13 & 3073 & 0 & 1 & $\mathbf{0}$ & -1 & GASOLINE \\
\hline 13 & 3087 & 0 & 1 & 0 & -1 & FLARES \\
\hline 13 & 3088 & 0 & 1 & $\mathbf{0}$ & -1 & TRAFFIC CONES \\
\hline 13 & 3089 & 0 & 1 & 0 & -1 & BARRICADES \\
\hline 13 & 3100 & 0 & 1 & $\mathbf{0}$ & -1 & CLOTHING \\
\hline 13 & 3110 & 0 & 1 & $\mathbf{0}$ & -1 & SHELTER \\
\hline 13 & 3200 & 0 & 1 & 0 & -1 & CROPS \\
\hline 13 & 3210 & 0 & 1 & 0 & -1 & LIVESTOCK \\
\hline 13 & 3211 & 0 & 1 & 0 & -1 & LIVESTOCK FEED \\
\hline 13 & 3220 & 0 & 1 & 0 & -1 & POULTRY \\
\hline 13 & 3221 & 0 & 1 & 0 & -1 & EGGS \\
\hline 13 & 3230 & 0 & 1 & 0 & -1 & FARM EQUIPMENT \\
\hline 13 & 3231 & $\mathbf{0}$ & 1 & 0 & -1 & TRACTOR \\
\hline 13 & 3240 & 0 & 1 & 0 & -1 & FERTILIZER \\
\hline 13 & 3425 & 0 & 1 & 0 & -1 & HAND SAWS \\
\hline 13 & 3433 & 0 & 1 & $\mathbf{0}$ & -1 & HEATERS \\
\hline 13 & 3464 & 0 & 1 & 0 & -1 & STEEL SHAPES FOR SHORING \\
\hline 13 & 3482 & 0 & 1 & 0 & -1 & MODULAR BUILDINGS \\
\hline$\overline{13}$ & 3483 & 0 & 1 & 0 & -1 & MOBILE OFFICES \\
\hline
\end{tabular}




\begin{tabular}{|c|c|c|c|c|c|c|}
\hline Major & Minor & Param & Pen & Line & Decltr & Attribute Description \\
\hline 13 & 3484 & 0 & 1 & 0 & -1 & OFFICE TRAILERS \\
\hline 13 & 3485 & 0 & 1 & 0 & -1 & STORAGE CONTAINERS \\
\hline 13 & 3495 & 0 & 1 & 0 & -1 & LADDER \\
\hline 13 & 3531 & 0 & 1 & 0 & -1 & DOZERS \\
\hline 13 & 3532 & 0 & 1 & 0 & -1 & LOADERS \\
\hline 13 & 3533 & $\mathbf{0}$ & 1 & $\mathbf{0}$ & -1 & TRACTOR \\
\hline 13 & 3534 & 0 & 1 & 0 & -1 & CRAWLER DOZER \\
\hline 13 & 3535 & 0 & 1 & 0 & -1 & CRANE \\
\hline 13 & 3536 & $\mathbf{0}$ & 1 & 0 & -1 & CRAWLER MOUNT CRANE \\
\hline 13 & 3537 & 0 & 1 & 0 & -1 & EXCAVATOR \\
\hline 13 & 3538 & 0 & 1 & 0 & -1 & SCRAPER/BLADE \\
\hline 13 & 3539 & 0 & 1 & $\mathbf{0}$ & -1 & GRADER \\
\hline 13 & 3540 & 0 & 1 & $\mathbf{0}$ & -1 & BACKHOE \\
\hline 13 & 3541 & $\mathbf{0}$ & 1 & 0 & -1 & BOOM TRUCK \\
\hline 13 & 3542 & 0 & 1 & 0 & -1 & ROLLER \\
\hline 13 & 3543 & 0 & 1 & 0 & -1 & TRACTOR TRAILER \\
\hline 13 & 3544 & 0 & 1 & 0 & -1 & FORK LIFT \\
\hline 13 & 3545 & 0 & 1 & 0 & -1 & CONVEYOR \\
\hline 13 & 3546 & $\mathbf{0}$ & 1 & $\mathbf{0}$ & -1 & BOBCAT \\
\hline 13 & 3551 & 0 & 1 & 0 & -1 & VIBRATORS \\
\hline 13 & 3552 & 0 & 1 & 0 & -1 & WELDER \\
\hline 13 & 3553 & 0 & 1 & $\mathbf{0}$ & -1 & JACK HAMMER \\
\hline 13 & 3554 & 0 & 1 & 0 & -1 & HAND HELD POWER SAWS \\
\hline 13 & 3555 & $\mathbf{0}$ & 1 & $\mathbf{0}$ & -1 & NAIL GUN \\
\hline 13 & 3556 & $\mathbf{0}$ & 1 & $\mathbf{0}$ & -1 & STEEL CUTTING EQUIPMENT \\
\hline 13 & 3557 & $\mathbf{0}$ & 1 & $\mathbf{0}$ & -1 & CONCRETE SAW \\
\hline 13 & 3558 & 0 & 1 & $\mathbf{0}$ & -1 & GRINDERS \\
\hline 13 & 3561 & 0 & 1 & 0 & -1 & DRILL \\
\hline 13 & 3562 & 0 & 1 & 0 & -1 & WATER PUMP \\
\hline 13 & 3563 & 0 & 1 & $\mathbf{0}$ & -1 & AIR COMPRESSOR \\
\hline 13 & 3564 & $\mathbf{0}$ & 1 & 0 & -1 & STEAM CLEANER \\
\hline 13 & 3565 & $\mathbf{0}$ & 1 & $\mathbf{0}$ & -1 & COMPACTOR \\
\hline 13 & 3579 & 0 & 1 & 0 & -1 & COPIERS \\
\hline 13 & 3581 & $\mathbf{0}$ & 1 & $\mathbf{0}$ & -1 & MOBILE ASPHALT PLANT \\
\hline 13 & 3582 & $\mathbf{0}$ & 1 & $\mathbf{0}$ & -1 & CRUSHING EQUIPMENT \\
\hline 13 & 3584 & $\mathbf{0}$ & 1 & 0 & -1 & ROCK CRUSHING SPREAD \\
\hline 13 & 3591 & 0 & 1 & 0 & -1 & ASPHALT \\
\hline 13 & 3592 & 0 & 1 & 0 & -1 & CONCRETE \\
\hline 13 & 3596 & 0 & 1 & 0 & -1 & SCALES \\
\hline 13 & 3601 & 0 & 1 & $\mathbf{0}$ & -1 & FENCING \\
\hline 13 & 3620 & 0 & 1 & 0 & -1 & GENERATOR \\
\hline 13 & 3648 & 0 & 1 & $\mathbf{0}$ & -1 & WARNING LIGHTS \\
\hline 13 & 3662 & 0 & 1 & $\mathbf{0}$ & -1 & FAX MACHINE \\
\hline 13 & 3663 & $\mathbf{0}$ & 1 & $\mathbf{0}$ & -1 & PORTABLE COMM. EOUIP. \\
\hline 13 & 3664 & $\mathbf{0}$ & 1 & 0 & -1 & BULL HORN \\
\hline 13 & 3685 & 0 & 1 & $\mathbf{0}$ & -1 & FLOODLIGHTS \\
\hline 13 & 3708 & 0 & 1 & 0 & -1 & SERVICE/TOW TRUCK \\
\hline 13 & 3713 & 0 & 1 & 0 & -1 & PICKUP TRUCK \\
\hline 13 & 3714 & 0 & 1 & 0 & -1 & FLATBED TRUCK \\
\hline 13 & 3715 & $\mathbf{0}$ & 1 & 0 & -1 & END DUMP TRUCK \\
\hline
\end{tabular}




\begin{tabular}{ccccccl} 
Maior & Minor & Param & Pen & Line & Decltr & Altribute Description \\
\hline 13 & 3716 & 0 & 1 & 0 & -1 & BOTTOM DUMP TRUCK \\
13 & 3717 & 0 & 1 & 0 & -1 & WATER TRUCK \\
13 & 3721 & 0 & 1 & 0 & -1 & FIRE TRUCK - PUMPER \\
13 & 3722 & 0 & 1 & 0 & -1 & FIRE TRUCK - TANKER \\
13 & 3723 & 0 & 1 & 0 & -1 & BRUSH TRUCK \\
13 & 3724 & 0 & 1 & 0 & -1 & FIRE FIGHTING EQUIP. \\
13 & 3725 & 0 & 1 & 0 & -1 & FIRE EXTINGUISHERS \\
13 & 3733 & 0 & 1 & 0 & -1 & AMBULANCE \\
13 & 3741 & 0 & 1 & 0 & -1 & SCHOOL BUS \\
13 & 3742 & 0 & 1 & 0 & -1 & BUSES \\
13 & 3744 & 0 & 1 & 0 & -1 & BUS W/HYDR LIFT \\
13 & 3745 & 0 & 1 & 0 & -1 & VANS \\
13 & 3746 & 0 & 1 & 0 & -1 & VAN W/HYDR. LIFT \\
13 & 3747 & 0 & 1 & 0 & -1 & VEHICLE FLEET \\
13 & 3750 & 0 & 1 & 0 & -1 & PORTABLE RAMPS \\
13 & 3760 & 0 & 1 & 0 & -1 & RAILROAD TRANSPORTATION \\
13 & 3770 & 0 & 1 & 0 & -1 & WATER TRANSPORTATION \\
13 & 3800 & 0 & 1 & 0 & -1 & PROTECTIVE EQUIPMENT \\
13 & 3801 & 0 & 1 & 0 & -1 & RESP. PROT. EQUIP/ADULT \\
13 & 3802 & 0 & 1 & 0 & -1 & RESP. PROT. EQUIP/CHIID \\
13 & 3803 & 0 & 1 & 0 & -1 & PROTECTIVE CLOTHING \\
13 & 3804 & 0 & 1 & 0 & -1 & AIR FILTERING EQUIP. \\
13 & 3805 & 0 & 1 & 0 & -1 & POSITIVE PRESSURE EQUIP. \\
13 & 3810 & 0 & 1 & 0 & -1 & DECONTAMINATION EQUIPMENT \\
13 & 3820 & 0 & 1 & 0 & -1 & MEDICAL SUPPLIES \\
13 & 3821 & 0 & 1 & 0 & -1 & ANTIDOTE \\
13 & 3822 & 0 & 1 & 0 & -1 & STRETCHERS \\
13 & 3823 & 0 & 1 & 0 & -1 & WHEEL CHAIRS \\
13 & 3824 & 0 & 1 & 0 & -1 & BEDS \\
13 & 3825 & 0 & 1 & 0 & -1 & PRESCRIPTION DRUGS \\
13 & 3826 & 0 & 1 & 0 & -1 & MEDICAL CARE/TREATMENT \\
13 & 3830 & 0 & 1 & 0 & -1 & VETERINARY SUPPLIES \\
13 & 3831 & 0 & 1 & 0 & -1 & VET. DIAGNOSTIC EQUIPMENT \\
13 & 3840 & 0 & 1 & 0 & -1 & LABORATORY EQUIPMENT \\
13 & 3993 & 0 & 1 & 0 & -1 & SIGNS
\end{tabular}




\begin{tabular}{|c|c|c|c|c|c|c|}
\hline Maior & Minor & Param & Pen & Line & Decitr & Atribute Description \\
\hline & & & & & & LAND USE \\
\hline 14 & 1001 & $\mathbf{0}$ & 1 & $\mathbf{0}$ & -1 & WATERSHED (ACRES) \\
\hline 14 & 1002 & $\mathbf{0}$ & 1 & $\mathbf{0}$ & -1 & ALKALI BOTTOM (ACRES) \\
\hline 14 & 1003 & $\mathbf{0}$ & 1 & 0 & -1 & ALKALI FLAT (ACRES) \\
\hline 14 & 1004 & 0 & 1 & $\mathbf{0}$ & -1 & DESERT ALKALII SAND (ACRES) \\
\hline 14 & 1005 & $\mathbf{0}$ & 1 & $\mathbf{0}$ & -1 & DESERT SALT FLAT (ACRES) \\
\hline 14 & 1006 & $\mathbf{0}$ & 1 & $\mathbf{0}$ & -1 & DESERT SILT FLAT (ACRES) \\
\hline 14 & 1007 & $\mathbf{0}$ & 1 & $\mathbf{0}$ & -1 & DESERT GRAVELLY LOAM (ACRES) \\
\hline 14 & 1008 & 0 & 1 & $\mathbf{0}$ & -1 & DESERT FLAT (ACRES) \\
\hline 14 & 1009 & $\mathbf{0}$ & 1 & $\mathbf{0}$ & -1 & DESERT LOAM (ACRES) \\
\hline 14 & 1010 & 0 & 1 & 0 & -1 & DESERT SAND (ACRES) \\
\hline 14 & 1011 & $\mathbf{0}$ & 1 & $\mathbf{0}$ & -1 & HIGH MOUNTAIN LOAM (ASPEN) (ACRES) \\
\hline 14 & 1012 & 0 & 1 & 0 & -1 & $\begin{array}{l}\text { HIGH MOUNTAIN STONY LOAM (CONIFER) } \\
\text { (ACRES) }\end{array}$ \\
\hline 14 & 1013 & 0 & 1 & 0 & -1 & $\begin{array}{l}\text { LOAMY BOTTOM (BASIN BIG SAGEBRUSH) } \\
\text { (ACRES) }\end{array}$ \\
\hline 14 & 1014 & 0 & 1 & $\mathbf{0}$ & -1 & MOUNTAIN GRAVELLY LOAM (ACRES) \\
\hline 14 & 1015 & 0 & 1 & 0 & -1 & $\begin{array}{l}\text { MOUNTAIN GRAVELING LOAM (OAK) } \\
\text { (ACRES) }\end{array}$ \\
\hline 14 & 1016 & $\mathbf{0}$ & 1 & $\mathbf{0}$ & -1 & MOUNTAIN LOAM (SHRUB) (ACRES) \\
\hline 14 & 1017 & 0 & 1 & $\mathbf{0}$ & -1 & $\begin{array}{l}\text { MOUNTAIN SHALLOWW LOAM (MOUNTANN } \\
\text { MAHOGANY) (ACRES) }\end{array}$ \\
\hline 14 & 1018 & 0 & 1 & 0 & -1 & MOUNTAIN ŚTONY LOAM (ACRES) \\
\hline 14 & 1019 & $\mathbf{0}$ & 1 & $\mathbf{0}$ & -1 & MOUNTAIN WINDSWEPT RIDGE (ÁCRES) \\
\hline 14 & 1020 & $\mathbf{0}$ & 1 & $\mathbf{0}$ & -1 & OOLTIC DUNES (ACRES) \\
\hline 14 & 1021 & $\mathbf{0}$ & 1 & $\mathbf{0}$ & -1 & SALTY SILT (ACRES) \\
\hline 14 & 1022 & 0 & 1 & 0 & -1 & $\begin{array}{l}\text { SEMIDESERT GRAVELLY LOAM (WYOMING } \\
\text { SAGEBRUSH) (ACRES) }\end{array}$ \\
\hline 14 & 1023 & 0 & 1 & 0 & -1 & $\begin{array}{l}\text { SEMIDESERT LOAM (WYOMING SAGEBRUSH) } \\
\text { (ACRES) }\end{array}$ \\
\hline 14 & 1024 & 0 & 1 & 0 & -1 & SEMIDESERT SAND (UTAH JUNIPER) (ACRES) \\
\hline 14 & 1025 & $\mathbf{0}$ & 1 & $\mathbf{0}$ & -1 & SEMIDESERT SHALLOW HARDPAN (ACRES) \\
\hline 14 & 1026 & $\mathbf{0}$ & 1 & $\mathbf{0}$ & -1 & $\begin{array}{l}\text { SEMIDESERT SHALLOW LOAM (UTAH } \\
\text { JUNIPER) (ACRES) }\end{array}$ \\
\hline 14 & 1027 & 0 & 1 & 0 & -1 & $\begin{array}{l}\text { SEMIDESERT STONY LOAM (BLANCK } \\
\text { SAGEBRUSH) }\end{array}$ \\
\hline 14 & 1028 & $\mathbf{0}$ & 1 & $\mathbf{0}$ & -1 & SEMIWET FRESH STREAMBANK (ACRES) \\
\hline 14 & 1029 & 0 & 1 & $\mathbf{0}$ & -1 & UPLAND GRAVELLY LOAM (ACRES) \\
\hline 14 & 1030 & 0 & 1 & $\mathbf{0}$ & -1 & UPLAND LOAM (ACRES) \\
\hline 14 & 1031 & $\mathbf{0}$ & 1 & $\mathbf{0}$ & -1 & $\begin{array}{l}\text { UPLAND SHALLOW HARDPAN } \\
\text { (JUNIPER-PINYON) (ACRES) }\end{array}$ \\
\hline 14 & 1032 & 0 & 1 & 0 & -1 & $\begin{array}{l}\text { UPLAND SHALLOW LOAM (JUNIPER-PINYON) } \\
\text { (ACRES) }\end{array}$ \\
\hline 14 & 1033 & 0 & 1 & $\mathbf{0}$ & -1 & $\begin{array}{l}\text { UPLAND STONY LOAM (JUNIPER-PINYON) } \\
\text { (ACRES) }\end{array}$ \\
\hline 14 & 1034 & $\mathbf{0}$ & 1 & 0 & -1 & WET SALINE MEADOW (ACRES) \\
\hline 14 & 1100 & $\mathbf{0}$ & $\mathbf{0}$ & $\mathbf{0}$ & -1 & $\begin{array}{l}\text { FARMLAND: RANGE, WATER SURFACE } \\
\text { AREA, ETC. (ACRES) }\end{array}$ \\
\hline 14 & 1200 & $\mathbf{0}$ & $\mathbf{0}$ & 0 & -1 & FARMLAND: PRIME (ACRES) \\
\hline 14 & 1300 & 0 & $\mathbf{0}$ & 0 & -1 & FARMLAND: UNDESIGNATED (ACRES) \\
\hline
\end{tabular}




\begin{tabular}{|c|c|c|c|c|c|c|}
\hline Major & Minor & Param & Pen & Line & Decltr & Attribute Descriotion \\
\hline 14 & 1400 & $\mathbf{0}$ & $\mathbf{0}$ & $\mathbf{0}$ & -1 & $\begin{array}{l}\text { FARMLAND: STATEWIDE IMPORTANCE } \\
\text { (ACRES) }\end{array}$ \\
\hline 14 & 1500 & $\mathbf{0}$ & 0 & $\mathbf{0}$ & -1 & FARMLAND: URBANIZED LAND (ACRES) \\
\hline 14 & 2010 & $\mathbf{0}$ & $\mathbf{0}$ & 0 & -1 & HORTICULTURE (ACRES) \\
\hline 14 & 2110 & $\mathbf{0}$ & 0 & 0 & -1 & IRRIGATED FIELD CROP (ACRES) \\
\hline 14 & 2111 & $\mathbf{0}$ & $\mathbf{0}$ & $\mathbf{0}$ & -1 & IRRIGATED CROP: GRAIN (BUSHELS/ACRES) \\
\hline 14 & 2112 & $\mathbf{0}$ & $\mathbf{0}$ & $\mathbf{0}$ & -1 & $\begin{array}{l}\text { NON-IRRIGATED CROP: GRAIN } \\
\text { (BUSHELS/ACRES) }\end{array}$ \\
\hline 14 & 2114 & $\mathbf{0}$ & $\mathbf{0}$ & $\mathbf{0}$ & -1 & VEGETABLES (ACRES) \\
\hline 14 & 2125 & $\mathbf{0}$ & $\mathbf{0}$ & $\mathbf{0}$ & -1 & IRRIGATED CROP: PÁSTURE (AUM) \\
\hline 14 & 2126 & $\mathbf{0}$ & 0 & 0 & -1 & NON-IRRIGATED CROP: PASTURE (AUM) \\
\hline 14 & 2131 & $\mathbf{0}$ & $\mathbf{0}$ & $\mathbf{0}$ & -1 & IRRIGATED CROP: ALFALFA (TONS/ACRES) \\
\hline 14 & 2132 & 0 & 0 & $\mathbf{0}$ & -1 & $\begin{array}{l}\text { NON-IRRIGATED CROP: ALFALFA } \\
\text { (TONS/ACRES) }\end{array}$ \\
\hline 14 & 2133 & $\mathbf{0}$ & $\mathbf{0}$ & $\mathbf{0}$ & -1 & IRRIGATED CROP: CORN (TONS/ACRE) \\
\hline 14 & 2134 & $\mathbf{0}$ & $\mathbf{0}$ & $\mathbf{0}$ & -1 & NON-IRRIGATED CROP: CORN (TONS/ACRE) \\
\hline 14 & 2210 & $\mathbf{0}$ & $\mathbf{0}$ & $\mathbf{0}$ & -1 & IRRIGATED SOD (ACRES) \\
\hline 14 & 2220 & 0 & $\mathbf{0}$ & 0 & -1 & IMPROVED PASTURE (ACRES) \\
\hline 14 & 2240 & $\mathbf{0}$ & $\mathbf{0}$ & $\mathbf{0}$ & -1 & IRRIGATED MEADOW (ACRES) \\
\hline 14 & 3000 & 0 & 1 & 0 & -1 & $\begin{array}{l}\text { RANGE HABITAT FOR UPLAND BIG GAME: } \\
\text { TOTAL (ACRES) }\end{array}$ \\
\hline 14 & 3010 & 0 & 1 & $\mathbf{0}$ & -1 & $\begin{array}{l}\text { RANGE HABITAT FOR UPLAND BIG GAME: } \\
\text { WINTER HABITAT (ACRES) }\end{array}$ \\
\hline 14 & 3020 & $\mathbf{0}$ & 1 & $\mathbf{0}$ & -1 & $\begin{array}{l}\text { RANGE HABITAT FOR UPLAND BIG GAME: } \\
\text { SUMMER IIABITAT (ACRES) }\end{array}$ \\
\hline 14 & 3030 & $\mathbf{0}$ & 1 & $\mathbf{0}$ & -1 & $\begin{array}{l}\text { RANGE HABITAT FOR UPLAND BIG GAME: } \\
\text { YEARLONG HABITAT(ACRES) }\end{array}$ \\
\hline 14 & 3040 & $\mathbf{0}$ & 1 & $\mathbf{0}$ & -1 & $\begin{array}{l}\text { RANGE HABITAT FOR UPLAND BIG GAME: } \\
\text { PRODUCTION }\end{array}$ \\
\hline 14 & 3500 & 0 & 1 & $\mathbf{0}$ & -1 & $\begin{array}{l}\text { RANGE HABITAT FOR UPLAND GAME BIRD: } \\
\text { TOTAL (ACRES) }\end{array}$ \\
\hline 14 & 3510 & $\mathbf{0}$ & 1 & $\mathbf{0}$ & -1 & $\begin{array}{l}\text { RANGE HABITAT FOR UPLAND GAME BIRD: } \\
\text { WINTER HABITAT (ACRES) }\end{array}$ \\
\hline 14 & 3520 & $\mathbf{0}$ & 1 & $\mathbf{0}$ & -1 & $\begin{array}{l}\text { RANGE HABITAT FOR UPLAND GAME BIRD: } \\
\text { SUMMER HABITAT (ACRES) }\end{array}$ \\
\hline 14 & 3530 & 0 & 1 & $\mathbf{0}$ & -1 & $\begin{array}{l}\text { RANGE HABITAT FOR UPLAND GAME BIRD: } \\
\text { YEARLONG HABITAT(ACRES) }\end{array}$ \\
\hline 14 & 3540 & 0 & 1 & 0 & -1 & $\begin{array}{l}\text { RANGE HABITAT FOR UPLAND GAME BIRD: } \\
\text { PRODUCTION }\end{array}$ \\
\hline 14 & 4000 & $\mathbf{0}$ & 1 & $\mathbf{0}$ & -1 & RANGE HABITAT FOR FISHERIES: TOTAL \\
\hline 14 & 4010 & 0 & 1 & $\mathbf{0}$ & -1 & $\begin{array}{l}\text { RANGE HABITAT FOR FISHERIES: } \\
\text { HABITAT (ACRES) }\end{array}$ \\
\hline 14 & 4020 & 0 & 1 & $\mathbf{0}$ & -1 & $\begin{array}{l}\text { RANGE HABITAT FOR FISHERIES: SUMMER } \\
\text { HABITAT (ACRES) }\end{array}$ \\
\hline 14 & 4030 & 0 & 1 & $\mathbf{0}$ & -1 & $\begin{array}{l}\text { RANGE HABITAT FOR FISHERIES: } \\
\text { YEARLONG HABITAT (ACRES) }\end{array}$ \\
\hline 14 & 4040 & $\mathbf{0}$ & 1 & $\mathbf{0}$ & -1 & $\begin{array}{l}\text { RANGE HABITAT FOR FISHERIES: } \\
\text { PRODUCTION }\end{array}$ \\
\hline
\end{tabular}




\begin{tabular}{|c|c|c|c|c|c|c|}
\hline Maior & Minor & Param & $\operatorname{Pen}$ & Line & Decitr & Attribute Deseriotion \\
\hline & & & & & & PROPERTY VALUES \\
\hline 15 & 1111 & $\mathbf{0}$ & 1 & $\mathbf{0}$ & -1 & PROPERTY VALUE (1000'S) \\
\hline 15 & 2111 & $\mathbf{0}$ & $\mathbf{0}$ & 0 & -1 & CROP VALUE: GRAIN ( $\$$ ÍN 100'S) \\
\hline 15 & 2112 & $\mathbf{0}$ & $\mathbf{0}$ & 0 & -1 & CROP VALUE: WHEAT (S IN 100'S) \\
\hline 15 & 2131 & $\mathbf{0}$ & $\mathbf{0}$ & $\mathbf{0}$ & -1 & CROP VALUE: ALFALFA ( $\$$ IN 100'S) \\
\hline 15 & 2132 & 0 & $\mathbf{0}$ & $\mathbf{0}$ & -1 & CROP VALUE: CORN (\$ IN 100'S) \\
\hline 15 & 2210 & $\mathbf{0}$ & $\mathbf{0}$ & 0 & -1 & $\begin{array}{l}\text { CROP VALUE: PASTURE (NEGÁTTVE S IN } \\
100^{\prime} \text { ') }\end{array}$ \\
\hline 15 & 2220 & $\mathbf{0}$ & $\mathbf{0}$ & 0 & -1 & CROP VALUE: MEADOW (\$ IN 100'S) \\
\hline 15 & 2240 & $\mathbf{0}$ & $\mathbf{0}$ & $\mathbf{0}$ & -1 & CROP VALUE: SOD (S IN 100'S) \\
\hline 15 & 3005 & 0 & 1 & $\mathbf{0}$ & -1 & VALUE OF CATTLE COMMODITY (\$ IN 100'S) \\
\hline 15 & 3110 & $\mathbf{0}$ & 1 & $\mathbf{0}$ & -1 & VALUE OF SHEEP, LAMB, WOOL (\$ IN 100'S) \\
\hline 15 & 3115 & $\mathbf{0}$ & 1 & 0 & -1 & VALUE OF OTHER LIVESTOCK ( $\$$ IN 100'S) \\
\hline 15 & 3120 & $\mathbf{0}$ & 1 & $\mathbf{0}$ & -1 & VALUE OF FEED GRAIN \\
\hline 15 & 3200 & $\mathbf{0}$ & 1 & $\mathbf{0}$ & -1 & VALUE OF HAY (\$100's) \\
\hline
\end{tabular}




\section{Appendix C IBS Icon Dictionary (Icon Control File)}

This appendix lists the attribute codes, names, graphic file names, and types of the standard graphic icons used in the IBS and specified in the IBS icon dictionary.

Icon Types. In the following table, the icon type is specified as F (facility icon), $\mathbf{R}$ (resource icon), or no type (general use icon).

\begin{tabular}{|c|c|c|}
\hline $\begin{array}{l}\text { Major } \\
\text { Attribute } \\
\text { Code } \\
\end{array}$ & $\begin{array}{c}\text { Minor } \\
\text { Attribute } \\
\text { Code } \\
\end{array}$ & Description \\
\hline 1 & 0410 & DAM \\
\hline 3 & 0120 & RECREATION AREA \\
\hline $\mathbf{s}$ & 1100 & TRAF CTRL PT \\
\hline 5 & 1101 & TRAF CIRL PT (INACTIVE) \\
\hline 7 & 0080 & POWER STATION \\
\hline 7 & 0100 & HYDROELECTRIC PLANT \\
\hline 7 & 0110 & CIVILIAN AIRPORT \\
\hline 7 & 0111 & AIRPORT \\
\hline 7 & 0112 & CTVILIAN AIR HUB \\
\hline 7 & 0115 & MILTTARY AIRPORT \\
\hline 7 & 0250 & TRANSPORTATION FACILITY \\
\hline 8 & $\mathbf{0 0 1 0}$ & CHURCH \\
\hline 8 & 0020 & SCHOOL \\
\hline 8 & 0030 & HOSPITAL \\
\hline 8 & 0031 & V.M.A.C. HOSPITAL \\
\hline 8 & 0032 & HOSPITAL (INACTTVE) \\
\hline 8 & 0181 & PUBLCC UTILTYY \\
\hline 8 & 0190 & INDUSTRIAL PARK \\
\hline 8 & 0195 & PRIVATE BUSINESS \\
\hline 8 & 0200 & EMERGENCY EQUTPMENT \\
\hline 8 & 0205 & NUCLEAR PLANT \\
\hline 8 & 0210 & HAZMAT SITE \\
\hline 8 & 0530 & SHELTER \\
\hline 8 & 0536 & SHELTER (INACTTVE) \\
\hline 8 & 0560 & POLICE STATION \\
\hline 8 & 0570 & FIRE STATION \\
\hline 8 & 0585 & GOVERNMENT BUILDING \\
\hline 8 & 0620 & E.O.C. \\
\hline 8 & 0621 & (INACTIVE) \\
\hline 8 & 0690 & FUEL TANK FARM \\
\hline 8 & 0710 & WATER SUPPLY \\
\hline 8 & 0770 & COMMUNICATIONS FACILITY \\
\hline 8 & 1400 & SIREN • ROTATING \\
\hline
\end{tabular}

\begin{tabular}{|c|c|}
\hline $\begin{array}{r}\text { File Name } \\
\text { MapeICONG. }\end{array}$ & $\frac{\text { Type }}{P}$ \\
\hline MAPSICONS:DAM.DMS & \\
\hline MAPSICONS:P_RECAREA.DMS & $\mathbf{F}$ \\
\hline MAPSICONS:F_TRAFFIC_A.DMS & $\mathbf{F}$ \\
\hline MAPSICONS:F_TRAFFIC_I.DMS & $\mathbf{F}$ \\
\hline MAPSICONS:POWSTA.DMS & $\mathbf{F}$ \\
\hline MAPSICONS:HYDRO.DMS & $\mathbf{P}$ \\
\hline MAPSICONS:AIRPORT.DMS & $\mathbf{F}$ \\
\hline MAPSICONS:AIRPORT.DMS & $\mathbf{F}$ \\
\hline MAPSICONS:AIRHUB.DMS & $\mathbf{P}$ \\
\hline MAPSICONS:MAIRPORT.DMS & $\mathbf{F}$ \\
\hline MAPSICONS:F_TRANSPORT.DMS & $\mathbf{F}$ \\
\hline MAPSICONS:CHURCH.DMS & $\mathbf{F}$ \\
\hline MAPSICONS:SCHOOL.DMS & $\mathbf{F}$ \\
\hline MAPSICONS:HOSPITAL_A.DMS & $\mathbf{F}$ \\
\hline MAPSICONS:VMAC.DMS & $\mathbf{P}$ \\
\hline MAPSICONS:HOSPITAL_I.DMS & $\mathbf{P}$ \\
\hline MAPSICONS:P_PUB_UTLDMS & $\mathbf{F}$ \\
\hline MAPSICONS:INDUST.DMS & $\mathbf{F}$ \\
\hline MAPSICONS:F_PRIV_BIZ.DMS & $\mathbf{F}$ \\
\hline MAPSICONS:EQUIP.DMS & \\
\hline MAPSICONS:NUCPLANT.DMS & $\boldsymbol{F}$ \\
\hline MAPSICONS:HAZMAT.DMS & \\
\hline MAPSICONS:SHELTER_A.DMS & $\mathbf{F}$ \\
\hline MAPSICONS:SHELTER_I.DMS & $\boldsymbol{F}$ \\
\hline MAPSICONS:POLICEDMS & $\mathbf{F}$ \\
\hline MAPSICONS:FIREDMS & $\mathbf{F}$ \\
\hline MAPSICONS:F_GOVT_BLDG.DMS & $\mathbf{F}$ \\
\hline MAPSICONS:EOC_A.DMS & $\mathbf{F}$ \\
\hline MAPSICONS:EOC_I.DMS & $\mathbf{F}$ \\
\hline MAPSICONS:FUELLDMS & $\mathbf{F}$ \\
\hline MAPSICONS:WATERDMS & $\boldsymbol{F}$ \\
\hline MAPSICONS:COMMFAC.DMS & $\mathbf{F}$ \\
\hline MAPSICONS:SIRENRDMS & $\mathbf{F}$ \\
\hline
\end{tabular}




\begin{tabular}{|c|c|c|}
\hline $\begin{array}{l}\text { Major } \\
\text { Attribute } \\
\text { Code }\end{array}$ & $\begin{array}{l}\text { Minor } \\
\text { Attribute } \\
\text { Code }\end{array}$ & Description \\
\hline 8 & 1401 & SIREN - NONROTATING \\
\hline 9 & 0050 & SPECLAL POPULATION \\
\hline 9 & 0430 & OTHER CONTROL POINT \\
\hline 9 & 0560 & EVACUATION SHELTER \\
\hline 9 & 0561 & RECEPTION CTR \\
\hline 9 & 0565 & RECEPTION CIR (INACTIVE) \\
\hline 9 & 0566 & DECON CENTER \\
\hline 9 & 0567 & DECON CENTER (INACITVE) \\
\hline 9 & 0570 & MORGUE \\
\hline 9 & 0571 & (INACITVE) \\
\hline 9 & 0700 & HURRICANE \\
\hline 9 & 0701 & TROPICAL STORM \\
\hline 9 & 0702 & TSUNAMI \\
\hline 9 & 0703 & EARTHQUAKE \\
\hline 9 & 0704 & FIRE \\
\hline 9 & 0705 & BLIZZARD \\
\hline 9 & 0706 & TORNADO \\
\hline 9 & 0707 & ILOOD \\
\hline 9 & 0708 & CIIY \\
\hline 9 & 0712 & MOBLE MEDICAL UNTT \\
\hline 9 & 0715 & PRIVATE RESIDENCE \\
\hline 9 & 0812 & ENVIRONMENT REPORT \\
\hline 9 & 0813 & WEATHER REPORT \\
\hline 9 & 0900 & NAPB TARGET \\
\hline 9 & 1006 & REFINERY \\
\hline 9 & 1007 & COAL MINE \\
\hline 9 & 1008 & COKE PRODUCER \\
\hline 9 & 1009 & NAT GAS PRESSURE PLANT \\
\hline 9 & 1010 & NAT GAS UNDERGROUND ST \\
\hline 9 & 1011 & PETROL IMPORT FACIIITY \\
\hline 9 & 1012 & PORT PACILITY \\
\hline 9 & 1013 & GRAIN STORAGE \\
\hline 9 & 1014 & FLOUR MILIS \\
\hline 9 & 1015 & FOOD DISTRIBUTION \\
\hline 9 & 1016 & POULTRY PRODUCTION \\
\hline 9 & 1017 & AGRICULTURE EO.C. \\
\hline 9 & 1018 & EGG PRODUCERS \\
\hline 9 & 1019 & POOD PRODUCTION \\
\hline 13 & 0001 & 1ST AID \\
\hline 13 & 0002 & BARRICAD \\
\hline 13 & 0003 & CARS \\
\hline 13 & 0004 & COMM EQ \\
\hline 13 & 0005 & CONST EO \\
\hline 13 & 0006 & DRY GOOD \\
\hline 13 & 0007 & EMERG EQ \\
\hline
\end{tabular}

\begin{tabular}{|c|c|}
\hline Name & \\
\hline MAPSICONS:SIRENNR.DMS & \\
\hline MAPSICONS:P_SPECPOP.DMS & \\
\hline MAPSICONS:OCONTROLDMS & \\
\hline MAPSICONS:EVAC_SHELTERDMS & \\
\hline MAPSICONS:P_RECEPCTR_A.DMS & \\
\hline MAPSICONS:P_RECEPCIR_U.DMS & \\
\hline MAPSICONS:DECON_A.DMS & \\
\hline MAPSICONS:DECON_I.DMS & \\
\hline MAPSICONS:MOROUE A.DIMS & \\
\hline MAPSICONS:MORGUE I.DMS & \\
\hline
\end{tabular}

MAPSICONS:HURRICANE.DMS

MAPSICONS:TROPSTORM.DMS

MAPSICONS:TSUNAMI.DMS;

MAPSICONS:QUAKEDMS

MAPSICONS:FIRE3.DMS

MAPSICONS:BLIZZARD.DMIS

MAPSICONS:TORNADO.DMS

MAPSICONS:FLOOD.DMS

MAPSTCONS:CTTY.DMS

MAPSICONS:P_MOBMED.DMS

MAPSICONS:F_PRIV_RES.IMMS

MAPSICONS:ENVIRONMEIVT.DMS

MAPSICONS:WEATHERDMS

MAPSICONS:TARGET.DMS

MAPSICONS:RERINERY.DMS

MAPSICONS:COAL.DMS

MAPSICONS:COKEDMS

MAPSICONS:NGPP.DMS

MAPSICONS:NGUS.DMS

MAPSICONS:PET.DMS

MAPSICONS:PORT.DMS

MAPSICONS:GRAIN.DMS

MAPSICONS:PMILLS.DMS;

MAPIICONS:P_FOOD_DIST.DMS

MAPSICONS:POLT.DMS

MAPSICONS:AEOC.DMS

MAPSICONS:EGGS.DMS

MAPSICONS:F_FOOD_PROD.DMS

MAPSICONS:R_1ST_AID_.DMS

MAPSICONS:R_BARRICAD.DMS

MAPSICONS:R_CARS_DMS

MAPSICONS:R_COMM_BOQ_DMS

MAPSICONS:R_CONST_IBO.DMS

MAPSICONS:R_DRY_GOOD.DMS

MAPSICONS:R_EMERG_EO.DMS

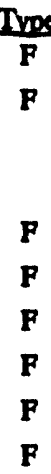

P

$\mathbf{P}$

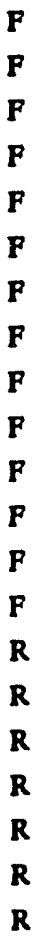


Appendix C: IBS Icon Dictionary (Icon Control File)

\begin{tabular}{|c|c|c|}
\hline $\begin{array}{l}\text { Major } \\
\text { Attribute } \\
\text { Code } \\
\end{array}$ & $\begin{array}{l}\text { Minor } \\
\text { Attribute } \\
\text { Code } \\
\end{array}$ & Description \\
\hline 13 & 0008 & POOD \\
\hline 13 & 0009 & GENERATR \\
\hline 13 & 0010 & HAND TL \\
\hline 13 & 0011 & HEAVY EQ \\
\hline 13 & 0012 & LOHTS \\
\hline 13 & 0013 & MEDIC BQ \\
\hline 13 & 0014 & OFFICE M \\
\hline 13 & 0015 & PAVE EO \\
\hline 13 & 0016 & PEOPLE \\
\hline 13 & 0017 & POWER TL \\
\hline 13 & 0018 & PREPAB B \\
\hline 13 & 0019 & RESCUE S \\
\hline 13 & 0020 & SCALPS \\
\hline 13 & 0021 & SIREN-NR \\
\hline 13 & 0022 & SIREN-R \\
\hline 13 & 0023 & TRANSPRT \\
\hline 13 & 0024 & TRUCKS \\
\hline 13 & 0025 & WATER \\
\hline 13 & 0026 & WEAPONS \\
\hline
\end{tabular}

File Nome

MAPSICONS:R_FOOD_DMS R MAPSICONS:R GENERATRDMS $R$ MAPSICONS:R_HAND_TI_DMS $R$ MAPSICONS: $R$ HEAVY EO.DMS $R$ MAPSICONS:R_LGHTS_DMS R MAPSICONS:R_MEDIC_BO.DMS R MAPSICONS:R_OFFICE_M.DMS $R$ MAPSICONS:R_PAVE_BO_DMS $R$ MAPSICONS:R_PEOPLE_DMS $R$ MAPSICONS:R_POWER_TLDMS $R$ MAPSICONS:R_PREFAB_B.DMS $R$ MAPSICONS:R RESCUE_S.DMS $R$ MAPSICONS:R_SCALES_DMS $R$ MAPSICONS: $R$ SIREN NR.DMS $R$ MAPSICONS: $R$ SIREN $R$.DMS $R$ MAPSICONS: $R$ TRANSPRT.DMS $R$ MAPSICONS:R TRUCKS .DMS $R$ MAPSICONS: R WATER DMS $R$ MAPSICONS:R_WEAPONS_DMS R 


\section{Appendix D Map Projections in IBS Map Displays}

Various map display utilities such as GEDIT, MPDISPLAY, and PLOTDMS give you the option to choose what type of map projection to use when displaying map data:

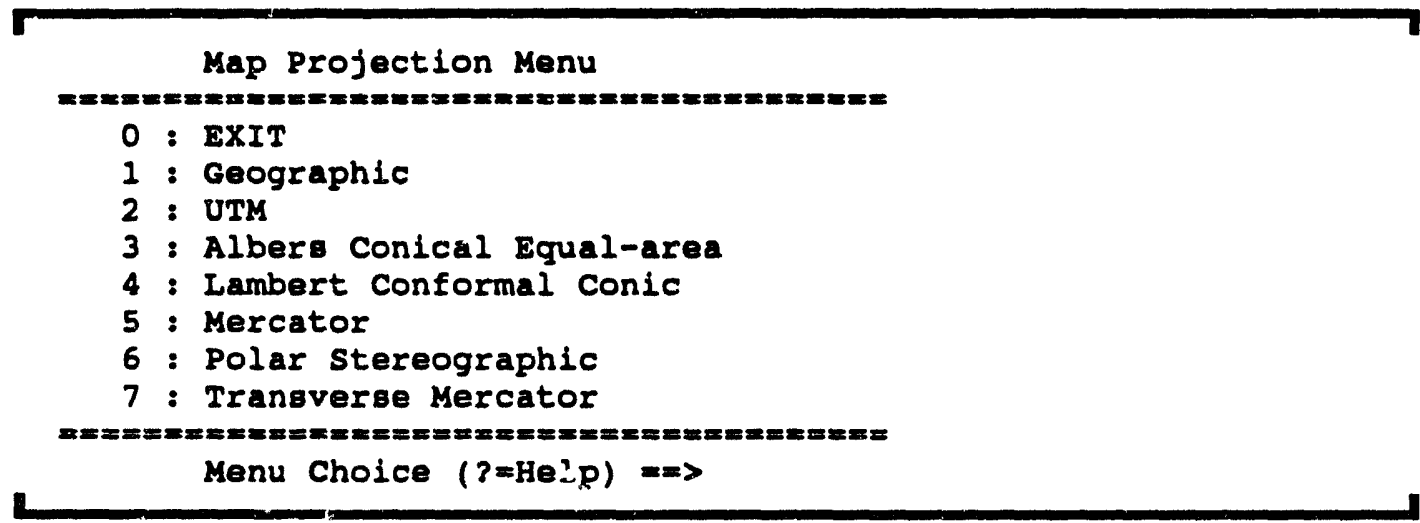

To choose one of the map projections, enter the corresponding number.

\section{Why use different map projections?}

Whenever map data from the earth's curved surface is to be represented by a fla: display, choices must be made about what kinds of distortions can be tolerated in that display. Will relative areas be correct? Will relative linear distances be correct? Will the display be continuous? Or will the display be broken like an orange peel that is sliced and pressed flat? The different map projections represent different solutions for what (and how much) map qualities are distorted.

\section{Choosing a Map Projection Type}

For most cases (up to 100-mile radius), you will probably want to enter 1 to select the "Geographic" projection. Enter a number ( 2 to 7 ) if you desire a projection other than Geographic.

The proper choice of projection depends on what kind of area is included and what the r:ojected map will be used for. In many cases there is no clear advantage to using one type of projection over another. Because a complete discussion of the 
different types of map projection is beyond the scope of this document, the following are very simplified descriptions of how the different map projections might be used.

The Geographic projection is just a straightforward projection of coordinates onto a regular X-Y plane. This projection is fine for small areas (say, up to a 100-mile radius) where the difference between a flat plane and the curved surface of the earth is not very large. The Geographic projection requires no special processing of coordinates, so it is the fastest projection. For most uses, especially for small areas and for applications where speed is important, the Geographic projection is adequate.

In general the Universal Transverse Mercator (UTM) and Transverse Mercator projections are useful for medium-sized areas (up to 1000-mile radius) or where the largest extent of the mapped area extends north-south rather than east-west. For larger areas, or where the largest extent of the mapped area extends east-west, you might use the Albers, Lambert, or Mercator projections. The Polar Stereographic projection might be the projection of choice for either of the polar areas of the globe. The following table summarizes these general applications.

Map Projection Type

\section{Geographic}

Universal Transverse Mercator (UTM) or Transverse Mercator

\author{
Albers Conical Equal-Area \\ or Lambert Conformal Conic \\ Polar Stereographic
}

Situations for Use

small areas (up to a 100-mile radius)

medium-sized areas (up to 1000-mile radius) or where the largest extent of the mapped area extends north-south rather than east-west

larger areas, or where the largest extent of the mapped area extends east-west

polar areas of the globe 


\section{Appendix $\mathrm{E}$ IBS Privilege Listings}

The PDMGR (Permiti.Dat Manager) utility is used by the database administrator or system manager to change IBS privileges for IBS users. The IBS privileges for all users are specified in the IBS authorization file (PERMITI.DAT). Most IBS programs then access PERMITI.DAT to see whether a specific user has privileges to use the programs or modules within the programs.

In the process of adjusting user privileges, PDMGR displays Permission Management screens similar to the following:

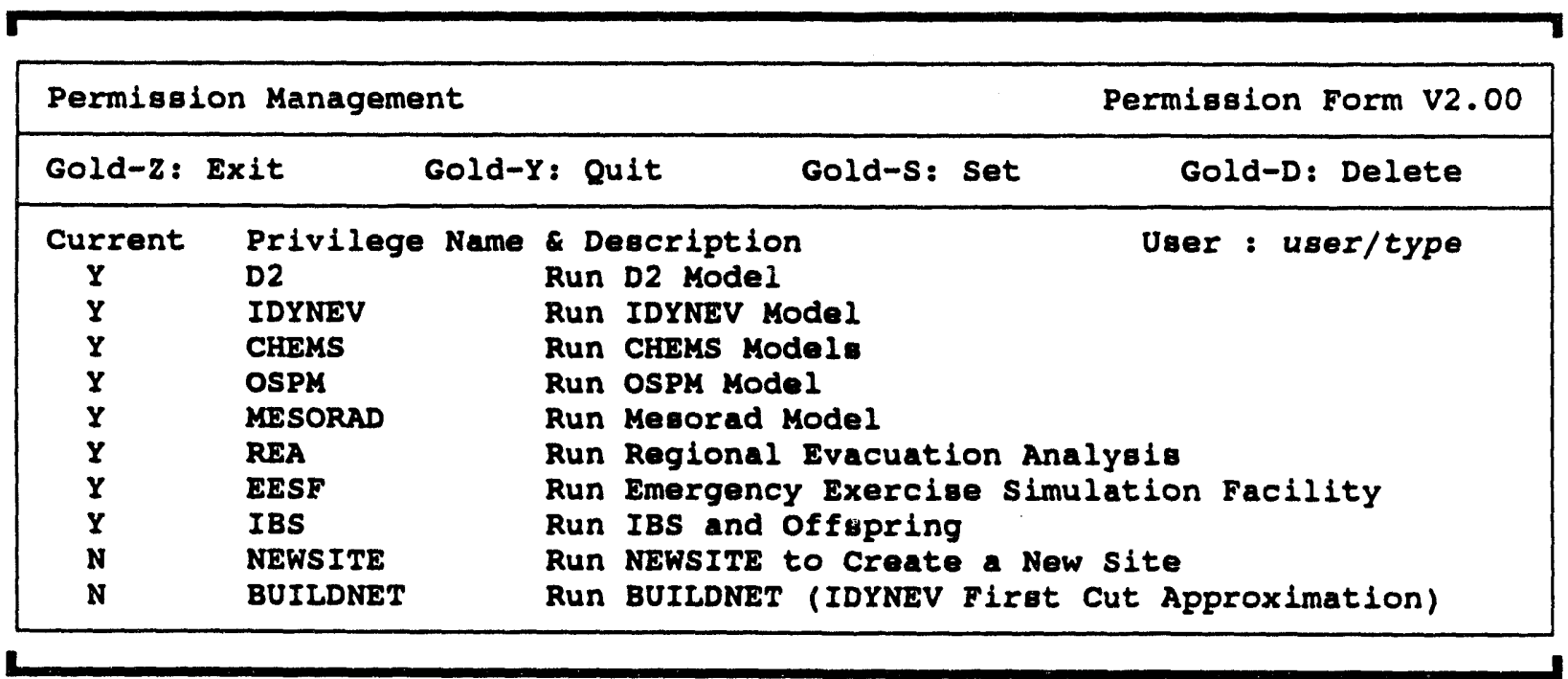

A Y (or $\mathbf{N}$ ) entered beside each privilege grants (or revokes) the privilege.

Use the arrow keys to move between fields. Press the up (or down) arrow key when on the first (or last) line of the form to show the previous (or next) ten privileges, if there are any.

Table E.1 lists the privileges (in the order they appear in PDMGR) along with some general programs and functions that require those privileges.

Table E.2 lists the PDMGR privileges according to the menu options of the CSEPP IBS program.

Table E.3 is a reverse list of the CSEPP IBS program menu options listed according to an alphabetical ordering of PDMGR privileges. 
Table E.1. IBS Privileges Listed in PDMGR

\begin{tabular}{|c|c|c|}
\hline IBS Privilege Name & Privilege Description in PI & Controls These Programs or Punctions \\
\hline $\begin{array}{l}\text { D2 } \\
\text { IDYNEV } \\
\text { CHEMS } \\
\text { OSPM } \\
\text { MESORAD } \\
\text { REA } \\
\text { EESF }\end{array}$ & $\begin{array}{l}\text { Run D2 Model } \\
\text { Run IDYNEV Model } \\
\text { Run CHEMS Models } \\
\text { Run OSPM Model } \\
\text { Run Mesorad Model } \\
\text { Run Regional Evacuntion Analyais } \\
\text { Run Emergency Exercice Simulation Facility }\end{array}$ & $\begin{array}{l}\text { <see Table E.3> } \\
\text { REA from IBSSH, <see Table E.3> } \\
\text { Update Case Data, Change Case/Site in CHEMS } \\
\text { OSPM NOGR } \\
\text { - unuced. } \\
\text { - unused - } \\
\text { - unused. }\end{array}$ \\
\hline $\begin{array}{l}\text { IBS } \\
\text { NEWSITE } \\
\text { BUILDNET } \\
\text { GEDIT } \\
\text { MPDISPLAY } \\
\text { DELSITE } \\
\text { NEWLOG } \\
\text { ARCHIVE }\end{array}$ & $\begin{array}{l}\text { Run IBS and Offopring } \\
\text { Run NEWSITE to Create a New Site } \\
\text { Run BUILDNET (IDYNEV First Cut Approx) } \\
\text { Run GEDIT (Graphical Editor) } \\
\text { Run MPDISPLAY (Map Display) } \\
\text { Run DELSITE to Delete \& Site } \\
\text { Run NEWLOG to Create New Log Journals } \\
\text { Run SITEBKP to Backup a Site }\end{array}$ & $\begin{array}{l}\text { IBS } \\
\text { NEWSITE } \\
\text { REA from IBSSH } \\
\text { - unused - } \\
\text { - unused - } \\
\text { DELSITE } \\
\text { NEWLOG } \\
\text { SITEBKP, <see Table E.3> }\end{array}$ \\
\hline $\begin{array}{l}\text { SW_MANAGER } \\
\text { WS_MANAGER } \\
\text { PER_MANAGER } \\
\text { POP_MANAGER } \\
\text { LOC_MANAGER } \\
\text { RA_MANAGER } \\
\text { RM_MANAGER } \\
\text { LGD'MANAGER } \\
\text { PD_ĒANAGER } \\
\text { CNX_MANAGER }\end{array}$ & $\begin{array}{l}\text { Create/send IBS to Onpost reports } \\
\text { Create/send Onpost to IBS reports } \\
\text { Edit Perconnel Database } \\
\text { Bdit Special Population Database } \\
\text { Edit People Location Databace } \\
\text { Edit Risk Area Analysis Database } \\
\text { Edit Facility \& Resource Database } \\
\text { Edit the Common Legend } \\
\text { Run PDMGR to Edit the Permissions Database } \\
\text { Edit the shared CaseIndex file }\end{array}$ & $\begin{array}{l}\text { <see Table E.3> } \\
\text { ONPOSTSIM, <see Table E.3> } \\
\text { RMMGR, <see Table E.3> } \\
\text { <see Table E.3> } \\
\text { LOCATE, DELPTDB } \\
\text { <see Table E.3> } \\
\text { RMMGR, IMPORT } \\
\text { <see Table E.3> } \\
\text { PDMGR } \\
\text { <see Table E.3> }\end{array}$ \\
\hline $\begin{array}{l}\text { D2_ANW } \\
\text { PLANNER } \\
\text { COMBINED_LOG } \\
\text { ADD_TO_LOG } \\
\text { CHG_OPER_SITE } \\
\text { RESTORE_SHARED } \\
\text { DECLARE_EVENT } \\
\text { C1_EDIT } \\
\text { D2_SELECT } \\
\text { IDYYNEV_SELECT }\end{array}$ & $\begin{array}{l}\text { Do D2 Animation } \\
\text { Do Planning Functions } \\
\text { Create the Combined Log } \\
\text { Annotate the Log Journal } \\
\text { Change the Operational Site } \\
\text { Restore Shares Dats from Backup } \\
\text { Declare an Event } \\
\text { Check in/out from the shared CaseIndex } \\
\text { Select D2 case } \\
\text { Select Evacuation case }\end{array}$ & $\begin{array}{l}\text { <see Table E.3> } \\
\text { REA from IBSSH, <see Table E.3> } \\
\text { <see Table E.3> } \\
\text { <see Table E.3> } \\
\text { OSPM_NOGR, REA, <see Table E.3> } \\
\text { <see Table E.3> } \\
\text { <see Table E.3> } \\
\text { <see Table E.3> } \\
\text { <see Table E.3> } \\
\text { REA from IBSSH, <see Table E.3> }\end{array}$ \\
\hline $\begin{array}{l}\text { IP_CREATE } \\
\text { IP_SELECT } \\
\text { IP_CHANGE } \\
\text { IP_USE } \\
\text { IP_VALIDATE } \\
\text { IP_LINK }\end{array}$ & $\begin{array}{l}\text { Create an IP } \\
\text { Select a new Common IP } \\
\text { Change the Common IP } \\
\text { Use (Checkoff) Tasbs in the Common IP } \\
\text { Validate an IP } \\
\text { Link an IP to D2 \& IDYNEV Cases }\end{array}$ & $\begin{array}{l}<\text { see Table E.3> } \\
\text { <see Table E.3> } \\
\text { <see Table E.3> } \\
\text { <see Table E.3> } \\
\text { <see Table E.3> } \\
\text { <see Table E.3> }\end{array}$ \\
\hline $\begin{array}{l}\text { COPY_TO_INFO } \\
\text { COPY_TO- } \\
\text { COPY_FROM }\end{array}$ & $\begin{array}{l}\text { Copy Data to Infomanager } \\
\text { Copy Data to Some Other User } \\
\text { Copy Data from Some Other User }\end{array}$ & $\begin{array}{l}<\text { see Table E.3> } \\
<\text { see Table E.3> } \\
<\text { see Table E.3> }\end{array}$ \\
\hline $\begin{array}{l}\text { EOC_ACTIVATION } \\
\text { SEAR्RCH_RESCUE } \\
\text { DAMAGE_ASSESS } \\
\text { EF }\end{array}$ & $\begin{array}{l}\text { Do EOC Activation Functions } \\
\text { Do Search \& Rescuc Functions } \\
\text { Do Demage Ascessment Punctions } \\
\text { Emergency Functions (1 of up wo 36) }\end{array}$ & $\begin{array}{l}<\text { see Table E.3> } \\
\text { <see Table E.3> } \\
\text { <see Table E.3> } \\
\text { <see Tabie E.3> }\end{array}$ \\
\hline
\end{tabular}


Table E2. Privileges Listed by Program \& by Menu Options in the IBS Program

These options for the IBS program Are controlled by

\begin{tabular}{|c|c|}
\hline These options for the IBS program & these privileges in PDMGR \\
\hline IBS (privilege set up for all IBS users) & IBS \\
\hline Message Board & - none - \\
\hline - Annotate Logs & ADD_TO_LOG \\
\hline$\rightarrow$ Create Common Log Report & COMBINED_LOG \\
\hline$\rightarrow$ Create IBS $\rightarrow$ Onpost Rpt & SW_MANAGER \\
\hline$\rightarrow$ Resend IBS $\rightarrow>$ Onpost Rpt & SW_MANAGER \\
\hline - VAX Mail & - none - \\
\hline Status Board & - none - \\
\hline$\rightarrow$ Archive & ARCHIVE \\
\hline - Archive IP Options & ARCHIVE \\
\hline - Check-In IP (planning mode) & $\begin{array}{l}\text { CI EDIT \& COPY_TO_INFO } \\
\text { \& IP_CREATE }\end{array}$ \\
\hline$\rightarrow$ Check-Out IP (planning mode) & $\begin{array}{l}\text { CI EDIT \& COPY_FROM } \\
\text { \& IP_CREATE }\end{array}$ \\
\hline - Continuous Map Update (operational mode) & - none - \\
\hline - Create New IP (planning mode) & IP_CREATE \\
\hline - Implementing Procedures & - none - \\
\hline$\rightarrow$ Change IP Notes (planning mode) & $E F(i)$ \& IP_CHANGE \\
\hline $\begin{array}{c}\text { - Change Tasks (for emergency function) } \\
\text { (planning mode) }\end{array}$ & $\begin{array}{l}E F(i) \\
\& \text { IP_CHANGE }\end{array}$ \\
\hline$\vec{\rightarrow} \rightarrow$ (Add New Agency/Position) (planning mode) & $\begin{array}{l}\text { EF(i) \& IP_CHANGE } \\
\text { \& PER_MĀNAGER }\end{array}$ \\
\hline$\rightarrow \rightarrow$ Actions (planning mode) & $E F(i) \&$ IP_CHANGE \\
\hline$\rightarrow-$ Resources (planning mode) & $E F(i) \&$ IP_CHANGE \\
\hline$\rightarrow \rightarrow$ Phase Display & IP_CHANGE \\
\hline$\rightarrow$ - Select New IP & IP_SELECT \\
\hline $\begin{array}{c}\text { - - Use Tasks (for emergency function) } \\
\text { (operational mode) }\end{array}$ & $E F(i) \&$ IP_USE \\
\hline$\rightarrow \rightarrow$ Actions (operational mode) & $E F(i) \&$ IP_USE \\
\hline$\rightarrow \rightarrow-$ Resources (operational mode) & $E F(i) \&$ IP_USE \\
\hline $\begin{array}{c}\rightarrow \rightarrow \text { (Adding a new Agency/Position) } \\
\text { (operational mode) }\end{array}$ & $\begin{array}{l}E F(i) \& \text { IP USE } \\
\text { \& PER_MĀNAGER }\end{array}$ \\
\hline - - View Tasks (for emergency function) & - none - \\
\hline
\end{tabular}


These options for the IBS program

$\rightarrow$ Reset IP

- Select Onpost Work Activity

- Validate IP

- View Onpost Activity -> Declare Event

- View Onpost Work Plan -> Select IP

Are controlled by these privileges in PDMGR

IP_CHANGE

WS_MANAGER

IP_VALIDATE

DECLARE_EVENT

IP SELECT

Personnel Management

- Add Agencies

- none -

- Add People

PER_MANAGER

- Add Positions

PER_MANAGER

$\rightarrow$ Delete Agencies

PER_MANAGER

- Delete People

PER_MANAGER

$\rightarrow$ Delete Positions

PER_MANAGER

$\rightarrow$ Modify Agencies

PER_MANAGER

$\rightarrow$ Modify People

PER_MANAGER

- Modify Positions

PER_MANAGER

PER MANAGER

Map Analysis (all functions) - none -

Resource Management

- Add Facilities

$\rightarrow-$ (Adding a new Person)

- none -

- Delete Facilities

RM_MANAGER

PER MANAGER

\& R̄M_MANAGER

$\rightarrow$ Modify Facilities

$\rightarrow \rightarrow$ (Adding a new Person)

RM_MANAGER

RM_MANAGER

PER MANAGER

\& RTM_MANAGER

- Assign Resources

RM_MANAGER

- Define Resources

RM_MANAGER

- Delete Resources

RM_MANAGER

- Modify Resources

RM_MANAGER

- Move Resources

RM MANAGER

Show Latest Onpost Track - none -

Refresh Latest Onpost Track - none -

Analyze D2 Track - none - 
These options for the IBS program

Are controlled by these privileges in PDMGR

Change D2 Case

D2 SELECT

Show D2 Description

- none -

Emergency Activities

- Damage Assessment

- none -

$\rightarrow$ Delete Contamination Area

- none -

- Delete National Defense Area

DAMAGE_ASSESS

DAMAGE_ASSESS

- Draw Contamination Area

DAMAGE_ASSESS

$\rightarrow$ - Draw National Defense Area

DAMAGE_ASSESS

- Direction/Control

$\rightarrow$ Add Facilities

- none -

$\rightarrow$ Modify Facilities

RM_MANAGER

- - Add Special Population

RM_MANAGER

POP_MANAGER

$\rightarrow$ Modify Special Population

POP_MANAGER

- - Risk Area Analysis

$\rightarrow \rightarrow$ Pick Risk Polygon

- none -

$\rightarrow \rightarrow$ Draw Wedge Risk Polygon

RA_MANAGER

- - D Draw New Risk Polygon

RA_MANAGER

- - Delete Drawn Risk Polygon

RA_MANAGER

RA_MANAGER

$\rightarrow$ - Save Current Risk Area

RA_MANAGER

- - - Add EP Zone (Name)

RA_MANAGER

$\rightarrow \rightarrow$ Add EP Zone (Pick)

RA_MANAGER

$\rightarrow$ - Delete EP Zone (Name)

RA_MANAGER

$\rightarrow \rightarrow$ Delete EP Zone (Pick)

RA_MANAGER

$\rightarrow$ - Select IP

IP_SELECT

- EOC Activation

$\rightarrow$ - Generate Call List

- none -

EOC_ACTIVATION

- Evacuation

- - Evac Route Overlays

- none -

SW_MANAGER

$\rightarrow \rightarrow$ Create Route Overlays

SW_MANAGER

- - - Delete Route Overlays

SW_MANAGER

- - - List Route Overlays

SW_MANAGER

- Execute Evac Model

IDYNEV 
These options for the IBS program

- Select Model Case

$\rightarrow$ Hazard Analysis

$\rightarrow$ Change Track Case

$\rightarrow$ Execute D2

- - Add Scenario Records (planning mode)

$\rightarrow-$ Delete Scenario Records (planning mode)

$\rightarrow-$ Modify Scenario Records (planning mode)

$\rightarrow \rightarrow$ Change Track Case

$\rightarrow-$ Copy Onsite D2 Input to Offsite

$\rightarrow \rightarrow$ Create New D2 Case

$\rightarrow-$ Create Scenario for Current D2 Case

- - - Modify D2 Case Input

- - Perform File Maintenance

- - Run Current D2 Case

- Search/Rescue

- Delete Search Areas

- Draw Search Areas

- Sheltering

- Add Facilities

$\rightarrow$ Modify Facilities

$\rightarrow$ - Add Location

\section{Setup}

- Backup/Restore Site Data

$\rightarrow$ Restore

- Change Site

- Change Operational Site

- Common Legend (operational mode)

- Job Environment

- Change D2 Case
Are controlled by these privileges in PDMGR

\section{IDYNEV_SELECT}

- none -

\section{D2_SELECT}

D2

D2 \& IP_LINK

D2 \& IP_LINK

D2 \& IP_LINK

D2 \& D2_SELECT

CI EDIT \& COPY_FROM \& D2

D2 \& D2_SELECT

D2 \& IP_LINK

D2 \& D2_SELECT

D2

D2

- none -

SEARCH_RESCUE

SEARCH_RESCUE

- none -

RM_MANAGER

RM_MANAGER

RM MANAGER

- none -

ARCHIVE (or none in planning mode)

ARCHIVE (or none in planning mode)

CHG_OPER SITE or PLANNER

CHG_OPER_SITE

LGD_MANAGER

- none -

D2_SELECT 
These options for the IBS program

Are controlled by

\begin{tabular}{|c|c|}
\hline These options for the IBS program & these privileges in PDMGR \\
\hline - Change Evac Case & IDYNEV_SELECT \\
\hline - - Change IP Case & IP_SELECT \\
\hline - - Copy Case Site & COPY_TO or COPY_FROM \\
\hline$\rightarrow \rightarrow$ General Copy Case Site & - none - \\
\hline$\rightarrow \rightarrow$ Check-In D2 & CI_EDIT \& COPY_TO \\
\hline$\rightarrow \rightarrow$ Check-Out D2 & CI_EDIT \& COPY_FROM \\
\hline$\rightarrow \rightarrow$ Check-In Evac & CI_EDIT \& COPY_TO \\
\hline$\rightarrow \rightarrow$ Check-Out Evac & CI_EDIT \& COPY_FROM \\
\hline$\rightarrow \rightarrow$ Check-In IP & CI_EDIT \& COPY_TO \\
\hline$\rightarrow \rightarrow$ Check-Out IP & CI_EDIT \& COPY_FROM \\
\hline$\rightarrow \rightarrow$ Copy Topo & $\begin{array}{l}\text { COPY TO \& COPY_FROM } \\
\& \text { COPY_TO_INFO- }\end{array}$ \\
\hline$\rightarrow \rightarrow$ Copy Pop Set & $\begin{array}{l}\text { COPY TO \& COPY_FROM } \\
\& \text { COPY_TO_INFO- }\end{array}$ \\
\hline$\rightarrow \rightarrow \rightarrow$ Delete Site & DELSITE \\
\hline Set Basemap & - none - \\
\hline Map Zoom & - none - \\
\hline Zoom In & - none - \\
\hline Zoom Out & - none - \\
\hline Refresh Screen & - none - \\
\hline
\end{tabular}


Table E3. IBS Program Menu Options Listed by Privilege

This Privilege in PDMGR

ADD_TO_LOO
ARCHIVE (or none in
planning mode)
ARCHIVE
CHG_OPER_SITE
CI_EDIT

COMBINED_LOG

COPY_FROM

COPY_TO
Controls Theac Options in the IBS Program

Moange Board -> Annotale Logs

Setup -> Backup/Rectore Site Date

Sexup -> Beckup/Reatore Site Data - > Reatore

Status Bourd -> Archive

Status Board -> Archive IP Options

Setup $->$ Change Site *

Setup $->$ Change Site $->$ Change Operational Site

Emergency Activitien - > Hazard Analysis -> Execute D2 -> Copy Onsite D2 Input to Offsite*

Setup $->$ Job Environment $->$ Copy Case Site $->$ Check-In Evec $\bullet$

Setup - > Job Environment - > Copy Case Site -> Check-Out Evec *

Setup -> Job Environment -> Copy Case Site -> Check-Out D2 *

Setup $->$ Job Environment $->$ Copy Case Site $->$ Chock-In IP *

Setup - $>$ Job Environment - $>$ Copy Case Site $->$ Check-Out IP *

Setup -> Job Environment -> Copy Case Site -> Check-In D2 *

Status Board -> Check-In IP * (planning mode)

Status Board $\rightarrow>$ Check-In IP * (planning mode)

Status Board $->$ Check-Out IP * (planning mode)

Stutus Board - > Check-Out IP * (planning mode)

Mearage Board - > Create Common Log Report

Emergency Activities -> Hezard Analysis - > Execute D2 -> Copy Onsite D2 Input to Offsite *

Setup $->$ Job Environment $->$ Copy Case Site

Setup -> Job Environment - > Copy Cawe Site $->$ General Copy Case Site *

Setup $->$ Job Environment $->$ Copy Case Site $->$ Check-Out D2 *

Setup -> Job Environment -> Copy Case Site -> Check-Out Evac *

Setup $->$ Job Environment $->$ Copy Case Site $->$ Chock-Out IP *

Setup $->$ Job Environment $->$ Copy Cace Site $->$ Copy Pop Set *

Setup $->$ Job Environment $->$ Copy Cace Site $->$ Copy Topo

Stutus Board -> Chock-Out IP * (planning mode)

Status Board -> Check-Out IP * (planning mode)

Setup -> Job Environment -> Copy Case Site

Setup -> Job Environment - > Copy Case Site - $>$ General Copy Cace Site *

Setup $->$ Job Environment - $>$ Copy Care Site - > Chock-In D2 *

Setup -> Job Environment - > Copy Care Site -> Check-In Evac *

Setup -> Job Environment - > Copy Case Sile - > Check-In IP *

Setup - > Job Environment - > Copy Case Site - > Copy Pop Set *

- This option requires other privileges aleo. 
Controls Thene Options in the IBS Progrem

COPY_TO_INFO
D2

Setup -> Job Environment - > Copy Case Site $>>$ Copy Topo *

Setup - > Job Environment - > Copy Case Site $->$ Copy Pop Set

Setup -> Job Environmeat - > Copy Case Sito - > Copy Topo *

Stutus Board -> Check-In IP * (plenning mode)

Status Board -> Check-In IP * (plenning mode)

Emergency Activities -> Hezard Analysis - > Execute D2

Emergency Activities - > Hezard Analysis - > Execute D2 -> Create Scenario for Current D2

Emergency Activities -> Hazard Analysis -> Execute D2 -> Add Scenario Records * (planning mode)

Emergency Activities - > Hazard Andysis - > Execute D2 - > Change Track Case *

Emergency Activities - > Hezard Analyais - > Execute D2 -> Create Now D2 Case *

Emergency Activities -> Hazard Analysis -> Execute D2 -> Perform File Maintenance

Emergency Activities - > Hazard Andysis - > Execute D2 -> Run Current D2 Case

Emergency Activities -> Hezard Analysis -> Execute D2 -> Delete Scenario Records * (planning mode)

Emergency Activities - > Hezard Analysis - > Execute D2 -> Modify Scenario Records * (planning mode)

Emergency Activitien -> Hezard Analysis -> Execute D2 -> Copy Onsite D2 Input to Offsite *

Emergency Activities -> Heznrd Analysis -> Execute D2 -> Modify D2 Case Input *

D2_SELECT

Change D2 Track Case

Emergency Activitiea - > Hazard Analysis -> Change Track Case

Emergency Activities -> Hazard Analysis - > Execute D2 -> Change Track Case *

Emergency Activities -> Hazard Analysis - > Execute D2 -> Create New D2 Case*

Emergency Activities -> Hazard Analysis - > Execute D2 -> Modify D2 Case Input *

Setup -> Job Environment -> Change D2 Case

DAMAGE_ASSESS Emergency Activities - $>$ Damage Aweament $->$ Delete Contamination Area

Emergency Activitios - > Demage Asecesment - > Delete National Defenee Area

Emergency Activities - > Demage Aseeasment -> Draw Contamination Area

Emergency Activities -> Damage Ascenoment -> Draw National Defence Ares

DECLARE_EVENT

Status Board - > Viow Onpont Activity - > Declare Event

DELSITE

Setup - > Job Environment - > Copy Case Site - > Delete Site

Emergency Function EF(i)

Status Board -> Implementing Procedures -> Change IP Notes * (planning mode)

Stacus Board -> Implementing Procedures -> Change Taks (for emergency function) * (planning mode)

Status Board -> Implementing Procedures -> Change Tasks -> Actions * (planning mode)

Status Board -> Implementing Procedures -> Change Tasks -> Resources * (planning mode)

Status Board -> Implementing Procedures -> Change Tasks - >

(Adding a new Agency/Position) * (planning mode)

- This option requires other privileges also. 
EOC_ACTIVATION

IBS

IDYNEV

IDYNEV_SELECT

IP_CHANGE

IP_CREATE

IP_SELECT

IP_LINK

IP_SELECT

IP_USE
Status Board - > Implemeating Procedures - > Use Takes (for emergency function) * (operational mode)

Stutus Board -> Implementing Procedures - > Use Tacks -> Actions * (Operational mode)

Status Board -> Implementing Procedures - > Uee Teske - > Resources * (operational mode)

Stutus Board -> Implementing Procedures - > Uee Takk -> (Adding a new Agency/Position) * (operational modo)

Emergency Activitiea -> EOC Activation - > Cenerate Call Lixt

IBS (privilege set up for all IBS ueers)

Emergency Activities - $>$ Evacuation - > Execute Evac Model

Emergency Activities -> Evecuation $->$ Select Model Case

Setup - > Job Environment - > Change Evac Case

Sturus Board -> Implementing Procedures -> Change IP Notes * (planning mode)

Stutus Board -> Implementing Procedures $->$ Change Takk * (planning mode)

Status Board -> Implementing Procedures - > Change Tasks -> (Adding a now Agency/Position) * (planning mode)

Status Board -> Implementing Procodures -> Change Taks (for emergency function) *

Status Board -> Implementing Procedures -> Change Taks -> Actions * (planning mode)

Status Board -> Implementing Procedures -> Change Tasks -> Resources * (planning mode)

Stutus Board -> Implementing Procodures -> Phuse Dieplay

Stutus Board -> Reece IP

Stutua Board -> Check-In IP * (planning mode)

Stutus Board -> Chock-Out IP * (plenning mode)

Status Board -> Create New IP (planning mode)

Emergency Activities -> Direction/Control -> Select IP

Emergency Activities -> Hezard Analysis - > Execute D2 -> Add Scenario Records * (planning mode)

Emergency Activities - > Hazard Analysis - > Execute D2 -> Create Scenario for Current D2 Case

Emergency Activities - > Hazard Amalyais -> Execute D2 -> Delete Scenario Records * (planning mode)

Emergency Activities -> Hazand Analysis -> Exocute D2 -> Modify Scenario Records * (planning mode)

Setup - > Job Environment -> Change IP Case

Statun Board -> Change Onpoat Work Plan -> Seloct IP

Status Board -> Implementing Procedures - > Select New IP

Status Board - > View Onpoat Work Plan -> Select IP

Status Board -> Implementing Procedures - > Use Takse (for emergency function) (operational mode)

Status Board -> Implementing Procedures - > Use Tacks - > Actions * (operational mode)

Status Board -> Implementing Procedures - > Use Teeks -> Resources * (operational mode)

- This option requires other privileges also. 
Scatus Board -> Implementing Procedures -> Use Tauke -> (Adding a now Agency/Position)* (operational mode)

IP_VALIDATE

LOD_MANAGER

PER_MANAGER

PLANNER

POP_MANAGER

RA_MANAGER

RM_MANAGER
Stutue Board -> Validate IP

Stutus Board $->$ Validate If

Sexup -> Common Logend (operational mode)

Perwonnel Management - > Add Agencies

Perwonnel Management - > Delele Agencies

Perwonnel Manugement - > Modify Agenciea

Perwonnel Management - > Add Poople

Perwonnel Management - > Delcte Pcople

Perwonnel Management - > Modify People

Perwonnel Menagemeat -> Add Positions

Perwonel Managemeat -> Delete Positions

Pereonnel Management - > Modify Positions

Rewource Management - $>$ Add Facilities - > (Adding a new Person) *

Reeource Management - > Modify Facilities - > (Adding a new Person) *

Status Board -> Implementing Procedures -> Change Take ->

(Adding a new Agency/Position) " (planning mods)

Status Board -> Implementing Procodures - > Une 'Tacks - > (Adding a new Agency/Position) * (operational mode)

Setup $->$ Change Site

Emergency Activities - > Direction/Control - > Add Special Population

Emergency Activitiea - > Direction/Control - > Modify Special Population

Emergency Activities - > Direction/Control - > Riek Area Analysis -> Add EP Zone (Name)

Emergency Activities - > Diroction/Control - > Riak Area Analysis -> Add EP Zone (Pick)

Emergency Activities - > Direction/Control - > Rink Area Analysis -> Delete Drawn Riak Polygon

Emergency Activitiee -> Direction/Control -> Rink Area Analysis -> Delete EP Zone (Name)

Emergency Activities - > Direction/Control -> Rink Area Analysis -> Delete EP Zone (Pick)

Emergency Activitien -> Direction/Control -> Rink Area Analysis -> Draw New Riak Polygon

Emergency Activities -> Direction/Control -> Risk Area Analysis -> Draw Wedge Risk Polygon

Emergency Activities - > Direction/Control -> Riek Area Analysis -> Pick Rick Polygon

Emergency Activities - > Direction/Control -> Riak Area Analysis - > Save Current Risk Area

Emergency Activities - > Direction/Control -> Add Facilities

Emergency Activities -> F'section/Control -> Mlodify Facilities

Emergency Activities - > Sheltering - $>$ Add Facilities

Emergency Activities - > Sheltering - > Modify Facilities

Emergency Activities - > Sheltering - > Add Location

* This option requires other privileges aleo. 
Resource Management - > Add Facilitice

Recource Management - > Add Facilitiea - > (Adding a now Pereon) •

Resource Management - > Delete Facilitiea

Resource Managemeat - > Modify Facilitiea

Resource Management - > Modify Facilities - > (Adding a now Pereon) *

Reeource Management - > Assign Rosources

Resource Manegement - > Define Rosources

Resource Management - > Delete Rewources

Reeource Management - $>$ Modify Resources

Recource Management - > Move Rewurces

SEARCH_RESCUE

Emergency Activities - > Search/Reacue - > Delete Search Areas

Emergency Activitiea - > Search/Rescue - > Draw Search Areas

SW_MANAGER

Emergency Activities -> Evacuation -> Evac Route Overlays

Emergency Activities -> Evacuation -> Evec Route Overlaya -> Create Route Overiays

Emergency Activitiea -> Evacuation $->$ Evec Route Overlays $\rightarrow>$ Delete Route Overlays

Emergency Activitiea - > Evecuntion -> Evac Route Overlays -> Liat Route Overlays

Measage Board -> Create IBS -> Onpoat Rpt

Mearage Bourd -> Resend IBS -> Onpout Rpt

WS_MANAGER

Stutus Board -> Select Onpost Work Activity

- This option requires other privileges alwo. 


\section{Appendix F Undocumented Utility Programs}

The following IBS utility programs are NOT documented in detail in Section 2 of this document because they are either 1) subprograms called from other IBS programs or 2) special-purpose programs intended for use only by system developers. These programs should NOT be removed from the system.

\section{Utilities Called by other IBS Programs}

Utility Program

ALLSITE.COM

CHGOSITE.EXE

CHKUSER.COM

DELTOPO.COM

FDISP.COM

FIXCTRLZLOG.COM

GETDISKS.COM

IBS SM.EXE

MAKEE T DAT.COM

MD AD̄D.EXE

MD DELETE.EXE

PD ADD.EXE

PD DELETE.EXE

PRËP LOG.COM

SORT MAIL.COM

SORT TF.COM

TRANSLOG.COM

VIEWEVENTLOG.COM
Short Description

Create list of locations of all known user sites

(ALLSITE.DAT)

Installed image: Change operational site

See if user account exists

Delete topography

Display file

Remove CTRL-Z from mail log

Get list of disks on the system

Installed image: Shared memory

Create list of DMS of TAC files in current directory

Add entry to Master Directory file

Delete entry from Master Directory file

Add entry to Permission file

Delete entry from Permission file

Sort $\log$ journal by date (reversed) and entry \#

Sort mail $\log$ by date (reversed) and entry \#

Sort TIGER files before conversion

Completely translate a logical name

Look at EVENT log 


\section{Utilities Intended for Use by IBS Developers}

Utility Program

COUNT CODE.EXE

DEDISPL̄AY.EXE

DEPRINT.EXE

DOCUMENTOR.EXE

DTBLGEN.EXE

LIMITXX2.COM

LIMITXX3.COM

MAKE HELP.EXE

MKBUĪLDEXE.COM

MKBUILDLIB.BIS

MKBUILDLIB.COM

MKTBLGEN.COM

SEE HELP.EXE

TBLG̈EN.EXE
Short Description

Count \# of lines in certain types of code files

Display a form

Print a form

Extract routine header, declaration, include files from code

Create form from TIN file

Create limit file for a topography

Create limit file for a topography

Create DIGCART help file

Create BUILDEXE file for current directory

Create BUILDLIB file for all directories

Create BUILDLIB file for current directory

Create TBLGEN file for current directory

Dump DIGCART help file

Create form from TIN file 
A

agency data 2.328

importing 2.177

ATTRIBDIC.BDX 2.11

used to create declutter input file 2.34

attribute codes $\mathbf{1 . 2 1}$

attribute dictionary vi, $1.10,1.20,1.21$

ASCII versus binary forms $2.8,2.11$

combining two dictionaries 2.76

contents of B.1

modifying 2.11

attributes

updating 2.383

adding 2.384

adding attributes to DMS objects 2.3

adding to map objects 2.110

attribute dictionary $\mathbf{B . 1}$

Attribute Functions in MPDISPLAY 2.244

Attribute Menu in GEDIT 2.108

Boolean logical expressions for 2.3

categorical and numeric 2.33

changing 2.385

conversion [octal, hexadecimal, decimal] 2.339

decluttering attributes $\mathbf{2 . 3 3}$

defined 2.103

deleting 2.385

deleting from map objects 2.110

frequency in a DMS file 2.9

lookup 2.111

major codes list B.1

minor code B.2

modifying 2.111

objects with no attributes 2.354

of map objects 2.109

parameter value B.2

replacing attribute codes 2.227

selection criteria for decluttering 2.69

statistics 2.355

updating system attributes 2.383

using for decluttering $\mathbf{2 . 3 3}$

audience for utilities guide vi

B

Background Programs 1.1

mail status checking 2.199

starting the EVENT program 2.65

stopping EVENT program 2.358

stopping MAIL_C program 2.359 backups of site data 2.345

C

Case Index file [CASEINDEX.DAT] 2.80

creating $\mathbf{2 . 7 8}$

listing cases for a site 2.195

color

viewing contents of 2.340

converting DMS objects to a single

color 2.15

composite strings 2.103

Conversion Utilities menu 2.173

converting data files

[see Data File Conversion Utilities] 1.9

coordinates

of a point on a map 2.236

switching coordinate systems in TAC

files 2.361

cover map 2.206, 2.208

D

D2 data

upgrading 2.79

upgrading D2 polygons 2.81

D2 dispersion model

executing a case 2.20

executing multiple cases 2.21

Data File Conversion

ASCII to attribute dictionary 2.8

ASCII to DMS 2.202

attribute dictionary to ASCII 2.11

DEM to TAC 2.52

DLG to DMS 2.53

DMS to ASCII 2.56

DMS to DLG 2.59

DMX ASCII to DMS 2.6

importing IBS data 2.177

sequential to random access 2.337

TAC to DMS 2.16

TIGER boundary data 2.366

TIGER population data 2.306

TIGER to DMS 2.371, 2.379

USGS to DMS 2.394

USGS100K to DMS 2.386

UTM <-to-> Long/Lat [TAC file] 2.361

Data File Conversion Utilities 1.1, 1.3

Data File Modification

adding attributes 2.3

adding decluttering attributes 2.33 
appending DMS files 2.5

changing TAA reference 2.360

changing TXT file reference 2.228

convert sequential file to random access 2.337

converting DMS objects to a singile color 2.15

creating a TAC file 2.204

extracting DMS data from a database 2.67

fixing DMS file limits 2.82

joining vector strings $2.191,2.196$

reducing number of points in a DMS

file $2.353,2.370$

replacing attributes 2.227

rescaling a DMS file 2.332

splitting off objects with no attributes 2.354

subdividing a DMS file 2.13

switching coordinate systems in TAC

files 2.361

Data File Modification Utilities 1.1

data files

Data File Conversion utilities 1.9

Data File Modification utilities 1.7

DEM 2.52

DLG 2.53, 2.59

DMS 1.7

DMX-formatted ASCII files 2.6

DTX files 2.60

importing to IBS database[s] 2.177

MESORAD RSP files 2.93

TAC 1.7, 2.361

TAC files for OSPM 2.204

TIGER 1.9, 2.371, 2.379

USGS 2.394

USGS100K 2.386

Data Management Guide, IBS iii, v

Database Administration

check icon validity $\mathbf{2 . 1 2}$

creating a Case Index file for a user site 2.78

creating a limit file $2.18,2.190$

creating a map database 2.205

creating multiple limit files 2.61

deleting People Tracking Database 2.45

editing an attribute dictionary 2.8, 2.11

limit file display 2.333

list of files for one topography 2.335

$\log$ journal files, fixing 2.83

log journal files, restarting 2.280 map of area covered by topography 2.342

new site creation 2.281

resource management 2.312, 2.321

site database deletion 2.46

site, duplicating $2.63,2.64$

site/restore site data backups 2.345

updating a map database $\mathbf{2 . 2 1 1}$

updating system attributes 2.383

Database Administration Utilities 1.1, 1.3

Database Quality Assurance

checking for empty DMS files 2.25

checking text [TXT] files 2.30

DMS file limits, checking 2.27

DMS file problems, checking 2.22

Database Quality Assurance [QA] Utilities 1.1

databases

People Tracking Database 2.45

site databases 2.281

DCLTABLES

fixing IBS verbs in 1.15

decluttering 2.33, 2.69

attribute input for 2.11

decluttering code B.2

example of 2.38

in MPDISPLAY 2.260

prccedure for applying decluttering attributes 234

default values and file names $\mathbf{2 . 2}$

DEM files

converting to TAC files 2.52

developer utilities F.2

Diagnostic and Reporting

attribute conversion [octal, hexadecimal, decimal] 2.339

attribute frequency in a DMS file 2.9

Case Index file, viewing $\mathbf{2 . 3 4 0}$

contents of TXT files 2.198

current terminal settings 2.344

displaying icons available 2.305

icon validity, checking 2.12

name of TXT file in DMS file header 2.336

reporting TAA file reference in TAC file 2.360

resource management data, checking 2.310

shared memory dump to file/screen 2.350

show terminal colors 2.341 
statistics for a DMS file 2.355

Summary Functions in MPDISPLAY 2.272

TAC file header contents 2.363

text labels of a DMS file 2.60

user environment 2.343

Diagnostic and Reporting Utilities 1.2, 1.3, $1.6,1.16$

Digital Mapping Structure [DMS] 1.17, 2.101

DLG files

converting from DMS 2.59

converting to DMS 2.53

DMS files 1.7, 1.17

adding decluttering attributes 2.33

appending one to another 2.5

checking file limits 2.27

checking for empty files 2.25

checking for problems in $\mathbf{2 . 2 2}$

checking TXT file references 2.30

conversion from TIGER 2.371, 2.379

converting objects to a single color 2.15

converting to ASCII 2.56

converting to DLG 2.59

creating 2.101, 2.202

displaying 2.299

editing 2.101, 2.241

extracting from a site database 2.67

fixing DMS file limits 2.82

graphic data structures 1.17, 2.102

joining vector strings in 2.191, 2.195

map database 1.17

modifying TXT reference 2.228

producing from TAC files 2.16

reducing number of points $2.353,2.370$

rescaling 2.332

statistics 2.355

statistics in MPDISPLAY 2.272

Store Region in GEDIT 2.138

Store Region in MPDISPLAY 2.269

subdividing 2.13

text data for 1.20

topography area coverage map $\mathbf{2 . 3 4 2}$

TXT file name in header $\mathbf{2 . 3 3 6}$

DMX files 2.6

documents

KERMIT documentation files 2.189

list of IBS documents iii

MYTAPE documentation file 2.279

DTX files 2.60
$\mathbf{E}$

electronic mail 2.201

mail list updating 2.219

remote mail list updating 2.226

elevation data

Edit Elevation Data in GEDIT 2.140

viewing in MPDISPLAY 2.253

Emergency Management and Planning

execute D2 dispersion model 2.20

resource maragement 2.312, 2.321

Emergency Management and Planning utilities 1.1

environment

show user environment 2.343

equipment needed 1.2

F

facility data $2.312,2.321,2.322$

importing 2.177

Facility Tracks data 2.314, 2.326

FACIITY TRACK.DAT 2.315, 2.326

file names

default 2.2

files

file formats $v$

$\mathbf{G}$

General System

converting UTM <-to-> Long/Lat

coordinates 2.395

copying files between IBS users 2.169

electronic mail 2.201

general file transfer 2.188

IBS Shell [IBSSH] 2.171

screen colors 2.369

tape file transfer [MYTAPE] 2.279

Tektronix terminal setup 2.338

General System Utilities 1.1

geo-political master file 2.69

graphic data structures $1.17,2.102$

Graphic GIS Utilities menu 2.175

Graphic Map Display and Editing

DMS file display 2.299

general map editing 2.101

map display 2.230

resource management 2.312

Graphic Map Display and Editing Utilities 1.1

Graphic Models menu 2.174

graphics editing 2.101 
H

how to use this guide vii

\section{I}

IBS program iii

IBS Programs menu 2.172

IBS Shell [IBSSH] 2.1

icon control file vi

icon dictionary [icon control file] 1.10, 1.16, 1.20

checking icons in $\mathbf{2 . 1 2}$

displaying dictionary icons 2.305

icons 1.18, 2.103

checking for validity 2.12

displaying 2.305

DMS data files for 1.20

enable/disable in MPDISPLAY 2.260

icon function in MPDISPLAY 2.25;

Icon menu in GEDIT 2.125

seed point 2.103

upgrading for resource management 2.91

IP [Implementing Procedure] upgrading IP data 2.80

italics in this guide viii

J

job position data, importing 2.177

JOB_ENV.DAT 2.292

joining vector strings 2.191, 2.196

journal files

[see log journal files] 2.83, 2.280

$\mathbf{K}$

known points data 2.314, 2.32?

importing 2.177

Known Polygons file

upgrading 2.81

L

limit file 1.10, 1.20

creating $2.18,2.190$

creating for a database 2.61

displaying 2.333

listing for one topography 2.335

saving as ASCII text 2.333

line segment 2.102

deleting in GEDIT 2.118

deleting in MPDISPLAY 2.252
LOCATE program 2.45, 2.65

log journal files 2.280

fixing 2.83

for EVENT processing 2.66

logical names

deassigning map database logical names 2.32

LOGIN.COM 2.292

M

mail 2.201

mail list updating $\mathbf{2 . 2 1 9}$

remote mail list updating 2.226

XMAIl enhancement 2.397

mail checking 2.199

stopping 2.359

MAII_SYSTEMS.DAT 2.226, 2.290

map coordinates

displaying in GEDIT 2.120

map data

data structures 2.102

DMS files 1.17

IBS map data 1.3

overview 1.17

TAC files 1.18

terminology 1.17

map database 1.17

components of 1.18

deassigning mapdb logical names $\mathbf{2 . 3 2}$

directories and files 1.19

extracting from 2.205

updating topography files $\mathbf{2 . 2 1 1}$

upgrading 2.84

map display

MPDISPLAY program 2.230

screen coordinates 2.105

summary statistics in MPDISPLAY 2.272

map editing

GEDIT program 2.101

Miscellaneous menu in GEDIT 2.136

map layer

defined 1.18

map projections vi

in GEDIT 2.115

types described D.2

MESORAD

RSP files 2.93

Message Board 2.201

Miscellaneous Utilities menu 2.174

Models Guide, IBS iii 
$\mathbf{N}$

Non-Graphic Models menu 2.175

$\mathbf{0}$

OFF TO ON.DAT 2.87

OFF'̄TIE database upgrading site directories 2.88

online documentation

for KERMIT 2.189

for MYTAPE 2.279

operational mode 2.281

operational site database 2.281 real and exercise $\mathbf{2 . 2 8 2}$

organization of Utilities Guide vi

OSPM 2.204

Outdoor Sound Propagation Model [OSPM] TAC files for 2.204

$\mathbf{P}$

pen numbers [colors] 2.341 displaying $\mathbf{1 . 1 6}$

People Tracking Database deleting old data 2.45

PERMITI.DAT 2.292, 2.293, E.1

personnel data $2.312,2.321,2.329$

importing 2.177

planning mode 2.281

planning site database 2.281

point 2.102

enable/disable in MPDISPLAY 2.260

point registration in GEDIT 2.136

statistics 2.355

polygons 2.102

complex 2.102

convert string to polygon in GEDIT

2.116

D2 2.81

damage assessment areas 2.81

deleting in GEDIT 2.119

enable/disable fill in MPDISPLAY

2.260

EPZ 2.81

form complex polygon in GEDIT 2.121

geometric manipulation in GEDIT 2.122

polygon editing in MPDISPLAY 2.252

Polygon Fill menu in GEDIT 2.151

risk areas 2.81

search/rescue 2.81

statistics 2.355

positions data 2.328 privileges

for using IBS 1.2

IBS menu options listed by privilege E.8

IBS privileges lists E.1

listed by program or menu option E.3

needed for IBS programs vii

privilege manager utility [PDMGR]

2.293

programs listed by privilege E.2

usertypes 2.293

process quotas 2.292

projections [map] D.1

Q

QA utilities for databases 1.12

quality assurance [QA] 1.12

quotas [process quotas] 2.292

$\mathbf{R}$

READDMS 2.56

reporting utilities

[see Diagnostic and Reporting Utilities] 1.16

resource data 2.323

resource management data 2.310, 2.312, 2.321

importing 2.171

redefining resources 2.325

undefining resources 2.324

$\mathbf{S}$

SCEN INDEX.DAT 2.80

SCEN_TABLE.DAT 2.80

screen colors 2.369

screen coordinates 2.105

screen parameters 2.338

Setup menu of IBS Shell 2.172

shared memory 1.16

shared memory block 1.14

dump to file/screen 2.350

initializing 2.351

setting variables 2.352

site

duplicating a site $2.63,2.64$

listing cases for a site 2.195

listing valid sites 2.86

site data

save/restore site data backups 2.345

site database

creating 2.281

deleting 2.46 
operational 2.281

planning 2.281

SITE.DAT 2.292

special populations 2.329

statistics

for a DMS file 2.355

Summary Functions in MPDISPLAY 2.272

[see Diagnostic and Reporting Utilities] 1.16

string 2.102

deleting in GEDIT 2.117

Insert menu in GEDIT 2.130

merge strings in GEDIT 2.135

Re-Color-Texture in GEDIT 2.154

string editing in MPDISPLAY 2.252

subpolygons 2.103

subprogram utilities F.1

System Administration menu 2.173

System Management

file directory tree, deleting $\mathbf{2 . 4 9}$

IBS privileges, setting 2.293

installing/deinstalling IBS images 2.187

listing valid disks, users, sites 2.86

mail list updating 2.219

map logical names, deassigning 2.32

new user accounts 2.289

remote mail list updating 2.226

shared memory, dump to file/screen 2.350

shared memory, initialize 2.351

shared memory, show/modify variables

2.352

user accounts, deleting 2.50

System Management Guide, IBS iii, v

System Management Utilities 1.1

System Upgrade

adding verbs to DCLTABLES 2.98

combining two attribute dictionaries

2.76

corresponding onpost/offpost sites 2.87

D2 data 2.79

file/directory protections $\mathbf{2 . 9 0}$

IP data $\mathbf{2 . 8 0}$

Known Polygons file 2.81

map databases 2.84

MESORAD RSP files 2.93

onpost/offpost modem link 2.99

permissions file 2.89

resource management icons 2.91

SETUP.COM 2.94 site databases 2.95

site directories 2.88

System Upgrade Utilities 1.1

SYSUAF.DAT 2.397

$\mathbf{T}$

TAA files 2360

TAC file utilities 1.7

TAC files 1.7, 1.18

changing reference to TAA file 2.360

converting from DEM files 2.52

converting to DMS vector contours 2.16

creating 2.204

data file 1.21

elevation data 2.140

header file 1.21

header [.TAC] contents 2.363

UTM <-to-> Long/Lat 2.361

Tektronix terminal

pen numbers [colors] 2.341

screen color setting 2.369

screen default parameters 2.338

showing current settings 2.344

text data 1.22

checking text [TXT] files 2.30

contents of TXT files 2.198

editing in GEDIT 2.157, 2.164

enable/disable in MPDISPLAY 2.260

listing text labels of a DMS file 2.60

name of TXT file in DMS file header 2.336

supporting DMS files 1.20

text attributes 2.103

tert point 2.103

text string manipulation in GEDIT

$2.157,2.164$

TIGER data

boundary data 2.366

conversion to DMS 2.371, 2.379

DMS files based on 2.196

population data 2.306

utilities for 1.9

topographic codes 1.21

topography

2-character codes for 1.21

area coverage map for 2.342

creating 2.5

defined 1.18

descriptions A.1

DMS data file 1.20

selecting in MPDISPLAY 2.266 
static and volatile A.1

topographic codes list A.1

topography control file 1.20

topography description file 1.20

TXT files 2.198

TXT reference in DMS files 2.228

typographic conventions in this guide viii

U

undocumented utilities F.1

undocumented utility programs vii, 1.3

user accounts

deleting 2.50

user environment 2.343

User Guide, IBS iii, v

user privileges 2.293

usertype 2.293

USGS

converting 100K DLG files to DMS 2.386

converting DLG files to DMS 2.53

converting DMS files to DLG 2.59

DEM files 2.52

DLG-to-DMS conversion 1.9

utilities

Background Programs 1.4

capsule descriptions 1.1

categories of 1.1, 1.3

Data File Conversion 1.9

Data File Modification 1.7

Database Administration 1.10

default values in $\mathbf{2 . 2}$

Diagnostic and Reporting 1.16

Emergency Management and Planning

1.4

examples in this guide 2.2

General System 1.5

Graphic Map Display and Editing 1.6

program descriptions 2.1

Quality Assurance [QA] 1.12

shell menu 2.171

starting and using 2.1

System Management 1.14

System Upgrade 1.15

undocumented utilities 1.3

what you need to know before using $\mathbf{1 . 2}$

V

visual conventions in this guide viii 
PNL-8370

Ver. 2.0

UC. 620

\section{DISTRIBUTION}

No. of

Copies

OFFSITE

2 DOE/Office of Scientific and Technical Information

COL Lawrence E. Skelly United States Army Nuclear and Chemical Agency

7500 Backlick Road, Building 5073

Springfield, VA 22150-3198

Mr. J. Thompson

Operations Support Directorate

HQ FEMA

5321 Riggs Road

Gaithersburg, MD 20882

John Bower

Bower Software Services

104 E. 36th Place

Kennewick, WA 99337
No. of

Copies

ONSITE

DQE Richland Operations Office

R. B. Goranson

33 Pacific Northwest Laboratory

B. M. Bailey (20)

M. J. Burford

T. R. Downing

S. W. Matsumoto (2)

S. L. Savelli

S. N. Stevens

J. R. Williams

C. Winters (2)

Publishing Coordination

Technical Report Files (3) 

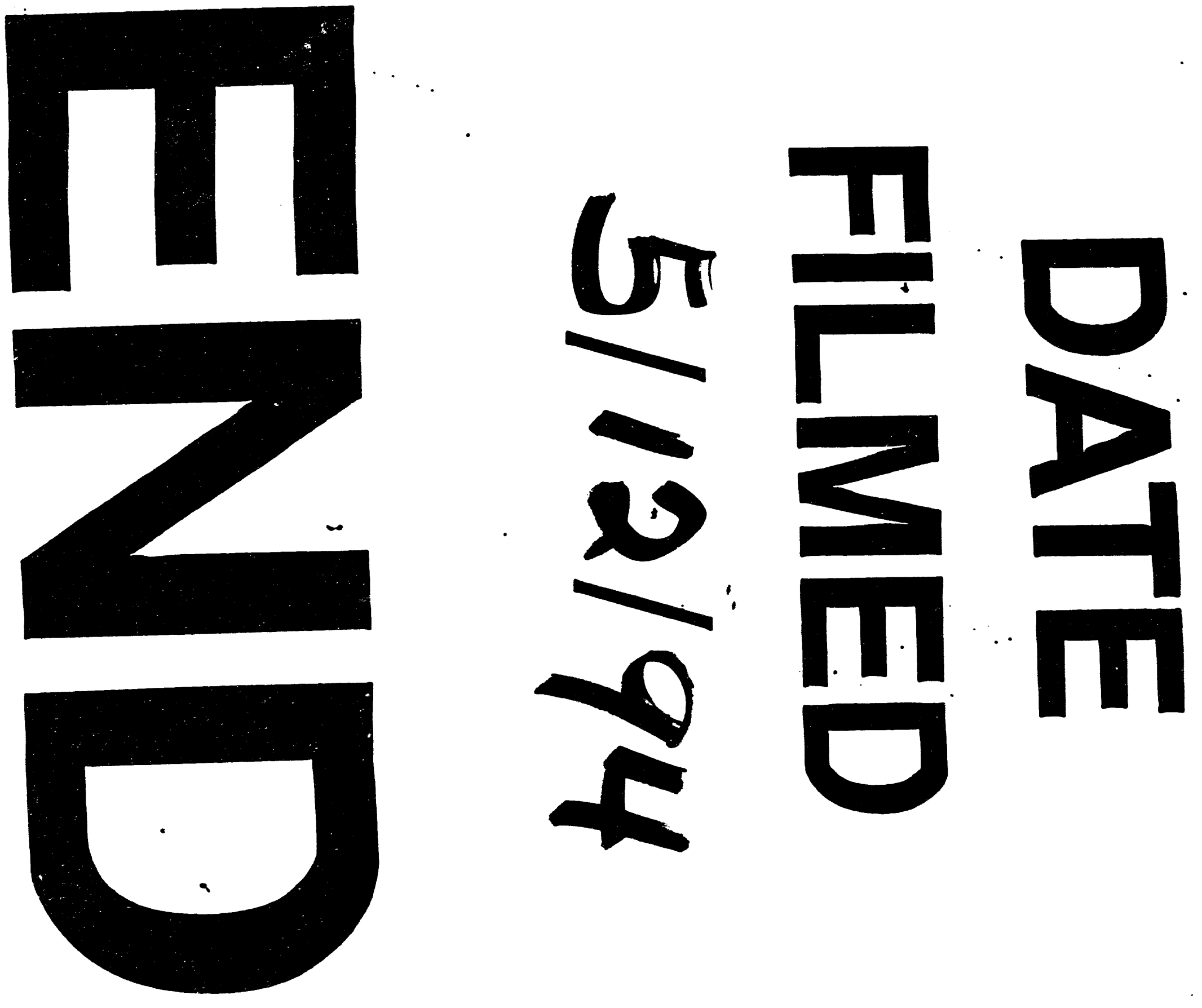
